\title{
Techniques for Studying Vocal Learning in Bottlenose Dolphins, Tursiops truncatus
}

\author{
by
}

\author{
Deborah Redish Fripp
}

B.S. Biological Sciences, Stanford University, 1992

Submitted to the Department of Biological Oceanography, in partial fulfillment of the requirements for the degree of

\section{Doctor of Philosophy}

at the

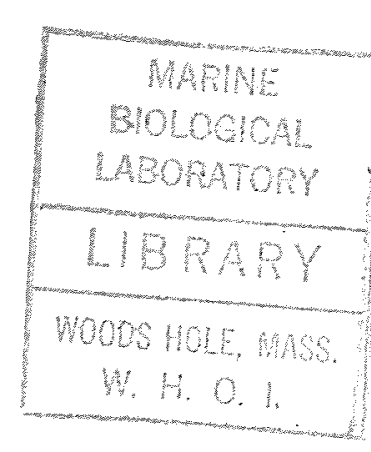

Massachusetts Institute of Technology

and the

\section{Woods Hole Oceanographic Institution}

February 1999

(C) Deborah M.R. Fripp 1999. All rights reserved.

The author hereby grants to MIT and WHOI permission to reproduce paper and electronic copies of this thesis in whole or in part and to distribute them publicly.

Signature of Author

Joint Program in Biological Oceanography

December 11, 1998

Certified by

Dr. Peter Lloyd Tyack

Thesis Supervisor, Associate Scientist, Department of Biological Oceanography

Accepted by

Dr. Mark Hahn

Chairman, Joint Committee on Biological Oceanography Massachusetts Institute of Technology/Woods Hole Oceanographic Institution 
This thesis is dedicated to my husband, Michael. 


\title{
Techniques for Studying Vocal Learning in Bottlenose Dolphins, Tursiops truncatus
}

\author{
by Deborah Redish Fripp \\ SubMitTed to THE DePARTMENT OF BIOLOGICAL OCEANOGRAPHY ON DECEMBER 11, 1998 IN PARTIAL \\ FULFILLMENT OF THE REQUIREMENTS FOR THE DEGREE OF DOCTOR OF PHILOSOPHY.
}

\begin{abstract}
The objective of this thesis is to develop the methods necessary for evaluating the role of learning in the natural whistle development of bottlenose dolphins. Bottlenose dolphins provide a unique opportunity to study social influences on vocal learning in a highly social non-human mammal. Vocal learning is critical for the development of human language but plays a much smaller role in the vocal development of most non-human terrestrial mammals. Preliminary evidence has indicated that the signature whistles of dolphin calves are modeled on the whistles in the calves' early environments and that the calves' social interactions influence the choice of model. The methods currently used to study the acoustic and social behavior of dolphins are insufficient to evaluate the role of learning in whistle development and the social influences on that development. The techniques necessary to perform such a study have therefore been developed and tested in this thesis.
\end{abstract}

The methods used to study vocal learning in various species were reviewed and a study of vocal learning appropriate to dolphins was designed. A strategy for sampling the dolphins' social and acoustic behavior was developed. To test the sampling strategy, and to provide data for the development of analysis techniques, a pilot study was performed on dolphin calves born in captivity. Focal samples of the social interactions of dolphin mothers and calves were taken over several months before and after the births of four calves, with simultaneous acoustic recordings during all focal sessions. A test of sampling times determined that five focal samples spaced throughout the day adequately represented the dolphins' behavior for the entire day. The interactions recorded during the focal samples were analyzed with loglinear analysis, multidimensional scaling, and hierarchical cluster analysis to determine the types of social relationships that occurred between the dolphins. For both calves and adults, three types of relationships were found. An analysis of a prolonged alloparenting incident demonstrated that the social relationship between mothers and calves was a care-giving relationship independent of their genetic relationship. Measures other than the total association were found to be necessary to the evaluation of the subtle relationships between the dolphins.

Methods for the quantitative analysis of the whistles produced by the dolphins were needed. Therefore, programs were developed to automatically detect and extract the whistles from the recordings in an unbiased manner. Several methods for categorizing whistles were compared and hierarchical cluster analysis of dynamic time warping of extracted contours was shown to perform well for comparing both stereotyped and un-stereotyped whistles. These techniques were then used to compare the early acoustic environments of the calves born in the pilot study. The early environments of the four calves were found to be distinctive. In particular, the putative signature whistle of each calf's mother made up a substantial proportion of the whistles in that calf's early environment. The combination techniques developed in this thesis for the analysis of the social and acoustic behavior of dolphins will allow a study of vocal learning in dolphin whistle development to be performed in a quantitative, unbiased manner.

Thesis Supervisor: Dr. Peter L. Tyack, Associate Scientist 


\section{ACKNOWLEDGEMENTS}

There are many people I need to thank for helping me with this thesis over the years. First of all, I need to thank my advisor, Peter Tyack. Peter has been a more supportive advisor than I could have hoped for. He was extremely helpful in all parts of the project, from planning to analysis to writing. I particularly appreciated his willingness to allow me to take this project in the direction of my interests. In addition, the speed with which he read and returned drafts to me was nothing short of amazing. Working with Peter has been an enlightening and enjoyable experience.

The second person I need to mention is my husband, Michael Fripp. I want to thank Michael for his infinite supply of patience, support, and help. Michael helped me in more ways than I could possibly name, from technical support to editing to simply being there when I needed to talk out a problem. I could not have done this without him. In addition to his help, I want to thank Michael for simply making my life fun.

I would also like to thank my committee members: Cindy Moss, Hal Caswell, Rachel Smolker, and Steve Pinker. I was lucky enough to have the chance to work with both Cindy Moss and Hal Caswell in my first two years of graduate school. From Cindy, I learned about the vagaries of sound analysis. From Hal, I learned computer programming. Both of these were essential in my later work. Rachel Smolker joined my committee late in the game but was nonetheless extremely helpful in the analysis phase of my work. I particularly want to thank her for being willing to come in so late. I would also like to thank Steve Pinker, my MIT committee member, for being willing to be on a committee so far out of his field. His questions were extremely useful in pointing me in the right direction. In addition, I would like to thank Heidi Sosik for chairing my thesis defense and Larry Madin for chairing my thesis proposal. They both did wonderful jobs at keeping the meetings running smoothly.

The majority of the work discussed in this thesis was performed at Kolmårdens Djurpark in Sweden. The staff at the Djurpark was helpful to me in many ways. In particular, I would like to thank Dr. Mats Amundin for his support and help. He was my advisor away from home for the six months that I was in Sweden. I would also like to thank his wife, Birgitta, for her help and support and for making me feel at home. In addition, I thank the staff at the dolphinarium, particularly the dolphin trainers and Susanne Adolfsson, the curator, for allowing me access to the animals and for helping with my recordings. I also need to thank Christer Blomqvist, Helena Erikson, and Anne-Sophie Gustavson for their friendship and support. In addition, I want to thank the dolphins at Kolmårdens for tolerating my presence and allowing me to observe them for six months.

Although the work discussed in this thesis comes primarily from Kolmårdens, I also had the chance to do work in a number of other locations. In particular, I spent four months studying the dolphins at SeaWorld of San Diego. I would like to thank the staff at SeaWorld for their help and for allowing me access to their animals. In addition, I did some work at the Hawk's Cay Dolphin Connection in the Florida Keys. I would especially like to thank Doug and Cheryl Messinger and the staff at the Dolphin Connection for making recordings for me for a long period of time. I would also like to thank them for showing me that there are captive facilities with a profound commitment to research. I also need to thank the staff at MarineLand of Florida, the Miami Seaquarium, and DolphinQuest Hawaii for allowing me to observe and record their animals and for their help in doing so.

I would like to thank the many people who helped me with specific areas of my thesis work. The ethogram was developed with help from Amy Samuels. Amy and her assistant, Cindy Flaherty, 
Acknowledgements

took time to teach me focal animal sampling. The computer programs in chapter 4 were written with a great deal of help from Jim Partan, Mark Johnson, and Rebecca Thomas. Other parts of the acoustic analysis took a great deal of help from John Buck and Matt Grund. The whistles used in the second part of chapter 4 come from studies by Laela Sayigh, Jen Miksis, and Janet McIntosh. I would also like to thank Laela Sayigh for having done the study that was the precursor to this thesis. In addition, I want to thank Tim Silva for the extended loan of his PAL VCR for acoustic analysis. I got specific help on statistical problems from Hal Caswell, Bonnie Ripley, and Vicki Starzcek. The pictures used in the thesis and in my oral defense come from a number of sources, including the web-site ArtToday, The Audubon Society Field Guide to North American Birds, WHOI graphics, the Kolmårdens Djurpark graphics department, and Michael Fripp. They were included following a great deal of scanning and manipulation by Michael Fripp.

In the last year of my thesis work, I developed a case of repetitive strain injury. In the process of dealing with this, I need to thank the JCBO, and particularly Mark Hahn, for allowing me an extra four months to finish. I also need to thank the Education office, and particularly Stella Callagee, for providing me with the funds to buy the ergonomic equipment I needed and for their support. Finally, I want to again thank my husband, Michael Fripp, for constantly reminded me to stretch.

A special thanks goes to my parents, Ginny and Joe Redish, for a lifetime of support and encouragement. They have always been supportive of my academic career and extremely helpful when I was uncertain of my direction. In particular, I want to thank my mother for teaching me to be organized in my planning and my writing, and my father for teaching me how to be a scientist. I also want to thank my father for starting me down this path by introducing me to the writing of Konrad Lorenz many years ago.

Finally, I would like to thank the staff and students of the Woods Hole Oceanographic Institution. Particularly, I want to thank the past and present members of the Tyack and Watkins labs for their help, support, and friendship over the course of my graduate career. The administrative staff of the biology department also deserves special thanks for their help over the years. I also want to thank my carpool-mates, Eli Hestermann and Shannon Bard, for making it possible to live with my husband and still make it to Woods Hole safely every morning. I cannot name all the people who have given me friendship, support, and help over the last six and a half years, but I would like to thank all of them nonetheless.

The first five years of my thesis work were funded by a pre-doctoral fellowship from the Howard Hughes Medical Institute. I particularly want to thank them for being willing to fund a project so different from what they normally fund. The WHOI Education office funded the following year and a half. My fieldwork was funded by two grants, from the Rinehart Coastal Research Center and the Ocean Ventures Fund. 


\section{TABLE OF CONTENTS}

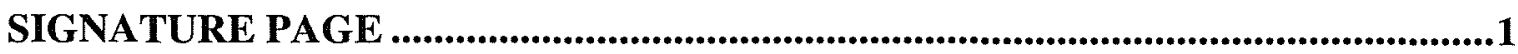

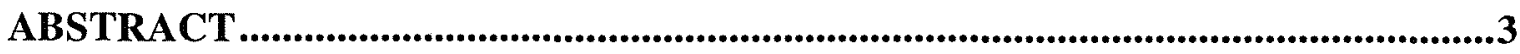

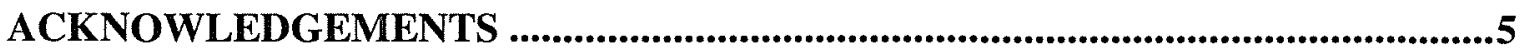

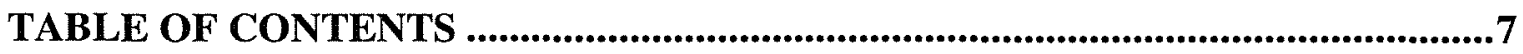

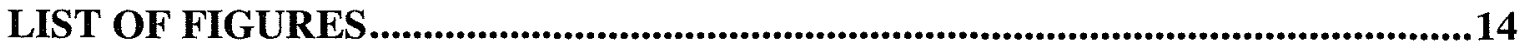

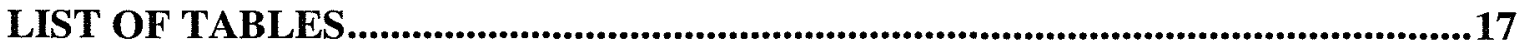

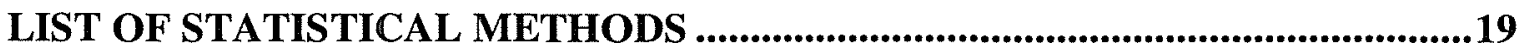

CHAPTER 1: INTRODUCTION ................................................................21

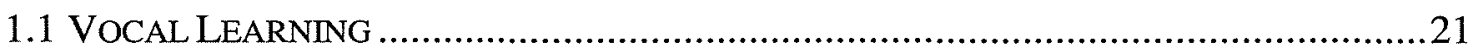

1.2 METHODS USED TO STUDY VOCAL LEARNING...............................................2 23

1.3 MECHANISMS OF VOCAL LEARNING ...........................................................29

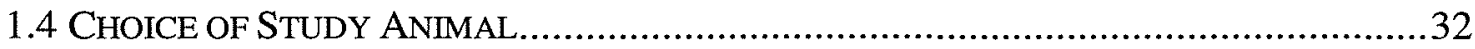

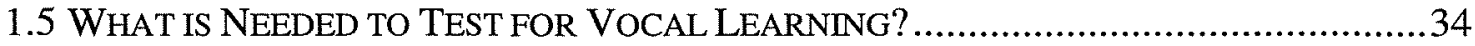

1.5.1 What is the Normal Adult Repertoire?....................................................... 34

1.5.2 What Time Period is Important? ............................................................ 35

1.5.3 Is the Whistle Repertoire Learned from Whistles in the Natal Environment? .36

1.5.4 Is the Calf Learning How to Produce Whistles or How to Use Them? .......... 38

1.5.5 What Aspect of the Environment is Important? ......................................... 38

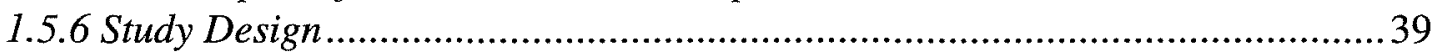

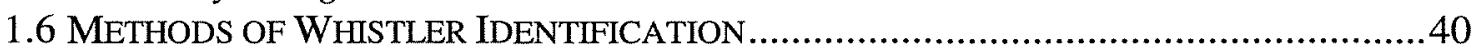

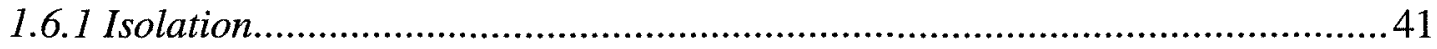

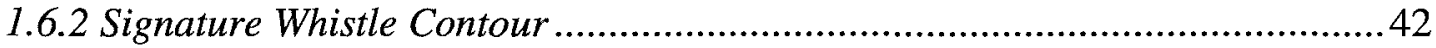

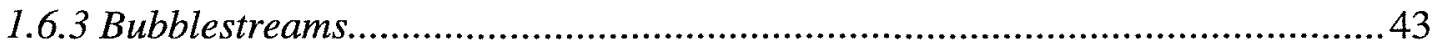

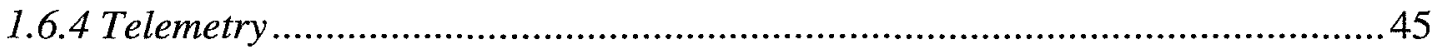

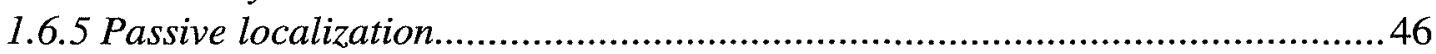

1.7 PREVIOUS WORK ON DOLPHIN WHISTLE DEVELOPMENT ..............................46

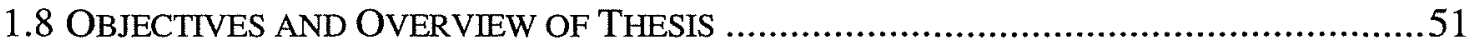

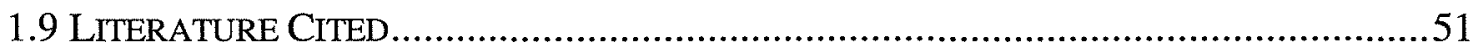

CHAPTER 2: SAMPLING METHODS .................................................................56

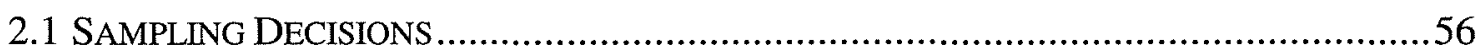

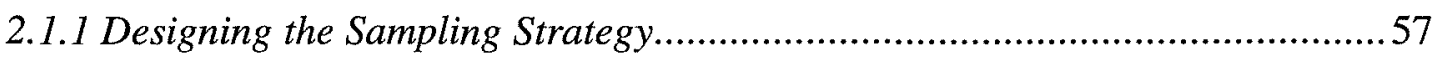

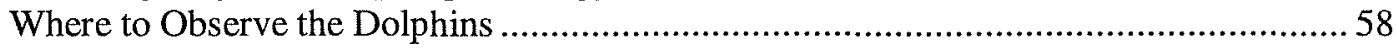

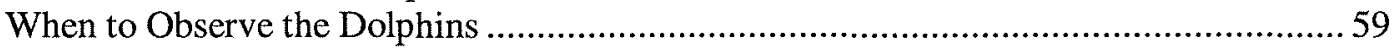

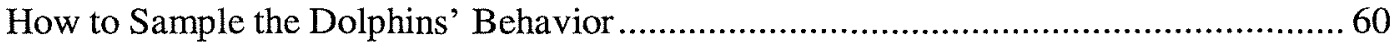


Table of Contents

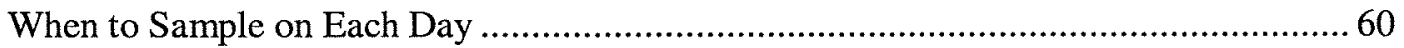

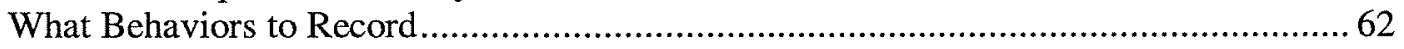

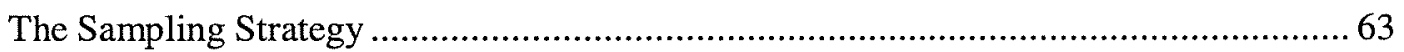

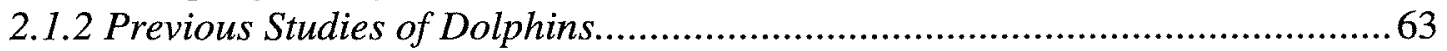

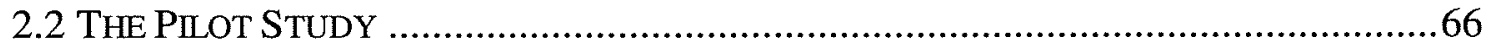

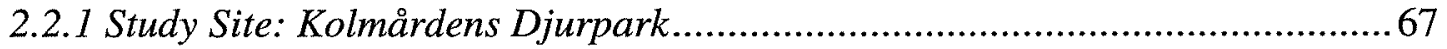

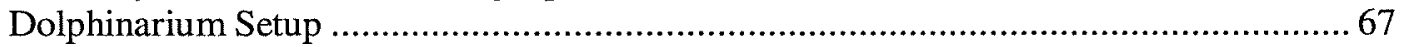

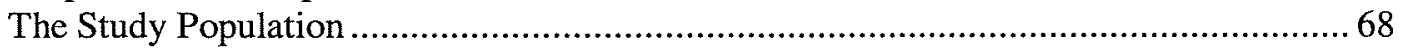

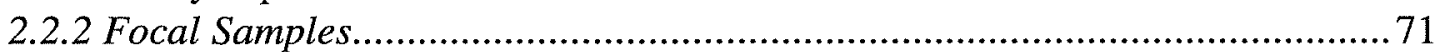

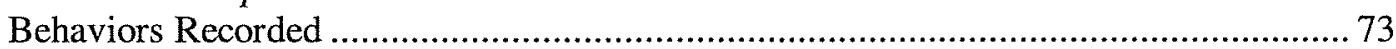

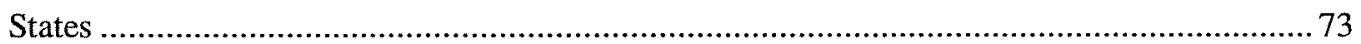

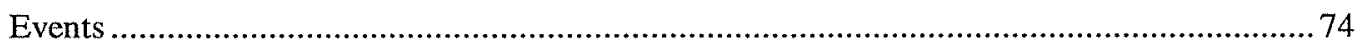

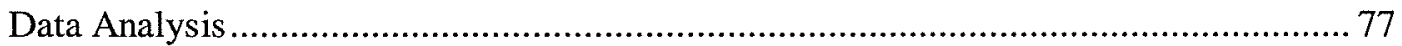

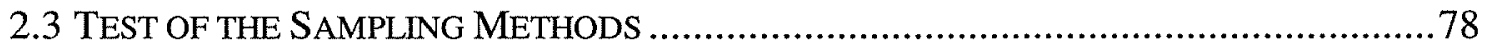

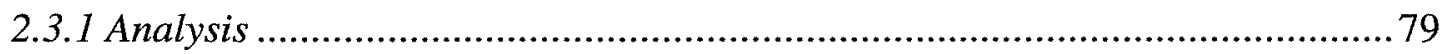

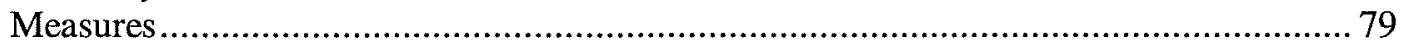

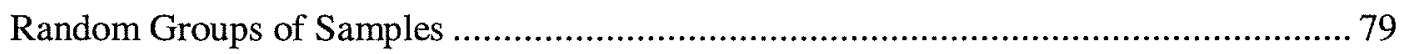

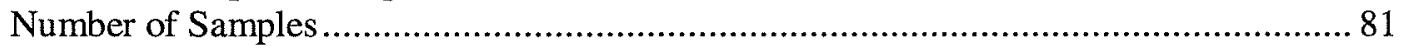

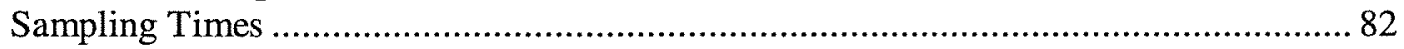

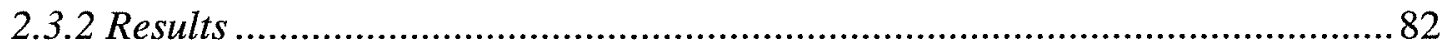

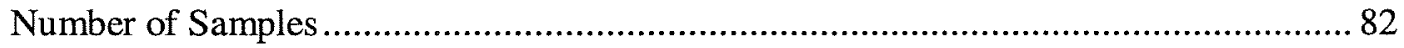

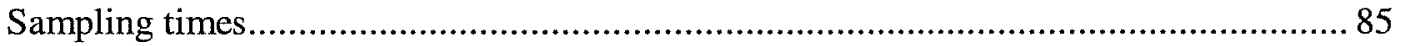

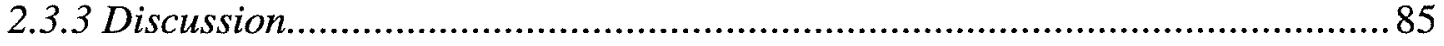

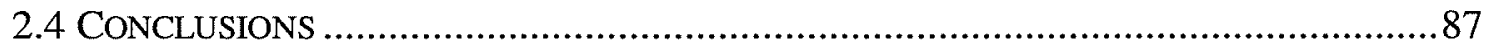

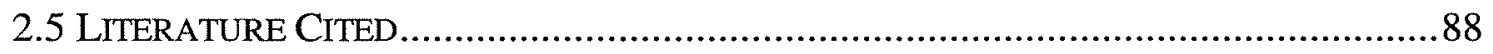

CHAPTER 3: SOCIAL RELATIONSHIPS.................................................92

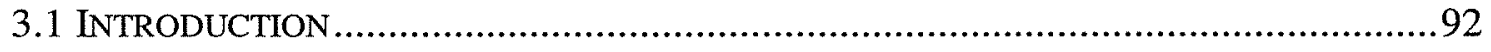

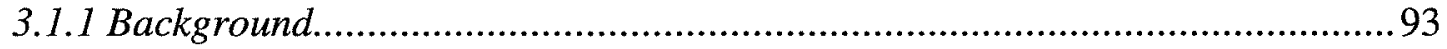

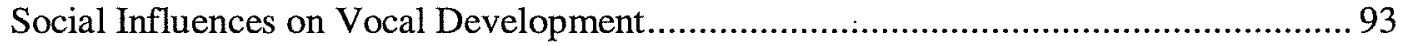

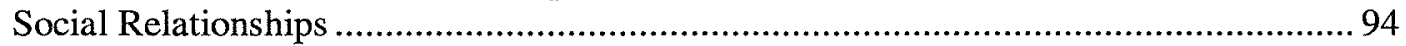

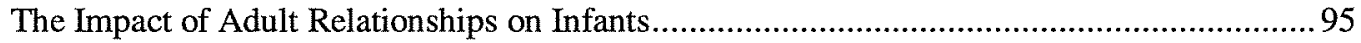

3.1.2 Cetacean Social Relationships .............................................................96

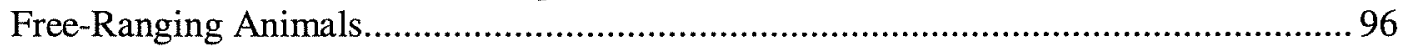

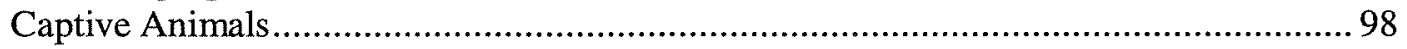

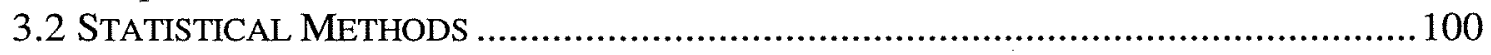

3.2.1 Characterizing Relationships ..................................................... 100

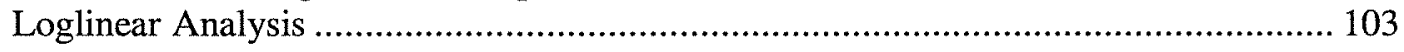

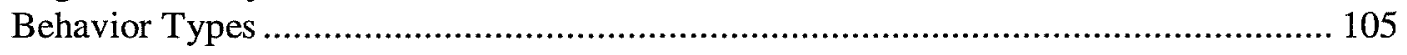

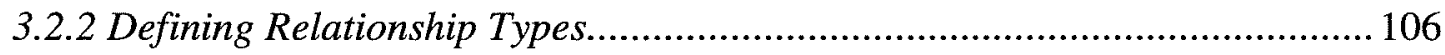

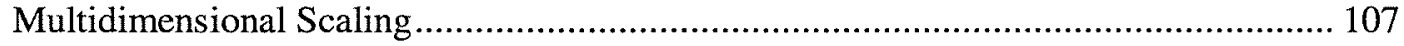

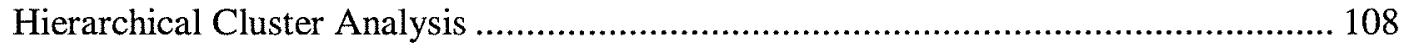

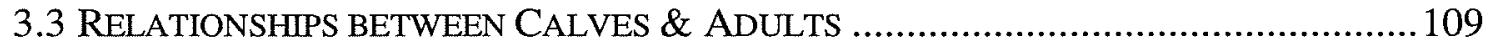

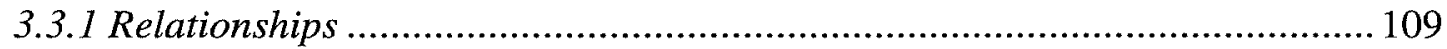

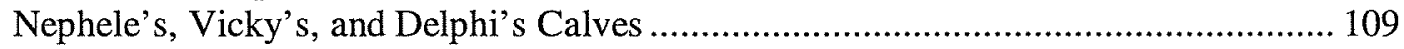

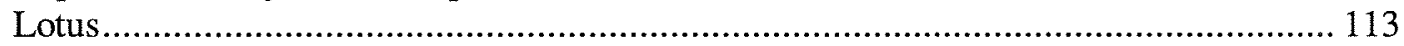




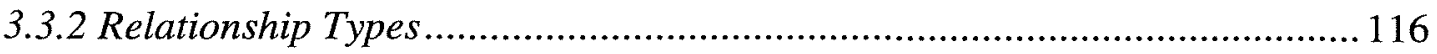

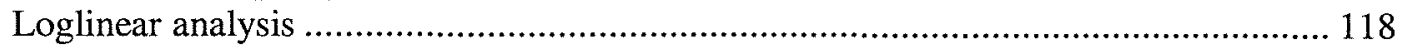

Multidimensional Scaling and Hierarchical Cluster Analysis ..................................... 119

3.3.3 Lotus' Later Weeks............................................................................... 122

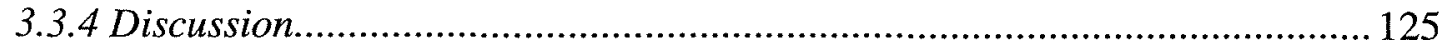

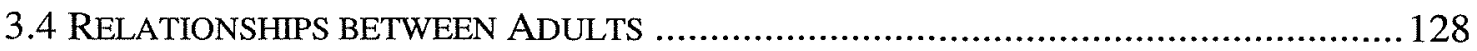

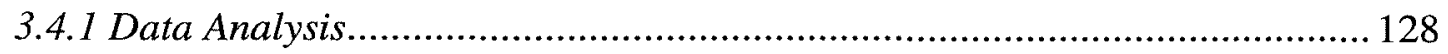

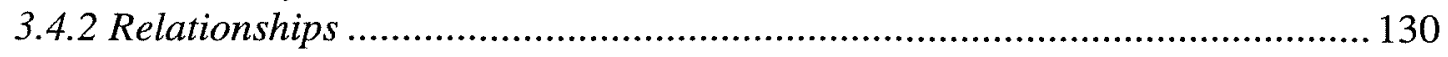

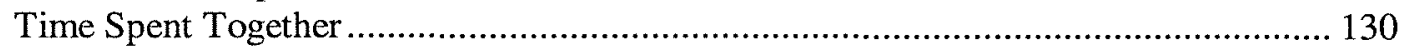

Agonistic and Affiliative Contact ......................................................................... 130

3.4.3 Relationship Types ............................................................................. 133

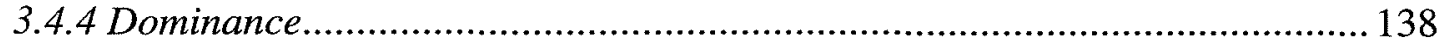

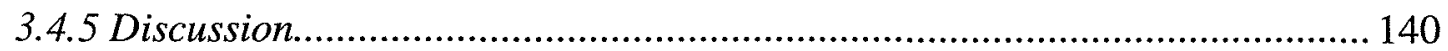

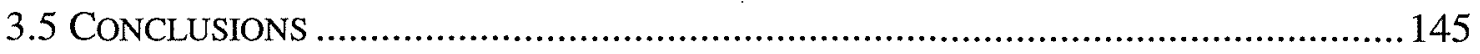

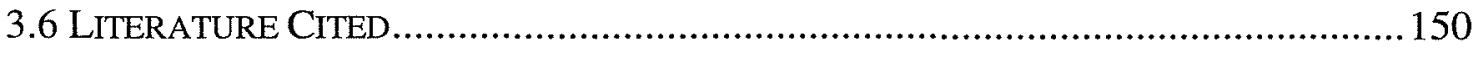

CHAPTER 4: ACOUSTIC ANALYSIS METHODS...............................................155

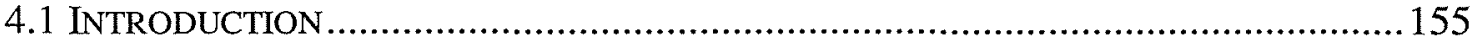

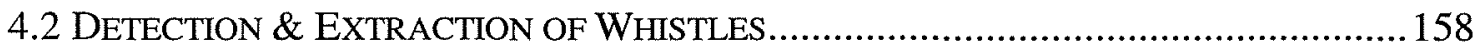

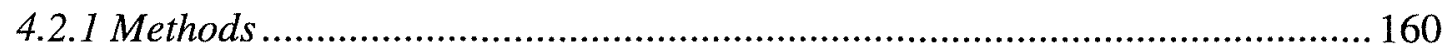

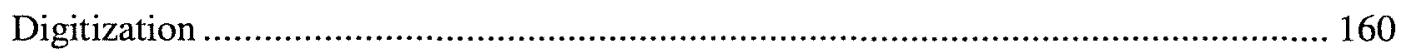

Detection and Extraction of Sounds ................................................................... 160

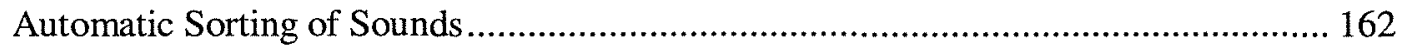

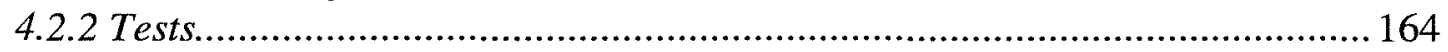

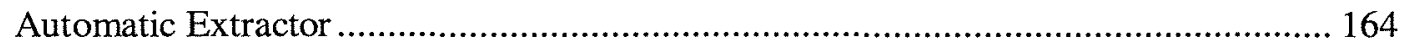

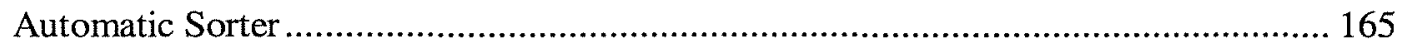

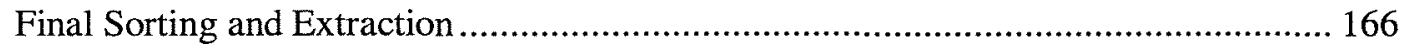

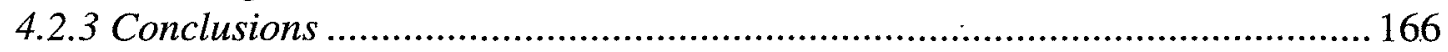

4.3 COMPARISON AND CATEGORIZATION OF WHISTLES ...........................................168

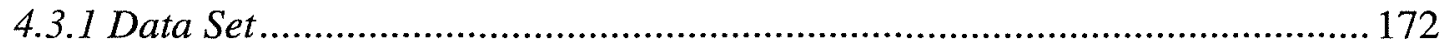

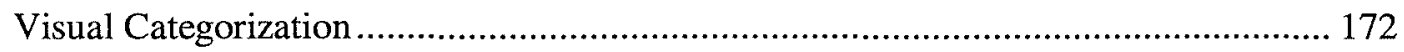

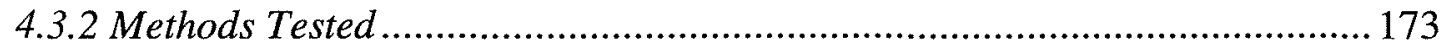

4.3.2.1 Feature Extraction................................................................................... 173

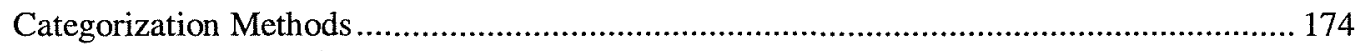

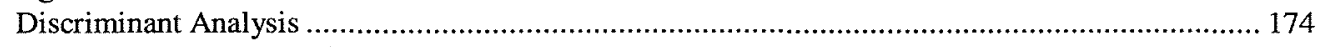

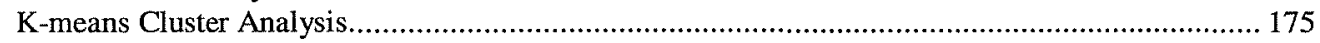

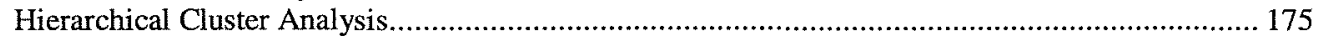

4.3.2.2 Contour Extraction...................................................................... 176

Comparison Methods............................................................................... 177

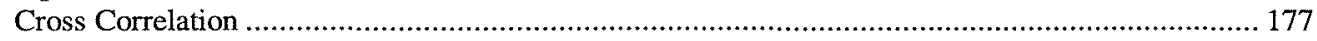

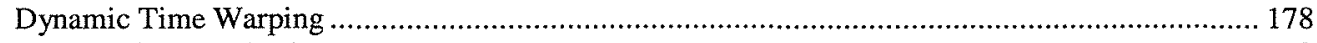

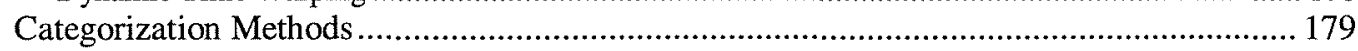

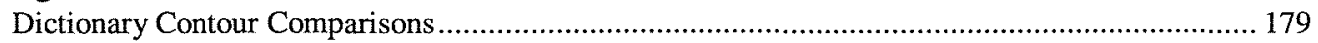

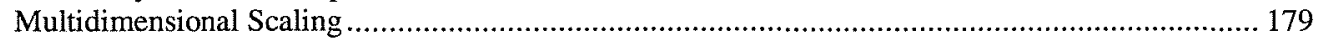

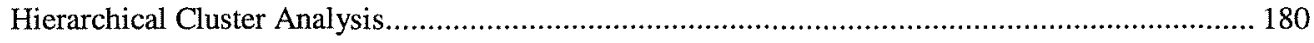

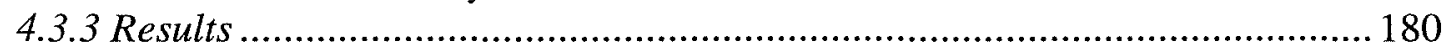

4.3.3.1 Feature Extraction...................................................................................... 180

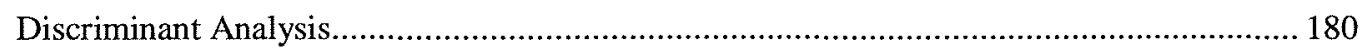


Table of Contents

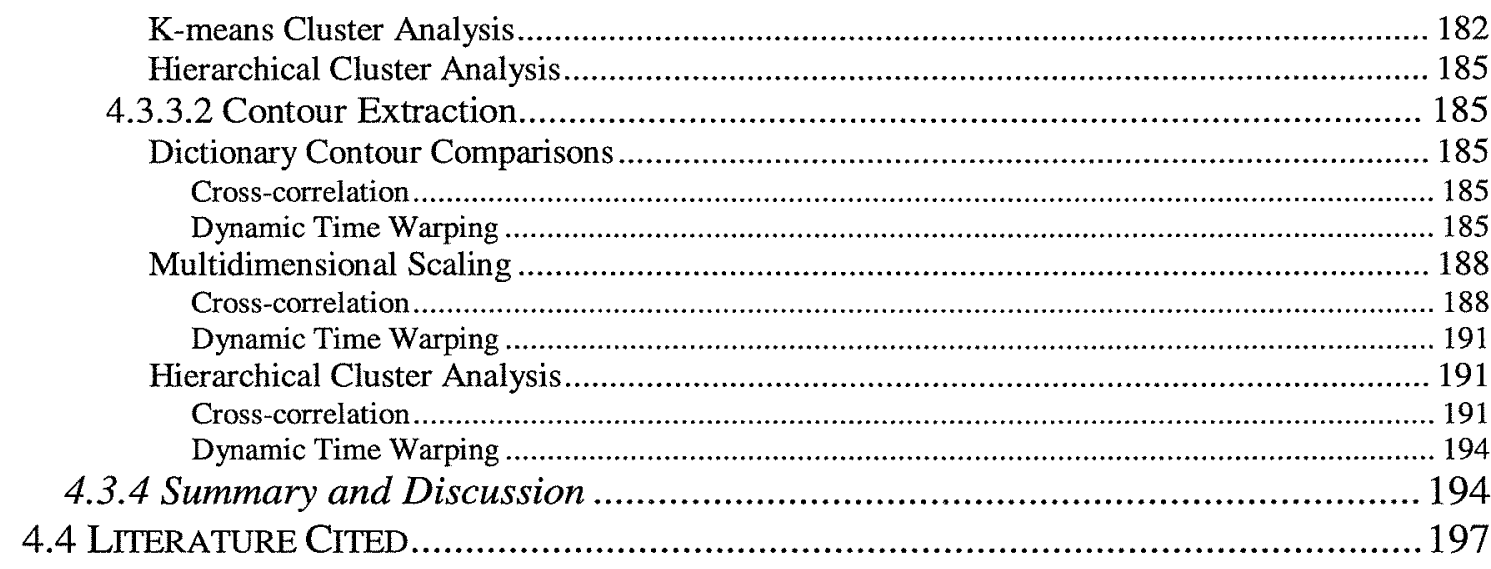

CHAPTER 5: CHARACTERIZING THE ACOUSTIC ENVIRONMENT .........199

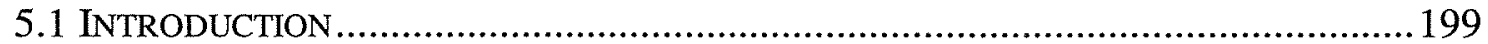

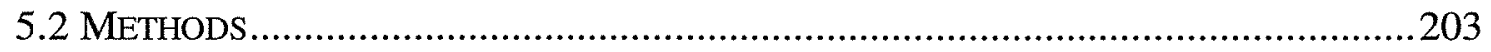

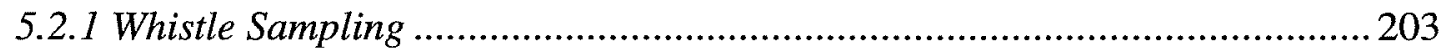

5.2.2 Contour Extraction ................................................................................... 206

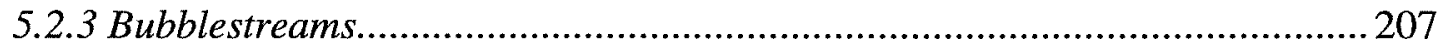

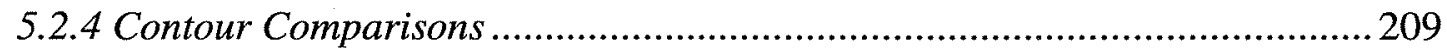

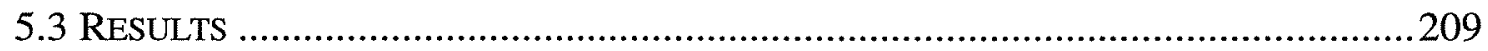

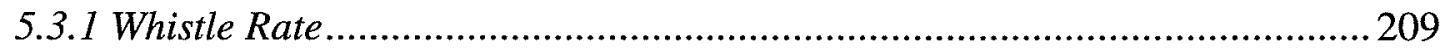

Statistical Analysis ...................................................................................... 212

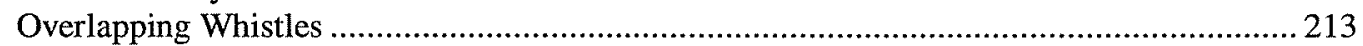

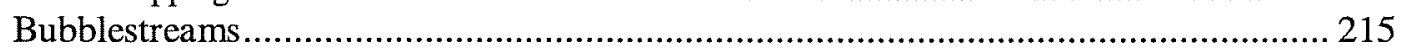

5.3.2 Determining Signature Whistles from Bubblestream-Whistles ..................... 219

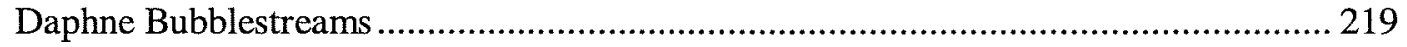

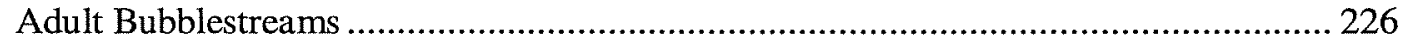

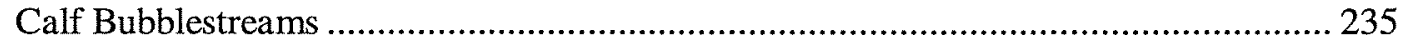

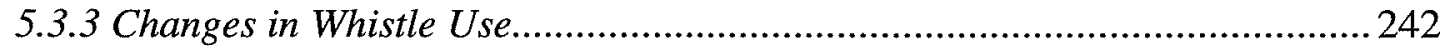

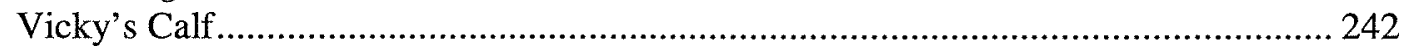

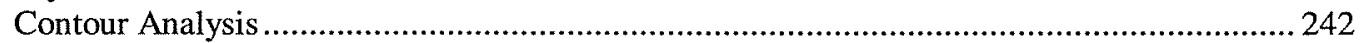

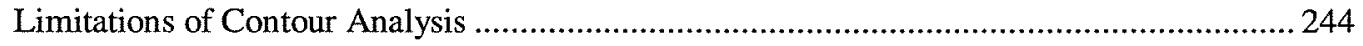

Signature Whistle Use ……………………………………………………………. 246

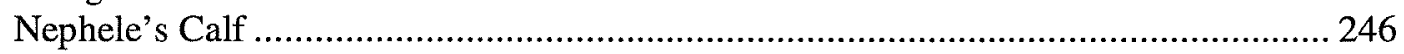

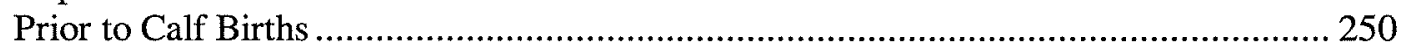

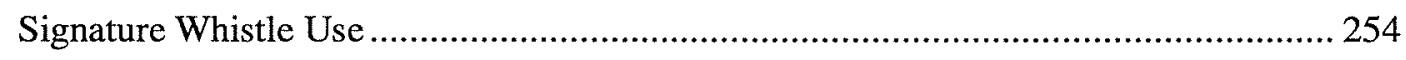

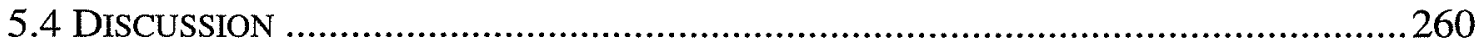

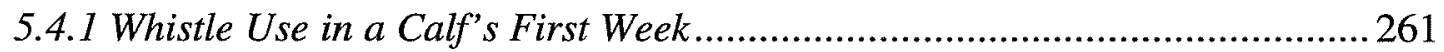

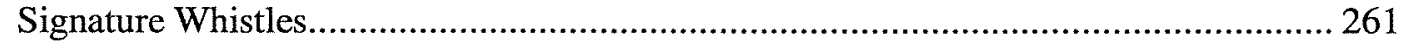

Lotus: The Difference between Acoustic and Behavioral Measures............................. 263

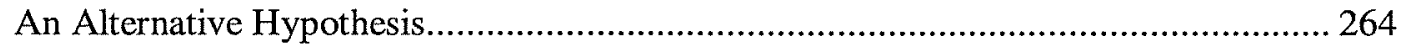

5.4.2 Methodological Issues........................................................................... 267

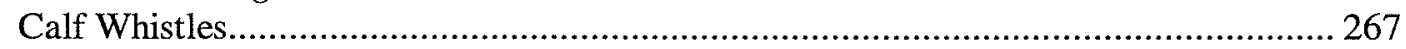

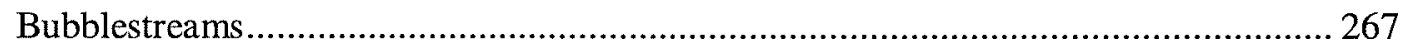

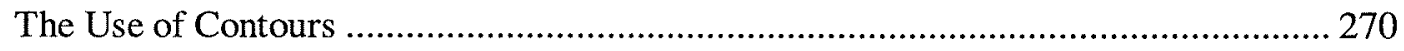


Dynamic Time Warping \& Hierarchical Cluster Analysis ....................................... 271

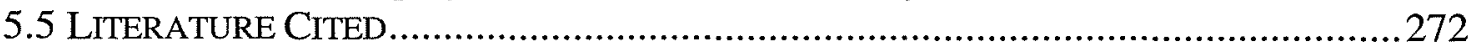

CHAPTER 6: SUMMARY AND CONCLUSIONS ........................................276

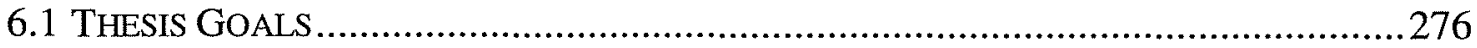

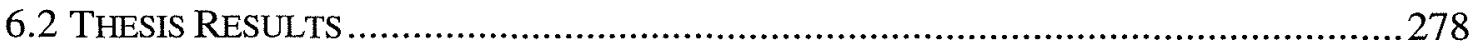

6.2.1 Techniques for Sampling Acoustic and Social Behavior ...........................278

6.2.2 Techniques for Evaluating Behavioral Data...........................................2.279

6.2.3 Techniques for Evaluating Acoustic Data........................................... 281

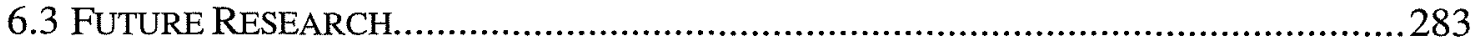

6.3.1 A Study of Vocal Learning in Whistle Development ................................. 283

6.3.2 Recent Advances in Passive Whistle Localization......................................284

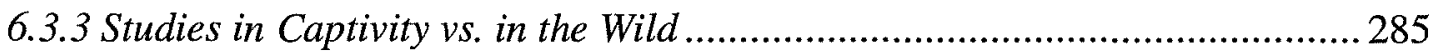

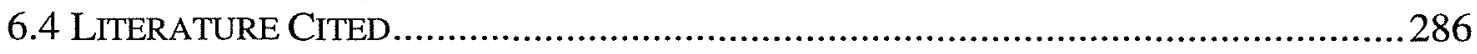

APPENDIX 1：BEHAVIORAL RECORDINGS...........................................289

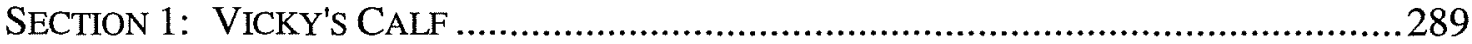

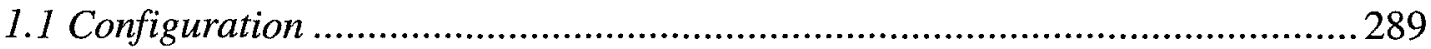

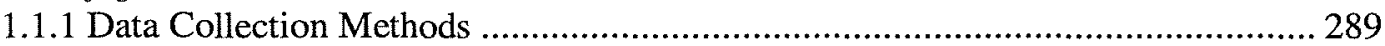

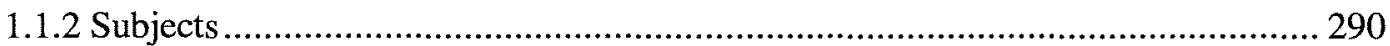

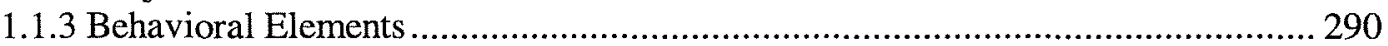

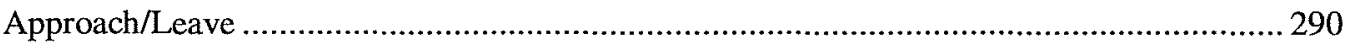

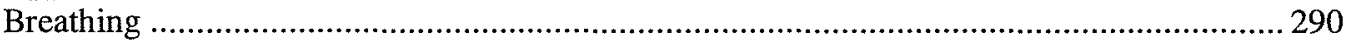

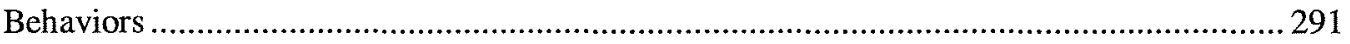

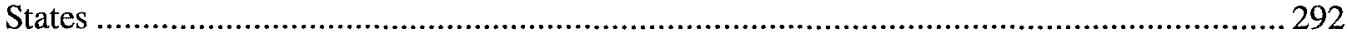

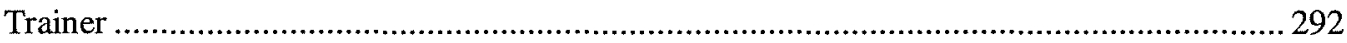

Other

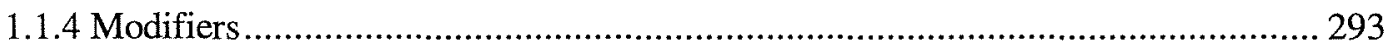

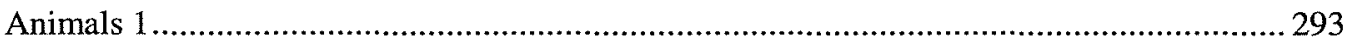

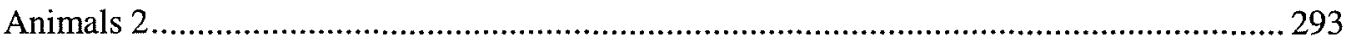

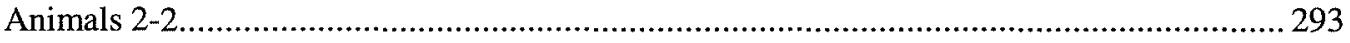

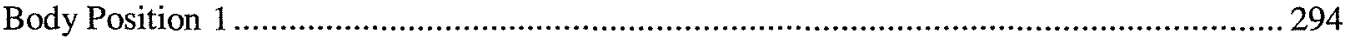

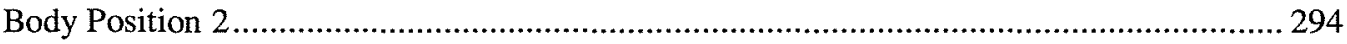

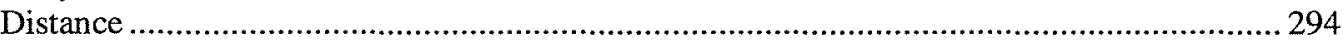

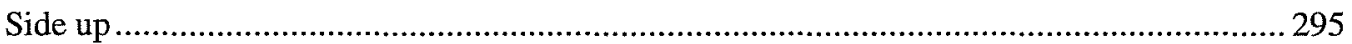

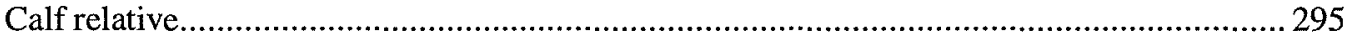

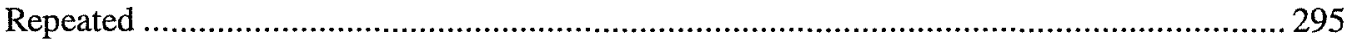

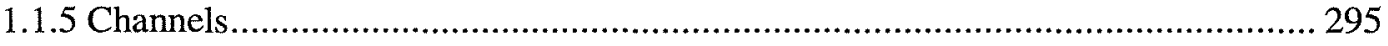

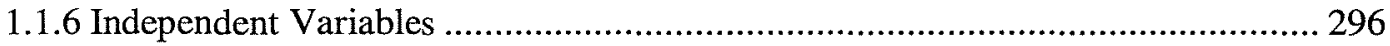

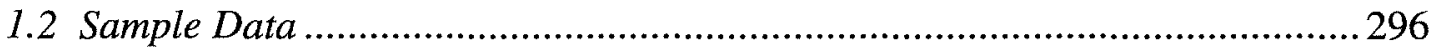

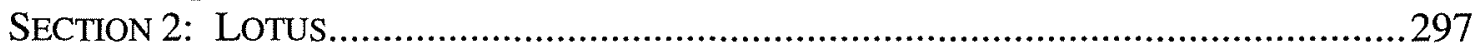

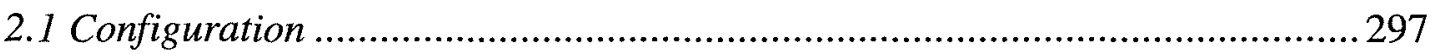

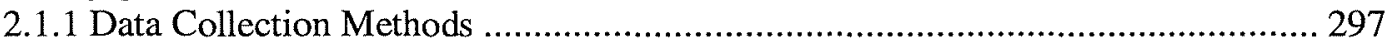

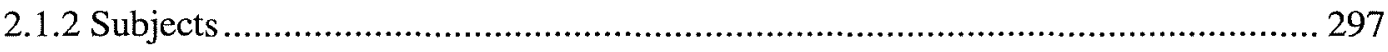

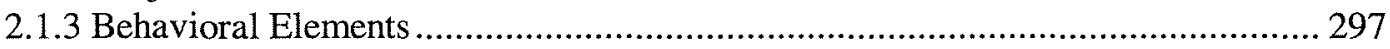

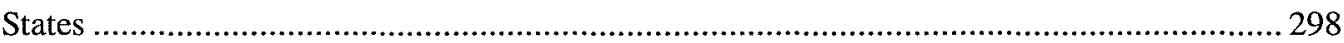

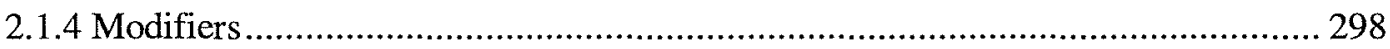

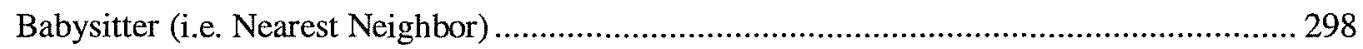


Table of Contents

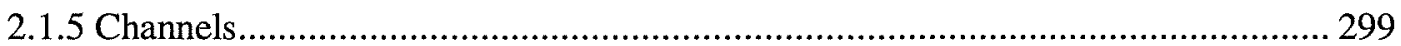

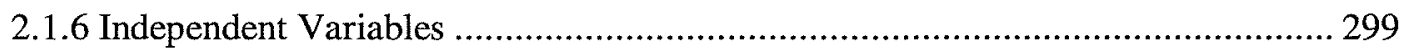

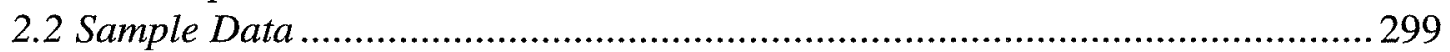

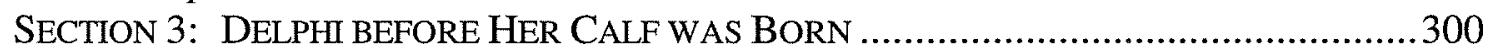

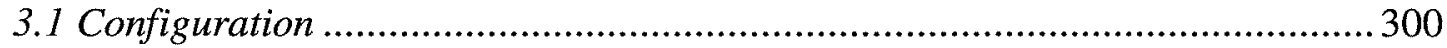

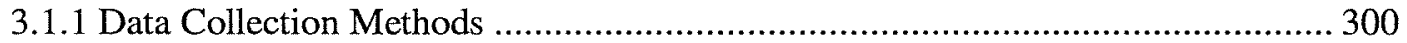

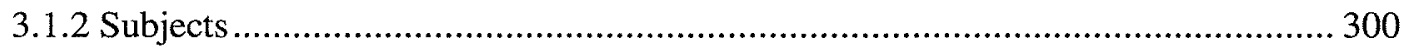

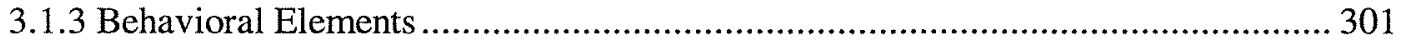

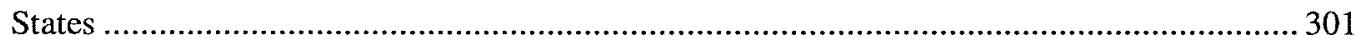

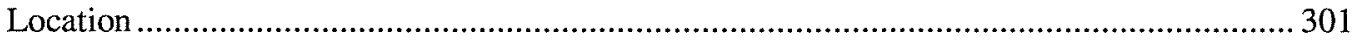

Other

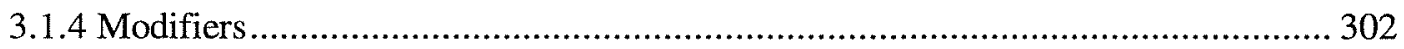

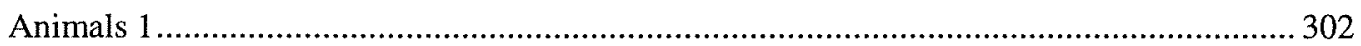

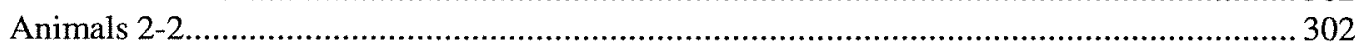

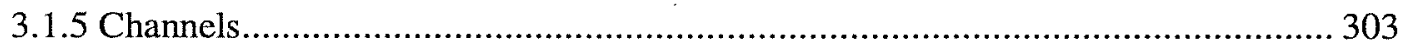

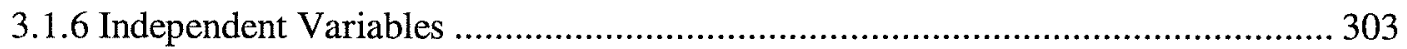

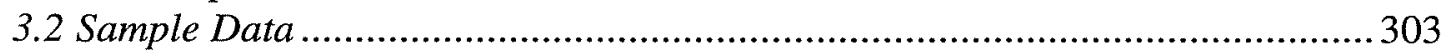

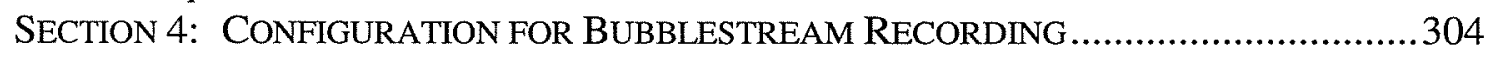

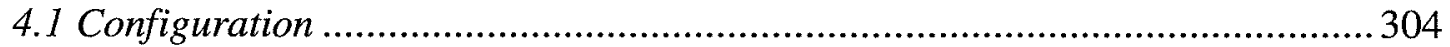

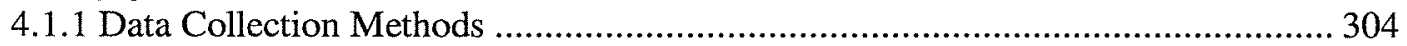

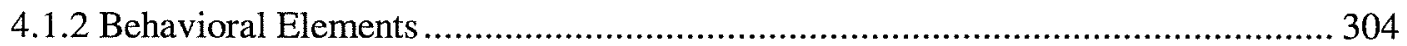

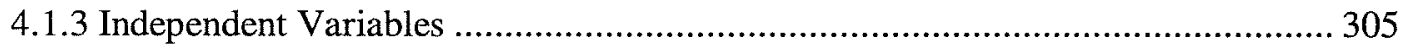

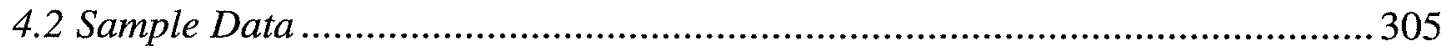

APPENDIX 2: COMPUTER PROGRAMS ...........................................................306

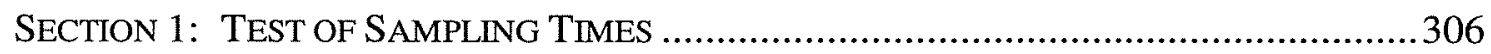

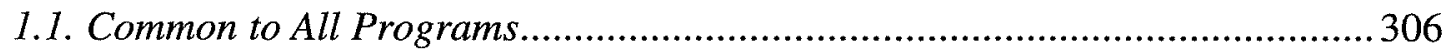

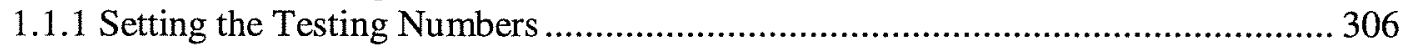

1.1.2 Calculation of Differences between Group Means and Overall Mean .................. 306

1.1.3 Calculation of Overall Mean and Standard Deviation ....................................... 307

1.2 Calculation of the Percent Deviation and Coefficient of Variation ................. 307

1.2.1 Calculation of Distribution Mean and Standard Deviation ....................................307

1.2.2 Calculation of Percent Deviation and Coefficient of Variation........................... 307

1.3 Comparison of Standard 5 Times ............................................................... 308

SECTION 2: PROGRAMS FOR WHISTLE EXTRACTION AND SORTING..........................309

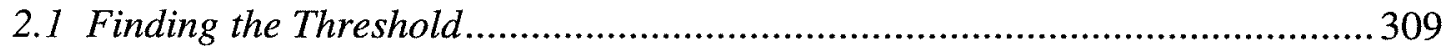

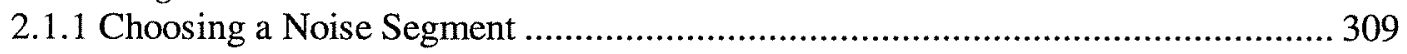

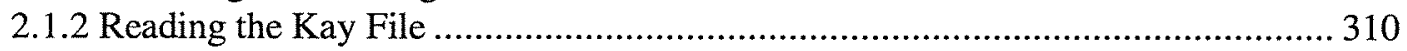

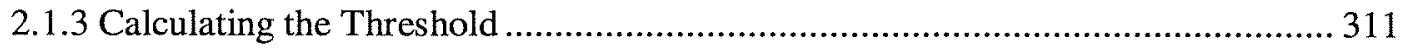

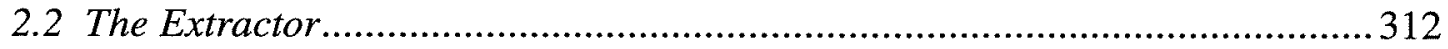

2.2.1 Extraction of Whistles from Multiple Files.................................................. 312

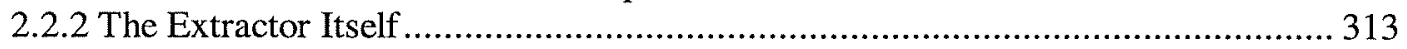

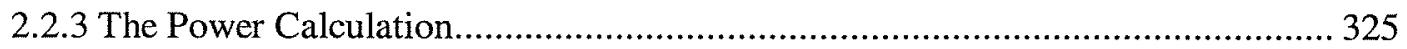

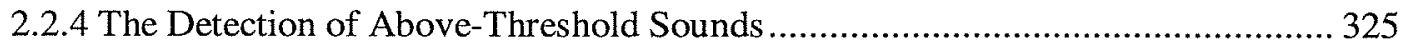

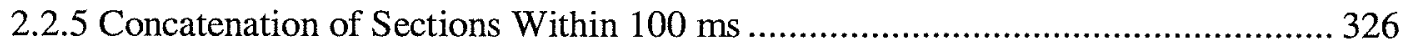

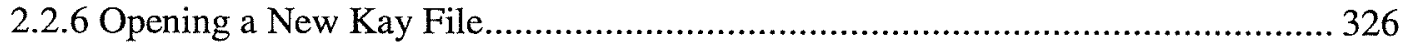

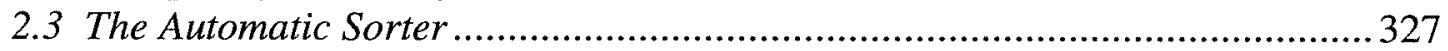




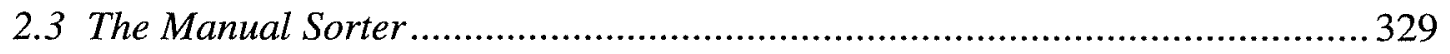

SECTION 3: PROGRAMS FOR CONTOUR COMPARISON..........................................336

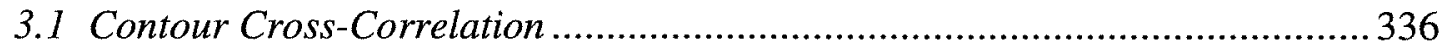

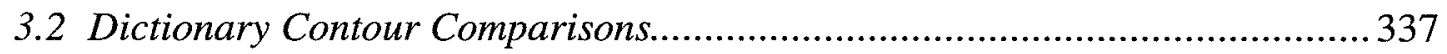

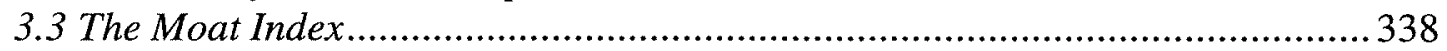

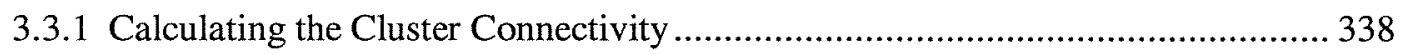

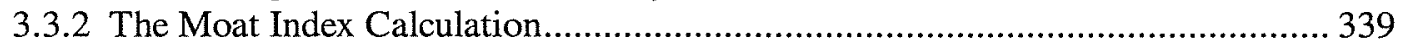

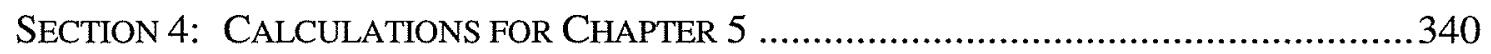

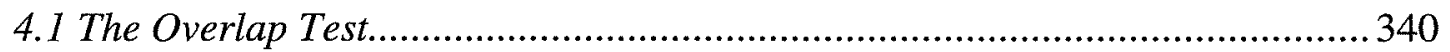

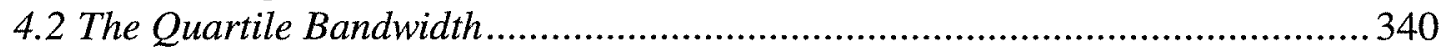

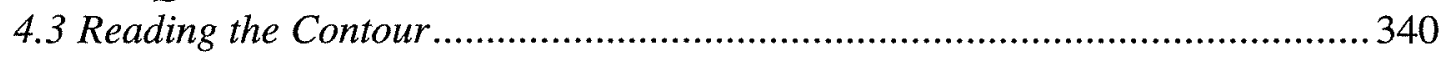

APPENDIX 3: PROGRAMS BY OTHER PEOPLE.............................................341

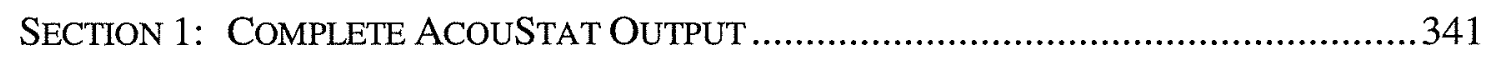

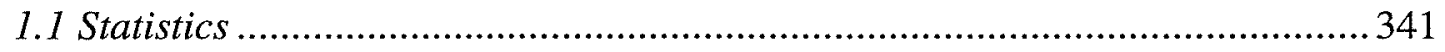

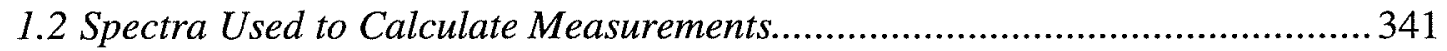

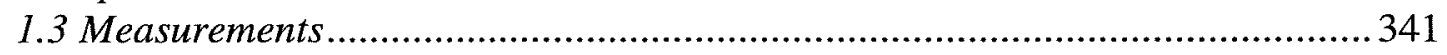

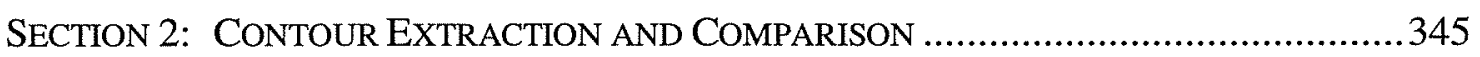

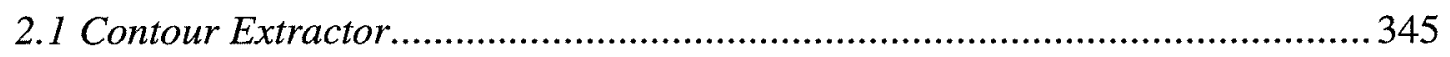

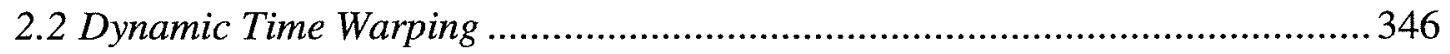

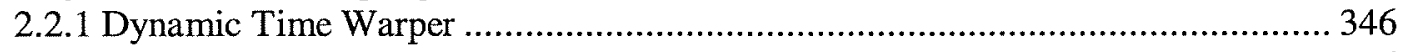

2.2.2 Program to Convert DTW Output into Matrix Form..................................... 346

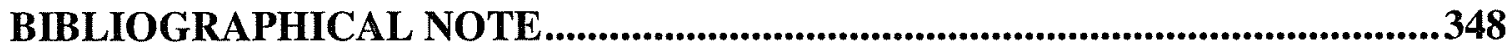




\section{LIST OF FIGURES}

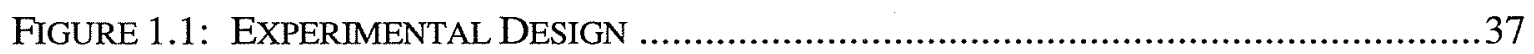

FIGURE 2.1: SCHEMATIC OF THE KOLMÅRDENS DJURPARK DOLPHINARIUM ...................68

FIGURE 2.2: ANALYSIS OF ONE BEHAVIORAL MEASURE ON ONE TEST DAY ..................80

Figure 3.1: ContingenCy TABLES OF BeHavioral Data ................................... 101

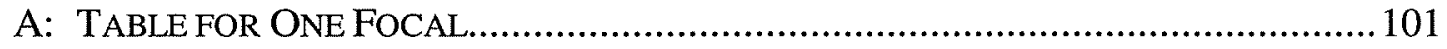

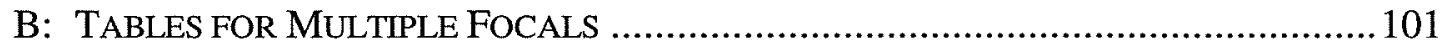

C: MUltiVARIABLE TABLE FOR MULTIPLE FoCALS ......................................... 101

Figure 3.2: Total Time In AsSOciation: First ThreE CALVES ......................... 110

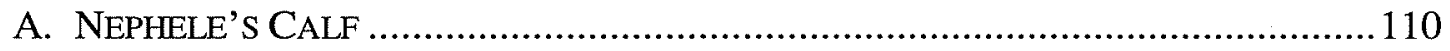

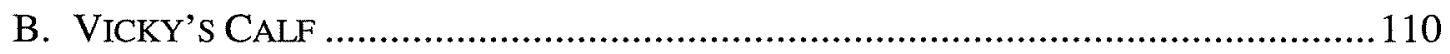

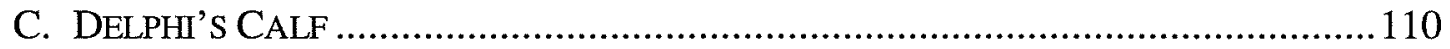

FIGURE 3.3: TOTAL TIME IN ASSOCIATION: LOTUS............................................... 114

FIGURE 3.4: CALF RELATIONSHIP TYPES ................................................... 120

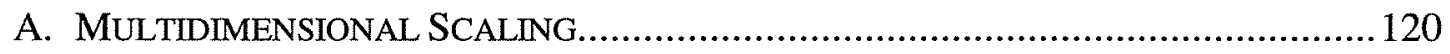

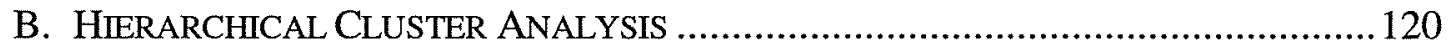

C. MULTIDIMENSIONAL SCALING OF TOTAL ASSOCIATION ONLY ........................121

FIGURE 3.5: TOTAL TIME LOTUS SPENT ALONE ( $>$ 1M AWAY FROM AN ADULT) ............. 124

FIGURE 3.6: LOTUS' SEXUAL PLAY WITH VICKY AND LOTTY ..................................... 124

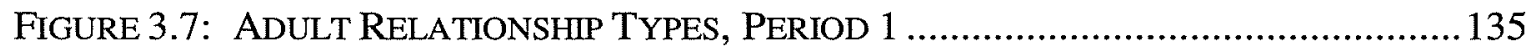

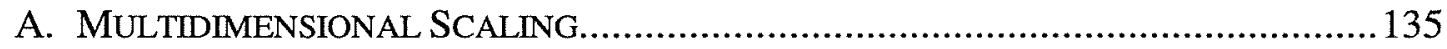

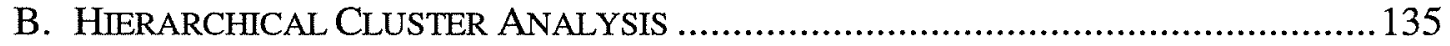

FIGURE 3.8: ADULT RELATIONSHIP TYPES, PERIOD 2 ........................................... 136

A. Multidimensional SCALING............................................................ 136

B. HIERARChICAL CluSTER ANALYSIS ............................................... 136

FIGURE 3.9: HIERARCHICAL ClUSTER ANALYSIS OF TOTAL ASSOCIATION ONLY .......... 137

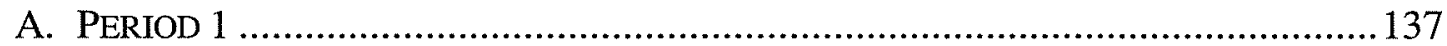

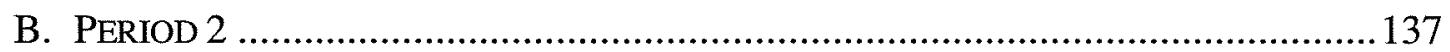

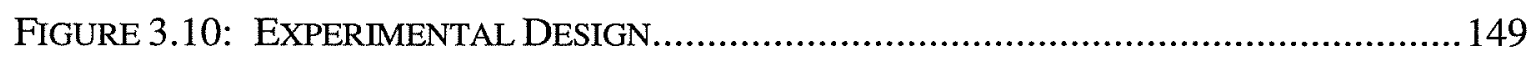

Figure 4.1: An Example of the Automatic Whistle Detection ......................... 161

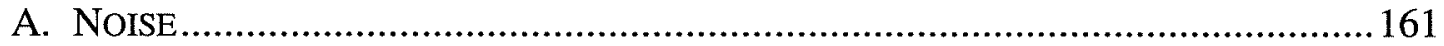

B. VOCALIZATION .............................................................................. 161

FIGURE 4.2: AN EXAMPLE OF CONTOUR EXTRACTION ........................................ 177

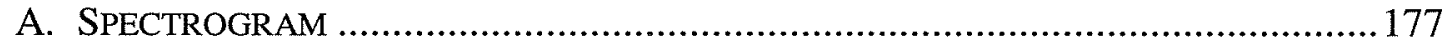

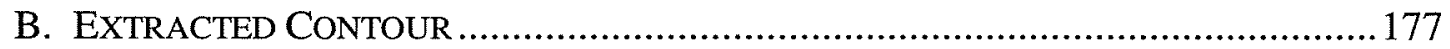

FIGURE 4.3: DYNAMIC TIME WARPING OF 2 CONTOURS .......................................... 178

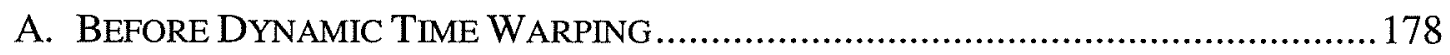

B. AFTER DYNAMIC TIME WARPING ......................................................... 178

FIGURE 4.4: DISCRIMINANT ANALYSIS OF EXTRACTED FEATURES ........................... 181 


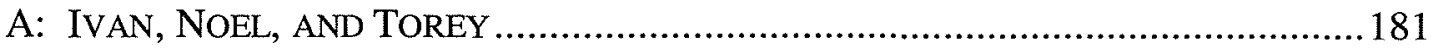

B: IVAN, NOEL, TOREY, AND BEBE .......................................................... 181

FIGURE 4.5: SUMMED F-RATIOS FOR K-MEANS ClUSTER ANALYSIS .......................... 183

Figure 4.6: HiERARCHICAL ClUSTER ANALYSIS OF EXTRACTED FEATURES.................184

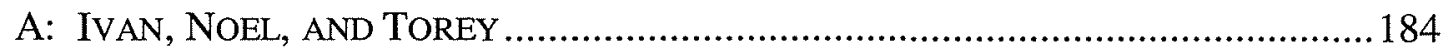

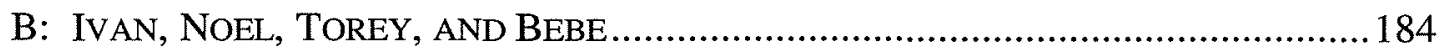

Figure 4.7: DiCTIONARY CONTOUR COMPARISONS, CONTOUR CROSS-CORRELATIONS 186

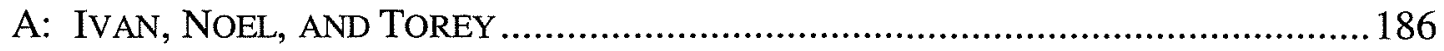

B: IVAN, NoEL, TOREY, AND BEBE, WITH CONTOUR B8 .................................... 186

C: IVAN, NOEL, TOREY, AND BEBE, WITH CONTOUR B11 ..................................186

FigURE 4.8: DICTIONARY CONTOUR COMPARISONS, DYNAMIC TIME WARPING............ 187

A: IVAN, NOEL, AND TOREY ............................................................. 187

B: IVAN, NOEL, TOREY, AND BEBE, WITH CONTOUR B8 ................................. 187

C: IVAN, NoEL, TOREY, AND BEBE, WITH CONTOUR B11 .................................. 187

Figure 4.9: MultidimEnSIONAL SCAling, Contour Cross-Correlations............. 189

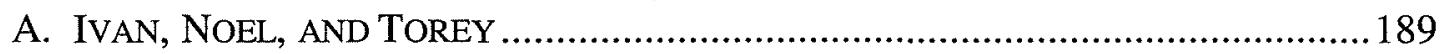

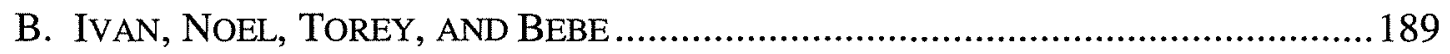

Figure 4.10: MulTIDIMENSIONAL SCALING, DYNAMIC TIME WARPING ..................... 190

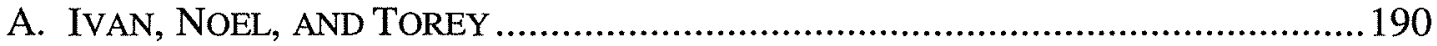

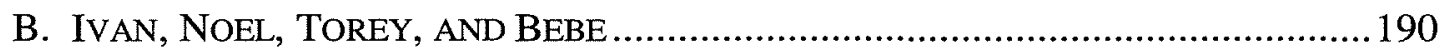

Figure 4.11: HiERARCHICAL Cluster ANALYSis, CONTOUR Cross-CORRELATIONS ... 192

A: IVAN, NOEL, AND TOREY ................................................................... 192

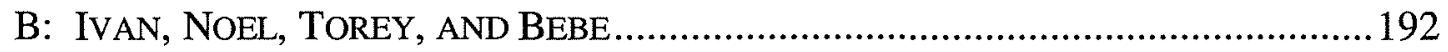

FIgURE 4.12: HiERARCHCAL CluSTER ANALYSIS, DyNAMIC TIME WARPING ............... 193

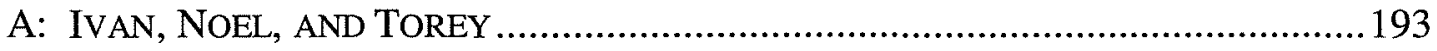

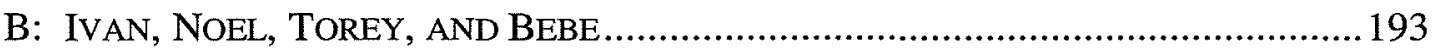

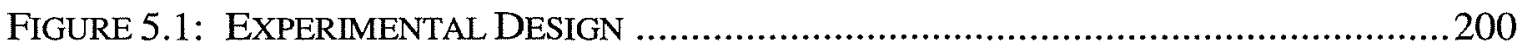

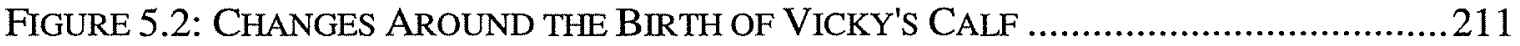

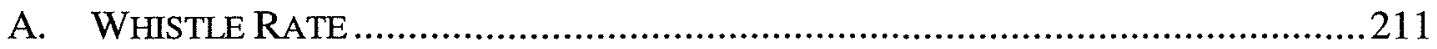

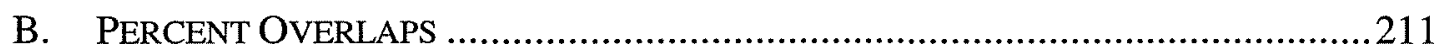

C. AVERAGE LENGTH OF USABLE CONTOURS .........................................211

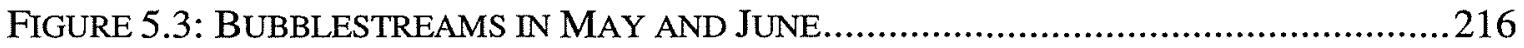

FIGURE 5.4: AMBIGUITY IN THE ASSIGNMENT OF BUBBLESTREAMS TO WHISTLES ..........218

FIGURE 5.5: EXAMPLES OF DAPHNE'S BUBBLESTREAM-WHISTLES .............................220

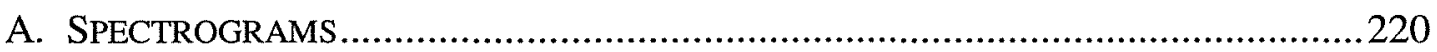

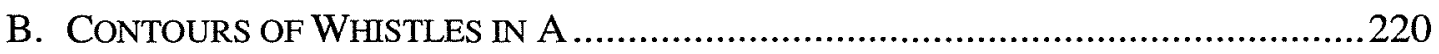

FIGURE 5.6: MULTIDIMENSIONAL SCALING OF DAPHNE's BUBBLESTREAM-WHISTLES ... 222 FIGURE 5.7: HIERARCHICAL CLUSTER ANALYSIS OF DAPHNE'S BUBBLESTREAM-WHISTLES

FiguRE 5.8: POSSIBLE DAPHNE SIGNATURE WHISTLES AMONG DAPHNE'S BUBBLESTREAM-

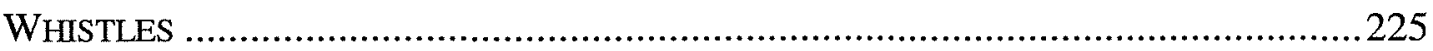

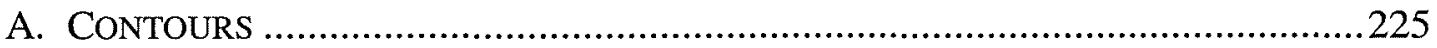

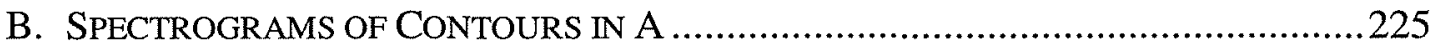


List of Figures

Figure 5.9: Contours of AdUlt BubBLESTREAM-WHISTLES ..............................227

FigURE 5.10: MULTIDIMENSIONAL SCALING OF THE ADULT BUBBLESTREAMS..............230

FIGURE 5.11: HIERARCHICAL ClUSTER ANALYSIS OF ADULT BUBbLESTREAMS.............231

FIGURE 5.12: COMPARISONS OF EXAMPLE ADULT BUBBLESTREAM-WHISTLES .............232

A. SPECTROGRAMS: NEPHELE COMPARISONS.............................................2232

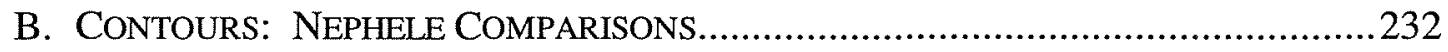

C. SPECTROGRAMS: VICKY COMPARISONS .................................................2233

D. CONTOURS: VICKY COMPARISONS ........................................................23

FIGURE 5.13: HIERARCHICAL ClUSTER ANALYSIS OF CALF BUBBLESTREAM-WHISTLES 236

FIGURE 5.14: EXAMPLES OF CONTOURS ...........................................................238

A. KNOWN CALF CONTOURS: EXAMPLES FROM LotUS AlONE..........................238

B. EXAMPLES OF CALF BUBbleSTREAM-WHISTLE CONTOURS...........................2238

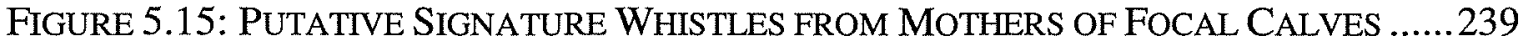

FIGURE 5.16: COMPARISON OF BUBBLESTREAM-WHISTLES WITH PUTATIVE SIGNATURE

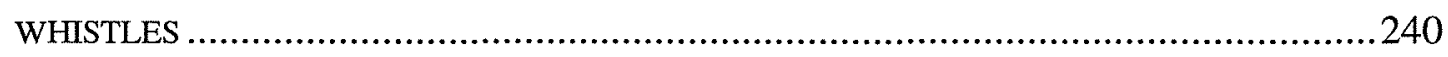

FIGURE 5.17: HCA OF THE WEEKS BEFORE AND AFTER VICKY'S CALF WAS BORN ........243

FIGURE 5.18: HCA OF THE WEEKS BEFORE AND AFTER NEPHELE'S CALF WAS BORN ....247

FIGURE 5.19: HCA OF THE FIRST WEEKS OF NEPHELE'S CALF AND VICKY'S CALF .........249

FIGURE 5.20: HCA OF A WEEK IN MARCH VS. THE WEEK BEFORE NEPHELE'S CALF ......251

FIGURE 5.21: HCA OF THE WEEKS BEFORE NEPHELE'S CALF VS. BEFORE VICKY'S CALF

Figure 5.22: Signature Whistle PRODUCTION By Mothers of FoCAl CALVES .......255

FIGURE 5.23: MOTHER'S SIGNATURE WHISTLES AS PERCENT OF TOTAL ......................257

FIGURE 5.24: SIGNATURE WHISTLE USE BY VICKY AND LOTTY .................................259

A. Signature WHISTLE Production ...................................................259

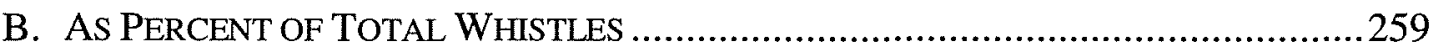




\section{LIST OF TABLES}

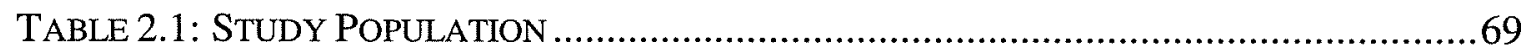

TABLE 2.2: NUMBER OF 10-MINUTE SAMPLES ON EACH FOCAL ANIMAL ........................72

TABLE 2.3: ACTIVITIES................................................................................. 74

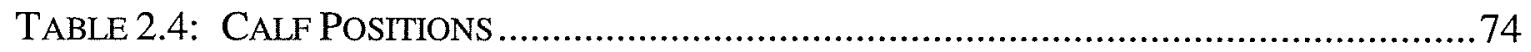

TABLE 2.5: BEHAVIORAL EVENTS ............................................................... 76

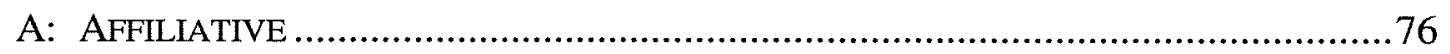

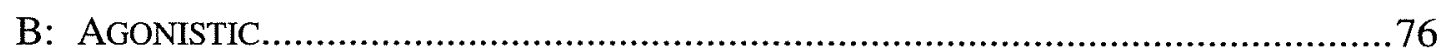

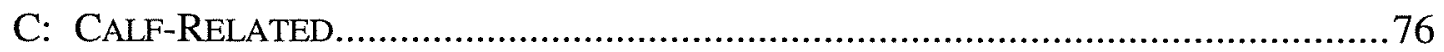

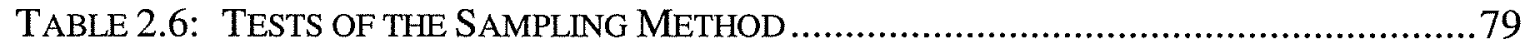

TABLE 2.7: RESULTS OF METHODS TEST, NUMBER OF SAMPLES:.............................83 AVERAGE COEFFICIENT OF VARIATION AND PERCENT DEVIATION FOR ALL MEASURES

TABLE 2.8: RESULTS OF METHODS TEST, SAMPLING TIMES: .....................................8 84

P-VALUES FOR STANDARD FIVE SAMPLES COMPARED TO FIVE RANDOM SAMPLES

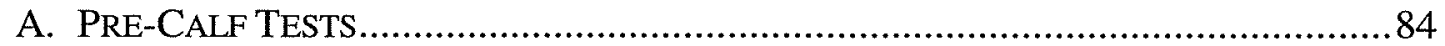

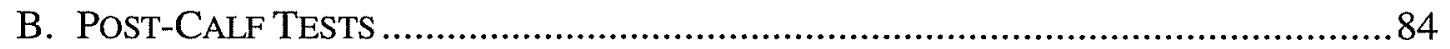

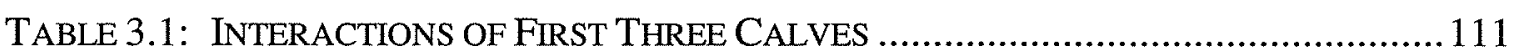

TABle 3.2: BIC VAlues From Loglinear Models of DATA FOR FIRST THREe Calves

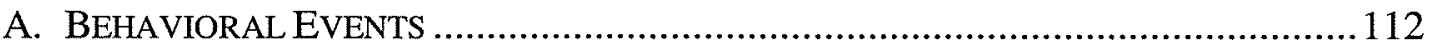

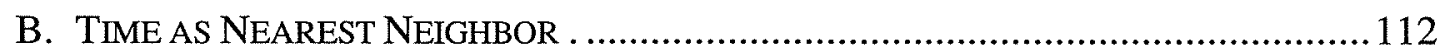

TABLE 3.3: LOTUS' INTERACTIONS ............................................................. 115

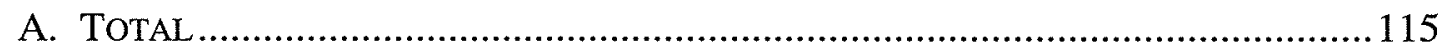

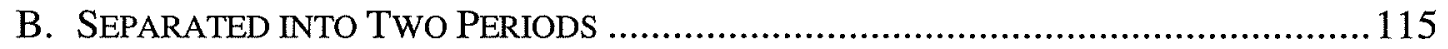

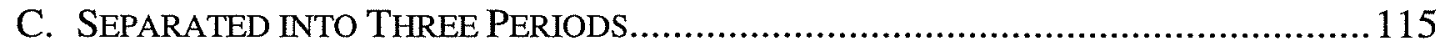

TABLE 3.4: HYPOTHESIZED CALF RELATIONSHIPS.............................................. 117

TABLE 3.5: BIC VALUES FROM LOGLINEAR MODELS OF DATA FOR ALL FOUR CALVES 119

TABLE 3.6: LOTUS' LATER WEEKS ............................................................. 123

TABLE 3.7: RULES FOR DECIDED VS. UNDECIDED AGONISTIC INTERACTIONS .............129

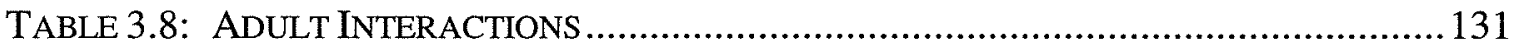

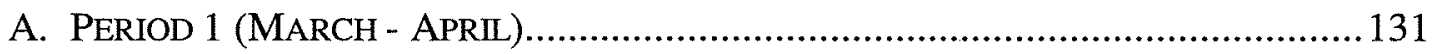

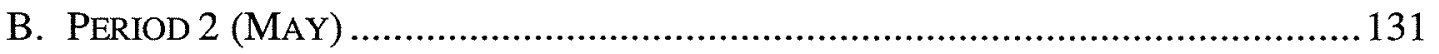

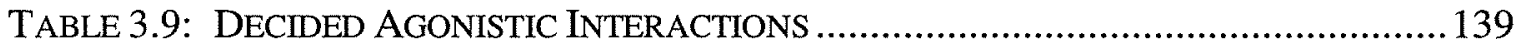

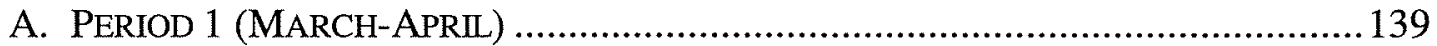

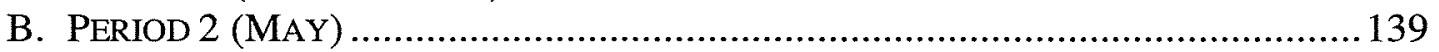

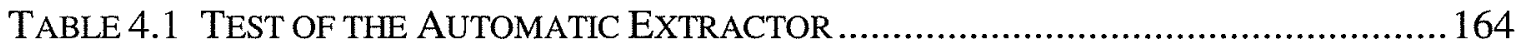

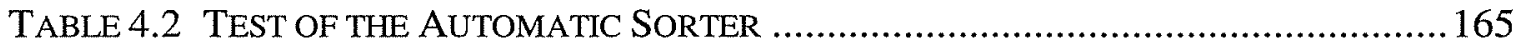

TABLE 4.3: MEASUREMENTS USED BY DISCRIMINANT ANALYSIS............................. 182

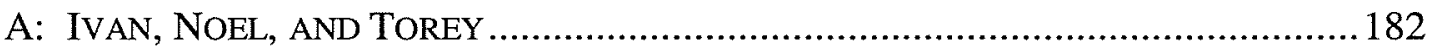

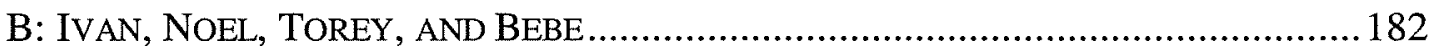


List of Tables

TABle 4.4: PERCENT ClassIFIEd CORRECTLY By DisCRIMINANT ANALYSIS.................182

TABLE 4.5: RESULTS OF K-MEANS ClUSTER ANALYSIS .......................................183

A: IVAN, NOEL, AND TOREY ......................................................... 183

B: IVAN, NOEL, TOREY, AND BEBE ........................................................ 183

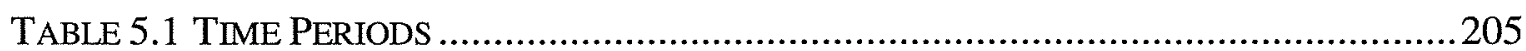

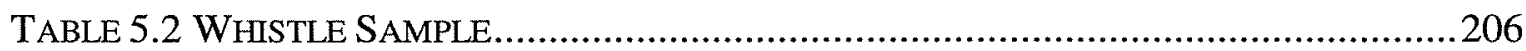

TABLE 5.3 USABLE BUBBLESTREAM-WHISTLES COLLECTED .....................................208

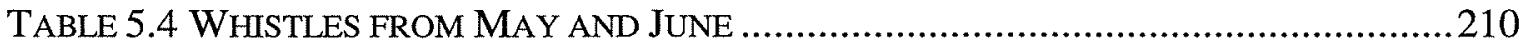

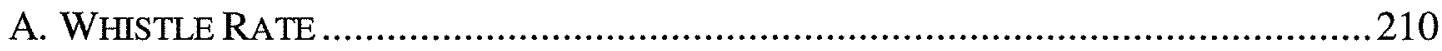

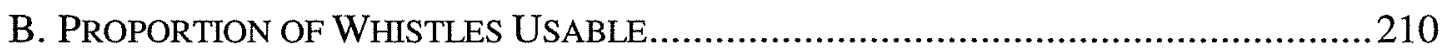

C. USABLE WHISTLES...........................................................................2 210

TABLE 5.5 RANDOM TRIALS OF PERCENT OVERLAPPING WHISTLES..........................215

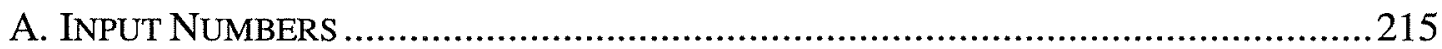

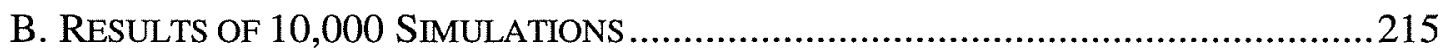

TABLE 5.6 BUBBLESTREAMS BY INDIVIDUAL IDENTITY ...................................217

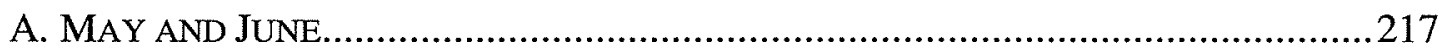

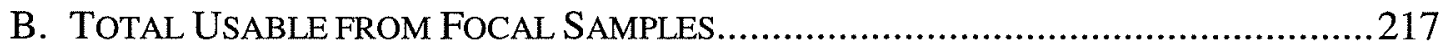

TABLE 5.7 SignATURE WHISTLE USE By TIME PERIOD .........................................2254

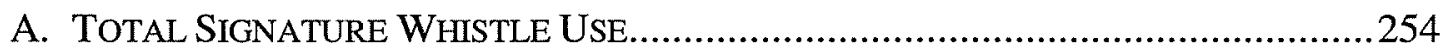

B. SigNATURE WHISTLE USE By INDIVIDUAL ............................................2254

TABLE 5.8 MOTHER's WhistLes AS PROPORTION OF THE ACOUSTIC ENVIRONMENT .....258 


\section{LIST OF STATISTICAL METHODS}

Because so many statistical methods were used in this thesis, a list is provided here for reference. The list is in alphabetical order.

1. Contour Cross-Correlation

Method for comparing contours by sliding them across each other and finding the offset where the correlation is maximized.

Used in Chapter 4.

Explained on page 177.

2. DICTIONARY CONTOUR COMPARISON (DCC)

Method for categorizing contours by comparing them to pre-defined dictionary contours and assigning each contour to the category with the most similar dictionary contour.

Used in Chapter 4.

Explained on page 179.

3. DISCRIMINANT ANALYSIS

Method of predicting group membership based on input variables. The group membership must be pre-defined and the discriminant analysis determines how well the input variables can separate the groups.

Used in Chapter 4.

Explained on page 174.

4. DYNAMIC TIME WARPING (DTW)

Method for determining the dissimilarity between two contours by aligning the features of the contours. DTW allows the non-uniform stretching of the time axis of one contour to match the other and then takes the normalized sum square frequency difference between the aligned contours.

Used in Chapters 4 and 5.

Explained on pages 178 and 209.

5. Hierarchical Cluster ANALysis (HCA)

Method of detecting natural groupings in data by connecting cases based on their similarity.

Statistics: moat index for determining number of clusters that maximizes the cluster cohesion.

Used in Chapters 3, 4, and 5.

Explained on pages 108, 175, 180, and 209. 
6. K-MEANS CLUSTER ANALYSIS

Method of separating cases into groups by their relative similarities. The desired number of groups is specified a priori and each case is placed into the group with the closest centroid.

Statistics: summed F-statistic used to determine optimal number of groups.

Used in Chapter 4.

Explained on page 175 .

7. LOGLINEAR ANALYSIS

Method for determining which of the variables or variable interactions in a contingency table has a significant influence on the data.

Statistics: Raferty's BIC. Negative $(<0)$ BIC measures means the model fits. The best model is the one with the lowest (most negative) BIC value.

Used in Chapters 3.

Explained on page 103.

\section{MultidimENSIONAL SCALING (MDS)}

Method of computing graphical equivalents to calculated similarities in order to be able to plot the points in space of reduced dimensionality ( 2 or $3 \mathrm{D}$ ) and compare the similarities visually.

Used in Chapters 3, 4, and 5.

Explained on pages 107, 179, and 209. 


\section{CHAPTER 1: INTRODUCTION}

\subsection{VOCAL LEARNING}

Learning plays an important role in the development of many communication systems, both human and non-human. Vocal learning is critical for the development of human language but plays a minor role in the vocal development of most non-human terrestrial mammals (Janik \& Slater 1997, Seyfarth \& Cheney 1997). Learning may be involved in the development of dolphin whistles, however (Tyack \& Sayigh 1997). Several studies have reported that bottlenose dolphin calves developed signature whistles matching acoustic models in their environment, including the whistles of unrelated animals and man-made whistles (Caldwell \& Caldwell 1979, Sayigh 1992, Tyack 1997). Adult and juvenile dolphins have also been shown to learn new sounds spontaneously and in trained situations (e.g. Caldwell \& Caldwell 1972, Richards et al. 1984).

Demonstrating that vocal learning plays a role in the development of natural vocalizations can be tricky, however. "Vocal learning within the natural repertoire can nevertheless be shown by rearing experiments if infants that were raised with different acoustic stimuli are found to match the sounds they heard in detail" (Janik \& Slater 1997, p.62). The main challenge in such a study is to find, or create, a situation where infants are raised with different acoustic stimuli. A common method has been to determine whether vocalizations develop normally when an infant is deprived of normal input (e.g. Marler 1970, Winter et al. 1973, Volman \& Khanna 1995). In a social species, however, depriving an infant of normal social or acoustic input may cause deficiencies in more areas than vocal output (e.g. West et al. 1997). Techniques for studying vocal learning in social species that incorporate the normal social environment of the species are needed. The objective of this thesis is to develop such techniques for studying vocal learning in the natural development of dolphin whistles.

The ability to produce the proper vocalization in the proper context is a matter of knowing how to produce the sound itself and knowing the appropriate context in which to use it. Demonstrating that the sounds infants use match the sounds they hear is not 
Chapter 1: Introduction

sufficient to distinguish between these two processes (Nelson 1997). Matching can be achieved by learning how to produce sounds or by learning which sounds to produce. Learning to use vocalizations in the proper context is a relatively common phenomenon among non-human mammals, particularly among primates (Seyfarth \& Cheney 1997). Other than humans, the best known example of animals that learn to produce new sounds is songbirds (e.g. Marler 1970, West \& King 1990). Learning to produce sounds is much less common among non-human terrestrial mammals (Janik \& Slater 1997). Marine mammals, and bottlenose dolphins in particular, have shown an ability to learn to produce new sounds that is unusual among non-human mammals (Caldwell \& Caldwell 1972, Richards et al. 1984, Ralls et al. 1985). Adult and juvenile animals of several species of marine mammals have learned to produce new sounds in captivity, imitating both human speech and computer generated whistles (e.g. beluga whales: Eaton 1979; harbor seals: Ralls et al. 1985; bottlenose dolphins: Caldwell \& Caldwell 1972, Richards et al. 1984, Reiss \& McCowan 1993). Bottlenose dolphins have been shown to be particularly adept at learning new sounds (e.g. Caldwell \& Caldwell 1972, Richards et al. 1984). The strong evidence for the ability of older dolphins to modify their vocalizations suggests that this type of learning might be used in the development of dolphins' natural vocalizations. Preliminary studies of vocal development have added support to this idea (e.g. Sayigh 1992, Tyack \& Sayigh 1997).

Studies of both birds and mammals have shown that social interactions play a role in the course of vocal development (Snowdon \& Hausberger 1997). Social input is essential to normal language development in humans (Locke \& Snow 1997). Animal studies of the social influences on learning vocal production have concentrated on songbirds (e.g. Immelmann 1969, Brown 1985, Margoliash et al. 1994, Hausberger et al. 1995), primarily because few non-human mammals have been clearly shown to learn to produce their vocalizations (Seyfarth \& Cheney 1997). Although it has been suggested that birdsong functions to maintain social bonds (e.g. Brown 1985, Hausberger et al. 1995), only a few studies of birdsong have investigated the "social bonds" between the birds (e.g. Brown 1985). On the other hand, the concept of social relationships has been 
well developed in the literature of on large mammals (e.g. primates: Seyfarth 1976, Seyfarth 1980, Altmann 1980, Smuts 1985; jackals: Moehlman 1987; elephants: Moss \& Poole 1983). Dolphins are highly social mammals with a social structure similar to some primates and elephants (e.g. dolphins: Wells 1991, elephants: Moss \& Poole 1983, primates: Cheney et al. 1986). Techniques for studying aspects of mammalian social structure, such as dominance hierarchies, have been successfully applied to dolphins (e.g. Samuels \& Gifford 1997). Studies of dolphins therefore provide a unique opportunity to combine the fields of mammalian social relationships and of vocal learning into powerful tools for investigating the social influences on vocal learning.

The objective of this thesis is to design a study of vocal learning in dolphin whistles that investigates both acoustic and social influences on learning, and to develop the techniques necessary to implement that study. I will first determine exactly what data are needed to thoroughly investigate whether learning is involved in the natural development of dolphin vocal communication and the impact of social interactions on that development. These issues will be discussed in this chapter. The techniques necessary to collect those data will be developed and tested in chapters two through five. Chapters two and three will discuss the sampling and evaluation of social interactions. Chapters four and five will cover the same issues for acoustic data. The final chapter will entail a discussion of how the techniques developed in this thesis can be used in the future to study vocal learning in dolphin whistle development.

\subsection{METHODS USED TO STUDY VOCAL LEARNING}

The first task in studying vocal learning is to determine whether learning plays any role in the development of natural communication in the species of interest. As was suggested by Janik and Slater (1997), vocal learning can be studied by comparing the vocal output of infants in different acoustic environments, meaning infants that heard different sounds as they developed. This has been done in the laboratory, by controlling the acoustic stimuli experienced by each infant (e.g. Baptista \& Petrinovich 1984, Winter et al. 1973), and in the wild, primarily by taking advantage of natural variation in the 
Chapter 1: Introduction

sounds produced by different groups (e.g. Hodun et al. 1981, Ford 1991). Studies of wild groups have to deal with the possibility that there is also genetic variation between the two groups (e.g. Morrice et al. 1994). Laboratory studies must contend with the difficulty of controlling the acoustic input without disrupting the infant's social development (e.g. West et al. 1997). A few studies have done this by quantifying the acoustic input an infant receives in a normal social setting. This design solves the problem of the infant's social development, and because the infant develops normally, also allows a more detailed investigation of the process of vocal learning.

One of the most common methods of studying vocal learning is to look at geographical variation in call structure caused by natural separations between subgroups of the same species (Janik \& Slater 1997). In Janik and Slater's (1997) review of mammalian vocal learning studies, $40 \%$ were of this type. The idea behind this is that if two groups are isolated from each other, infants born into each group will primarily be exposed to the calls of that group. One explanation for geographical variation in call structure is that the infants are learning the sounds to which they are exposed. For instance, geographical variation in dialects of birdsong is generally interpreted as evidence for vocal learning (Kroodsma \& Baylis 1982). A major caveat of this method, however, is the possibility that the subgroups may be genetically distinct as well. If they are, the infants may be inheriting their distinctive call patterns rather than learning them. Therefore, the amount of interbreeding that occurs between the groups must be determined. Studies of seals, for instance, often indicate that there is significant fidelity to breeding sites (e.g. Morrice et al. 1994). This means that while call structure may have a learned component, testing for dialects cannot be used as evidence for learning. Similarly, studies of orcas have found dialectical differences between pods, and a learned component to vocal development has been suggested (Ford 1991). The breeding behavior of the pods is not known, however, so learned components cannot be distinguished from inherited ones. This situation is common, where studies find geographical differences in calls but are not able to distinguish between possible inherited and learned components in call development (e.g. Wang Ding et al. 1995, Mitani et al. 
1992, Green 1975). In some cases, the calls of hybrids can help. In a study of saddleback tamarins, Hodun et al. (1981) found that hybrid animals produced the calls of the group they grew up with, suggesting a strong learned component. Hybrid gibbons, on the other hand, develop great calls intermediate between those of their parents, indicating a strong genetic component (Brockelman \& Shilling 1984).

Another common method for studying vocal learning is to control the infant's acoustic input in the laboratory. One way this is often done is by removing all acoustic stimulation from some infants. Some studies actually remove all relevant stimuli from the infants by hand-raising them in isolation from other animals. This was very common in the early studies of birdsong (e.g. Marler 1970). One problem with these studies is that infants "might simply need auditory input of some non-specific sort to develop normal calling behavior" (Janik \& Slater 1997, p.63). A study where the infant is kept in complete acoustic isolation cannot discount this possibility (e.g. Esser 1994, Romand \& Ehret 1984). An extension of studying animals in complete isolation is to give them acoustic input only, with no social input. Many early studies of birdsong only gave fledglings a tape of song to listen to (e.g. Marler 1970, see Nelson 1997). Others gave the infants a live tutor that they could only interact with across a wire mesh (e.g. Baptista \& Petrinovich 1984).

A major problem with all of these studies is the assumption that a social animal can develop normally when raised in social isolation. Early studies of primates showed that isolation caused generalized deficits in their behavioral development (Harlow \& Harlow 1962). While isolation studies showed that birds could learn to sing in this way, few investigated whether they knew when to sing (West et al. 1997). A recent study of male cowbirds tested whether birds that develop normal vocalizations in socially impoverished environments are able to use those vocalizations properly (West et al. 1997). Male cowbirds sing to females in order to gain copulations. Some of the songs they sing elicit precopulatory displays, such as wing-strokes, from the females, and a normal male will increase the production of those songs (West \& King 1988). Males raised in impoverished social settings produced a proper song but rarely sang to females 
Chapter 1: Introduction

(Freeberg et al. 1995). In some cases, the songs of these males elicited precopulatory displays from the females but the males did not increase the production of those songs and often moved away from the displaying females (West et al. 1997). Male cowbirds raised with canaries actually sang more to canaries than to female cowbirds, in spite of the fact that the canaries fled and the female cowbirds produced precopulatory displays (Freeberg et al. 1995, West et al. 1997). Interestingly, subsequent exposure to a normal social setting, including adult males successfully singing to and copulating with females, rehabilitated the socially impoverished males so that they used the song properly in the following year (Freeberg et al. 1995). Social input was essential to the normal development of male cowbirds in this study. Therefore, abnormal vocal development in animals raised in social isolation may be a symptom of more generalized deficits, rather than evidence for vocal learning in normal development.

Another common technique for controlling the auditory environment is to deafen the infants and then raise them in a normal social environment. As with isolation, deafening was a common method in the early birdsong studies (e.g. Nottebohm \& Nottebohm 1971). However, deafening has the same problem as isolation in that infants may simply need non-specific auditory input to develop vocal behavior. Although deafening cannot clearly show that learning is involved in vocal development, it can show that learning is not involved. For instance, deafening squirrel monkeys did not prevent them from developing normal vocalizations (Winter et al. 1973). Occasionally, rather than the infants being deafened, the mothers are muted (Winter et al. 1973). This way the infants receive acoustic input from each other but no adult models. However, the invasiveness of both these techniques make them inappropriate for many species. In addition, while this method is useful for determining whether learning plays a role at all, such an extreme treatment does not allow the study of influences on such learning (see section 1.3). For that, the factors influencing development need to be studied when the infants develop normally.

One way to do this is to allow the infant to develop in a normal social setting while clearly determining what sounds it is hearing. The influences on vocal 
development can then be understood by comparing the infant's vocalizations to the sounds it heard. However, if the infant is raised by its biological mother, or by a group that it is genetically related to, distinguishing the importance of learning from inherited influences may be difficult. One solution to this problem is to cross-foster the infant so that it is brought up by unrelated animals. If the vocalizations of the infant's natal and foster groups are different enough, the relative importance of learning and inheritance can be determined by which group the infant's vocalizations match. It is very important that the two groups use different sounds, however. The results of a between-species crossfostering with two species of macaques were inconclusive because the two species' vocalizations were too similar (Masataka \& Fujita 1989, Owren et al. 1992). This method has been very successful at demonstrating that birds learn the structure of their song (Nelson 1997, Baptista \& Gaunt 1997). Many studies have been done where young birds were raised with unrelated birds, both conspecific and allospecific, and developed vocalizations that matched those of the birds they were raised with (e.g. Baptista \& Gaunt 1997, Clayton 1988). However, cross-fostering studies are complicated by the possibility of the infant hearing its parents' vocalizations before it is born. There is evidence that both human infants and ducklings can hear well before birth and that their prenatal auditory experience influences their postnatal preferences (Querleu et al. 1989, Gottlieb 1988). Therefore, while an infant's preference for the vocalizations of its foster group clearly show learning, a preference for the vocal pattern of the natal group may not necessarily indicate a strong genetic component.

In some situations, cross-fostering of the infants is not actually necessary. For instance, the acoustic frequency of the echolocation calls of greater horseshoe bats is a function of age (Jones \& Ransome 1993). The frequency an infant hears therefore depends on the age of its mother. Jones and Ransome (1993) compared echolocation calls of infants in their first few weeks and found that the acoustic frequency of those calls was significantly correlated with the age of the mother. Mothers over the age of five years had significantly lower-frequency echolocation calls and so did their infants. 
Chapter 1: Introduction

Because the mothers' frequency changed as she aged, the genetic relatedness of the mother and infant did not influence the conclusions in this case.

In spite of its usefulness, cross-fostering is not always an option. Because husbandry concerns usually outweigh scientific ones in the breeding of cetaceans in captivity, planned cross-fostering experiments cannot be easily performed with cetaceans. Unplanned situations do arise sometimes, such as when an infant is orphaned or found stranded on the beach (e.g. Kastelein et al. 1990, Tyack 1997). An infant in that situation is often fostered to an unrelated conspecific female. In such instances, the development of the infant's vocalizations can be evaluated in light of the vocalizations produced by its foster mother. These opportunities are rare, however, and such orphans are often housed with a single female (e.g. Tyack 1997), which is an unusual situation for dolphin calves to be in (Wells 1991). In addition, sounds the infant heard before it was orphaned might influence the development of its vocalizations. For these reasons, cross-fostering is not an ideal method in this species.

The ideal study of vocal learning depends on the normal social structure of the species in question. In species where infants are normally raised by solitary mothers, determining the sounds the infant hears will generally not allow genetic influences to be distinguished from learning, except in unusual circumstances such as Jones and Ransome's (1993) bats. The best method for studying this type of animal may be carefully constructed cross-fostering studies. In species where infants are normally raised in a social group, such as dolphins or primates, a study where the infant's environment is quantified as the infant grows up with its own mother may be preferable. In such a study, however, the genetic relationship between all the animals in the group must be known so that genetic factors can be taken into account. If most of the animals in the group are not related to the infant, comparing the sounds the infant produces to the sounds the infant hears may be sufficient to demonstrate vocal learning. Quantifying the infant's acoustic environment, whether in its natal group or in a foster group, will also allow a more detailed investigation of the factors that influence vocal learning. 


\subsection{MECHANISMS OF VOCAL LEARNING}

The development of a vocal repertoire is partly a matter of learning to make the sounds (production) and partly of deciding which sounds to make (usage). An animal's adult repertoire will reflect the results of both of these processes (Nelson 1997). Demonstrating that infants raised in different acoustic environments match the sounds they heard is not sufficient to differentiate between learning production and learning usage. All vocalizations produced by all infants may have acoustic structures largely determined by innately specified motor patterns and the adult repertoire may be determined by selective attrition based on auditory input (learning usage) rather than selective acquisition (learning production) (see e.g. Nelson 1997). For example, human infants babble some phonemes that are not found in their natal language (Locke 1993). The phonemes not in the infant's native language are lost as language development proceeds, by selective attrition of the phonemes the infant does not hear. A more detailed analysis of the course of vocal development and the social setting in which development occurs is needed to distinguish between learning production and learning usage.

One method that has been used to demonstrate learning usage is to look for the selective loss of sounds in response to novel social settings. For example, male field sparrows (Spizella pusilla) arrive at a new territory with a repertoire of two to four songs, and preferentially keep only the one that best matches their neighbor's (Nelson 1997). Yearling buntings (Passerina cyanea) captured from the wild and exposed to live tutors preferentially retained and modified pre-existing syllables to match those of the tutor (Margoliash et al. 1994). The implication of these results is that if infants are learning usage, more types of vocalization should be seen in the infant's early repertoire than in the later, adult repertoire. This is known as "overproduction", where the infants produce more types of vocalizations than they will use as adults (Nelson 1997). A test for overproduction of vocalizations by infants can help determine whether learning is occurring by selective attrition or selective acquisition. However, a simple comparison of the vocal repertoires of animals when they are very young to their vocal repertoires when they are older may not be a valid test for overproduction. In many cases, the maturation 
Chapter 1: Introduction

process may need to be accounted for as well. For instance, very young dolphins produce whistles that are substantially different from adult whistles (Caldwell \& Caldwell 1979). Calves begin to produce adult-like whistles in their first few months. Overproduction can be tested for at this point, when the calves are capable of producing adult-like whistles.

An example of how a test for overproduction would help distinguish learning production from learning usage comes from the unique signature whistles of bottlenose dolphins. Some researchers have suggested that dolphin calves learn to produce their signature whistles (Tyack \& Sayigh 1997). The evidence cited for this is generally that calves match the signature whistles of dolphins they grew up with (Caldwell \& Caldwell 1979, Tyack \& Sayigh 1997). Such evidence cannot distinguish learning production from learning usage, however. An investigation of overproduction could. If the calves were learning which whistles to use, rather than how to produce the whistles, the calves should produce many different possible signature whistles early on and reduce that to their one signature whistle later. In the most extreme version of this model, all calves should produce all possible signature whistles early on and each calf should choose its signature whistle from that pool of possible whistles that they all produce. This type of overproduction does not appear to occur with dolphin signature whistles (Sayigh 1992, Tyack \& Sayigh 1997). Because every dolphin's signature whistle is at least slightly different from every other dolphin's signature whistle, a single calf could not produce all possible signature whistles for all dolphins. However, the calves could all produce a few basic contour types, learn which one to keep, and then improvise on that to create a unique signature whistle for itself. This does not appear to occur either (Sayigh 1992, Tyack \& Sayigh 1997). Therefore, dolphin calves probably are learning to produce their signature whistles, rather than learning which whistles to use as their signature whistles.

Since few mammals have been shown to learn the production of vocalizations (Janik \& Slater 1997, Seyfarth \& Cheney 1997), most studies looking at the learning of sound production involve birds. The most commonly used method to investigate the mechanisms by which animals learn to produce sounds is to give the animal a choice of models and look for selective acquisition of certain sounds. In most cases, this consists 
of giving a young bird a choice of tutors, usually one it can see or interact with and one it can only hear. Commonly, the tutor that the young bird can see and interact with is a bird from another species which sings a different song than the youngster's species sings. There are many examples of this type of study where the young bird matches the model produced by the tutor it can see, even though it is then learning an allospecific song (Baptista \& Gaunt 1997). For example, indigo buntings raised in this manner preferentially learned songs from the tutor that they could see (Payne \& Payne 1997), and bullfinches raised by canaries learned canary song, even though they could also hear bullfinch song (Baptista \& Gaunt 1997). An interesting modification of this type of study was done by Clayton (1988), where zebra finches could actually interact with two types of tutors: a zebra finch singing a Bengalese finch song and a Bengalese finch singing a zebra finch song. The young birds learned from the zebra finch, even though they were learning Bengalese finch song. This preference was demonstrated to be tutor-specific, not song-specific, by testing a similar situation where both tutors were zebra finches, one singing a zebra finch song and one a Bengalese finch song. In this situation, the youngsters showed no preference for one song over the other (Clayton 1988).

Another method used to investigate mechanisms of learning production is to examine vocal production in different social settings. For example, starlings raised by humans imitated human sounds only if the human was the starling's main social companion (West et al. 1983). If the starling was housed with other birds and did not interact socially with humans, it imitated the other birds but not the humans. This study was done in a yoked design so that the latter starling could hear all the sounds heard by the starling interacting with the human (West et al. 1983).

To understand the process of vocal learning, then, the acoustic environments of multiple infants must be quantified over the course of development, as must the social setting in which development takes place. Infants that experience different acoustic environments are necessary to demonstrate that each infant only matches the model from its own environment. To tease apart the various aspects of vocal learning, the aspects of social contact that are important need to be determined. Is the mere presence of the 
Chapter 1: Introduction

companion enough or are specific types of social interaction necessary? Hausberger (1997) found that the repertoire of adult starlings varied with the size and composition of the social group. Sharing of songs between adults in a social group was strongly influenced by affiliative contact between the adults. The different social interactions human children had with each member of their family influence the course of each child's vocal development as well (e.g. Plomin \& Daniels 1987, Jouanjean-L'Antoëne 1997). These results suggest that both the overall social environment, i.e. who is in the social group, and the specific social interactions each infant has with each member of the group are important and need to be quantified in addition to the acoustic environment.

\subsection{CHOICE OF STUDY ANIMAL}

The species and vocalization to study must be determined before the study can be designed. As was discussed in section 1.2, the social structure of the species will have a profound influence on the design of the study. Marine mammals, and bottlenose dolphins in particular, have shown an ability to learn new sounds that is unusual among nonhuman mammals (Seyfarth \& Cheney 1997, Tyack \& Sayigh 1997). The demonstrated ability of adult bottlenose dolphins to learn sounds (e.g. Caldwell \& Caldwell 1972, Richards et al. 1984) suggests that vocal learning might be involved in the development of their natural vocalizations as well. Preliminary studies of vocal development also indicate that this may be the case (Sayigh 1992, Tyack \& Sayigh 1997). Dolphins are therefore a promising species for studying vocal learning.

Narrow-band, frequency modulated whistles are one of the three main types of vocalizations dolphins produce (Caldwell et al. 1990). The other two are short broadband clicks used for echolocation, and broad-band sounds generally termed "burst-pulse" sounds. Both whistles and burst-pulse sounds appear to be used for communicative purposes (Caldwell et al. 1990, Overstrom 1983), but only whistles have been studied in detail (e.g. Caldwell et al. 1990, McCowan \& Reiss 1995a). Several studies have shown that dolphins can imitate computer-generated whistles and, in some cases, may associate the whistles with specific objects. Caldwell and Caldwell (1972) reported that a juvenile 
male dolphin spontaneously mimicked synthetic whistles being used in his training. Richards et al. (1984) trained an adult female to imitate novel synthetic whistles and use them to label objects that were shown to her. Reiss and McCowan (1993) presented a group of dolphins with a keyboard so that when a key was pressed, a synthetic whistle was played and the dolphins received a specific object. The two juvenile males in the group imitated the whistles after only a few exposures and most often produced them when in actual contact with the associated object. However, the pre-exposure repertoires of these dolphins was not quantified so this study cannot distinguish between the dolphins selectively using sounds already in their repertoires and the dolphins learning new sounds. Studies of whistle development indicate that many calves, especially those born in captivity, develop whistles that are different from their mothers' whistles (Sayigh et al. 1995, Sayigh 1992). In fact, several studies have reported calves producing whistles similar to unrelated animals in their pool (Caldwell \& Caldwell 1979, Sayigh 1992, see section 1.7). This evidence, combined with the evidence that older dolphins are capable of learning new whistles, suggests that learning may be involved in the natural ontogeny of dolphin whistles.

Bottlenose dolphins are being successfully bred in captivity, where clear underwater viewing is often possible. This allows in-depth studies of social behavior and development to be done in a situation where all the possible influences can be taken into account. Because of husbandry concerns, planned cross-fostering experiments cannot be done with dolphins. However, calves in captivity are generally housed with the group of dolphins, most of whom they are not related to. This is a major advantage of captive groups over wild groups. Wild dolphin calves grow up in a nursery group with their mothers, several other adult females and the calves of those females (Wells 1991). However, there is some evidence that some of the females in these groups are related (Duffield \& Wells 1991). Captive groups are more often composed of unrelated animals. Because learning from a related dolphin is difficult to distinguish from inheriting a call from that dolphin, unrelated associates are important to a study of vocal learning. 
Chapter 1: Introduction

Captive groups of dolphins have several other advantages as well. Dolphins collected from the same geographical region are often transported to different aquariums. This means that the dolphins in two aquariums, who therefore have no acoustic contact, are not genetically distinct. This situation, dolphins that have completely separate acoustic environments but are not genetically distinct, is almost impossible to achieve in the wild, but it essential to a study of vocal learning. In addition, it is possible to record all the whistle types a calf in captivity hears and to know all the dolphins that calf interacts with. Neither of these is possible with calves in the wild. Dolphins born in captivity have the opportunity to learn from unrelated dolphins in a situation where all the influences on their whistle development can be quantified. Calves born in different aquariums can be compared because they share a genetic history but not an acoustic environment. Captive groups of dolphins are therefore ideally set up for an investigation of the role of learning in whistle development.

\subsection{WHAT IS NEEDED TO TEST FOR VOCAL LEARNING?}

What then is needed for a complete test of whether learning is involved in the development of dolphin whistles? Altmann (1974) states that "an unambiguous formulation of the research question is a prerequisite" for making decisions about what data to collect and how to collect it (p228). The question of interest is "How do the acoustic and social environments experienced by a young dolphin influence that dolphin's vocal repertoire of whistles?" This question includes a series of more specific questions.

\subsubsection{What IS THE NORMAL ADULT REPERTOIRE?}

Before development can be evaluated, a clear picture of the normal adult repertoire of whistles is needed. To determine the normal adult repertoire, whistles need to be recorded from adults in multiple social groups. With those data, the repertoires of multiple adults can be compared to determine whether all adults have the same repertoire of whistles. Current evidence indicates that this is not the case. When dolphins are 
isolated, each adult produces a "signature" whistle that is specific to that dolphin (Caldwell et al. 1990, Janik \& Slater 1998). In other situations, adults appear to share some whistle types but not all (Tyack 1986, McCowan \& Reiss 1995a). In fact, there is evidence that the repertoires of adult dolphins changed over time (Tyack 1986, Smolker \& Pepper in press). If the animals' adult repertoire is not fixed, development can never be said to be truly "complete" and a "normal adult repertoire" cannot be defined. The underlying question, which is at what point in the study should the animals' repertoires be compared, becomes more complicated. One possible answer is to use the repertoire at the point that it begins to resemble the repertoires of adults in the population. For signature whistles, this occurs when the dolphin primarily produces a single stereotyped whistle when isolated (Sayigh 1992). This generally occurs between 3 months and 2 years from most dolphins (Caldwell \& Caldwell 1979, Sayigh 1992). At this point, most other whistles are also comparable to adult whistles in overall structure. Therefore, the dolphins' repertoires can be compared to their early environments when they are approximately two years old.

\subsubsection{WHAT TIME PERIOD IS IMPORTANT?}

A conservative estimate of the time period that might influence the calf would be the first few years, starting at, or shortly before, birth. The whistles a calf hears starting at birth may not actually be sufficient. Sound is known to travel into the human uterus, both from the mother and from external sources, and the acoustic environment of a human fetus affects its auditory and vocal development after birth (Querleu et al. 1989). Dolphin calves are precocious in many sensorimotor skills (McBride \& Kritzler 1951), and seem to be able to hear at birth. Since the water-to-tissue barrier is less difficult for sound to cross than the air-to-tissue barrier (Pierce 1991), dolphin calves can probably hear the sounds in their mother's environment during the last weeks before birth. In addition, dolphin calves begin whistling shortly after birth (McBride \& Kritzler 1951), and some produce whistles in the first week that are similar to what will be their signature 
whistles (Tyack \& Sayigh 1997). Therefore, an analysis of the prenatal acoustic environment of the calf may be necessary.

What part of the period from before birth through the first few years is important cannot be known a priori. In some species, such as songbirds, there is a critical period early in the bird's life during which an auditory template is developed (Nelson 1997). This period actually occurs several months before the bird begins to sing. This is not the case with dolphins as dolphin calves begin whistling at birth (McBride \& Kritzler 1951). However, calves may stop learning long before whistle development is complete. The timing of whistle development may also vary from calf to calf (e.g. Sayigh 1992). This should be possible to determine from a study covering the first two years.

\subsubsection{IS THE WHISTLE REPERTOIRE LEARNED FROM WHISTLES IN THE NATAL} ENVIRONMENT?

To answer this question, several pieces of data are needed: 1) the dolphin's repertoire at two years, 2) the whistles produced in its environment in its first two years, and 3 ) the whistles produced in another environment. If vocal learning is occurring, each dolphin's whistles should match those of its natal environment, and not those of other environments. Figure 1.1 shows two pools in separate locations where calves have been born. Each calf lives in a pool with his mother and at least one unrelated adult dolphin. If the calves are learning their whistles, each calf's whistles must match a model from the whistles produced in his own pool. Calf 1 , therefore, must produce a whistle (Whistle 1C) that matches Whistle $1 \mathrm{M}$ or $1 \mathrm{U}$, and does not match Whistle $2 \mathrm{M}$ or $2 \mathrm{U}$. If Calf 1 's whistle matches Whistle $2 \mathrm{M}$ or $2 \mathrm{U}$, he could not have learned his whistle because these whistles were not produced in his acoustic environment. Equivalently, Calf 2 should match Whistle $2 \mathrm{M}$ or $2 \mathrm{U}$ and not Whistle $1 \mathrm{M}$ or $1 \mathrm{U}$. Each calf's whistles must therefore be compared to potential models from his own acoustic environment and from the acoustic environments of calves in other pools. The models should all come from environments that include calves in case whistle use changes when calves are present. 
Chapter 1: Introduction

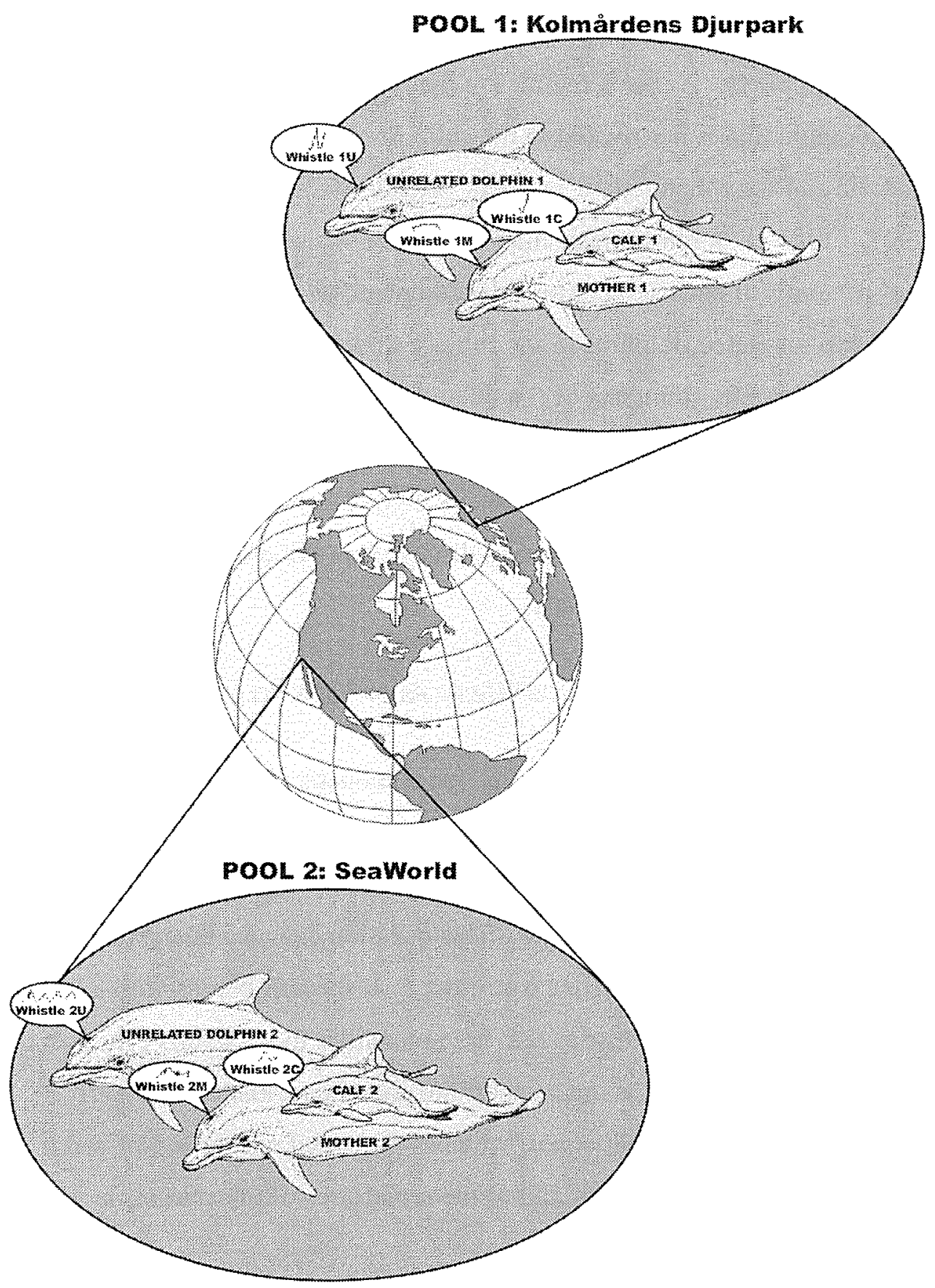

Figure 1.1: Experimental Design

The two locations were chosen for demonstration purposes only. Similarly, the contours were used as examples: they do not represent contours of dolphins in those actual locations. To demonstrate vocal learning, each calf's whistles must be shown to match the whistles produced by unrelated dolphins in the calf's own pool. Whistle $1 \mathrm{C}$, produced by Calf 1 , should therefore match Whistle $1 \mathrm{U}$ and not Whistle $1 \mathrm{M}$ or Whistles $2 \mathrm{U}$ or $2 \mathrm{M}$. Whistle $2 \mathrm{C}$ should match Whistle $2 \mathrm{U}$ and not Whistle $2 \mathrm{M}$ or Whistles $1 \mathrm{U}$ or $1 \mathrm{M}$. (The dolphin picture was purchased from ArtToday.) 
Chapter 1: Introduction

If Calf 1 's whistle matches Whistle $1 \mathrm{M}$, produced by his mother, learning cannot be distinguished from inheritance. This is the reason that each calf's social group must include at least one dolphin that is not related to the calf. Previous evidence indicates that few calves born in captivity match their mothers' whistles (Caldwell \& Caldwell 1979, Sayigh 1992). Calf 1 is therefore more likely to match Whistle $1 \mathrm{U}$ than Whistle 1M. The whistle repertoires of each subject must be compared to multiple models produced by related and unrelated dolphins in the subject's own early acoustic environment and in the early environments of the other subjects from other locations. To demonstrate that the whistle repertoire is learned, each subject's repertoire should match models produced by unrelated dolphins in the subject's own environment and not models that were only produced in other locations. As was discussed previously, artificial captive groups are perfect for this kind of study.

\subsubsection{IS THE CALF LEARNING HOW TO PRODUCE WHISTLES OR HOW TO USE THEM?}

The comparison of the adult repertoire to the natal acoustic environment is not sufficient to differentiate between learning production and learning usage. A more detailed analysis of the calf's whistles as the repertoire is developing and changing is needed to distinguish between these possibilities. This question can also be expressed "Is the calf selectively learning or losing whistles over time?" If the calf begins with a large

repertoire and selectively loses those whistles that are not similar to the adults', the calf is learning usage. If the calf begins with a small repertoire and adds whistles to it over time, it is learning production. A comparison of whistles produced when the calf was a few months old to its repertoire at two years and to the repertoires of the animals in its natal social group will best answer this question.

\subsubsection{WHAT ASPECT OF THE ENVIRONMENT IS IMPORTANT?}

If the dolphins' whistle repertoire includes whistles similar to certain models and not others, those models must have been more salient to the calf. Are these the whistles that are heard most often in the pool, or are they whistles made by adults with whom the 
calf had specific types of interactions? To answer these questions, the whistle repertoires of each animal in the social group are needed, as well as the interactions between the calf and each animal in the group. The specific interactions can include association, affiliative contact, or agonistic interactions, for example. These need to be carefully specified beforehand in order to know what to record. The combination of interactions can be expressed in terms of what kind of social relationship the calf had with each member of its group. In addition, all the whistles in the environment need to be recorded over time to determine how often various sounds were heard. In a captive group, for instance, the whistle used by the trainers to call the dolphins might be a very salient stimulus to both the adult dolphins and their calves.

\subsubsection{STUDY DESIGN}

A single study can be performed to collect the data to answer all the questions. The data that need to be collected are

1) the whistles produced by the calf throughout the sampling period,

2) the whistles heard by the calf,

3) the whistles produced by all the dolphins the calf interacted with, and

4) the interactions between the calf and those dolphins.

These data need to be collected for at least one infant from each of several social groups (see Figure 1.1). The vocalizations of each social group and the interactions of each infant need to be recorded throughout the sampling period. As was discussed above, the sampling period should include the months before the infant is born that it is capable of hearing in-utero. During this time, the whistles heard by the calf's pregnant mother and the interactions of the mother with the other dolphins in the group should be recorded. To separate the whistles into vocalizations produced by each animal in the group, all the whistles produced need to be recorded in such a way that the source can be identified. Simultaneous recordings can be made over the sampling period of

1) all the whistles heard in the pool,

2) the source of each whistle, and 
Chapter 1: Introduction

3) the interactions of each calf (or pregnant mother).

Once these data are collected, the acoustic and social environments can be evaluated. First, the whistles need to be separated out and compared to each other. Unbiased methods for sampling whistles from recordings and comparing the individual whistles to each other are essential. The rate that each calf interacted socially with each dolphin in its group must also be calculated in an unbiased manner. These interaction rates then need to be compared so that the relationship between each pair of individuals can be determined. The calf's repertoire as it changes over time can then be compared to the repertoires of animals in the group with whom the calf had different relationships, as well as to the repertoires of animals from other groups. If vocal learning is involved in whistle development, the calf's whistle repertoire should become more similar to the repertoires of adults in its natal social group than adults in other groups. The mechanisms by which this is occurring can be elucidated from the data on how the calf's whistle repertoire changed over time and the calf's interactions with its poolmates.

\subsection{METHODS OF WHISTLER IDENTIFICATION}

Studies of vocal learning in dolphins are complicated by the difficulty of identifying who is vocalizing. Since dolphins can vocalize without producing a visible sign, and since our ability for directional hearing does not work underwater, determining which animal is whistling in a group is very difficult. As we saw in section 1.5 , to study the development of natural communication the ability to attribute vocal repertoires to individual animals is essential. A variety of solutions to this technical problem have been used or are being developed for dolphin vocalizations. Two of these methods collect a sample of identified whistles by sub-sampling a particular set of the whistles. Either whistles are collected in particular settings or only whistles that are associated with particular behaviors are used. Neither of these has been clearly shown to produce a sample that is representative of the animal's entire repertoire. The other two methods utilize promising new technologies but neither is currently ready to use for studying vocal 
development. A robust method for identifying vocalizers to study whistle development is therefore still undeveloped but is necessary to be able to perform such a study properly.

\subsubsection{ISOLATION}

One solution to obtaining whistles from known individuals is to collect whistles from isolated animals. When dolphins are isolated, as much as $94 \%$ of the whistles each animal's produces are of one particular frequency contour, or pattern of frequency change over time (Tyack \& Sayigh 1997). This contour is unique to each individual, and is referred to as the dolphin's "signature whistle" (Caldwell \& Caldwell 1965). Signature whistles recorded from wild dolphins in isolation settings have been shown to develop by approximately one year of age and be stable throughout the dolphin's lifetime, over multiple decades (Sayigh et al. 1990).

A recent study has suggested that signature whistles are primarily used as contact calls when animals are separated and are less common in undisturbed social settings (Janik \& Slater 1998). Recordings were made of four dolphins in a captive setting where the dolphins could voluntarily move into a smaller pool. A hydrophone was placed in each pool so it was possible to tell which pool each whistle came from. Whistles were classified first without taking context into account, and then whistle use was compared by context. Four stereotyped whistles were found, and each individual produced one of the four when it was alone in the smaller pool. That whistle was labeled as that individual's signature whistle. Signature whistles were always the most common single whistle type produced when an animal was alone and made up between $30 \%$ and $90 \%$ of the whistles produced in that situation. When one of the dolphins was in the small pool, the signature whistles of the remaining three animals made up more than $50 \%$ of the whistles heard in the main pool. However, signature whistles made up less than $2.5 \%$ of the whistles heard when the entire group was together. These results suggest that signature whistles are used primarily when animals are out of sight of each other. Signature whistle use as a contact call was also reported between wild mothers and calves by Smolker et al. (1993). These results indicate that signature whistles represent only a portion of the entire whistle 
Chapter 1: Introduction

repertoire. However, a study of vocal learning in the development of signature whistles in particular would be interesting. Preliminary evidence suggests that learning is involved in signature whistle development (Sayigh 1992, Tyack \& Sayigh 1997). Isolation would be useful in such a study to determine the animals' signature whistles.

\subsubsection{SigNATURE WHISTLE CONTOUR}

Some researchers have taken advantage of the signature whistle's unique contour to identify whistlers. Because the signature whistle's contour is unique to each individual, all whistles with the contour of a dolphin's signature whistle could be assigned to that animal. This technique is not only useful in isolation settings because signature whistle use has also been reported in some social settings (Tyack 1986, Sayigh 1992). Sayigh (1992) reported that approximately $50 \%$ of the whistles recorded from a free-ranging group of dolphins match the signature whistle of one of the animals in the group. One study of two captive dolphins found that $50-70 \%$ of the whistles they produced when together were signature whistles (Tyack 1986). This is very different from the $2.5 \%$ reported by Janik and Slater (1998). Janik and Slater (1998) have two explanations for this discrepancy. They report that in situations where the animals were disturbed, such as when people were present or feeding was delayed, signature whistle use increased dramatically. The use of telemetry devices in Tyack's (1986) study, as well as the presence of trainers and researchers, may have influenced the whistle use of those animals. Janik \& Slater (1998) also suggest that whistle use in a captive group, where no animals are out of sight, might be different from a wild group. In captivity, most animals in one pool are usually within visual and acoustic range of the whistler. In the wild, dolphins are more likely to be out of visual range but within hearing distance of the whistling dolphin.

Tyack's (1986) study discovered a problem with assigning signature whistles to dolphins by contour, however: dolphins sometimes imitate each others' signature whistles. In this study, whistlers were identified by a device attached to the dolphin's melon (see section 1.6.4). Tyack (1986) found that $25 \%$ of the occurrences of each 
dolphin's signature whistle were actually produced by the other animal. This kind of "signature whistle imitation" has been seen in other situations as well. When wild dolphins are temporarily captured (Wells 1991), some of the non-signature whistles recorded from each animal are the signature whistles of other dolphins (Tyack \& Sayigh 1997). This is particularly true with pairs or "coalitions" of males but has also been reported among groups of females (Tyack 1993). Signature whistle matching has also been reported for free-ranging animals in the wild (Janik 1998). Signature whistle imitation calls into question the practice of assigning whistles to individuals by the whistle contour. Since dolphins imitate each other's signature whistles, not all the whistles that are recorded with a dolphin's signature contour were necessarily produced by that dolphin. Signature whistle contour is therefore not a reliable method for identifying whistlers.

\subsubsection{BUBBLESTREAMS}

Another method that has been used to identify whistlers is to take advantage of behavioral cues. In particular, some whistles are produced concurrently with a small stream of bubbles from the blowhole, which allows an observer to tell which animal is producing these whistles. Some researchers use only these whistles as their sample set (e.g. McCowan 1995, Herzing 1996). The advantage of this method over isolation whistles is that bubblestream whistles can be collected in a normal social setting. Based on the results of Janik and Slater's (1998) study, the sample of whistles obtained in this manner would be expected to be more variable and have fewer signature whistles than whistles recorded in isolation. The limitation of this method is that it can only be used in settings with good underwater visibility because bubblestreams are very difficult to observe from above the water's surface.

The assumption of this technique is that whistles produced in this manner are representative of the dolphin's entire repertoire, but this has yet to be clearly demonstrated. The only study done to date to test this assumption was performed by McCowan (1995). The rate of production of different whistle types was compared for 20 
Chapter 1: Introduction

whistles produced without bubblestreams and 57 produced with bubblestreams.

McCowan (1995) concluded that bubblestream whistles were representative because a $\chi^{2}$ test showed no difference between the samples. Since non-bubblestream whistles are easy to collect, the reason the sample of non-bubblestream whistles was so small is not clear, nor is the reason the two samples were not of the same size. In addition, the data on how the whistle types were determined, or how many types were used, were not presented. For a $\chi^{2}$ test to be valid, the expected values in all cells need to be at least five (Devore 1995). With only 20 whistles, that means there should be no more than four whistle types. Fifteen whistle types were described in McCowan's (1995) later analysis. If 15 whistle types were used in this test, the $\chi^{2}$ was not valid. The results of this test are therefore questionable.

Using bubblestreams to identify whistlers, McCowan and Reiss (1995a) performed a study of whistle use in three captive populations. The repertoires of ten adult dolphins were determined. The dolphins used 29 whistle types: 11 that were produced by more than one animal and 18 that were unique to individual animals. Of the 11 produced by multiple animals, 5 were produced by animals from different social groups. From these results, McCowan and Reiss (1995a) conclude that a dolphin's normal repertoire consists of some whistles that are unique to that dolphin, some that the dolphin shares with the other members of its current social group, and some whistles that are shared by all dolphins. This analysis was done using $\mathrm{K}$-means cluster analysis (McCowan 1995). K-means cluster analysis separates cases, in this case contours, into a pre-selected number of clusters. The analysis can be repeated on different numbers of clusters but the method used by McCowan (1995) to determine the optimal number of clusters is unclear. Therefore, it is unclear how robust the 29 whistle types are (McCowan \& Reiss 1995a). Since the classification into whistle types is the core of their conclusions, this problem poses a serious concern in the interpretation of their results. 


\subsubsection{TELEMETRY}

Another technique that has been used to identify whistlers is to put a telemetry device on a free-swimming animal. Two types of telemetry have been attempted: activating light-emitting diodes in response to sounds made by the animal and actually recording the sounds produced by the animal. Tyack (1986) used a device called a "vocalight" that lit up when it detected a sound. Each animal in the study wore one and several observers recorded how many LED's on each animal's vocalight were lit during each whistle. In this way, which animal was whistling could be determined. This process was very labor intensive and, as was discussed above, the need for real-time observations by several observers may have disturbed the animals. Tyack and Recchia (1991) developed a data logger that was placed on each animal and stored the level and frequency of detected sounds for later analysis. Early work using these with beluga whales met with some technical difficulties, however, and was discontinued (C.A. Recchia personal communication).

Recently, tags with small DAT recorders built into them have been designed and tested on several species with considerable success (elephant seals: Fletcher et al. 1996, dolphins: Nowacek et al. 1998). Some of these include hydrophones housed in suctioncups to acoustically couple them to the animal and increase the recording level (Nowacek et al. 1998). The whistles produced by the animal carrying the tags are therefore much louder than the ambient noise. Preliminary tests of these devices on free-ranging animals suggest that the animal resumes normal activity within a few hours (Nowacek et al. 1998). The devices are designed to passively release from the animal and can reliably be recovered at sea. The problem with this type of device is the need to physically put it on the animal. In the wild, equipping either a mother or calf with such a device requires temporarily capturing both mother and calf. Calves less than one year old are not captured in capture-release studies (e.g. Wells 1991), for fear of injuring the calves. Even in captivity a telemetry device would be difficult to place on a young calf because of the possibility of injuring the calf. Captive studies might be possible, however, by designing a tag to put on the mother that would also record whistles produced by the calf. Although 
Chapter 1: Introduction

these tags are not likely to be useful for studies of vocal development in calves, this is a promising technology for a variety of studies involving adult dolphins.

\subsubsection{PASSIVE LOCALIZATION}

In some situations, passive localization of sounds with an array of hydrophones has been used to identify vocalizers. The position of the whistler is determined by differences in the time of arrival at the dispersed hydrophones of the array. This has been successfully done using four to six hydrophones in a captive environment (Freitag \& Tyack 1993) or three hydrophones in channels in the wild (Janik 1998). In many captive settings the calculations are complicated by the reverberations from the pool walls, so a solution may have to be separately worked out for each pool arrangement (Freitag \& Tyack 1993). Alternatively, the animal's location can be determined by the phase shift caused by the angle of arrival at a linear array (Clark 1980, Miller \& Tyack 1998). For these techniques to be useful, whistle locationalization must be coupled with observations of animal locations. A rigid linear array can be towed behind a boat during behavioral observations of wild animals (Miller \& Tyack 1998). In some cases, real-time analysis of caller locations may even be possible. However, current protocols combining localization with visual observations of the animals' positions are not accurate enough to distinguish the whistles of a mother and calf who are swimming very close to each other. Therefore, passive localization may not be useful for studies of vocal development in its current state. However, this is the most promising technology currently under development to solve the problem of identifying whistlers in a study of vocal development.

\subsection{PREVIOUS WORK ON DOLPHIN WHISTLE DEVELOPMENT}

To investigate the possible role of learning in the development of signature whistles, comparisons have been made between signature whistles of animals and the

signature whistles they might have heard as infants. A study comparing whistles of wild 
mothers and calves found that $74 \%$ of the calves did not develop whistles similar to those of their mothers (Sayigh et al. 1995). A son was more likely to have a signature whistle that was somewhat similar to his mother's than a daughter was. Several studies have reported calves developing whistles similar to the whistles of unrelated animals.

Caldwell and Caldwell (1979) reported that a male calf raised with seven bottlenose and two Pacific white-sided dolphins developed a whistle similar to the more vocal whitesided dolphin's. An orphaned calf raised by an unrelated foster mother actually changed her signature whistle between the ages of one and six months to a whistle that closely resembled her foster mother's signature whistle (Tyack \& Sayigh 1997). A longitudinal study of three captive-born calves found that two of the calves developed whistles that most closely resembled the whistle used by the human trainers (Tyack \& Sayigh 1997). None of these studies looked at the social interactions that might have led to such modeling, however. In fact, both calves in the final study were recorded producing their signature whistles in their first week of life. Without a thorough analysis of all the whistles produced by the calves in their early weeks, these studies cannot distinguish between learning by selective acquisition and learning by selective attrition.

One study that did look at the social interactions involved in signature whistle development is Sayigh (1992). Sayigh (1992) followed four free-ranging calves as their whistles developed and recorded both whistle use and association patterns. Two calves developed signature whistles within the first two months that were similar to their mothers' signature whistles. These two were found in smaller groups and heard fewer whistles, of which a higher proportion were their mothers', than the other two calves. The other two calves took longer to develop their whistles and developed whistles that were less like their mothers' whistles. The calf that took the longest, and developed the whistle least similar to her mother's, was exposed to the highest rate of whistling and the lowest proportion of whistles from her mother. This study suggests that several factors may be involved in determining the course and timing of signature whistle development, and that acoustic exposure and number of associations are the most important of these. Calves exposed to a higher proportion of whistles from their mother, such as the 
Chapter 1: Introduction

orphaned calf raised with only her foster mother (Tyack \& Sayigh 1997), tend to develop whistles more similar to their mothers, and to develop them more quickly.

Using bubblestreams to identify whistlers, McCowan and Reiss (1995b) performed a study of whistle development in three captive populations. Eight infants were studied for their first year. None of the infants produced a totally adult-like repertoire at the end of their first year, suggesting that whistle development was not yet complete. However, since only one of the 28 adult whistle types was produced by all the adults in the study, it might be difficult to determine exactly what an "adult-like" repertoire would be. The infants produced a total of 128 whistle types: 34 shared and 94 unique to individual animals. 1281 whistles from the calves were analyzed but 845 of these were of only two types. There were only 436 whistles in the other 126 types, or less than four whistles per type on average. Exactly how distinct the categories actually were is therefore unclear. In addition, these whistle types were determined by k-means cluster analysis in the same way that the study of adult whistle repertoires determined types (McCowan 1995, see section 1.6.3). The robustness of the number of whistle types is therefore questionable in this study as well. Of the 34 shared types, 11 were also shared with the adults. However, only ten of these were whistle types that were shared by adults and calves in the same social group. Three of the 11 shared types were produced by calves in different social groups from the adults who produced that type. In fact, one "shared" whistle type was produced by only one adult and then by a calf in a different social group. Therefore, even if the calves were learning some of the whistle types from the adults, they were clearly not learning others. In addition, the McCowan and Reiss (1995b) do not clearly indicate whether all the whistle types shared between adults and calves were recorded from the adults before they were recorded from the calves.

Only one of these studies was designed to investigate the social setting in which development was occurring (Sayigh 1992). None were designed to be able to distinguish between the types of learning that might be occurring. The one study which did investigate the social setting was performed on free-ranging dolphins and therefore 
limited in what could be observed (Sayigh 1992). A similar study of captive animals would allow the social and acoustic environment of the calf to be sampled more precisely. However, captive studies must be interpreted with caution as the social and acoustic environment experienced by a calf might not reflect the normal environment of a free-ranging calf. In particular, groups of dolphins in the wild are fluid and change voluntarily while groups in captivity are determined by human handlers (Wells 1991). However, previous evidence indicates that the social behavior of captive dolphins is similar to wild dolphins (Samuels \& Gifford 1997). In fact, these static, predetermined captive groupings are exactly what makes the captive environment so useful for vocal learning studies. Because the dolphins do not choose their poolmates, a researcher studying two calves in different captive facilities knows that these calves never spent time with the same dolphins. A researcher studying calves in the wild cannot know this unless the calves come from widely separated locations. Calves from widely separated locations may also have different genetic backgrounds, however, which calves in captivity generally do not. A conclusive demonstration of vocal learning in bottlenose dolphins may therefore only be possible by studying calves born captivity.

In most of the studies discussed here and in section 1.6, whistles were extracted from the recordings and compared to each other manually. Manual extraction of whistles is very time consuming and cannot be guaranteed to produce an unbiased sample. Whistles in these studies were then compared and categorized by human judges (e.g. Sayigh 1992, Janik \& Slater 1998). Reliability tests showed judges ratings to be highly reliable (Sayigh 1992), but there are several problems with visual categorization. First, visual categorization is severely limited by the number of comparisons that can be reliable performed in a reasonable amount of time, which severely limits the sample size. Comparisons done by computer, on the other hand, do not suffer from that problem since computers can do a very large number of comparisons in a small amount of time. In addition, it is not possible to know what features of a sound human judges are using, even if all the judges come to the same decisions. A comparison made by computer allows a more explicit understanding of what features are being compared. However, both types 
Chapter 1: Introduction

of comparison need to be tested against the dolphins' responses to the various sounds. Playback studies are therefore necessary to determine what features the dolphins use to categorize sounds. However, playback studies using the judges' categorizations will not clearly show what features the dolphins are using to categorize the sounds because what features the judges used is not clear. Overall, computer comparisons are preferable because they are explicit, fast, and capable of dealing with large samples.

One set of studies that did use computers to compare the whistles in a sample was McCowan \& Reiss (1995a,b), the method for which is described by McCowan (1995). There are some problems with this method, however. As in the other studies, the whistles in these studies were extracted from the recordings manually. Twenty evenly spaced frequency measurements were then manually extracted from the fundamental frequency. The absolute duration of the whistles, although measured, was not used in the categorization procedures. McCowan (1995) does not indicate the range of durations that she recorded but Caldwell et al. (1990) reported whistles that varied in duration from as short as $60 \mathrm{~ms}$ to as long as $5.4 \mathrm{~s}$. While there is some evidence for time-dilation of whistles (e.g. Buck \& Tyack 1993), equating the durations over such a great range seems questionable. In fact, some researchers have suggested that very short whistles should be treated differently from longer whistles (Caldwell \& Caldwell 1970). The 20 frequency measurements for each pair-wise combination of whistles were then correlated by computer and the correlation matrix was subject to principal component analysis and $\mathrm{k}$ means cluster analysis (McCowan 1995). Exactly how the number of clusters for the kmeans cluster analysis was decided on is not clear (see section 1.6.3). The results of this categorization were cross-validated using discriminant analysis. Unfortunately, the results were not compared to the results of any techniques used in previous studies so whether this technique gives similar results to techniques that have been used by other researchers cannot be evaluated. Janik (in press) compared a similar method to the visual analysis performed in Janik and Slater (1998). He found that the results of the computer analysis did not match the visual ones exactly, although the results were similar. More detailed tests and comparisons of this and other techniques are therefore necessary. 


\subsection{OBJECTIVES AND OVERVIEW OF THESIS}

The objective of this thesis is to design the techniques necessary to perform studies of vocal learning on dolphin whistles. I have therefore developed and tested unbiased methods for sampling and comparing dolphin whistles, and for sampling and comparing social relationships between dolphins. Methods for identifying who is whistling are being developed elsewhere and will not be discussed in this thesis (see section 1.6). To achieve this objective, a pilot study was performed on a captive population of dolphins. The sampling protocols are described in chapter 2. Focal samples of the social interactions of individual animals were taken over several months before and after the births of four calves. Simultaneous acoustic recordings were made during all focal sessions. Some of the choices made in the sampling design were tested, and those tests are described in chapter 2 as well. The focal sample data were then used to develop methods for comparing interactions and determining social relationships (chapter 3). To sample whistles in an unbiased manner, programs for the automatic detection and extraction of whistles were developed (chapter 4). Chapter 4 also presents a comparison of several methods for categorizing whistles. The results of using the automatic extraction and quantitative comparison methods to determine the early acoustic environments of the calves in the pilot study are presented in chapter 5. Finally, how these methods and results can be used in the future to study vocal learning in dolphin whistle development is discussed in chapter 6 .

\subsection{LITERATURE CITED}

Altmann, J., 1974. “Observational study of behavior: Sampling methods." Behaviour, 49, pp. $227-267$. Altmann, J., 1980. Baboon mothers and infants. Harvard University Press, Cambridge, MA.

Baptista, L.F. and Gaunt, S.L.L., 1997. "Social interaction and vocal development in birds." Social influences on vocal development, eds. C.T. Snowdon and M. Hausberger. Cambridge University Press, New York, NY. pp. 23-40.

Baptista, L.F. and Petrinovich, L., 1984. "Social interaction, sensitive phases, and the song template hypothesis in the white-crowned sparrow." Animal Behaviour, 32, pp. 172-181.

Brockelman, W.Y. and Shilling, D., 1984. "Inheritance of stereotyped gibbon calls." Nature, 312, pp.634636

Brown, E.D., 1985. "The role of song and vocal imitation among common crows (Corvus brachyrhynchos)." Zeitschrift fur Tierpsychologie, 68, pp. 115-136. 
Chapter 1: Introduction

Buck, J.R. and Tyack, P.L., 1993. "A quantitative measure of similarity for Tursiops truncatus signature whistles." Journal of the Acoustical Society of America, 94, pp. 2497-2506.

Caldwell, M.C. and Caldwell, D.K., 1965. "Individualized whistle contours in bottlenosed dolphins (Tursiops truncatus)." Science, 207, pp. 434-435.

Caldwell, M.C. and Caldwell, D.K., 1970. "Etiology of the chirp sounds emitted by the Atlantic bottlenosed dolphin: A controversial issue." Underwater Naturalist, 6, pp. 6-8.

Caldwell, M.C. and Caldwell, D.K., 1972. "Vocal mimicry in the whistle mode by an Atlantic bottlenosed dolphin." Cetology, 9, pp. 1-8.

Caldwell, M.C. and Caldwell, D.K., 1979. "The whistle of the Atlantic bottlenose dolphin (Tursiops truncatus) - ontogeny." Behavior of marine animals, vol. 3 Cetaceans, eds. H.E. Winn and B.L. Olla. Plenum, New York, NY. pp. 369-401.

Caldwell, M.C., Caldwell, D.K., and Tyack, P.L., 1990. "Review of the signature-whistle hypothesis for the Atlantic bottlenose dolphin." The Bottlenose Dolphin, eds. S. Leatherwood and R.R. Reeves. Academic Press Inc., New York, NY. pp. 199-234.

Cheney, D., Seyfarth, R., and Smuts, B., 1986. "Social relationships and social cognition in nonhuman primates." Science, 234, pp. 1361-1366.

Clark, C.W., 1980. "A real-time direction finding device for determining the bearing to the underwater sounds of Southern Right Whales, Eubaleana australis." Journal of the Acoustical Society of America, 68, pp. 508-511.

Clayton, N.S., 1988. "Song tutor choice in zebra finches and Bengalese finches: The relative importance of visual and vocal cues." Behaviour, 104, pp. 281-299.

Devore, J.L., 1995. Probability and Statistics for Engineering and the Sciences. Duxbury Press, Boston, MA.

Duffield, D.A. and Wells, R.S., 1991. "The combined application of chromosome, protein, and molecular data for the investigation of social unit structure and dynamics in Tursiops truncatus." Reports to the International Whaling Commission, Special issue 13, pp. 155-169.

Eaton, R.L., 1979. "A beluga whale imitates human speech." Carnivore, 2(3), pp. 22-23.

Esser, K.H., 1994. "Audio-vocal learning in a non-human mammal: The lesser spear-nosed bat Phyllostomus discolor." Neuroreport, 5, pp.1718-1720.

Fletcher, S., Le Boeuf, B.J., Costa, D.P., Tyack, P.L., and Blackwell, S.B., 1996. "Onboard acoustic recording from diving northern elephant seals." Journal of the Acoustical Society of America, 100 , pp. 2531-2539.

Ford, J.K.B., 1991. "Vocal traditions among resident killer whales (Orcinus orca) in coastal waters of British Columbia." Canadian Journal of Zoology, 69, pp. 1454-1483.

Freeberg, T.M., King, A.P., and West, M.J., 1995. "Social malleability in cowbirds (Molothrus ater artemisiae): Species and mate recognition in the first 2 years of life." Journal of Comparative Psychology, 109, pp. 357-367.

Freitag, L.E. and Tyack, P.L., 1993. "Passive acoustic localization of the Atlantic bottlenose dolphin using whistles and echolocation clicks." Journal of the Acoustical Society of America, 93(4), pp. 21972205.

Gottlieb, G., 1988. "Development of species identification in ducklings: XV. Individual auditory recognition." Animal behaviour, 41, pp.953-963.

Green, S., 1975. "Dialects in Japanese monkeys: Vocal learning and cultural transmission of localespecific vocal behavior?" Z. Tierpsychology, 38, pp. 304-314.

Harlow, H.F. and Harlow, M.K., 1962. "Social deprivation in monkeys." Scientific American, 207, pp. 136-146.

Hausberger, M., 1997. "Social influences on song acquisition and sharing in the European starling (Sturnus vulgaris)." Social influences on vocal development, eds. C.T. Snowdon and M. Hausberger. Cambridge University Press, New York, NY. pp. 128-156.

Hausberger, M., Richard, M.A., Henry, L., Lepage, L., and Schmidt, I., 1995. "Song sharing reflects the social organization in a captive group of European starlings (Sturnus vulgaris)." Journal of Comparative Psychology, 109, pp. 222-241. 
Herzing, D.L., 1996. "Vocalizations and associated underwater behavior of free-ranging Atlantic spotted dolphins, Stenella frontalis and bottlenose dolphins, Tursiops truncatus." Aquatic Mammals, 22.2, pp. 61-79.

Hodun, A., Snowdon, C.T., and Soini, P., 1981. "Subspecific variation in the long calls of the tamarin, Saguinus fuscicollis." Z. Tierpsychology, 57, pp. 97-110.

Immelmann, K., 1969. "Song development in the zebra finch and other estrildid finches." Bird Vocalizations, ed. R.A. Hinde. Cambridge University Press, Cambridge, UK. pp. 64-74.

Janik, V.M. and Slater, P.J.B., 1997. "Vocal learning in mammals." Advances in the Study of Behavior, 26, pp. 59-99.

Janik, V.M. and Slater, P.J.B., 1998. "Context-specific use suggests that bottlenose dolphin signature whistles are cohesion calls." Animal Behavior, 56: 4, pp. 829-838.

Janik, V.M., 1998. Functional and organizational aspects of vocal repertoires in bottlenose dolphins, Tursiops truncatus. Ph.D. Dissertation, University of St. Andrews.

Janik, V.M., in press. "Pitfalls in the categorization of behaviour: a comparison of dolphin whistle classification methods." Animal behaviour.

Jones, G. and Ransome, R.D., 1993. "Echolocation calls of bats are influenced by maternal effects and change over a lifetime." Proceeds of the Royal Society of London, Series B, 252, pp. 125-128.

Jouanjean-L'Antoëne, A., 1997. "Reciprocal interactions and the development of communication and language between parents and children." Social influences on vocal development, eds. C.T. Snowdon and M. Hausberger. Cambridge University Press, New York, NY. pp. 312-327.

Kastelein, R.A., Dokter, T., and Zwart, P., 1990. "The suckling of a Bottlenose dolphin calf (Tursiops truncatus) by a foster mother, and information on transverse birth bands." Aquatic Mammals, 16(3), pp. 134-138.

Kroodsma, D.E., and Baylis, J.R., 1982. "Appendix: A world survey of evidence for vocal learning in birds." Acoustic communication in birds, volume 2, eds. D.E. Kroodsma, and E.H. Miller. Academic Press, New York, NY. pp. 311-337.

Locke, J.L. and Snow, C., 1997. "Social influences on vocal learning in human and nonhuman primates." Social influences on vocal learning, eds. C.T. Snowdon and M. Hausberger. Cambridge University Press, New York, NY. pp. 274-92.

Locke, J.L., 1993. The child's path to spoken language. Harvard University Press, Cambridge, MA.

Margoliash, D., Staicer, C.A., and Inoue, S.A., 1994. "The process of syllable acquisition in adult indigo buntings (Passerina cyanea)." Behaviour, 131, pp. 39-64.

Marler, P., 1970. "A comparative approach to vocal learning: Song development in white-crowned sparrows." Journal of Comparative Physiology and Psychology, 71, pp. 1-25.

Masataka, N. and Fujita, K., 1989. "Vocal learning of Japanese and rhesus monkeys." Behaviour, 109, pp. 191-199.

McBride, A.F. and Kritzler, H., 1951. "Observations of pregnancy, parturition, and postnatal behaviour in the bottlenose dolphin." Journal of Mammology, 32 (3), pp. 251-265.

McCowan, B. and Reiss, D., 1995a. "Quantitative comparison of whistle repertoires from captive adult bottlenose dolphins (Delphinidae, Tursiops truncatus): a re-evaluation of the signature whistle hypothesis." Ethology, 100, pp. 194-209.

McCowan, B. and Reiss, D., 1995b. "Whistle contour development in captive-born infant bottlenose dolphins (Tursiops truncatus): Role of learning." Journal of Comparative Psychology, 109(3), pp. 242-260.

McCowan, B., 1995. "A new quantitative technique for categorizing whistles using simulated signals and whistles from captive bottlenose dolphins (Delphinidae, Tursiops truncatus)." Ethology, 100, pp. 177-193.

Miller, P.J. and Tyack, P.L., 1998. "A small towed beamforming array to identify vocalizing resident killer whales (Orcinus orca) concurrent with focal behavioral observations." Deep Sea Research, 45, pp.1389-1405.

Mitani, J.C., Hasegawa, T., Gros-Louis, J., Marler, P., and Byrne, R., 1992. "Dialects in wild chimpanzees?" American Journal of Primatology, 27, pp. 233-243.

Moehlman, P.D., 1987. "Social organization in jackals." American Scientist, 75, pp. 366-375. 
Chapter 1: Introduction

Morrice, M.G., Burton, H.R., and Green, K., 1994. "Microgeographic variation and songs in the underwater vocalisation repertoire of the Weddell seal (Leptonychotes weddellii) from the Vestfold Hills, Antarctica." Polar Biology, 14, pp. 441-446.

Moss, C.J. and Poole, J.H., 1983. "Relationships and social structure of African elephants." Primate social relationships, an integrated approach, ed. R.A. Hinde. Sinauer Associates, Inc., Sunderland, MA. pp. 314-24.

Nelson, D.A., 1997. "Social interaction and sensitive phases for song learning: A critical review." Social influences on vocal development, eds. C.T. Snowdon and M. Hausberger. Cambridge University Press, New York, NY. pp. 7-22.

Nottebohm, F. and Nottebohm, M.E., 1971. "Vocalizations and breeding behaviour of surgically deafened ring doves (Streptopelia risoria)." Animal behaviour, 19, pp. 313-327.

Nowacek, D.P., Tyack, P.L., Wells, R.S., and Johnson, M.P., 1998. "An onboard acoustic data logger to record biosonar of free-ranging bottlenose dolphins." Proceeds of the 16th ICA and 135th Meeting of the ASA, Seattle, WA, June 1998.

Overstrom, N.A., 1983. "Association between burst-pules sounds and aggressive behavior in captive Atlantic bottlenosed dolphins (Tursiops truncatus)." Zoo Biology, 2, pp. 93-103.

Owren, M.J., Dieter, J.A., Seyfarth, R.M., and Cheney, D.L., 1992. "“Food" calls produced by adult female rhesus (Macaca mulatta) and Japanese ( $M$. fuscata) macaques, their normally-raised offspring, and offspring cross-fostered between species." Behaviour, 120, pp. 218-231.

Payne, R.B. and Payne, L.L., 1997. "Field observations, experimental design, and the time and place of learning bird songs." Social influences on vocal development, eds. C.T. Snowdon and M. Hausberger. Cambridge University Press, New York, NY. pp. 57-84.

Pierce, A.D., 1991. Acoustics: An introduction to its physical principles and applications. Acoustical Society of America, Woodbury, NY.

Plomin, R. and Daniels, D., 1987. "Why are children in the same family so different from one another?" Behavioral and Brain Sciences, 10, pp. 1-60.

Querleu, D., Renard, X., Boutteville, C., and Cripin, G., 1989. "Hearing by the human fetus?" Seminars in Perinatology, 13, pp. 409-420.

Ralls, K., Fiorelli, P., and Gish, S., 1985. "Vocalizations and vocal mimicry in captive harbor seals, Phoca vitulina." Canadian Journal of Zoology, 63, pp. 1050-1056.

Reiss, D. and McCowan, B., 1993. "Spontaneous vocal mimicry and production by bottlenose dolphins (Tursiops truncatus): Evidence for vocal learning." Journal of Comparative Psychology, 107(3), pp. 301-312.

Richards, D.G., Wolz, J.P., and Herman, L.M., 1984. "Vocal mimicry of computer-generated sounds and vocal labeling of objects by a bottlenose dolphin, Tursiops truncatus." Journal of Comparative Psychology, 98(1), pp. 10-28.

Romand, R. and Ehret, G., 1984. "Development of sound production in normal, isolated, and deafened kittens during the first postnatal months." Developmental psychobiology, 17, pp.629-649.

Samuels, A. and Gifford, T., 1997. "A quantitative assessment of dominance relations among bottlenose dolphins." Marine Mammal Science, 13, pp. 70-99.

Sayigh, L.S., 1992. Development and functions of signature whistles of free-ranging bottlenose dolphins, Tursiops truncatus. Ph.D. Dissertation, MIT/WHOI Joint Program, WHOI 92-37.

Sayigh, L.S., Tyack, P.L., Wells, R.S., and Scott, M.D., 1990. "Signature whistles of free-ranging bottlenose dolphins Tursiops truncatus: Stability and mother-offspring comparisons." Behavioral Ecology and Sociobiology, 26, pp. 247-260.

Sayigh, L.S., Tyack, P.L., Wells, R.S., Scott, M.D., and Irvine, A.B., 1995. "Sex difference in whistle production in free-ranging bottlenose dolphins, Tursiops truncatus." Behavioral Ecology and Sociobiology, 36, pp. 171-177.

Seyfarth, R.M. and Cheney, D.L., 1986. "Vocal development in vervet monkeys." Animal behaviour, 34, pp. 1640-1658.

Seyfarth, R.M. and Cheney, D.L., 1997. "Some general features of vocal development in nonhuman primates." Social influences on vocal development, eds. C.T. Snowdon and M. Hausberger. Cambridge University Press, New York, NY. pp. 249-274. 
Seyfarth, R.M., 1976. "Social relationships among female baboons." Animal behaviour, 24, pp. 917-938.

Seyfarth, R.M., 1980. "The distribution of grooming and related behaviors among adult female vervet monkeys." Animal behaviour, 28, pp. 798-813.

Smolker, R.A., Mann, J., and Smuts, B.B., 1993. "Use of signature whistles during separations and reunions by wild bottlenose dolphin mothers and infants." Behavioral Ecology and Sociobiology, 33, pp. 393-402.

Smolker, R.S. and Pepper, J., in press. "Whistle convergence among allied male bottlenose dolphins." Ethology.

Smuts, B.B., 1985. Sex and friendship in baboons. Gruyter, New York.

Snowdon, C.T. and Hausberger, M., 1997. Social influences on vocal development. Cambridge University Press, New York, NY.

Snowdon, C.T., Elowson, A.M., and Roush, R.S., 1997. "Social influences on vocal development in new world primates." Social influences on vocal development, eds. C.T. Snowdon and M. Hausberger. Cambridge University Press, New York, NY. pp. 234-248.

Tyack, P.L. and Recchia, C.A., 1991. "A datalogger to identify vocalizing dolphins." Journal of the Acoustical Society of America, 90, pp. 1668-1671.

Tyack, P.L. and Sayigh, L.S., 1997. "Vocal learning in cetaceans." Social influences on vocal development, eds. C.T. Snowdon and M. Hausberger. Cambridge University Press, New York, NY. pp. 208233.

Tyack, P.L., 1986. "Whistle repertoires of two bottlenosed dolphins, Tursiops truncatus: Mimicry of signature whistles?" Behavioral Ecology and Sociobiology, 18, pp. 251-257.

Tyack, P.L., 1993. "Animal language research needs a broader comparative and evolutionary framework." Language and communication: Comparative perspectives, eds. H.L. Roitblat, L.M. Herman, and P. Nachtigall. Erlbaum, Hillsdale, NJ. pp. 115-152.

Tyack, P.L., 1997. "Development and social functions of signature whistles in bottlenose dolphins Tursiops truncatus." Bioacoustics, 8, pp. 21-46.

Volman, S.F. and Khanna, H., 1995. "Convergence of untutored song in group-reared zebra finches (Taeniopygia guttata)." Journal of Comparative Psychology, 109, pp. 211-221.

Wang Ding, Wursig, B., and Evans, W.E., 1995. "Whistles of bottlenose dolphins: Comparisons among populations." Aquatic Mammals, 21(1), pp. 65-77.

Wells, R.S., 1991. "The role of long-term study in understanding the social structure of a bottlenose dolphin community." Dolphin Societies, eds. K. Pryor and K.S. Norris. University of California Press, Berkeley, CA. pp. 199-235.

West, M.J. and King, A.P., 1988. "Female visual displays affect the development of male song in the cowbird." Nature, 334, pp. 244-246.

West, M.J. and King, A.P., 1990. "Mozart's Starling." American Scientist, 78, pp. 106-114.

West, M.J., King, A.P., and Freeberg, T.M., 1997. "Building a social agenda for the study of bird song." Social influences on vocal development, eds. C.T. Snowdon and M. Hausberger. Cambridge University Press, New York, NY. pp. 41-56.

West, M.J., Stroud, A.N., and King, A.P., 1983. "Mimicry of the human voice by European starlings (Sturnus vulgaris): The role of social interaction." Wilson Bulletin, 95, pp. 635-640.

Winter, P., Handley, P., Ploog, D., and Schott, D., 1973. "Ontogeny of squirrel monkey calls under normal conditions and under acoustic isolation." Behaviour, 47, pp. 230-239. 


\section{CHAPTER 2: SAMPLING METHODS}

\subsection{SAMPLING DECISIONS}

A quantitative characterization of infants' acoustic and social environments is essential to any study of vocal learning. The infant's vocalizations need to be compared to the sounds it heard to determine whether the vocalizations might be learned (see chapter 1, and Figure 1.1, for a more thorough discussion of these issues). If the infant's vocalizations match the vocalizations it heard from unrelated animals, and do not match the vocalizations heard by other infants in other environments, the infant most likely learned its vocalizations from the sounds in its environment (see Figure 1.1). This requires the acoustic environment of each infant to be quantitatively characterized. Infants' social interactions with other animals have been shown to influence the course of vocal development in many species (e.g. Snowdon \& Hausberger 1997; birds: West et al. 1997; humans: Locke \& Snow 1997; primates: Seyfarth \& Cheney 1997). Therefore, to understand the factors influencing vocal learning, the infant's social environment must be characterized as well.

Bottlenose dolphin whistles are ideally suited to this sort of vocal learning study. Dolphin calves live with their mothers in groups of females (Wells 1991). In the wild, some of these females are related to the calf, but the dolphins also associate with unrelated animals (Duffield \& Wells 1991, Wells 1991). In captivity, calves are generally housed with their mothers in groups of unrelated dolphins. Calves both in captivity and in the wild therefore have the opportunity to hear the sounds of unrelated animals. Previous evidence suggests that calves are more likely to match the whistles of unrelated adults than their mothers' whistles (e.g. Caldwell \& Caldwell 1979, Sayigh et al. 1990, Sayigh 1992). Because the whistle repertoire of each dolphin is different, the acoustic environment experienced by different calves will be different (Caldwell et al. 1990). Whistles of dolphin calves can therefore be compared both to the whistles from the calves' own environments and to whistles from the early acoustic environments of other calves (see Figure 1.1). To study vocal learning in dolphins, the acoustic and social 
environments experienced by several calves must be quantified. This requires that the whistles heard by the calves and the interactions the calves have with other dolphins are recorded while the calves' whistles are developing. Those recordings must then be evaluated to develop a complete picture of the calves' early environments.

Recording and evaluating whistles and behaviors 24 hours a day is impractical, however, particularly over the entire course of development for several calves. Therefore, the whistles and behaviors need to be sampled. The decisions made in designing a strategy for sampling sound and behavior have a profound impact on the conclusions that can be reached (Altmann 1974). For instance, samples that focus on the behavior of one individual will not allow conclusions to be drawn about the relative locations of all the animals in the group. Conversely, scans of all the animals in the group will not yield information about the subtle behaviors of one or two individuals. The sampling strategy must therefore be carefully considered and explicitly laid out. The decisions involved in designing a sampling strategy for a study of vocal learning in bottlenose dolphins will be discussed in this chapter. The strategy will then be implemented in a pilot study and some of the decisions will be explicitly tested.

\subsubsection{DESIGNING THE SAMPLING STRATEGY}

There are many ways to sample acoustic and behavioral data. The methods used will have a significant impact on the conclusions that can be reached. For instance, if rare behaviors are noted every time they occur but common behaviors only marked some of the time, the occurrence of rare behaviors can be evaluated but the actual rate of common behaviors cannot (e.g. Bateson 1974, see Altmann 1974). This is an appropriate strategy if the issue of interest is what type of behaviors the animals display but not if the issue of interest is how often behaviors occur relative to each other. In a study of vocal learning, the relative rate at which each calf interacts with each dolphin in the group is important, so this method would not be appropriate. The most appropriate methods for each study therefore depend on the exact issues being addressed (Altmann 1974). For 
Chapter 2: Sampling Methods

this reason, the sampling methods must be chosen carefully and the choices described explicitly. This process involves a wide array of decisions about what to record and when, including who to observe, how many times to observe them, when to start observing, when to stop observing, what behaviors to record, and how to record them. The sampling methods must be tailored to the question being addressed. This section will describe the decisions to be made for sampling the acoustic and social environments of dolphin calves. The strategy chosen must allow the environments of multiple calves to be compared and the dolphins' social relationships to be understood. Ultimately, question of interest is how the calves' whistle repertoires compare to their acoustic environments and what social factors influenced that.

\section{Where to Observe the Dolphins}

Many of the sampling decisions depend on whether the study is being conducted with captive or free-ranging animals. With free-ranging animals, samples cannot always be started at the same time each day and each calf cannot be recorded on every day. Samples can only be taken when a calf is found and conditions allow that calf to be followed. Observing dolphins in the wild also limits the number of behaviors that can be observed, because the dolphins can generally only be observed when they are at or near the surface (e.g. Sayigh 1992 but see Nowacek et al. 1995, Dudzinski 1996). The amount of time a dolphin spends near the surface of the water may depend on the activity the dolphin is engaged in (Mann 1999). Differences in the proportion of time each animal spends in each activity may therefore introduce some unexpected biases into the data. A great deal more detail of the behaviors and interactions can be recorded in a study performed in a captive facility with good underwater visibility where the animals are easy to follow. In addition, it is difficult to know all the animals a free-ranging calf might interact with, or all the types of whistles it might hear. This makes quantifying all the possible influences on the calf's whistle development problematic. Even though all the interactions of a captive calf might not be recorded, all the possible interactors are known, and the genetic and social relationship between the calf and each dolphin in the group can be evaluated. There are, of course, tradeoffs to studying dolphins in captivity. 
Some behaviors, such as foraging, will be very different from the wild. However, comparisons of the behavior of captive and free-ranging dolphins have shown their social behavior to be similar (Samuels \& Gifford 1997). For studies investigating vocal development, the benefits of being able to record the details of the interactions and to control the calf's possible interactors outweigh the concerns that some interactions might be different from the wild. The study designed here will therefore be performed on calves born in captivity.

\section{When to Observe the Dolphins}

To decide when during its life each calf needs to be observed, the time period over which the whistles develop must be known. However, even if the extent of this period is known, what part of this period is important to the development cannot be known a priori. The sounds produced and interactions between animals may change over time. Therefore, a study where each calf is recorded only once or twice risks missing the influential period. A longitudinal study covering the entire developmental period, where the behavior of each calf is recorded on many days, would better uncover the influences on whistle development. In later studies, the time could be shortened if the influential period can be determined, or if some periods can be determined to not be important to whistle development. Previous studies have shown that most calves' signature whistle development, at least, is complete by the end of the first year, and all calves have signature whistles by the end of their second year (Caldwell \& Caldwell 1979, Sayigh 1992). In addition, calves are likely to be able to here in utero for several months before birth (see chapter 1). Since sounds heard in utero are known to influence vocal development in humans and ducks (Gottlieb 1988, Querleu et al. 1989), the mothers should be recorded for several months before the calves are born. The calves in this study will therefore be studied from several months prior to birth until the end of their first year. 
Chapter 2: Sampling Methods

\section{How to Sample the Dolphins' Behavior}

To quantify the possible social influences on whistle development, the social relationship each calf has with each dolphin in the group needs to be established (see section 1.3). Social relationships between animals are brought to light by the interactions those animals have with each other (Hinde 1976). The interactions each calf has with each dolphin in the group must therefore be observed and recorded. The relationships the calves' mothers have with other dolphins may also influence the calves, especially in a species such as dolphins where calves spend most of their time with their mothers (McBride \& Kritzler 1951, Wells 1991; see chapter 3 for a more thorough analysis of this issue). The interactions calves and their mothers have with each other and with other dolphins can be recorded systematically by focal animal sampling of mother-calf pairs. Focal animal sampling is a technique where a specific animal is carefully observed and all the behaviors of that animal are recorded (Altmann 1974). This is done to prevent a bias toward flashy, obvious behaviors and to ensure that subtle behaviors are not missed. Focal sampling can also be performed on very small groups, such as a mother-infant pair, if the members of the group spend most of their time in very close proximity to each other (Altmann 1974). Dolphin mothers and calves can be expected to spend most of their time within a few meters of each other (McBride \& Kritzler 1951, Wells 1991). The social interactions each calf and its mother have with each other and all the other dolphins in the group will therefore be recorded by focal animal sampling.

\section{When to Sample on Each Day}

To prevent a bias toward certain behavior types, the time that samples will begin and end for each focal must be decided before the sampling is started. This prevents observers from starting samples ad hoc when interesting behaviors occur or waiting to end them until the interesting behaviors are finished. A pre-made decision on when to finish sampling also prevents the observers from ending samples early because nothing of interest is happening. These types of mistakes can lead to an overestimation of how frequently certain types of behaviors occur (Altmann \& Altmann 1970). Even in the wild, where samples cannot always be started at the same time every day, the rules for 
when samples are started and how long they run must be explicitly stated (Altmann 1974). In the wild, rules must also be established for how to choose which calf will be sampled on each day. In captive studies, the actual time that samples are started can be decided a priori and consistently followed. All the calves can be sampled on every day and the rules governing the order of the samples can be decided a priori. For some studies in captivity, observing the dolphins when they have not yet been disturbed by the presence of people is preferable. In this case, samples might need to be performed early in the morning before people arrive. To study vocal development, however, a clear picture of all the influences on the calf is needed. In order to obtain a complete picture, behaviors need to be sampled from a variety of situations. Samples should be spaced throughout the day to include a fuller set of contexts, as well as the possibility of diurnal changes in behavior. In addition, for longitudinal comparisons of the changes in behavior that might occur over the course of development to be valid, equal length samples that start and end at the same time on each observation day are preferable.

The appropriate length of each sample depends on a number of factors. It partly depends on the types of behaviors being recorded and the usual length of an interaction. Samples should be long enough to cover entire interactions but separated enough during the day that the behavior in each sample is independent of the previous sample. However, the appropriate length also depends on practical concerns. How long can a single observer observe before mistakes begin to be made? This depends on how many behaviors are being recorded and how many calves are being observed at each location. If three or four calves are being observed on each day, less time can be devoted to each calf than can be when only one calf is being observed. However, the samples must be the same length for all the calves in the study, regardless of the size of their respective social groups. Sample length should therefore be determined based on the practical concerns of the location with the most calves to observe at one time. In this study, ten-minute focal animal samples will be performed at five times spaced throughout the day. Ten minutes is long enough to cover most behavioral interactions of dolphins (Samuels \& Gifford 
1997). Whether five samples adequately represent the behavior of the entire day will be explicitly tested (see section 2.3 ).

\section{What Behaviors to Record}

The final sampling decisions involve what behaviors to record and how to record them. This decision is heavily influenced by where the study is done and what can be seen in each situation. Observations of behavior can be made in much more detail in captivity than in the wild. In captivity, the activities and associations of the animals can be recorded continuously during the entire sample. In the wild, activities and associations can only be recorded when the animals are visible at or near the surface, which is unlikely to be the case throughout the entire sample (Sayigh 1992). If the study is performed in captivity, a continuous record of the activity, associations, and interactions of the animals can be taken. The limitation is how quickly and accurately an observer can record the behaviors, not how many of the behaviors can be seen. Observations that are recorded onto tape or directly into a computer may allow more behaviors to be recorded than hand-written records (see Martin \& Bateson 1986). Commercial computer programs exist which facilitate both the recording and the analysis of the behaviors (e.g. Noldus 1991, Elsberry \& Blackwood 1995). While computers may be problematic when samples are being taken on a small boat at sea, they are very useful for the poolside samples taken in captivity.

The behaviors that are to be recorded need to be carefully defined before sampling starts. Each behavior also has to be classified as either an event, where only the occurrence of the behavior is recorded, or a state, where the duration of the interaction is also recorded. Associations and activities, such as when two animals swim or rest together, should be recorded as states if possible. Other behaviors, such as when one dolphin hits another, occur almost instantaneously and can be recorded as events. Still others, such as rubbing or nursing, occur over short periods of time. With these behaviors, whether the actual time taken or only the occurrence of the behavior is important needs to be decided. In all cases, all parties involved in the interaction must be recorded, in order to be able to distinguish relationships between particular animals. 
When it is appropriate, such as for aggressive behaviors, the directed-ness of the behavior, i.e. who is the sender and who the receiver, should also be recorded.

The Sampling Strategy

In summary, to investigate the possibility of vocal learning in bottlenose dolphins and the social and acoustic influences on whistle development, a longitudinal study of several calves born into several captive groups needs to be performed. The interactions of each mother-calf pair with all the animals in the group should be systematically recorded using focal animal samples of a specified length, starting at predetermined times each day. Samples should be spread out over the entire day to include a representative set of contexts. Carefully defined behaviors should be continuously recorded directly onto a computer during each sample. Because vocal development also requires the analysis of vocalizations, acoustic recordings should be taken simultaneously with the focal samples. Simultaneous acoustic and behavioral recordings will ensure that any biases in the sampling will be the same in the social and acoustic samples and therefore allow the social and acoustic environments to be compared.

\subsubsection{PREVIOUS STUDIES OF DOLPHINS}

The sampling done in studies of free-ranging dolphins has changed considerably in the past few decades. Until recently, research on free-ranging dolphins, and other cetaceans, was largely driven by management considerations and therefore focused on population-level studies (Samuels \& Tyack in press). The research possibilities broadened dramatically in the 1960 s, when scientists discovered they could identify individual animals by natural markings (IWC 1990) or with visible tags placed on temporarily captured dolphins (Irvine et al. 1982). Individual identification was first used with bottlenose dolphins to achieve better estimates of population parameters such as fecundity, life-span, and home ranges (e.g. Wells \& Scott 1990, Irvine et al. 1981). In the process of this, long-term studies were set up to monitor population changes (e.g. Wells 1991). As background information became available on more animals, researchers 
Chapter 2: Sampling Methods

realized that individual identification could also be used to determine the dolphins' social structure by simply recording how often known animals were seen together (e.g. Wells et al. 1987).

While overall population parameters and social structure could be investigated at the same time, studying individual behavior required different sampling strategies. Achieving good estimates of population parameters, or social structure, requires census techniques that sample as many animals as possible (Samuels \& Tyack in press). Studies of individual behavior, on the other hand, require staying with an individual for a long period of time. These two requirements are at odds with each other, which means that studies of individual behavior generally have to be done separately from population-level studies. For a long time, the behavioral studies that were done focused on groups rather than individuals (Mann 1999). The most common sampling strategies were $a d$ lib. and focal group sampling, where the activities of all animals in the group are recorded (Mann 1999, e.g. Shane 1990a). While the behavior of animals in a group can be measured systematically with methods such as scan sampling (Altmann 1974), focal group sampling lacks the rigor of those techniques. Therefore, focal group sampling tends to overestimate noticeable activities, such as foraging, while underestimating subtler activities (Mann 1999).

Focal sampling techniques (Altmann 1974) for studying individual cetacean behavior were first used to study the spatial relationships of right whale mothers and calves (Taber \& Thomas 1982). However, focal studies of free-ranging animals posed some difficulties. In particular, researchers "recognized that the validity of conclusions about the behavior of these animals depended on the quality of the background information" that was available (Wells 1991, p201). In early focal studies, "all too often, [the] known animals interacted with identifiable animals of unknown age or sex" (Wells 1991, p205). Before detailed focal studies could be done, therefore, detailed background information had to be obtained on as much of the population as possible. Since some of the population studies have been ongoing for almost 30 years (e.g. Wells 1991), enough background now exists to be able to perform focal studies on these animals (e.g. Sayigh 
1992, Smolker et al. 1993). Focal-individual sampling of free-ranging dolphins has now been used to investigate a number of topics, including mother-infant separations (Smolker et al. 1993), male alliance formation (Connor et al. 1992), and signature whistle development (Sayigh 1992).

Behavioral sampling of captive dolphins has followed a slightly different path than studies of free-ranging dolphins. Research on captive dolphins began in earnest when Marine Studios was established in 1938 (Samuels \& Tyack in press).

The visibility and accessibility of small cetaceans at aquaria provided opportunities for close-up viewing and hands-on experimentation, thus attracting many scientists to investigate the intricacies of cetacean social behavior, sensory systems, and communication. Early descriptive studies form the basis of much of what is known today about the social behavior of small cetaceans. (Samuels \& Tyack in press, $\mathrm{p} 3$ ).

In the 1950s, successful breeding programs in captive dolphin groups made observations of maternal behavior and calf development possible for the first time (e.g. McBride \& Kritzler 1951, Tavolga \& Essapian 1957). Most of the behavioral studies were qualitative and descriptive (e.g. McBride \& Kritzler 1951, Tavolga \& Essapian 1957), and behaviors were not studied in a systematic manner (see Altmann 1974). Therefore, while these studies gave scientists a good idea of the types of behaviors the dolphins engaged in, the scientists could not describe the relative rates at which the behaviors occurred. Similarly, many subtle behaviors, such as female aggression, were missed by these early descriptive studies (Samuels \& Gifford 1997).

Since that time, studies of behavior have become rarer in captive facilities (Kleiman 1992). One major reason for this is that zoo research is now generally driven by interests in collection management and wildlife conservation (Kleiman 1992). This means that most of the research done in zoos and aquaria is aimed at successful husbandry and breeding, concentrating on genetic and medical studies rather than behavioral ones. The behavioral studies that are done tend to be driven by very specific, applied questions related to successful breeding (e.g. Kastelein et al. 1990). Very recently, however, a few scientists have used captive animals to look at other topics such 
Chapter 2: Sampling Methods

as aggression (Östmann 1991), dominance (Samuels \& Gifford 1997), and even the use of vocalizations (Janik \& Slater 1998). The most recent of these studies have used systematic techniques such as focal animal sampling (e.g. Samuels \& Gifford 1997).

Behavioral studies of dolphins most often involve dolphins from only one location and rarely include both individual behavior and acoustic recordings. Studies in captivity rarely include dolphins from more than one aquarium (but see e.g. McCowan \& Reiss $1995 a, b)$. One reason for this is that many studies are done by aquarium staff on the animals at their facility, generally in order to increase breeding success or survival (e.g. Kastelein et al. 1990). For outside scientists, studies at multiple locations can also be problematic because getting permission and sufficient funding to work with the animals at multiple locations can be difficult. Studies of free-ranging dolphins at multiple locations are equally rare (but see e.g. Shane 1990b). Instead, the researchers are often involved in all the aspects of a long-term study at a specific location, including studying both population parameters and individual behavior (e.g. Wells 1991). Few studies include both individual behavior and acoustic recordings. The difficulty in identifying which animal is vocalizing may discourage some researchers (see section 1.6). In spite of this difficulty, a few scientists have combined systematic behavioral and acoustic recordings to investigate topics such as social influences on signature whistle development (Sayigh 1992), the contextual use of vocalizations (McCowan \& Reiss 1995b, Janik \& Slater 1998), and whistle convergence in male alliances (Smolker \& Pepper in press). Nonetheless, quantitative, longitudinal studies of individual behavior and vocalizations at multiple locations remain rare among studies of bottlenose dolphins. This type of study is necessary to investigate issues such as vocal learning.

\subsection{THE PILOT STUDY}

To test the sampling strategy laid out in section 2.1.1, a study was performed with calves born in one captive group. This study will also provide sample data for the development of analysis techniques in later chapters. The study was conducted at Kolmårdens Djurpark, just outside Norrköping, Sweden, from March 1 through August 
12, 1995. Four calves were born during the study, three of whom died at 6 to 10 days of age (see Table 2.1). The study continued until the surviving calf was 10 weeks old.

Although it was unlucky for this study, the $75 \%$ calf mortality that occurred during this study does not represent the overall calf survival for this dolphinarium. Nine calves were born at Kolmårdens Djurpark between 1994 and 1998. This includes one calf born before this study began. Four of these were still alive in 1998. DeMaster and Drevenak (1988) calculate the annual survival rate (ASR) of animals based on the number of animals that have died weighted by the number of days animals have survived. The ASR of calves younger than one year born at 57 captive institutions between 1975 and 1984 was 0.6 (DeMaster \& Drevenak 1988). The ASR of calves born at Kolmårdens Djurpark between 1994 and 1998 is actually 0.7. Wells \& Scott (1990) calculated the ASR of wild dolphins calves in Sarasota Bay, Florida at 0.8 between 1980 and 1987. While this appears greater than captive dolphin calves, wild studies may miss calves that die younger than a few weeks old because these calves may never be seen by the researchers. If calves had to survive more than seven days to be counted, the ASR of calves born at Kolmårdens Djurpark would be 0.85 .

\subsubsection{STUDY SITE: KOLMÅRDENS DJURPARK}

\section{Dolphinarium Setup}

The dolphinarium at the Kolmårdens Djurpark consisted of three pools separated by gates (Figure 2.1). These gates provided barriers to movement but not to vision or sound. There were two main pools, the Lagoon and the Show Pool, which were connected by a smaller Holding Pool. The Lagoon was a $2800 \mathrm{~m}^{3}$ pool with two contiguous sections, one three meters deep and one six meters deep. All the dolphins in this study were housed in the Lagoon, and all four calves were born there. In front of the six-meter deep section was a wall of windows allowing public viewing (Figure 2.1). These windows gave observers a clear view of all the behaviors and interactions of the animals in the Lagoon. 


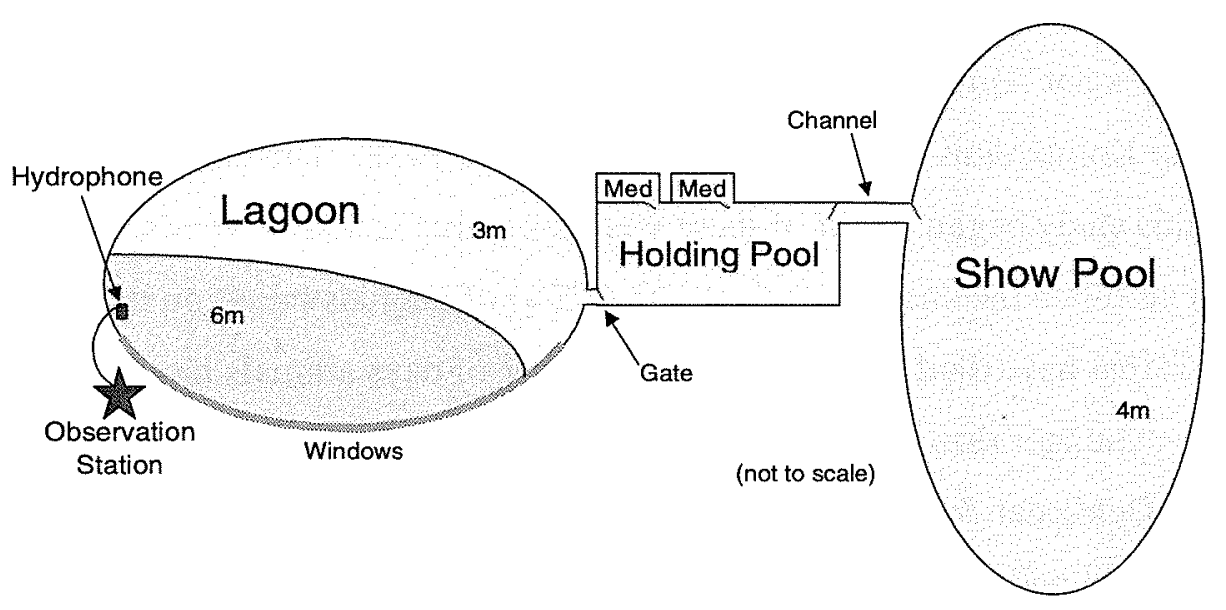

The Show Pool was a second large pool with a uniform depth of 4 meters, where most of the non-breeding animals were housed. Public shows were held there, and the animals were trained almost continually. Shows could be clearly heard in the Lagoon, both in the air and through the water. The two large pools were connected by a smaller Holding Pool, which had two small medical pools attached to it (Figure 2.1). The Lagoon was connected to the Holding Pool by a single gate, which was sometimes open before the calves were born, but was always closed to the calves. The connection between the Show Pool and the Holding Pool was a channel that could be gated at either end but was often left open. The two ends of the Holding Pool were never open at the same time, however. For part of the study, animals were housed in the Holding Pool with both ends closed (see Table 2.1).

\section{The Study Population}

Four females and their calves housed in the Lagoon were the subjects of this study. Table 2.1 shows the dolphins that were in the Lagoon at any time during the study. Four other animals (three females, one male) were in the Show Pool for the 
Table 2.1: Study Population

Highlighted cells indicate focal animals.

\begin{tabular}{|c|c|c|c|c|}
\hline Animal & Sex & Age/Birth Date & Mother & Location \\
\hline Nephele & $\mathrm{F}$ & $\begin{array}{r}10 \text { years } \\
\text { b. } 1985\end{array}$ & & $\begin{array}{l}\text { Lagoon: through May } 22 \\
\text { Holding: May } 22 \text { to August } 8 \\
\text { Lagoon: August } 8 \text { to August } 12\end{array}$ \\
\hline Nephele's calf & $\mathbf{M}$ & $\begin{array}{r}\text { born April 25, } 1995 \\
\text { died May 1, } 1995\end{array}$ & Nephele & Lagoon: April 25 to May 1 \\
\hline Vicky & $\mathrm{F}$ & $\begin{array}{l}21 \text { years } \\
\text { b. } 1974\end{array}$ & & $\begin{array}{l}\text { Show: through March } 6 \\
\text { Lagoon: March } 6 \text { to August } 12\end{array}$ \\
\hline Vicky's calf & $M$ & $\begin{array}{l}\text { born May } 22,1995 \\
\text { died May 30, } 1995 \\
\end{array}$ & Vicky & $\begin{array}{r}\text { Lagoon: May } 22 \text { to May } 30 \\
\text { dead body to June } 3 \\
\end{array}$ \\
\hline Delphi & $\mathrm{F}$ & $\begin{array}{l}10 \text { years } \\
\text { b. } 1985\end{array}$ & & $\begin{array}{l}\text { Lagoon: through May } 22 \text {, and } \\
\text { May } 24 \text { to June } 8 \\
\text { Holding: May } 22 \text { to } 24, \text { June } 8 \text { to Aug. } 12\end{array}$ \\
\hline Delphi's calf & $\mathrm{M}$ & $\begin{array}{r}\text { born May 29, } 1995 \\
\text { died June 7, } 1995 \\
\end{array}$ & Delphi & $\begin{array}{r}\text { Lagoon: May } 29 \text { to June } 7 \\
\text { dead body to June } 8\end{array}$ \\
\hline Lotty & $F$ & $\begin{array}{c}13 \text { years } \\
\text { b. } 1982 \\
\end{array}$ & & Lagoon: March 1 to August 12 \\
\hline Lotus & $\mathrm{M}$ & born June 4, 1995 & Lotty & $\begin{array}{l}\text { Lagoon: June } 4 \text { to August } 12 \\
\text { except June 9: alone in small pool }\end{array}$ \\
\hline Sharky & $\mathrm{F}$ & $\begin{array}{l}13 \text { years } \\
\text { b. } 1982\end{array}$ & & $\begin{array}{l}\text { Lagoon: through April } 25 \\
\text { Show: April } 25 \text { to August } 12\end{array}$ \\
\hline Daphne & $F$ & $\begin{array}{l}7 \text { months } \\
\text { b. Nov. 1, } 1994\end{array}$ & Sharky & $\begin{array}{l}\text { Lagoon: through April } 25 \\
\text { Show: April } 25 \text { to August } 12\end{array}$ \\
\hline Vindy & $\mathrm{F}$ & $\begin{array}{l}4 \text { years, b. } 1991 \\
\text { died July } 13,1995\end{array}$ & Vicky & $\begin{array}{l}\text { Show/Holding: through July } 10 \\
\text { Lagoon: July } 10 \text { to } 13\end{array}$ \\
\hline
\end{tabular}

duration of the study. Four females gave birth during this period: Nephele, Vicky, Delphi, and Lotty. They and their calves were the study subjects. Sharky and her sevenmonth-old daughter, Daphne, were present for part of the time but were not study subjects. Since the first three calves born in this study died before they were two weeks old, they were never named. They will be referred to here as their mother's calf (e.g. Nephele's calf). With the exception Daphne, who was not a focal subject, all the calves were male and all the adults were female, so calves are referred to as "he" and adults as "she" throughout this thesis. Only one of the four calves, Nephele's, was healthy during his first week. The behavior of the other three may have been influenced by the fact that they all became very sick. During the first two weeks of each calf's life, the public was not allowed into the viewing area around the Lagoon. 
The first calf was born in April and the other three were born within two weeks of each other in late May. Nephele's calf was born on April 25, 1995 (Table 2.1). He was healthy for six days but died in an aggressive encounter between Nephele and Delphi on May 1. Sharky and Daphne were moved out of the Lagoon early on the day Nephele's calf was born. Vicky's calf was born on May 22, 1995 (Table 2.1). He lived for eight days before he died of an acute infectious disease on May 29. Delphi and Nephele were in the Lagoon when Vicky's calf was born but were taken out a few hours later. Delphi was returned to the Lagoon two days later, but Nephele remained in the Holding Pool until shortly before the end of the study in August. Delphi's calf was born on the afternoon of May 29, 1995, shortly before Vicky's calf died. He lived for nine days before dying of an acute infectious disease on June 7 .

Lotty's calf, Lotus, was the only calf to survive his first two weeks, but his first week was rather unusual. Lotus was born to Lotty on June 4, 1995 (Table 2.1). As soon as Lotus was born, Vicky swam up to him. Before Lotty could turn around, Lotus had gone to the surface with Vicky. Lotus appeared to be riding next to Vicky's dorsal fin without swimming, a behavior common to neonatal dolphins (Cockroft \& Ross 1990, Norris \& Dohl 1980). Lotty made no obvious attempt to reclaim him, and Lotus remained with Vicky for the next five days. Vicky appeared to still be lactating, having lost her own calf only six days earlier. Delphi's calf died when Lotus was four days old (Table 2.1). During his fifth day, Lotus got very sick, and at the end of that day, the trainers at the Djurpark separated him from the adults and held him alone in a small pool, not connected to the other pools. They kept him in that pool until the evening of his sixth day, giving him antibiotics, antibodies (IgG previously taken from his father), and iron (Dextran: Fe 3+), as well as feeding him several times during the day. That evening, they returned Lotus to the Lagoon with only Vicky in it. Lotty was being kept in the Holding Pool at the time but was allowed back into the Lagoon a few hours later when Vicky began to ignore Lotus. From that time on, Lotus remained primarily with Lotty, although Vicky was in the Lagoon with them. This arrangement remained until July 10 when Vindy, Vicky's four year old daughter who had been ill for several days, was moved into 
the Lagoon. Vindy died three days later. After this, Lotus remained in the Lagoon with only Vicky and Lotty until Nephele was introduced on August 8. Nephele remained in the Lagoon with Lotus, Vicky, and Lotty through the end of the study. The study was concluded when Lotus was ten weeks old, on August 12, 1995.

\subsubsection{FOCAL SAMPLES}

Based on the sampling decisions discussed in section 2.1.1, focal animal samples (Altmann 1974) were performed daily on the pregnant females for 8 to 13 weeks before the calves were born, and on the mother-calf pairs for up to 10 weeks after the calves were born (Table 2.2). The calves that died were sampled approximately daily for their entire lives. Focal samples were ten minutes long and spaced throughout the day, starting at approximately 9 a.m., 10:30 a.m., 1:30 p.m., 4:10 p.m., and 6 p.m. When more than one animal was being observed, each session consisted of a sequence of ten-minute samples, one on each focal animal. To ensure that the samples for different focal animals were comparable, animals were observed in the same order for all five sessions on each day and this order was rotated daily. When samples were only being collected on only one animal, those samples were begun at the specified times on every day. Simultaneous acoustic recordings from a hydrophone in the Lagoon were made during all the focal sessions.

The observation times were chosen to be representative of the different contexts the animals experienced, including before their first feeding of the day, during feeding/training sessions, during shows in the Show Pool, with and without people in the observation area, and after the trainers had left for the day. There were no shows in the Lagoon during this study, but training sessions were held there every day at erratic times. Since the sampling times were chosen to represent the different contexts experienced by the dolphins, the time observed in each context may not be weighted properly in proportion to the actual time spent in those contexts. However, the timing of conditions such as training and people in the observation area varied enough from day to day that the 
Chapter 2: Sampling Methods

TAble 2.2: Number of 10-Minute SAMPLes on EACH Focal ANimal When the calves were alive, the focal animal was the calf.

\begin{tabular}{|c|l|c|c|}
\hline Focal Adult & Time period & Samples & Days \\
\hline \multirow{2}{*}{ Nephele } & Before Calf Born & 167 & 43 \\
\cline { 2 - 4 } & Nephele's Calf & 27 & 6 \\
\hline \multirow{2}{*}{ Vicky } & Before Calf Born & 252 & 60 \\
\cline { 2 - 4 } & Vicky's Calf & 35 & 7 \\
\hline \multirow{2}{*}{ Lelphi } & Before Calf Born & 316 & 74 \\
\cline { 2 - 4 } & Delphi's Calf & 41 & 9 \\
\hline & Before Calf Born & 338 & 79 \\
\cline { 2 - 4 } & Lotus' First Week & 22 & 5 \\
\cline { 2 - 4 } & Lotus & 298 & 60 \\
\hline
\end{tabular}

†During Lotus' first week, Lotus was swimming with Vicky and focal samples were taken on Lotty separately from Lotus.

chosen times likely covered the actual time spent in each context better than expected. Additionally, no night sessions were conducted. However, for the first two weeks after each birth, the dolphins were observed by the staff 24 hours a day. The dolphins' association patterns did not change obviously during the night on those days that I performed the overnight observations (personal observation). The choices of the times of day sampled and the number of samples taken per day were tested on several days (section 2.3).

Dolphins were observed from a station set up by a corner of the Lagoon next to one of the viewing windows (the star in Figure 2.1). Several cameras and monitors were set up to allow viewing of areas not visible from the station so that, with the exception of a very small area, the entire Lagoon could be seen from the station. Behavioral observations were recorded using The Observer 3.0 (Noldus) on an IBM Thinkpad 755Cs. Examples of the Observer configurations and data files can be found in Appendix 1. The hydrophone was placed in a corner of the pool near the observation station, where the dolphins could not reach it (see Figure 2.1). Recordings were made using an HTI hydrophone and a Radio Design Labs STM2 preamplifier, onto one channel of a Panasonic VHS, PAL-format, stereo VCR. The second channel was used for voice comments. 


\section{Behaviors Recorded}

Several types of behaviors need to be recorded to determine the social relationships between animals. First, the amount of time the two animals spend together must be recorded. At the same time, the activity the animals are engaged in while spending time together may be important to the type of relationship they have. Both the occurrence of an association and the amount of time the dolphins spend in a particular activity are important. Therefore, the associations and activity of the focal animals should be recorded as "states", where both the start time and the duration of the behavior are recorded. In this way, the proportion of time that the focal spends with another dolphin can be determined. The social interactions between dolphins are an important part of their relationship as well. These interactions include agonistic interactions made up of aggressive and submissive displays, and affiliative interactions made up of gentle touches. Each behavior within such an interaction can be recorded as an "event", where only the occurrence of the behavior is important. The duration of the behaviors in such interactions is likely to be less important to the dolphins' relationship. Behaviors performed by the focal animal when the focal is alone are less likely to directly impact the focal's relationship with other dolphins and therefore do no need to be recorded.

\section{$\underline{\text { States }}$}

The associations and activities of the focals were recorded continuously as states, so both the occurrence and the duration of these behaviors were recorded. Activities are listed and defined in Table 2.3. Two animals were recorded as associates, and defined as "neighbors", if they swam or rested in the same direction within a meter of each other. A chain rule was applied to this so that an animal swimming within a meter of an animal within a meter of the focal was defined as the focal's neighbor as well. When a calf was swimming with other animals, the closest dolphin in the group to the calf by distance was defined as his "nearest neighbor". The position of the calf relative to his nearest neighbor was also recorded (Table 2.4), as was his distance to his mother if she was not his nearest neighbor. If he was swimming with only one other animal, she was automatically his nearest neighbor. If he was swimming equidistant between two animals, they were both 
TABLE 2.3: ACTIVITIES

\begin{tabular}{|l|l|}
\hline Activity & Definition \\
\hline Alone & Swim or rest more than a meter away from any other animal. \\
\hline Swim Together & $\begin{array}{l}\text { Swim within one meter of another animal in the same direction, } \\
\text { chain rule applies. }\end{array}$ \\
\hline Rest Together & Rest within one meter of another animal, chain rule applies. \\
\hline Socialize & $\begin{array}{l}\text { Interact with another animal while not swimming or resting, } \\
\text { generally agonistic interactions. }\end{array}$ \\
\hline Train & $\begin{array}{l}\text { Interact with trainer in training setting, adults only, } \\
\text { considered neighbors if both animals are trained by one trainer. }\end{array}$ \\
\hline
\end{tabular}

Table 2.4: Calf Positions

\begin{tabular}{|l|l|}
\hline Position & Definition \\
\hline Next To & Swim by the side or dorsal fin, between pectorals and peduncle. \\
\hline Slipstream & $\begin{array}{l}\text { "Next to" with flukes not beating, receiving hydrodynamic lift } \\
\text { from the adult and not expending energy. }\end{array}$ \\
\hline Under & $\begin{array}{l}\text { Underneath the adult, generally beneath the mammary region } \\
\text { but can be as far forward as between the pectorals. }\end{array}$ \\
\hline In Front Of & Forward of the pectoral fins or around the head. \\
\hline Behind & Behind the peduncle or around the tail. \\
\hline
\end{tabular}

considered his nearest neighbors, although this occurred infrequently. If he was swimming more than a meter away from any other animal, he was considered to be "alone" and to have no nearest neighbor. The distance from the calf to his mother was recorded at these times, except for Lotus in his first five days the distance to Vicky, not Lotty, was recorded. Distances were estimated by eye. A new code was entered every time there was a change in any of the measures, and the time of occurrence of the change was automatically recorded by the program. The amount of time spent in each activity or association was calculated by the program from the recorded times.

\section{$\underline{\underline{E v e n t s}}$}

All behaviors that occurred during interactions between the focal animals and the other animals in the Lagoon were recorded continuously. The time of occurrence of these behavioral events was automatically recorded by the program when the code was entered. The behavioral events in this ethogram are listed and defined in Table 2.5. They 
were separated into three categories: affiliative contact (Table 2.5A), agonistic interactions (Table 2.5B), and calf-related behaviors (Table 2.5C).

The affiliative behaviors all involve gentle contact between two animals, including rubs, touches, and swimming in contact, which appear to be affiliative in nature (Mann \& Smuts in prep). Each discrete touch by one animal to a second animal was recorded as a separate event. The duration of these behaviors was not marked but rubs and nuzzles usually lasted one to three seconds and contact swims two to ten seconds (personal observation). In rare cases, these behaviors were prolonged, lasting more than ten seconds, but these cases made up less than $1 \%$ of the total. The body parts involved in all affiliative interactions were recorded. Because which dolphin initiated affiliative contact often could not be determined, affiliative behaviors were not considered to be directed from one dolphin to another. Mount and nuzzle were exceptions to this, however. The dolphin initiating both mount and nuzzle could generally be determined and was recorded.

Agonistic interactions involve behaviors known to be either aggressive or submissive in nature (Table 2.5B, Samuels \& Gifford 1997). Of the behaviors recorded, only flee and flinch were submissive behaviors. The rest were aggressive, consisting mostly of threats. Aggressive physical contact, such as hitting or biting, was never observed in this study. Although every attempt was made to record the specific behaviors, the rapid combination of many behaviors often overwhelmed my ability to record the individual behaviors. Therefore, "general threat" was recorded when an animal was making multiple threat behaviors too quickly for each behavior to be recorded separately (Table 2.5B) . Because the specific behaviors were not recorded in every instance, agonistic behaviors were analyzed as complete interactions rather than as individual behaviors. Unlike affiliative contact, agonistic behaviors were directed from one dolphin to a second and the initiator of each behavior was recorded. When both dolphins threatened each other simultaneously, the threat was recorded as "mutual" (Table 2.5B). Although most interactions occurred when the dolphins were within a few 
Chapter 2: Sampling Methods

TABle 2.5: Behavioral Events

A: AFFiliative

Note: In all cases, the body parts involved were recorded.

\begin{tabular}{|l|l|}
\hline Behavior & Definition (A = actor, $\mathbf{R}=$ recipient) \\
\hline Rub & Gentle moving contact between dolphins. \\
\hline Contact Swim & Stationary contact while swimming. \\
\hline Casual Touch & Brief contact between dolphins. \\
\hline Nuzzle & Rub of A's rostrum to any part of R's body. \\
\hline Mount & $\begin{array}{l}\text { Rub or casual touch, sometimes with an erection, of A's genital } \\
\text { or ventral area to any part of } \mathbf{R} \text { 's body, often accompanied by } \\
\text { some thrusting action. }\end{array}$ \\
\hline
\end{tabular}

B: AgONISTIC

\begin{tabular}{|c|c|c|}
\hline Type & Behavior & Definition $(A=$ actor, $R=$ recipient $)$ \\
\hline \multirow[t]{7}{*}{ Aggressive } & Head Jerk & Sharp nod of A's head in R's direction. \\
\hline & Jaw Clap & $\begin{array}{l}\text { Sharp closing of A's jaws in R's direction while } \\
\text { A produces a loud sound. }\end{array}$ \\
\hline & Mouth Open & $\begin{array}{l}\text { Opening of A's the mouth widely in } \mathbf{R} \text { 's } \\
\text { direction. }\end{array}$ \\
\hline & General Threat & $\begin{array}{l}\text { A combination of the threats listed above, } \\
\text { usually involving thrashing of the body, burst- } \\
\text { pulse sounds and bubble-blowing. }\end{array}$ \\
\hline & Distant Threat & General threat from a distance of more than $3 \mathrm{~m}$ \\
\hline & $\begin{array}{l}\text { Mutual Threat (or } \\
\text { Distant Threat) }\end{array}$ & $\begin{array}{l}\text { General (or distant) threat made simultaneously } \\
\text { by } \mathbf{A} \text { and } \mathbf{R} \text {. }\end{array}$ \\
\hline & Chase & A swimming after $\mathbf{R}$ quickly. \\
\hline \multirow[t]{2}{*}{ Submissive } & Flee & A swimming away from $\mathbf{R}$ quickly. \\
\hline & Flinch & $\begin{array}{l}\text { A pulling sharply away from } \mathbf{R} \text {, in response to a } \\
\text { threat or to } \mathbf{R} \text { swimming by. }\end{array}$ \\
\hline
\end{tabular}

C: Calf-Related

\begin{tabular}{|l|l|}
\hline Behavior & Definition ( $\mathbf{A}=$ actor, $\mathbf{R}=$ recipient) \\
\hline $\begin{array}{l}\text { Nurse } \\
\text { (calves) }\end{array}$ & $\begin{array}{l}\text { Suckling from mammary region, calves only. These are actual } \\
\text { suckles, not suckling bouts, and may occur several times in a row. }\end{array}$ \\
\hline $\begin{array}{l}\text { Retrieve } \\
\text { (adults) }\end{array}$ & $\begin{array}{l}\text { Swimming up to a calf who is alone and beginning to swim with him, } \\
\text { adults only. This is different from a simple approach. }\end{array}$ \\
\hline
\end{tabular}

meters of each other, the recipients of threats made from a greater distance could still be clearly determined. These threats were recorded as "distant threats" (Table 2.5B).

The final behavior group consisted of two behaviors that specifically involve calves (Table 2.5C). Nursing was recorded as a discrete event each time a calf locked on 
to an adult's mammary. Unsuccessful attempts to lock on were also recorded. The duration of suckling was not recorded but was on the order of 5 to 10 seconds in most cases (personal observation). A retrieve serves to return a wayward calf to the apparent safety of swimming with an adult. It usually consists of an adult forcing the calf to come along with her, either by swimming past him quickly or by herding him in a certain direction. This was only performed by adults toward calves and was distinct from a simple approach (see Appendix 1).

\section{Data Analysis}

Behavioral events were analyzed in several ways to determine the rate per minute of each behavior type in each focal sample. The two calf-related behaviors, nurse and retrieve, were analyzed separately. Affiliative and agonistic behaviors were analyzed by category, not by individual behavior type. All affiliative behaviors (see Table 2.5A) between each pair of animals were added together to determine the total affiliative contact between the animals. The total number of occurrences of affiliative behaviors, nursing, and retrieves was calculated for each sample (The Observer 3.0, Noldus). Agonistic contact between animals was analyzed as agonistic interactions rather than separate behaviors. This was done because the total number of individual behaviors could not be determined when a "general threat" was recorded. An interaction began with the first recording of an agonistic behavior (see Table $2.5 \mathrm{~B}$ ) and ended when the focal was recorded as alone or as interacting with a different animal. The next recording of an agonistic behavior, which could be a distant threat, was considered the beginning of a new interaction. The total number of interactions was calculated manually. The rate per minute of nursing, retrieves, affiliative behaviors, and agonistic interactions in each sample was calculated by dividing the total number of occurrences of each behavior type by the total time observed in that sample (Excel 5.0 for Windows, Microsoft). The total time observed was calculated from the total time spent observing (generally 10 minutes) minus the time the focal animal could not be seen from the observation station (the Observer). Loss of the focal occurred infrequently, generally for only a few seconds at a time, and was usually caused by poor water clarity. 
Chapter 2: Sampling Methods

The time spent in each behavioral state was expressed as a proportion of the total time and as the number of minutes per sample. The amount of time each focal associated with each dolphin in the Lagoon was summed across all activities and other neighbors (The Observer, Excel). This was expressed as a percent of the total time observed for each sample. For the calves, the amount of time the calf spent with each adult as his nearest neighbor was summed across calf positions in minutes for each sample (The Observer, Excel). The total number of minutes each calf spent with each nearest neighbor was separated into time when other adults were also swimming with them and time when no other adults were with them. The rate per sample of each of these was then calculated in minutes divided by the total time observed. Statistical analysis of all behaviors was done in Excel, Matlab 4.2 for Windows (Mathworks), and Systat 7.0 for Windows (SPSS). The analysis of the acoustic data is discussed in chapter 4.

\subsection{TEST OF THE SAMPLING METHODS}

On several days during the course of the study, samples were taken at short regular intervals throughout the day, starting in the early morning and continuing into the evening. These were done to test whether the five samples taken at the standard times (see above) were sufficient to represent the entire day, under the assumption that the complete test represented the day adequately. Four tests were performed (Table 2.6), two before the calves were born (pre-calf), and two after (post-calf). The sampling protocols were the same as on the non-testing days (section 2.2.2). The first test was on the day before Vicky was moved into the Lagoon and therefore did not include Vicky. The second test included all four adults and therefore consisted of the fewest sessions. The third test was the most extensive one, performed when Lotus was six weeks old and consisting of 30 samples. A fourth test was performed when Lotus was nine weeks old, the day before Nephele was re-introduced into the Lagoon. 
Table 2.6: Tests of The Sampling Method

\begin{tabular}{|c|c|c|c|c|c|}
\hline Date & $\begin{array}{c}\text { Samples } \\
\text { per Focal }\end{array}$ & Focals & $\begin{array}{c}\text { Start } \\
\text { Time }\end{array}$ & $\begin{array}{c}\text { End } \\
\text { Time }\end{array}$ & $\begin{array}{c}\text { Approximate } \\
\text { Interval }\end{array}$ \\
\hline March 5 & 11 & Delphi, Nephele, Lotty & $8 \mathrm{am}$ & $5 \mathrm{pm}$ & $1 \mathrm{hour}$ \\
\hline March 25 & 9 & Delphi, Nephele, Lotty, Vicky & $7: 30 \mathrm{am}$ & $5 \mathrm{pm}$ & $40 \mathrm{~min}$. \\
\hline July 20 & 30 & Lotus & $6: 45 \mathrm{am}$ & $9: 15 \mathrm{pm}$ & $30 \mathrm{~min}$. \\
\hline August 7 & 15 & Lotus & $8: 15 \mathrm{am}$ & $7 \mathrm{pm}$ & $45 \mathrm{~min}$. \\
\hline
\end{tabular}

\subsubsection{ANALYSIS}

\section{Measures}

Each of the behavior types listed above were tested: nursing, retrieves, affiliative contact, agonistic interactions, total association, and the time as the calf's nearest neighbor. Agonistic interactions were only used in the pre-calf tests because agonism around calves was rare. Calf-related behaviors and time as the calf's nearest neighbor were only used in the post-calf tests. As before, time as the calf's nearest neighbor was separated into with and without other adults present. Each measure was calculated separately for each sample of the testing days. The four testing days were analyzed separately.

\section{Random Groups of Samples}

To compare the results of using different numbers of samples and samples taken at different times of day, random groups of samples were generated (Figure 2.2A; Appendix 2). Random sets of samples can be used to determine how the results of taking a certain number of samples depended on what time of day the samples were taken. Each measure was analyzed separately. First, the measure was calculated for each sample on the testing day (Figure 2.2A: 1). Then, $\mathbf{M}$ samples were randomly chosen from the total of $\mathbf{N}$ samples taken on that day, for all $\mathbf{M}$ from 1 to $\mathbf{N}$ (Figure 2.2A: 2). For each $\mathbf{M}$, 1000 such random groups were generated (Figure 2.2A: 3). The mean of the behavioral measure was calculated for each group (Figure 2.2A: 4). These means formed a distribution of group means for each value of $\mathbf{M}$ (Figure 2.2A: 5). 


\section{Chapter 2: Sampling Methods}

\section{FIgURE 2.2: ANALYSIS OF ONE BEHAVIORAL MEASURE ON ONE TEST DAY}

A total of $\mathbf{N}$ samples were taken in this test.

Note: Normal distribution curves are shown here for demonstration purposes only. The distributions in this study were not normal. Rather, they were skewed toward 0 because negative numbers were impossible.

A. Generation of random groups of samples:

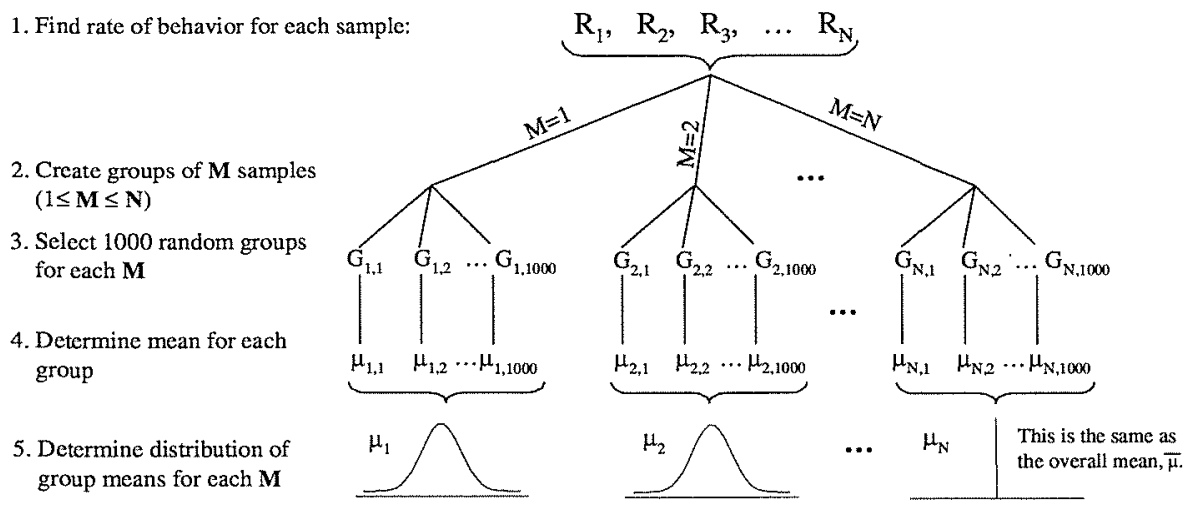

B. Characterization of the distribution of group means for $\mathbf{M}$ samples:

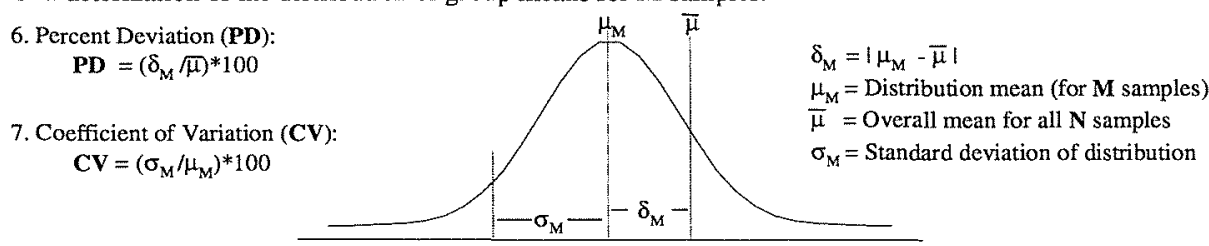

C. Evaluation of the standard 5 samples:

8. Take all groups from $M=5$ samples

9. Determine the mean $(\mu)$ for each group

10. Determine the mean deviation $(\delta)$ of each group from the overall mean (ii)

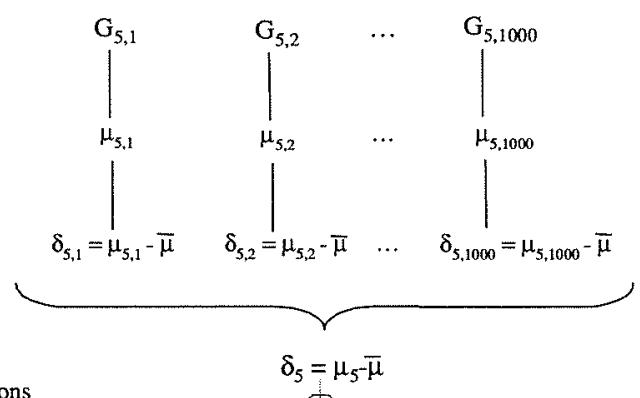

11. Determine the distribution of the mean deviations

12. Determine mean deviation for the five standard samples, $\mathrm{d}_{5}=\left|\mathrm{m}_{5} \bar{\mu}\right| \quad\left(\mathrm{m}_{5}\right.$ is the mean for the standard samples $)$

13. Determine the proportion (P) of groups with mean deviations $(\delta)$ whose absolute value is greater than $\mathrm{d}_{\mathrm{s}}$ 


\section{Number of Samples}

To compare the results of using each $\mathbf{M}$ number of samples, the distributions of group means were characterized and compared. Two statistics were used: the percent deviation (PD) and the coefficient of variation (CV) (Figure 2.2B). The percent deviation (PD) is a measure of how different the mean of the distribution is from the true mean, assumed to be equivalent to the mean of using all $\mathbf{N}$ samples. The $\mathbf{P D}$ is therefore the difference between the distribution mean, $\mu_{\mathrm{M}}$, and the overall mean, $\bar{\mu}$, as a percent of the overall mean (Figure 2.2B: 6). PD's were considered to be non-significant if they were less than $5 \%$.

The coefficient of variation $(\mathbf{C V})$ is a measure of the width of the distribution. It is calculated by expressing the standard deviation as a percent of the mean (Figure 2.2B: 7). In this way, the standard deviations of two distributions can be compared. If these distributions had been normal, which they were not, one standard deviation would encompass $68 \%$ of the cases (Chapra \& Canale 1988). This means that $32 \%$ of the cases would be more than one standard deviation away from the mean. If one standard deviation is $100 \%$ of the mean, or the $\mathrm{CV}$ is $100 \%$, in a normal, and therefore symmetric, distribution, $16 \%$ of the cases would be at least twice the mean and $16 \%$ would be zero or less. The distributions in this test were not normal, partly because negative values are impossible. However, if the $\mathbf{C V}$ is $100 \%$, a reasonable proportion of the values will still be either zero or twice the mean. Similarly, if the distribution were normal, $95 \%$ of the cases would be within two standard deviations of the mean (Chapra \& Canale 1988). If $100 \%$ of the mean was two standard deviations, rather than one, only $5 \%$ of the cases would be zero or twice the mean. This proportion can be considered insignificant. Therefore, $\mathbf{C V}$ 's were considered acceptably small if they were $\mathbf{5 0 \%}$ or less, so that only a small proportion of the cases would be either twice the mean or zero. If the CV was less than $50 \%$, two standard deviations were less than $100 \%$ of the mean. For each testing day, the $\mathbf{P D}$ and $\mathbf{C V}$ for each number of samples (M) were averaged over all the behavioral measures taken on that day. This allowed the different numbers of samples to be compared on all the test days. 
Chapter 2: Sampling Methods

\section{Sampling Times}

To determine whether the five times used on all other days (see above) were representative of the entire day, the result of using those five samples was compared to the results from using any other five samples from each testing day. For this purpose, a mean deviation, $\delta$, was calculated for each random group of five samples (Figure 2.2C, see Appendix 2). The mean deviation was calculated by subtracting the overall mean from the group mean (Figure 2.2C: 8-10). A distribution of mean deviations was calculated for each measure from the 1000 randomly selected sample groups of five samples ( $M=5$, Figure 2.2C: 11). The mean deviation of the five standard times was then compared to this distribution $\left(\mathrm{d}_{5}\right.$, Figure $\left.2.2 \mathrm{C}: 12\right)$. A p-value was calculated based the proportion of groups with a greater absolute value of the mean deviation than the standard sample (Figure 2.2C: 13; Appendix 2).

\subsubsection{RESULTS}

\section{Number of Samples}

The average percent deviation from the overall mean (PD) and the average coefficient of variation $(\mathbf{C V})$ both decreased as the number of samples used increased (Table 2.7). For the post-calf tests, the PD was never greater than 5\%, suggesting that almost any number of samples was representative of the whole day on average. The measures for the pre-calf tests were more variable than for the post-calf tests (Table 2.7). For the pre-calf tests, the PD was greater than 5\% when only one or two samples were used on March 5. The coefficient of variation (CV) for one sample was more than $100 \%$ in all cases. The CV for one sample in the pre-calf tests was almost $200 \%$ and for two samples was still more than $100 \%$. This indicates that the variability of one or two samples is too great to get an accurate measurement of the behaviors that occur. For the post-calf tests, five samples brought the $\mathbf{C V}$ to approximately 50\% (Table 2.7). For the pre-calf tests, six or seven samples were needed. To cut the $\mathbf{C V}$ in half, to $25 \%$, eight to ten samples needed to be taken in the pre-calf tests and ten to fifteen in the post-calf tests. This difference may partly be caused by the small number of samples taken in the two 
Table 2.7: Results of Methods Test, Number of Samples: Average Coefficient of Variation and Percent Deviation for All Measures Standard number of samples (5) is highlighted. Pre-calf: March 5 and March 25. Post-calf: July 20 and Aug 7.

\begin{tabular}{|c|c|c|c|c|c|c|c|c|}
\hline \multirow{2}{*}{$\begin{array}{c}\text { No. of } \\
\text { Samples }\end{array}$} & \multicolumn{4}{|c|}{ Average Percent Deviation (PD) } & \multicolumn{4}{|c|}{ Average Coefficient of Variation (CV) } \\
\hline & March 5 & March 25 & July 20 & August 7 & March 5 & March 25 & July 20 & August 7 \\
\hline 1 & 6.19 & $\overline{4.70}$ & 2.55 & 1.83 & 211.36 & 196.34 & 124.99 & 135.31 \\
\hline 2 & 5.47 & 3.60 & 2.67 & 2.03 & 146.90 & 129.92 & 87.40 & 91.82 \\
\hline 3 & 2.15 & 2.35 & 2.52 & 1.19 & 110.67 & 97.90 & 69.24 & 72.11 \\
\hline 4 & 2.45 & 1.84 & 1.91 & 1.60 & 89.34 & 76.80 & 59.58 & 58.77 \\
\hline 5 & 2.11 & 1.50 & 1.11 & 1.36 & 73.35 & 61.88 & 51.91 & 50.87 \\
\hline 6 & 1.79 & 1.25 & 1.36 & 1.33 & 60.99 & 48.73 & 46.35 & 43.73 \\
\hline 7 & 1.27 & 0.87 & 1.17 & 0.95 & 51.81 & 36.81 & 42.43 & 38.75 \\
\hline 8 & 0.98 & 0.59 & 0.85 & 0.49 & 41.19 & 24.32 & 38.87 & 33.56 \\
\hline 9 & 0.86 & 0.00 & 1.07 & 0.51 & 31.89 & 0.00 & 35.88 & 29.15 \\
\hline 10 & 0.58 & & 0.58 & 0.41 & 21.14 & & 32.86 & 25.41 \\
\hline 11 & 0.00 & & 0.91 & 0.60 & 0.00 & & 30.61 & 21.56 \\
\hline 12 & & & 0.71 & 0.45 & & & 28.18 & 17.99 \\
\hline 13 & & & 0.50 & 0.44 & & & 26.48 & 13.88 \\
\hline 14 & & & 0.48 & 0.22 & & & 25.22 & 9.38 \\
\hline 15 & & & 0.63 & 0.00 & & & 23.32 & 0.00 \\
\hline 16 & & & 0.49 & & & & 21.67 & \\
\hline 17 & & & 0.73 & & & & 20.69 & \\
\hline 18 & & & 0.50 & & & & 19.04 & \\
\hline 19 & & & 0.51 & & & & 17.83 & \\
\hline 20 & & & 0.40 & & & & 16.64 & \\
\hline 21 & & & 0.36 & & & & 15.40 & \\
\hline 22 & & & 0.37 & & & & 13.93 & \\
\hline 23 & & & 0.32 & & & & 12.88 & \\
\hline 24 & & & 0.33 & & & & 11.76 & \\
\hline 25 & & & 0.17 & & & & 10.54 & \\
\hline 26 & & & 0.30 & & & & 9.07 & \\
\hline 27 & & & 0.20 & & & & 7.76 & \\
\hline 28 & & & 0.17 & & & & 6.10 & \\
\hline 29 & & & 0.08 & & & & 4.37 & \\
\hline 30 & & & 0.00 & & & & 0.00 & \\
\hline
\end{tabular}

pre-calf tests. Random groups of eight samples taken from a pool of nine will have a considerable amount of overlap, which will decrease the coefficient of variation. This analysis suggests that at least 5 samples are needed for the $\mathbf{P D}$ to be consistently less than 5\% and the $\mathbf{C V}$ less than 50\%. 
Chapter 2: Sampling Methods

Table 2.8: Results of Methods Test, SAMPling Times:

P-values for Standard Frve Samples Compared to Five Random SAMPles

Cells with $\mathrm{p}<0.1$ are highlighted.

\section{A. Pre-Calf Tests}

N/A indicates Vicky was not present during the March 5 test.

Blank cells indicate measures with no non-zero entries.

\begin{tabular}{|c|c|c|c|c|c|c|c|}
\hline \multirow[b]{2}{*}{ Focal } & \multirow[b]{2}{*}{ Interactor } & \multicolumn{2}{|c|}{ Total Association } & \multicolumn{2}{|c|}{ Agonistic Interactions } & \multicolumn{2}{|c|}{ Affiliative Behaviors } \\
\hline & & March 5 & March 25 & March 5 & March 25 & March 5 & March 25 \\
\hline \multirow[t]{5}{*}{ Delphi } & Daphne & 0.35 & 0.45 & & 1.00 & & 0.46 \\
\hline & Lotty & 0.11 & 0.74 & & 1.00 & & \\
\hline & Nephele & 0.14 & 0.17 & & 0.45 & 1.00 & \\
\hline & Sharky & 0.36 & 0.71 & & 1.00 & & \\
\hline & Vicky & N/A & 0.18 & N/A & 1.00 & N/A & \\
\hline \multirow{5}{*}{ Lotty } & Daphne & 0.86 & 0.48 & 0.48 & & 0.58 & 1.00 \\
\hline & Delphi & 0.08 & 0.35 & & & & \\
\hline & Nephele & 0.11 & 0.34 & & & 0.37 & \\
\hline & Sharky & 0.93 & 0.63 & 0.58 & 1.00 & 0.43 & 0.44 \\
\hline & Vicky & N/A & 0.34 & N/A & & N/A & 0.44 \\
\hline \multirow[t]{5}{*}{ Nephele } & Daphne & 0.09 & 0.39 & 1.00 & & & 1.00 \\
\hline & Delphi & 0.65 & 0.33 & & & & \\
\hline & Lotty & 0.71 & 0.28 & & & 1.00 & \\
\hline & Sharky & 0.51 & 0.35 & 1.00 & 1.00 & & \\
\hline & Vicky & N/A & 0.16 & N/A & & N/A & \\
\hline \multirow[t]{5}{*}{ Vicky } & Daphne & N/A & 0.65 & N/A & 1.00 & N/A & \\
\hline & Delphi & N/A & 0.29 & N/A & & N/A & \\
\hline & Lotty & N/A & 0.63 & N/A & & N/A & 1.00 \\
\hline & Nephele & N/A & 0.38 & N/A & & N/A & \\
\hline & Sharky & N/A & 0.44 & N/A & 0.16 & N/A & 1.00 \\
\hline
\end{tabular}

\section{B. Post-Calf Tests}

\begin{tabular}{|c|c|c|c|c|}
\hline \multicolumn{2}{|l|}{ Behavior type: } & Dolphins & July 20 & August 7 \\
\hline \multirow{3}{*}{\multicolumn{2}{|c|}{ Total Association }} & Lotty-Vicky & 0.43 & 0.76 \\
\hline & & Lotus-Lotty & 0.14 & 0.20 \\
\hline & & Lotus-Vicky & 0.58 & 0.69 \\
\hline \multirow{2}{*}{\multicolumn{2}{|c|}{ Time Alone }} & Lotty & 0.13 & 0.39 \\
\hline & & Lotus & 0.23 & 0.13 \\
\hline \multirow{2}{*}{\multicolumn{2}{|c|}{ Affiliative Contact }} & Lotus-Lotty & 0.73 & 0.67 \\
\hline & & Lotus-Vicky & 0.02 & 0.09 \\
\hline \multicolumn{2}{|l|}{ Nursing } & Lotus-Lotty & 0.26 & 0.54 \\
\hline \multicolumn{2}{|l|}{ Retrieves } & Lotus-Lotty & 0.03 & 0.62 \\
\hline \multirow[t]{4}{*}{ Lotus' nearest neighbor: } & No other adults & Lotty & 0.75 & 0.46 \\
\hline & & Vicky & 0.08 & 0.04 \\
\hline & With other adults & Lotty & 0.79 & 0.57 \\
\hline & & Vicky & 0.49 & 0.10 \\
\hline
\end{tabular}




\section{Sampling times}

For most of the measures, the results from the standard five samples used on all other days were not significantly different from the distribution of five randomly chosen samples (Table 2.8A-B). For a few measures, however, the p-value was less than 0.1 , which means that fewer than $10 \%$ of the means of randomly selected sample groups were more different from the overall mean than the standard sample (highlighted cells in Table 2.8). These were generally measures where the behaviors were not evenly distributed throughout the day. In these cases, the bulk of the observations occurred in one or two of the samples so a random selection of five samples might include none, or all, of the observations of the behavior. However, only two of these behaviors occurred in this fashion in more than one test. These were affiliative contact between Lotus and Vicky, and the time Vicky was Lotus' nearest neighbor with no other adults were present (Table 2.8B). In neither case was the p-value less than 0.05 on both days. The standard five samples are therefore reasonable choices for when to sample.

\subsubsection{DISCUSSION}

How well a selected group of samples represents the day depends on how the behaviors are distributed in the day. If all the instances of a behavior occur during a short period of time, a small number of samples or unevenly distributed samples might not accurately depict the pattern with which the behaviors occur. From the percent deviation, one or two samples might appear to be sufficient. However, the percent deviation is only an indication of how well that number of samples performs on average. The coefficient of variation indicates that any particular two samples are highly likely to yield a mean that is significantly different from the overall mean. To be guaranteed a mean close to the overall mean, more samples are needed. The results of this test indicate that between five and ten samples are sufficient in most circumstances. For most measures, five samples are sufficient. However, for more variable measures, more than five samples might be preferable. For some measures, six or seven samples were needed for the standard deviation to be less than $50 \%$ of the mean. If the $\mathrm{CV}$ is greater than $50 \%$, a 
significant proportion of the group means will be either twice the actual mean or zero. As the standard deviation approaches $100 \%$ of the mean, the probability that a randomly selected group of samples has a mean of zero increases, even though the behavior was seen in some samples during the day. In such a case, the observer could conclude that the behavior did not occur at all on that day, which would be incorrect.

However, practical considerations also need to be taken into account in generating a sampling protocol. The number of samples that can reasonably be taken in a day depends on how many animals are being sampled and how many observers are available. If samples are ten minutes long, five samples of one animal only take 50 minutes per day. Five samples of four animals, on the other hand, take 200 minutes, or 3.3 hours per day. Both of these can be reasonably done in a day, even by only one observer. Ten samples of one animal take 1.6 hours. Ten samples of four animals, on the other hand, take 6.6 hours per day. Fifteen samples of four animals means ten hours of data recording per day. For any given day, ten hours is possible, but for a longitudinal study, the same sampling schedule needs to be performed every day for several months. Ten hours a day every day can be fatiguing even for several observers. The number of animals and the number of observers at each location of the study are therefore important to consider before the number of samples is decided. However, the same number of samples must be taken in all locations, so the maximum possible number of animals that might need to be observed at the same time at a single location must be known.

One issue that was not explicitly tested in this study was the length of the samples. All samples taken in this study were ten minutes long. In most cases, five tenminute samples were sufficient to represent the entire day. In those cases where five samples were not sufficient, a more even coverage of the day was necessary. Longer samples would not solve the problem in those cases. Ten well-spaced ten-minute samples would likely do a better job of representing the entire day than five twentyminute samples. As was discussed in section 2.1.1, in some studies samples should be taken early in the morning, before the dolphins are disturbed by the presence of people. In that case, fewer, longer samples might be preferable. However, for studying vocal 
learning, a clear picture of the overall situation the calf experiences is necessary, suggesting that more, shorter samples are preferable.

\subsection{CONCLUSIONS}

To test the hypothesis that learning is involved in the development of dolphin whistles, a study needs to be designed to record several calves from different social groups as their whistles develop. The whistles produced by the calf, the whistles heard by the calf, and the calf's interactions all need to be recorded in a systematic manner. The best method for this is focal animal samples of mother-calf pairs (Altmann 1974) performed at set times daily for the duration of whistle development. This analysis suggests that five to ten samples per day are sufficient to cover the day if they are spread out over the entire day. The appropriate sample length was not tested but ten minutes, as used here, appears to be adequate. Having established the proper sampling strategies, methods for analyzing the acoustic and behavioral recordings, and for extracting whistles from the acoustic recordings, need to be developed. This is the goal of chapters 3 to 5 .

Explicit discussions of all the sampling decisions made are important because these decisions can affect the results that can be presented. For instance, Bateson (1974) mentions very few of his sampling decisions. One of the few decisions he does mention is the decision to pay more attention to rare behaviors than to common ones. This means that he could not address the actual rate of common events and could not be sure of the relationships he saw. Reports of cetacean studies commonly omit some of the important decisions. Early studies often did not discuss behavioral sampling methods at all (e.g. McBride \& Kritzler 1951, Tavolga \& Essapian 1957). Studies that are more recent often leave out important points as well. Most papers discuss the sampling technique used, i.e. focal or scan or ad lib., but many do not mention how they decided to start sampling (e.g. Herzing 1996, Connor et al. 1992, Bel'kovich 1991, Shane 1990a). This detail can be very important to evaluating the possible biases in the study (see e.g. Altmann \& Altmann 1970). Many papers also to fail to mention how long the samples were (e.g. Herzing 1996, Connor et al. 1992, Bel'kovich 1991), which can be important to being 
Chapter 2: Sampling Methods

able to compare studies. The implicit assumption of these papers is that such decisions do not influence the conclusions, but this may be a faulty assumption (Altmann 1974). All the sampling decisions have the potential to make a difference to the interpretation of the results and need to be stated explicitly.

One of the most important sampling decisions is where to do the study, particularly whether to do it in captivity or in the wild. This decision is particularly important because it affects many of the other decisions that need to be made. There are several advantages to working in captivity. One is the ability to quantify all the possible social interactors that might influence a calf's development. Another major advantage is the ability to see subtle behaviors and interactions that are often missed in the wild. As will be shown in chapter 3 , this allows researchers to investigate some very subtle questions about how dolphins interact. Another advantage is the ability to observe animals in multiple locations. The cost of setting up observations in more than one location in the wild can be prohibitive. This is less of a problem in captivity where good underwater viewing areas are often already set up. For a study of vocal learning, the ability to observe calves in several locations is extremely important to the ability to compare environments. Showing that a calf's whistles match the whistles from his early environment is not sufficient to demonstrate vocal learning. The converse, that the calf's whistles do not match the whistles from other calves' early environments, is also necessary (see Figure 1.1). There are, of course, caveats to working in captivity, but comparisons of the behavior of captive and free-ranging dolphins have shown most of the social behavior to be comparable (Samuels \& Gifford 1997).

\subsection{LITERATURE CITED}

Altmann, J., 1974. "Observational study of behavior: Sampling methods." Behaviour, 49, pp. $227-267$. Altmann, S.A. and Altmann, J., 1970. Baboon ecology: African field research. University of Chicago Press, Chicago, IL.

Bateson, G., 1974. "Observations of a cetacean community." Mind in the Waters, ed. J. McIntyre. Charles Scribner's Sons, New York,NY. pp. 146-69.

Bel'kovich, V.M., 1991. "Herd structure, hunting, and play: Bottlenose dolphins in the black sea." Dolphin Societies: Discoveries and Puzzles, eds. K. Pryor and K.S. Norris. University of California Press, Berkeley, CA. pp. 17-78. 
Caldwell, M.C. and Caldwell, D.K., 1979. "The whistle of the Atlantic bottlenose dolphin (Tursiops truncatus) - ontogeny." Behavior of marine animals, vol. 3 Cetaceans, eds. H.E. Winn and B.L. Olla. Plenum, New York, NY. pp. 369-401.

Caldwell, M.C., Caldwell, D.K., and Tyack, P.L., 1990. "Review of the signature-whistle hypothesis for the Atlantic bottlenose dolphin." The Bottlenose Dolphin, eds. S. Leatherwood and R.R. Reeves. Academic Press, Inc., New York, NY. pp. 199-234.

Chapra, S.C. and Canale, R.P., 1988. Numerical methods for engineers. McGraw Hill, New York, NY.

Cockcroft, V.G. and Ross, G.J.B., 1990. "Observations on the early development of a captive bottlenose dolphin calf." The Bottlenose Dolphin, eds. S. Leatherwood and R.R. Reeves. Academic Press, New York, NY. pp. 461-85.

Connor, R.C., Smolker, R.A., and Richards, A.F., 1992. "Two levels of alliance formation among male bottlenose dolphins (Tursiops sp.)." Proceeds of the National Academy of Science, USA, 89, pp. $987-990$.

DeMaster, D.P. and Drevenak, J.K., 1988. "Survivorship patterns in three species of captive cetaceans." Marine Mammal Science, 4, pp. 297-311.

Dudzinski, K.M., 1996. Communication and behavior in the Atlantic spotted dolphins (Stenella frantalis): Relationships between vocal and behavioral activities. Ph.D. Dissertation, Texas A\&M University.

Duffield, D.A. and Wells, R.S., 1991. "The combined application of chromosome, protein, and molecular data for the investigation of social unit structure and dynamics in Tursiops truncatus." Reports to the International Whaling Commission, Special issue 13, pp. 155-169.

Elsberry, W.R. and Blackwood, D.J. 1995. "Cetacean behavior event recording by multiple observers." 11th Biennial Conference on the Biology of Marine Mammals, Orlando, FL, December 1995.

Gottlieb, G., 1988. "Development of species identification in ducklings: XV. Individual auditory recognition." Animal behaviour, 41, pp.953-963.

Herzing, D.L., 1996. "Vocalizations and associated underwater behavior of free-ranging Atlantic spotted dolphins, Stenella frontalis and bottlenose dolphins, Tursiops truncatus." Aquatic Mammals, 22.2, pp. 61-79.

Hinde, R.A., 1976. "Interactions, relationships, and social structure." Man, 11, pp. 1-17.

International Whaling Commission, 1990. Report of the International Whaling Commission, Special Issue 12: Individual recognition in cetaceans., Cambridge, UK.

Irvine, A.B., Scott, M.D., Wells, R.S., and Kaufmann, J.H., 1981. "Movements and activities of the Atlantic bottlenose dolphin, Tursiops truncatus, near Sarasota, Florida." Fisheries Bulletin, 79, pp. 671-688.

Irvine, A.B., Wells, R.S., and Scott, M.D., 1982. "An evaluation of techniques for tagging small odontocete cetaceans." Fisheries Bulletin, 80, pp. 135-143.

Janik, V.M. and Slater, P.J.B., 1998. "Context-specific use suggests that bottlenose dolphin signature whistles are cohesion calls." Animal Behavior, 56: 4, pp. 829-838.

Kastelein, R.A., Dokter, T., and Zwart, P., 1990. "The suckling of a Bottlenose dolphin calf (Tursiops truncatus) by a foster mother, and information on transverse birth bands." Aquatic Mammals, 16(3), pp. 134-138.

Kleiman, D.G., 1992. "Behavior research in zoos: Past, present, and future." Zoo Biology, 11, pp. 301312.

Locke, J.L. and Snow, C., 1997. "Social influences on vocal learning in human and nonhuman primates." Social influences on vocal learning, eds. C.T. Snowdon and M. Hausberger. Cambridge University Press, New York, NY. pp. 274-92.

Mann, J. and Smuts, B.B., in prep. "Behavioural development of wild bottlenose dolphin newborns."

Mann, J., 1999. "Behavioral sampling methods for cetaceans: A review and critique." Marine Mammal Science, 15, pp.102-122.

Martin, P. and Bateson, P., 1986. Measuring behaviour: An introductory guide. Cambridge University Press, Cambridge, UK. 
Chapter 2: Sampling Methods

McBride, A.F. and Kritzler, H., 1951. "Observations of pregnancy, parturition, and postnatal behaviour in the bottlenose dolphin." Journal of Mammology, 32 (3), pp. 251-265.

McCowan, B. and Reiss, D., 1995a. "Quantitative comparison of whistle repertoires from captive adult bottlenose dolphins (Delphinidae, Tursiops truncatus): a re-evaluation of the signature whistle hypothesis." Ethology, 100, pp. 194-209.

McCowan, B. and Reiss, D., 1995b. "Whistle contour development in captive-born infant bottlenose dolphins (Tursiops truncatus): Role of learning." Journal of Comparative Psychology, 109(3), pp. 242-260.

Noldus, L.P.J.J., 1991. "The Observer: a software system for collection and analysis of observational data." Behavior Research Methods, Instruments \& Computers, 23, pp. 415-429.

Norris, K.S. and Dohl, T.P., 1980. "The structure and functions of cetacean schools." Cetacean behavior, ed. L.M. Herman. Wiley, New York, NY. pp. 149-209.

Nowacek, D.P., Lange, W.N., Wells, R.S., and Tyack, P.L., 1995. "A new method for studying cetacean behavior and acoustics: Overhead video combined with underwater audio." 11th Biennial Conference on the Biology of Marine Mammals, Orlando, FL, December 1995.

Östmann, J., 1991. "Changes in aggressive and sexual behavior between two male bottlenose dolphins (Tursiops truncatus) in a captive colony." Dolphin Societies, eds. K. Pryor and K.S. Norris. University of California Press, Berkeley, CA. pp. 305-17.

Querleu, D., Renard, X., Boutteville, C., and Cripin, G., 1989. "Hearing by the human fetus?" Seminars in Perinatology, 13, pp. 409-420.

Samuels, A. and Gifford, T., 1997. "A quantitative assessment of dominance relations among bottlenose dolphins." Marine Mammal Science, 13, pp. 70-99.

Samuels, A. and Tyack, P., in press. "Flukeprints: A history of studying cetacean societies." Cetacean societies: Field studies of whales and dolphins, eds. J. Mann, R. Connor, P.L. Tyack, and H. Whitehead. University of Chicago Press, Chicago, IL.

Sayigh, L.S., 1992. Development and functions of signature whistles of free-ranging bottlenose dolphins, Tursiops truncatus. Ph.D. Dissertation, MIT/WHOI Joint Program, WHOI 92-37.

Sayigh, L.S., Tyack, P.L., Wells, R.S., and Scott, M.D., 1990. "Signature whistles of free-ranging bottlenose dolphins Tursiops truncatus: Stability and mother-offspring comparisons." Behavioral Ecology and Sociobiology, 26, pp. 247-260.

Seyfarth, R.M. and Cheney, D.L., 1997. "Some general features of vocal development in nonhuman primates." Social influences on vocal development, eds. C.T. Snowdon and M. Hausberger. Cambridge University Press, New York, NY. pp. 249-74.

Shane, S.H., 1990a. "Behavior and ecology of the bottlenose dolphin at Sanibel Island, Florida." The Bottlenose Dolphin, eds. S. Leatherwood and R.R. Reeves. Academic Press, San Diego, CA. pp. 245-66.

Shane, S.H., 1990b. "Comparison of bottlenose dolphin behavior in Texas and Florida, with a critique of methods for studying dolphin behavior." The Bottlenose Dolphin, eds. S. Leatherwood and R.R. Reeves. Academic Press, San Diego, CA. pp. 541-58.

Smolker, R.A., Mann, J., and Smuts, B.B., 1993. "Use of signature whistles during separations and reunions by wild bottlenose dolphin mothers and infants." Behavioral Ecology and Sociobiology, 33, pp. 393-402.

Smolker, R.S. and Pepper, J., in press. "Whistle convergence among allied male bottlenose dolphins." Ethology.

Snowdon, C.T. and Hausberger, M., 1997. Social influences on vocal development. Cambridge University Press, New York, NY.

Taber, S. and Thomas, P., 1982. "Calf development and mother-calf spatial relationships in southern right whales." Animal Behavior, 30, pp. 1072-1083.

Tavolga, M.C. and Essapian, F.S., 1957. "The behaviour of the bottlenose dolphin Tursiops truncatus: Mating, pregnancy, parturition and mother-infant behaviour." Zoologica, 421, pp. 11-31. 
Wells, R.S. and Scott, M.D., 1990. "Estimating bottlenose dolphin population parameters from individual identification and capture-release techniques." Individual recognition of cetaceans: Use of photographic identification and other techniques to estimate population parameters, eds. P.S. Hammond, S.A. Mizroch, and G.P. Donovan. Report of the International Whaling Commission, Special Issue \#12, Cambridge,UK. pp. 407-15.

Wells, R.S., 1991. "The role of long-term study in understanding the social structure of a bottlenose dolphin community." Dolphin Societies, eds. K. Pryor and K.S. Norris. University of California Press, Berkeley, CA. pp. 199-235.

Wells, R.S., Scott, M.D., and Irvine, A.B., 1987. "The social structure of free-ranging bottlenose dolphins." Current Mammology, ed. H.H. Genoways. Plenum Press, New York, NY. pp. 247305 .

West, M.J., King, A.P., and Freeberg, T.M., 1997. "Building a social agenda for the study of bird song." Social influences on vocal development, eds. C.T. Snowdon and M. Hausberger. Cambridge University Press, New York, NY. pp. 41-56. 


\section{CHAPTER 3: SOCIAL RELATIONSHIPS}

\subsection{INTRODUCTION}

Bottlenose dolphins provide a unique opportunity to study social influences on vocal learning in a highly social non-human mammal. Social input is essential to normal language development in humans (Locke \& Snow 1997), and studies of both birds and mammals have shown that social interactions play a role in the course of their vocal development (Snowdon \& Hausberger 1997). Animal studies of the social influences on learning vocal production have concentrated on songbirds (e.g. Immelmann 1969, Brown 1985, Margoliash et al. 1994, Hausberger et al. 1995). In fact, birdsong has been discussed as a means of maintaining social bonds (e.g. Brown 1985, Hausberger et al. 1995). However, only a few studies of birdsong have investigated in detail the "social bonds" between the birds (e.g. Brown 1985). In contrast, the concept of social relationships has been well developed in the mammalian literature (e.g. primates: Seyfarth 1976, Altmann 1980, Seyfarth 1980, Smuts 1985; jackals: Moehlman 1987; elephants: Moss \& Poole 1983).

Dolphins are highly social mammals with a social structure similar to some primates and elephants (dolphins: Wells 1991, primates: Cheney et al. 1986, elephants: Moss \& Poole 1983). Techniques for studying aspects of mammalian social structure, such as dominance hierarchies, have been successfully applied to dolphins (e.g. Samuels \& Gifford 1997). Bottlenose dolphins have repeatedly shown an ability to learn to produce new sounds (e.g. Richards et al. 1984) that is unusual among non-human mammals (Janik \& Slater 1997, Seyfarth \& Cheney 1997). There is evidence that they use this ability in their natural vocal development (Tyack \& Sayigh 1997). Studies of dolphins therefore provide a unique opportunity to combine the fields of vocal learning and mammalian social relationships into powerful tools for investigating the social influences on vocal learning.

This chapter is concerned with strategies for determining the social relationships of dolphins. Techniques derived both from studies of non-human social relationships (e.g. Smuts 1985) and from human sociology (e.g. Goodman 1978) will be applied to the 
analysis of dolphin interactions. The behavioral data collected in the pilot study at Kolmårdens Djurpark (see chapter 2) will be used in this chapter to explore methods of analyzing interactions to define relationships and of categorizing those relationships into types. Many studies of cetacean social relationships have concentrated on association patterns (e.g. Whitehead 1997, Wells et al. 1987, Heimlich-Boran 1986), but association patterns will only tell part of the story. In this chapter, relationships will be based on multiple measures, including affiliative contact, agonistic interactions and calf-related behaviors (see chapter 2). These interactions will be evaluated with a number of multivariate statistical techniques, including loglinear analysis, multidimensional scaling, and hierarchical cluster analysis, to determine relationships and categorize those relationships into types.

\subsubsection{BACKGROUND}

\section{Social Influences on Vocal Development}

Social interactions have clearly been shown to have a profound impact on the development of birdsong (Snowdon \& Hausberger 1997). Early studies of song development involved young birds raised in isolation listening to song on tape (e.g. Marler 1970). These youngsters could only learn a species-specific song and only did so during a short sensitive period (e.g. Marler 1970). By contrast, when young songbirds were given a live tutor to learn from, their repertoires expanded considerably (e.g. Baptista \& Petrinovich 1984). They could even learn allo-specific song from the live tutor over an extended sensitive period (e.g. Baptista \& Petrinovich 1984). In later studies, young birds were given a choice of tutors, one they could see or interact with and one they could only hear. In many cases, the tutor the young birds could interact with sang an allo-specific song while the tutor they could hear sang the song of the youngsters' own species. In almost every case, the young birds chose the tutor they could see, even though they were learning allo-specific songs when they could also hear conspecific songs (e.g. Clayton 1988, Payne \& Payne 1997, see Baptista \& Gaunt 1997 for a review). 
Chapter 3: Social Relationships

Investigations of the influence of the social setting in which birds are raised have also found that social interactions play an important role in vocal learning. For instance, starlings raised by humans imitated human sounds only if the human was the starling's main social companion (West et al. 1983). Several researchers have suggested that sharing songs functions to maintain social bonds (e.g. Brown 1985, Hausberger et al. 1995). The sharing of songs and syllables in birds has been related to aggressive interactions (e.g. indigo buntings: Margoliash et al. 1994), affiliative contact (e.g. common crows: Brown 1985), provisioning (e.g. zebra finches: Immelmann 1969), and proximity (e.g. European starlings: Hausberger et al. 1995).

Social interactions have also been shown to impact the development of mammalian vocalizations (Seyfarth \& Cheney 1997). Vocal development includes knowing how to produce the sounds, knowing when to use the sounds, and knowing how to respond to the sounds of others. Although few mammals have been shown to learn to produce their vocalizations, many species of primates learn to use and respond to vocalizations (Seyfarth \& Cheney 1997). For example, infant vervet monkeys living in areas with higher concentrations of starlings learn to recognize starling alarm calls more quickly (Hauser 1988). Another example is the "wrr" vocalizations of vervets, which are produced in inter-group encounters and differ in acoustic structure depending on the context (Hauser 1989). Infants' use of these vocalizations becomes more precise over time. Infants exposed to many inter-group encounters, and therefore to many wrrs, learn to use wrrs in the appropriate contexts more quickly than infants with less exposure (Hauser 1989). Similarly, cross-fostered Japanese and rhesus macaque infants learned to respond correctly to their foster mothers' vocalizations, even though those vocalizations were used differently from the infant's own species' vocalizations (Seyfarth \& Cheney 1997). The social interactions between animals are therefore an important part of the process of vocal development.

\section{Social Relationships}

If an animal remembers previous interactions with a particular individual, repeated interactions can be used to define "social relationships" between animals. 
Interactions of one type can affect how other types of interactions occur and can have long-term consequences (Cheney et al. 1986). When animals interact over a period of time, how they interact at one time may influence how they interact at a later time (Hinde 1983). For instance, elephants exchange elaborate greetings when they meet other elephants but only when they meet elephants they have spent a great deal of time with in the past (Moss \& Poole 1983). Similarly, which male baboons will associate with a new mother can be predicted from which males groomed and mated with that female previously (Altmann 1980). These repeated patterns of interaction describe a relationship between the animals (Hinde 1976, Whitehead 1997). Because the interactions between animals in particular age-sex classes tend to follow a few patterns, these relationships can be classified into types (Hinde 1976, Whitehead 1997). For instance male and female baboons that mate, groom, and spend time together are often described as "consorts" (Hinde 1976). Elephants who spend time together, rest together, rub together, and exchange elaborate greetings are said to form a "bond group" (Moss \& Poole 1983). Because infants have a choice of animals to learn vocalizations from, determining relationship types is important to the study of vocal learning. By defining a set of relationship types, each potential "tutor" can be classified into a relationship with the infant. Comparing the tutors chosen by a number of infants will help elucidate what types of social contact affect the process of vocal learning.

The Impact of Adult Relationships on Infants

Because many young mammals have one primary caretaker, often the youngster's mother (Gittleman 1985), the relationships between adults may be important to understanding the relationships young animals have with adults. The relationships between the mother and other animals can affect the relationships those animals have with the infant. In many species, the mother's dominance rank will influence how other animals interact with the infant (e.g. Altmann 1980). In cercopithecine primates and spotted hyenas, for instance, infants inherit a rank directly below their mothers, often above other adults (Samuels et al. 1987, Frank 1986). This rank will influence what kind 
Chapter 3: Social Relationships

of interactions both the mother and the infant have with other adults. Female baboons, for example, form coalitions with females who have neighboring ranks (Seyfarth 1976).

The relationships animals have with the mother can also influence the opportunities those animals have to interact with the infant. Dolphin calves, for instance, spend most of their time with their mothers (McBride \& Kritzler 1951, Wells 1991, Mann \& Smuts 1998). If access to the calf is controlled by the mother, another dolphin's opportunities to interact with the calf may depend on the dolphin's relationship with the mother. For instance, a particularly close relationship between an adult and the mother might lead to opportunities for alloparenting (e.g. Dudok van Heel \& Meyer 1974). Alloparenting is the situation where an infant spends time with an adult without its mother present. This type of situation could allow the adult to have interactions with the calf that might not occur when the mother was present. Alternatively, if the infant is with the mother most of the time, behaviors other animals direct to the mother may be received by the infant as well. The mother's reaction to specific other animals may also influence the infant's reaction to those animals and thereby the infant's relationships with them. Therefore, to understand why young animals have specific interactions, and therefore relationships, with adults, the relationship those adults have with the infant's mother or primary caretaker must be understood as well.

\subsubsection{CETACEAN SOCIAL RELATIONSHIPS}

\section{Free-Ranging Animals}

Association patterns are the primary measure used to define the social relationships of wild cetaceans (e.g. Whitehead 1997, Wells et al. 1987, Heimlich-Boran 1986). In most studies, an association index is calculated for each pair of animals in the study based on how often those animals are sighted together compared to how often they are each sighted separately (e.g. Whitehead 1997, Smolker et al. 1992, Wells et al. 1987). These indices vary from 0 to 1 , where 0 indicates that the animals were never seen together. To determine the association patterns of the entire population, a matrix of the association indices for all the animals is generated. The most common method of 
analyzing these matrices has been by visual inspection of the matrix itself (e.g. Wells $e t$ al. 1987), or with an association diagram or sociogram where connections are drawn between animals based on their association indices (e.g. Heimlich-Boran 1986, Smolker et al. 1992). In this way, male and female dolphins were shown to have different patterns of association (Wells et al. 1987, Smolker et al. 1992). Some adult males associate very strongly with one or two other males in stable groups lasting several years (Smolker et al. 1992). Associations between adult females are more fluid but each adult female tends to associate most with a few other adult females (Wells et al. 1987, Smolker et al. 1992). The strongest female associations are not as strong as the strongest male ones, or as stable, but some female-female associations do last over multiple years (Smolker et al. 1992). Associations between mothers and calves are very strong for at least three years, with association indices averaging 0.96 (Wells et al. 1987). Associations between adult males and adult females are less frequent and tend to be dependent on the female's reproductive state (Wells et al. 1987, Connor et al. 1992).

Association matrices have also been analyzed with a variety of multivariate statistical techniques. One common method is multidimensional scaling, which plots the animals in space so that the distance between animals is based on their association index (e.g. Smolker et al. 1992, see Whitehead 1997). Animals that associate more often with each other are plotted close together, allowing groups to be determined. Using this technique, Smolker et al. (1992) found that stable groups of female dolphins associated consistently over several years. This technique is most useful when the groups are distinct, however. If the group members also commonly associate with members of other groups, the boundaries between the groups can be difficult to determine with multidimensional scaling (Whitehead 1997). Another common method is hierarchical cluster analysis (e.g. Ballance 1990, Heimlich-Boran 1993, see Whitehead 1997). In hierarchical cluster analysis, animals are connected based on their association patterns in a hierarchical manner to create a clustering tree where all the animals are connected at some level. Cluster analysis was used to demonstrate that subgroups of preferred associates exist within pods of orcas (Heimlich-Boran 1988), and to demonstrate stable 
Chapter 3: Social Relationships

associations in pods of pilot whales (Heimlich-Boran 1993). As with multidimensional scaling, hierarchical cluster analysis is most useful when groups are distinct. Whitehead (1997) analyzed association patterns by their temporal stability, using lagged interaction rates to determine how long animals continue to interact. He could therefore distinguish groups based on long-term as well as short-term associations.

All of these analyses depend on a measure of association. However, the definitions of an association, or "group", used in studies of wild dolphins vary considerably (e.g. Smolker et al. 1992, Wells et al. 1987, Bigg et al. 1990, Pryor \& Shallenberger 1991). Some are extremely imprecise, with a group defined as animals in "apparent association, moving in the same direction and often, but not always, engaged in the same activity" (Shane 1990, p247), or animals that "usually stayed closer to each other than to animals from other groups" (Bel'kovich et al. 1991, p19; Pryor \& Shallenberger 1991). This type of imprecision can make studies difficult to replicate. Other researchers have used more quantitative methods to define groups. Some define associations between animals as animals that are both seen in the same photograph (e.g. Bigg et al. 1990, Ballance 1990). Researchers using this method must tackle the problem that animals surfacing together may not be equivalent to animals associating and that animals that are associating may not all surface together. Photographic methods also effectively define groups as dolphins surfacing within a certain distance of each other, based on the field of view of the camera. Other studies use more precise definitions, such as animals swimming within 10 meters (Smolker et al. 1992, Connor et al. 1992) or 100 meters (Wells et al. 1987) of each other. However, the most appropriate distance to use in such a situation is not clear.

\section{Captive Animals}

In captive studies, the "groups" are artificially created because humans decide which dolphins share pools with each other. Most definitions of association used in wild studies are therefore not useful in captivity, because all animals within the pool would be considered in association. Instead, association can be measured as animals within a much shorter distance of each other (e.g. Chirighin 1987, Reid et al. 1995). A study of distance 
criteria for associations of captive beluga whales suggested that one body length, or approximately 3 meters, was an appropriate measure for distinguishing different association patterns (Recchia 1994). In addition to association, the number of times each animal in a pair approaches or leaves the other animal can be recorded in captive settings. Because such subtle behaviors can be measured, a subtler set of relationships can be investigated than when association is defined by group membership only. The approaches and leaves can be analyzed to determine which member of the pair is responsible for maintaining proximity (Hinde \& Atkinson 1970). For instance, Reid et al. (1995) found that young captive calves were very seldom far from their mothers and were never responsible for maintaining proximity. Chirighin (1987) found that the responsibility for proximity shifted from the mothers to the calves as the calves reached about one year of age.

Few researchers have used measures other than association to look at social relationships between cetaceans. In a few cases, dominance relationships have been evaluated (e.g. Samuels \& Gifford 1997), but the dominance hierarchies have not been compared to other interactions between the animals. Several studies have investigated the function of behaviors (e.g. Dudzinski 1996, Samuels \& Gifford 1997) or the use of vocalizations in conjunction with those behaviors (e.g. Overstrom 1983, Herzing 1996). Few of these studies have looked at how these behaviors varied between pairs of animals or how the behaviors correlated with other types of interactions. Several studies have found, however, that animals who associate also form alliances in agonistic encounters with less familiar animals (Connor et al. 1992, Samuels \& Gifford 1997). Connor et al. (1992) found that free-ranging males who associated a great deal also helped each other in herding females, potentially for mating purposes.

In addition, some studies, particularly with captive groups, have looked at the types of interactions that occur between animals with known, or presumed, relationships, such as mothers and their calves (e.g. McBride \& Kritzler 1951, Smolders 1986, Kastelein et al. 1990, Peddemors et al. 1992). Most of these are anecdotal accounts of births (e.g. Dudok van Heel \& Meyer 1974, Amundin 1986, Smolders 1986, Peddemors 
Chapter 3: Social Relationships

et al. 1992), but some studies have looked at the development of specific behaviors, primarily nursing, breathing, and proximity maintenance (e.g. Chirighin 1987, Reid et al. 1995, Mann \& Smuts in prep). Several studies have reported infants spending time with adults other than their mothers (e.g. McBride \& Kritzler 1951, Leatherwood 1977, Mann \& Smuts 1998) and a few of these reported the calves nursing from those animals (e.g. Messinger et al. 1996). Only a few studies have looked at affiliative contact between animals, particularly in relation to other measures (Dudzinski 1996, Mann \& Smuts in prep).

\subsection{STATISTICAL METHODS}

The interactions and associations between the dolphins at Kolmårdens Djurpark were recorded with focal animal sampling as described in chapter 2 (section 2.2). The behaviors recorded in that pilot study were used to determine the social relationships between each focal dolphin and the other dolphins in the pool. These relationships were then categorized into types using multivariate statistics to group the relationships by the interactions that made up each relationship. This analysis was done first for the relationships the focal calves had with the adults and then for the relationships between the adults before the calves were born.

\subsubsection{CHARACTERIZING RELATIONSHIPS}

For a behavior to be useful in differentiating between social relationships, the frequency with which a dolphin performs that behavior must depend on which individual the dolphin is interacting with. The first step in distinguishing social relationships is therefore determining the behaviors that vary by which pair of dolphins is interacting. The relationships between the dolphins can then be characterized by how often these behaviors are performed when a particular pair is interacting. The behaviors can then be used to classify the relationships into types where each relationship type is characterized by certain types of interactions.

The behaviors that vary by which pair of dolphins is interacting can be determined by considering the data to be categorical. The behaviors of the focal dolphin 
FIGURE 3.1: CONTINGENCY TABLES OF BEHAVIORAL DATA

In this example, the focal dolphins are different from the interactor dolphins. The focal dolphins do not interact with each other.

A. TABLE FOR ONE FOCAL

\begin{tabular}{|c|c|c|c|}
\hline FOCAL 1 & \multicolumn{3}{|c|}{ INTERACTOR } \\
\hline BEHAVIOR & DOLPHIN 1 & DOLPHIN 2 & DOLPHIN 3 \\
\hline TYPE 1 & $X_{11}$ & $x_{12}$ & $X_{1,3}$ \\
\hline TYPE 2 & $x_{21}$ & $X_{22}$ & $X_{23}$ \\
\hline TYPE 3 & $x_{31}$ & $x_{32}$ & $X_{33}$ \\
\hline
\end{tabular}

B. TABLES FOR MULTIPLE FOCALS

\begin{tabular}{|c|c|c|c|c|c|}
\hline & & FOCAL 3 & \multicolumn{3}{|c|}{ INTERACTOR } \\
\hline & & BEHAVIOR & DOLPHIN 1 & DOLPHIN 2 & DOLPHIN 3 \\
\hline & FOCAL 2 & \multicolumn{3}{|c|}{ INTERACTOR } & $x_{1: 3}$ \\
\hline & \multirow[t]{2}{*}{ BEHAVIOR } & DOLPHIN 1 & DOLPHIN 2 & DOLPHIN 3 & $x_{2,3}$ \\
\hline FOCAL 1 & & \multicolumn{2}{|l|}{ INTERACTOR } & $x_{17}$ & \multirow[t]{5}{*}{$x_{33}$} \\
\hline BEHAVIOR & DOLPHIN I & DOLPHIN 2 & DOLPHIN 3 & $x_{23}$ & \\
\hline TYPE 1 & $x_{11}$ & $\mathrm{x}_{12}$ & $x_{13}$ & $x_{3,3}$ & \\
\hline TYPE 2 & $\mathrm{X}_{21}$ & $\mathrm{X}_{22}$ & $\mathrm{X}_{23}$ & & \\
\hline TYPE 3 & $x_{31}$ & $x_{32}$ & $x_{33}$ & & \\
\hline
\end{tabular}

C. Multivariable TABle For Multiple Focals

\begin{tabular}{|c|c|c|c|c|}
\hline \multirow{2}{*}{ FOCAL } & BEHAVIOR & \multicolumn{3}{|c|}{ INTERACTOR } \\
\cline { 2 - 5 } & DOLPHIN 1 & DOLPHIN 2 & DOLPHIN 3 \\
\cline { 2 - 5 } & TYPE 1 & $\mathrm{X}_{111}$ & $\mathrm{X}_{112}$ & $\mathrm{X}_{113}$ \\
\cline { 2 - 5 } & TYPE 2 & $\mathrm{X}_{121}$ & $\mathrm{X}_{122}$ & $\mathrm{X}_{123}$ \\
\hline \multirow{2}{*}{ FOCAL 2 } & TYPE 3 & $\mathrm{X}_{131}$ & $\mathrm{X}_{132}$ & $\mathrm{X}_{133}$ \\
\cline { 2 - 5 } & TYPE 1 & $\mathrm{X}_{211}$ & $\mathrm{X}_{212}$ & $\mathrm{X}_{213}$ \\
\cline { 2 - 5 } & TYPE 2 & $\mathrm{X}_{221}$ & $\mathrm{X}_{222}$ & $\mathrm{X}_{223}$ \\
\hline FOCAL 3 & TYPE 3 & $\mathrm{X}_{231}$ & $\mathrm{X}_{232}$ & $\mathrm{X}_{233}$ \\
& TYPE 1 & $\mathrm{X}_{311}$ & $\mathrm{X}_{312}$ & $\mathrm{X}_{313}$ \\
& TYPE 2 & $\mathrm{X}_{321}$ & $\mathrm{X}_{322}$ & $\mathrm{X}_{323}$ \\
\cline { 2 - 5 } & TYPE 3 & $\mathrm{X}_{331}$ & $\mathrm{X}_{332}$ & $\mathrm{X}_{333}$ \\
\hline
\end{tabular}




\section{Chapter 3: Social Relationships}

can be categorized in two different ways: 1) by what type of behavior it is (e.g. affiliative, agonistic, etc.), and 2) by which dolphin the focal is interacting with. The behaviors can then be laid out in a contingency table with behavior type and interactor as the categories (Figure 3.1A). A similar table can be made for each focal (Figure 3.1B). In order to be able to see the interactions of all the focals simultaneously, the tables are put together to form a single, multivariable contingency table (Figure 3.1C). The categories, in this case focal animal, behavior type, and interacting dolphin, are called the "variables" of the contingency table.

The number in each cell of the contingency table (e.g. $X_{123}$, see Figure 3.1C) is the number of times that focal (Focal 1) engaged in that behavior (Type 2) while interacting with that dolphin (Dolphin 3). If a focal animal performed one type of behavior more than another, the number of behaviors in each cell will depend on which behavioral category the cell represents. For instance, if Focal 1 performs Behavior Type 1 more often than Type 2, the numbers in the Behavior Type 1 cells of Focal 1 's section of the table will be greater than the numbers in the Behavior Type 2 cells of Focal 1's section. Behavior is then said to have an effect on the data. If the focal had more interactions with one dolphin than with another, the number in each cell will depend on which interacting dolphin the cell represents and interactor has an effect on the data. If the focal performs Behavior Type 1 more with Dolphin 1 than Dolphin 2 and Type 2 more with Dolphin 2 than Dolphin 1, the number in each cell depends both on which behavior type and which interacting dolphin the cell represents. Behavior type and interactor are then said to have an interaction effect on the data. If how often a certain behavior is performed depends on the relationship between the dolphins, the numbers of behaviors will depend both on which focal is performing the behavior and which dolphin the focal is interacting with. This would be seen as an interaction effect of focal animal and interacting dolphin. Therefore, behaviors that demonstrate an interaction effect between focal and interactor are useful in differentiating social relationships. These are the behaviors of interest to this study. 
Loglinear Analysis

With three variables, there are seven possible effects: Focal $(\mathbf{F})$; Interactor $(\mathbf{I})$; Behavior (B); the interaction of Focal and Behavior (FB); the interaction of Focal and Interactor (FI); the interaction of Interactor and Behavior (IB); and the three-way interaction of Focal, Interactor, and Behavior (FIB). Groups of behaviors that will aid in distinguishing relationships will show an interaction between Focal and Interactor (FI) or a three-way interaction of Focal, Interactor, and Behavior (FIB). Other effects may be present in the data as well, but the presence of other effects would not change the usefulness of the behaviors in distinguishing relationships. To determine which of these possible effects have a significant influence on the data, the multivariable contingency table can be analyzed using loglinear models (Systat 7, SPSS Inc. 1997; Knoke \& Burke 1980). In loglinear analysis, a model is created to take into account all the possible effects, and a new table is generated based on the expectations of the model. The expected table is then compared to the observed data to see how well the model fits. The counts in the expected table of a loglinear model are calculated by multiplying the geometric mean of all the cells in the observed table by parameters representing the variables and their interactions. The parameters are calculated from the odds that a randomly selected behavior will fall into a certain cell or category. The odds of being in cell $\mathrm{A}$, for instance, is the number of behaviors in cell A divided by the total number not in cell $\mathrm{A}^{1}$

A model including all possible effects of the variables and their interactions is called a saturated model. The expected table of a saturated model always matches the observed table exactly. To determine which parameters have a significant effect on the observed variation, unsaturated models can be generated where certain variables or interactions have no influence. This is done by setting the parameters for those effects equal to 1 . These models have degrees of freedom equal to the number of parameters

\footnotetext{
${ }^{1}$ Cells with a count of zero cause problems to this procedure because calculating the odds could necessitate dividing by zero. A small number, 0.5 , is added to all cells before the odds are calculated to circumvent this problem (Knoke \& Burke 1980). Because the numbers in the cells are counts of behaviors and therefore integers, 0.5 is half the smallest number that could represent an actual behavior.
} 
Chapter 3: Social Relationships

that were set to 1 . The fit of these models to the data can be tested with a likelihood ratio. $^{2}$ If the expected counts in most cells are greater than five, the likelihood ratio has a $\chi^{2}$ distribution. If the expected counts are not greater than five, the $\mathrm{p}$-value from the $\chi^{2}$ distribution may not be accurate (Colgan \& Smith 1978). Raferty's BIC measure ${ }^{3}$ (Raferty 1986) is better for determining which model best describes the data when some cells have very small expected frequencies. The BIC measure is designed to make a tradeoff between the likelihood ratio (how well the model fits) and the degrees of freedom (how parsimonious the model is) and therefore allow a direct comparison between models. The interpretation of the BIC does not depend on the distribution of the data, and is therefore not sensitive to small expected frequencies. If the $\mathrm{BIC}$ is negative, the model in question is preferable to the saturated model in that the fit to the data is equivalent and the model in question is more parsimonious than the saturated model. If a model without a particular effect fits the data, or is preferable to the saturated model, that interaction does not have a significant impact on the data. When comparing several models, the best model is the one with the lowest (most negative) BIC value.

The loglinear models used here were all hierarchical. This means that if a higher order effect is present in the model (e.g. a Focal-Interactor interaction), all possible lower-order effects of those variables (in this case a Focal effect and an Interactor effect) must also be included. The models can therefore be designated by the highest order effects included for each variable. The saturated model for a Focal-InteractorBehavior comparison would be denoted FIB, which would indicate that the three-way interaction (FIB), all two-way interactions (FI, FB, IB), and all single variable effects (F, I, B) are included. A model that included only a Focal-Interactor interaction and a single variable effect of Behavior would be designated FI, B. Because the model is hierarchical, the presence of the FI parameter implies that the single variable effects of both F (Focal) and I (Interactor) are included as well, so this model includes the

${ }^{2} L^{2}=2 \sum f_{i j} \ln \left(f_{i j} / F_{i j}\right), f_{i j}=$ observed count, $F_{i j}=$ expected count. 
parameters for FI, F, I, and B. The model designated simply FI, on the other hand, would include an effect of the Focal-Interactor interaction (FI), and the single variable effects of both Focal (F) and Interactor (I), but no effect of Behavior (B).

\section{Behavior Types}

The parameters of a loglinear model depend on the categorical nature of the data. The model therefore requires that each count be independent and each behavior only fall into one category. Therefore, if interactor is a category, behaviors that occur simultaneously with two interactors cannot be used because they will be counted in two categories. The total association between animals is an example of this type of measure. Focal animals can be swimming with two other animals at the same time. This time is counted under both interactors (see chapter 2). Total association, therefore, cannot be analyzed using loglinear models.

The requirement of independence also means that the use of loglinear models to analyze behavioral states is problematic. If the data are represented in minutes, for instance, each count represents a single minute. The design of loglinear analysis assumes that each minute falls completely into one category and is independent of all other minutes. If the data were sampled using 1/0 sampling (see Altmann 1974), each minute would be assigned to a single category and these assumptions would be valid. Similarly, if the states were recorded using point sampling (see Altmann 1974), each point sample would be a count and the data would be both independent and categorical. However, if the data were collected continuously, a given minute may include time spent in multiple states. In that case, the parts of the minute would be counted in more than one category. Alternatively, a behavioral state could encompass more than one minute, in which case the minutes might not be independent. Changing the time scale used in the analysis could solve both of these problems. For instance, if the accuracy of the recording was several seconds, the analysis could be done in tenths of minutes ( 6 seconds) rather than minutes.

${ }^{3} B I C=L^{2}-(d f) \log N, d f=$ degrees of freedom, $N=$ total sample size (number of occurrences). For large $\mathrm{N}, \mathrm{BIC}$ is approximately $-2 * \log (\mathrm{B})$, where $\mathrm{B}$ is the probability that this model is preferable divided by the probability that the saturated model is preferable. 
Chapter 3: Social Relationships

Most tenths would likely fall into only one category. However, the total count will change depending on the unit of time used. Since the parameters of the model depend heavily on the total count, the preferred model could change as well. This problem will be demonstrated with an analysis of behavioral states in the following section.

On account of these issues, only behavioral events were analyzed using loglinear analysis. These included affiliative, agonistic, and calf-related behaviors (see section 2.2.2). The behavioral events were represented as the total number of events that occurred. Calf-related behaviors were only used in the analysis of calf relationships (section 3.3). Agonistic interactions were only used for adult relationships (section 3.4) because agonism with calves was rare. The calf analysis therefore included only affiliative and calf-related behaviors. The calf-related behaviors, nurse and retrieve, were analyzed as separate behaviors rather than as a single category.

\subsubsection{DEFINING RELATIONSHIP TYPES}

Loglinear models can be used to distinguish between social relationships by determining which behaviors vary depending on who is interacting. The relationship between a particular pair of dolphins can then be characterized by how often that pair performs those behaviors when interacting with each other. The next step is to use those behaviors to classify the relationships into types. Two methods were used for this analysis: multidimensional scaling (MDS) and hierarchical cluster analysis (HCA). MDS is a method plotting cases in space based on their relative ranks on each measurement (see Figure 3.4A). HCA is a method of detecting natural groupings in data by connecting cases based on their similarity (see Figure 3.4B).

For both analyses, all the interactions of each pair of dolphins were tabulated (see Table 3.8). While loglinear analysis relies on odds, allowing counts to represent different amounts of time, MDS and HCA compare the numbers themselves. Numbers must therefore represent equivalent time periods. For this reason, rates per sample were used for all measures. Events were represented as rate of occurrence per sample, and states as minutes per sample. Both were averaged over all samples. Because averages were used, 
behavioral states, representing the average duration spent in that state, could be included. In addition, because the interactions between pairs do not have to be mutually exclusive, total association could be included, also represented as minutes per sample. The behavioral measures used were the same as in the loglinear analysis, with the addition of total association and for the calves, the time each adult was the calf's nearest neighbor, divided into time with and without other adults present (see section 2.2.2). However, all the measurements used in MDS and HCA need to be equivalent. Therefore, the measurements for each behavior type were standardized. This was done by converting each measurement to a z-score, by first subtracting the mean for that behavior type and then dividing by the standard deviation. When this has been done, each behavior type had a mean of 0 and a standard deviation of 1 .

Each point on the MDS plot, or branch of the HCA tree, therefore represents a relationship between a pair of dolphins, not an individual dolphin, and is determined by all the interactions of that pair. Each relationship is a "case" in the analysis. If the relationships can be categorized into types, relationships of the same type will be placed close together on the MDS and HCA plots. The grouping of cases on these plots can therefore be used to define relationship types.

\section{Multidimensional Scaling}

When the values for a number of measurements are used to determine the similarity between cases in MDS, a method known as "unfolding" is used in Systat (Torgerson 1958). In this method, the distance between cases is determined from their relative ranks on the scales of the various measurements. Because only the ranks are used, this procedure is a "non-metric" version of MDS. First the variables are plotted relative to each other and the midpoints between those variables are calculated. The cases are then placed among the variables based on the ranking of each variable for each case. From this, a preliminary set of coordinates is computed. A stress measure is calculated by comparing the computed distances between cases to the actual distances between the cases for all the variables. The distances between the points are then shifted 


\section{Chapter 3: Social Relationships}

iteratatively to minimize the stress. To make the plots easier to read, only the points representing cases are shown, with the points representing the variables excluded.

\section{Hierarchical Cluster Analysis}

For the HCA, the "similarity" between cases was calculated by taking the Euclidean distance (multivariate root mean-squared distance) normalized by sample size (the Euclidean distance option in Systat). The clustering algorithm first links the cases with the smallest distance. Cases are then joined to clusters, and clusters to each other, in hierarchical order of their similarity, to form a clustering tree (see Figure $3.3 \mathrm{~B}$ ). When clusters are to be joined, the distance between the two clusters is computed by averaging the distances between all pairs where one case is in each of the clusters (the average linkage option).

To determine how many clusters to divide the trees into, a moat index was calculated for each possible number of clusters (in Matlab and Excel, see Appendix 2). The moat index is the average cluster cohesion for a given number of clusters. The cluster cohesion is calculated by subtracting the maximum distance between cases within a cluster from the minimum distance between cases in that cluster and cases outside that cluster (Podos et. al. 1992) ${ }^{4}$. This index was calculated for every possible number of clusters, from 1 to the number of cases, and the number of clusters used was the one that maximized the moat index.

${ }^{4} M_{n}=\frac{\sum_{i=1}^{n}\left[\min \left(B_{i}\right)-\max \left(W_{i}\right)\right]}{n}$, for $n$ clusters. $B=$ distance between cases in the cluster and cases outside the cluster, $W=$ distance between cases within the cluster. 


\subsection{RELATIONSHIPS BETWEEN CALVES \& ADULTS}

\subsubsection{RELATIONSHIPS}

Nephele's, Vicky's, and Delphi's Calves

The first three calves born in this study died within ten days of birth. Focal samples were taken on all three throughout their lives, with the calves themselves as the focal animals. All three spent a great deal of time with their mothers and very little with any other adult (Figure 3.2A-C). None of the calves was ever alone with an adult that was not his mother, nor did any spend more than a few seconds with a nearest neighbor that was not his mother (Table 3.1). The calf-related behaviors, nursing and retrieve, occurred only between calves and their mothers. The calves also had a great deal of affiliative contact with their mothers, and almost none with any other adult (Table 3.1). Overall, the calves interacted a great deal with their mothers and very little with other adults. The interactions each calf had with each adult might therefore be explained by the adult's genetic relationship, i.e. mother or not-mother, with the calf.

This hypothesis was tested with two sets of loglinear models. First, the null hypothesis that the interactions did not depend on any relationship was tested. Loglinear analysis was performed using focal calf, behavior type, and adult interactor as variables. If the null hypothesis was correct, an interaction effect between calf and adult should not be necessary. If the calves did have different relationships with different adults, no model without that interaction should fit. As might be expected, all three variables had an effect, demonstrating that there is inter-individual variation for both calves and adults and variation in how often different behavior types occur (Table 3.2A). However, no model that did not also include an interaction between interacting adult and focal calf fit the data (Table 3.2A; best fitting model: AC, CB). The null hypothesis can therefore be rejected: all the behaviors tested varied by which calf was interacting and which adult the calf was interacting with. In addition, the solutions to all the models that included this adult-calf interaction were not unique, meaning that there were multiple ways of designing the model to achieve the same solution. This could 


\section{Chapter 3: Social Relationships}

Figure 3.2: Total Time In Association: First Three Calves

(Mean of the 5 samples on each day, \pm standard error.)

Nephele was moved from the pool before Vicky's calf and Delphi's calf were born.

\section{A. NEPHELE'S CALF}

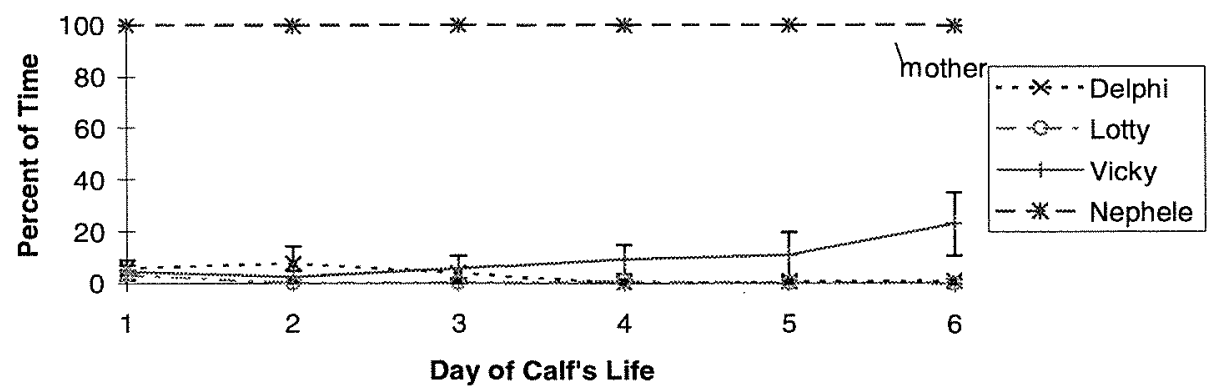

\section{B. VICKY'S CALF}

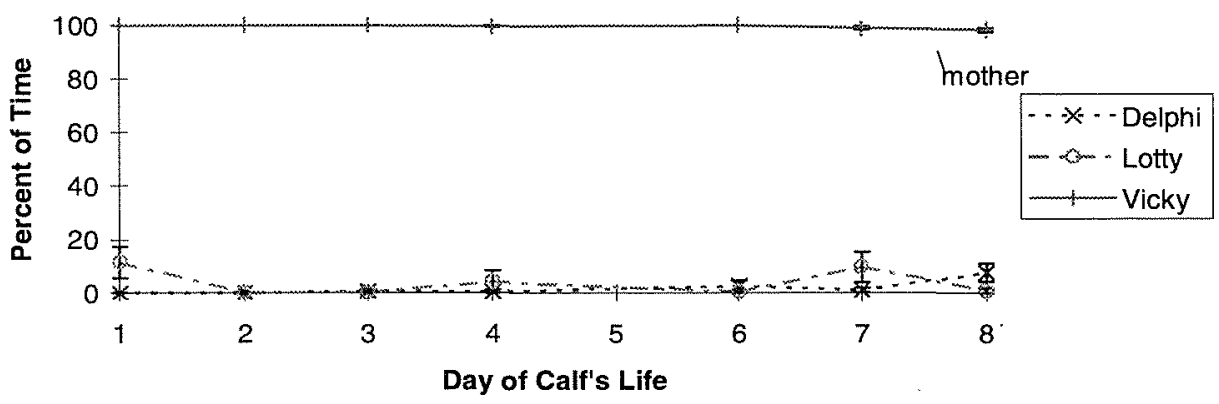

C. DELPHI'S CALF

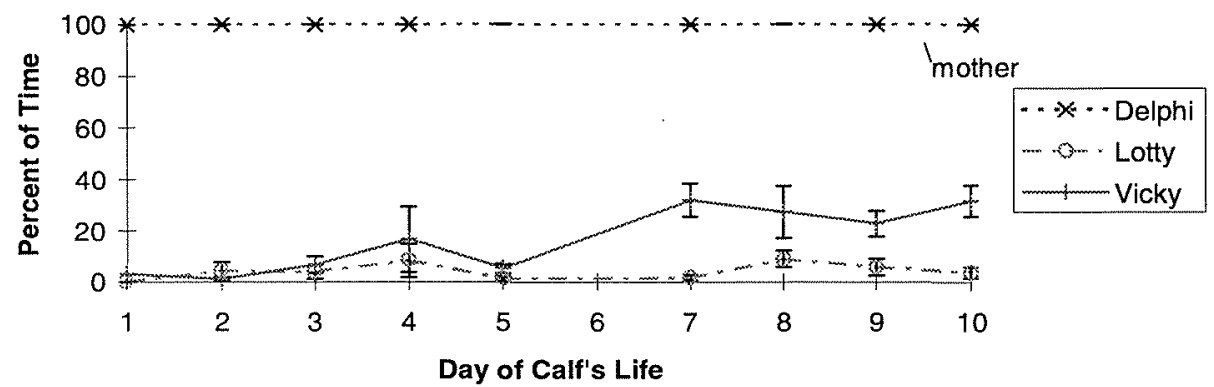




\section{TABLE 3.1: INTERACTIONS OF FIRST THREE CALVES}

Total Association is presented in percent of the total time, nearest neighbor data as minutes per sample (each sample was 10 minutes), and event data as number of occurrences per sample. Highlighted cells indicate the calf's interactions with his mother. Blank cells were counted as "structural zeros" and not used in the loglinear models. Nephele was moved from the pool before Vicky's and Delphi's calves were born.

\begin{tabular}{|l|l|c|c|c|c|}
\hline Calf & Behavior type & Nephele & Vicky & Delphi & Lotty \\
\hline Nephele's Calf & Total Association & $100.0 \%$ & $9.2 \%$ & $3.2 \%$ & $0.7 \%$ \\
$(\mathrm{~N}=27)$ & Nearest Neighbor, no other adults & 8.9 & 0 & 0 & 0 \\
& Nearest Neighbor, other adults & 1.1 & 0 & 0 & 0 \\
& Affiliative Contact & 7.6 & 0 & 0 & 0 \\
& Nursing & 2.1 & 0 & 0 & 0 \\
& Retrieves & 2.4 & 0 & 0 & 0 \\
\hline Vicky's Calf & Total Association & & $99.5 \%$ & $1.8 \%$ & $3.9 \%$ \\
$(\mathrm{~N}=35)$ & Nearest Neighbor, no other adults & & 9.1 & 0 & 0 \\
& Nearest Neighbor, other adults & & 0.8 & 0.003 & 0 \\
& Affiliative Contact & & 20.7 & 0 & 0 \\
& Nursing & & 0.8 & 0 & 0 \\
& Retrieves & & 0.9 & 0 & 0 \\
\hline Delphi's Calf & Total Association & & 0 & $7.3 \%$ & 0.9 \\
$(\mathrm{~N}=41)$ & Nearest Neighbor, no other adults & & 0 & 1.9 & 0 \\
& Nearest Neighbor, other adults & & 0.1 & 1.0 & 0 \\
& Affiliative Contact & & 0 & 2.3 & 0 \\
& Nursing & & 0 & 0.7 & 0 \\
\hline
\end{tabular}

occur if two variables being used in the analysis were correlated. Since each calf only had one mother, and each mother only one calf, the adult and calf variables would be correlated if the variation depended primarily on whether the adult was the calf's mother.

The second loglinear analysis tested this hypothesis. Each adult was coded as to whether or not she was each calf's mother, and loglinear analysis was performed as before using this relationship in place of adult. If that relationship did explain the differences in the amount of interaction between the calves and the various adults, an interaction effect between mother and calf should not be needed. The values used for the non-mother category for each calf was the sum of the values for all the adults in that category. Again, all variables had an effect but now the interaction between adult relationship and calf could be discounted (Table 3.2B; best fitting model: CB, M). This confirms the hypothesis that the variation in how often each calf performed each behavior when interacting with a particular adult depended primarily on whether the adult was that calf's mother. 
Chapter 3: Social Relationships

TABle 3.2: BIC VAlues From LoglineAR Models of DATA For FIRST THREe CAlves Letters represent model designations (see section 3.2.1). All models are hierarchical. Highlighted cells indicate the preferred model.

\section{A. BeHAVIORAL EVENTS}

$\mathbf{X}=$ Adult Code $; \mathrm{C}=$ Calf; $\mathrm{B}=$ Behavior. ${ }^{\mathrm{t}}$ The solutions to these models are not unique.

\begin{tabular}{|c|c|c|}
\hline MODEL & $\mathrm{X}=$ ADUIT & $\begin{array}{l}\text { X }=\text { RELATIONSHIP: } \\
\text { MOTHER/NON-MOTHER }\end{array}$ \\
\hline $\mathrm{XC}, \mathrm{XB}, \mathrm{CB}$ & $-55.3^{t}$ & -27.0 \\
\hline $\mathrm{XC}, \mathrm{XB}$ & $-81.2^{\ddagger}$ & 44.6 \\
\hline $\mathrm{XC}, \mathrm{CB}$ & $-98.3^{7}$ & -39.4 \\
\hline $\mathrm{XB}, \mathrm{CB}$ & 1643.7 & -39.7 \\
\hline $\mathrm{XB}, \mathrm{C}$ & 1693.1 & 106.5 \\
\hline $\mathrm{CB}, \mathrm{X}$ & 1683.4 & -51.5 \\
\hline $\mathrm{XC}, \mathrm{B}$ & $-18.1^{\ddagger}$ & 32.8 \\
\hline $\mathrm{X}, \mathrm{C}, \mathrm{B}$ & 1756.2 & 20.6 \\
\hline
\end{tabular}

\section{B. TIME AS NeAREst NeIGHBOR}

$\mathrm{A}=$ Adult; $\mathrm{C}=$ Calf; $\mathrm{B}=$ Behavior. ${ }^{\ddagger}$ The solutions to these models are not unique.

\begin{tabular}{|l|c|c|c|c|}
\hline MODEL & HoURS & TEN MiNuTES & MINUTES & TENTHS OF MINUTES \\
\hline ACB (Saturated) & $0^{\ddagger}$ & $0^{\ddagger}$ & $0^{\ddagger}$ & $-64.6^{\ddagger}$ \\
$\mathrm{AC}, \mathrm{AB}, \mathrm{CB}$ & $-10.3^{\ddagger}$ & $-16.6^{\ddagger}$ & $-25.3^{\ddagger}$ & $-69.6^{\ddagger}$ \\
$\mathrm{AC}, \mathrm{AB}$ & $-15.4^{\ddagger}$ & $-24.9^{\ddagger}$ & $-38.0^{\ddagger}$ & $-77.2^{\ddagger}$ \\
$\mathrm{AC}, \mathrm{CB}$ & $-17.3^{\ddagger}$ & $-27.3^{\ddagger}$ & $-42.0^{\ddagger}$ & $-91.1^{\ddagger}$ \\
$\mathrm{AB}, \mathrm{CB}$ & -10.4 & 56.6 & 947.8 & $10,044^{\ddagger}$ \\
$\mathrm{AB}, \mathrm{C}$ & -15.2 & 50.5 & 955.3 & $10,236^{\ddagger}$ \\
$\mathrm{CB}, \mathrm{A}$ & -17.1 & 48.0 & 951.3 & $10,232^{\ddagger}$ \\
$\mathrm{AC}, \mathrm{B}$ & $-22.9^{\ddagger}$ & $-35.1^{\ddagger}$ & $-42.1^{\ddagger}$ & $97.4^{\ddagger}$ \\
$\mathrm{A}, \mathrm{C}, \mathrm{B}$ & -22.7 & 40.3 & 958.2 & $10,429^{\ddagger}$ \\
$\mathrm{AB}$ & -22.2 & 119.6 & 2,075 & 22,192 \\
$\mathrm{CB}$ & -23.1 & 240.1 & 2,624 & 28,012 \\
$\mathrm{AC}$ & $-19.6^{\ddagger}$ & $15.7^{\ddagger}$ & $572.7^{\ddagger}$ & $6,305^{\ddagger}$ \\
$\mathrm{C}, \mathrm{A}$ & -21.7 & 165.5 & 2,671 & 28,347 \\
$\mathrm{~A}, \mathrm{~B}$ & -29.9 & 109.0 & 2,077 & 22,384 \\
$\mathrm{C}, \mathrm{B}$ & -28.7 & 149.0 & 2,631 & 28,210 \\
$\mathrm{C}$ & -25.9 & 198.6 & 3,237 & 34,417 \\
$\mathrm{~B}$ & -34.1 & 142.1 & 2,643 & 28,455 \\
$\mathrm{~A}$ & -27.1 & 158.6 & 2,684 & 28,592 \\
\hline
\end{tabular}

To demonstrate the problem of using loglinear analysis on continuous duration data, the nearest neighbor data for the first three calves was analyzed using loglinear analysis. As before, the time each adult spent as each calf's nearest neighbor was divided into time with other adults in the group and time without any other adults in the group. The counts in each cell of the table were the total number of minutes spent in each 
behavioral state. The results of changing the time unit to tenths of minutes, tens of minutes, and hours were then compared (Table 3.2B). The first line of the table indicates the BIC value for the saturated model. Because the saturated model should have no degrees of freedom, this number should be 0 . The non-zero result that occurred for tenths of minutes may be a result of poor convergence of the model, i.e. that the expected frequencies of the saturated model did not exactly match the observed frequencies. Highly skewed loglinear models, where some numbers are much larger than others, often have difficulty converging on a solution (Colgan \& Smith 1978). The large numbers that occurred in this test may have prevented the model from properly converging.

The highlighted cells in this table indicate the preferred model. When minutes or tens of minutes are used, the preferred model is $\mathbf{A C , ~ B}$. This result is similar to the results of the loglinear analysis of the behavioral events. However, when the data is divided into hours, rather than minutes, the preferred solution includes only an effect of behavior (B) with adult and calf having no effect on the data at all. There is a clear progression of an increasing number of significant effects as the time unit decreases and the sample size increases. The time unit chosen would therefore heavily influence the conclusions that would be drawn from these data. However, the pattern of interest in the data has not actually changed, only the size of the difference has changed. Since the data were collected as continuous durations, the proper time unit is not obvious. Therefore, the proper conclusion to draw from the analysis is not obvious, and loglinear models cannot be used to analyze duration.

\section{Lotus}

Lotus' interactions with the adults in his environment did not mirror those of the three calves who had come before him (Figure 3.3). He interacted quite a bit more with adults other than his mother, Lotty, and quite a bit less with his mother in his first week than the others had. For these purposes, only Lotus' first three weeks were analyzed to make the data comparable to the other three calves, none of whom lived more than ten days (Lotus' later weeks are analyzed below). As was discussed in chapter 2, Lotus had 
FIGURE 3.3: Total TIME IN AsSOCIATION: LotuS

(Mean of the 5 samples on each day, \pm standard error.) Delphi was moved from the pool when Lotus was 6 days old. On that day, Lotus was alone with the trainers.

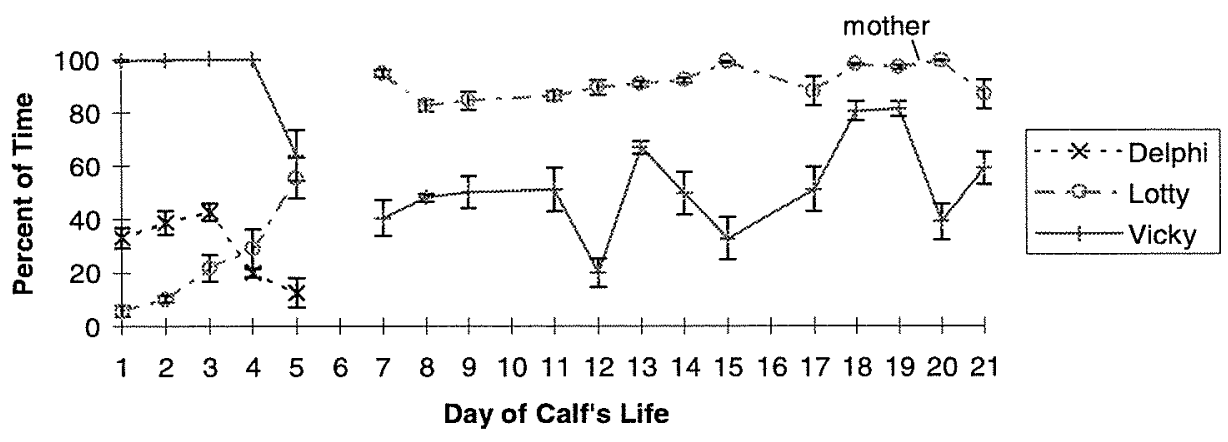

an unusual first week. As soon as Lotus was born, Vicky swam up to him. Before his mother, Lotty, could turn around, Lotus had gone to the surface with Vicky. Lotty made no obvious attempt to reclaim him, and Lotus remained with Vicky for the next five days. On his fifth day, he became very sick and was removed from the Lagoon by the Kolmårdens Djurpark staff. He remained with them for a full day before being returned to the Lagoon. Shortly after Lotus was returned, Vicky appeared to lose interest in him and he returned to his mother, Lotty. The sixth day therefore appears to be a break-point in his association patterns, as can be seen from Figure 3.3. Before day 6, Lotus spent most of his time with Vicky; after, he spent most of his time with his mother, Lotty.

When Lotus' interactions with the adults are summed over the entire three weeks, he does not appear to interact predominantly with one adult as the earlier calves did with their mothers (Table 3.3A). However, if Lotus' interactions are separated into the two time periods discussed, before and after the sixth day, there is one adult in each time period who interacted with Lotus more than the other adults did (Table 3.3B). As expected from the association patterns, this was Vicky before day 6 and Lotty after. A closer look at Figure 3.3 reveals that on day 5, Lotus spent less time with Vicky, and more time with Lotty, than he did on days 1 through 4 . When the data from day 5 are separated from the data from days 1 through 4 , a pattern even more similar to the earlier calves' behavior emerges (Table 3.3C). On days 1 to 4 , Lotus had interactions very 
TABLE 3.3: LotUS' INTERACTIONS

All numbers are represented as in Table 3.1. Delphi was moved from the pool when Lotus was 6 days old, so days 7-21 are not included in the calculations of Delphi's total interactions. Lotus was alone with trainers on his 6th day, so that day is also not included in the calculations of total interactions. Highlighted cells indicate Lotus' interactions with his caregiver (see text). Blank cells were counted as "structural zeros" and not used in the model.

A. TOTAL

\begin{tabular}{|l|l|c|c|c|}
\hline Time period & Behavior type & Vicky & Lotty & Delphi \\
\hline Days 1-21 & Total Association & $64.0 \%$ & $72.8 \%$ & $29.5 \%$ \\
(N=88) & Nearest Neighbor, no other adults & 1.7 & 3.5 & 0.2 \\
& Nearest Neighbor, other adults & 2.2 & 2.3 & 0.1 \\
& Affiliative Contact & 10.3 & 29.5 & 0.1 \\
& Nursing & 0.9 & 1.7 & 0.04 \\
& Retrieves & 0.4 & 0.7 & 0.2 \\
\hline
\end{tabular}

B. SEParated into Two Periods

\begin{tabular}{|c|c|c|c|c|}
\hline Time period & Behavior type & Vicky & Lotty & Delphi \\
\hline $\begin{array}{l}\text { Days } 1-5 \\
(\mathrm{~N}=23)\end{array}$ & $\begin{array}{l}\text { Total Association } \\
\text { Nearest Neighbor, no other adults } \\
\text { Nearest Neighbor, other adults } \\
\text { Affiliative Contact } \\
\text { Nursing } \\
\text { Retrieves }\end{array}$ & $\begin{array}{c}92.9 \% \\
4.7 \\
4.4 \\
30.8 \\
3.0 \\
1.0 \\
\end{array}$ & $\begin{array}{c}24.2 \% \\
0.4 \\
0.1 \\
0.1 \\
0.04 \\
0.3 \\
\end{array}$ & $\begin{array}{c}29.5 \% \\
0.2 \\
0.1 \\
0.1 \\
0.04 \\
0.2 \\
\end{array}$ \\
\hline $\begin{array}{l}\text { Day } 7-21 \\
(N=65)\end{array}$ & $\begin{array}{l}\text { Total Association } \\
\text { Nearest Neighbor, no other adults } \\
\text { Nearest Neighbor, other adults } \\
\text { Affiliative Contact } \\
\text { Nursing } \\
\text { Retrieves }\end{array}$ & $\begin{array}{c}53.0 \% \\
0.6 \\
1.5 \\
3.0 \\
0.2 \\
0.2\end{array}$ & $\begin{array}{c}91.5 \% \\
4.6 \\
3.3 \\
40.0 \\
2.3 \\
0.9 \\
\end{array}$ & \\
\hline
\end{tabular}

C. Separated into Three Periods

\begin{tabular}{|l|l|c|c|c|}
\hline Time period & Behavior type & Vicky & Lotty & Delphi \\
\hline Days 1-4 & Total Association & $99.9 \%$ & $16.7 \%$ & $33.7 \%$ \\
& Nearest Neighbor, no other adults & 5.2 & 0 & 0 \\
& Nearest Neighbor, other adults & 4.8 & 0 & 0.01 \\
& Affiliative Contact & 33.9 & 0 & 0 \\
& Nursing & 2.9 & 0 & 0 \\
& Retrieves & 1.2 & 0 & 0 \\
\hline Day 5 & Total Association & $64.6 \%$ & $54.5 \%$ & $12.9 \%$ \\
(N=5) & Nearest Neighbor, no other adults & 3.1 & 1.9 & 0.8 \\
& Nearest Neighbor, other adults & 2.8 & 0.5 & 0.3 \\
& Affiliative Contact & 19.6 & 0.4 & 0.6 \\
& Nursing & 3.2 & 0.2 & 0.2 \\
& Retrieves & 0 & 1.6 & 1.0 \\
\hline Days 7-21 & Total Association & $53.0 \%$ & $91.5 \%$ & \\
(N=65) & Nearest Neighbor, no other adults & 0.6 & 4.6 & \\
& Nearest Neighbor, other adults & 1.5 & 3.3 & \\
& Affiliative Contact & 3.0 & 40.0 & \\
& Nursing & 0.2 & 2.3 & \\
& Retrieves & 0.2 & 0.9 & \\
\hline
\end{tabular}




\section{Chapter 3: Social Relationships}

similar to the other three calves': many interactions with one adult, in this case Vicky, and almost none with any other adult. On day 5 and after day 6, he still had more interactions with one particular adult than with the other adults: Vicky on day 5 and Lotty after day 6 . However, in these periods, he had more interactions with all the adults than any of the previous three calves had with anyone other than their mothers.

To test the hypothesis that Lotus' interactions with the adults changed over time, these data were tested against loglinear models with adult, behavior, and time period as variables. Lotus' first three weeks were divided into three time periods for this analysis (see Table 3.3C). Because loglinear analysis uses the odds of being in various categories to calculate the parameters, the categories do not have to represent the same amount of time. If the hypothesis that Lotus' interactions with the different adults varied by time period is correct, an interaction effect between adult and time period should be required to fit the data. The best fitting model for the behavioral event data includes an interaction between adult and behavior and one between adult and time period (AB, AT: BIC = 11.8). No model without an interaction between adult and time period (AT) fit the data set. The adult-time period interaction means that the behaviors tested varied by both which time period the behavior occurred in and which adult Lotus was interacting with. This confirms the hypothesis that Lotus' interactions with the adults, and therefore his relationships with them, changed over the three time periods.

\subsubsection{RELATIONSHIP TYPES}

The change in Lotus' interactions with the various adults around days 5 and 6 suggests that classifying the adults as mother or not-mother, as was done with the previous three calves, may not be sufficient to explain the observations. A social equivalent of "mother", which does not have to be the calf's biological mother, might be preferable. This hypothesized relationship type will be called "caregiver" (Table 3.4). Caregivers are the adults that the calves spend most of their time with. All four calves had many interactions with their caregivers, which are highlighted in Tables 3.1 and 3.3. The first three calves' caregivers were their mothers. Lotus' caregiver was Vicky for the 
TABLE 3.4: HYPOTHESIZED CALF RELATIONSHIPS.

N/A: That adult was not present in the Lagoon during that period.

\begin{tabular}{|ll|c|c|c|c|}
\hline Calf & Time Period & Nephele & Vicky & Delphi & Lotty \\
\hline Nephele's & Entire Life & Caregiver & Poolmate & Poolmate & Poolmate \\
Vicky's & Entire Life & N/A & Caregiver & Poolmate & Poolmate \\
Delphi's & Entire Life & N/A & Poolmate & Caregiver & Poolmate \\
Lotus & Days 1 to 4 & N/A & Caregiver & Poolmate & Poolmate \\
& Day 5 & N/A & Caregiver & Associate & Associate \\
& Days 7 to 21 & N/A & Associate & N/A & Caregiver \\
\hline
\end{tabular}

first 5 days and Lotty subsequently. All the other adults can then be classified as "noncaregivers". The first three calves had almost no interactions with these adults. Lotus, on the other hand, had interactions with non-caregivers starting on day 5 (Table 3.3C). This suggests a third relationship type, unique to Lotus in this study, which shall be called "associate" (Table 3.4). Associates are adults other than the caregiver with whom the calf interacts and who spends some time as the calf's nearest neighbor.

These hypotheses were tested in several ways. All three hypotheses were first tested with loglinear models and then by plotting the relationships relative to each other with MDS and HCA. First, the null hypothesis that the genetic relationship (mother) is sufficient for all four calves, as it was for the first three calves, was tested with loglinear models. This is unlikely to be the case since Lotus' interactions with the adults changed over time (see previous section) but his mother (Lotty) did not. Next, the hypothesis that the social equivalent to mother, caregiver, accounts for the variation in calf interaction was tested with loglinear models. After this, all three relationship types were tested against loglinear models. As before, it is the interaction between adult relationship and calf that is important here. If being the calf's mother is sufficient to explain the difference in calf behavior, a mother-calf interaction effect should not be necessary to fit the data. The same holds true for all the relationships tested here: if the hypothesized relationships account for all the variation, a relationship-calf interaction effect should not be necessary. Finally, all the relationships were plotted using multidimensional scaling and hierarchical cluster analysis, as described in section 3.2.2, to determine whether the three hypothesized relationship types separated from each other with those techniques. 
Chapter 3: Social Relationships

\section{Loglinear analysis}

To test the null hypothesis, the interactions of all four calves were compared to loglinear models where the adults were classified as mother or not-mother for each calf. Lotus' mother was Lotty in all time periods. The preferred model included an interaction between calf and behavior and one between relationship (mother) and calf (CB, RC; Table 3.5). No model with out the latter interaction (RC) fit the data (Table 3.5). This interaction suggests that the four calves interacted with their mothers in different ways. The null hypothesis can therefore be rejected: the genetic relationship is not sufficient. Next, the hypothesis that the social equivalent of mother, "caregiver", accounted for the variation was tested. For each calf, the count used for each category was the sum of the counts for all the adults in that category in any time period. The best fitting model had all three dyadic interactions (RB, RC, CB; Table 3.5). Almost all the simpler models could be rejected (Table 3.5), although a model without the relationshipbehavior interaction also fit ( $\mathbf{R C}, \mathbf{C B}$; Table 3.5). The presence of a relationship-calf interaction in all these models indicates that "caregiver" is not sufficient. Another relationship, such as associate, needs to be defined.

The hypothesis that the variation would be accounted for by this third relationship type was therefore tested. All adults were coded as caregiver, associate, or poolmate (Table 3.4). Caregivers were defined above. Associates were defined as animals that spent at least one minute as the calf's nearest neighbor. Poolmates were all other animals. Again, for each calf the count used for each category was the sum of the counts for all the adults in that category in any time period. The best model included an interaction between relationship and behavior and one between calf and behavior (RB, CB; Table 3.5), although a model without the relationship-behavior interaction also fit (CB, R; Table 3.5). The lack of relationship-calf interactions (RC) in these models means that these three relationships account for most of the variation seen. In addition, the solutions to the models that included an interaction between relationship and calf were not unique. As before, this indicates that these variables may have been correlated, possibly because only Lotus had associates. 
TABLE 3.5: BIC VAlues FRom Loglinear Models of Events For All Four Calves $\mathbf{R}=$ Relationship; $\mathrm{C}=\mathrm{Calf} ; \mathrm{B}=$ Behavior. Letters represent model designations (see 3.2.1). All models are hierarchical. Highlighted cells indicate the preferred model. ${ }^{\ddagger}$ These solutions are not unique.

\begin{tabular}{|c|c|c|c|}
\hline MODEL & $\begin{array}{l}\mathbf{R}=\text { MoTmER } / \\
\text { NoT-MoTHER }\end{array}$ & $\begin{array}{l}\mathrm{R}=\text { CAREGIVER/ } \\
\text { NOT-CAREGIVER }\end{array}$ & $\begin{array}{c}\mathrm{R}=\text { Caregiver/ } \\
\text { PoOLMATE/ASSOCIATE }\end{array}$ \\
\hline $\mathrm{RC}, \mathrm{RB}, \mathrm{CB}$ & -47.9 & -48.8 & $-47.7^{\ddagger}$ \\
\hline $\mathbf{R C}, \mathbf{R B}$ & 74.4 & 68.2 & $66.3^{\ddagger}$ \\
\hline $\mathrm{RC}, \mathrm{CB}$ & -48.1 & -26.7 & $-40.7^{\ddagger}$ \\
\hline $\mathrm{RB}, \mathrm{CB}$ & 704.2 & 66.5 & -63.5 \\
\hline RB, C & 811.4 & 173.8 & 53.5 \\
\hline $\mathrm{CB}, \mathbf{R}$ & 688.9 & 78.8 & -53.5 \\
\hline RC, B & 59.1 & 80.5 & $66.1^{\ddagger}$ \\
\hline $\mathbf{R}, \mathrm{C}, \mathrm{B}$ & 796.1 & 186.0 & 53.3 \\
\hline
\end{tabular}

\section{Multidimensional Scaling and Hierarchical Cluster Analysis}

The relationships between the calves and adults were plotted relative to each other with multidimensional scaling (MDS) and hierarchical cluster analysis (HCA). All the measurements, including total association, were included after being standardized to $\mathrm{z}^{-}$ scores (as described in section 3.2.2). Both the MDS and HCA separated caregivers from associates and poolmates (Figure 3.4A,B). However, although the caregivers were different from the poolmates and associates, they did not form a single, cohesive group. This is particularly clear from the HCA (Figure 3.4B). The moat index, which measures the cohesiveness of clusters, indicates that there were significant differences in how each caregiver interacted with her calf. Interestingly, while Lotus' relationship with Vicky on day 5 was separated from his other caregiver relationships, his relationships with Vicky on days 1 to 4 and Lotty on days 7 to 21 were clustered together. As has been mentioned before, Lotus' interactions with Vicky on day 5 were unusual. The close clustering of his other two caregivers indicates that these two relationships were equivalent. Lotus' "caregiver" relationship was therefore completely transferred from Vicky to Lotty after day 6.

The poolmates formed a cohesive group in both analyses, but the associates did not (Figure 3.4A,B). In fact, all three associate relationships were separated from each other by the moat index (Figure 3.4B). This suggests that "associate", while generally different from "poolmate", may not represent a single relationship type. In both analyses, 


\section{Chapter 3: Social Relationships}

\section{FIGURE 3.4: CALF RELATIONSHIP TYPES}

The focal animals are listed first in italics. In all cases, the calves were the focal animals.

A. Multidimensional Scaling

Stress (a measure of the fit) $=0.03$; Proportion of variance accounted for $=0.65$

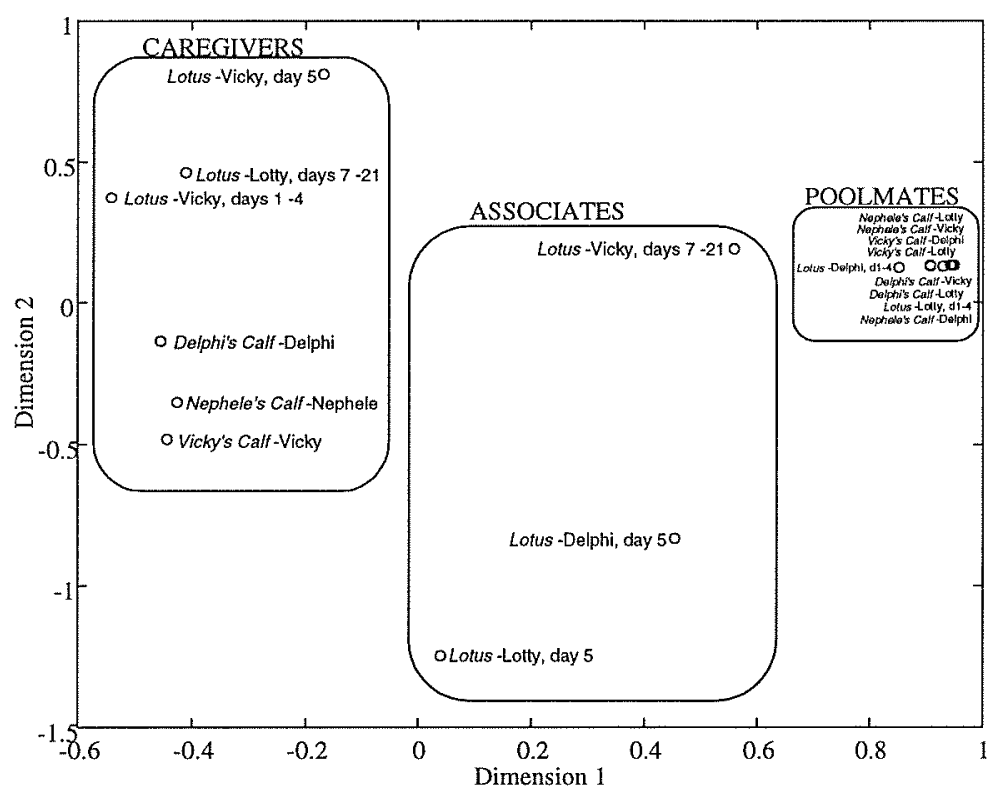

\section{B. HierarchicAl Cluster ANAlysis}

The dotted line through the clusters represents the moat index.

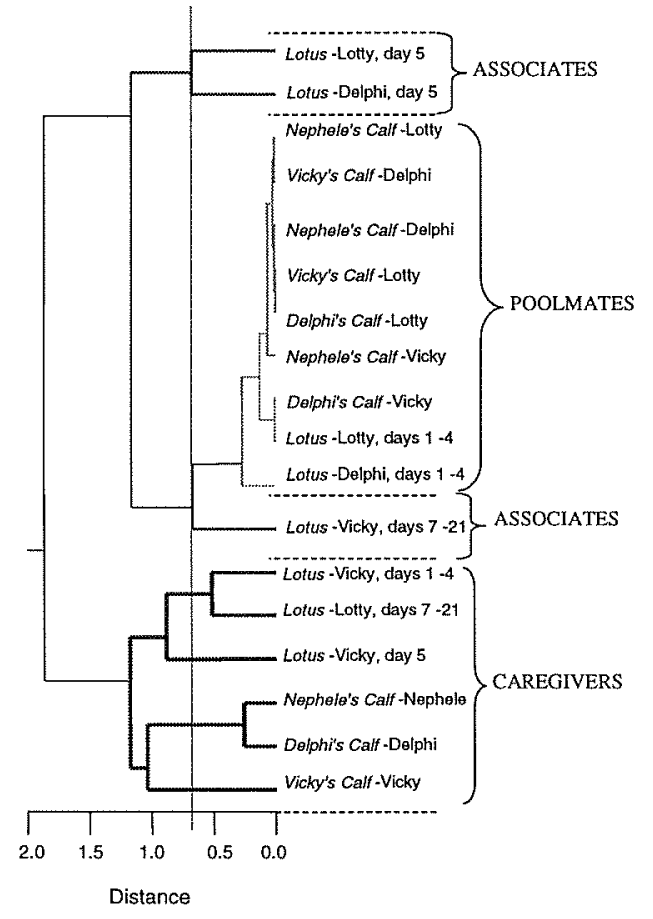




\section{Hierarchical Cluster Analysis of Total Association Only}

The dotted line through the clusters represents the moat index.

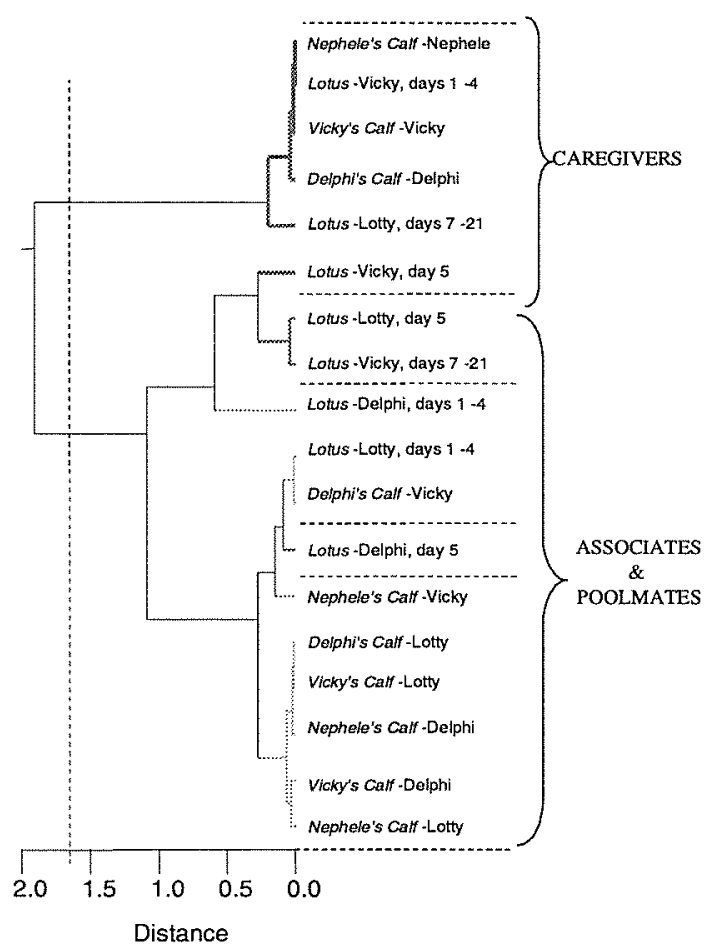

Lotus' relationship with Vicky between days 7 and 21 was closer to the poolmates than to the other associates. This may be due to the small number of times Vicky retrieved Lotus in this period (see Table 3.3C). Because all the variables were normalized, each variable has the same weight in the MDS and HCA as every other variable. Therefore, the smaller number of retrieves is equally weighted with the larger amount of affiliative contact and with the nursing (see Table 3.3C). However, there is no way to know which behaviors are most important to the dolphins themselves. By this analysis, two of the three hypothesized relationships appear to accurately represent the subtle bonds created by association, affiliative contact, nursing, and calf-protection behaviors such as retrieve and swimming as the calf's nearest neighbor. The third, associate, may represent a number of intermediate relationship types.

Because studies of free-ranging dolphins commonly use total association as the only measure (e.g. Smolker et al. 1992, Wells et al. 1987), a comparison of the results presented here to the results of using only total association would be interesting. When 
only total association was used, several aspects of the pattern were obscured. In particular, the poolmates were no longer a coherent group that could be separated from the associates (Figure 3.4C). In addition, the putative caregiver relationship between Lotus and Vicky on day 5 no longer groups with the other caregivers. This suggests that total association was reflecting a different process than the hypothesized relationships. An example of this was Lotus and Delphi. Their total association was unusually high on days 1 to 4 (Table 3.3C), and using only total association in the HCA moved their relationship during that period toward the group of associates. Conversely, their total association on day 5 was relatively low, and their relationship on day 5 moved into the group of poolmates when only total association was included. The likely cause for this, however, was the presence of Delphi's calf. During Lotus' first four days, Delphi's calf was alive, and Delphi and her calf swam with Vicky and Lotus a great deal. On Lotus' fifth day, Delphi's calf was dead and Delphi stopped swimming with Vicky and Lotus. However, the association between Delphi and Lotus was likely a byproduct of the association between Delphi and Vicky. In wild dolphins, females in similar reproductive condition, such as with young calves, are typically found swimming together (Wells 1991). Total association in this case may therefore be an indication of the adults' relationship with each other and not a good indication of the adults' relationships with the calves.

\subsubsection{LOTUS' LATER WEEKS}

In order to ensure that the data on Lotus were comparable to the data on the previous calves, none of whom lived for more than ten days, only the data for Lotus' first 21 days were used in the foregoing analysis. However, samples were taken on Lotus through his 70th day (Table 3.6). To determine whether calves' interactions with adults change as a calf ages, Lotus' interactions with Vicky and Lotty were evaluated to determine whether they remained consistent over time. The number of minutes per sample that Lotty and Vicky each spent as Lotus' nearest neighbor changed very little between days 7-21 and days 22-70 (Tables 3.3, 3.6). 
TABLE 3.6: LOTUS' LATER WEEKS

All measures are presented in the same manner as in previous tables.

\begin{tabular}{|l|l|c|c|}
\hline Time period & Behavior type & Vicky & Lotty \\
\hline Days 22-70 & Total Association & $40.9 \%$ & $82.9 \%$ \\
$(\mathrm{~N}=210)$ & Nearest Neighbor, no other adults & 0.6 & 4.6 \\
& Nearest Neighbor, other adults & 1.3 & 1.8 \\
& Affiliative Contact & 2.1 & 14.9 \\
& Nursing & 0 & 0.4 \\
& Retrieves & 0 & 0.5 \\
\hline
\end{tabular}

The behavioral events that occurred between Lotus and the adults did change, however. In particular, the number of interactions Lotus had with both adults decreased over the two periods. Most notably, although Lotus had affiliative contact with Vicky in both time periods, he only had calf-related interactions with her before day 21 . In addition, the amount Lotus nursed and his affiliative contact with his mother decreased considerably. In fact, loglinear models of these data, with adult (Lotty vs. Vicky), behavior, and time period (days 7-21 vs. days 22-70) as variables, did not converge. This means that the statistical package (Systat) could not determine a solution that fit the constraints of the model, such as matching the category totals. This was probably caused by the lack of calf-related interactions between Lotus and Vicky in the second period. Because of the large number of nursing events and retrieves between Lotus and Lotty between days 22 and 70, compared to none between Lotus and Vicky, these models were too highly skewed for the loglinear analysis to handle. Models using only calf-related interactions, without the affiliative contact, did not converge either. A model using only the affiliative contact data, and therefore without behavior as a variable, did converge but only the saturated model, including an interaction between adult and time period, fit the data $(\mathbf{A}, \mathbf{T}: \mathrm{BIC}=45.7)$. A model including all three behavior types but only Lotty's interactions converged but again, only the saturated model fit $(B, T: B I C=20.4)$. These results suggest that, although the time the two adults spent as Lotus' nearest neighbor did not change over time, the behavioral interactions Lotus had with both adults did change over time.

In addition, the total amount of time Lotus spent with both adults decreased between days 7 and 70 . In his first three weeks, Lotus spent $92 \%( \pm \mathrm{SE} 2 \%)$ of his time 
Figure 3.5: Total TIME LotUs SPENT AlONE (> 1M AWAY FROM AN ADULT)

(Mean of the 5 samples on each day, \pm standard error.)

On day 6, Lotus was in a separate pool with the trainers.

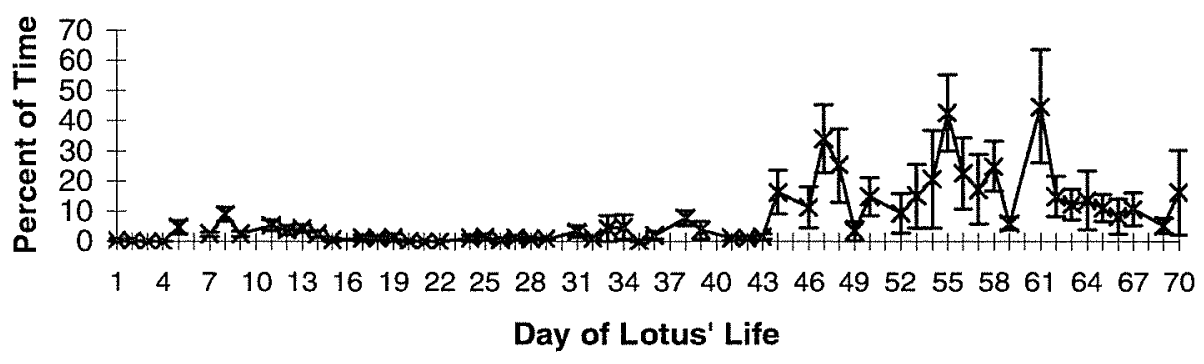

FIGURE 3.6: LOTUS' SEXUAL PLAY WITH VICKY AND LOTTY

(Mean of the 5 samples on each day, \pm standard error.)

On day 6, Lotus was in a separate pool with the trainers.

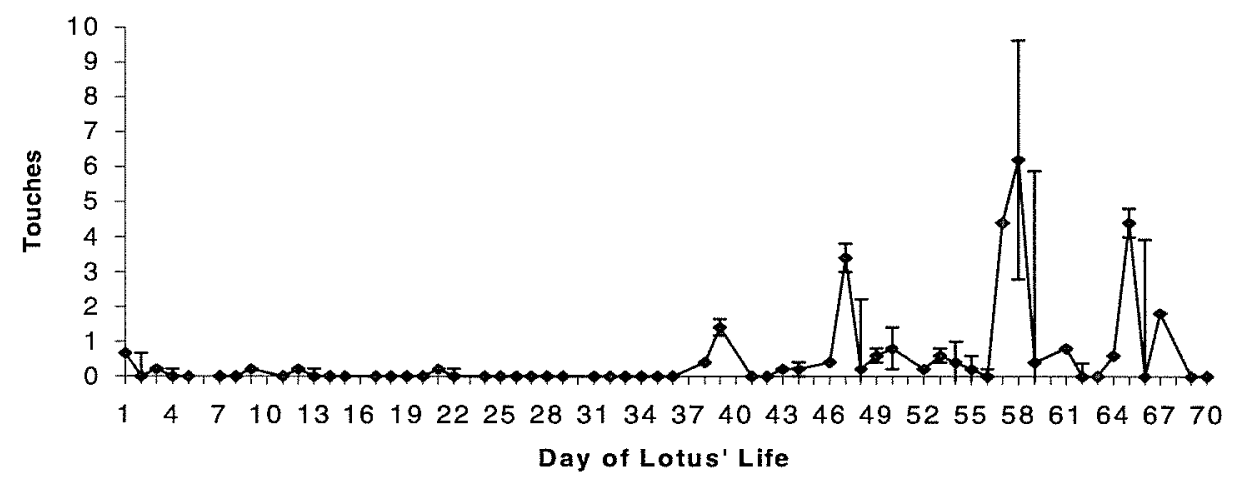

with his caregiver. In the last three weeks of the study, he spent only $81 \%( \pm \mathrm{SE} 3 \%)$ of his time with his caregiver. Concurrent with this decrease was a marked increase in the time Lotus spent alone ( $>1 \mathrm{~m}$ away from any adult), starting at about 36 days (Figure 3.5). These were indications of Lotus' growing independence. At about the same time, he began to play sexually with both adults (Figure 3.6). Sexual play was defined as Lotus rubbing his genital region on the adult, often with an erection accompanied by thrusting movements. However, he very seldom rubbed against the adults' genital or ventral regions. More often, he rubbed against their sides or peduncles. These results, taken together, suggest that calves do change how they interact with adults as they age. 


\subsubsection{DISCUSSION}

These analyses demonstrate how relationship types between two groups of animals, in this case calves and adults, can be determined from their interactions and association. A number of multivariate statistical techniques were used to show that adults could be separated into three relationship types: caregiver, associate, and poolmate, based on association, affiliative interactions, nursing, and calf-protection behaviors. For most calves, the caregiver is the biological mother, but Lotus' unusual situation allowed us to separate the relationship from the individual and the social relationship from the genetic one. The HCA also showed that there was individual variation in how each caregiver interacted with her calf. While the caregiver relationship appeared to be a single relationship type, the associate relationship did not. While associates interacted with calves more than poolmates did, they did not all interact with calves in the same way. The specific interactions that made up both the associate and caregiver relationship types changed slightly as the calf grew up and gained a measure of independence.

Interestingly, these relationships were not apparent if only the total association between animals was included in the analysis. This is important because most of the studies of the social relationships of wild dolphins rely solely on association (e.g. Wells 1991, Smolker et al. 1992). In this analysis, other measures such as affiliative contact and behaviors specific to calves were needed to separate the adults' relationships with the calves from the adults' relationships with each other. When these other measures were included, whether or not total association was included made very little difference to the results. However, the specific relationship types defined in this study may not be generalizable to other calves for two reasons: only one of the calves (Lotus) survived past ten days, and only one (Nephele's) was healthy. Nonetheless, these relationships offer insight into the kinds of bonds calves can have with adults and what measures might be important to determining calves' social relationships. These techniques can be combined to create a powerful set of tools for defining relationship types and classifying specific relationships between animals. The relationships determined in this way can then be used to classify potential tutors and investigate the process of vocal learning. 
Chapter 3: Social Relationships

In spite of the unusual circumstances, the relationships seen here are similar to previous reports of calves' interactions with adults. Most reports on calf behavior indicate that calves spend most of their time in their first weeks very close to their mothers (e.g. McBride \& Kritzler 1951, Mann \& Smuts in prep), and rub with their mothers often (Mann \& Smuts in prep). Reports of calves spending time with females other than their mothers, as Lotus did with his associates, have been very common since the earliest studies of calf behavior (e.g. McBride \& Kritzler 1951, Tavolga \& Essapian 1957). This type of "alloparenting" occurs in many species (Riedman 1982). Primate infants spend anywhere from $1 \%$ (chimpanzees) to $60 \%$ (langurs) of their time with allomothers (Nicolson 1987). Other species for which alloparenting has been reported include African elephants (Lee 1987), orcas (Haenal 1986), seals (Riedman 1990) and a variety of terrestrial carnivores (Riedman 1982). Dolphin calves in captivity are often reported swimming with other females while their mothers perform (Leatherwood 1977) or eat (Leatherwood 1977, Gurevich 1977, Tavolga \& Essapian 1957). Mann and Smuts (1998) found that in the first month, wild calves spent only $2.5 \%$ of their time more than 10 meters from their mothers. A third of this was in association with another dolphin, but that other dolphin was often another calf. Nursing from allomothers, as Lotus did from his associates, has also been reported in many species (e.g. bighorn sheep: Hass 1990; African elephants: Lee 1987; Northern elephant seals: Riedman \& Le Boeuf 1982; bottlenose dolphins: Messinger et al. 1996). Allomaternal nursing is more commonly reported in studies of captive animals than free-ranging ones (Packer et al. 1992). In species that give birth to one offspring at a time as dolphins do, allomaternal nursing often involves females that have recently lost young (Packer et al. 1992).

There are several possible explanations for why only Lotus had interactions that can be classified as alloparental. In some species, the amount of time infants spend with alloparents increases over the first month of life (e.g. langurs: Vogel 1984). Primate mothers sometimes do not allow alloparenting until the infant is old enough to grip properly (Hrdy 1976). The time wild dolphin calves spent with dolphins other than their mothers increased from less than $1 \%$ in the first month to more than $8 \%$ in the second 
(Mann \& Smuts 1998). Therefore, the first three calves might have had associates had they lived longer. Lotus' unusual situation might also have contributed to the difference. Having a caregiver who was not his biological mother may have made Lotus more accessible to prospective associates. That one of these associates was actually his biological mother is unlikely to be coincidental. In addition, Vicky's role as Lotus' associate after Lotty became his caregiver may have been related to Vicky's previous role as Lotus' caregiver.

Alloparenting appears to be motivated by a number of factors. In some species alloparental care is given preferentially to related infants, suggesting the behavior is driven by kin selection (e.g. Hass 1990, bighorn sheep). In others, reciprocal alloparenting may occur (e.g. Stanford 1992, capped langurs). In many species, alloparents are often nulliparous females, who appear to gain experience in maternal care through this behavior (e.g. primates: Hrdy 1976; birds: Riedman 1982; orcas: Waite 1988; Northern elephant seals: Riedman \& Le Boeuf 1982; bottlenose dolphins: Mann \& Smuts 1998). Another class of adults that is over-represented among alloparents is nearterm pregnant and postpartum females (e.g. langurs: Hrdy 1977; Northern elephant seals: Riedman \& Le Boeuf 1982; bottlenose dolphins: McBride \& Kritzler 1951). Several researchers have suggested that these females are hormonally "primed" to be more responsive to young infants (Hrdy 1977, Riedman \& Le Boeuf 1982), which may be adaptive if it also increases the chance the female responds properly to her own infant. All of the females in this study were either in late-term pregnancy, postpartum, or had recently lost a very young calf. The alloparenting that occurred is therefore likely to have been related to that reproductive condition.

The five days Vicky was Lotus' caregiver also represents an example of alloparenting, since Vicky was not Lotus' mother. This type of prolonged alloparenting has been reported in a number of species (baboons: Shopland \& Altmann 1987; Northern elephant seals: Riedman \& Le Boeuf 1982; bottlenose dolphins: Dudok van Heel \& Meyer 1974). In some cases, the allomother kept the infant until it starved to death (e.g. Shopland \& Altmann 1987). In other cases, where the allomother was lactating, she 
Chapter 3: Social Relationships

actually adopted the infant (e.g. Marsden \& Vessey 1968, Riedman 1990). There are multiple reports of adult dolphins trying, sometime successfully, to take a newborn calf from its mother (e.g. Prescott 1977, Shallenberger \& Kang 1977, Thurman \& Williams 1986). In one case that is remarkably similar to Lotus', an older female took a calf as soon as it was born (Dudok van Heel \& Meyer 1974). Although the mother tried to retrieve him several times, the calf was only returned to her when the trainers took him away from the other female. Whether such an attempt succeeds seems to be related to the relative dominance of the two females involved. When the mother is subordinate, she often loses her calf (e.g. Shallenberger \& Kang 1977, Dudok van Heel \& Meyer 1974), but when the mother is dominant, a competition for a calf can end with the mother keeping her calf (Shallenberger \& Kang 1977). This pattern has been reported in other species as well (primates: Hrdy 1976, Altmann 1980; seals: Riedman \& Le Boeuf 1982). Dudok van Heel and Meyer (1974) also note that the two females involved in the prolonged alloparenting were "very attached" (p14). In this case, the allomother may have been able to take the calf because of her previous affiliative relationship with the mother. The possibilities for alloparenting, then, might be dependent on the relationship between the adults before the calf is born.

\subsection{RELATIONSHIPS BETWEEN ADULTS}

\subsubsection{DATA ANALYSIS}

To find out how the relationships between the adults influenced the adults' relationships with the calves, the relationships between the adults before the calves were born need to be determined. For this purpose, the data from the focal samples of the pregnant dolphins were used. As was discussed in chapter 2, the calves were born in two groups. One calf, Nephele's, was born at the end of April. The other three were born at the end of May. Before the first calf was born, there were six dolphins in the group, all females: Delphi, Lotty, Nephele, Vicky, Sharky, and Daphne (see Table 2.1). Delphi, Lotty, Nephele, and Vicky were pregnant during this time and were therefore the focal 
TABLE 3.7: RULES FOR DECIDED VS. UNDECIDED AGONISTIC INTERACTIONS

(see Samuels \& Gifford 1997) Aggressive and submissive behaviors are defined in Table 2.5B.

\begin{tabular}{|cc|cc|}
\hline Action by Dolphin A & Action by Dolphin B & Type & Winner \\
\hline Aggression & Aggression & Undecided & Neither \\
& Aggression \& Submission & Undecided & Neither \\
& Neutral & Undecided & Neither \\
& Submission & Decided & Dolphin A \\
& & & \\
Aggression \& Submission & Aggression \& Submission & Undecided & Neither \\
& Neutral & Undecided & Neither \\
& Submission & Undecided & Neither \\
& & & \\
Submission & Neutral & Decided & Dolphin B \\
& Submission & Undecided & Neither \\
\hline
\end{tabular}

animals. When Nephele's calf was born, Sharky and Daphne were moved out of the pool (see Table 2.1). After Nephele's calf died, four adults remained: Delphi, Lotty, Nephele, and Vicky. Delphi, Lotty, and Vicky were still pregnant and were now the focal animals. Because of this change in the group, the analysis of the adult relationships was divided into two periods: Period 1 (P1) extended from the beginning of the study until the day before Nephele's calf was born (March 1 to April 24, 1995), and Period 2 (P2) included from the day after Nephele's calf died until the day before Vicky's calf was born (May 2 to May 21, 1995). The relationships of each focal were determined based on the interactions she had with each other member of the group during her focal sessions. The relationship between Sharky and Daphne, who are mother and daughter, could not be determined because neither was a focal.

As was done with the calves, the adults' interactions were analyzed with loglinear models to determine which behaviors varied depending on which focal adult was interacting and which dolphin the focal was interacting with (see section 3.2.1). For this analysis, the total number of agonistic interactions and affiliative behaviors between animals were analyzed. The cells where animals would be interacting with themselves (e.g. Delphi as focal and Delphi as interacting animal) were counted as structural zeros and not included in the models. The rates of affiliative behaviors and agonistic interactions, as well as the total association between animals, were then standardized and analyzed with MDS and HCA (see section 3.2.2) to determine relationship types. 


\section{Chapter 3: Social Relationships}

Because the dominance rank of the mother can influence an infant's interactions, dominance relationships between animals were also evaluated. Agonistic interactions were classified as decided or undecided based on the criteria from Samuels and Gifford (1997). For an interaction to be decided, one animal had to perform submissive behaviors and not aggressive ones while the other did not perform submissive ones (Table 3.7). Dominance relationships were determined based only on the decided interactions between animals.

\subsubsection{RELATIONSHIPS}

Time Spent Together

In general, the focal animals spent less than $10 \%$ of their time with each of the other dolphins in the Lagoon (Table 3.8). For each focal, however, there were a few animals she spent more time with. Nephele and Delphi spent $82 \%$ of their time alone, but the majority of the time they spent swimming with other animals, they were with each other (Table 3.8). This was true in both periods. In the first period (P1), Vicky spent $68 \%$ of her time alone. During the time she was not alone, she was usually swimming with either Lotty or Sharky (Table 3.8A), or often both. Vicky's habits changed in the second period (P2), however. In this period, she spent only $50 \%$ of her time alone, and now she spent $36 \%$ of her time swimming with Nephele, as well as $20 \%$ with Lotty (Table 3.8B). Lotty only spent $49 \%$ of her time alone in the first period. The rest of the time she spent with Vicky, Sharky, or Daphne (Table 3.8A). When Sharky and Daphne were moved out of the Lagoon, Lotty spent more time alone (73\%). Most of the time she was not alone, she was swimming with Vicky (Table 3.8B).

\section{Agonistic and Affiliative Contact}

Overall, both affiliative contact and agonism were rare between most adults (Table 3.8). Most pairs engaged in less than one affiliative behavior in ten samples, but a few pairs averaged around one affiliative behavior per sample. In $\mathrm{P} 2$, these pairs actually engaged in more than one affiliative behavior per sample (Table 3.8B). In some instances, these were the same animals whose total association was also high: Delphi and 
TABLE 3.8: ADULT INTERACTIONS

Total association is presented in percent of the total time. Affiliative behaviors and agonistic interactions are presented as number per sample.

A. PERIOD 1 (MARCH - APRIL)

\begin{tabular}{|l|l|c|c|c|}
\hline Focal & Relationship with & Total Association & $\begin{array}{c}\text { Affiliative } \\
\text { Behaviors }\end{array}$ & $\begin{array}{c}\text { Agonistic } \\
\text { Interactions }\end{array}$ \\
\hline Delphi & Lotty & $7.5 \%$ & 0 & 0.01 \\
& Vicky & $7.9 \%$ & 0 & 0.01 \\
& Nephele 168$)$ & $13.2 \%$ & 1.0 & 0.02 \\
& Sharky & $6.6 \%$ & 0.02 & 0.1 \\
& Daphne & $5.5 \%$ & 0.1 & 0.1 \\
\hline Lotty & Delphi & $5.3 \%$ & 0 & 0 \\
(N=168) & Vicky & $23.1 \%$ & 0.8 & 0.04 \\
& Nephele & $6.9 \%$ & 0.1 & 0.02 \\
& Sharky & $30.3 \%$ & 0.3 & 0.5 \\
& Daphne & $29.5 \%$ & 1.1 & 0.2 \\
\hline Nephele & Delphi & $12.7 \%$ & 0.9 & 0.02 \\
(N=167) & Lotty & $7.3 \%$ & 0.1 & 0.01 \\
& Vicky & $8.0 \%$ & 0.01 & 0.01 \\
& Sharky & $6.2 \%$ & 0.01 & 0.1 \\
& Daphne & $4.9 \%$ & 0.2 & 0.1 \\
\hline Vicky & Delphi & $6.3 \%$ & 0.01 & 0.04 \\
(N=138) & Lotty & $20.1 \%$ & 0.8 & 0.04 \\
& Nephele & $6.9 \%$ & 0 & 0.01 \\
& Sharky & $15.7 \%$ & 0.6 & 0.4 \\
& Daphne & $7.7 \%$ & 0.1 & 0.1 \\
\hline
\end{tabular}

B. PERIOD 2 (MAY)

\begin{tabular}{|l|l|c|c|c|}
\hline Focal & Relationship with & Total Association & $\begin{array}{c}\text { Affiliative } \\
\text { Behaviors }\end{array}$ & $\begin{array}{c}\text { Agonistic } \\
\text { Interactions }\end{array}$ \\
\hline Delphi & Lotty & $2.9 \%$ & 0.01 & 0.01 \\
$(\mathrm{~N}=88)$ & Vicky & $3.6 \%$ & 0.01 & 0.1 \\
& Nephele & $15.5 \%$ & 2.5 & 0.2 \\
\hline Lotty & Delphi & $3.6 \%$ & 0 & 0.02 \\
$(\mathrm{~N}=88)$ & Vicky & $19.6 \%$ & 1.4 & 0.01 \\
& Nephele & $8.5 \%$ & 0.1 & 0.2 \\
\hline Vicky & Delphi & $3.8 \%$ & 0.03 & 0.03 \\
$(\mathrm{~N}=88)$ & Lotty & $19.9 \%$ & 1.8 & 0 \\
& Nephele & $35.8 \%$ & 0.6 & 0.06 \\
\hline
\end{tabular}

Nephele, Lotty and Vicky, and Lotty and Daphne. However, a few pairs who had high total association had an intermediate amount of affiliative contact, one behavior every two or three samples in general (Table 3.8). These were Lotty and Sharky, Vicky and Sharky, and Vicky and Nephele in P2. Vicky and Nephele are a particularly interesting case: they exchanged more than 50 affiliative touches in P2, a striking increase from the one affiliative touch they exchanged in all of P1. 


\section{Chapter 3: Social Relationships}

Agonism was even less common. No pair had an agonistic interaction more than once every two samples. Agonism followed a very different pattern from affiliative contact, however. While some pairs did have more interactions than others, in each period there was one dolphin that accounted for the majority of the interactions with all the others. In P1, Sharky accounted for the majority of the agonistic interactions for all four focal animals, though more than half her interactions were with Lotty (Table 3.8A). For all four focals, the second most common partner for agonistic interactions in P1 was Daphne. As with Sharky, Daphne had more agonistic interactions with some dolphins than with others, and she had the most with Lotty. In P2, Nephele was involved in 41 interactions out of 52 that were recorded. She accounted for the majority of agonistic interactions with all three of the focal animals, although most of her interactions were with Lotty and Delphi (Table 3.8B).

For a behavior type to be useful in determining relationships, the focal animals must perform that behavior with some animals more than with others. To determine whether the focal animals' affiliative and agonistic contact varied in this way, the observed frequency of interaction for each period was compared to loglinear models. First, to determine whether the two behaviors varied in the same way, the two behavior types were tested together. No model simpler than the saturated model (Focal-BehaviorInteractor) fit the data in either period (FB, FI, BI: P1 BIC $=24.5 ; \mathrm{P} 2 \mathrm{BIC}=39.3$ ). This means that the focal animals interacted with different animals differently but did not interact agonistically and affiliatively with the same animals. The two behavior types were therefore analyzed separately.

When the affiliative contact between animals was analyzed by loglinear models, no model simpler than the saturated model (Focal-Interactor) fit the data in either period (F,I: P1 BIC = 1236.7; P2 BIC = 585.7). This means that in both periods, affiliative contact varied both by focal and by who the focal was interacting with. Agonism, on the other hand, does not appear to be as useful. When tested with loglinear models, agonism varied by dolphin but did not depend on who that dolphin was interacting with. In P1, the best fitting loglinear model for the agonistic interactions included separate effects of 
the focal and the interactor but no interaction effect $(\mathbf{F}, I: B I C=-22.4)$. In P2, the best fitting model depended only on the interactor, without any effect of which focal animal was involved $(\mathbf{I}: \mathrm{BIC}=-8.0)$. In both cases, the models did not require an interaction between focal and interactor, which means that the number of agonistic interactions did not depend on which two animals were interacting with each other. This confirms the observation that agonistic interactions depended more on single dolphins than pairs of dolphins.

\subsubsection{RELATIONSHIP TYPES}

Relationship types can be defined when animals interact in similar ways with different interactors. In this study, affiliative contact and time animals spent in association varied in a manner that allowed relationships between adults to be differentiated. In both time periods, the pairs Vicky/Lotty and Delphi/Nephele each spent a lot of time together and had a large number of affiliative interactions. When Daphne was in the pool, she also associated a great deal and had many affiliative interactions with Lotty. In the same period, Sharky spent a lot of time with Vicky and Lotty and had an intermediate number of affiliative interactions with them. In the second period, Vicky and Nephele began to spend time together and have some affiliative contact, although not as much as Vicky and Lotty did.

Therefore, in the same way that three relationships were hypothesized for the calves (see section 3.3), three relationships can be hypothesized for the adults. Some pairs interact affiliatively and spend time together: Vicky/Lotty, Delphi/Nephele, and Lotty/Daphne. These might be called "affiliates". Some pairs spend time together and interact affiliatively to a lesser degree than affiliates: Vicky/Sharky and Lotty/Sharky in P1, and Vicky/Nephele in P2. These might be termed "associates". All other pairs have very few affiliative interactions and spend less than $10 \%$ of their time together. As with the calves, these might be called "poolmates". To test these hypotheses, multidimensional scaling and hierarchical cluster analysis were performed with these data. 
Chapter 3: Social Relationships

As with the calves, the results of the MDS and HCA depended on what measures were included. In P1, the three relationship types separated best when total association, affiliative contact, and agonism were all included (Figure 3.7A,B). With these measures, the three relationship types separated very clearly in the MDS and by the moat index in the HCA. Interestingly, without agonism, affiliates and associates did not separate well. This suggests that Sharky's relationships with Lotty and Vicky were characterized by a large amount of agonism in addition to some affiliative contact. This result is consistent with the result of the loglinear analysis that agonism in P1 depended both on interactor and on focal. In $\mathrm{P} 2$, the best separation between the three relationships was achieved by including only total association and affiliative contact without agonism (Figure 3.8A,B). Once again, these analyses clearly separated all three relationship types. When agonistic interactions were added to the analyses, the affiliates and associates were more difficult to separate. This may be an indication that Nephele's agonistic interactions were more spread out among her partners than Sharky's were (see Table 3.8A,B).

As was the case with the calves, when only total association was included, several aspects of the relationships were no longer apparent (Figure 3.9A,B). In P1, the affiliates and associates were completely mixed and some of the affiliates and associates were not separated from the poolmates by the moat index. In P2, the affiliates, associates, and poolmates each formed separate groups, but the affiliates were not separated from the poolmates by the moat index. As with the calves, total association may reflect a different process than the affiliative relationships hypothesized here. For instance, the fact that Lotty and Daphne were affiliates might have resulted in Lotty and Sharky being associates because Sharky is Daphne's mother. In fact, when only total association is used for the HCA of P1, Lotty's relationships with Daphne and Sharky cluster very close to each other, even though Lotty's relationship with Sharky was characterized by far fewer affiliative interactions than her relationship with Daphne. Therefore, measures other than association are necessary to show the subtle differences in relationships between dolphins. 
FIGURE 3.7: ADULT RELATIONSHIP TYPES, PERIOD 1

The focal animals are listed first in italics.

\section{A. Multidimensional SCALING}

Stress (a measure of the fit) $=0.03$; Proportion of variance accounted for $=0.8$

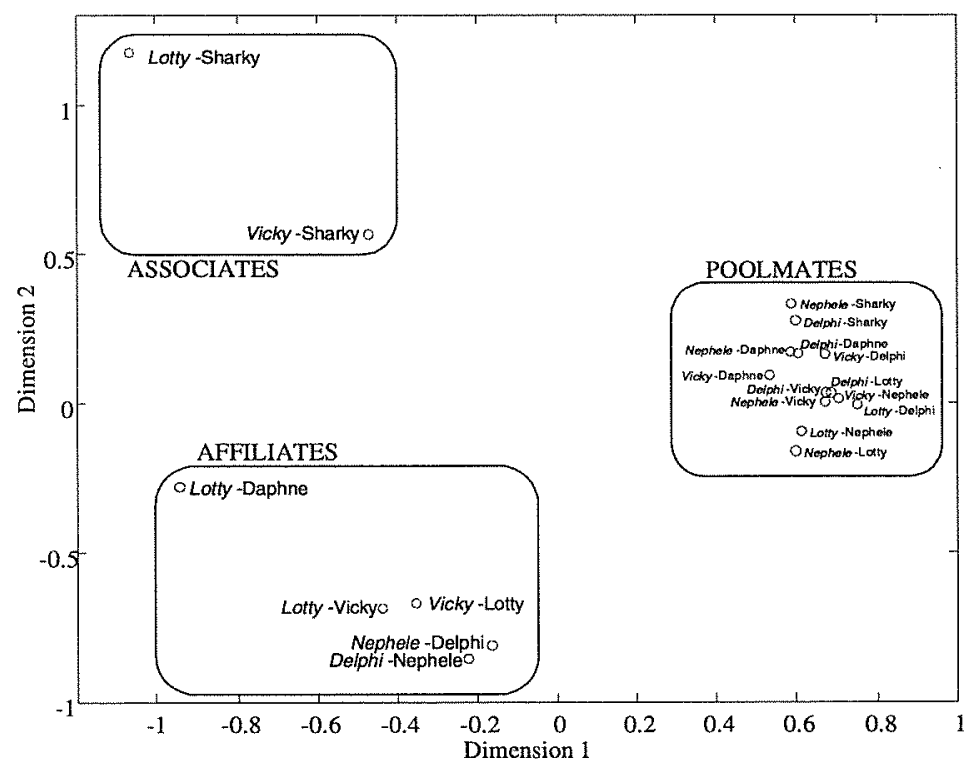

\section{B. Hierarchical Cluster Analysis}

The dotted line through the clusters represents the moat index.

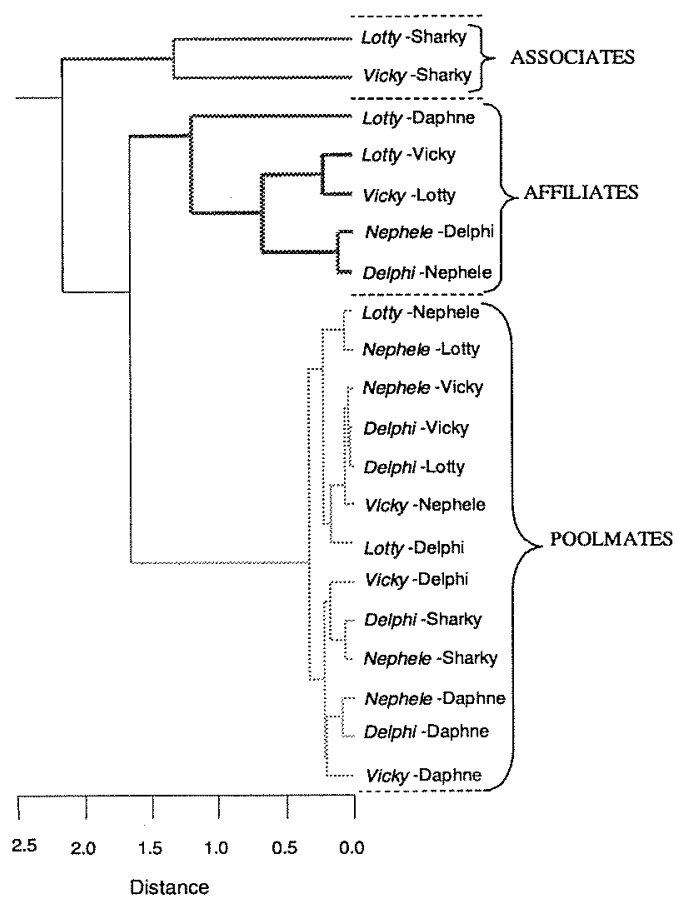




\section{Chapter 3: Social Relationships}

\section{FIGURE 3.8: ADULT RELATIONSHIP TYPES, PERIOD 2}

Agonistic interactions are not included. The focal animals are listed first in italics.

A. Multidimensional SCALING

Stress (a measure of the fit) $=0$; Proportion of variance accounted for $=1$.

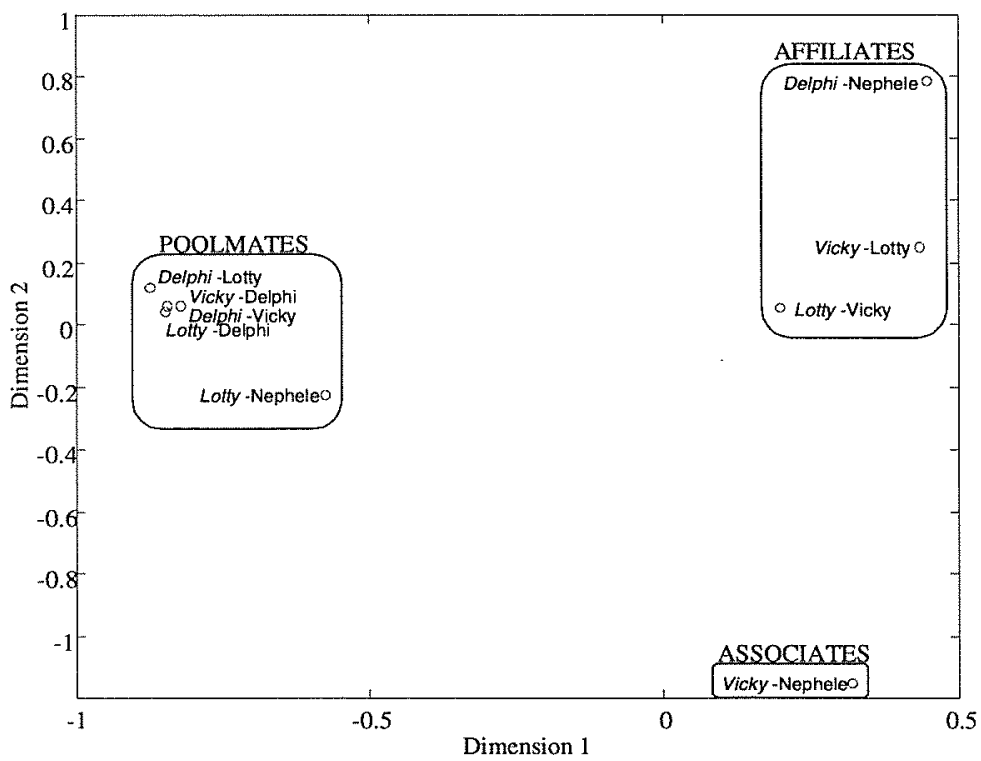

B. HiERARChICAL Cluster ANALYSIS

The dotted line through the clusters represents the moat index.

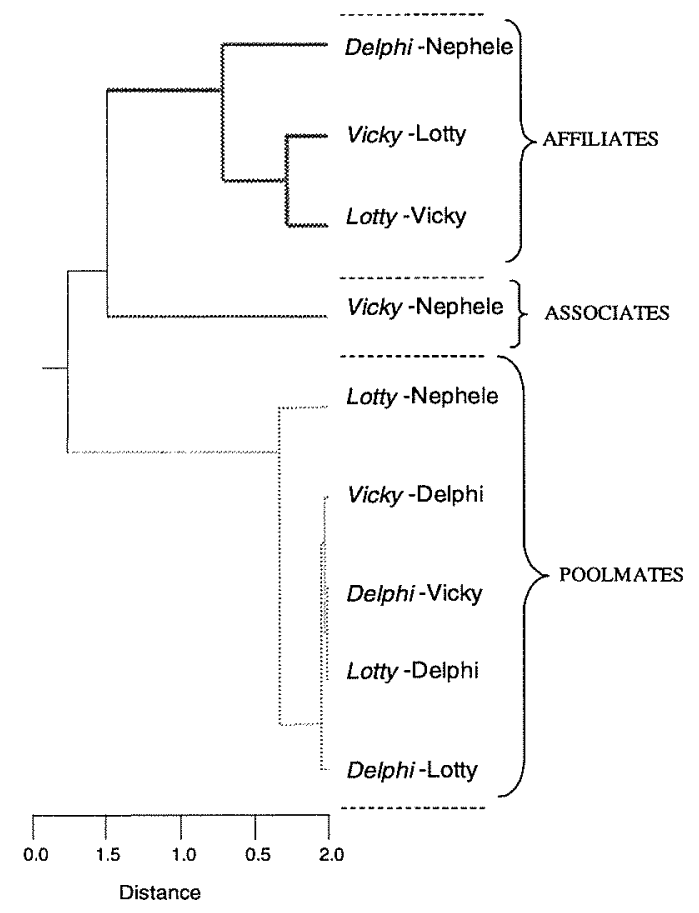


FIGURE 3.9: HIERARCHICAL ClUSTER ANALYSIS OF TOTAL ASSOCIATION ONLY The dotted line through the clusters represents the moat index.

A. PERIOD 1

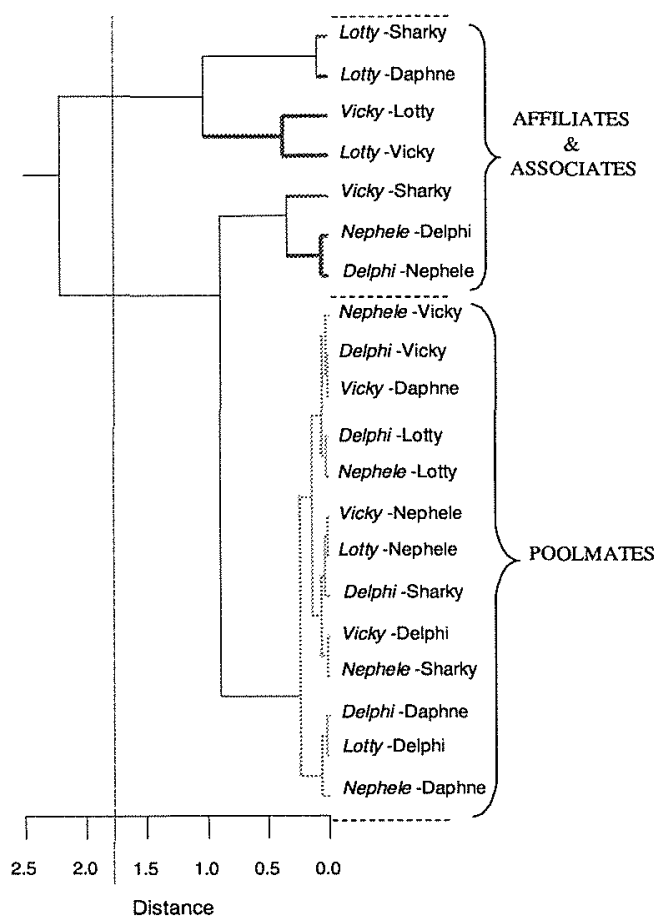

B. PERIOD 2

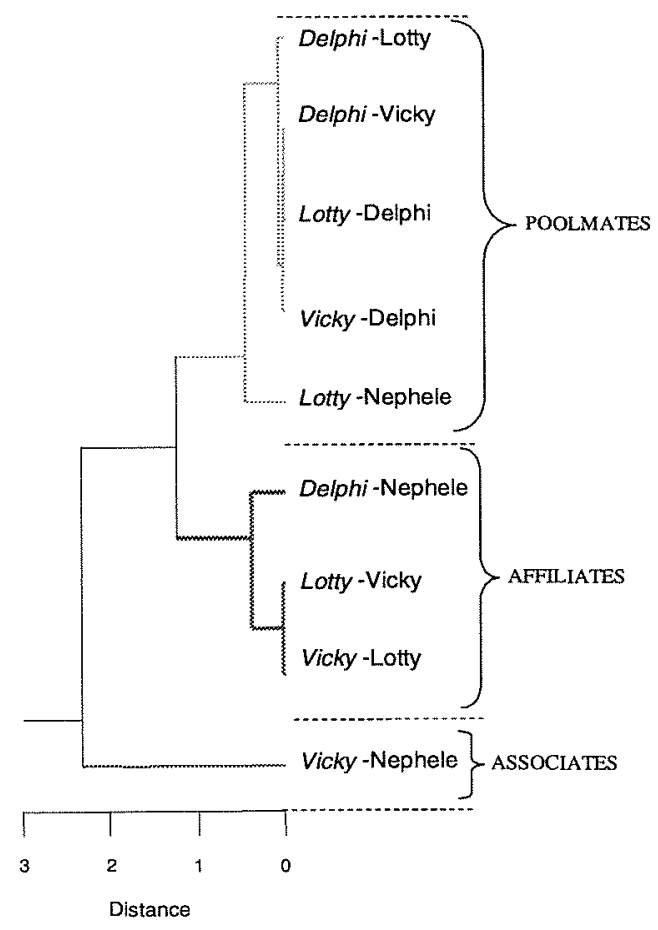


Chapter 3: Social Relationships

\subsubsection{DOMINANCE}

In addition to the relationship types defined by affiliative contact and total association, dominance relationships can have a profound influence on the interactions both of adults and of their calves. Dominance is determined by decided agonistic interactions only. Of the 323 agonistic interactions in the two periods, only $31 \%$ were decided. Decided interactions occurred only once every 10 samples in P1, and once every 8 samples in $\mathrm{P} 2$. Undecided interactions were much more common in P1, occurring once every 3 samples, but much less common in P2, occurring only once every 14 samples. Because of the scarcity of decided interactions between some pairs, some aspects of the dominance hierarchy could not be determined (Table 3.9). There were several pairs of animals where no decided interactions were recorded and a few where only one or two interactions were recorded. In very few cases did one animal win all the interactions; the reversal rate was $26 \%$ in P1 and $30 \%$ in P2. For instance, Lotty and Nephele had 13 decided agonistic interactions in P2 (Table 3.9B). Lotty won more of these than Nephele (7/13) so Lotty could be considered the winner in this pair. However, the reversal rate, i.e. the proportion of interactions won by the putative loser, in this case Nephele, was $46 \%$. In addition, with only one exception, decided interactions were always interspersed with undecided ones. This exception is Vicky/Daphne: on the day Vicky was introduced into the Lagoon, she had one undecided interaction with Daphne. After that, they had several agonistic interactions, but Vicky won all of them. All other pairs of animals had undecided interactions throughout the study. The prevalence of reversals and undecided interactions may indicate that the hierarchy was in flux, possibly because Nephele and Delphi were first introduced to the other animals only a few weeks before the study began.

The putative dominance hierarchy for $\mathrm{P} 1$ is shown on the right of Table 3.9A. Vicky is at the top of the hierarchy, having won almost all of her decided interactions (Table 3.9A). However, she had only one interaction with Nephele, and no interactions at all with Lotty or Delphi. Sharky is next, having won against everyone except Vicky. Because Sharky is dominant to Lotty and Delphi and subordinate to Vicky, Vicky is 
TABLE 3.9: DECIDED AGONISTIC INTERACTIONS

A. PERIOD 1 (MARCH-APRIL)

* Neither Sharky nor Daphne was a focal so their interactions cannot be assessed.

\begin{tabular}{|c|cccccc|c|}
\hline Winner & Vicky & Sharky & Lotty & Nephele & Delphi & Daphne & Dominance Hierarchy \\
\hline Vicky & - & 5 & 0 & 1 & 0 & 9 & Vicky \\
Sharky & 1 & - & 7 & 6 & 3 & $*$ & Sharky \\
Lotty & 0 & 4 & - & 1 & 0 & 9 & Lotty-Nephele-Delphi \\
Nephele & 0 & 3 & 1 & - & 2 & 9 & Daphne \\
Delphi & 0 & 1 & 0 & 2 & - & 8 & \\
Daphne & 0 & $*$ & 6 & 3 & 0 & - & \\
\hline
\end{tabular}

B. PERIOD 2 (MAY)

\begin{tabular}{|c|cccc|}
\hline & \multicolumn{4}{|c|}{ Loser } \\
Winner & Vicky & Lotty & Nephele & Delphi \\
\hline Vicky & - & 0 & 2 & 4 \\
Lotty & 0 & - & 7 & 0 \\
Nephele & 2 & 6 & - & 10 \\
Delphi & 0 & 1 & 1 & - \\
\hline
\end{tabular}

hypothesized to be dominant to Lotty and Delphi as well, although they never interacted.

A triangular dominance hierarchy could have existed among some of these animals, however. A linear hierarchy assumes that because Vicky was dominant to Sharky and Sharky to Lotty, Vicky was also dominant to Lotty. A triangular dominance hierarchy would occur if Lotty were actually dominant to Vicky, so a triangle of dominance exists, with Vicky dominant to Sharky who is dominant to Lotty who is dominant to Vicky. Dominance ranks between Lotty, Delphi, and Nephele could not be determined at all. Delphi and Nephele had no interactions, and in the other two pairs, each partner won exactly half the time (Table 3.9A). This may indicate that these three had equivalent dominance ranks or that their dominance relationships were in flux. The clearest case was Daphne, the 7-month old, who was subordinate to everyone. She did win 9 interactions but 6 of these were instances when she was with her mother, Sharky. In all 9 cases, the interactions were with animals Sharky was dominant to.

No hierarchy could be determined for P2 because there was a clear winner in only two of the six pairs: Vicky and Nephele were both clearly dominant to Delphi (Table 3.9B). A hierarchy could not be clearly set up between Vicky, Nephele, and Lotty, however. Vicky and Lotty had no interactions in this period, as in the previous one. 
Chapter 3: Social Relationships

Vicky and Nephele interacted 4 times but each won $50 \%$ of the interactions. Lotty and Nephele interacted 13 times, but although Lotty won more than Nephele, the reversal rate was $46 \%$. Although Nephele was dominant to Delphi and Lotty may have been dominant to Nephele, Delphi won the only decided interaction she had with Lotty (Table 3.9B). This may indicate that a triangular hierarchy existed between them, where Lotty was dominant to Nephele who was dominant to Delphi who was dominant to Lotty. Alternatively, it may indicate that these three had no clear hierarchy, as appeared to be the case in P1.

\subsubsection{DISCUSSION}

These analyses demonstrate how relationships between adult females can be determined from their interactions and association. First, loglinear analysis was used to show that the amount of affiliative contact that occurred in this group depended on which pair of animals was interacting but agonism depended more on single dolphins than on pairs of dolphins. Affiliative contact and total association, which also depended on which pair of animals was associating, could therefore be used to determine relationships. How these relationships separated into types with MDS or HCA depended on which measures were included, as it did with the calves. Three levels of relationships were found based on multiple measures: affiliate, associate, and poolmate. In one of the periods, including agonistic interactions in the analysis helped to separate the relationship types, in the other it hindered the separation. In both periods, affiliative contact and total association were both needed to bring out the three types. Including only total association in the analysis obscured that pattern. This is significant because most analyses of relationships in wild bottlenose dolphins are solely based around the association between the animals (e.g. Wells et al. 1987, Smolker et al. 1992). An exploration of subtler behaviors, such as affiliative contact, yielded a different result in this case than the analysis of association patterns.

An analysis of both the affiliative relationships and the dominance relationships of pregnant females is useful to the study of vocal learning in calves because the 
relationships those females have with other adults may influence the relationships their calves have with the same adults. For instance, Lotty's relationship with Vicky before Lotus was born may have influenced Lotus' subsequent relationship with Vicky. Lotty may have only allowed Vicky near her during the birth because they were affiliates. Alternatively, if Vicky was dominant to Lotty, her dominant status may have made Lotty less likely to attempt to retrieve Lotus from her. In addition, if sounds heard prenatally influence vocal development, the mother's relationships when she is pregnant could impact the vocalizations heard and therefore the vocal development. However, while the analysis of pregnant dolphins is useful to the study of vocal learning, the relationships of pregnant dolphins may not be generalizable to non-pregnant dolphins. The results of this study may not be generalizable for another reason: two of the dolphins had only recently been introduced to the rest of the group. Nephele and Delphi were brought to Sweden together from a dolphinarium in Germany only a few months before the beginning of the study and were only introduced into the communal pool a few weeks before the study began. Their relationship, therefore, may have been a result of being familiar with each other in an unfamiliar situation. This is also a likely explanation for the difficulty determining a clear dominance hierarchy. The dominance relationships between some of the animals may still have been unsettled.

The introduction of two groups to each other has been shown to change behavior in a number of species. In Samuels and Gifford's (1997) study of captive bottlenose dolphins, during a short period where two groups of females were introduced to each other for the first time, agonism increased from one interaction every 167 minutes to one every 24 minutes. In the first period of the present study, shortly after Nephele and Delphi were introduced to the group, agonistic interactions occurred between adults approximately once every 32 minutes. In a comparison between two captive groups of chimpanzees, one established and one newly formed, agonism between females was much higher in the newly formed group (Baker \& Smuts 1994). As the females in the new group became familiar with each other, such dominance struggles decreased (Baker \& Smuts 1994). In the second period of the present study, agonism decreased from one 


\section{Chapter 3: Social Relationships}

interaction every 32 minutes to one every 51 minutes. This may be due to the dolphins becoming more familiar with each other. In Baker and Smuts' (1994) study, some other behaviors changed as well. For instance, affiliative contact following agonism, also known as reconciliation, was rare between the female chimpanzees when the colony was well established but frequent in the newly formed group (Baker \& Smuts 1994).

The difficulty in establishing a clear linear dominance hierarchy could be partly caused by the newness of the group but several factors suggest that the data could not have been used to determine a definitive linear hierarchy in any case. Appleby (1983) investigated the probability that linear hierarchies occur by chance in data of dominance interactions, and suggested that interactions between animals could appear to indicate a linear hierarchy when they are actually occurring randomly. Appleby (1983) suggests that for groups smaller than 6 animals, such as the group of 4 in P2, there is a very high probability that a linear hierarchy will appear by chance, and for groups of 6 , as in P1 here, a confident level of linearity can only be achieved if fewer than 2 relationships are missing. In P1, at least 5 dominance relationships were missing, 6 if the relationship between Daphne and Sharky is included. In addition, Hausfater (1975) states that a linear hierarchy is the result "of consistency and transitivity of agonistic relations" and therefore the consistency of winners is an important consideration when evaluating a hierarchy (p20). The reversal rate in the present study was quite high in both periods, and undecided interactions were very common. Both of these are indications of inconsistent dominance relations.

In a study where there were enough interactions to determine a linear hierarchy among female dolphins, that hierarchy was stable over time (Samuels \& Gifford 1997). Dominance was related to the age of the female, with older females dominant to younger ones. Stable hierarchies are common among females of many species (e.g. hyenas: Frank 1986; baboons: Altmann 1980; chimpanzees: Baker \& Smuts 1994). In some species, daughters actually inherit the rank of their mothers (hyenas: Frank 1986; baboons: Samuels et al. 1987). Because of this, young animals can actually win encounters with older, larger animals who are subordinate to the youngster's mother (Cheney et al. 1986). 
This happened a number of times in the present study with the juvenile, Daphne. Daphne was subordinate to all the dolphins in the pool but still won some agonistic interactions against dolphins who appeared to be subordinate to Daphne's mother, Sharky. The rate of agonism between females in Samuels and Gifford's (1997) study was very low, with only one interaction every 167 minutes of observation. Low rates of agonism in a stable hierarchy are common in other species a well (e.g. Frank 1986, Altmann 1980, Baker \& Smuts 1994). In Samuels and Gifford's (1997) study, the rate of agonism was higher with the juvenile female in the group than with other adult females. This was true of the present study as well. In P1, the focals had 0.011 interactions per minute with Daphne and only 0.008 per minute with each of the other adults. This was comparable to the 0.018 per minute with the juvenile and 0.002 per minute between the adults found by Samuels and Gifford (1997). The overall rate of agonism decreased between the two periods but this was primarily due to the removal of the juvenile, Daphne. The mean rate per partner in P2 was 0.007 per minute, very close to the rate between adults in P1.

The patterns of affiliative interaction and association between the females in this group were reminiscent of previous studies of both bottlenose dolphins and other mammals. Female dolphins in the wild associate with other females who are in a similar reproductive condition (Wells 1991). In general, these groups are fluid and most females are seen together at some point, but certain associations between females are more persistent then others (Wells 1991). Each female is seen with certain other females a majority of the time. These "bands" can range in size from 2 females and their offspring to 13 or more females and their offspring (Wells 1991). In such bands, some of the females are known to be kin (Duffield \& Wells 1991) and females often return to their natal bands (Wells 1991). However, the genetic relationships between all the females in these groups are not known, and some of the females in these groups may not be related to each other. In captivity, females have been seen to ally themselves in agonistic encounters with long-term associates who are not kin (Samuels \& Gifford 1997). While coalitions during agonistic encounters were not recorded in the present study, long-term associates were more likely to associate and engage in affiliative interactions than 
dolphins that had known each other for shorter time periods. An example of this is Delphi and Nephele, who had come from Germany to Sweden together shortly before the study and were each others' only affiliates.

The general social structure of some primates is very similar to dolphins, where females often stay in their natal groups and males do not (Cheney et al. 1986, Wells 1991). These primates develop distinct relationships with each other based on their previous interactions, as well as their age, sex, and rank (Cheney et al. 1986). These long term bonds are maintained with a combination of competitive and affiliative interactions and can contribute to the reproductive success of the individuals (Cheney et al. 1986). Such associates often support each other in agonistic interactions with other animals. Associations and grooming behavior in female baboons are related to the females' dominance rank and to their reproductive state. For instance, lactating females with young infants tend to have more associates (Seyfarth 1976). Females with adjacent ranks groom each other more and were more likely to form coalitions during agonistic encounters than other females (Seyfarth 1976). Rank could not be determined in the present study but affiliates were not generally in adjacent ranks in the putative dominance hierarchy. Vicky, for instance, was two steps above her affiliate Lotty in the putative P1 ranking.

Male-female associations in baboons are generally longer-term than femalefemale associations (Altmann 1980, Smuts 1985). Altmann (1980) found that she could usually predict which males associated with a new mother by looking at which males mated, groomed, and associated with her before her baby was born. Similarly, male baboons that groom with females are also commonly those females' neighbors when not grooming (Smuts 1985). As was seen with female dolphins in the present study, Smuts (1985) found two levels of male-female associations: affiliates, males who both associated and groomed with the females, and associates, males who associated with the females but did not groom with them. For the most part, neither of these partners were the female's kin. Smuts (1985) suggests that associates might be pairs of animals in transition, in the process of either forming or losing an affiliation. Although male-female 
interactions are often different from female-female interactions (e.g. Smuts 1985, Smolker et al. 1992), similar processes could be occurring in both situations. For example, Vicky and Nephele may have had a similar transitional relationship in the present study, as both the association and affiliative contact between them increased dramatically between the two periods. There is also evidence for several species that primates recognize the bonds that exist between other animals (Cheney et al. 1986).

The social structure of African elephants also has similarities to dolphins (Moss \& Poole 1983). Females remain in their natal group, which is made up of several related females and their offspring. Males leave the group upon reaching sexual maturity. Family groups of females associate with each other in the rainy season depending on food availability (Moss \& Poole 1983). Each family is sighted most often with two to five other families in what is known as a "bond group." The quality of interactions between members of bond groups is substantially different from their interactions with other elephants (Moss \& Poole 1983). Members of bond groups greet each other with a very elaborate ceremony, intermingle freely, rub each other, and lean on each other (Moss \& Poole 1983). Their interactions with other elephants tend to be "brief and perfunctory" and their greetings simple (Moss \& Poole 1983, p322). This same phrase could be used quite accurately to describe the interactions between "poolmates" in the present study. There is evidence that the members of the different elephant families in a bond group may be related to each other, suggesting that elephant social relationships are driven by kinship, as may be the case with some wild dolphins (Wells 1991).

\subsection{CONCLUSIONS}

This chapter has demonstrated that a combination of several statistical methods is necessary to combine the interactions between animals into relationships and relationship types. Loglinear analysis was originally developed for use with the categorical data gathered in sociology (Goodman 1978), but as this analysis shows, it can be very useful for the analysis of interactions between animals. In particular, loglinear modeling can be used to determine what types of behaviors co-vary and whether animals interact 
differently with different partners. However, loglinear models cannot be used to analyze behavioral states when absolute duration is recorded, because that data is not truly categorical. In addition, loglinear analysis is of limited use in determining which pairs of animals interact in particular ways, which is necessary to categorize the relationships between animals into types. Hierarchical cluster analysis (HCA) and multidimensional scaling (MDS) can both be used for this purpose. In this analysis, the results of the MDS and HCA were very similar. However, each statistical technique has advantages and disadvantages. Neither MDS nor HCA can clearly demonstrate whether the variation is controlled by relationships between animals or by the behavior of specific animals, as loglinear analysis can. On the other hand, both MDS and HCA can separate relationships into types, which loglinear analysis cannot. MDS can be difficult to interpret because boundaries between groups are not always obvious but is useful for showing how some relationships are intermediate between other relationships. HCA shows the separation of relationships into types more clearly but does not show the gradient of types as well as MDS. The combination of these methods is therefore most useful for translating interactions between animals into relationships and then into relationship types. It is important to remember, however, that some structure may come from artifacts of the methods. Especially with small sample sizes, random data may have structure in cluster analysis or MDS (Whitehead 1997). Methods for determining whether this is the case, such as Monte Carlo analysis where simulated data is tested with the same statistical methods, can be used in conjunction with statistical analysis to rule out this possibility (Whitehead 1997).

In this chapter, the relationship types defined depended on whether measures other than the total association between the animals were included. The association patterns of animals painted a slightly different picture than their affiliative interactions. This is important because most of the studies on the relationships of wild dolphins depend entirely on their association patterns (e.g. Wells 1991). The current analysis suggests that the relationships determined by association patterns may not tell the whole story. In some cases, the association between two dolphins is actually a reflection of 
each dolphin's separate relationship with a third dolphin, rather than their relationship with each other. This was seen in the present study in the large amount of association between Lotty and Sharky, which may have been more a reflection of Lotty and Sharky's separate relationships with Daphne than their relationship with each other. One reason that wild studies often rely on association patterns is that behaviors such as rubbing and nursing can be very difficult to observe in the wild (Whitehead 1997, but see e.g. Mann \& Smuts 1998). However, when the analysis of calf relationships was done with $50 \%$ of the affiliative contact and nursing lost, and no retrieves at all, the results were equivalent. The results of the adult relationships were also equivalent with $50 \%$ of the affiliative contact lost. Therefore, even if not all of the interactions that occur can be recorded, recording some of the interactions is sufficient, as long as the interactions are recorded in a systematic and unbiased manner (see chapter 2). This analysis suggests that a clear understanding of the relationships between dolphins requires recording interactions such as affiliative contact and nursing, as well as subtler measures of association such as who is the calf's nearest neighbor.

The ability to observe behaviors such as rubbing and nursing is one of the advantages of studying dolphins in captivity rather than in the wild. Another advantage is the ability to know all the dolphins that the calf has an opportunity to interact with. Because this is possible, the relationship each calf has with every dolphin he has ever met can be determined. For a study of vocal learning, the ability to completely quantify a calf's social environment in this way is essential. Such a complete quantification of the calf's social environment would be impossible in a study of free-ranging calves because there is no way to know all the dolphins a free-ranging calf has ever interacted with. In addition, calves in captivity can be observed on a regular schedule starting at, or even before, birth. Few free-ranging calves are observed before they are a few weeks old (Wells et al. 1987). In fact, since dolphin births are never actually observed in the wild, most studies of free-ranging dolphins determine calves' mothers by which animal they are seen to associate with most often (e.g. Wells et al. 1987). One particularly interesting effect of this is that the relationships between Vicky, Lotty, and Lotus would not have 


\section{Chapter 3: Social Relationships}

been clear in the wild. If Lotus had been observed in his first five days, Vicky would have been assumed to be his mother. The change in caregiver from Vicky to Lotty would then have been interpreted as the beginning of an alloparenting event, or perhaps even a kidnapping, rather than the end of an alloparenting event. Additional genetic analyses would have been necessary to discover the error. The caveat to studying dolphins in captivity is that captive behavior occurs in an unnatural setting and may be different from the behavior of free-ranging animals. Therefore, studies of wild dolphins are necessary to validate the results of captive studies. However, comparisons of the behavior of dolphins in captivity to that of free-ranging dolphins have found dolphins' social behavior to be similar in both settings (Samuels \& Gifford 1997).

The analyses in this chapter demonstrate how relationships between animals can be determined from their interactions and associations. Determining relationship types is important to the study of vocal learning. In a social setting, calves have a choice of tutors to learn from (Figure 3.10). By defining a set of relationship types, each potential tutor can be classified into a relationship with the calf. Comparing the tutors chosen by a number of calves will help elucidate what types of social contact are important to the process of vocal learning. An understanding of the relationships of the calf's primary caregiver may also be important to understanding the relationships of the calf. First, the calf's relationships may be influenced by his caregiver's. In one case where a calf was kept away from its mother by an alloparent, the allomother and biological mother were reported to have a close social bond before the calf was born (Dudok van Heel \& Meyer 1974). Similarly, the fact that Vicky and Lotty were affiliates before Lotus was born might been one of the reasons Vicky was able to take Lotus away from Lotty so quickly. The caregiver's relationships may therefore influence the access the calf has to other animals. Alternatively, if vocal development is influenced by sounds heard prenatally, the relationships the mother has before the calf is born may play a direct role in the calf's vocal development. If the mother has a physiological reaction to the presence of another 


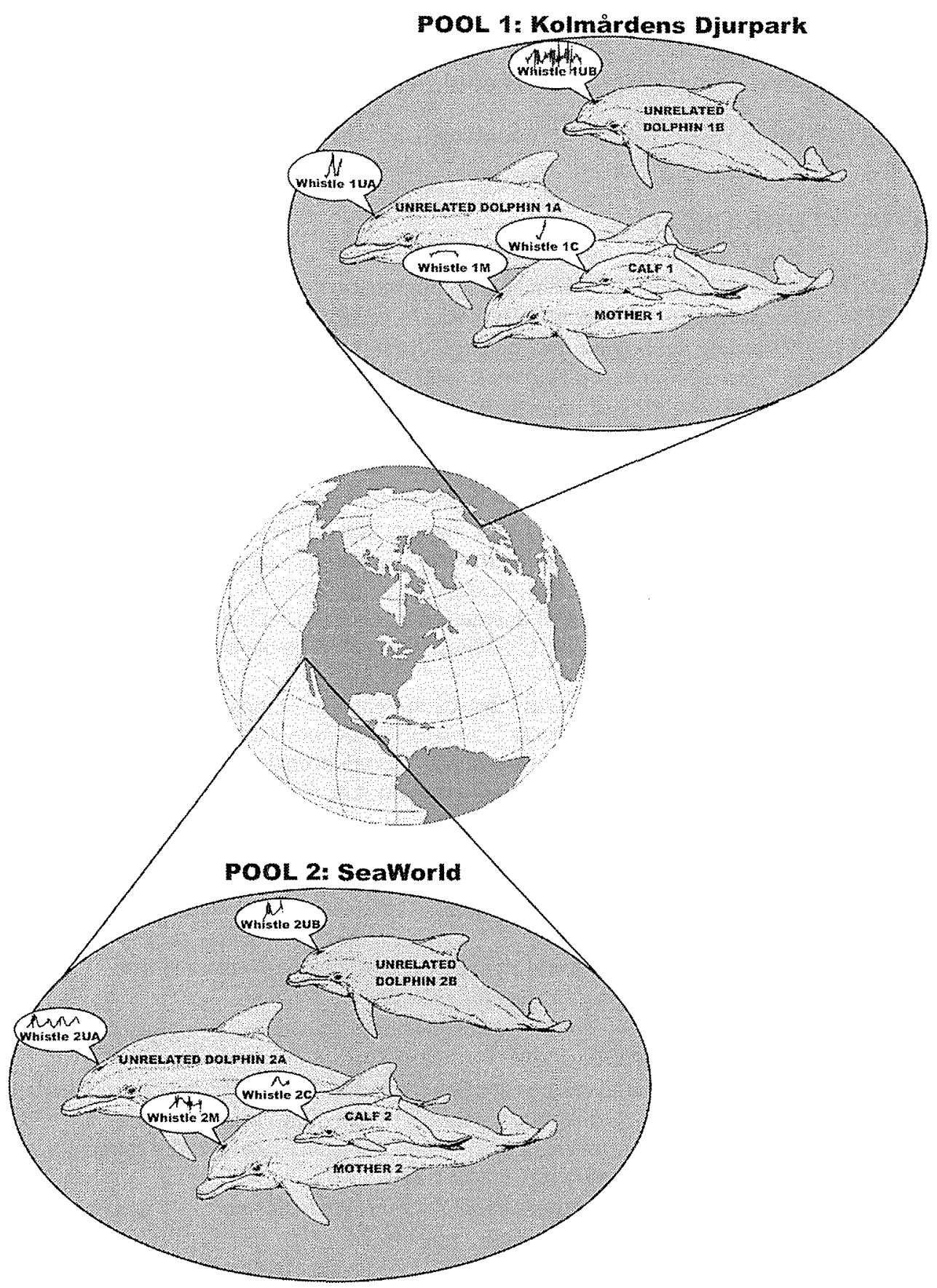

FIGURE 3.10: EXPERIMENTAL DESIGN

The two locations were chosen for demonstration purposes only. Similarly, the contours were used as examples: they do not represent contours of dolphins in those actual locations. Each calf's whistle should be compared to the whistles of all adults in both pools. If the whistles are learned, the calf's whistle should only match the whistles of the unrelated adults in his pool. The social relationships both the calf and his mother have with those adults will help determine what social factors influence vocal learning. (The dolphin picture was purchased from ArtToday.) 
Chapter 3: Social Relationships

dolphin, for instance, that reaction could influence the salience to the fetus of the sounds that dolphin produces. An example of this could be an increase or decrease in the mother's stress level in response to the vocalizations of other dolphins.

\subsection{LITERATURE CITED}

Altmann, J., 1974. "Observational study of behavior: Sampling methods." Behaviour, 49, pp. 227-267.

Altmann, J., 1980. Baboon mothers and infants. Harvard University Press, Cambridge, MA.

Amundin, M., 1986. "Breeding the bottle-nosed dolphin, Tursiops truncatus, at the Kolmården Dolphinarium." International Zoo Yearbook, 24/25, pp. 263-271.

Appleby, M.C., 1983. "The probability of linearity in hierarchies." Animal Behaviour, 31, pp. 600-608.

Baker, K.C. and Smuts, B.B., 1994. "Social relationships of female chimpanzees: Diversity between captive social groups." Chimpanzee cultures, eds. R.W. Wrangham, W.C. McGrew, F.B.M. deWaal, and P.G. Heltne. Harvard University Press, Cambridge, MA. pp. 227-42.

Ballance, L.T., 1990. "Residence patterns, group organization, and surfacing associations of bottlenose dolphins in Kino Bay, Gulf of California, Mexico." The bottlenose dolphin, eds. S. Leatherwood and R.R. Reeves. Academic Press, Inc., Boston, MA. pp. 267-84.

Baptista, L.F. and Gaunt, S.L.L., 1997. "Social interaction and vocal development in birds." Social influences on vocal development, eds. C.T. Snowdon and M. Hausberger. Cambridge University Press, New York, NY. pp. 23-40.

Baptista, L.F. and Petrinovich, L., 1984. "Social interaction, sensitive phases, and the song template hypothesis in the white-crowned sparrow." Animal Behaviour, 32, pp. 172-181.

Bel'kovich, V.M., 1991. "Herd structure, hunting, and play: Bottlenose dolphins in the black sea." Dolphin Societies: Discoveries and Puzzles, eds. K. Pryor and K.S. Norris. University of California Press, Berkeley, CA. pp. 17-78.

Bigg, M.A., Olesiuk, P.F., Ellis, G.M., Ford, J.K.B., and Balcomb III, K.C., 1990. "Social organization and genealogy of resident killer whales (Orcinus orca) in the coastal waters of British Columbia and Washington State." Report of the International Whaling Commision, Special Issue 12, pp. 383-404.

Brown, E.D., 1985. "The role of song and vocal imitation among common crows (Corvus brachyrhynchos)." Zeitschrift fur Tierpsychologie, 68, pp. 115-136.

Cheney, D., Seyfarth, R., and Smuts, B., 1986. "Social relationships and social cognition in nonhuman primates." Science, 234, pp. 1361-1366.

Chirighin, L., 1987. "Mother-calf spatial relationships and calf development in the captive bottlenose dolphin (Tursiops truncatus)." Aquatic Mammals, 13, pp. 5-15.

Clayton, N.S., 1988. "Song tutor choice in zebra finches and Bengalese finches: the relative importance of visual and vocal cues." Behaviour, 104, pp. 281-299.

Colgan, P.W. and Smith, J.T., 1978. "Multidimensional contingency table analysis." Quantitative ethology, ed. P.W. Colgan. Wiley \& Sons, New York. pp. 145-74.

Connor, R.C., Smolker, R.A., and Richards, A.F., 1992. "Two levels of alliance formation among male bottlenose dolphins (Tursiops sp.)." Proceeds of the National Academy of Science, USA, 89, pp. 987-990.

Dudok van Heel, W.H. and Meyer, M.M., 1974. "Birth in dolphins (Tursiops truncatus, Mont.) in the Dolphinarium Harderwijk, Netherlands." Aquatic Mammals, pp. 11-22.

Dudzinski, K.M., 1996. Communication and behavior in the Atlantic spotted dolphins (Stenella frantalis): Relationships between vocal and behavioral activities. Ph.D. Dissertation, Texas A\&M University. 
Duffield, D.A. and Wells, R.S., 1991. "The combined application of chromosome, protein, and molecular data for the investigation of social unit structure and dynamics in Tursiops truncatus." Reports to the International Whaling Commission, Special issue 13, pp. 155-169.

Frank, L.G., 1986. "Social organization of the spotted hyaena (Crocuta crocuta). II. Dominance and reproduction." Animal behaviour, 34, pp. 1510-1527.

Gittleman, J.L., 1985. "Functions of communal care in mammals." Evolution: Essays in honour of John Maynard Smith, eds. P.J. Greenwood, P.H. Harvey, and M. Slatkin. Cambridge University Press, Cambridge, UK. pp. 187-208.

Goodman, L.A., 1978. Analyzing qualitative/categorical data: Log-linear models and latent structure analysis. Abt Books, Cambridge, MA.

Gurevich, V.S., 1977. "Post natal behaviour of an Atlantic bottlenose dolphin calf." Breeding Dolphins: present status, suggestions for the future, eds. S.H. Ridgeway and K. Benirschke. US Marine Mammal Commission Report No. MMC-76-/07, Washington, DC. pp. 168-75.

Haenal, N., 1986. "General notes on the behavioural ontogeny of Puget Sound Killer Whales and the occurrence of allomaternal behaviour." Behavioural Biology of Killer Whales, eds. B.C. Kirkeold and J.S. Lockard. Alan Liss, Inc, New York, NY. pp. 285-300.

Hass, C.C., 1990. "Alternative maternal-care patterns in two herds of bighorn sheep." Journal of Mammology, 71(1), pp. 24-35.

Hausberger, M., Richard, M.A., Henry, L., Lepage, L., and Schmidt, I., 1995. "Song sharing reflects the social organization in a captive group of European starlings (Sturnus vulgaris)." Journal of Comparative Psychology, 109, pp. 222-241.

Hauser, M.D., 1988. "How infant vervet monkeys learn to recognize starling calls: The role of experience." Behaviour, 105, pp. 187-201.

Hauser, M.D., 1989. "Ontogenetic changes in the comprehension and production of vervet monkey (Cercopithecus aethiops) vocalizations." Journal of Comparative Psychology, 103(2), pp. 149158.

Hausfater, G., 1975. "Dominance and reproduction in baboons (Papio cynocephalus)." Contributions to primatology, eds. H. Kuhn, W.P. Luckett, C.R. Noback, A.H. Schultz, D. Starch, and F.S. Szalay. S. Karger, New York, NY.

Heimlich-Boran, J.R., 1993. Social organisation of the short-finned pilot whale, Globicephala macrorhynchus, with special reference to the comparative social ecology of delphinids. Ph.D. Dissertation, Cambridge University.

Heimlich-Boran, S., 1986. "Cohesive relationships among Puget Sound killer whales." Behavioral biology of killer whales, eds. B.C. Kirkevold and J.S. Lockard. Alan R. Liss, Inc., New York. pp. 251-84.

Heimlich-Boran, S.L., 1988. Association patterns and social dynamics of killer whales (Orcinus orca) in Greater Puget Sound. MA Thesis, San Jose State University.

Herzing, D.L., 1996. "Vocalizations and associated underwater behavior of free-ranging Atlantic spotted dolphins, Stenella frontalis and bottlenose dolphins, Tursiops truncatus." Aquatic Mammals, 22.2, pp. 61-79.

Hinde, R.A. and Atkinson, S., 1970. "Assessing the roles of social partners in maintaining mutual proximity, as exemplified by mother-infant relations in rhesus monkeys." Animal behaviour, 18, pp. 169-176.

Hinde, R.A., 1976. "Interactions, relationships, and social structure." Man, 11, pp. 1-17.

Hinde, R.A., 1983. "A conceptual framework." Primate social relationships, an integrated approach, ed. R.A. Hinde. Sinauer Associates, Inc., Sunderland, MA. pp. 1-7.

Hrdy, S.B., 1976. "Care and exploitation of nonhuman primate infants by conspecifics other than the mother." Advances in the Study of Behavior, 6, pp. 101-158.

Hrdy, S.B., 1977. The Langurs of Abu. Harvard University Press, Cambridge, MA.

Immelmann, K., 1969. "Song development in the zebra finch and other estrildid finches." Bird Vocalizations, ed. R.A. Hinde. Cambridge University Press, Cambridge, UK. pp. 64-74. 
Chapter 3: Social Relationships

Janik, V.M. and Slater, P.J.B., 1997. "Vocal learning in mammals." Advances in the Study of Behavior, 26, pp. 59-99.

Kastelein, R.A., Dokter, T., and Zwart, P., 1990. "The suckling of a Bottlenose dolphin calf (Tursiops truncatus) by a foster mother, and information on transverse birth bands." Aquatic Mammals, 16(3), pp. 134-138.

Knoke, D. and Burke, P.J., 1980. Log-linear models. Sage publications, Beverly Hills, CA.

Leatherwood, S., 1977. "Some preliminary impressions on the numbers and social behaviour of freeswimming bottlenose dolphin calves." Breeding Dolphins: present status, suggestions for the future, eds. S.H. Ridgeway and K. Benirschke. US Marine Mammal Commission Report No. MMC-76-/07, Washington, DC. pp. 168-75.

Lee, P.C., 1987. "Allomothering among African elephants." Animal behaviour, 35, pp. 278-291.

Locke, J.L. and Snow, C., 1997. "Social influences on vocal learning in human and nonhuman primates." Social influences on vocal learning, eds. C.T. Snowdon and M. Hausberger. Cambridge University Press, New York, NY. pp. 274-92.

Mann, J. and Smuts, B.B., 1998. "Natal Attraction: Kidnapping, allocare, and separations among wild Bottlenose dolphin (Tursiops truncatus) mothers and infants." Animal behaviour, 55, pp. 10971113.

Mann, J. and Smuts, B.B., in prep. "Behavioural development of wild bottlenose dolphin newborns."

Margoliash, D., Staicer, C.A., and Inoue, S.A., 1994. "The process of syllable acquisition in adult indigo buntings (Passerina cyanea)." Behaviour, 131, pp. 39-64.

Marler, P., 1970. "A comparative approach to vocal learning: Song development in white-crowned sparrows." Journal of Comparative Physiology and Psychology, 71, pp. 1-25.

Marsden, H.M. and Vessey, S.H., 1968. "Adoption of an infant green monkey within a social group." Communications in Behavioral Biology, Part A, 2, pp. 275-279.

McBride, A.F. and Kritzler, H., 1951. "Observations of pregnancy, parturition, and postnatal behaviour in the bottlenose dolphin." Journal of Mammology, 32 (3), pp. 251-265.

Messinger, C., Messinger, D., and Reddy, M., 1996. "Spontaneous lactation in a five-year-old bottlenose dolphin, Tursiops truncatus." Marine Mammals: Public Display and Research.

Moehlman, P.D., 1987. "Social organization in jackals." American Scientist, 75, pp. 366-375.

Moss, C.J. and Poole, J.H., 1983. "Relationships and social structure of African elephants." Primate social relationships, an integrated approach, ed. R.A. Hinde. Sinauer Associates, Inc., Sunderland, MA. pp. 314-24.

Nicolson, N., 1987. "Mothers, infants and adult females." Primate Societies, eds. B.B. Smuts, D.L. Cheney, R.M. Seyfarth, R.W. Wrangham, and T. Struhsaker. University of Chicago Press, Chicago, IL. pp. 330-42.

Overstrom, N.A., 1983. "Association between burst-pules sounds and aggressive behavior in captive Atlantic bottlenosed dolphins (Tursiops truncatus)." Zoo Biology, 2, pp. 93-103.

Packer, C., Lewis, S., and Pusey, A., 1992. "A comparative analysis of non-offspring nursing." Animal behaviour, 43(2), pp. 265-281.

Payne, R.B. and Payne, L.L., 1997. "Field observations, experimental design, and the time and place of learning bird songs." Social influences on vocal development, eds. C.T. Snowdon and M. Hausberger. Cambridge University Press, New York, NY. pp. 57-84.

Peddemors, V.M., Fothergill, M., and Cockcroft, V.G., 1992. "Feeding and growth in a captive-born bottlenose dolphin Tursiops truncatus." South African Journal of Zoology, 27(2), pp. 74-80.

Podos, J., Peters, S., Rudnicky, T., Marler, P., and Nowicki, S., 1992. "The organization of song repertoires in song sparrows: Themes and variations." Ethology, 90, pp. 89-106.

Prescott, J.H., 1977. "Comments on captive births of Tursiops truncatus at Marineland of the Pacific (19571972)." Breeding dolphins: Present status, suggestions for the future, eds. S.H. Ridgway and K. Benirschke. Report No. MMC-76/07 to the US Marine Mammal Commission, Washington, DC. pp. 71-6. 
Pryor, K. and Shallenberger, I.K., 1991. "Social structure in spotted dolphins (Stenella attenuata) in the tuna purse seine fishery in the eastern tropical Pacific." Dolphin Societies: Discoveries and puzzles, eds. K. Pryor and K.S. Norris. University of California Press, Berkeley, CA. pp. 161-98.

Raferty, A.E., 1986. "Choosing models for cross-classifications." American Sociological Review, 51, pp. 145-146.

Recchia, C.A., 1994. Social behaviour of captive belugas, Delphinapterus leucas. Ph.D. Dissertation, Woods Hole Oceanographic Institution \& Massachusetts Institute of Technology, Woods Hole, MA.

Reid, K., Mann, J., Weiner, J.R., and Hecker, N., 1995. "Infant development in two aquarium bottlenose dolphins." Zoo Biology, 14, pp. 00-00.

Richards, D.G., Wolz, J.P., and Herman, L.M., 1984. "Vocal mimicry of computer-generated sounds and vocal labeling of objects by a bottlenose dolphin, Tursiops truncatus." Journal of Comparative Psychology, 98(1), pp. 10-28.

Riedman, M., 1990. The pinnipeds: Seals, sea lions, walruses. University of California Press, Berkeley, CA.

Riedman, M.L. and Le Boeuf, B.J., 1982. "Mother-pup separation and adoption in Northern elephant seals." Behavioral Ecology and Sociobiology, 11, pp. 203-215.

Riedman, M.L., 1982. "The evolution of alloparental care and adoption in mammals and birds." Quarterly Review of Biology, 57, pp. 405-435.

Samuels, A. and Gifford, T., 1997. "A quantitative assessment of dominance relations among bottlenose dolphins." Marine Mammal Science, 13, pp. 70-99.

Samuels, A., Silk, J.B., and Altmann, J., 1987. "Continuity and change in dominance relations among female baboons." Animal behaviour, 35, pp. 785-793.

Seyfarth, R.M. and Cheney, D.L., 1997. "Some general features of vocal development in nonhuman primates." Social influences on vocal development, eds. C.T. Snowdon and M. Hausberger. Cambridge University Press, New York, NY. pp. 249-74.

Seyfarth, R.M., 1976. "Social relationships among female baboons." Animal behaviour, 24, pp. 917-938.

Seyfarth, R.M., 1980. "The distribution of grooming and related behaviors among adult female vervet monkeys." Animal behaviour, 28, pp. 798-813.

Shallenberger, E.W. and Kang, I., 1977. "Dolphin births at Sea Life Park." Breeding dolphins: Present status, suggestions for the future, eds. S.H. Ridgway and K. Benirschke. Report No. MMC-76/07 to the US Marine Mammal Commission, Washington, DC. pp. 77-84.

Shane, S.H., 1990. "Behavior and ecology of the bottlenose dolphin at Sanibel Island, Florida." The Bottlenose Dolphin, eds. S. Leatherwood and R.R. Reeves. Academic Press, Boston, MA. pp. 245-66.

Shopland, J.M. and Altmann, J., 1987. "Fatal intragroup kidnapping in yellow baboons." American Journal of Primatology, 13, pp. 61-65.

Smolders, J., 1986. "Pre- and post-natal behavioural problems of a Tursiops female conceived in the ocean." Aquatic Mammals, 12(3), pp. 80-82.

Smolker, R.A., Richards, A.F., Connor, R.C., and Pepper, J.W., 1992. "Sex differences in patterns of association among Indian Ocean bottlenose dolphins." Behaviour, 123(1/2), pp. 38-69.

Smuts, B.B., 1985. Sex and friendship in baboons. Gruyter, New York.

Snowdon, C.T. and Hausberger, M., 1997. Social influences on vocal development. Cambridge University Press, New York, NY.

Stanford, C.B., 1992. "Costs and benefits of allomothering in wild capped langurs (Presbytis pileata)." Behavioral Ecology and Sociobiology, 30, pp. 29-34.

Tavolga, M.C. and Essapian, F.S., 1957. "The behaviour of the bottlenose dolphin Tursiops truncatus: Mating, pregnancy, parturition and mother-infant behaviour." Zoologica, 421, pp. 11-31.

Thurman, G.D. and Williams, M.C., 1986. "Neonatal mortality in two Indian Ocean bottlenose dolphins bred in captivity." Aquatic Mammals, 12(3), pp. 83-86.

Torgerson, W.S., 1958. Theory and methods of scaling. John Wiley \& Sons, Inc., New York, NY. 


\section{Chapter 3: Social Relationships}

Tyack, P.L. and Sayigh, L.S., 1997. "Vocal learning in cetaceans." Social influences on vocal development, eds. C.T. Snowdon and M. Hausberger. Cambridge University Press, New York, NY. pp. 208-33.

Vogel, C., 1984. "Pattern of infant transfer within two troops of common langurs (Presbytis entellus) near Jodphur: Testing hypotheses concerning benefits and risks." Current Primate Researches, eds. M.L. Roonwal, S.M. Mohnot, and N.S. Rathore. University of Jodphur Department of Zoology, Jodphur, India. pp. 361-80.

Waite, J.M., 1988. Alloparental care in killer whales: Orcinus orca. Masters Thesis, University of California, Santa Cruz, CA.

Wells, R.S., 1991. "The role of long-term study in understanding the social structure of a bottlenose dolphin community." Dolphin Societies, eds. K. Pryor and K.S. Norris. University of California Press, Berkeley, CA. pp. 199-235.

Wells, R.S., Scott, M.D., and Irvine, A.B., 1987. "The social structure of free-ranging bottlenose dolphins." Current Mammology, ed. H.H. Genoways. Plenum Press, New York, NY. pp. 247-305.

West, MJ, Stroud, A.N., and King, A.P., 1983. "Mimicry of the human voice by European starlings (Sturnus vulgaris): The role of social interaction." Wilson Bulletin, 95, pp. 635-640.

Whitehead, H., 1997. “Analyzing animal social structure." Animal behaviour, 53, pp. 1053-1067. 


\section{CHAPTER 4: ACOUSTIC ANALYSIS METHODS}

\subsection{INTRODUCTION}

A study of vocal development requires the quantitative characterization of infants' acoustic environments. To study vocal learning, the vocalizations of infants must be compared to the vocalizations in their own acoustic environments and in the acoustic environments of other infants (see Figures $1.1 \& 3.10$ ). A demonstration of vocal learning requires that the infants match the vocalizations made in their own environments and do not match those made only in the environments of other infants (see Figures $1.1 \&$ 3.10). This requires the quantitative comparison of the acoustic environments of multiple infants. The quantitative comparison of acoustic environments involves several steps: 1) sampling the sounds in each environment, 2) extracting those sounds from the recordings, and 3) comparing the sounds to each other. The first step, sampling, involves deciding when and how to record the sounds. Strategies for sampling sounds were discussed in chapter 2 . This chapter is concerned with the second and third steps: extracting and comparing sounds. In dolphin research, both tasks have traditionally been done by hand (e.g. Tyack 1986, Caldwell et al. 1990, Janik \& Slater 1998). However, manual extraction and comparison of whistles is an extremely time consuming process with many possibilities for introducing biases. Automatic, computerized extraction and comparison of whistles can solve most of those problems and significantly increase the possible sample size. The goal of this chapter is to develop and test automatic, computerbased methods for extracting dolphin whistles from recordings and comparing those whistles to each other.

Extracting whistles is an essential task in the characterization of an acoustic environment, and a potentially very time consuming one. In the sampling protocol designed in chapter 2 , the sounds made in the pool were recorded onto tape at the same time that behavioral samples were taken. In this design, all the sounds made in the pool were recorded for the entire duration of the behavioral sample. Within that sample, there will be some whistles, but there will also be time without whistles. The first step, therefore, is to find the whistles on the tape and extract them. Manually searching 
Chapter 4: Acoustic Analysis Methods

through large amounts of tape to find whistles is extremely time-consuming. Having found the whistles, making sure that they are all extracted consistently can be difficult. Before starting to extract whistles, a series of decisions must be made. Some examples of these decisions include 1) the minimum amplitude for a whistle to be included, 2) the shortest sound that will count as a "whistle", and 3) how much time there can be between two sections of sound for them to be considered part of the same whistle. Most studies where whistles are extracted by hand do not even mention these decisions (e.g. Tyack 1986, Sayigh et al. 1995, Herzing 1996). Once the decisions have been made, each sound on the tape must be evaluated to determine whether it fits the requirements. The measurement entailed in making these determinations for every whistle can make a timeconsuming task even more time-consuming. More importantly, ensuring that the decisions are made consistently every time a whistle is extracted can be difficult, particularly if the decisions were not explicitly laid out before starting the extraction. If the decisions are not made consistently, the data may be biased toward certain types of sounds. A computer program in which these decisions are pre-programmed can solve that problem.

Automatic extraction can also increase the number of whistles that can be extracted. Because manually extracting whistles is time consuming, the sample size achieved by manual extraction is often small (e.g. Tyack 1986, McCowan \& Reiss 1995, Sayigh et al. 1995). Sayigh et al. (1990) state that "Because each animal typically emitted hundreds to thousands of whistles in a recording session, it was prohibitively time consuming to make a spectrogram of every whistle" (p251). This problem adds another decision to the list: which whistles to select. This process has the potential to add a serious bias if not carefully done. An automatic, computer-based extractor allows far more whistles to be included in the sample and ensures that any bias in choosing whistles is at least consistent in all whistles. The decisions can be made before the extraction begins and every sound treated equally based on the parameters set up in the program. Because the parameters are pre-programmed, the extractor can be tested to ascertain what biases might exist. A method for automatically extracting whistles from 
tapes will be developed and tested in this chapter. The programs for this were written with the help of Mark Johnson, Jim Partan, and Rebecca Thomas (see Appendix 2 for the text of the programs).

As with automatic extraction, automatic comparison and categorization of whistles can solve many of the problems that arise with manual comparison. Whistles that are extracted by hand are then often compared by human judges (e.g. Sayigh et al. 1995, Janik \& Slater 1998). Although judges' ratings have been shown to be reliable (Sayigh 1992, Janik 1998), using human judges severely restricts the number of whistles that can be used. The problem is that each judge can only compare a certain number of spectrograms before becoming fatigued. To make all the pairwise comparisons for a sample of a thousand whistles, a million pairwise comparisons need to be made. If a judge can compare a hundred pairs of whistles, ten thousand judges would be needed. Finding ten thousand judges is, of course, impractical. Because computers can do many calculations quickly, an automatic, computer-based comparison algorithm can solve this problem. Some researchers have used another method to increase their samples size: rather than performing pairwise comparisons, they sort the whistles into categories based on the overall shape of the whistle (e.g. Tyack 1986, Caldwell et al. 1990, Janik \& Slater 1998). One problem with this method is that the exact features of the sounds a human judge is using for the categorization can be difficult to ascertain. Comparisons made by computer allow for a more explicit understanding of the features being compared. Automatic, computerized comparisons are therefore preferable because they are explicit, fast, and capable of dealing with very large samples.

While few studies have used automatic extraction of sounds, a number of recent studies have used computer-based categorization methods (e.g. extraction: Sturtivant \& Datta 1995, Mellinger \& Clark 1997; categorization: Buck \& Tyack 1993, McCowan 1995, Smolker \& Pepper in press). However, many of these studies use different methods on data that were collected or extracted in different manners. This makes comparing studies, or determining which method is best suited for a given problem, difficult. Few studies compared multiple methods or tested the outcome of the methods 
Chapter 4: Acoustic Analysis Methods

against manual categorization (but see Nowicki \& Nelson 1990, Janik in press). This chapter will address that problem. A number of methods for automatically comparing and categorizing whistles will be tested and compared to each other and to manual categorization.

\subsection{DETECTION \& EXTRACTION OF WHISTLES}

Automatic detection of whistles is a complicated task because there are many sounds recorded in the presence of dolphins that are not whistles, including other types of vocalizations and transient noises. The whistles must be separated from these other sounds. If the sounds of interest have fixed time-frequency characteristics, as do the sounds of some birds and mysticetes, a program can be written to search for a sound with a certain spectrogram or waveform (Clark et al. 1987, Mellinger \& Clark 1997). Dolphin whistles, however, are too variable for that strategy to work. To find dolphin whistles, an automatic algorithm must look for all the sounds with a certain amplitude relative to the recording noise, or signal-to-noise ratio, and then determine which are whistles (e.g. Sturtivant \& Datta 1995). What makes whistles different from other dolphin sounds is that they are tonal and narrow-band. One method for determining which sounds are whistles is therefore to search for narrow-band sounds and only extract those sounds (Sturtivant \& Datta 1995). With this method, all the cuts are narrow-band, and most of them should be whistles. In any recording, however, there will be narrow-band noises that will be detected by the program. The cuts will therefore have to be sorted by hand to pick out the ones with whistles. In addition, some whistles may overlap broadband sounds, particularly since dolphins can produce whistles and broadband sounds simultaneously (Lilly \& Miller 1961). Without an extremely advanced filter, this method will miss those sounds. An alternative is to cut all sounds with a certain signal-to-noise ratio and sort them afterwards. This is the path taken in this chapter. All the sounds whose amplitude was greater than a set threshold were extracted from the tapes and the whistles were sorted out with a separate program. 
To go from sounds recorded onto tape to a usable set of whistle cuts requires a number of steps. The acoustic recordings made as part of the pilot study at Kolmårdens Djurpark (see chapter 2, section 2.2) were used to develop and test the automatic methods for this process. The sounds were recorded onto one channel of PAL-format VHS videotapes during the ten-minute behavioral focal samples (see chapter 2.2.2, Figure 2.1). Because this is an analog medium, the sounds needed to be digitized into the computer before the whistles could be extracted from the raw data. In addition, the timing of the whistles within the ten-minute focal sample was not known. Therefore, the entire ten minutes of each focal sample was digitized. The result was a file with ten minutes of sounds produced in the pool. The whistles were then extracted from these files in several steps. First, a threshold was determined based on a selected section of noise in the file, and all the sounds whose amplitude was above that threshold were detected and extracted from the file. Those sounds were then sorted based on their bandwidth to separate the narrow-band whistles from all other, broadband sounds. The programs for both tasks were written in Matlab 5.0 (Mathworks) for Linux (Red Hat 4.2), and the full programs can be found in Appendix 2. Because some burst-pulse sounds have most of their energy in a few frequencies and some narrow-band noises had greater amplitude than the threshold, not all the sounds classified as possible whistles by the sorter actually were whistles. A final manual sorting was therefore necessary to separate the whistles from the non-whistles. This combined process decreased the time needed to extract a sample of whistles considerably over manual extraction. It also allowed many more whistles to be extracted in a more consistent manner than would be possible with manual extraction. The outcome of both the automatic extractor and the automatic sorter were compared to manual extraction and sorting of the same data set to determine possible biases in the process. 
Chapter 4: Acoustic Analysis Methods

\subsubsection{METHODS}

\section{Digitization}

The ten-minute segments that corresponded to the focal samples were digitized into an IBM-compatible computer. The sounds were played back on a Samsung SV300W videocassette recorder. They were then filtered with a Frequency Devices 9002 programmable filter with a high-pass filter set at $2 \mathrm{kHz}$ and a post-filter gain of $5 \mathrm{x}$. The 2 $\mathrm{kHz}$ high-pass filter was necessary to eliminate low frequency noise from the water filtration system. Low-pass, anti-aliasing filtering was not needed on these recordings because the recorder had an upper-frequency cutoff around $30 \mathrm{kHz}$. Sounds were digitized at $80 \mathrm{kHz}$ by an analog-to-digital conversion board made by Dalanco Spry (model 250).

\section{Detection and Extraction of Sounds}

Sounds to be extracted from the digitized file were detected by comparing the power of the waveform to a pre-determined threshold (in Matlab, see Appendix 2). For each sample, the power output was determined as follows:

$$
\operatorname{Output}(i)=[\operatorname{Input}(i)]^{2}+0.9 \times \operatorname{Output}(i-1)
$$

Input $(i)$ was the amplitude of the $i^{\text {th }}$ digitized sample and Output(0) was defined to be 0 . The final term in the equation is a roll-off term to minimize the detection of short noise spikes. With this term, samples with high amplitudes also had high power output only if they were also preceded by samples with high amplitude (Figure 4.1). The output memory (0.9) was determined by trial and error to be the value that performed best with these data.

The threshold was determined based on a section of noise that was hand-selected from each file. Early work used a section of $200 \mathrm{~ms}$. However, the outcome of those extractions suggested that $200 \mathrm{~ms}$ was not enough to adequately represent the noise in the file. Therefore, later extractions used a section of noise that was 1 second long. The 


\section{Figure 4.1: AN EXAMPLe OF The AUtOMAtic Whistle Detection}

The voltage represents the amplitude of the sound. The power was calculated by the extractor (see text).

Figure A is a short ( $<50 \mathrm{~ms}$ ) section of noise. Figure B is a longer section $(>1 \mathrm{~s})$ containing a vocalization.

\section{A. NoISE}

The green line on the output is the threshold determined from the first 500 samples of this section. By this threshold, the sounds in this example would be considered noise and not extracted.
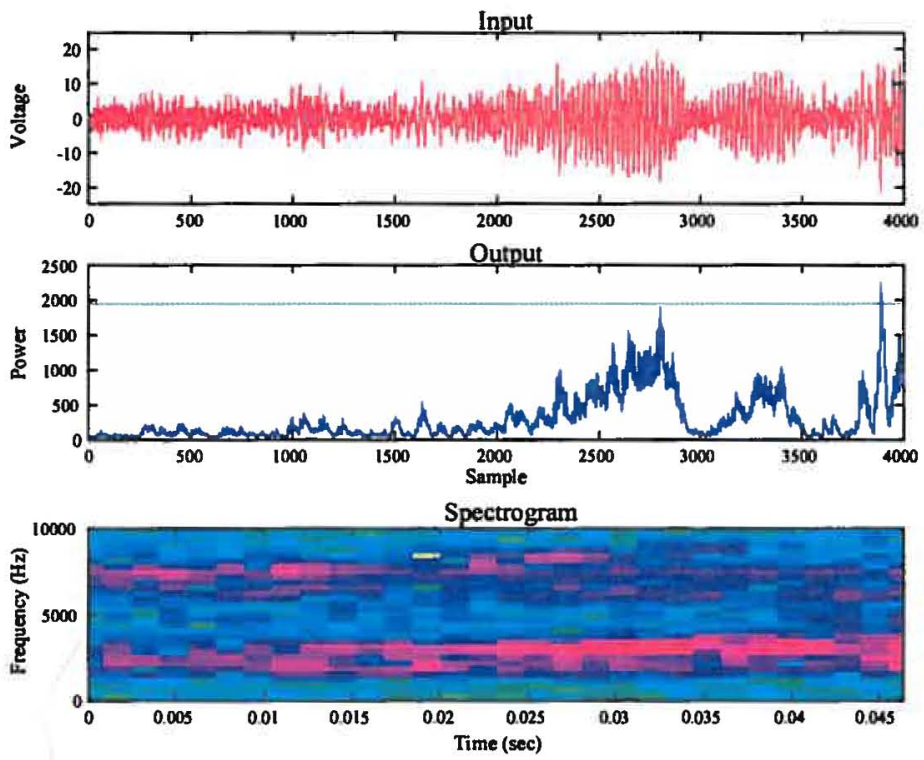

\section{B. VOCALIZATION}

The green line is a representative threshold. This broadband, burst-pulse call would have been extracted.
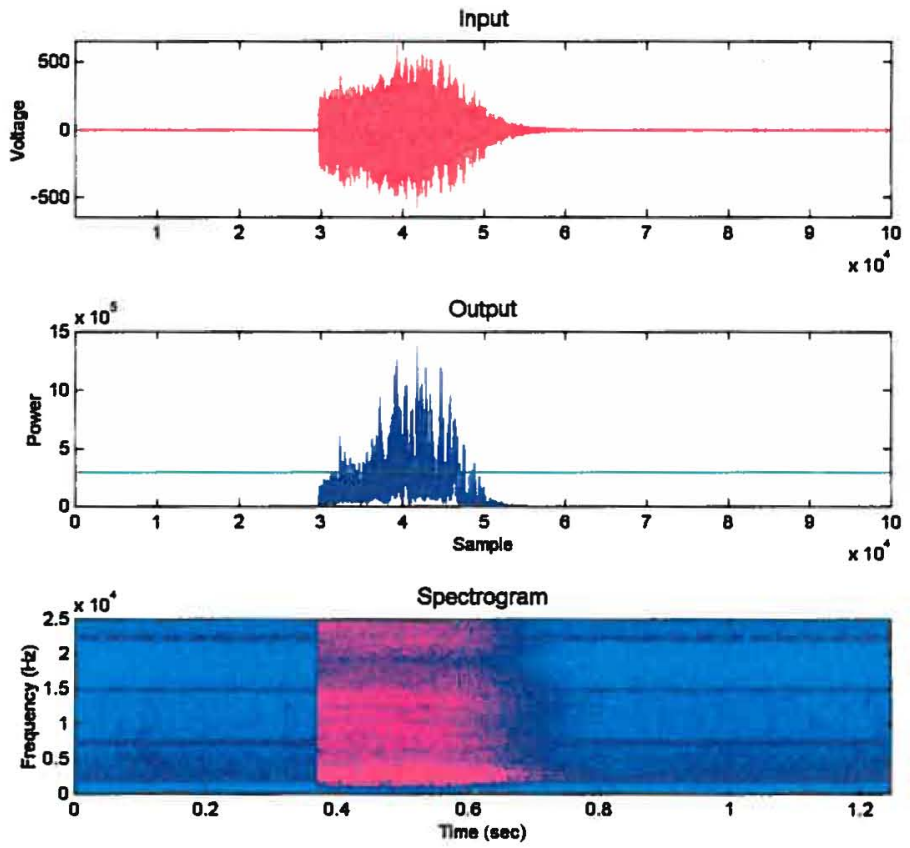
Chapter 4: Acoustic Analysis Methods

noise segments were chosen to be representative of the background noise in the file while lacking any vocalizations. The threshold for each file was calculated as follows:

$$
\text { Threshold }=5 \times \operatorname{stdev}(\text { Output }(\text { noise }))+\text { mean }(\text { Output(noise })) \text {. }
$$

As with the output memory, 5 standard deviations above the mean of the noise was determined by trial and error to perform best with these data. The power output of each digitized sample was compared to this threshold to determine whether that sample was "above-threshold" (Figure 4.1).

Sounds whose power output was greater than the threshold were automatically extracted from the files. The maximum allowed separation between sections was $100 \mathrm{~ms}$. This means that samples that were above the threshold were combined into one sound if the separation between them was less than $100 \mathrm{~ms}$. This value was determined by measurements of previously manually-extracted whistles, also from Kolmårdens Djurpark. In the manual extraction, whistle-like sounds that had up to $100 \mathrm{~ms}$ of silence in them had been considered to still be a single whistle, while sounds with more than 100 $\mathrm{ms}$ of silence had been divided into two whistles. The minimum duration for a sound to be extracted by the automatic extractor was $50 \mathrm{~ms}$. Sections that were above the threshold were therefore extracted if they were at least $50 \mathrm{~ms}$ long. With a sampling rate of $80 \mathrm{kHz}$, the minimum length for a sound to be extracted was therefore 4000 samples. As with the maximum separation, the minimum duration $(50 \mathrm{~ms})$ was determined from the minimum duration of previously manually-extracted whistles from Kolmårdens Djurpark.

\section{Automatic Sorting of Sounds}

One of the major differences between whistles and other sounds made by dolphins is the bandwidth. Although whistles are frequency-modulated, each section of the whistle is narrow-band, while most burst-pulse sounds and echolocation clicks are broadband (Caldwell et al. 1990). Extracted sounds were therefore sorted using an automatic measurement of bandwidth (in Matlab, see Appendix 2). The measure used was spectral concentration, which measures how many frequency bins of the spectrogram must be included to reach $50 \%$ of the total amplitude. Although the measure 
is called "concentration" (Fristrup \& Watkins 1994), the value will actually be lower for sounds with more concentrated energy. For instance, narrow-band sounds, whose energy is highly concentrated in a few frequency bins, will have a lower value of the spectral concentration measure than broad-band sounds, whose energy is less concentrated.

The spectral concentration was calculated for each time bin of the spectrogram (FFT size 256) for the frequency bins between 4 and $20 \mathrm{kHz}$. Each frequency bin encompassed $312.5 \mathrm{~Hz}$. In order to prevent the inclusion of short-term narrow-band noise, sounds were required to have low spectral concentration in two adjacent time bins. Each time bin was $3.2 \mathrm{~ms}$ long, so two bins encompassed $6.4 \mathrm{~ms}$. A time bin was considered to be narrow-band if its spectral concentration and that of the following bin were both less than 3 bins, or approximately $1 \mathrm{kHz}$. Because the concentration in each time bin was calculated separately, the energy in the adjacent time bins did not have to be concentrated in the same frequency bins. Therefore, sounds with rapid frequency modulation, within $6 \mathrm{~ms}$, were not excluded by this requirement. However, some whistles were so closely followed or preceded by broad-band sounds that the two sounds were not separated by the extraction program. In fact, a single dolphin can produce whistles and broadband sounds simultaneously (Lilly \& Miller 1961). Therefore, whistles were often found overlapping broadband sounds. Whistles that actually overlapped broadband sounds could not be separated and were therefore excluded. Less than $10 \%$ of the whistles found by manual extraction and sorting were of this type (see test of sorter, section 4.2.2). Whistles that were produced within $100 \mathrm{~ms}$ of a broadband sound without actually overlapping it were not separated by the extractor but could be separated later. In order not to exclude those whistles, only $10 \%$ of the time bins in a sound file's spectrogram had to be narrow-band for that file to be identified as a possible whistle. If a file were one second long, for instance, there would be 312 time bins. For the file to be considered a possible whistle, 31 of these bins, or at least $100 \mathrm{~ms}$, would have to have low spectral concentration and be adjacent to a bin with low spectral concentration. 
Chapter 4: Acoustic Analysis Methods

\subsubsection{TESTS}

TABLe 4.1 Test OF THE AUtOMATIC EXTRactor

\begin{tabular}{|l|c|c|c|c|}
\hline & May 27 & June 2 & June 5 & Total \\
\hline Cuts made by extractor & 1266 & 1503 & 1441 & 4210 \\
\hline Cuts with whistles (percent) & $279(22 \%)$ & $467(31 \%)$ & $206(14 \%)$ & $952(23 \%)$ \\
\hline Whistles identified by extractor & 336 & 614 & 257 & 1207 \\
\hline Whistles extracted manually & 311 & 576 & 179 & 1066 \\
\hline $\begin{array}{l}\text { Whistles not found manually } \\
\text { (percent of extractor's whistles) }\end{array}$ & $25(7 \%)$ & $38(6 \%)$ & $78(30 \%)$ & $141(12 \%)$ \\
\hline
\end{tabular}

Automatic Extractor

To test the accuracy of the extractor, sounds were extracted by hand from three recording sessions: one on May 27, 1995, one on June 2, 1995, and one on June 5, 1995. Manual extraction was done using CSIG, a spectrographic analysis program written by Kurt Fristrup with built-in noise and gain compensation (Watkins et al. 1992). The results of this extraction were compared to the automatic extractor for the same sessions (Table 4.1). The extractor made a total of 4210 cuts from the three sessions (Table 4.1). These cuts were sorted manually to determine how many whistles had been found by the extractor. Manual sorting was done so that the extractor was tested separately from the automatic sorter. Of the cuts, $952(23 \%)$ contained whistles. Since some of the files contained more than one whistle, a total of 1207 whistles were found by the extractor. Manual extraction resulted in 1066 whistles cut from the three sessions (Table 4.1). All of the whistles found by hand were also found by the extractor, but an average of $12 \%$ of the whistles found by the extractor were not found by hand (Table 4.1). One reason for this may be that the maximum separation of $100 \mathrm{~ms}$ was not always followed exactly in the manual extraction. Therefore, some of the whistles that were found manually were divided into multiple whistles by the extractor. The other whistles found by the extractor but not by the manual extraction were low amplitude, flat whistles that may have been missed when the files were analyzed visually. This suggests that the extractor is more sensitive to some types of whistles than manual extraction. 
TABle 4.2 Test OF THE AUtOMATIC SORTER

\begin{tabular}{|l|c|c|c|}
\hline & March 14 & April 20 & Total \\
\hline Total sounds & 1640 & 1339 & 2979 \\
\hline \hline Whistles identified by eye & 111 & 360 & 471 \\
\hline Whistles correctly identified by sorter & $107(96 \%)$ & $316(88 \%)$ & $423(90 \%)$ \\
\hline Whistles missed by sorter & $4(4 \%)$ & $44(12 \%)$ & $48(10 \%)$ \\
\hline \hline Non-whistles identified by eye & 1529 & 979 & 2508 \\
\hline Non-whistles correctly identified by sorter & $1390(91 \%)$ & $955(98 \%)$ & $2345(93 \%)$ \\
\hline Sounds identified as possible whistles & 246 & 340 & 586 \\
\hline Incorrect positive identifications & $139(56 \%)$ & $24(7 \%)$ & $163(28 \%)$ \\
\hline
\end{tabular}

\section{Automatic Sorter}

In the previous test, only $23 \%$ of the cuts made by the automatic extractor contained whistles. The reason for this is that, as was mentioned before, the extractor was designed to detect any sound whose power is greater than the threshold. The program did not differentiate between types of sounds, so both narrow-band whistles and broad-band burst-pulse and echolocation calls were extracted. The cuts made by the extractor must therefore be sorted before they can be used. This is the reason that the automatic sorter was created. To test the accuracy of the sorting program, sounds automatically extracted from two recording sessions, one on March 14, 1995 and one on April 20, 1995, were sorted manually. The results were compared to the performance of the automatic sorter (Table 4.2). In total, 2979 sound files were tested. Of these, 471 were qualitatively determined to be whistles and 2508 to be non-whistles. The automatic sorter correctly identified $90 \%$ (423) of the whistles and 93\% (2345) of the non-whistles. An examination of the $48(10 \%)$ whistles incorrectly identified as non-whistles by the program showed that the sorter missed short whistles, low amplitude whistles, and whistles that overlapped loud noises. These were whistles whose amplitude was not great enough relative to the surrounding noise to dominate the spectral concentration. The sorter also missed whistles that were completely overlapping broadband sounds that were louder than the whistles. 
Chapter 4: Acoustic Analysis Methods

\section{Final Sorting and Extraction}

Of the sounds identified by the automatic sorter as possible whistles, $28 \%$ (163) were not whistles (see Table 4.2). Because of this, the sounds identified as possible whistles by the sorting program had to be sorted again by hand (see Appendix 2). This procedure is likely to further bias the sample against very short, very low amplitude, or very noisy whistles. This will be especially true of low-frequency whistles that overlap the most common noise frequencies, which are approximately 2 to $5 \mathrm{kHz}$ for these recordings. In addition, some extracted files had to be further extracted because noise or broad-band sounds between or near the whistles prevented the automatic extractor from properly separating the whistles. I attempted to use the same rules for separation and minimum duration for the manual extraction procedure that were used by the automatic extractor. Whistles that overlapped other whistles were also excluded because the available analysis methods would not have been able to separate them (see below).

\subsubsection{CONCLUSIONS}

The extraction and the two sorting procedures yielded a usable set of whistles, that was very similar to the sample produced by careful manual extraction. The automatic extractor found more whistles than manual extraction did. In particular, the extractor picked up some low amplitude, short whistles that were missed by manual extraction. The automatic sorter missed only $10 \%$ of the whistles found by the extractor. Those that were missed were almost all short, low amplitude, or noisy, generally the type of whistles that the extractor had found that manual extraction had not. In addition, to characterize the acoustic environment, whistles need to be compared to each other. All the currently available comparison methods require that some information about the whistle be extracted from the spectrogram (see section 4.3). This requires a certain signal-to-noise ratio. Therefore, if a whistle were too low amplitude relative to the surrounding noise for the automatic sorter to distinguish it from the noise, the available analysis techniques would not be able to separate it either. For this reason, many of the 
sounds incorrectly excluded by the sorter are likely to have been excluded from later analysis anyway.

This process took a third of the time it took to extract the data manually. The extraction and the automatic sorting can be done by the computer without human supervision. On each day, the cuts made from the sessions digitized on the previous day can be manually sorted at the same time that new sessions are being digitized. This allows the time to be used efficiently. These programs therefore cut a great deal of time out of the process of whistle extraction. Since time is one of the limiting factors in the ability to create a large data set, these programs are enormously useful. While the sorting is slightly biased against very short, low amplitude, or noisy sounds, the extractor appears to be more sensitive to those sounds than manual extraction. The data set extracted by these programs is therefore equivalent to the one that would have been extracted manually, but includes many more whistles than would have been possible to include in a reasonable amount of time by manual extraction.

Less than a quarter of the cuts made by the automatic extractor contained whistles. Many of the cuts contained only noise. There are several reasons for this. In order to include whistles with breaks in them, the extractor was designed to combine segments with as much as $100 \mathrm{~ms}$ of space between the sounds. In addition, in order to include very short whistles, the minimum duration was only $50 \mathrm{~ms}$. Therefore, two short noise spikes that occurred within $100 \mathrm{~ms}$ of each other were combined and cut by the extractor. The underlying problem was the signal-to-noise ratio. The recordings made in the pilot study were very noisy and some of the whistles had very low amplitudes. In addition, the noise changed over the ten minutes of each segment. This occurred because in order to keep the hydrophone away from the dolphins, it was positioned near the edge of the pool. Therefore, the background noise was heavily influenced by the movement of the water at the edge of the pool, as well as several nearby filtration devices, which did not operate in a consistent manner. To improve the performance of the extractor, future versions should employ an automatic noise-extraction procedure that allows the threshold 
Chapter 4: Acoustic Analysis Methods

to change over time. Future versions could also use more sophisticated detection algorithms to differentiate whistles from non-whistles before they are extracted.

The automatic sorter had similar problems: more than a quarter of the cuts identified as "possible whistles" were simply noise. This is again partly due to the signalto-noise ratio. Because every cut, with or without whistles, had a great deal of noise in the low frequencies, as well as a few bands of noise in the high frequencies, the sorter was designed to only consider the frequencies between 4 and $20 \mathrm{kHz}$. The extractor on the other hand, considered all frequencies available (2 to $40 \mathrm{kHz}$ ). Noise that was concentrated below $4 \mathrm{kHz}$ was therefore listed as a "possible whistle." Similarly, echolocation clicks with very little energy below $20 \mathrm{kHz}$ was sometimes considered to be narrow-band as well, and listed as a "possible whistle." A more sophisticated version of the extractor could solve these problems by making the sorting process unnecessary.

The problems with both the extractor and the sorter were partly caused by the need to extract all possible whistles, including very short ones and very low amplitude ones. This is necessary in a study of vocal development to get a clear picture of all the possible influences on the calf. In a different study, with a different focus, finding all the whistles might not be necessary. If only the high amplitude, clear, long whistles are desired, such as in a study concentrating on signature whistles, the settings of the extractor and sorter could be changed to solve some of the problems experienced here.

\subsection{COMPARISON AND CATEGORIZATION OF WHISTLES}

The previous section describes how a usable set of whistles can be acquired by automatically extracting sounds from recordings. In order to compare the acoustic environments of multiple calves, these whistles must now be compared and grouped into categories. The most common method of doing this has been to use human judges (e.g. Tyack 1986, Moore \& Ridgeway 1995, Sayigh et al. 1995, Herzing 1996, Janik \& Slater 1998). Although the recordings used in these studies were digitized into a computer, the sounds of interest were manually extracted and then compared and categorized by visual inspection of the spectrograms. Statistical categorization is preferable to visual 
categorization for several reasons, including the ability to handle large sample sizes and to explicitly understand the physical features being used in the categorization. The latter ability is important partly because the perceptual features used by the dolphins to categorize whistles are not known. Testing for perceptual features requires a clear understanding of the physical features used to create the categories being tested.

Some studies have been done using statistical methods to compare and categorize whistles and other types of vocalizations (e.g. Nowicki \& Nelson 1990, Buck \& Tyack 1993, Fristrup \& Watkins 1994, McCowan 1995). The most common methods used for this have been the categorization of extracted features such as duration and bandwidth (e.g. Fristrup \& Watkins 1994), and the comparison and categorization of extracted frequency-time contours (e.g. Buck \& Tyack 1993, McCowan 1995). However, very few studies have compared multiple methods using a single data set (but see Nowicki \& Nelson 1990, Janik in press). Comparing the different methods used in different studies is therefore difficult. The objective of this section is to determine what method is best suited for the comparison of dolphin calves' acoustic environments. Therefore, a sample data set of dolphin whistles was categorized with several different categorization methods, including visual categorization, and the results of these categorizations were compared to each other.

Some of the studies using visual categorization have acknowledged the limitations of the technique. In some cases, multiple judges were used to limit the subjectivity of the measurement (e.g. Sayigh et al. 1995, Janik \& Slater 1998). Sayigh (1992) performed a reliability analysis on the visual categorization by 74 judges. Her results showed that this analysis was highly reliable between judges $(R=0.95)$. The subjectivity of categorization by visual inspection of spectrograms can therefore be factored out. There are still several disadvantages to visual comparison and categorization, however. The number of whistles that can be compared by human judges in a reasonable amount of time is limited, which severely restricts the sample size. In addition, the exact features human judges are using to make the categorization cannot be known. Statistical categorization is, therefore, 
preferable because it allows an explicit understanding of the features used in the categorization, as well as being able to handle a much larger sample.

One of the most common statistical methods used for categorization is to automatically extract acoustic features from the sounds (e.g. Nowicki \& Nelson 1990, Fristrup \& Watkins 1994, Weilgart \& Whitehead 1997). In most cases, these are features such as duration, bandwidth, and energy measurements. These measurements are then used to categorize the sounds with one of a number of different statistical techniques, including k-means cluster analysis (Weilgart \& Whitehead 1997), k-means cluster analysis combined with principal component analysis (Nowicki \& Nelson 1990, McCowan 1995), hierarchical cluster analysis (Fristrup \& Watkins 1994), and discriminant analysis (Recchia 1994). A few studies of birdsong have compared sounds by correlating the spectrograms and categorizing the sounds with multidimensional scaling (e.g. Clark et al. 1987, Nowicki \& Nelson 1990). However, spectrogram correlation is sensitive to the FFT size and to noise in the spectrogram and is therefore not appropriate for some types of recordings (Khanna et al. 1997).

One of the most common methods for comparing dolphin whistles is to extract a frequency "contour" from the spectrogram (e.g. Buck \& Tyack 1993, McCowan 1995, Smolker \& Pepper in press). A contour is a pattern of frequency modulation over time extracted by taking the frequency bin with the greatest energy from the spectrogram. This has been done both by taking the highest amplitude frequency in each time bin of the spectrogram (Buck \& Tyack 1993) and by taking the frequency at set points throughout the whistle (McCowan 1995). In the latter method, twenty evenly spaced frequency measurements were manually extracted from the fundamental frequency. The absolute duration of the whistles, although measured, was not used in the McCowan (1995) categorization procedure. McCowan (1995) does not indicate the range of durations that she recorded but Caldwell et al. (1990) reported whistles that varied in duration from as short as $60 \mathrm{~ms}$ to as long as $5.4 \mathrm{~s}$. While there is some evidence for time-dilation of whistles (e.g. Buck \& Tyack 1993), equating the durations over such a great range seems questionable (Janik 1998). In fact, some researchers have suggested 
that very short whistles should be treated differently from longer whistles (Caldwell \& Caldwell 1970).

Several comparison and categorization methods have been used with these extracted contours. Contours that were normalized for duration (McCowan 1995) were then correlated using the 20 frequencies as measurements. The correlation matrix was then subjected to principal component analysis and k-means cluster analysis (McCowan 1995). Other researchers have used similar techniques for determining contours and then used hierarchical cluster analysis to categorize the whistles (Smolker \& Pepper in press). Contours extracted without normalizing for duration were compared using a dynamic time warping algorithm that correlates the contours after stretching one of the contours to fit the other (Buck \& Tyack 1993). These whistles were categorized by choosing dictionary contours and classing each whistle in the group with the dictionary contour most similar to it ("dictionary contour comparisons": DCC, Buck \& Tyack 1993). The problem with DCC analysis is that it requires examples of the categories to be selected before the analysis is begun, which is not always possible. The DCC method was compared to visual categorization and found to perform equivalently. Few other methods have been compared to each other or to visual categorization. Janik (in press) used a method similar to McCowan's (1995) method and compared it to the visual analysis performed in Janik and Slater (1998). He found that the results of McCowan's (1995) method did not match the visual ones exactly, although the results were similar.

Each of the papers discussed above not only used a different statistical technique but also used a unique data set gathered or extracted in a different manner. Very few of the papers compared different methods with the same data set (but see Nowicki \& Nelson 1990, Janik in press). There is a need, therefore, to test multiple statistical methods using a single data set and to compare the results to each other and to visual analysis. This section describes such a test, categorizing a single data set with nine different comparison and categorization techniques and comparing the results to visual analysis. Both feature extraction and contour extraction were tested. Extracted features were categorized by discriminant analysis, $\mathrm{k}$-means cluster analysis and hierarchical cluster analysis. 
Chapter 4: Acoustic Analysis Methods

Contours were extracted by the Buck and Tyack (1993) method and compared with cross-correlation and with dynamic time warping (Buck \& Tyack 1993). Both comparisons were then categorized by dictionary contour comparisons, multidimensional scaling, and hierarchical cluster analysis. The results of all the analyses were compared to visual categorization.

\subsubsection{DATA SET}

The data consisted of identified whistles from four dolphins at the Miami Seaquarium. The data were collected by Janet McIntosh on a Realistic hi-fi VHS recorder and Scotch T-120 cassettes or a Sony TCD3M stereo cassette recorder and Maxell UDXLII tapes with a modified U.S. Navy sonobuoy hydrophone mounted in either the Top Deck pool or the Flipper pool of the Seaquarium. The frequency response was limited by the hydrophone in both systems and was approximately $100-15000 \mathrm{~Hz}$. Recordings were made in a variety of situations. Dolphins in Top Deck were viewed either from a floating platform or from an underwater window during recording sessions. Animals in the Flipper pool were observed from a dock during recordings. Vocalizing dolphins were identified by blowhole movement synchronized with the onset of whistle production, bubble-streams from the blowhole during whistle production, or whistles audible at the surface of the water that could be localized in air (Tyack et al. in prep).

Whistles were extracted from the recordings by Jennifer Miksis. Between 25 and 35 whistles were analyzed from each animal (Ivan 27, Noel 28, Torey 34, Bebe 26). Spectrograms were created on a Kay Elemetrics Corp. Model 5500 Digital Signal Processing System with an upper frequency limit of $32 \mathrm{kHz}$ and a dynamic range setting of $42 \mathrm{~dB}$, digitizing at $81920 \mathrm{~Hz}$. Because the number of identified whistles from the animals was limited, all identified whistles were used and no established sampling protocol was needed. The data in this set are not expected to include the entire repertoire of any of the animals, much less provide a representative sample. The data selected were merely a convenient set for the test being performed. 


\section{Visual Categorization}

Examination of the spectrograms showed that whistles from three of the four animals (Ivan, Noel, and Torey) were individually-distinctive, stereotyped whistles. The contours made by the three different animals differed from each other, but each dolphin's whistles were quite stereotyped in contour, although they varied somewhat in duration and number of loops. These whistles could therefore be categorized qualitatively by which individual produced them. The whistles from the fourth animal, Bebe, varied quite a bit in contour. When Bebe's whistles were added to the data set, the data became more difficult to categorize qualitatively. Each analysis was first performed on the whistles from Ivan, Noel, and Torey and then repeated adding Bebe's whistles.

\subsubsection{METHODS TESTED}

Whistles were compared in two ways: by extracting acoustic features from the spectrogram and by extracting a frequency contour from the spectrogram. The extracted contours were compared in two ways: by cross-correlation and by dynamic time warping (DTW; Buck \& Tyack 1993). Each of the comparisons was then categorized in several ways. The extracted features were categorized using discriminant analysis, k-means cluster analysis, and hierarchical cluster analysis (HCA). Both contour comparison methods were categorized by dictionary contour comparisons (DCC; Buck \& Tyack 1993), with multidimensional scaling (MDS), and with hierarchical cluster analysis (HCA). The results of all techniques were compared to each other and to visual categorization.

\subsubsection{Feature Extraction}

Acoustic features were extracted from the spectrograms (FFT size 256, no overlap) using a program called AcouStat, written for DOS by Kurt Fristrup (Fristrup and Watkins 1992). The program was designed to extract acoustic features that describe specific aspects of the sound. The calculation of features takes into account the relative amplitude of signals in the spectrogram and is therefore insensitive to recording artifacts such as the sensitivity of the hydrophone. The calculations are also adjusted to 
Chapter 4: Acoustic Analysis Methods

compensate for noise in the spectrogram. The program calculates 120 different measurements (see Appendix 3 for the entire list). Using all 120 measurements would have severely over-fit these analyses. Therefore, only a subset of the measurements was used. The measurements to use were chosen by the discriminant analysis to best separate the groups (see below).

\section{Categorization Methods}

\section{Discriminant Analysis}

Discriminant analysis is a method of predicting group membership based on input variables, in this case the measurements from AcouStat. This analysis requires group membership to be defined beforehand and then attempts to separate the groups as well as possible using linear combinations of the input measurements. For the purpose of this analysis, whistles were categorized by the individual producing the whistle. For this analysis to be valid, there must be several cases in each group for each measurement used (Tabachnick \& Fidell 1983). Because the smallest group had only 26 cases, only five of the AcouStat measurements were used. Which five were included was determined by the discriminant analysis.

The analysis was done in Systat 7.0 (SPSS) for Windows 3.11 (Microsoft) using the forward stepwise option. This method sequentially adds measurements to the analysis based on an F-statistic that calculates how much of the variance between the groups is accounted for by each measurement. In this way, the subset of measurements that best separates the groups is found. At each step, the measure that accounted for the most variance is added to the subset. The F-statistics are then re-calculated to determine how much of the left-over variance is accounted for by each of the measurements that are left. The first five measurements added were used in this analysis and in the subsequent cluster analyses (see below).

Discriminant functions, linear combinations of the included measurements, are then calculated to optimally separate the groups. The number of discriminant functions calculated is one less than the number of groups being separated. Cases are plotted by their discriminant function scores. From these functions, classification functions are 
determined. Each case is classified in the group for which its value of the classification function is the largest. To cross-validate the results, a jackknifed classification is performed, leaving out one case at a time.

\section{K-means Cluster Analysis}

$\mathrm{K}$-means cluster analysis is a method of separating cases into groups (Systat). The desired number of groups is specified $a$ priori, and how well the data divide into a particular number of groups can be tested. The analysis algorithm separates the cases by maximizing the variation between clusters relative to the variation within clusters. This is done by finding the case farthest from the centroid of the group and designating that case as the centroid of a second group. The distances between cases are computed by taking 1 minus the Pearson product-moment correlation coefficient between the cases (the Pearson distance option), and cluster centroids are calculated from these distances. Cases are then re-assigned to the cluster with the nearest centroid. Clusters are split in this manner until the requested number of clusters is reached. The input data used were the five AcouStat measurements determined by the discriminant analysis. For each input variable, an F-ratio is determined by comparing the between-cluster mean square (sum of squares divided by degrees of freedom) to the within-cluster mean square. The algorithm maximizes this F-ratio for all the variables. The sum of the F-ratios can therefore be used as an indicator of how well the specified number of groups fit the data. The best number of groups is the one that maximizes the F-ratio (Nowicki \& Nelson 1990).

\section{$\underline{\text { Hierarchical Cluster Analysis }}$}

Hierarchical Cluster Analysis is a method of detecting natural groupings in data by connecting cases based on their similarity. The five AcouStat measurements determined by the discriminant analysis were input into this analysis (in Systat) and the normalized Euclidean distance between each pair of points was calculated from those measurements. The clustering algorithm first links the closest points. Cases are then joined to clusters, and clusters to each other, in hierarchical order of their similarity to form a clustering tree (see Figure 4.7). When clusters are to be joined, the distance between the two clusters is computed by first determining the distances between each 
case in one cluster and all the cases in the other cluster. The distance between the two clusters is calculated by averaging those distances for all cases in the first cluster (the average linkage option).

To determine how many clusters to divide the clustering tree into, a moat index was calculated for each possible number of clusters (Matlab 4.2 (Mathworks) for Windows and Excel 5.0 (Microsoft), see Appendix 2). The moat index is the average cluster cohesion for a given number of clusters. The cluster cohesion is calculated by subtracting the maximum distance between cases within a cluster from the minimum distance between cases in that cluster and cases outside that cluster ${ }^{5}$ (Podos et al. 1992). This index was calculated for every possible value of $n$, from 1 to the number of cases, and the value used was the $n$ that maximized the index.

\subsubsection{Contour Extraction}

Frequency contours were automatically extracted from the whistles using a program written in C for Linux by John Buck (see Appendix 3, Buck \& Tyack 1993). The contour is extracted by taking the frequency with the highest amplitude in each time block of the spectrogram after noise compensation (Figure 4.2). The peak frequency, $f(m)$, for each time block of the spectrogram, $X(m, k)$ where $m$ represents time blocks and $k$ frequency blocks, is calculated as follows:

$$
f(m)=\frac{f_{s}}{N} \max _{k}|X(m, k)| .
$$

In this formula, $f_{s}$ is the sampling frequency and $N$ is the block length used to calculate the spectrogram. For this analysis, spectrograms were produced using an FFT size of 512 with no overlap. The sampling frequency was $81920 \mathrm{~Hz}$. A built-in feature of the extraction program makes sure that it is extracting the fundamental and not the upper harmonics by looking for peaks at half and one-third of the peak initially detected.

${ }^{5} M_{n}=\frac{\sum_{i=1}^{n}\left[\min \left(B_{i}\right)-\max \left(W_{i}\right)\right]}{n}$, for $n$ clusters. $W$ is the distance between cases within the cluster and $B$ is the distance between cases in that cluster and cases outside that cluster (Podos et al. 1992). 
FIGURE 4.2: AN EXAMPLE OF CONTOUR EXTRACTION

\section{A. SPECTROgRaM}

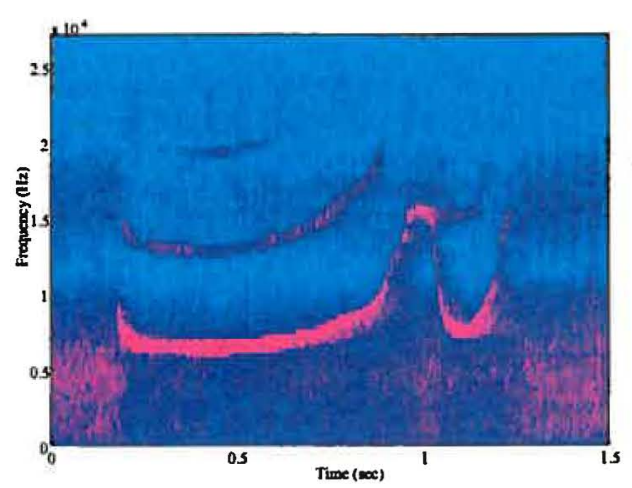

B. EXTRACTEd Contour

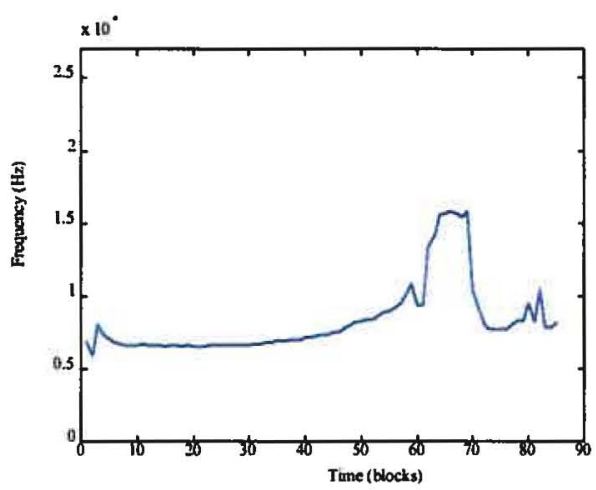

\section{Comparison Methods}

\section{Cross Correlation}

Each pair of contours was cross-correlated using a built-in function in Matlab 5.0 for Linux (see Appendix 2). In this procedure, the vectors of the contours were slid across each other and correlated at each offset. The maximum value of the crosscorrelation vector represents the offset where the two contours were best aligned. At each offset, the cross-correlation was calculated as follows:

$$
c_{x y}(m)=\sum_{n=0}^{N-|m|-1}[x(n) \times y(n+m)] .
$$

For each pair of contours, the maximum of the cross-correlation vector was found and then normalized by the maximums of the auto-correlation vectors, $c_{x x}$ and $c_{y y}$. The final correlation between the contours was therefore

$$
c=\frac{\max \left(c_{x y}\right)}{\sqrt{\max \left(c_{x x}\right) \times \max \left(c_{y y}\right)}} .
$$

This was calculated for each pair of contours to form a correlation matrix between all the contours. 
Chapter 4: Acoustic Analysis Methods

Figure 4.3: DyNAMic Time Warping of 2 Contours

Reproduced with permission from Buck and Tyack 1993.

A. Before Dynamic Time Warping

Two contours from similar whistles overlaid.

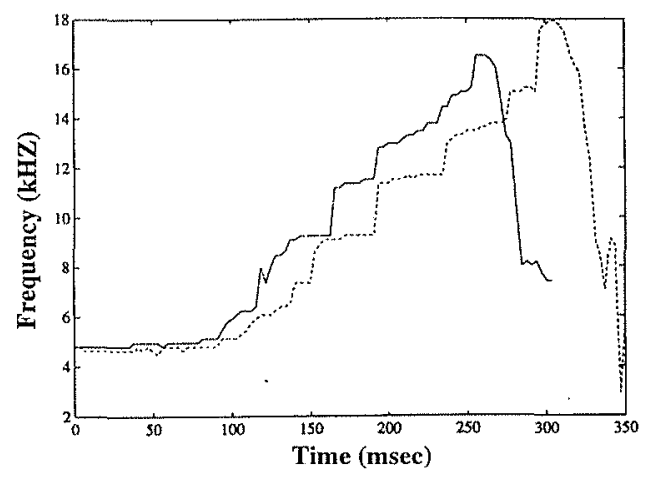

B. After Dynamic Time Warping The solid contour was "warped" to align its features with the dashed contour.

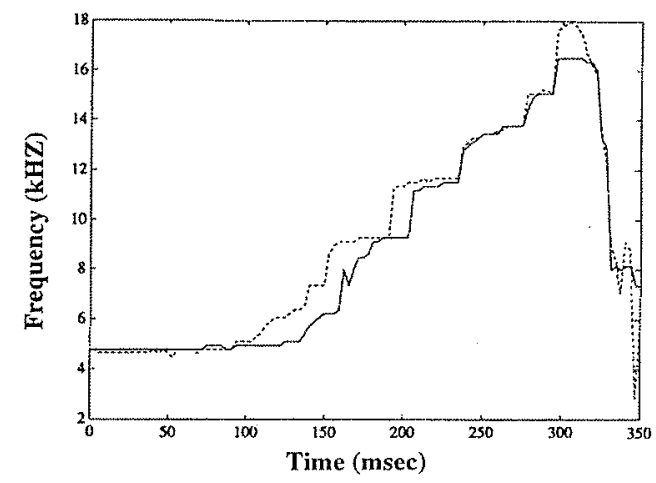

Dynamic Time Warping

Dynamic time warping (DTW) proceeds by first aligning the features of two contours (Buck \& Tyack 1993). To do this, the algorithm allows the non-uniform stretching of the time axis of one contour to match the other (Figure 4.3). The dissimilarity is then calculated by taking the normalized sum square frequency difference between the aligned contours:

$$
D(x, y)=\frac{1}{N} \min _{w}\left[\sum_{n=0}^{N-1}[x(n)-y(w(n))]^{2}\right],
$$

where $w$ is the warping function. Two contours whose durations differ by more than a factor of two are considered to be infinitely dissimilar. For the purposes of this analysis, infinity was converted to $10^{9}$, which is an order of magnitude greater than any other measurement made by the program. The DTW was done by a program written in $\mathrm{C}$ for Linux by John Buck (see Appendix 3). The results were converted into matrix form with a program written in Perl for Linux by Jim Partan (Appendix 3). 


\section{Categorization Methods}

\section{Dictionary Contour Comparisons}

The first method by which contours were categorized was dictionary contour comparisons (DCC), where a "dictionary contour" was defined for each group and the whistles were classified based on their similarity to each dictionary contour (Buck \& Tyack 1993). For each of the four animals, the contour that had the least noise contamination was selected as the dictionary contour. Because the fourth animal's whistles were so variable, two dictionary contours were chosen from her whistles for comparison purposes. Three dictionary sets were therefore used: one with only the first three animals and two that also included one of the two dictionary contours for the fourth animal. Each contour was classified in the group with the most similar dictionary contour. For each contour, six comparisons were made: one to each of the three dictionary sets using each of the two comparison methods, cross-correlation and DTW. All comparisons were done in Matlab 4.2 for Windows (see Appendix 2).

\section{Multidimensional Scaling}

Multidimensional scaling (MDS) is a method of computing graphical equivalents to calculated similarities in order to be able to plot the points in space of reduced dimensionality ( 2 or 3D) and compare the similarities visually. In this case, the input matrices were the cross-correlation and DTW matrices from the contour comparisons. MDS proceeds by first calculating a new matrix using a function of the ranks of the similarities. The analysis was done in Systat using the Guttman rank loss function ${ }^{6}$, which is a non-metric version of MDS. The non-metric version was used because the DTW violates the triangle inequality (distance $[A C] \leq \operatorname{distance}[A B]+\operatorname{distance}[B C]$ ), which is an assumption of metric MDS. An initial set of coordinates in $p$ dimensions is

${ }^{6} c_{i j}=1-\frac{r_{i j}}{\frac{n(n-1)}{2}} \cdot r_{i j}$ are the ranks of the input dissimilarities and $n$ is the number of points. The diagonal elements of this matrix are $c_{i i}=1-\sum_{j} r_{i j}$, summing the ranks over the entire row. 
Chapter 4: Acoustic Analysis Methods

computed by taking the first $p$ eigenvectors of the new matrix. For this analysis, the MDS was performed in three dimensions. These coordinates are normalized to have a centroid of 0 and a dispersion of 1 . A coefficient of alienation is calculated by comparing the ranks of the computed distances to the ranks of the original dissimilarities. MDS then iteratatively shifts the interpoint distances to minimize the coefficient of alienation. The final configuration is normalized so that the extreme values are 1.

\section{Hierarchical Cluster Analysis}

Hierarchical cluster analysis (HCA) was done on the contour comparisons in the same way that it was done on the AcouStat measurements (see section 4.3.2.1). However, instead of calculating the Euclidean distance between points, the analysis (in Systat) used the input similarities and dissimilarities as the distances between points (the Pearson distance option). Because the distance between cases is needed for this analysis, cross-correlation similarities were converted to dissimilarities by calculating 1 minus the similarity. For the analysis of the DTW, the centroid linkage method was used instead of the average method. Centroid linkage uses the average value of all objects in the cluster as a reference for calculating the distance to other cases. All other aspects of the analysis were the same as described above. The moat index was calculated as before. For the moat calculation, the distances in the DTW data were transformed to the log of the distance.

\subsubsection{RESULTS}

\subsubsection{Feature Extraction}

\section{Discriminant Analysis}

The plots resulting from the discriminant analysis of extracted features are shown in Figure 4.4. The measurements selected and the F-statistics associated with them are listed in Table 4.3. The direct classification of Ivan, Noel, and Torey's whistles classified the whistles correctly $96 \%$ of the time, and the jackknifed analysis $94 \%$ of the time (Table 
Figure 4.4: Discriminant ANalysis of Extracted Features

A: IVAN, NOEL, AND TOREY

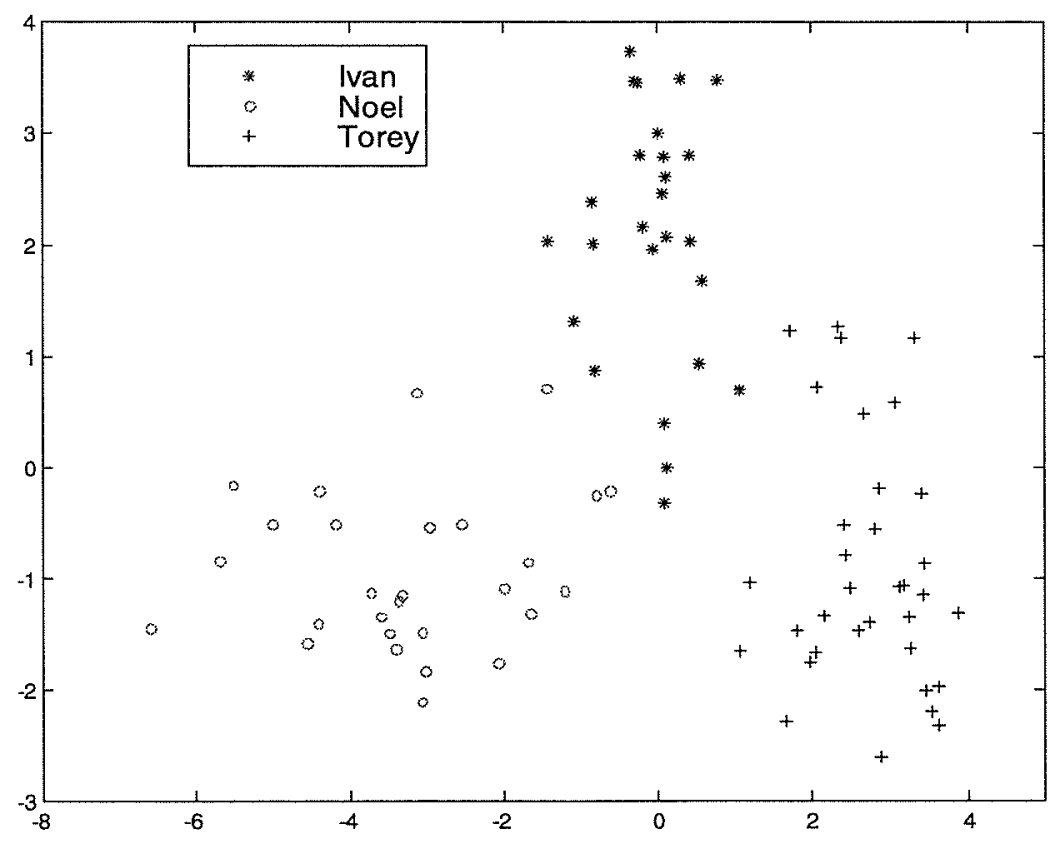

I

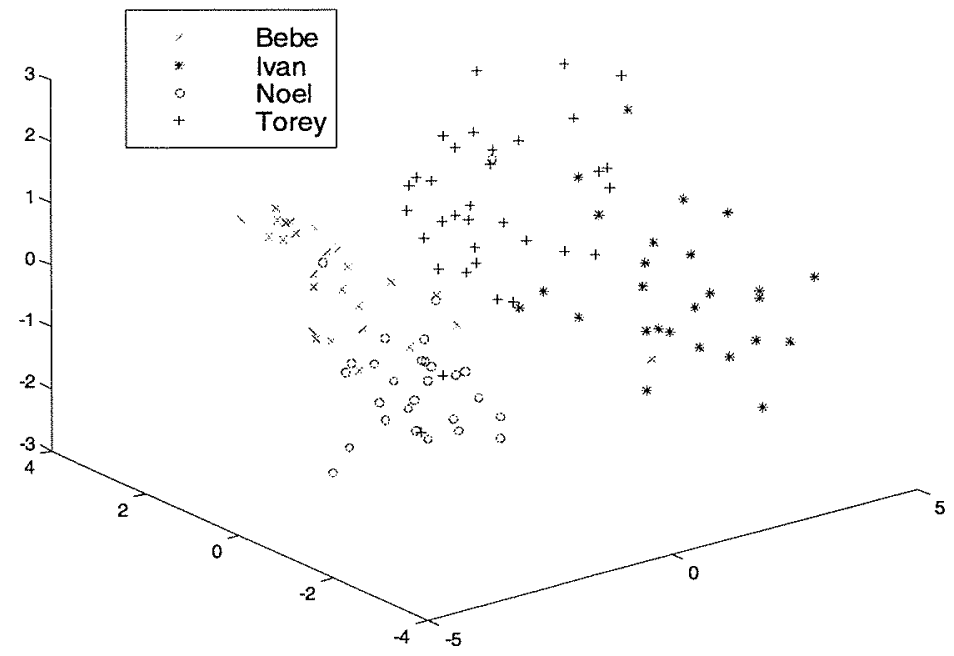


Chapter 4: Acoustic Analysis Methods

Table 4.3: Measurements Used By Discriminant Analysis

A: IVAN, NOEL, AND TOREY

\begin{tabular}{|l|l|r|}
\hline Measurement & Explanation & F-statistic \\
\hline TSCONC7 & Concentration of 75\% of the total spectrum & 57.862 \\
TFMEDR & Correlation between time and median frequency & 50.369 \\
TSUPP5 & Upper frequency of 50\% of average power spectrum & 34.674 \\
FMEDASYM & Asymmetry of median frequency (median-lower/upper-lower) & 27.031 \\
MSCONC7 & Concentration of 75\% of modal spectrum & 16.426 \\
\hline
\end{tabular}

B: IVAN, NOEL, TOREY, AND BEBE

\begin{tabular}{|l|l|c|}
\hline Measurement & Explanation & F-statistic \\
\hline MAXFLAT & Maximum length of flat section (less than x FM) & 85.118 \\
SWPFRAC & Fraction of blocks with non-zero energy that have different modal & 34.303 \\
& frequencies & 27.728 \\
FMODASYM & Asymmetry of modal frequency & 25.144 \\
TFMEDR & Correlation between time and median frequency & 10.698 \\
\hline AFM5MOD & Mode of 50\% of median frequency contour weighted by amplitude & \\
\hline
\end{tabular}

Table 4.4: Percent Classified Correctly By Discriminant Analysis

\begin{tabular}{|l|c|c|c|c|}
\hline \multirow{2}{*}{$\begin{array}{l}\text { Whistle } \\
\text { Identification }\end{array}$} & \multicolumn{2}{|c|}{ Ivan, Noel, \& Torey Only } & \multicolumn{2}{c|}{ Ivan, Noel, Torey \& Bebe } \\
\cline { 2 - 5 } & First Run & Jackknifed & First Run & Jackknifed \\
\hline Ivan & $100 \%$ & $96 \%$ & $89 \%$ & $89 \%$ \\
\hline Noel & $89 \%$ & $89 \%$ & $96 \%$ & $96 \%$ \\
\hline Torey & $97 \%$ & $97 \%$ & $82 \%$ & $76 \%$ \\
\hline Bebe & & & $85 \%$ & $85 \%$ \\
\hline
\end{tabular}

4.4). The worst classification was done on Noel's whistles, only classifying $89 \%$ of the whistles correctly. When Bebe's whistles were added, the direct classification only classified $88 \%$ of the whistles correctly, and the jackknifed $86 \%$ (Table 4.4). The worst classification when Bebe's whistles were included was on Torey's whistles (76\% jackknifed, Table 4.4). The best classification was on Noel's whistles, now classifying $96 \%$ of them correctly. Bebe's whistles changed the way the whistles were classified and actually interfered with the classification of Ivan's and Torey's whistles. However, discriminant analysis did a relatively good job at separating these whistles into types.

\section{K-means Cluster Analysis}

The summed F-ratios for dividing the data into 2 to 20 clusters, as well as 30,40 , and 50 clusters, are shown in Figure 4.5. For the first data set, with only Ivan's, Noel's, 


\section{Figure 4.5: Summed F-RATios For K-MEANS Cluster ANAlysis}

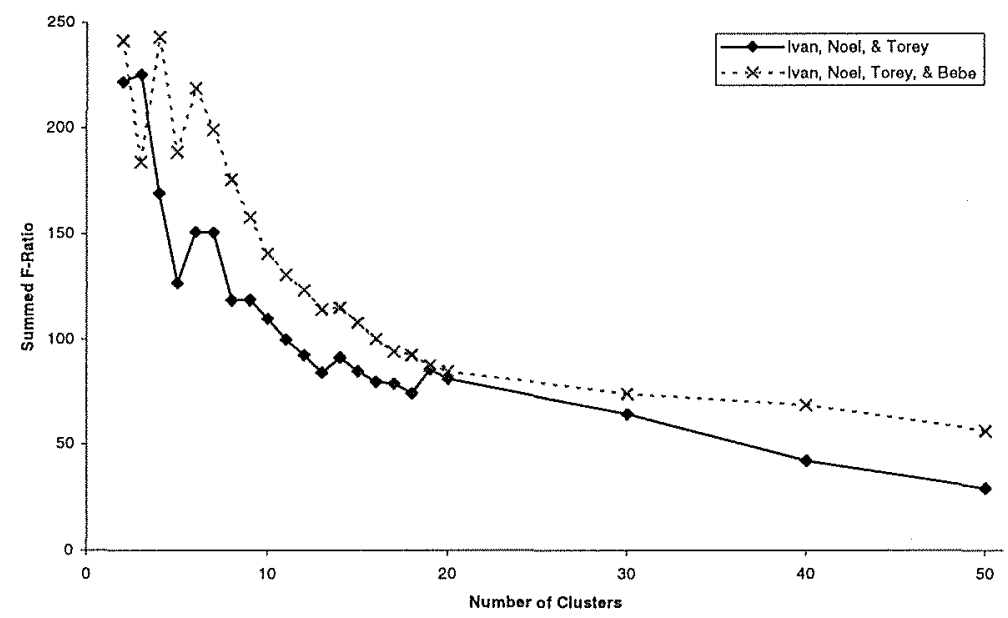

TAble 4.5: Results of K-MEAns Cluster ANALysis

A: IVAN, NoEL, AND TOREY

\begin{tabular}{|c|c|c|c|}
\hline Cluster & Ivan & Noel & Torey \\
\hline 1 & $26(96 \%)$ & $4(14 \%)$ & $34(100 \%)$ \\
\hline 2 & $1(4 \%)$ & $21(75 \%)$ & \\
\hline 3 & & $3(11 \%)$ & \\
\hline Total & $27(100 \%)$ & $28(100 \%)$ & $34(100 \%)$ \\
\hline
\end{tabular}

B: IVAN, NOEL, TOREY, AND BEBE

\begin{tabular}{|c|c|c|c|c|}
\hline Cluster & Ivan & Noel & Torey & Bebe \\
\hline 1 & $26(96 \%)$ & $1(4 \%)$ & $12(35 \%)$ & $1(4 \%)$ \\
\hline 2 & $1(4 \%)$ & $16(57 \%)$ & $14(41 \%)$ & $8(31 \%)$ \\
\hline 3 & & $4(18 \%)$ & $3(9 \%)$ & $10(38 \%)$ \\
\hline 4 & & $6(21 \%)$ & $5(15 \%)$ & $7(27 \%)$ \\
\hline Total & $27(100 \%)$ & $28(100 \%)$ & $34(100 \%)$ & $26(100 \%)$ \\
\hline
\end{tabular}

and Torey's whistles, three clusters maximized the F-ratio. This clustering grouped most of each animal's whistles together but did not separate Ivan's whistles from Torey's (Table 4.5A). For both Ivan and Noel, there were a few whistles that did not cluster with the others but this is to be expected because the whistles were not perfectly stereotyped. The maximum F-ratio for the data set with all four animals' whistles was at four clusters (Figure 4.5). However, although Ivan's whistles were clustered in the same way as before, the whistles of the other three were spread out over all four clusters (Table 4.5B). 
Chapter 4: Acoustic Analysis Methods

Figure 4.6: Hierarchical Cluster ANalysis OF EXtracted Features

The dotted line indicates the moat index.

A: IVAN, NOEL, AND TOREY

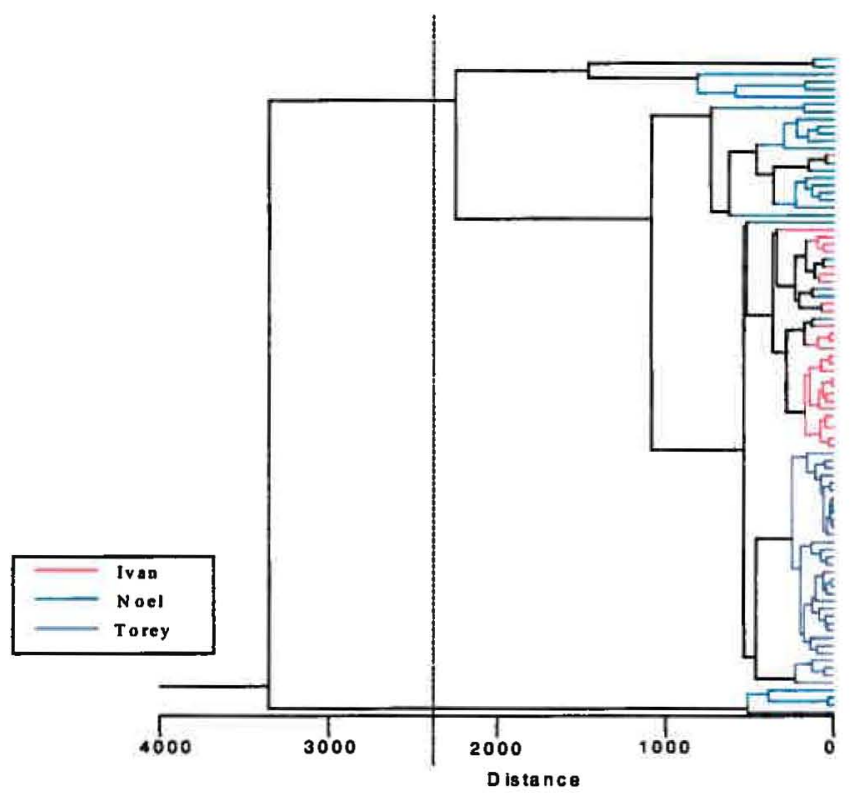

B: IVAN, NOEL, TOREY, AND BEBE

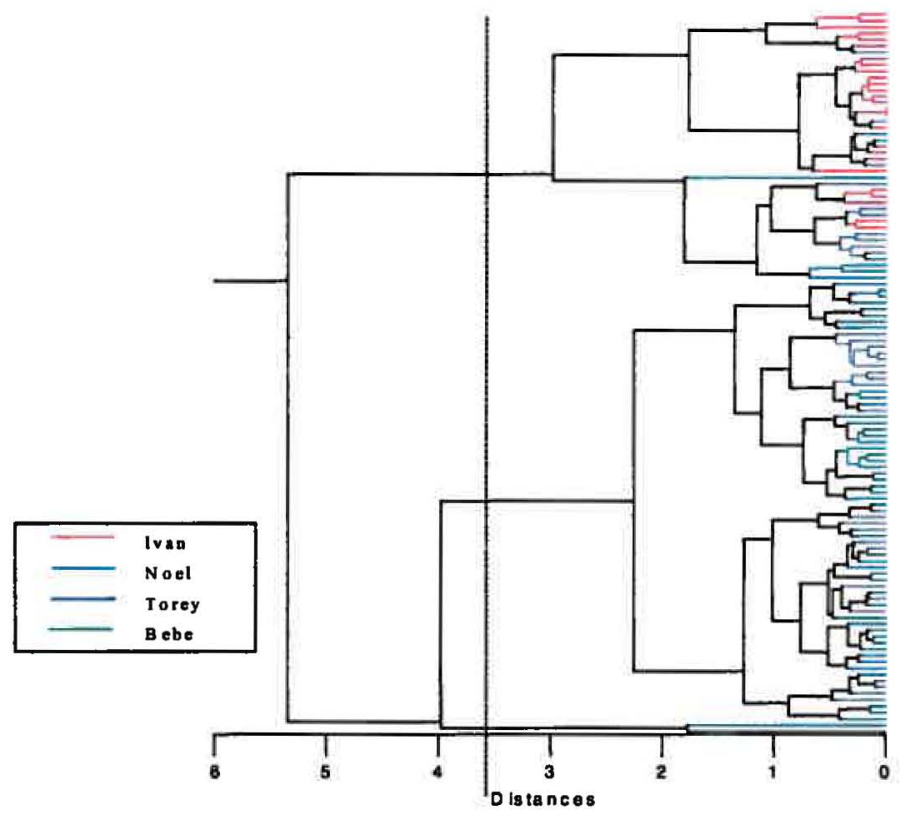


K-means cluster analysis did a poor job of separating the whistles, particularly when Bebe's less stereotyped whistles were included.

\section{Hierarchical Cluster Analysis}

The cluster trees from the HCA of the extracted features are shown in Figure 4.6. The branches of the trees are color-coded by which animal produced each whistle. The dotted lines indicate the cut-off calculated by the moat index. The analysis of the first three animals separated their whistles from each other but the moat index did not separate the three groups into distinct clusters (Figure 4.6A). When Bebe's whistles were added, once again, the separation of the first three became confused (Figure 4.6B). As with the k-means cluster analysis, the HCA of extracted features did a poor job of separating these whistles into groups. In fact, the non-stereotyped whistles interfered with the separation of stereotyped whistles in all the analyses of the extracted features.

\subsubsection{Contour Extraction}

\section{Dictionary Contour Comparisons}

\section{Cross-correlation}

When only the whistles from the first three animals were compared by crosscorrelation to the dictionary contours from those three animals, $88 \%$ of the contours were correctly classified (Figure 4.7A). When contour B8, the first dictionary contour from Bebe, was added to the dictionary set, and Bebe's whistles were added to the comparison set, only $71 \%$ of the contours were correctly classified (Figure 4.7B). This difference is the result of very poor identification of Bebe's whistles: only 19\% of her whistles were correctly identified. However, Bebe's whistle should not be expected to classify very well because they were not very stereotyped. This procedure still classified $88 \%$ of the whistles from Ivan, Noel, and Torey correctly. The addition of contour B8 to the dictionary set did not interfere with the correct classification of the other animals' whistles. Figure $4.7 \mathrm{C}$ shows, however, that using a different dictionary contour from Bebe, B11, did interfere with the correct classification of some of the other whistles. The correct classification of Noel's, Torey's, and Bebe's whistles did not change from the 
Chapter 4: Acoustic Analysis Methods

\section{Figure 4.7: Dictionary Contour Comparisons, Contour Cross-}

\section{CORRELATIONS}

Each column represents the whistles produced by that dolphin. "Incorrect" means that whistle was incorrectly assigned to another dolphin's category.

\section{A: IVAN, NOEL, AND TOREY}

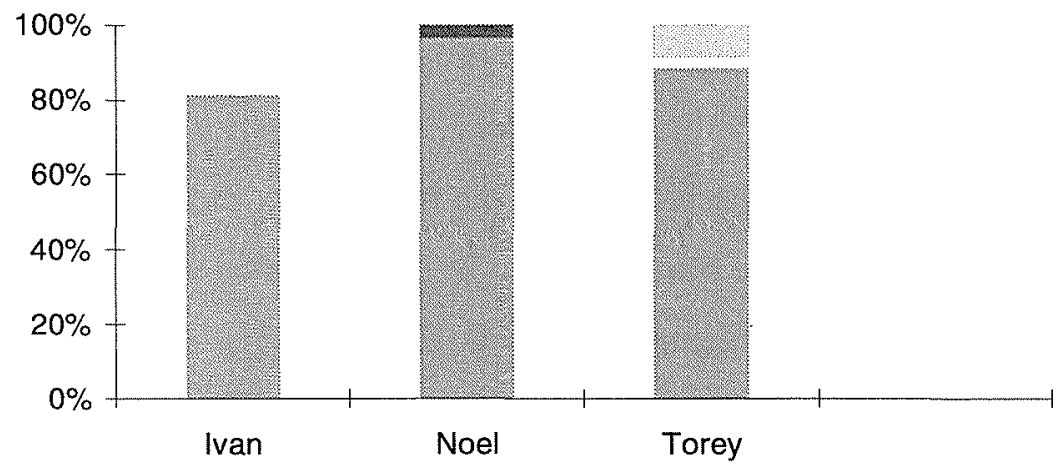

B: IVAn, NoEl, Torey, AND BEBE, WITH Contour B8

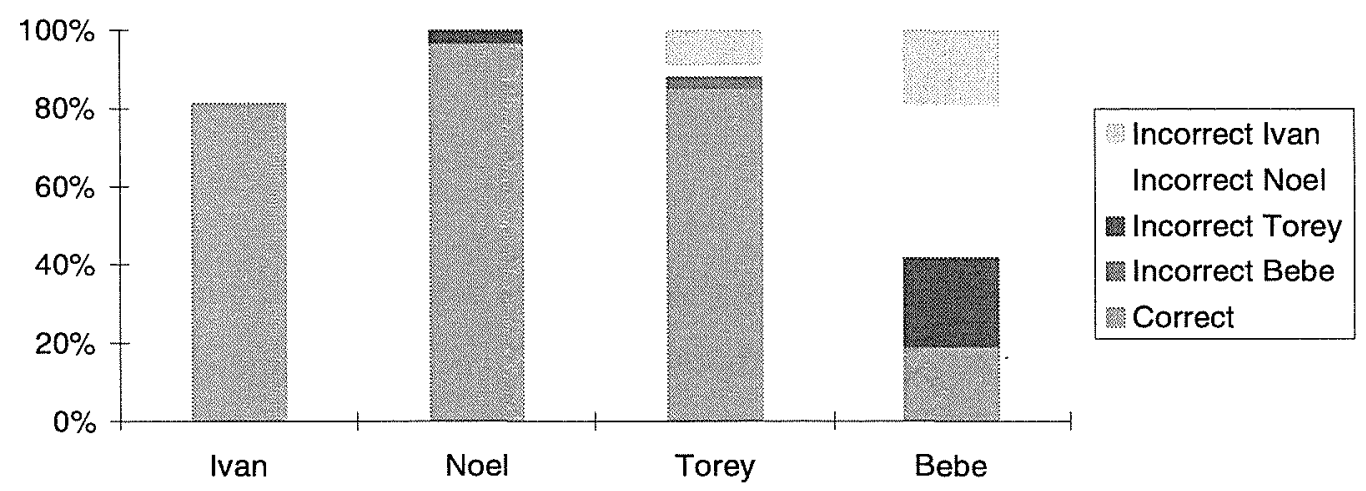

C: IVAn, Noel, Torey, AND Bebe, With Contour B11

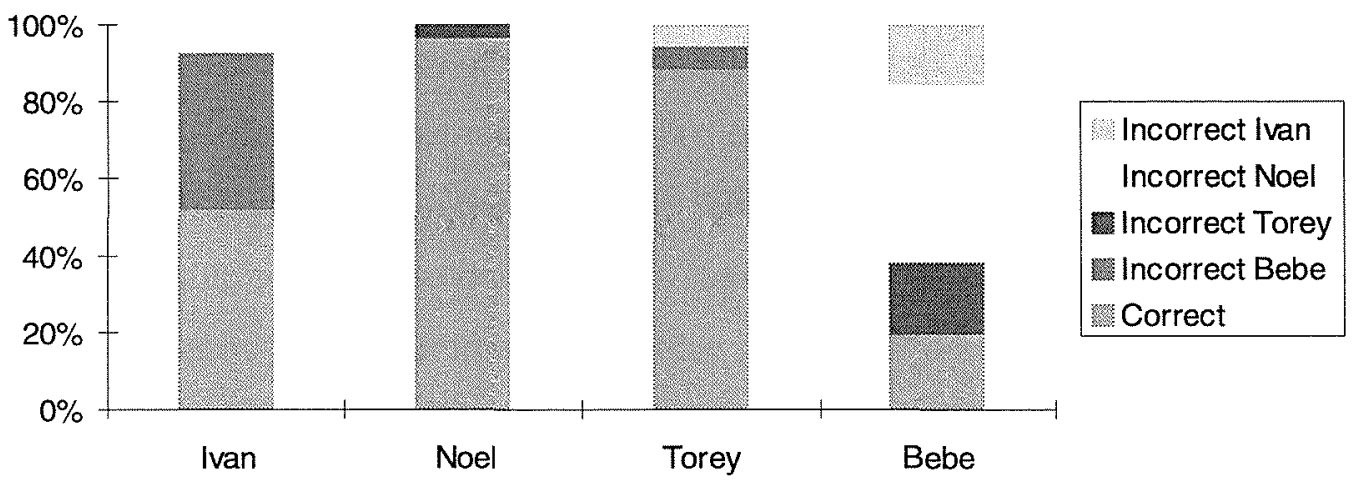


Figure 4.8: Dictionary Contour Comparisons, Dynamic Time Warping Each column represents the whistles produced by that dolphin. "Incorrect" means that whistle was incorrectly assigned to another dolphin's category.

\section{A: IVAN, NOEL, AND TOREY}
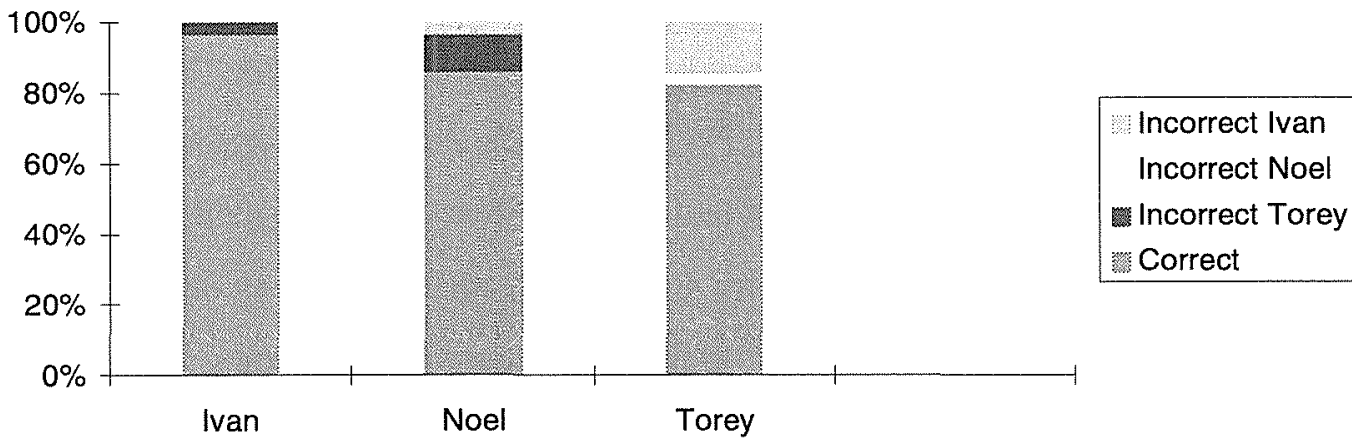

B: IVAN, Noel, ToRey, AND Bebe, With Contour $B 8$

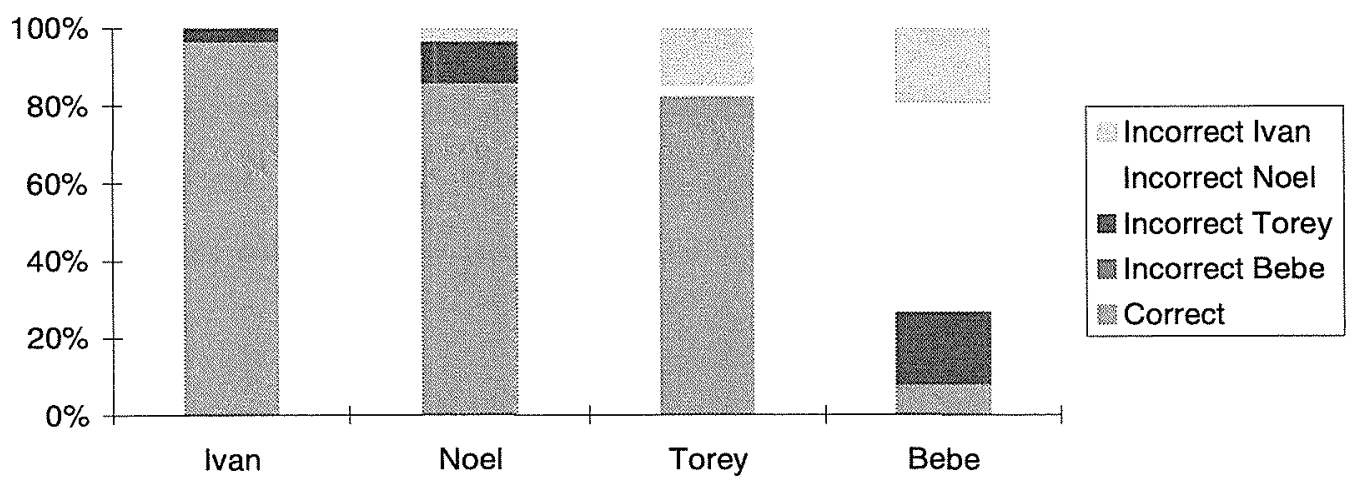

C: IVAn, Noel, Torey, AND Bebe, With Contour B11

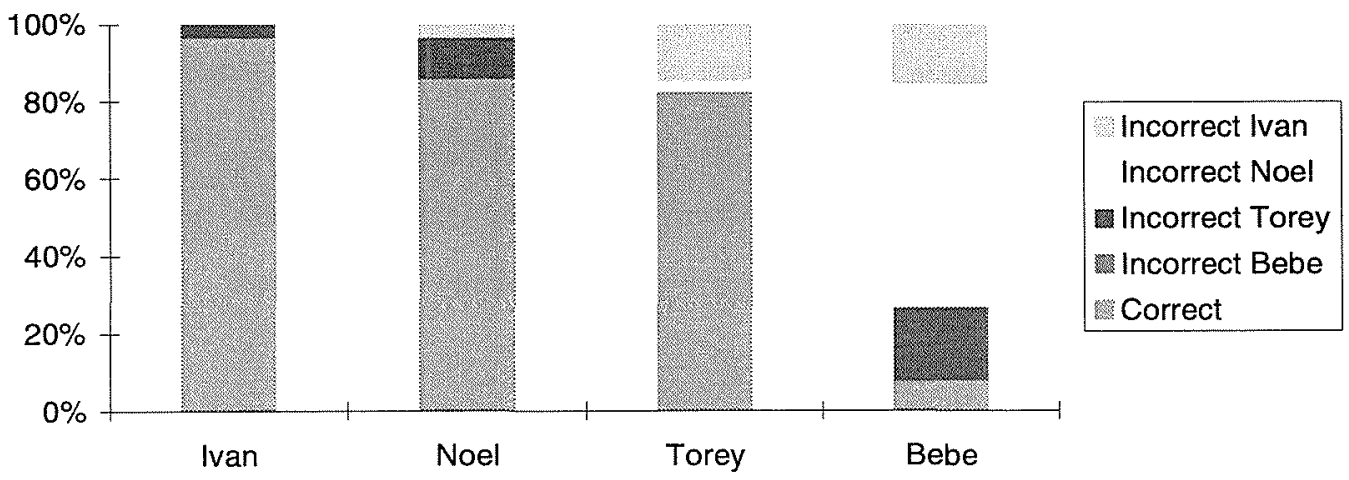


previous case, but in this case, only $50 \%$ of Ivan's whistles were classified correctly. Which dictionary contours are used, therefore, can significantly change how well this kind of comparison classifies a group of whistles.

\section{Dynamic Time Warping}

As with the cross-correlation, when only the whistles from the first three animals were compared to dictionary contours by DTW, $87 \%$ of the contours were correctly classified (Figure 4.8A). When contour B8 and Bebe's whistles were added, only 69\% of the contours were correctly classified (Figure 4.8B). This difference is again the result of very poor identification of Bebe's whistles: only $8 \%$ of her whistles were correctly identified with this method. As before, Bebe's whistle should not be expected to classify very well because they were not very stereotyped. This analysis still classified $87 \%$ of the whistles from Ivan, Noel, and Torey correctly. In this case, changing Bebe's dictionary contour to B11 did not change the percent of correct classifications (Figure 4.8C). Unlike with the cross-correlation, neither of Bebe's whistles in the dictionary set interfered with the correct classification of the other animals' whistles. The DCC of the DTW did a relatively good job of separating the stereotyped whistles into groups even after the addition of unstereotyped whistles.

\section{Multidimensional Scaling}

\section{Cross-correlation}

The MDS plots from the contour cross-correlation analysis are shown in Figure 4.9. These figures are 2-dimensional projections of 3-dimensional analyses. The whistles from Ivan, Noel, and Torey separated from each other relatively well (Figure 4.9A). As with the DCC, Bebe's whistles did not cluster together in this analysis, although they were somewhat separated from most of the whistles of the other animals (Figure 4.9B). However, both with and without Bebe's whistles, it would be difficult to determine how to cluster these plots if the points were not already labeled. 


\section{Figure 4.9: Multidimensional Scaling, Contour Cross-Correlations}

\section{A. IVAN, NOEL, AND TOREY}

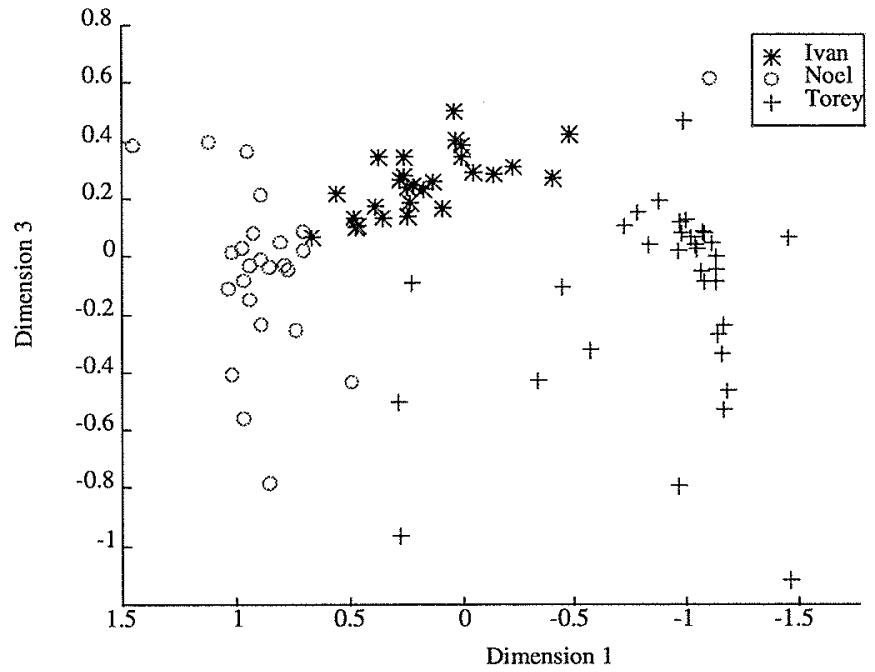

\section{B. IVAN, NoEL, TOREY, AND BEBE}

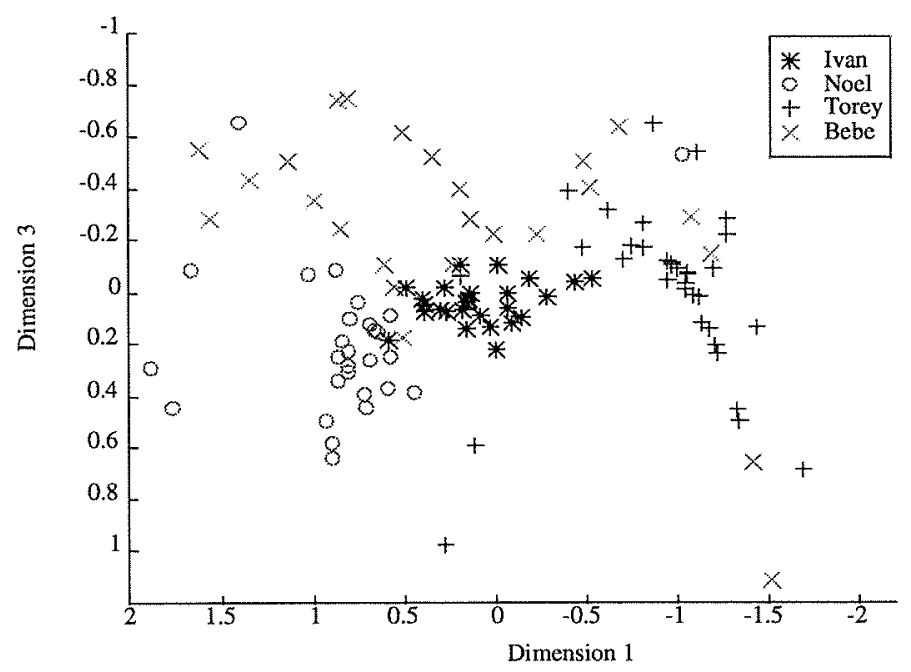


Chapter 4: Acoustic Analysis Methods

Figure 4.10: Multidimensional Scaling, Dynamic Time WarPing

\section{A. IVAN, NOEL, AND TOREY}

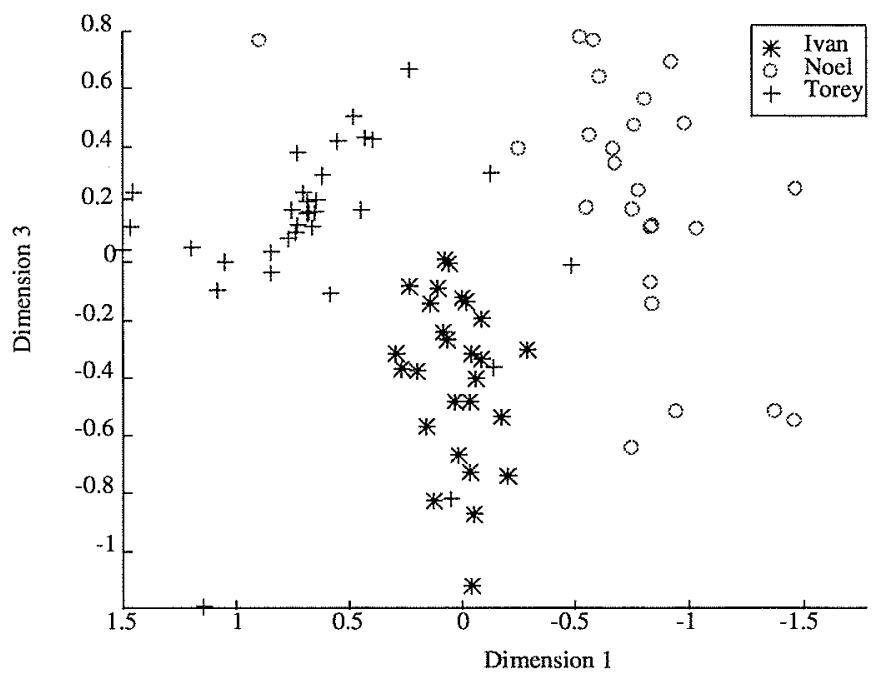

\section{B. IVAN, NoEL, TOREY, AND BEBE}

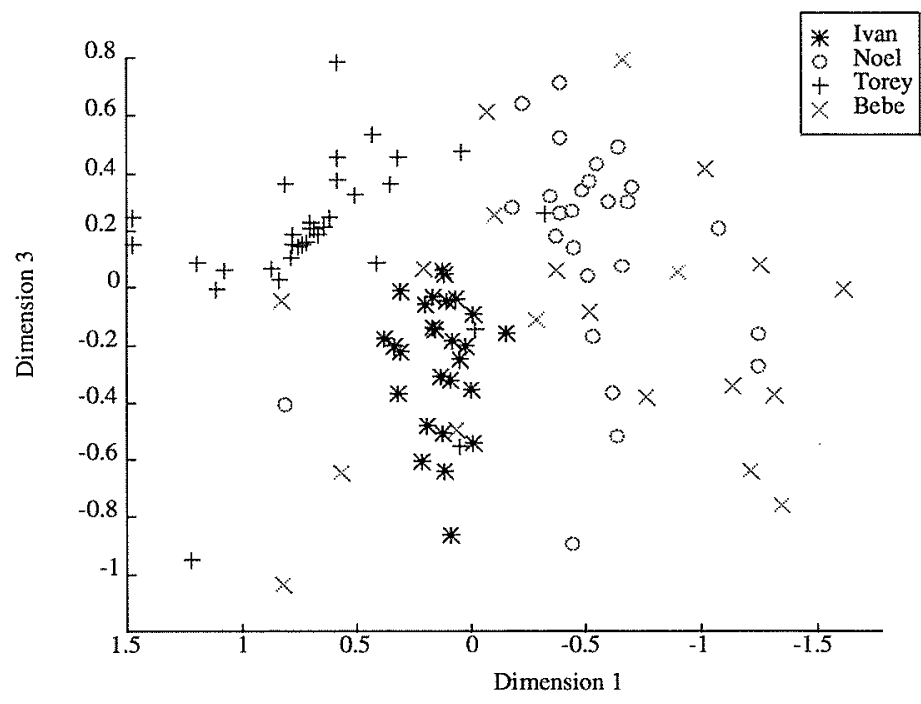




\section{Dynamic Time Warping}

The plots from the MDS of the DTW are shown in Figure 4.10. Again, these are 2-dimensional projections of 3-dimensional analyses. The whistles of the first three animals clearly separated from each other (Figure 4.10A). Separating these clusters might even be possible if the points were not labeled. However, in this case, Bebe's whistles not only did not cluster together, they did not separate from the whistles of the other animals at all (Figure 4.10B). In fact, they overlapped the clusters formed by all three of the other animals' whistles. Without labels, the points would be impossible to separate into clusters after the addition of Bebe's whistles. The non-stereotyped whistles from Bebe interfered with the separation of the more stereotyped whistles in this analysis. Therefore, while MDS of DTW is a useful method for separating stereotyped whistles, it breaks down when trying to separate stereotyped whistles from non-stereotyped ones.

\section{Hierarchical Cluster Analysis}

\section{Cross-correlation}

The cluster trees resulting from the HCA of contour cross-correlations are presented in Figure 4.11. As in Figure 4.6, the branches are color-coded by which animal produced each whistle, and the dotted lines indicate the cut-off calculated by the moat index. The whistles of Ivan, Noel, and Torey each clustered separately, but the moat index did not separate the group of Ivan's whistles from Noel's whistles (Figure 4.11A). An analysis of only the whistles in that cluster, however, did separate the whistles from the two animals into two clusters. When Bebe's whistles were added to this analysis, they did not cluster together as well as the whistles of the other animals (Figure 4.11B). Clustering is not expected from non-stereotyped whistles, however. Although some of Bebe's whistles clustered with the whistles of the other three animals, they did not interfere with the clustering of those whistles. However, when all four animals' whistles were included, the moat index no longer separated any of the groups into distinct clusters (Figure 4.11B) 
Chapter 4: Acoustic Analysis Methods

Figure 4.11: Hierarchical Cluster ANalysis, Contour Cross-CorRelations The dotted line indicates the moat index.

A: IVAN, NOEL, AND TOREY

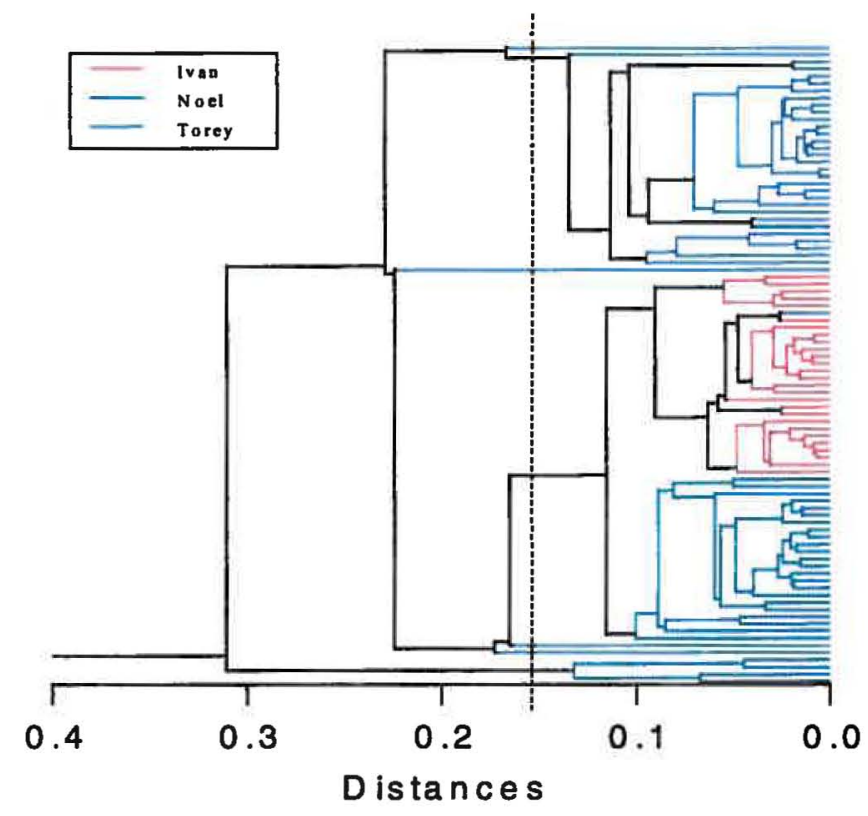

B: IVAN, NOEL, TOREY, AND BEBE

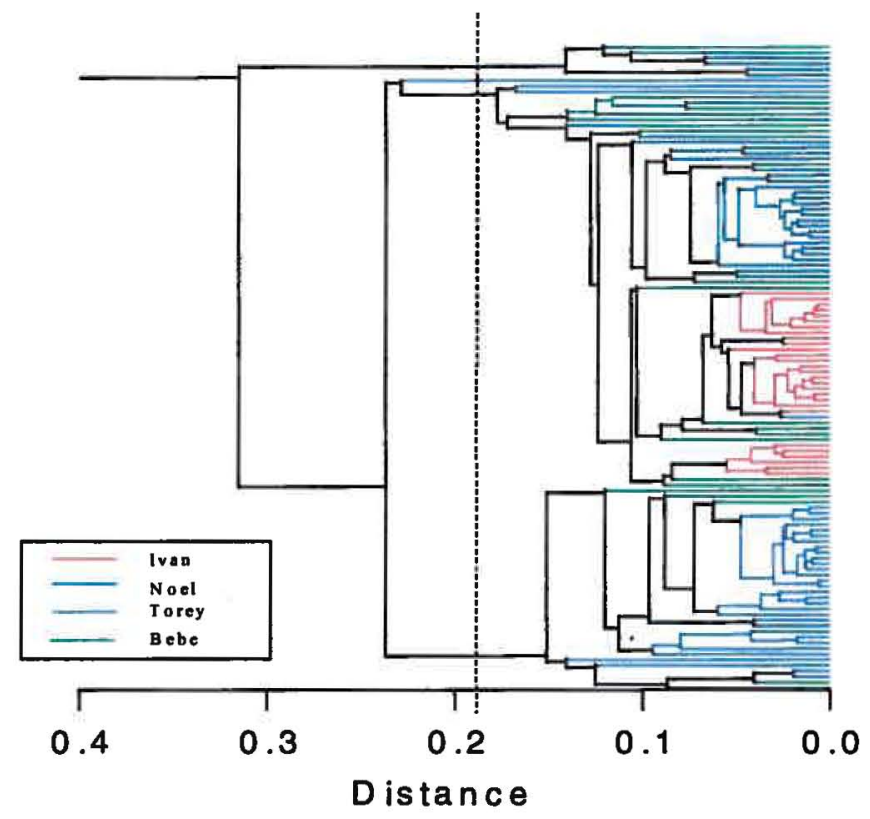


Figure 4.12: Hierarchical Cluster ANalysis, DYNamic Time WarPING The dotted line indicates the moat index.

\section{A: IVAN, NOEL, AND TOREY}

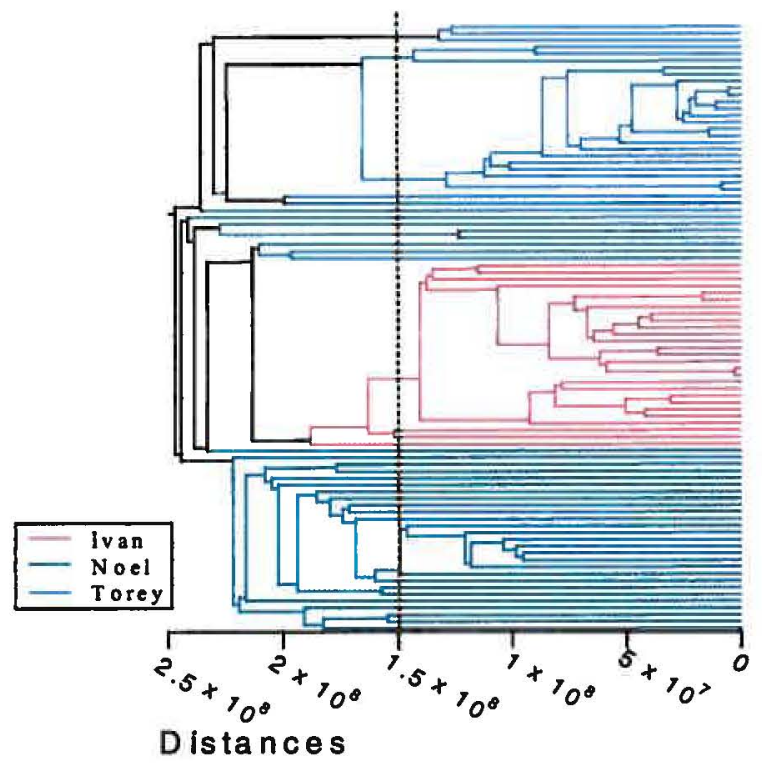

B: IVAN, NOEL, TOREY, AND BEBE

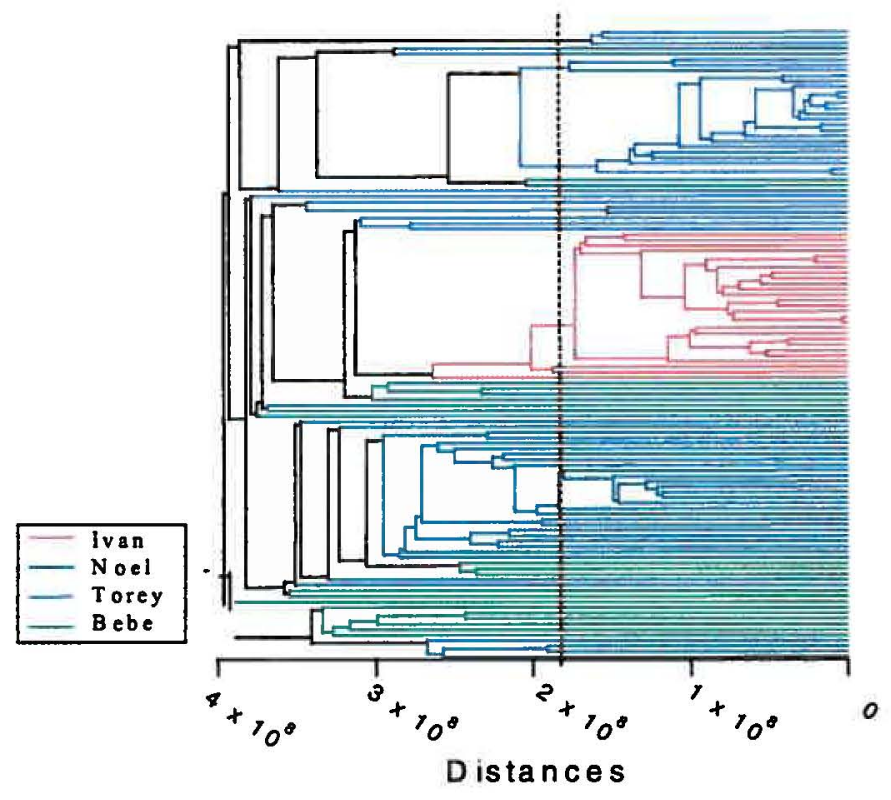


Chapter 4: Acoustic Analysis Methods

\section{Dynamic Time Warping}

The results of the HCA of the DTW are presented in Figure 4.12. Again, the branches are color-coded and the dotted lines indicate the cut-off calculated by the moat index. Ivan, Noel, and Torey's whistles each clustered separately from the others. In this case, the moat index separated each animal's whistles from those of the other animals (Figure 4.12A). The number of clusters formed by each animal varied quite a bit. Noel's whistles separated into 19 clusters, only one of which had more than one whistle in it. Ivan's whistles, on the other hand, formed only 5 clusters, while Torey's whistles formed 12 , four of which had more than one whistle. Overall, 36 clusters were formed, 6 with more than one whistle. When Bebe's whistles were added to this analysis, the results were much the same (Figure 4.12B). Her whistles did not cluster together, and were entirely grouped into clusters of single whistles. The clustering of the other three animals' whistles by the moat index did not change, so Bebe's whistles did not interfere with the clustering of the other whistles

\subsubsection{SUMMARY AND DISCUSSION}

Two types of data sets were tested here, a set consisting of entirely stereotyped whistles and a set that included stereotyped and non-stereotyped whistles. Most of the methods tested here worked relatively well on the stereotyped whistles. However, the more variable, non-stereotyped whistles interfered with the separation of stereotyped whistles by almost all of these methods. The method that best separated both stereotyped and non-stereotyped whistles was hierarchical cluster analysis (HCA) of dynamic time warping (DTW) of contours. This method was robust to the addition of non-stereotyped whistles and very successful at separating stereotyped whistles into categories. The moat index appears to be a good method of determining category boundaries in HCA. This method did not create a single group out of the whistles from each animal but this may be partly because not all the whistles were exactly alike. A test of this method with a larger data set might help determine exactly how the whistles are being separated. 
HCA is also preferable to some of the other methods, such as discriminant analysis or dictionary contour comparisons (DCC), because it does not require categories to be defined beforehand. For the task set out in this chapter, separating whistles into categories to compare acoustic environments, HCA of extracted contours appears to be a good choice. Because computers now have a great deal of computational power, this method is preferable over visual comparison because far more whistles can be compared and categorized. The best method depends on the task at hand, however. If the task at hand is to discover what acoustic features best separate known categories, discriminant analysis or DCC of DTW are good options.

Of the three ways of comparing whistles that were tested, the DTW did the best job of clustering the stereotyped whistles with most of the statistical methods. When extracted features were used, non-stereotyped whistles interfered with the separation of stereotyped whistles with almost all the techniques. With contours, the cross-correlation was not as robust to the choice of dictionary contour as the DTW was. The crosscorrelation did not perform as well as the DTW with the MDS or HCA either. DTW of extracted contours is therefore the preferable way to compare whistles for all the tasks, unless acoustic features other than the frequency contour are of particular interest. The dynamic time warping (DTW) reflects the intuition of many researchers that signature whistles are robust to small changes in duration (Tyack 1986, Buck \& Tyack 1993).

The only study that has compared multiple methods for categorizing dolphin whistles tested only three methods: visual categorization, McCowan's (1995) k-means cluster analysis of principle components analysis, and HCA of contour cross-correlation (Janik in press). Neither type of cluster analysis gave results that were exactly equivalent to the visual analysis in that study. In fact, in that study, the k-means cluster analysis was more similar to the visual analysis than the HCA was. The difference between the results of that study and this one may partly be due to the use of contour cross-correlation. In the present study, cross-correlation did not perform as well as DTW for clustering stereotyped whistles. The linkage method used for the HCA may also make a difference. Janik (in press) used the average linkage method, the same method used here for the 
cross-correlation. The analysis of the DTW used centroid linkage, however. This was done because the average linkage method did a poor job of clustering the large numbers that the DTW provides. The linkage method used has a profound impact on the results (De Ghett 1978). A study similar to the one presented here comparing different linkage methods for HCA is necessary to determine which method is best suited for categorizing dolphin whistles.

To know the proper way to categorize whistles, determining how the dolphins themselves categorize the whistles is necessary, of course. For instance, a study of whistle use in different contexts demonstrated that some whistle parameters, including duration, were influenced by context (Janik et al. 1994). Because the way the dolphins categorize whistles is not currently known, perceptual studies need to be performed. However, to design such a study, testable hypotheses are needed. Perceptual studies based on classification by human judges will not clearly demonstrate what features the dolphins are using. Because the physical features used in statistical categorization are more clearly known, perceptual studies based on statistical categorization will better indicate the perceptual features by which the dolphins categorize the sounds.

In conclusion, the ability to categorize large numbers of sounds is important to ability to study vocal learning. The acoustic environments in which several calves whistles developed need to be compared. A small sample is not sufficient to explore this issue. Methods are therefore necessary for the extraction, comparison, and categorizion of large numbers of whistles. These have been developed in this chapter. Using these methods, large numbers of whistles can be automatically extracted and categorized from recordings made during the development of calves' whistles. Once all the whistles recorded are categorized, the acoustic environments experienced by the calves can be compared. The whistle repertoires of the calves in the study can then be compared to their own acoustic environments and to the acoustic environments of other calves. If the calves are learning their whistles, each calf's repertoire should only include whistle types produced in its early acoustic environment (see Figures $1.1 \& 3.10$ ). It should not include 
whistle types produced in other acoustic environments that were not produced the calf's own environment.

\subsection{LITERATURE CITED}

Buck, J.R. and Tyack, P.L., 1993. "A quantitative measure of similarity for Tursiops truncatus signature whistles." Journal of the Acoustical Society of America, 94 (5), pp. 2497-2506.

Caldwell, M.C. and Caldwell, D.K., 1970. "Etiology of the chirp sounds emitted by the Atlantic bottlenosed dolphin: A controversial issue." Underwater Naturalist, 6, pp. 6-8.

Caldwell, M.C., Caldwell, D.K., and Tyack, P.L., 1990. "Review of the signature-whistle hypothesis for the Atlantic bottlenose dolphin." The Bottlenose Dolphin, eds. S. Leatherwood and R.R. Reeves. Academic Press, Inc., New York, NY. pp. 199-234.

Clárk, C.W., Marler, P., and Beeman, K., 1987. "Quantitative analysis of animal vocal phonology: An application to swamp sparrow song." Ethology, 76, pp. 101-115.

De Ghett, V.J., 1978. "Hierarchical cluster analysis." Quantitative Ethology, ed. P.W. Colgan. John Wiley \& Sons, New York, NY. pp. 115-44.

Fristrup, K.M. and Watkins, W.A., 1994. "Marine animal sound classification." Woods Hole Oceanographic Institution Technical Report No. 94-13, Woods Hole, MA.

Herzing, D.L., 1996. "Vocalizations and associated underwater behavior of free-ranging Atlantic spotted dolphins, Stenella frontalis and bottlenose dolphins, Tursiops truncatus." Aquatic Mammals, 22.2, pp. 61-79.

Janik, V.M. and Slater, P.J.B., 1998. "Context-specific use suggests that bottlenose dolphin signature whistles are cohesion calls." Animal Behavior, 56: 4, pp. 829-838.

Janik, V.M., 1998. Functional and organizational aspects of vocal repertiores in bottlenose dolphins, Tursiops truncatus. Ph.D. Dissertation, University of St. Andrews.

Janik, V.M., Dehnhardt, G., and Todt, D., 1994. "Signature whistle variations in a bottlenose dolphin, Tursiops truncatus." Behavioral Ecology and Sociobiology, 35, pp. 243-248.

Janik, V.M., in press. "Pitfalls in the categorization of behaviour: a comparison of dolphin whistle classification methods." Animal Behaviour.

Khanna, H., Gaunt, S.L.L., and McCallum, D.A., 1997. "Digital spectrographic cross-correlation: Tests of sensivity." Bioacoustics, 7, pp. 209-234.

Lilly, J.C. and Miller, A.M., 1961. "Sounds emitted by the Bottlenose Dolphin: The audible emissions underwater and in the air of captive dolphins are remarkably complex and varied." Science, 133, pp. 1689-1693.

McCowan, B. and Reiss, D., 1995. "Quantitative comparison of whistle repertoires from captive adult bottlenose dolphins (Delphinidae, Tursiops truncatus): a re-evaluation of the signature whistle hypothesis." Ethology, 100, pp. 194-209.

McCowan, B., 1995. "A new quantitative technique for categorizing whistles using simulated signals and whistles from captive bottlenose dolphins (Delphinidae, Tursiops truncatus)." Ethology, 100, pp. 177-193.

Mellinger, D.K. and Clark, C.W., 1997. "Methods for automatic detection of mysticete sounds." Marine and Freshwater Behavioral Physiology, 29, pp. 163-181.

Moore, S.E. and Ridgway, S.H., 1995. "Whistles produced by common dolphins from the Southern California Bight." Aquatic Mammals, 21, pp. 55-63.

Nowicki, S. and Nelson, D.A., 1990. "Defining natural categories in acoustic signals: Comparison of three methods applied to 'chick-a-dee' call notes." Ethology, 86, pp. 89-101.

Podos, J., Peters, S., Rudnicky, T., Marler, P., and Nowicki, S., 1992. "The organization of song repertoires in song sparrows: Themes and variations." Ethology, 90, pp. 89-106.

Recchia, C.A., 1994. Social behaviour of captive belugas, Delphinapterus leucas. Ph.D. Dissertation, Woods Hole Oceanographic Institution \& Massachussets Institute of Technology, Woods Hole, MA. 
Chapter 4: Acoustic Analysis Methods

Sayigh, L.S., 1992. Development and functions of signature whistles of free-ranging bottlenose dolphins, Tursiops truncatus. Ph.D. Dissertation, MIT/WHOI Joint Program, WHOI 92-37.

Sayigh, L.S., Tyack, P.L., Wells, R.S., and Scott, M.D., 1990. "Signature whistles of free-ranging bottlenose dolphins Tursiops truncatus: Stability and mother-offspring comparisons." Behavioral Ecology and Sociobiology, 26, pp. 247-260.

Sayigh, L.S., Tyack, P.L., Wells, R.S., Scott, M.D., and Irvine, A.B., 1995. "Sex difference in whistle production in free-ranging bottlenose dolphins, Tursiops truncatus." Behavioral Ecology and Sociobiology, 36, pp. 171-177.

Smolker, R.S. and Pepper, J., in press. "Whistle convergence among allied male bottlenose dolphins." Ethology.

Sturtivant, C. and Datta, S., 1995. "Techniques to isolate dolphin whistles and other tonal sounds from background noise." Acoustic Letters, 18, pp. 189-193.

Tabachnick, B.G. and Fidell, L.S., 1983. Using multivariate statistics. Harper \& Row Publishers, New York, NY.

Tyack, P.L., 1986. "Whistle repertoires of two bottlenosed dolphins, Tursiops truncatus: Mimicry of signature whistles?" Behavioral Ecology and Sociobiology, 18, pp. 251-257.

Tyack, P.L., Fristrup, K., and McIntosh, J., in prep. "Ontogeny of signature whistles in three captive bottlenose dolphins, Tursiops truncatus."

Watkins, W.A., Fristrup, K., Daher, M.A., and Howald, T., 1992. SOUND database of marine animal vocalizations: Structure and operations. Woods Hole Oceanographic Institution Technical Report No. 92-31, Woods Hole, MA.

Weilgart, L. and Whitehead, H., 1997. "Group-specific dialects and geographical variation in coda repertoire in South Pacific sperm whales." Behavioral Ecology and Sociobiology, 40, pp. 277285. 


\section{CHAPTER 5: CHARACTERIZING THE ACOUSTIC ENVIRONMENT}

\subsection{INTRODUCTION}

In a social species such as dolphins, vocal development must be studied in a normal social setting (see chapter 1). This is necessary for the social and vocal development of the calves to proceed normally. To investigate the role of learning in vocal development, each calf's whistles need to be compared to the whistles in the calf's prenatal and postnatal environment. If learning plays a role in whistle development, each calf's whistles should match the whistles in its prenatal or postnatal environments. To demonstrate vocal learning, each calf's whistles must be shown both to match whistles produced in that calf's early environment by unrelated dolphins and not to match whistles produced in the early environments of other calves that were not heard by this calf (Figure 5.1). If the calf's whistles match the whistles of a related dolphin, such as its mother, vocal learning cannot be distinguished from inheritance. Similarly, vocal learning cannot be clearly established if the calf matches both whistles it heard and whistles heard by other. calves but not by this calf (e.g. if Calf 1 in Figure 5.1 matches Whistles $1 \mathrm{U}$ and $2 \mathrm{U}$ ). Therefore, to investigate the role of learning in whistle development, calves' whistles need to be compared to the whistles from their own early environments and from the early environments of other calves. Quantitative techniques for sampling and comparing whistles in an unbiased manner were developed in chapters 2 and 4 . Those techniques will be used in this chapter to compare the early environments of the four calves born in the pilot study at Kolmårdens Djurpark.

In order to establish that a calf is matching the whistles of unrelated dolphins and not of related dolphins, the whistle repertoires of each dolphin must be known. A number of methods for determining which dolphin produced each whistle have been explored (see chapter 1.6 for a complete discussion of these techniques). None of these techniques is currently useful to studies of vocal learning. One method that has been used to identify whistlers in a study of vocal learning is to limit the whistle sample to whistles produced concurrently with a stream of bubbles (McCowan \& Reiss 1995). Although McCowan 


\section{Chapter 5: Characterizing the Acoustic Environment}

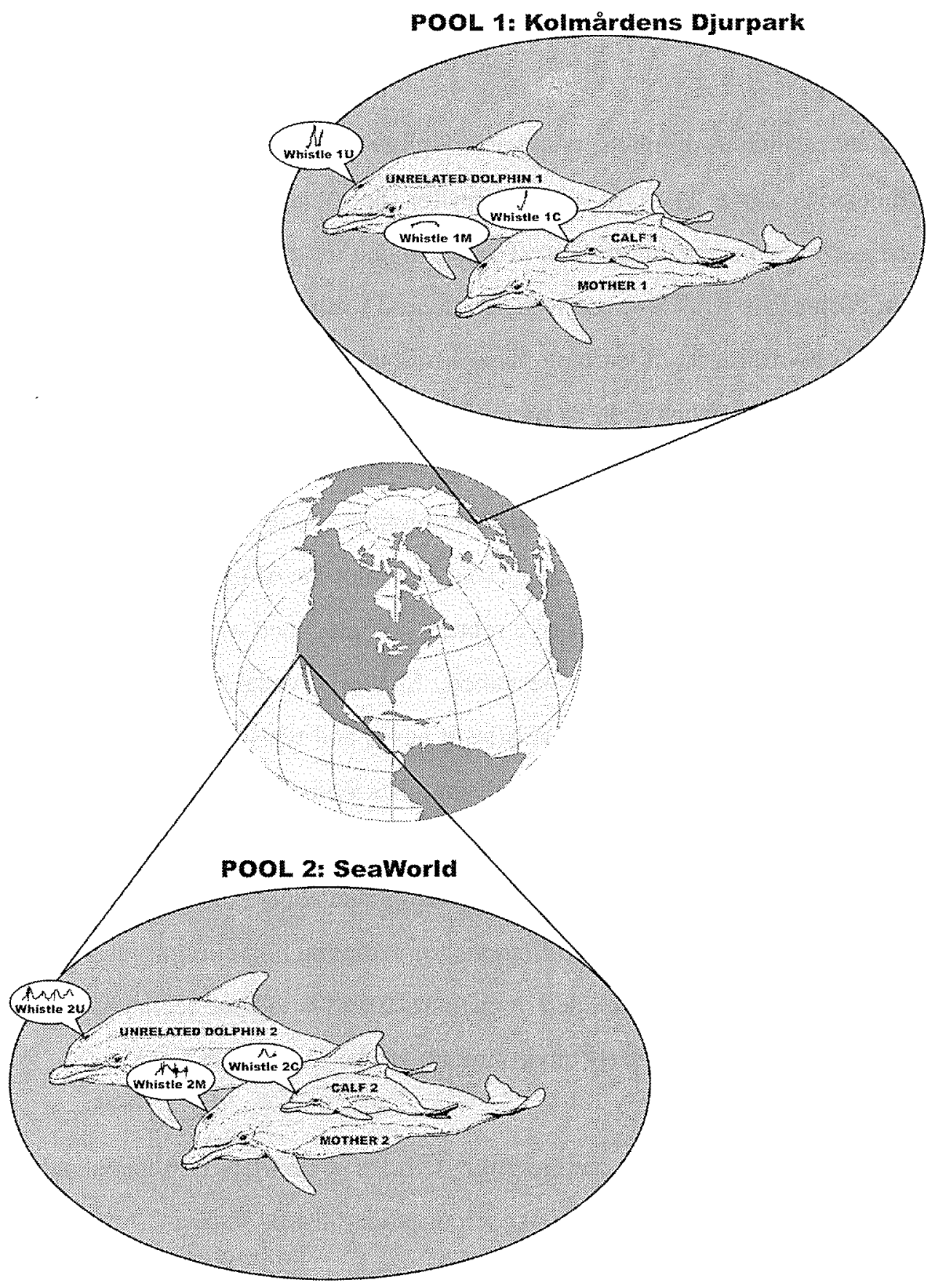

FIGURE 5.1: EXPERIMENTAL DESIGN

The two locations were chosen for demonstration purposes only. Similarly, the contours were used as examples: they do not represent contours of dolphins in those actual locations. To demonstrate vocal learning, each calf's whistles must be shown to match the whistles produced by unrelated dolphins in the calf's own pool. Whistle 1C, produced by Calf 1 , should therefore match Whistle $1 \mathrm{U}$ and not Whistle $1 \mathrm{M}$ or Whistles $2 \mathrm{U}$ or $2 \mathrm{M}$. Whistle $2 \mathrm{C}$ should match Whistle $2 \mathrm{U}$ and not Whistle $2 \mathrm{M}$ or Whistles $1 \mathrm{U}$ or $1 \mathrm{M}$. (The dolphin picture was purchased from ArtToday.) 
(1995) states that she has tested whether whistles associated with such "bubblestreams", "bubblestream-whistles", are representative of the entire whistle repertoire, the validity of her test is unclear. In particular, she does not present data on how many whistle types she used. If she used the same number of types in the test as she found in her later analysis, her sample size was too small for her $\chi^{2}$ test to be valid (McCowan 1995). Unfortunately, other researchers have since used bubblestream-whistles as their sole sample based on this test (e.g. Herzing 1996). The assumption made by both McCowan (1995) and those who have come after her is that bubblestream-whistles are representative of the dolphins' entire whistle repertoire. Because her test is questionable, the validity of that assumption needs to be investigated. Bubblestream-whistles were collected from the recordings made in the pilot study. These whistles will be evaluated to determine whether a representative sample of the dolphins' whistles could be achieved by only using bubblestream-whistles.

No other methods for identifying whistlers that are appropriate and accurate enough for a study of vocal learning are currently available. Therefore, if bubblestreamwhistles cannot be used to identify whistlers in an unbiased manner, there is currently no adequate technique to determine the identity of the whistlers. Without the ability to identify whistlers, it is not possible to establish whether a calf is learning from related dolphins or unrelated dolphins. However, even without the ability to determine which dolphin produced each whistle, the whistles produced in the early acoustic environments of two calves can be compared. As an example, Whistles $1 \mathrm{U}$ and $1 \mathrm{M}$ in figure 5.1 can be compared to Whistles $2 \mathrm{U}$ and $2 \mathrm{M}$, even though Whistle $1 \mathrm{U}$ cannot be positively identified as coming from Unrelated Dolphin 1 and not from Mother 1. To establish that learning was involved in the development of a calf's whistles, identified whistles must eventually be recorded from that calf. These whistles, preferably whistles recorded when the calf is a few years old, need to be compared to the whistles recorded from that calf's own early environment and from the early environments of other calves. In figure 5.1, Calf 1 's whistle (Whistle 1C) needs to be compared to the whistles from his pool (Whistles $1 \mathrm{U}$ or $1 \mathrm{M}$ ) and to the whistles from Pool 2 (Whistles $2 \mathrm{U}$ or $2 \mathrm{M}$ ). To show that Calf 1 learned 
his whistle, Whistle $1 \mathrm{C}$ must match the whistles from Pool 1 (1U or $1 \mathrm{M})$ and not the whistles from Pool 2 ( $2 \mathrm{U}$ or $2 \mathrm{M}$ ). In the pilot study, only one of the four calves survived its first two weeks. Therefore, whistles could not be recorded from the calves when they were a few years old. However, the whistles produced in the first weeks of each of the four calves can be compared. In this way, we will be able to quantitatively investigate the differences in the early acoustic environments of four calves born in the same pool within a few weeks of each other. Vocal learning can only be established if there are differences in the early environments of the calves being compared (e.g. in Figure 5.1, if Whistles $1 \mathrm{U}$ and $1 \mathrm{M}$ are different from $2 \mathrm{U}$ and $2 \mathrm{M}$ ). In addition, the acoustic environments from the calves' first weeks will be compared to the acoustic environments previous to the births. This will allow a more detailed investigation of the changes in the calves' acoustic environments.

In this chapter, whistles from different times in the pilot study will be compared by dynamic time warping (DTW) of contours and hierarchical cluster analysis (HCA) as described in chapter 4 . With the moat index, the HCA can divide the whistles into clusters or categories. In this study, the moat index was a good measure of the overall similarity of the whistles in the comparison but was not a robust means of separating whistles into categories. The HCA is therefore more useful for achieving an overall comparison of two acoustic environments than for determining the whistle "types" produced in each environment. However, the ability to categorize whistles into "types" may not be absolutely necessary to the ability to compare acoustic environments, or even to compare calf whistles to those environments. DTW and HCA give quantitative measurements of the relative similarity of two groups of whistles. This type of analysis is very different from the traditional analysis of dolphin whistles where whistles are categorized by their contour into signature whistles or variant whistles (e.g. Tyack 1986, Sayigh 1992, Janik \& Slater 1998). This traditional, qualitative, method has been very useful for analyzing the signature whistles of dolphins (e.g. Tyack 1986, Janik \& Slater 1998). In fact, visual categorization by contour will be used in this chapter to evaluate signature whistle use by 
some of the adults in the group. However, in most studies of signature whistles, all whistles that were not identified as a dolphin's signature whistle were grouped into a few, very general, "variant" whistle categories (e.g. Tyack 1986, Janik \& Slater 1998). The traditional methods generally did a poor job of evaluating variant whistle use. Because HCA allows all the whistles to be compared quantitatively, the variant whistles used in two environments can be evaluated along with the signature whistles.

\subsection{METHODS}

\subsubsection{WHISTLE SAMPLING}

Four calves were born in the course of the pilot study (see Table 2.1 in chapter 2). The first was born to Nephele in late April. The second calf was born to Vicky in late May. Delphi's calf was born a week after Vicky's calf. Lotus, Lotty's calf, was born several days after Delphi's. To explore the adults' whistle use around the time of each birth, whistles were extracted from recordings made in the week before and the week after each calf was born. Because three of the calves were born within a week of each other, two of the four "week-before" periods coincided with the "week-after" period for an earlier calf. To investigate whether the week immediately prior to the birth of a calf was different from other periods without calves, whistles were also extracted from a week in late March, a month before the first calf, Nephele's, was born. The time periods used were therefore as follows (section labels are in bold):

1. A week in late March, a month before Nephele's calf was born (March),

2. The week before Nephele's calf was born (Before Nephele's calf),

3. The week after Nephele's calf was born (With Nephele's calf),

4. The week before Vicky's calf was born (Before Vicky's calf),

5. The week after Vicky's calf was born, which is the week before Delphi's calf was born (With Vicky's calf),

6. The week after Delphi's calf was born, which is the week before Lotus was born (With Delphi's calf), 
Chapter 5: Characterizing the Acoustic Environment

7. The week after Lotus was born (divided into With Delphi's calf/Lotus, Lotus alone, With Lotus - see below).

In total, recordings from 26 days between March 21 and June 10 were analyzed (Table 5.1). The days are divided into nine periods, labeled by which calves were present on those days (Table 5.1). For instance, the week after Nephele's calf was born, when Nephele's calf was alive, is labeled "With Nephele's calf." Vicky's calf died the day that Delphi's calf was born so the week after Delphi's calf was born is labeled simply "With Delphi's calf." The week after Lotus was born is divided into 3 sections because Delphi's calf died in the middle of that week. The days when both calves were alive are labeled "With Delphi's calf/Lotus." The day after Delphi's calf died, Lotus was separated by the Kolmårdens Djurpark staff for medical treatment. He was held in an acoustically isolated pool for approximately 24 hours. This day is therefore labeled "Lotus Alone." The last day from which whistles were digitized was June 10, the day after Lotus was returned to the main pool. At that point, Lotus was the only calf left in the group. This section is therefore labeled "With Lotus." The time periods before the calves were born are labeled by which calf was born at the end of the week (Before Nephele's calf, Before Vicky's calf), except the week in March which is a month before any of the calves were born (see Table 5.1).

In addition to the births, the composition of the group changed over these periods (Table 5.1, see chapter 2). In particular, Sharky and Daphne were moved into the adjacent pool when Nephele's calf was born, and Nephele was moved into the adjacent pool when Vicky's calf was born. The animals in this adjacent pool were separated from the study animals by mesh gates. Although the social contact between the animals was limited, the pools were acoustically connected. Some whistles from the animals that had been moved out of the group could still be heard clearly in the recordings, and some of them may have ended up in the whistle sample.

On each day, only the recordings made simultaneously with focal animal samples were used. A total of 3775.75 minutes ( 62 hours, 55.75 minutes) were digitized from 378 
Table 5.1 Time Periods

\begin{tabular}{|c|c|c|c|c|c|}
\hline Time Period & Dates & $\begin{array}{c}\text { Samples } \\
\left(\text { Sessions }^{8}\right)\end{array}$ & Minutes & Adults Present & Calves Present \\
\hline March & $\begin{array}{l}\text { March } 21 \\
\text { March } 23 \\
\text { March } 25 \\
\text { March } 27 \\
\text { March } 29 \\
\end{array}$ & $80(20)$ & 800 & $\begin{array}{l}\text { Vicky, Lotty, } \\
\text { Nephele, Delphi, } \\
\text { Sharky }\end{array}$ & Daphne $^{\dagger}$ \\
\hline Before Nephele's calf & $\begin{array}{l}\text { April } 18 \\
\text { April } 24\end{array}$ & $32(8)$ & 320 & $\begin{array}{c}\text { Vicky, Lotty, } \\
\text { Nephele, Delphi, } \\
\text { Sharky }\end{array}$ & Daphne $^{\dagger}$ \\
\hline With Nephele's calf & $\begin{array}{l}\text { April } 25 \\
\text { April } 28 \\
\text { April } 29\end{array}$ & $52(13)$ & 515.75 & $\begin{array}{l}\text { Vicky, Lotty, } \\
\text { Nephele, Delphi }\end{array}$ & Nephele's calf \\
\hline Before Vicky's calf & $\begin{array}{l}\text { May } 16 \\
\text { May } 18 \\
\text { May } 20 \\
\text { May } 21\end{array}$ & $60(15)$ & 600 & $\begin{array}{l}\text { Vicky, Lotty, } \\
\text { Nephele, Delphi }\end{array}$ & none \\
\hline With Vicky's calf & $\begin{array}{l}\text { May } 22 \\
\text { May } 24 \\
\text { May } 28 \\
\text { May } 29 \\
\end{array}$ & $60(15)$ & 600 & $\begin{array}{l}\text { Vicky, Lotty, } \\
\text { Delphi }\end{array}$ & Vicky's calf \\
\hline With Delphi's calf & $\begin{array}{c}\text { May } 30 \\
\text { June } 1 \\
\text { June } 2\end{array}$ & $40(15)$ & 400 & $\begin{array}{l}\text { Vicky, Lotty, } \\
\text { Delphi }\end{array}$ & Delphi's calf \\
\hline $\begin{array}{c}\text { With } \\
\text { Delphi's calf/Lotus }\end{array}$ & $\begin{array}{l}\text { June } 4 \\
\text { June } 6 \\
\text { June } 8\end{array}$ & $45(15)$ & 450 & $\begin{array}{l}\text { Vicky, Lotty, } \\
\text { Delphi }\end{array}$ & $\begin{array}{l}\text { Delphi's calf, } \\
\text { Lotus }\end{array}$ \\
\hline Lotus alone & June 9 & $4(4)$ & 40 & none & Lotus $^{\ddagger}$ \\
\hline With Lotus & June 10 & $5(5)$ & 50 & Vicky, Lotty & Lotus $^{\ddagger}$ \\
\hline Total & 26 Days & $378(110)$ & 3775.75 & 5 adults & 5 calves \\
\hline
\end{tabular}

${ }^{8} \mathrm{~A}$ recording session consisted of one focal sample on each focal.

${ }^{\dagger}$ Daphne was 7 months old and not a subject of this study.

'Lotus was Lotty's calf.

focal samples in 110 recording sessions (Table 5.1). A recording session consisted of one focal sample on each focal animal because sounds were recorded continuously during each sampling session (see chapter 2). The uneven number of minutes was caused by a hydrophone failures in two of the focal samples. Recordings were digitized at $80 \mathrm{kHz}$. Sounds were then extracted by the automatic extraction and sorting procedure described in chapter 4 (section 4.2). More than 200,000 cuts were made, yielding more than 20,000 
Chapter 5: Characterizing the Acoustic Environment

TABLe 5.2 Whistle SAMPLE

\begin{tabular}{|c|c|c|c|}
\hline Section & Total Whistles & Usable Contours & Sub-sample \\
\hline March & $>1,138^{\S}$ & $>250^{\S}$ & 250 \\
\hline Before Nephele's calf & $>330^{\ddagger}$ & 266 & 250 \\
\hline With Nephele's calf & $>524^{\ddagger}$ & 405 & 250 \\
\hline Before Vicky's calf & 636 & 452 & 250 \\
\hline With Vicky's calf & 6,327 & 4162 & 250 \\
\hline With Delphi's calf & 6,439 & 3933 & 250 \\
\hline With Delphi's calf/Lotus & 5,568 & 3804 & 250 \\
\hline Lotus alone (June 9) & 250 & 245 & 245 \\
\hline Lotus (June 10) & 519 & 365 & 250 \\
\hline Total & $>21,731^{\ddagger \S}$ & $>13,882^{\S}$ & 2,245 \\
\hline
\end{tabular}

${ }^{\$}$ Not all the whistles from this section were saved, nor were all made into contours.

${ }^{\ddagger}$ Not all the overlapping whistles from these sections were saved.

whistles (Table 5.2). Files containing whistles were separated into files with single whistles and files with two or more whistles overlapping. Overlapping whistles cannot be separated by the contour extractor and were therefore excluded from later analysis. In order to determine the total whistle rate, files from May and June containing overlapping whistles were saved. The number of whistles in each of these files was counted and added to the number of files containing single whistles to determine the total number of whistles collected (see Table 5.4). Because overlapping whistles from March and April were not saved in this manner, the total whistle rate cannot be determined for those periods (see Table 5.2).

\subsubsection{CONTOUR EXTRACTION}

The files containing single whistles were converted into contours using the program described in chapter 4 (section 4.3.2.2, Buck \& Tyack 1993). The FFT size was 512 samples per block, with a step-size of 512 as well. Each FFT block therefore contained $6.4 \mathrm{~ms}$ of sound and covered a frequency band $156 \mathrm{~Hz}$ wide. The spectrograms were filtered to reduce the interference of noise, with a low frequency cutoff of $4 \mathrm{kHz}$, a high frequency cutoff of $22 \mathrm{kHz}$, and a band-pass filter which excluded 15.15 to 16.05 
$\mathrm{kHz}$ (see e.g. Figure 5.5). The $4 \mathrm{kHz}$ lower cutoff eliminated most of the low frequency pump noise without cutting off the lower edge of most whistles. The upper frequency cutoff, $22 \mathrm{kHz}$, was higher than most whistles and eliminated several high-frequency tonal bands (see e.g. Figure 5.5A, lower left). Contours were checked to confirm that these cutoffs had not cut off part of the whistle. In those few cases where the cutoffs were not appropriate, they were shifted as necessary. The $15-16 \mathrm{kHz}$ band-pass filter was necessary to compensate for the presence of a video monitor, which produced a 15-16 $\mathrm{kHz}$ tone near the hydrophone input (see e.g. Figure 5.5A, lower left). Beyond this frequency filtering, contours were not individually altered. Within the allowable frequency limits, noise and reverberations sometimes caused spikes in the contour (see e.g. Figure 5.5B, upper right). Most of these spikes represented single, relatively isolated points. Spikes were particularly common when whistles crossed the $15-16 \mathrm{kHz}$ excluded range (see e.g. contours \#4 \& \#5 in Figure 5.9A,B). However, not excluding this range caused much larger spikes in other sections of the contour. Spikes in the contour were allowed as long as more points fell along the whistle's contour than off it. Noise spikes did not appear to affect the analysis results. However, when an insufficient proportion of the points (approximately 50\%) fell on the whistle's contour, the contour was excluded from the analysis. The final sample was more than 13,000 usable contours (Table 5.2).

\subsubsection{BUBBLESTREAMS}

Dolphins produce a small stream of bubbles in conjunction with some whistles. These "bubblestreams" are sometimes the only means of identifying which dolphin produced a whistle. However, bubblestreams have never been clearly shown to produce an unbiased sample of the whistles used by the dolphins. Only one test has been performed investigating whether bubblestreams produce an unbiased sample of whistles (McCowan 1995), and the validity of that test is questionable (see above). Therefore, to investigate the dolphins' use of bubblestreams, all bubblestreams produced by the animals were recorded. Bubblestreams produced by focal animals during focal samples 
Chapter 5: Characterizing the Acoustic Environment

Table 5.3 Usable Bubblestream-Whistles Collected

\begin{tabular}{|c|c|c|c|c|}
\hline Section & Adults & Daphne & Calves & Total \\
\hline Focal Samples & 30 & 17 & 124 & 171 \\
\hline $\begin{array}{c}\text { Additional Focal } \\
\text { Samples }\end{array}$ & 18 & 16 & 0 & 34 \\
\hline $\begin{array}{c}\text { Additional from } \\
\text { Nephele's calf }\end{array}$ & 0 & 0 & 151 & 151 \\
\hline Total & 48 & 33 & 275 & 356 \\
\hline
\end{tabular}

were marked in the focal animal samples. Bubblestreams produced by non-focal animals were recorded $a d$ lib. and marked with a microphone on the second channel of the tape. The time within the focal sample that these latter bubblestreams occurred was recorded on the Observer (Noldus) while the sample was being digitized (see Appendix 1). When the whistles were extracted, the time from the beginning of the focal sample to the extraction of the whistle was recorded by the automatic extractor. The times recorded for whistles were matched to the times recorded for bubblestreams to determine which whistles were associated with the bubblestreams. These whistles will be referred to as "bubblestreamwhistles."

The final bubblestream sample was 356 usable contours from bubblestreamwhistles (Table 5.3). From the focal samples digitized for the general sample of whistles (see Table 5.1), 171 bubblestream-whistles were found among the usable contours (Table 5.3). Contours from bubblestream-whistles were separated from the general group of whistles and classified as produced by an adult, a calf, or Daphne. Daphne's whistles were separated because she was neither an adult nor a focal calf. In an attempt to increase the sample of bubblestreams, focal samples from several additional days were digitized and extracted. Focal samples were used from March 14, March 19, and April 20, yielding an additional 34 bubblestreams from the adults and Daphne (Table 5.3). In addition, a section of tape from April 25 containing a large number of bubblestreams from Nephele's calf was also digitized and extracted. This section was not from a focal sample from this study but was part of a focal study on Nephele's calf being conducted by the staff at Kolmårdens Djurpark. 


\subsubsection{CONTOUR COMPARISONS}

Pairwise comparisons between contours were made by dynamic time warping (DTW) and categorized by hierarchical cluster analysis (HCA) as described in chapter 4 (section 4.3.2.2). The maximum sample size was limited by the HCA program (Systat), which could not handle more than 1101 cases. The usable contours were therefore randomly sub-sampled (Matlab). 250 contours were taken from each section, except Lotus Alone (Table 5.2). Only 245 usable contours were produced in the Lotus Alone section and all of those were used in the final sample. All the contours for bubblestreamwhistles were also used. The total sample of contours used in the analysis, including the 356 bubblestreams, was 2,601 . Hierarchical cluster analyses were done in the manner described in chapter 4 for DTW (see section 4.3.2.2), using centroid linkage and the Pearson distance option. Cluster diagrams were copied into CorelDraw 8.0 (Corel) where the lines were color-coded by section (see e.g. Figures $5.11 \& 5.17$ ). In a few cases, twodimensional multidimensional scaling (MDS) was used for illustration purposes (see e.g. Figure 5.6). MDS was done as described in chapter 4 (section 4.3.2.2), using the Guttman rank loss function, a non-metric version of MDS.

\subsection{RESULTS}

\subsubsection{WHISTLE RATE}

The total number of whistles collected, including overlapping whistles, was recorded for the periods in May and June, from the week before Vicky's calf was born through the end of Lotus' first week. A striking change occurred after Vicky's calf was born: the mean whistle rate increased by an order of magnitude (Table 5.4A). The averages for the section labeled With Calves do not include the period Lotus Alone because no adults were present during that period. The rate increased slowly after Vicky's calf was born but then remained steady at the higher value through the first week 
Chapter 5: Characterizing the Acoustic Environment

TABLE 5.4 Whistles From MAY AND JUNE

Before Calves includes the section Before Vicky's calf only. With Calves includes the sections With Vicky's calf, With Delphi's calf, and With Lotus, but does not include Lotus Alone or With Nephele's calf.

\section{A. Whistle Rate}

\begin{tabular}{|c|c|c|c|c|c|}
\hline Section & Total Whistles & $\begin{array}{c}\text { Number of } \\
\text { Minutes }\end{array}$ & Rate/Min $^{\dagger}$ & \% Overlaps $^{\dagger}$ & $\begin{array}{c}\text { Average } \\
\text { Length (ms)*† }\end{array}$ \\
\hline Before Calves & 636 & 600 & 1.1 & $6 \%$ & 426.3 \\
\hline With Calves & 18852 & 1500 & 12.6 & $28 \%$ & 663.0 \\
\hline Total & 19488 & 2100 & 9.3 & $28 \%$ & 645.6 \\
\hline
\end{tabular}

* Average length refers to usable whistles only (see Table 5.2).

${ }^{\dagger}$ With Calves significantly greater than Before Calves by T-test, $\mathrm{p}<0.001$

B. Proportion of Whistles Usable

\begin{tabular}{|c|c|c|c|c|}
\hline Section & $\begin{array}{c}\text { Usable } \\
\text { Whistles }\end{array}$ & $\%$ Usable & $\begin{array}{c}\text { Non-overlapping } \\
\text { Whistles }\end{array}$ & $\begin{array}{c}\text { \% Non-overlapping } \\
\text { Usable }\end{array}$ \\
\hline Before Calves & 452 & $71 \%$ & 600 & $75 \%$ \\
\hline With Calves & 12264 & $65 \%$ & 13348 & $92 \%$ \\
\hline Total & 12716 & $65 \%$ & 13948 & $91 \%$ \\
\hline
\end{tabular}

C. USABle Whistles

\begin{tabular}{|c|c|c|c|}
\hline Section & Usable Whistles & Rate per Minute & Average Duration (ms) \\
\hline Before Nephele's Calf & 266 & 0.8 & $456.7^{\dagger}$ \\
\hline With Nephele's Calf & 405 & 0.8 & $517.9^{\dagger}$ \\
\hline \hline Before Vicky's Calf & 452 & 0.75 & 426.3 \\
\hline With Vicky's Calf & 4,162 & 6.9 & 691.5 \\
\hline With Delphi's Calf & 3,933 & 9.8 & 668.9 \\
\hline With Delphi's Calf/Lotus & 3,804 & 8.45 & 625.7 \\
\hline Lotus Alone & 245 & 6.1 & 507.2 \\
\hline With Lotus & 365 & 7.3 & 661.4 \\
\hline Total & 13,632 & 4.6 & 646.5 \\
(Before/With) $^{8}$ & $(718 / 12,914)$ & $(0.8 / 6.3)$ & $(437.5 / 658.4)$ \\
\hline
\end{tabular}

${ }^{\S}$ With does not include Lotus Alone.

${ }^{\dagger}$ Difference not significant by a T-test, $p=0.08$ 


\section{Figure 5.2: Changes Around the Birth of Vicky's Calf}

\section{A. Whistle Rate}

Mean of all focal samples on each day, \pm standard error.

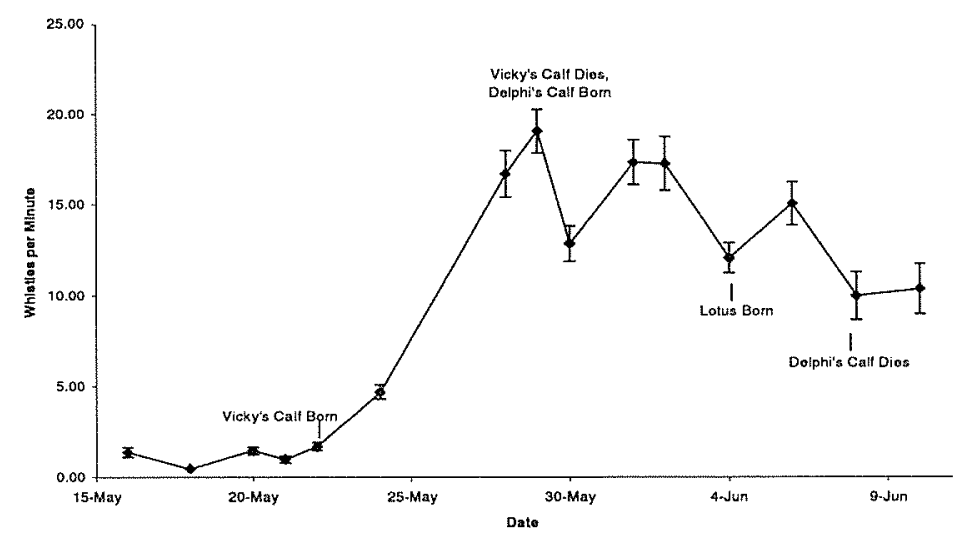

\section{B. Percent Overlaps}

Mean of all focal samples on each day, \pm standard error.

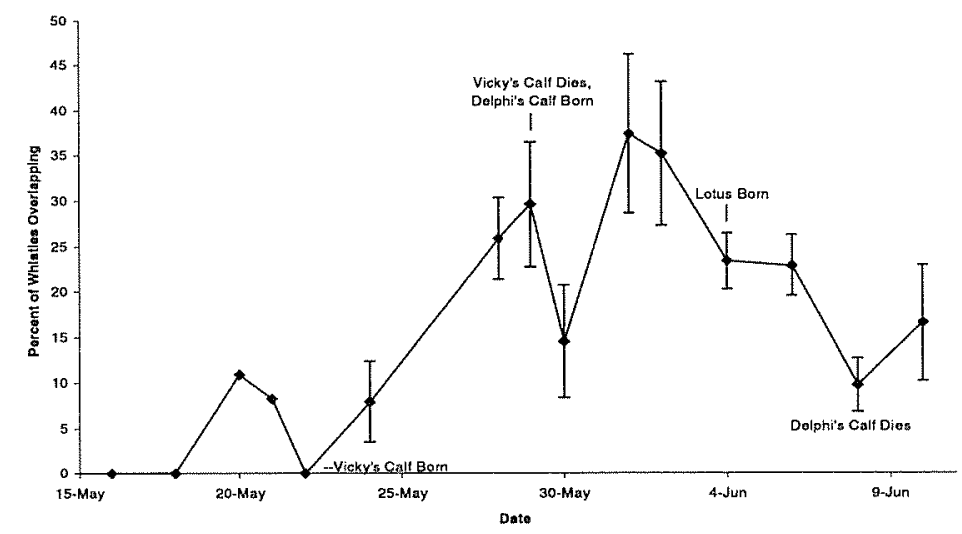

\section{Average Length of Usable Contours}

Mean of all contours from each day, \pm standard error.

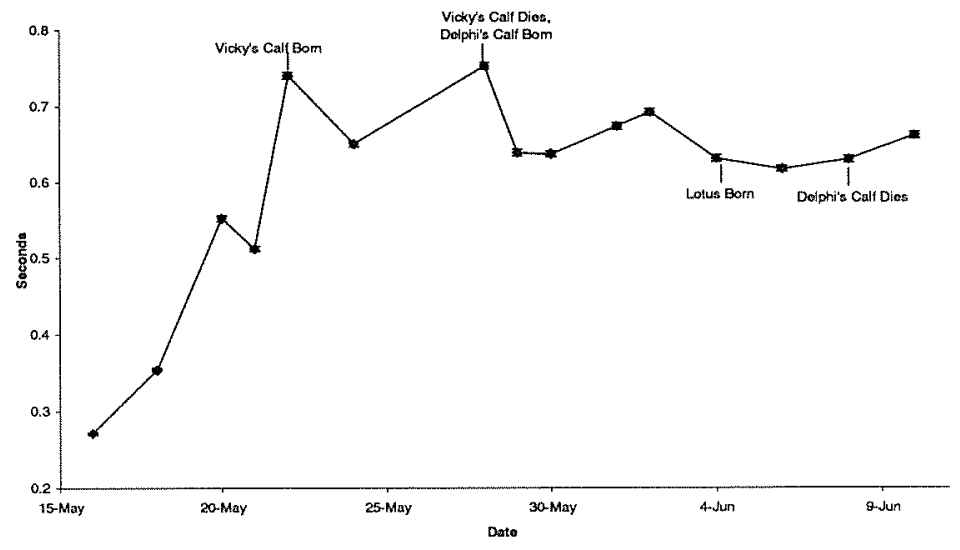


Chapter 5: Characterizing the Acoustic Environment

of all three calves born in this period (Figure 5.2A). Several other parameters of the whistles changed as well: the average length of usable contours increased by more than $200 \mathrm{~ms}$, and the percent of whistles that overlapped other whistles increased from $6 \%$ to $28 \%$ (Figure 5.2B,C; Table 5.4A). The percent of the non-overlapping whistles that could be turned into usable contours also increased, from $75 \%$ to $92 \%$ (Table 5.4B). These results suggest that both the whistles produced and the timing of whistle production changed after Vicky's calf was born.

\section{Statistical Analysis}

Statistical analysis was performed to determine whether the changes that occurred after Vicky's calf was born were significant. First, the period Before Vicky's Calf was compared to all the With Calf periods combined (With Vicky's Calf, With Delphi's calf, With Delphi's calf/Lotus, With Lotus) by T-tests. Three measures were tested: whistle rate per sample, overlap rate per sample, and average contour length. For all three measures, the mean With Calves was significantly larger than the mean Before Vicky's Calf $(\mathrm{p}<0.001$; Table 5.4A). This indicates that the rate of whistling, the rate at which whistles overlapped, and the average length of those whistles all increased significantly after Vicky's calf was born.

Interestingly, the increases in whistle rate and whistle length do not appear to have occurred after Nephele's calf was born. Although the absolute number of whistles from this period was not collected, an estimate of the whistle rate can be achieved from the usable contours (Table 5.4C). The increase after Vicky's calf was born is still apparent, with the rate changing from 0.75 to 6.9 usable whistles per minute (Table 5.4C). When Nephele's calf was born, the rate did not change, remaining at 0.8 usable whistles per minute in both periods (Table 5.4C). Because the overlapping whistles from this time period were not saved, this discrepancy may be caused by an even greater increase in the number of overlapping whistles during Nephele's calf's first week. The average length of usable contours also increased significantly after Vicky's calf was born (Table 5.4C). The 
average length increased somewhat after Nephele's calf was born but that increase was not significant (T-test: $\mathrm{p}=0.08$; Table 5.4C).

Because all three measures increased slowly after Vicky's calf was born (Figure 5.2), an ANOVA was performed on each measure with the time periods separated rather than combined (Before Vicky's calf, With Vicky's calf, With Delphi's calf, With Delphi's calf/Lotus, With Lotus). The changes in all three measures were significant $(p<0.001)$, particularly the increase from Before Vicky's Calf to all the periods with calves (Tukey tests, $\mathrm{p}<0.001$ ). The only exceptions to this were the differences in both whistle and overlap rate between the periods Before Vicky's calf and With Lotus, which were not significant (Tukey tests, whistle rate: $p=0.065$, overlap rate: $p=0.665$ ). This may be partly due to the small number of samples (5) in the With Lotus section. In addition, there were significant differences in both whistle and overlap rate between the periods With Vicky's calf and With Delphi's calf (Tukey test, whistle rate: $p=0.003$, overlap rate: $p=0.011$ ). This indicates that the slow increases in rate from Before Vicky's Calf to With Vicky's Calf to With Delphi's calf were all significant (see Figure 5.2A,B). There were also significant differences in the average contour length between the period With Delphi's calf/Lotus and the periods With Delphi's calf and With Vicky's calf (Tukey tests, $\mathrm{p}<0.001$; see Table 5.4C, Figure 5.2C). This indicates that the slight decrease in contour length after Lotus was born was significant as well (Figure 5.2C).

\section{Overlapping Whistles}

The percent of whistles that overlapped other whistles increased from $6 \%$ to $28 \%$ after Vicky's calf was born (Table 5.4A, Figure 5.2). The average length of usable whistles also increased by more than $200 \mathrm{~ms}$ (Table 5.4A). Most of the overlapping whistles had sufficient signal-to-noise ratios to have been usable had they not been overlapping. The average contour length is therefore a fair measure for the length of the overlapping whistles. However, even with the longer whistles, and assuming at least 100 ms between the whistles, almost 800 whistles could fit into a ten-minute sample without 
Chapter 5: Characterizing the Acoustic Environment

overlapping. The average number of whistles per sample found after Vicky's calf was born was 126.

The increase in overlapping whistles could indicate that the dolphins were more likely to whistle in response to each other after the calves were born than before. However, even if the whistles simply occurred randomly with respect to each other, the increased rate of whistling could combine with the increased whistle length to cause the increased rate of overlapping. To discriminate between these two possibilities, a distribution of overlap rates was generated by randomly placing whistles within 600 seconds (10 minutes) and determining what percentage overlapped (Matlab, see Appendix 2). 10,000 of such random samples were generated using the average number of whistles for each time period (Table 5.5A: 11 before and 126 after). Overlapping was defined as being within the average usable contour length for that time period (Table 5.5A: $426 \mathrm{~ms}$ before, $663 \mathrm{~ms}$ after). A p-value was generated by determining the proportion of samples in the distribution with a greater value than the observed value. The mean overlap rate for 126 whistles was $13 \%$ (range $5 \%-25 \%$, Table 5.5B). As is obvious from the range, none of the 10,000 cases had an overlap rate as high as the observed rate (of $28 \%$, so $\mathrm{p}=0$, Table 5.5B). The observed overlap rate before the calves were born, on the other hand, was not significant $(\mathrm{p}=0.075$, Table $5.5 \mathrm{~B}$ ), even though the mean simulated overlap rate for this period was only $0.7 \%$ (range $0 \%-18 \%$, Table 5.5B). These results indicate that the increased overlap rate was not simply caused by the increase in whistle rate or whistle length. The whistles were randomly timed with respect to each other before the calves were born but were not randomly timed after the calves were born. The results of the simulations indicate that the dolphins were more likely to produce whistles close together in time after the calves were born than before. There are two possible explanations for this: after the calves were born, all the dolphins were more likely to whistle in response to the same event, such as an action by a calf, or after the calves were born, the dolphins were more likely to whistle in response to other dolphins whistling. 
Table 5.5 Random Trials of Percent Overlapping Whistles

A. INPUT NUMBERS

\begin{tabular}{|c|c|c|c|}
\hline Section & Number of Whistles & Average Duration & Observed \% Overlap \\
\hline Before & 11 & $426 \mathrm{~ms}$ & $6 \%$ \\
\hline After & 126 & $663 \mathrm{~ms}$ & $28 \%$ \\
\hline
\end{tabular}

B. RESULTS OF 10,000 SimULATIONS

\begin{tabular}{|c|c|c|c|c|c|}
\hline \multirow{2}{*}{ Section } & \multicolumn{2}{|c|}{$\%$ Overlap } & Number $>$ Observed & Percent $>$ Observed & P-value \\
\cline { 2 - 6 } & Mean & Range & Oben & $7.5 \%$ & 0.075 \\
\hline Before & $0.7 \%$ & $0 \%-18 \%$ & 750 & $0 \%$ & 0.000 \\
\hline After & $13 \%$ & $5 \%-25 \%$ & 0 & 0 \\
\hline
\end{tabular}

\section{Bubblestreams}

Bubblestream-whistles were rare in this study. In the period around the birth of Vicky's calf, 203 bubblestream-whistles were found. Bubblestream-whistles occurred at a rate of less than 1 every ten minutes and made up only $1 \%$ of the 19,488 whistles found during this period (Table 5.6A, Figure 5.3). The majority of the bubblestream-whistles were made by calves. Only 35 were made by adults. The bubblestream-whistles produced by adults, therefore, constituted approximately $0.2 \%$ of all the whistles produced and on average occurred once an hour (Table 5.6A, Figure 5.3). The 168 bubblestream-whistles produced by the calves only constituted $0.9 \%$ of the whistles produced (Table $5.6 \mathrm{~A}$, Figure 5.3). Calf bubblestream-whistles occurred once per ten-minute focal sample. On average, $70 \%$ (range 33\% - 92\%) of the bubblestream-whistles produced usable contours, but bubblestream-whistles remained only $1.2 \%$ of the usable contours from these periods (Table 5.6A).

When the bubblestream-whistles are broken down by individual, there are even fewer to work with (Table 5.6, Figure 5.3). Each adult present when Vicky's calf was born averaged approximately one bubblestream-whistle every 3 hours (Table 5.6A). In fact, in the entire sample, including bubblestream-whistles added from extra focal samples, only 2 usable bubblestream-whistles were found from Nephele and only 4 from Sharky (Table 5.6B). On average, the adults produced fewer than 10 usable 


\section{Figure 5.3: Bubblestreams in MAY AND June}

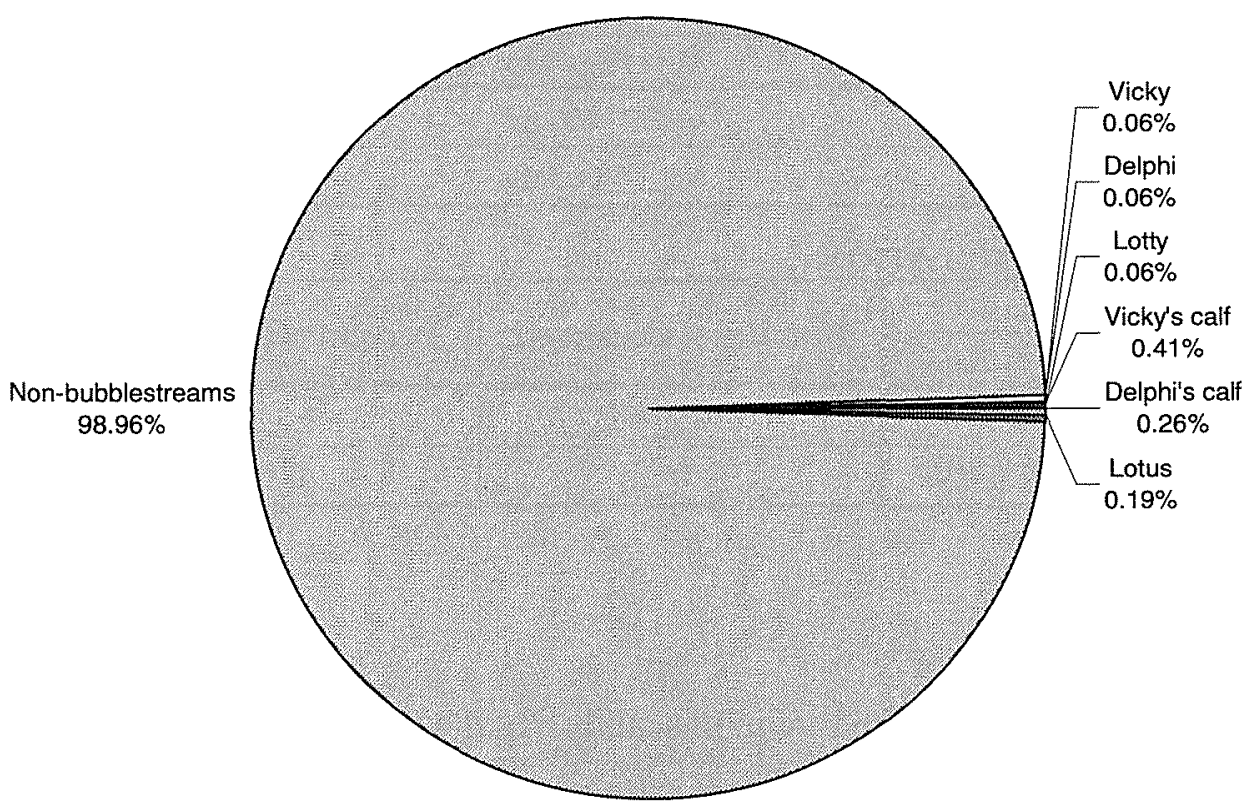

bubblestream-whistles and none of the adults produced more than 16, in more than 26 days of recordings (Table 5.6B). Bubblestream-whistles were even rarer in the periods before the calves were born. Of the 636 whistles collected from 10 hours of recordings the week before Vicky's calf was born, none were associated with bubblestreams.

The rarity of bubblestream-whistles strongly suggests that using bubblestreams as the sole whistle sample, as some researchers have (e.g. McCowan 1995, Herzing 1996), is ill-advised. Using rare whistles is problematic for two reasons. One is a practical problem: generating a reasonable sample size can take a very long time. More important, however, is the problem of possible biases in the sample. Since dolphins can obviously whistle without producing a bubblestream, whistles produced with bubblestreams are by definition different from other whistles. The rarity of the bubblestream display raises concerns about whether whistles produced with bubblestreams are rarer whistle types than whistles produced without bubblestreams.

In addition, another issue arises when bubblestreams are used to identify whistlers: properly identifying which whistle to associate with the bubblestream can be difficult. In this study, the imprecision in timing when bubblestreams were marked was 
TABLE 5.6 BUBBLESTREAMS BY INDIVIDUAL IDENTITY

\begin{tabular}{|c|c|c|c|}
\hline Dolphin & Total & Rate per Minute ${ }^{8}$ & Usable Contours \\
\hline Vicky & $12(0.06 \%)$ & 0.006 & $11(0.09 \%)$ \\
\hline Lotty & $11(0.06 \%)$ & 0.005 & $8(0.06 \%)$ \\
\hline Delphi & $12(0.06 \%)$ & 0.006 & $4(0.03 \%)$ \\
\hline Total Adult & $35(0.2 \%)$ & 0.017 & $23(0.2 \%)$ \\
\hline Vicky's calf & $80(0.4 \%)$ & 0.13 & $61(0.5 \%)$ \\
\hline Delphi's calf & $50(0.3 \%)$ & 0.06 & $34(0.3 \%)$ \\
\hline Lotus & $38(0.2 \%)$ & 0.08 & $29(0.2 \%)$ \\
\hline Total Calf & $168(0.9 \%)$ & 0.11 & $124(1 \%)$ \\
\hline Total bubblestreams & $203(1.0 \%)$ & 0.097 & $147(1.2 \%)$ \\
\hline Total whistles & 19,488 & 9.28 & 12,351 \\
\hline
\end{tabular}

${ }^{8}$ Rate per minute was calculated for adults and the total from the total minutes before and after the calves, but for the calves from only the time when that calf was alive. The total calf rate was calculated from the total time for all three calves.

B. TOTAL USABLE FROM FOCAL SAMPLES

\begin{tabular}{|c|c|}
\hline Dolphin & Usable Contours \\
\hline Vicky & 14 \\
\hline Lotty & 12 \\
\hline Delphi & 16 \\
\hline Nephele & 2 \\
\hline Sharky & 4 \\
\hline Total Adult & 48 \\
\hline Daphne & 34 \\
\hline Nephele's calf & 1 \\
\hline Vicky's calf & 61 \\
\hline Delphi's calf & 34 \\
\hline Lotus & 29 \\
\hline \hline Total Calf & 159 \\
\hline Total bubblestreams & $208^{\dagger}$ \\
\hline \hline Total whistles & $>14033^{\S}$ \\
\hline
\end{tabular}

${ }^{8}$ Not all the whistles from March were saved. Some bubblestreams may have been lost this way as well.

${ }^{\dagger}$ This number does not include the extra 151 Nephele's calf bubblestreams from non-focal samples. 
Figure 5.4: Ambiguity in the Assignment of Bubblestreams to Whistles A bubblestream from Vicky's calf was recorded 416 seconds into the focal sample. These four whistles were recorded within two seconds of that recording. The labels refer to the time within the focal sample that the whistles began, to the nearest half-second.
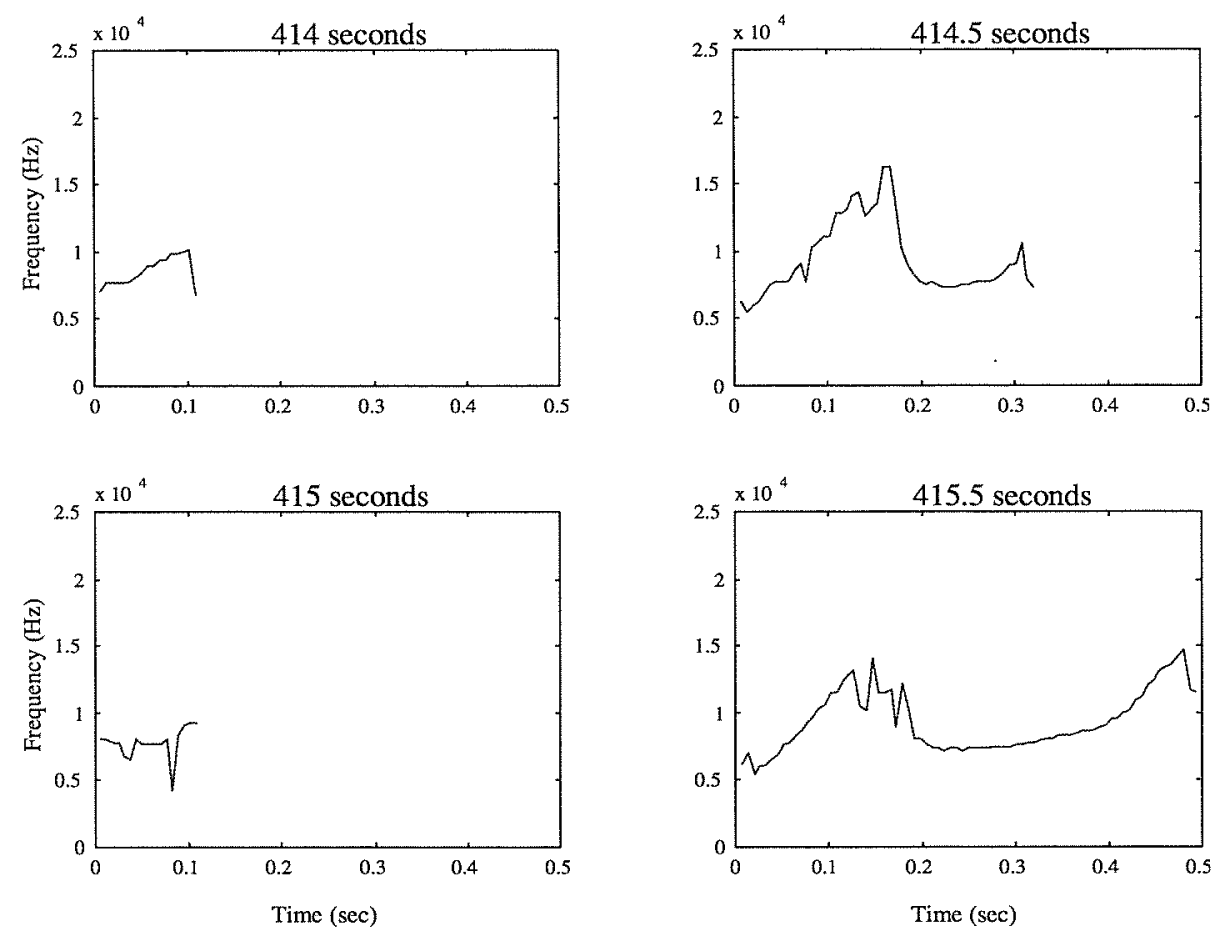

as much as several seconds. The start time of the whistles was only recorded to the nearest half-second at best. This is not a major problem when whistles are rare, as was the case before the calves were born in this study. If there is only one whistle within a few seconds of the bubblestream mark, the identification is not difficult. However, when there are several whistles per second, as occurred after the births, identifying which of the whistles to associate with the bubblestream can be problematic (Figure 5.4). The four whistles in figure 5.4 were produced within two seconds of each other. They are labeled with the time they began, to the nearest half-second within the focal sample (focal samples are 600 seconds long). A single bubblestream was recorded from Vicky's calf at 416 seconds. The whistle can be assumed to have occurred before the bubblestream was actually recorded but when within the previous few seconds the bubblestream-whistle occurred is unclear. The contours of these four whistles are extremely different (Figure 
5.4). A very different picture of the calf's ability to whistle will be achieved from assigning the bubblestream to one of the whistles on the right than to one of the whistles on the left.

\subsubsection{DETERMINING SignATURE WHISTLES FROM BUbBLESTREAM-WHISTLES}

\section{Daphne Bubblestreams}

Although bubblestream whistles are rare, they are the only identified whistles in this sample. The possibility that the dolphins' signature whistles can be determined from these identified whistles is therefore worth examining. Thirty-three bubblestreamwhistles were recorded from Daphne in March, when she was 7 months old. These whistles varied considerably in both duration and frequency modulation (Figure 5.5). The average duration of Daphne's bubblestream-whistles was $400 \mathrm{~ms}$, and the whistles varied from less than $60 \mathrm{~ms}$ to more than 1 second in length (Figure 5.5). In this chapter, spectrograms will be used to show the details of the whistles, while contours will be used to more clearly illustrate the relative durations of the whistles and to show the input data for the quantitative comparisons. Therefore, in order to maximize the resolution of the spectrograms, the time axis on each spectrogram matches the duration of the whistle, and the time axes on two spectrograms are not comparable. The time axes on all contours in a figure, on the other hand, are the same to make the contours easier to compare (e.g. Figure 5.5B). This means that the time axes on the contours are different from the axes on the corresponding spectrograms (e.g. compare Figures 5.5A and 5.5B).

Daphne's bubblestream-whistles also varied in the amount of frequency modulation (Figure 5.5). One measure sometimes used to describe frequency modulation is the number of "loops" (Caldwell et al. 1990). A "loop" is a pattern of frequency modulation that is repeated in a single whistle. The whistle in the upper right of figure $5.5 \mathrm{~A}$, for example, has four loops. The whistle on the lower left of figure 5.5, on the other hand, has no loops. Whistles such as the upper left example in figure 5.5A are sometimes said to have one loop because the pattern of frequency modulation seen 
Chapter 5: Characterizing the Acoustic Environment

\section{FIGURE 5.5: EXAMPLES OF DAPHNE'S BUBBLESTREAM-WHISTLES}

\section{A. SPECTROGRAMS}

Spectrograms are not scaled relative to each other. The time axes on the four spectrograms are therefore different from each other.
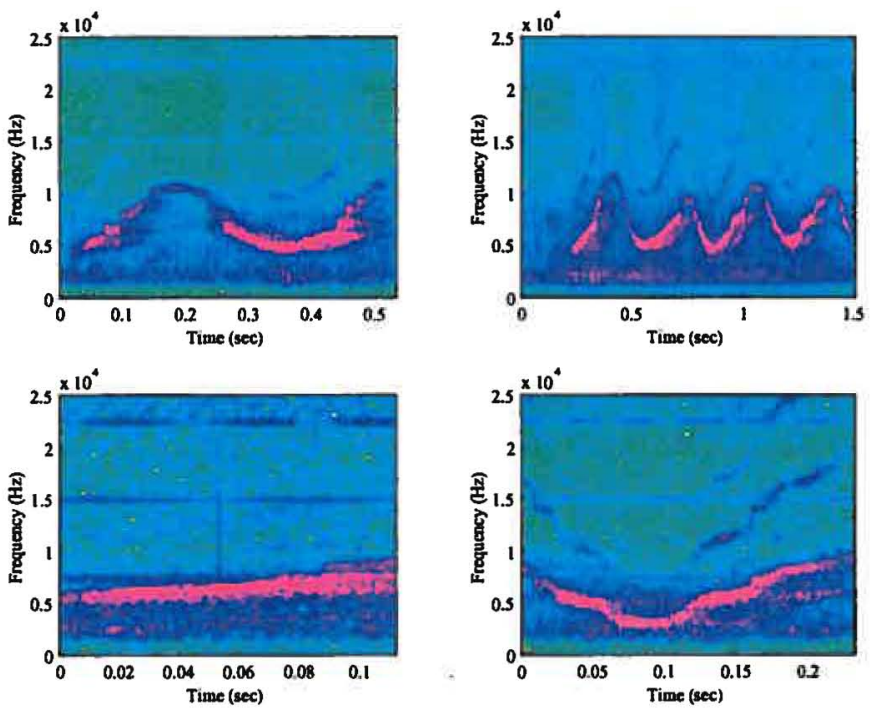

\section{B. Contours of Whistles IN A}

Contours are in the same order as the spectrograms in A. Contours are scaled relative to each other, and are therefore on different time axes than the corresponding spectrograms in $\mathrm{A}$
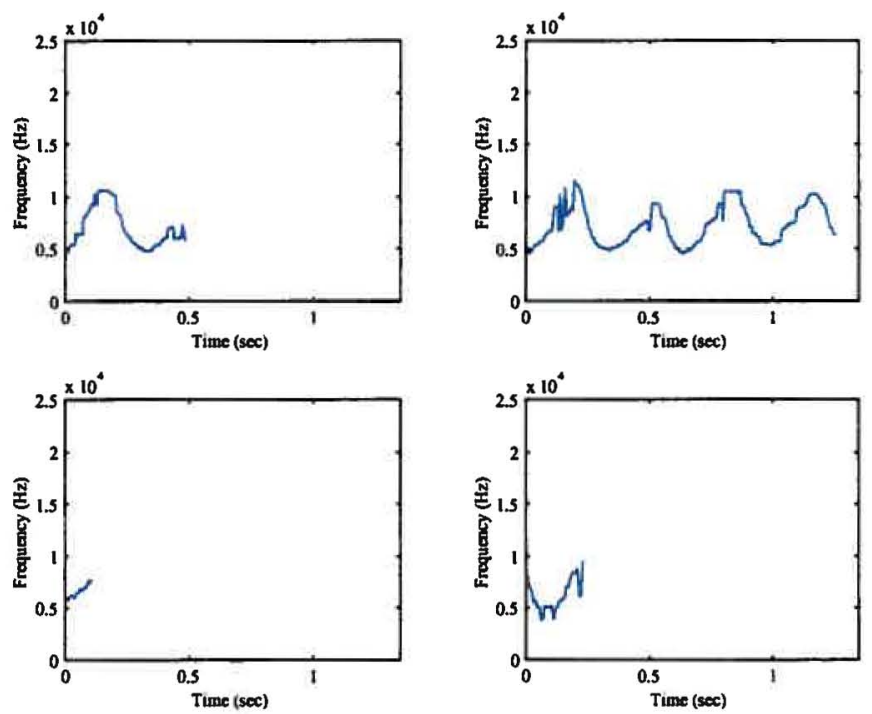
resembles the loops of other, multi-loop whistles (compare the upper contours in Figure 5.5B).

The contours of Daphne's 33 bubblestream-whistles were compared to each other with dynamic time warping (DTW). To visualize the similarity between the contours, $2 \mathrm{~d}$ multidimensional scaling (MDS) was performed, as described in chapter 4 (Figure 5.6). The contours on the MDS show the range of duration and frequency modulation. The dimensions of MDS are a combination of the factors that influence the DTW. The first dimension appears to be mostly based on duration. Although the DTW normalizes the duration when comparing contours, DTW comparisons are limited to contours whose durations are within a factor of two of each other. For instance, the contours labeled 1 $(A \cup \wedge)$ and $2(A, \cap 9)$ on the MDS (Figure 5.6) would be compared by the DTW by aligning the ends and warping the centers to match each other. Any difference in duration between these two contours becomes irrelevant with DTW. In the same way, contours 3 (I) and 4 ( ) would be aligned by the DTW. However, a comparison between contour $1(A \cup)$ and contour $3($ ) would not be allowed. The dynamic time warper defines the dissimilarity between these two contours to be infinite, which is translated to $10^{9}$ for these analyses. The greatest non-infinite value is approximately $5 \times 10^{8}$. In the same way, the dissimilarity between the single-loop contour $5(12)$ and multi-loop contour $1\left(H_{A}\right)$ is also infinite $\left(10^{9}\right)$ because contour 1 is more than twice as long as contour 5. Therefore, whistles that appear to be the same contour with a different number of loops are often not compared by DTW. The second dimension of the MDS appears to be based on the amount of frequency modulation and the number of loops (Figure 5.6). Since DTW compares the frequencies of two whistles, separation based on frequency structure is expected. The MDS positions the contours relative to each other in a manner that is intuitively logical. However, MDS gives no indication of where the boundaries between groups of contours should be drawn. Rather, contours on an MDS plot appear to form a continuum from short to long and from unmodulated to highly frequency modulated. 
FIGURE 5.6: MULTIDIMENSIONAL SCALING OF DAPHNE'S BUBBLESTREAM-WHISTLES Contours are scaled relative to each other. The coefficient of alienation $(\mathrm{CoA})$, a measure of how different the solution is from the original matrix, is 0.08 . High CoA's indicate poor fit. The proportion of variance (RSQ) accounted for by the solution is 0.97 .

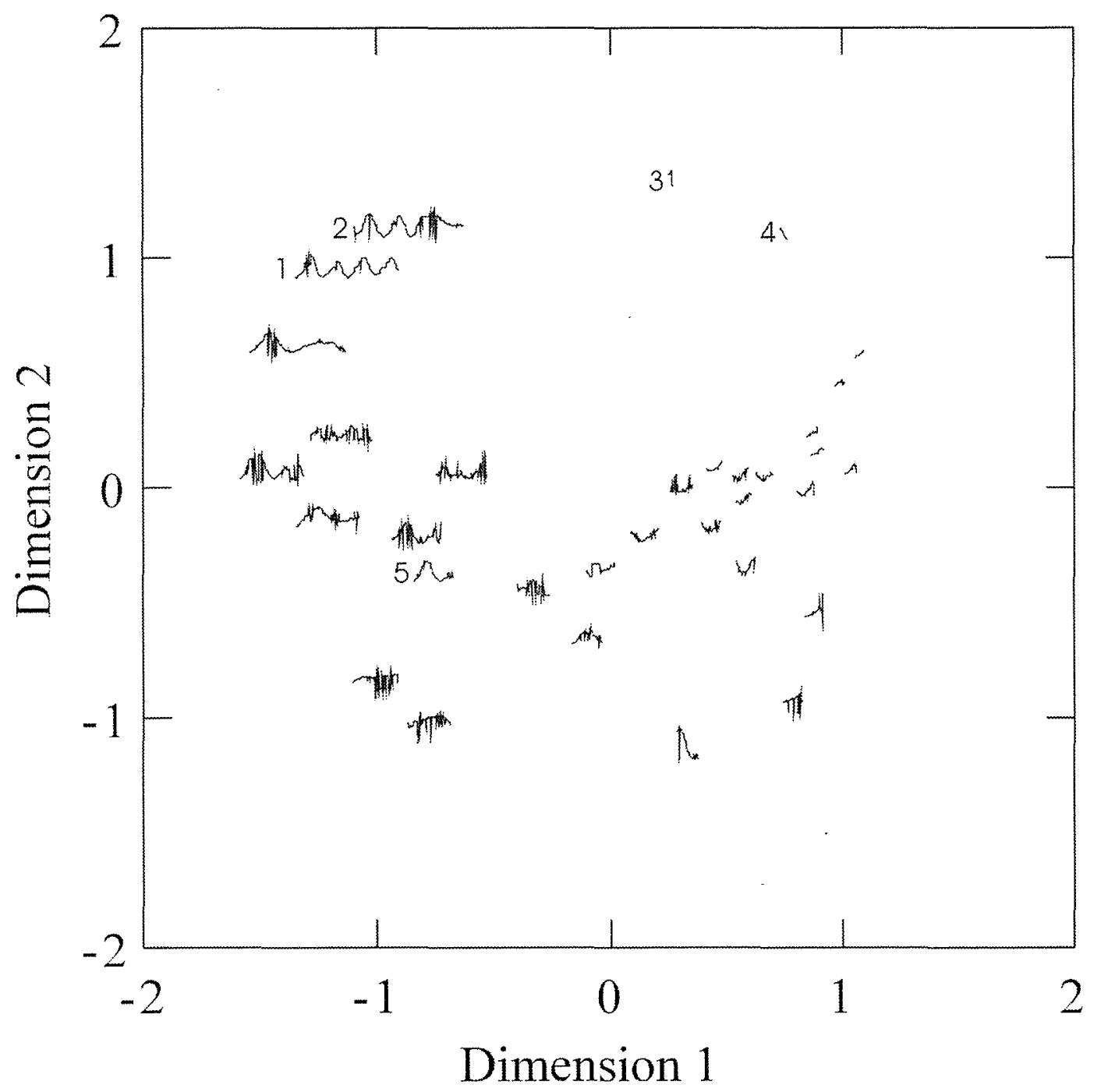

Hierarchical cluster analysis (HCA) separates these contours in a similar way to MDS (Figure 5.7). The horizontal line farthest to the left of this plot represents the place where all 33 cases (contours) are connected. From this point, the tree separates into two branches at a time, by similarity. The farther right two lines are separated, the more similar the cases those lines represent are. The ends of the lines on the far right represent 
FIGURE 5.7: HIERARCHICAL ClUSTER ANALYSIS OF DAPHNE's BUBBLESTREAM-WHISTLES Contours are scaled relative to each other. Dotted vertical line indicates the moat index ( 6 clusters). Contours at the moat index represent the average contour for that cluster. Average contours are scaled relative to each other, but not relative to the original contours.

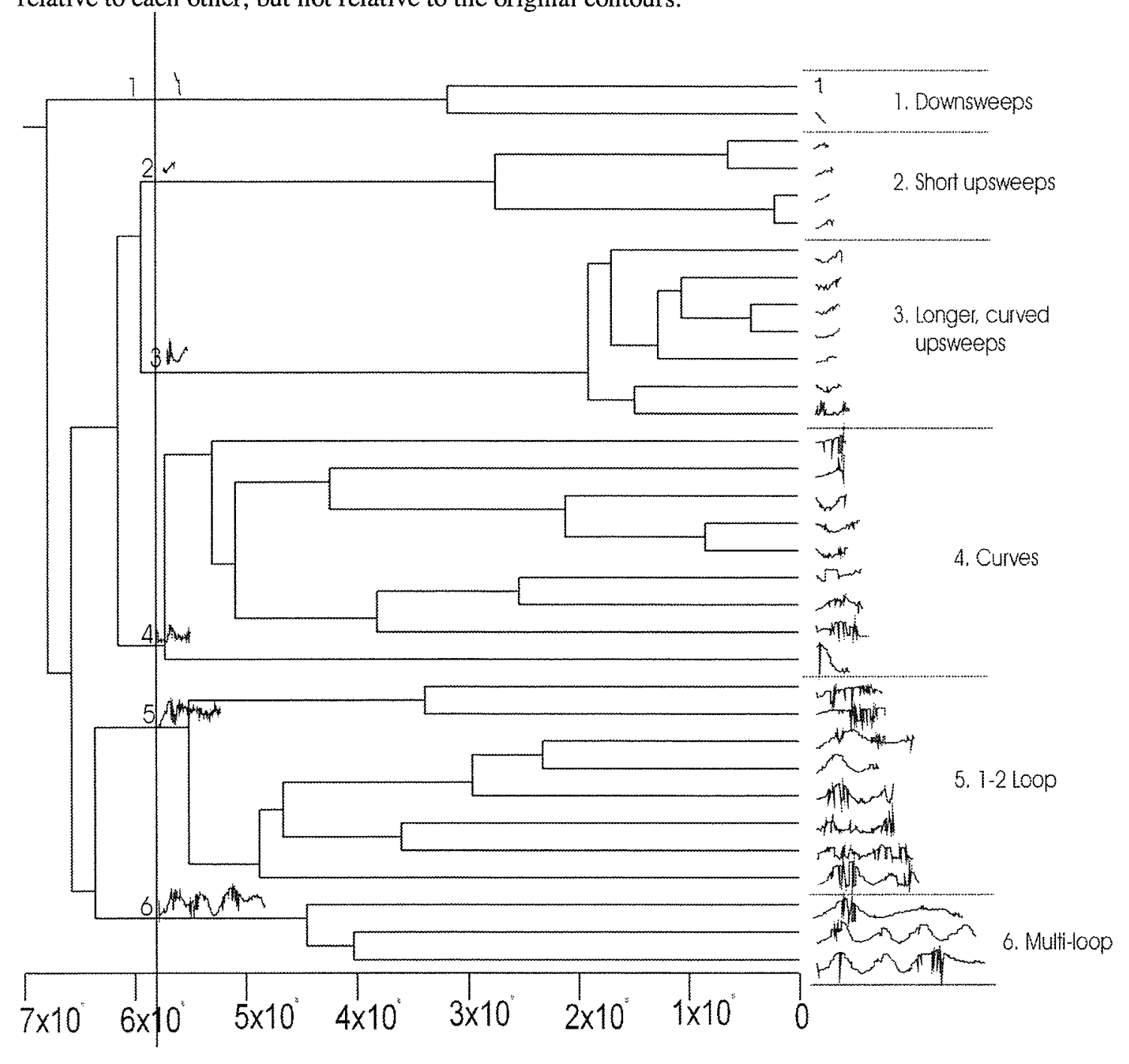

\section{Distances}

the separate cases, each labeled with the appropriate contour. The distance through the lines of the tree from one contour to another indicates the distance (dissimilarity) between those contours. A short distance between the contours indicates that the contours are similar to each other. The dotted vertical line represents the number of clusters that maximizes the moat index, a measure of how similar cases in a single cluster are compared 
Chapter 5: Characterizing the Acoustic Environment

to cases in different clusters (see chapter 4). The moat index is the number of clusters that maximizes within-cluster similarity compared to between-cluster similarity. The numbers at the moat index are cluster labels. The contours just to the right of the moat index line represent the average contour for the cluster. The average contours were determined by averaging all the contours in each cluster, without dynamic time warping which aligns the features as well as possible. Therefore, the contours may not have aligned perfectly to form the average contour. The average contour merely demonstrates how the contours in the cluster come together. The contours on this plot, and on the MDS, demonstrate how the noise in some spectrograms creates spikes in the contours. However, both the MDS and HCA clustered the whistles by overall contour, in spite of the noise spikes. This indicates that DTW is robust to some noise in the contours.

Unlike the MDS, the HCA indicates how contours can be separated from each other. The similarity between cases can be seen by examining the distance through the tree between the cases. The seven cases (contours) in cluster 3, for instance, are closer to each other than the nine cases in cluster 4 . This indicates that the cases of cluster 3 are more similar to each other than the cases in cluster 4 are. The distance to the next connection to the left of the moat index demonstrates the similarity between the clusters. For instance, the next connection to the left of the cutoff point for cluster 3 is between cluster 3 and cluster 2 . This indicates that these two clusters are closely related to each other. Cluster 1 , which is the last cluster to connect to the tree, has the least similarity with the other five clusters. These kinds of similarities are more apparent with the HCA than with the MDS. The two cases in cluster 1 , for instance, are contours 3 and 4 from the MDS (Figure 5.6). The separation between these two contours and the rest of the contours in this sample is clearer in the HCA than in the MDS.

An examination of the contours in each cluster of the HCA suggests a possibility for the contour of Daphne's signature whistle. As with the MDS, the HCA separates the contours by duration and amount of frequency modulation (Figure 5.7). Unlike the MDS, the moat index of the HCA suggests a way of separating the contours into groups, or 
Figure 5.8: Possible DAPHNE Signature Whistles AMONG DAPHNE'S BubBLESTREAMWHISTLES

\section{A. Contours}

Contours are scaled relative to each other. $\mathrm{C}=$ central loop, $\mathrm{T}=$ terminal loop.
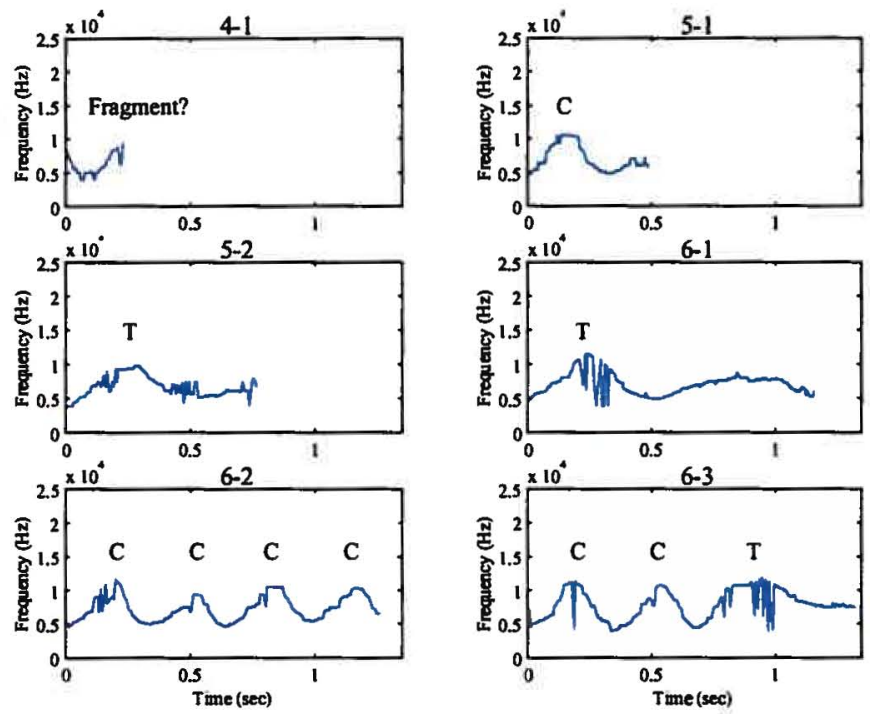

\section{B. SPECTROGRAMS OF CONTOURS IN A}

Spectrograms are not scaled relative to each other, or to the contours.
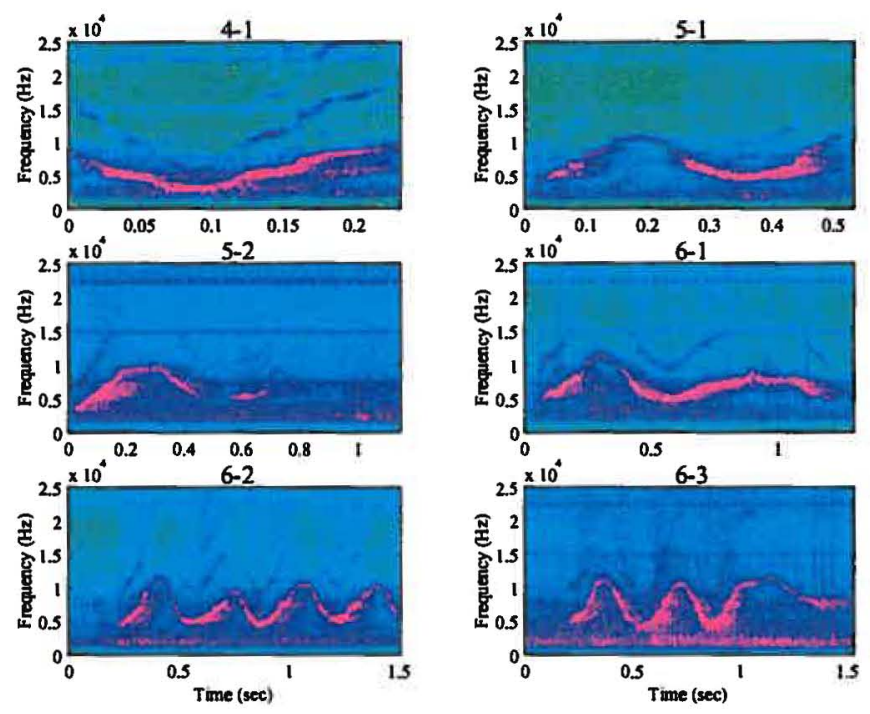
Chapter 5: Characterizing the Acoustic Environment

straight upsweeps, and curved upsweeps. Cluster 6 consists of three long, multi-loop whistles. Many of the contours in clusters 4 and 5 appear to be fragments or single loop variations of the whistles in cluster 6 (Figure 5.8). The whistles in cluster 6 may therefore represent variations of Daphne's signature whistle. Signature whistles have been reported having more than one type of loop, such as initial loops, central loops and terminal loops (e.g. Caldwell et al. 1990). Daphne's whistle appears to have a distinct terminal loop but no distinct initial loop. For instance, contour 6-3 (Figure 5.8) may be Daphne's signature whistle with 2 central loops and a terminal loop, while contour 6-2 has four central loops. Contours 5-2 and 6-1, on the other hand, appear to be only a terminal loop, and contour 5-1 a single central loop. Contour 6-1 has a second possible loop that cannot be clearly classified. The contours in cluster 6 may therefore represent the multi-loop versions of Daphne's signature whistle while cluster 5 represents the single-loop versions. Again, the spectrograms in figure 5.8B are enlarged to show the detail of the whistles and therefore have different time axes than the corresponding contours in figure $5.8 \mathrm{~A}$.

The contours in cluster 4, such as contour 4-1 (Figure 5.8), may represent fragments of a central loop of the signature whistle. A contour representing a fragment of a loop could occur in two ways. Dolphins have been recorded producing whistles identified as fragments of their signature whistles (e.g. Tyack 1986). In this case, however, these fragments could also be artifacts of the automatic extractor (see chapter 4). If Daphne sometimes produces her signature whistle with breaks of more than 100 ms, the whistles on either side of the break would be counted as two separate whistles by the extractor. Because only one bubblestream would have been recorded at that time, only one of the whistles would be in this group. The other half of the signature whistle would be in the general group of whistles.

\section{Adult Bubblestreams}

To see whether the adults' signature whistles could be determined in a similar fashion, the 48 bubblestream-whistles collected from the adults were analyzed in the 


\section{Figure 5.9: CONTOURS OF ADULT BUBblESTREAM-WHISTLES}

A. SHARKY
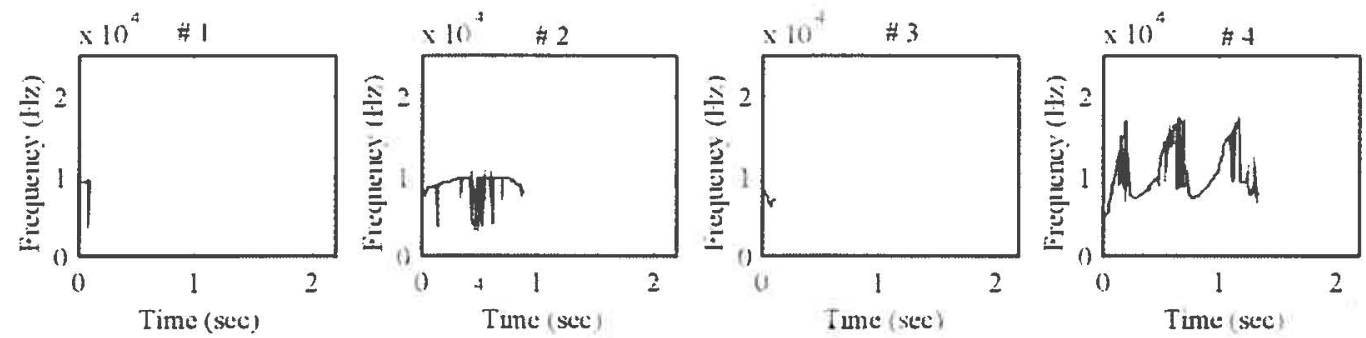

B. NEPHEI.E
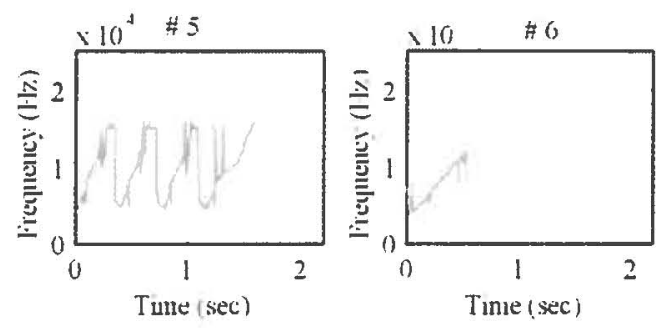

C. DEI.PHI
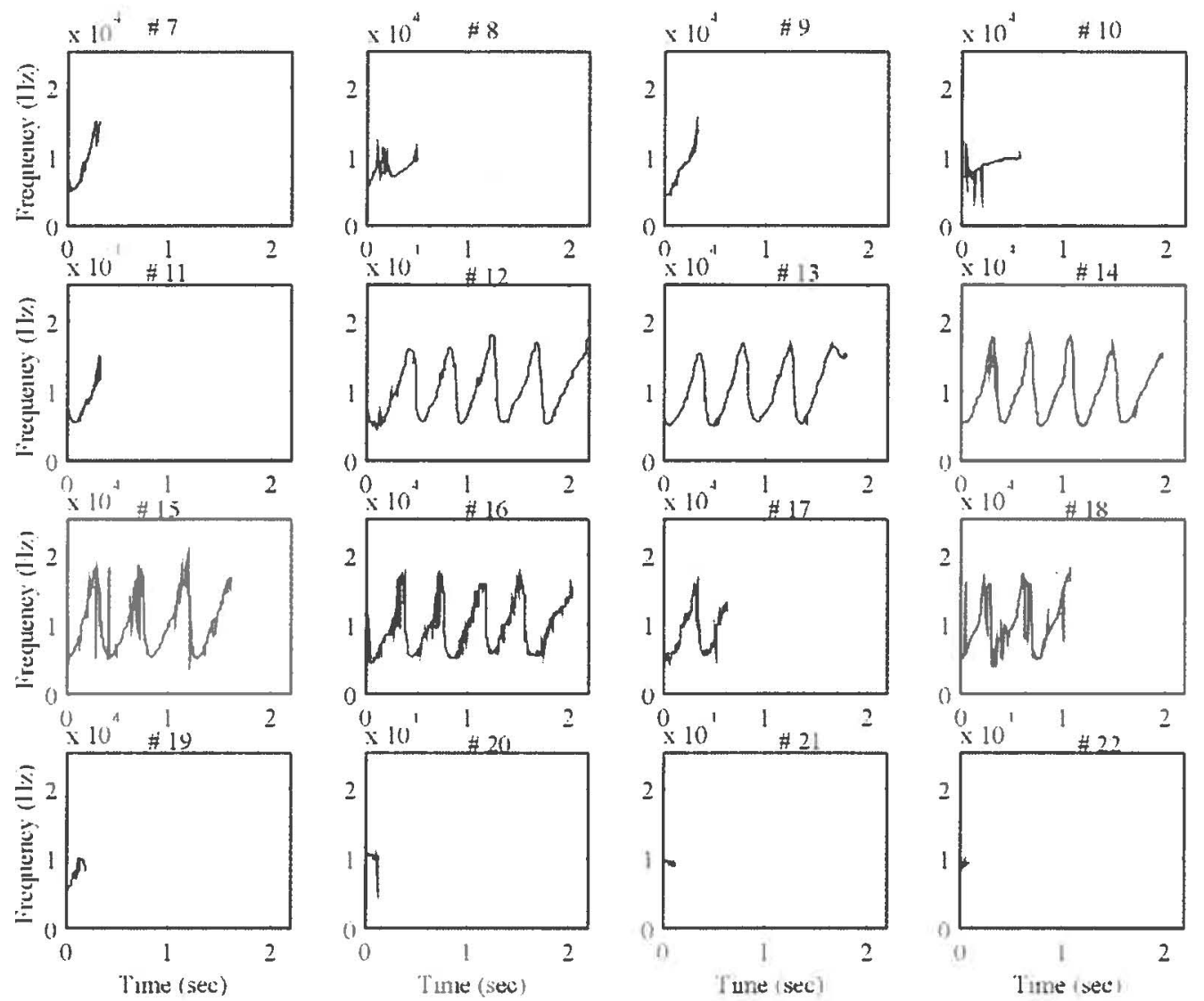


\section{Chapter 5: Characterizing the Acoustic Environment}

D. VICKY
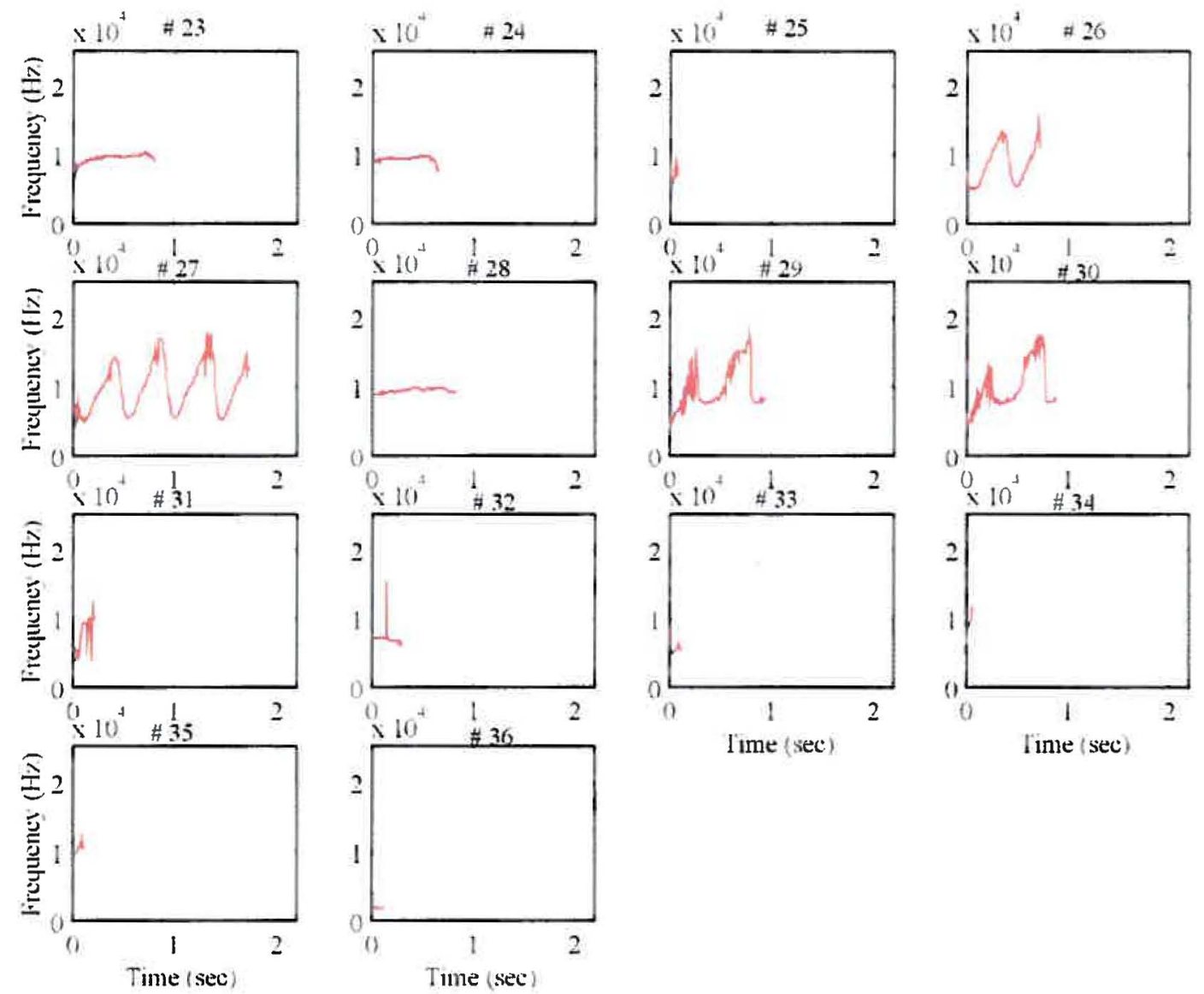

E. LOTIY
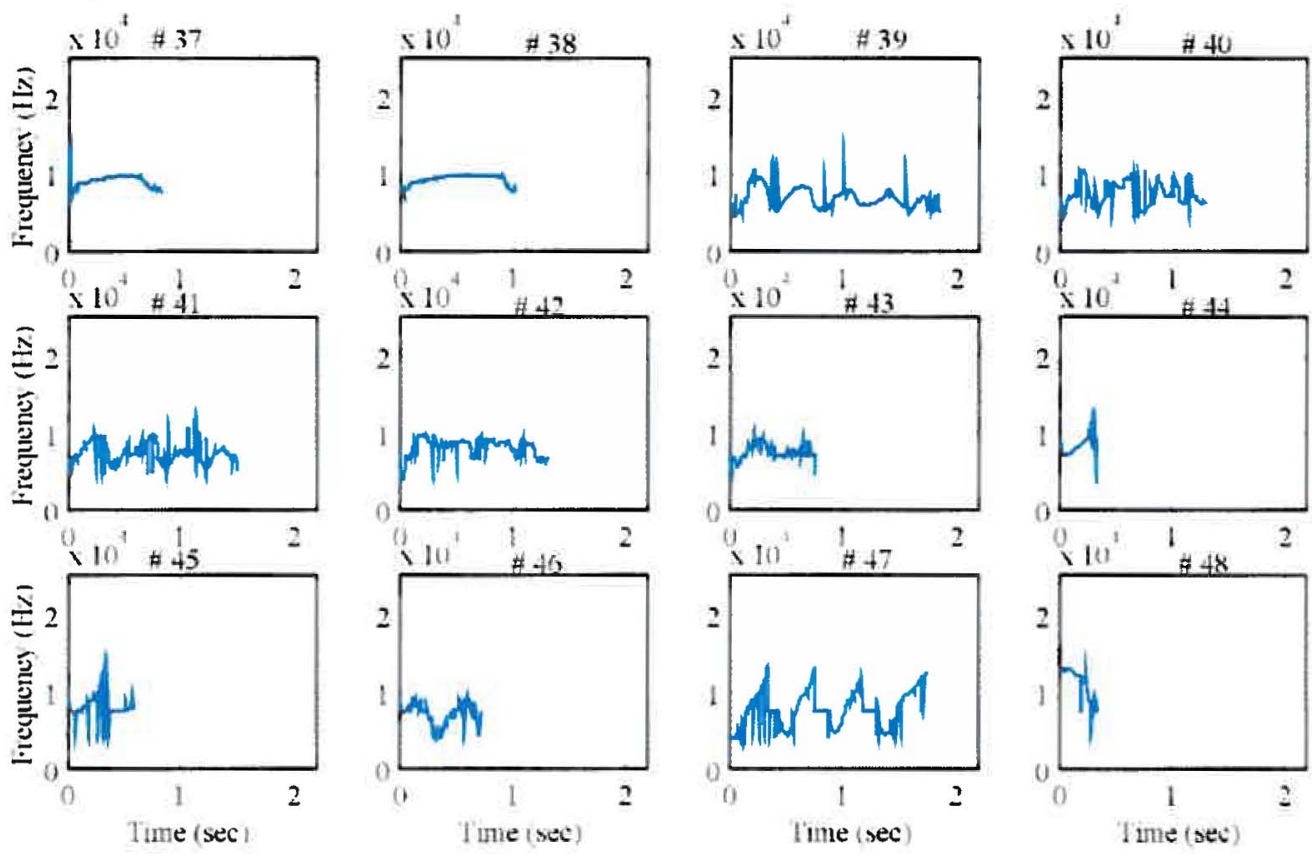
same way Daphne's bubblestream-whistles had been. The adults' bubblestream-whistles varied considerably in duration, from less than $60 \mathrm{~ms}$ to more than 2 seconds, averaging $800 \mathrm{~ms}$ (Figure 5.9). They also varied in frequency structure. These contours were analyzed with DTW, MDS and HCA in the same way that Daphne's bubblestreamwhistles were (Figures $5.10 \& 5.11$ ). Because the bubblestream-whistles were produced by several different adults, the contours on these plots are color-coded by which adult produced the bubblestream. As was the case with Daphne's bubblestream-whistles, the MDS and HCA of these whistles are quite comparable. Both analyses grouped the contours by duration and loop structure (Figures $5.10 \& 5.11$ ). The difference between these analyses is that the HCA gives a better idea of how the contours separate into groups. In this case, the moat index separates the contours into 40 clusters (Figure 5.11). Many of the clusters have only one contour in them but some have as many as 5 contours. Because only 13 of the contours are in clusters containing more than one contour, the connections between clusters are more useful than the moat index in determining the similarity between whistles (Figure 5.11). The clusters group together first by the amount of frequency modulation and then by duration (Figure 5.11). Again, both of these measures are built into the DTW. The large, numbered sections only separate the contours into general categories (upsweeps, single loops, etc.). Within those sections, contours are separated into subsections. In sections 3 and 4, for instance, the labeled subsections separate the contours by the amount and type of frequency modulation. The colors of the lines match the colors of the contours to represent which dolphin produced the bubblestream-whistle. The connections between cases or clusters are also colorcoded if all the cases in the group were produced by the same dolphin. Colored connector lines therefore represent sections of contours all produced by a single dolphin. In almost all cases, the contours in the subsections were produced by multiple dolphins. This is particularly apparent with the 3 to 4 loop contours in section 4. Many of these were produced by Delphi but several with very similar contours were produced by Vicky and Nephele (Figure 5.12 A-D). For this reason, adult bubblestream-whistles cannot be used to determine the signature whistles of the adults. Three examples of multi-loop whistles 
Chapter 5: Characterizing the Acoustic Environment

FigurE 5.10: MULTIDIMENSIONAL SCALING OF THE ADULT BUBBLESTREAMS

Contours are scaled relative to each other. Coefficient of Alienation (measure of fit) $=0.08$, Proportion of variance accounted for $(\mathrm{RSQ})=0.97$.

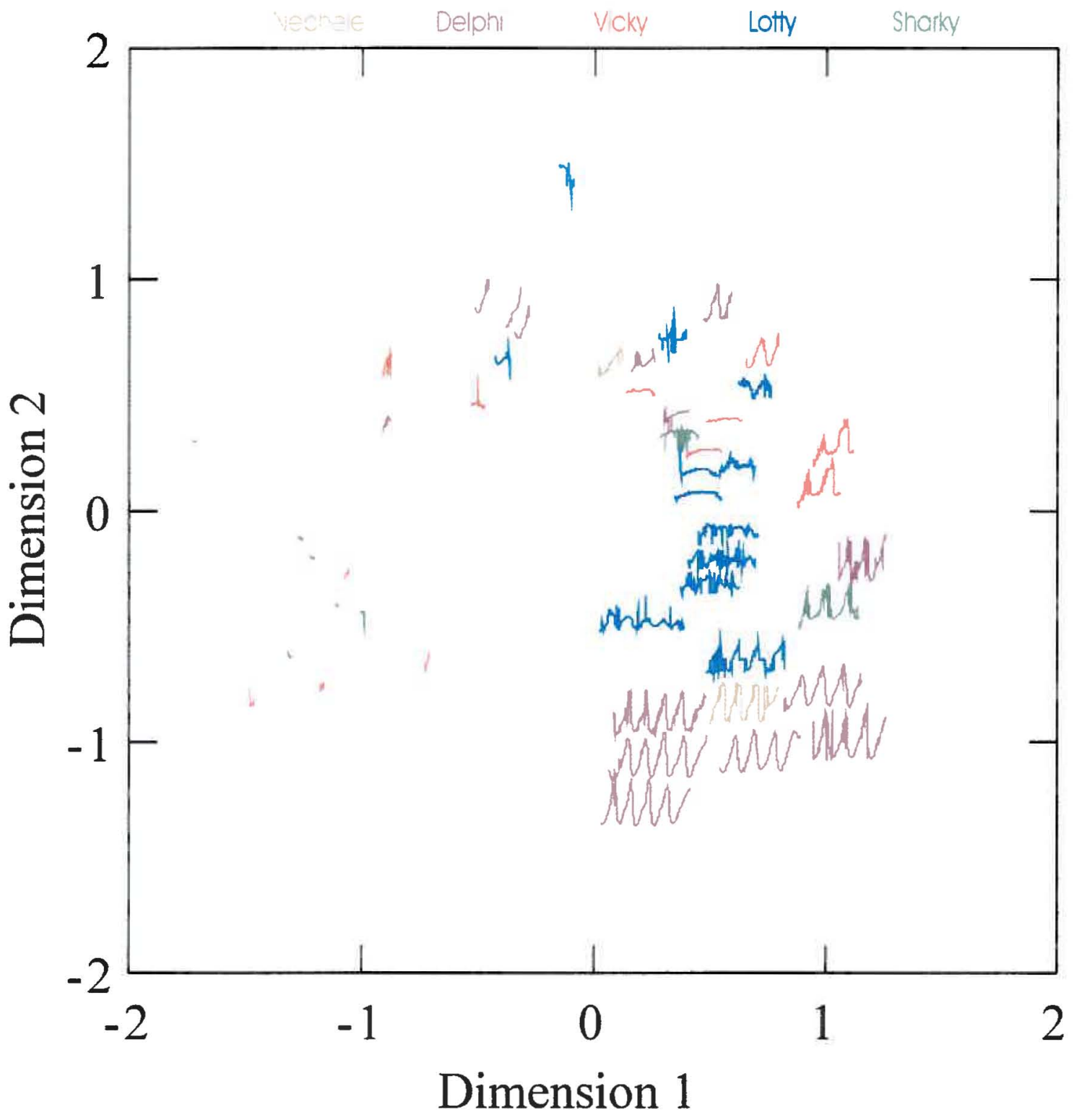


Chapter 5: Characterizing the Acoustic Environment

\section{Figure 5.11: HierarchicAl Cluster ANALYSis of ADUlt BubBlestreams}

Contours are scaled relative to each other. Vertical dotted line indicates the moat index ( 40 clusters).

Horizontal dotted lines represent the separation between the sections labeled to the left. These sections are for demonstration only. Because the moat index is much higher than four, the four sections are not statistically significant.

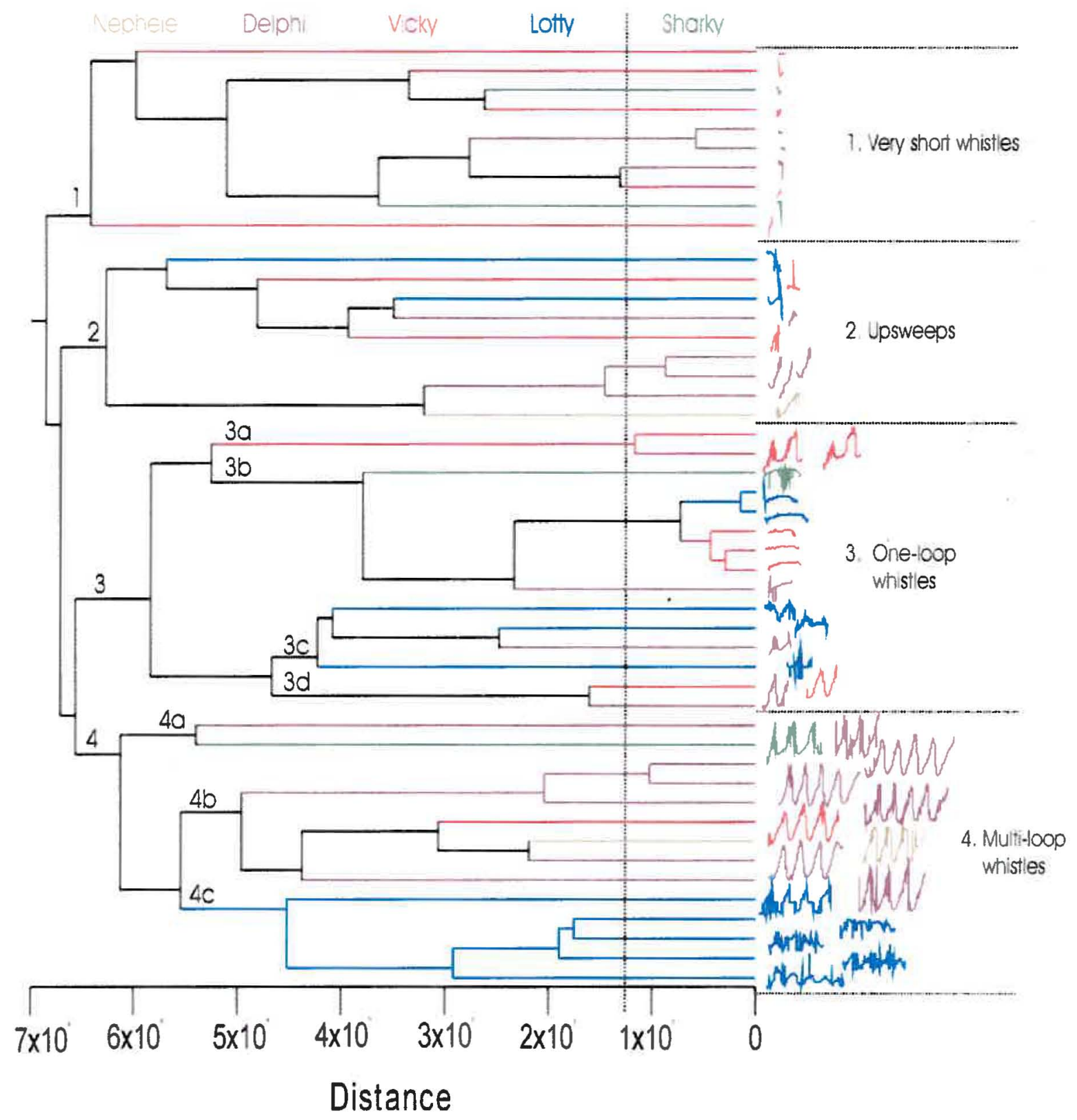


Chapter 5: Characterizing the Acoustic Environment

FigurE 5.12: COMPARISONS OF EXAMPLE ADULT BUBBLESTREAM-WHISTLES

A. SPECTROGRAMS: NEPHELE COMPARISONS
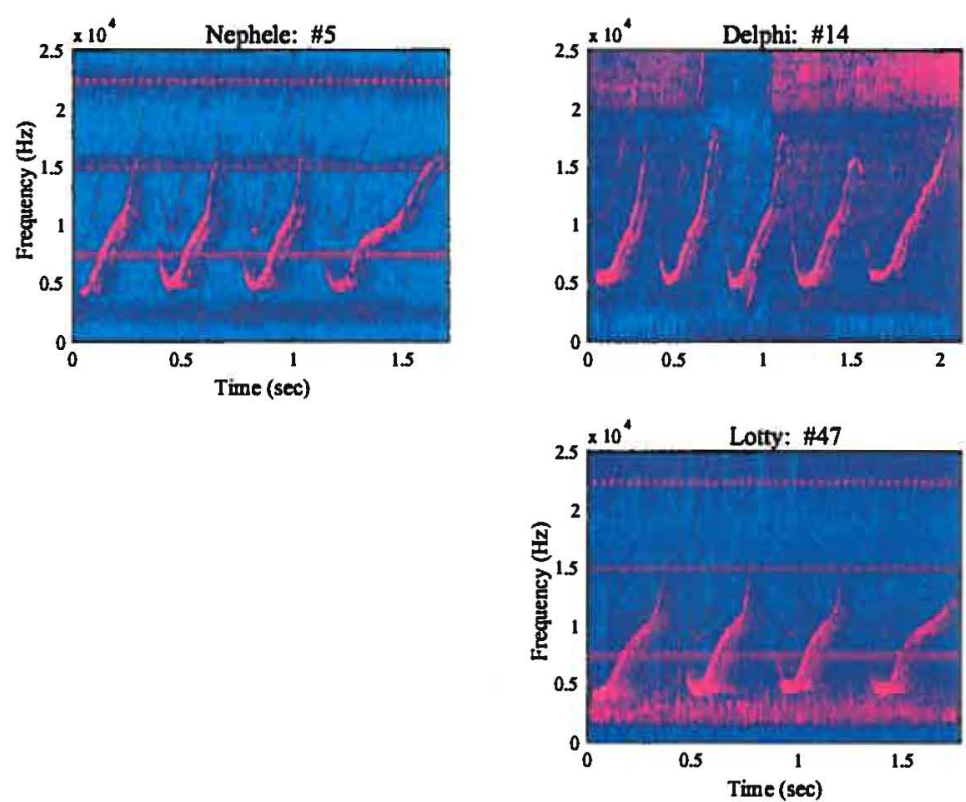

B. Contours: NePHELE COMPARISONS
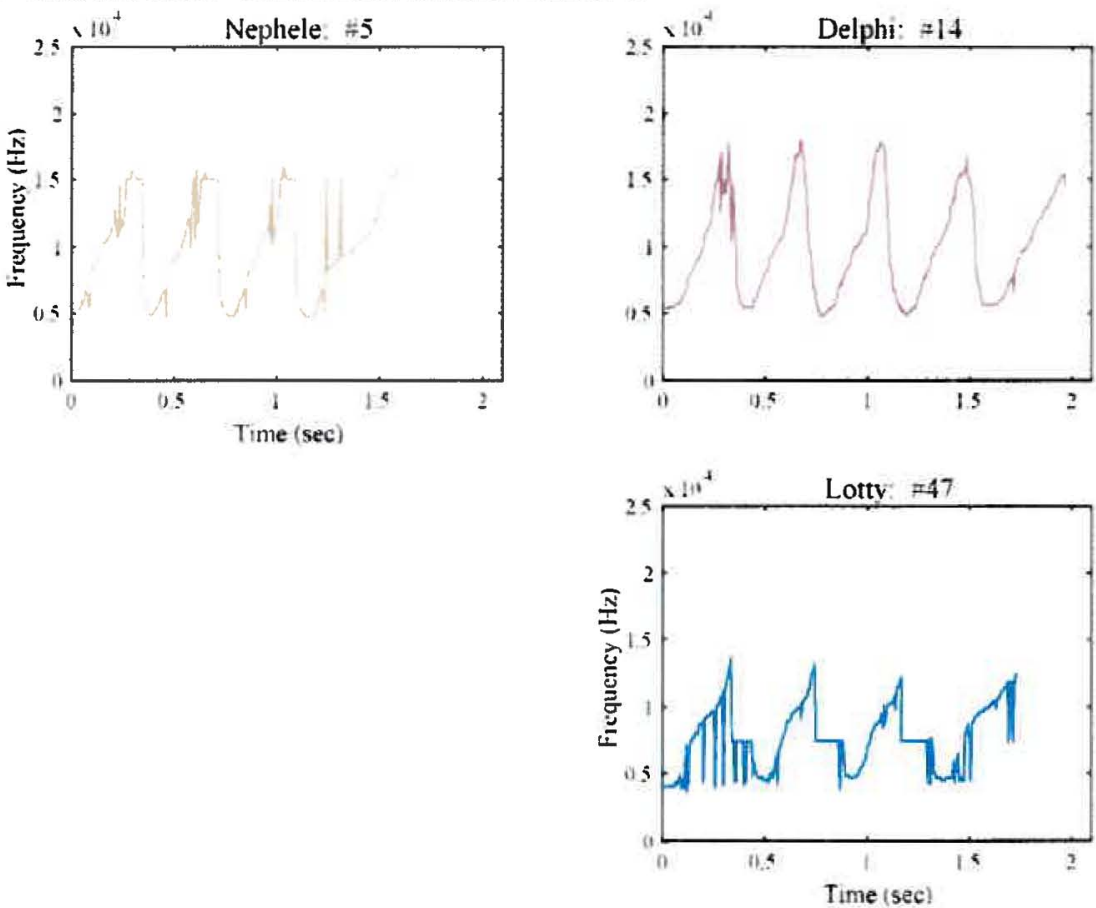
C. SPECTROGRAMS: VICKY COMPARISONS
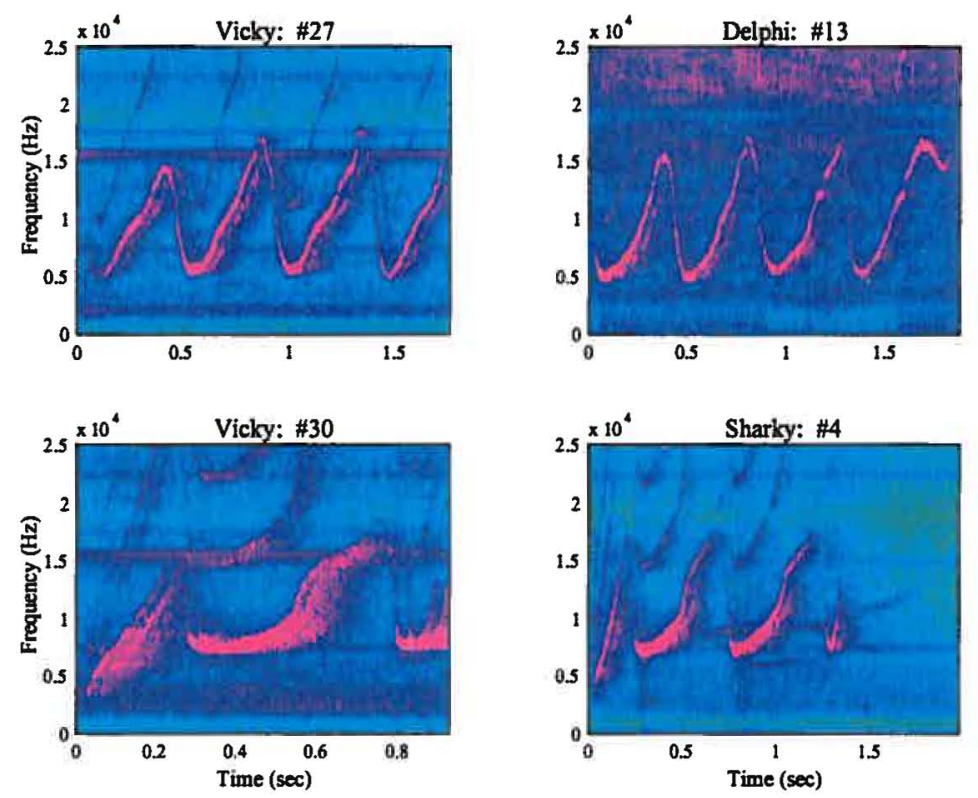

D. CONTOURS: VICKY COMPARISONS
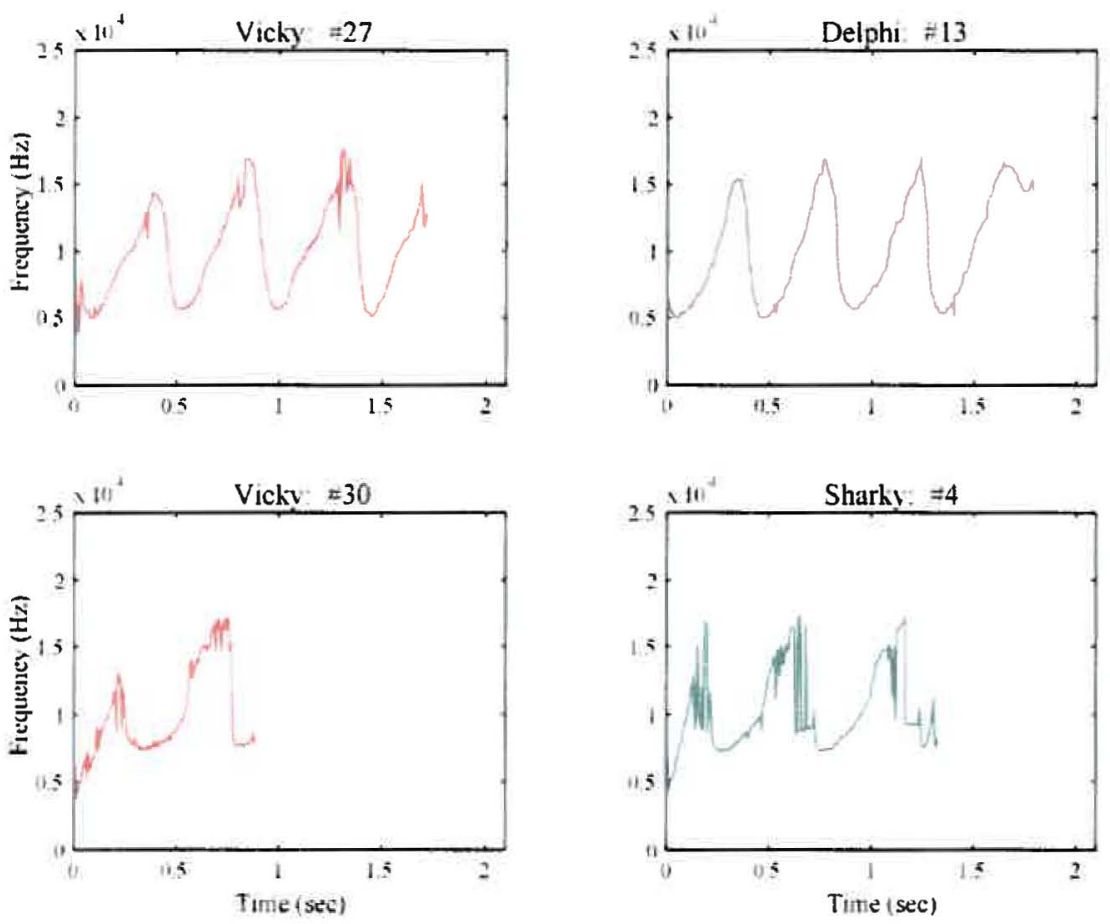
Chapter 5: Characterizing the Acoustic Environment

are shown in figure 5.12 (A-D), each of which was produced by at least two different dolphins. In addition, two multi-loop whistles from Vicky are shown (Figure 5.12C), with very different contours (Figure 5.12D). No single contour could be assigned as a signature whistle to any of the adults from their bubblestream-whistles.

Some of the clustering of the multi-loop whistles is due to the fact that some of the fine structure of the whistles is lost when contours are extracted from them (see Figure 5.12A,B). For instance, the difference between whistles \#5 and \#14, produced by Nephele and Delphi respectively, is clearer from the spectrograms (Figure 5.12A) than from the contours (Figure 5.12B). Nephele's whistle has sharper upsweeps, with a slight bump in the upsweep and with the corresponding down-sweeps almost always missing. Delphi's whistle, on the other hand, has a smoother upsweep that curves over into the down-sweep. However, because the gap between the upsweeps of Nephele's whistle is filled in by the contour, these differences become less apparent in the contours (Figure 5.12B). Similarly, whistle \#47 from Lotty appears very similar to whistle \#5 from Nephele in the spectrogram but very different in the contour (Figure 5.12 A, B). This is one of the disadvantages of working with contours. However, working with spectrograms is impractical, particularly with the low signal-to-noise ratio in these spectrograms. When spectrograms with low signal-to-noise ratios are cross-correlated, the noise is even more difficult to compensate for than when contours are crosscorrelated.

Viewed in another way, this analysis demonstrates the biases that bubblestreams introduce into a sample. Particularly, bubblestream-whistles are biased by the time they were produced. The whistles in the sections produced primarily by one dolphin were often produced very close in time. In several sections, all the whistles were produced within the same focal sample. In fact, contours \#12 to \#15 from Delphi (Figure 5.9C), all of which clustered relatively close together on the HCA (Figure 5.11), were originally extracted into a single file by the automatic extractor. This means that all four occurred within a few seconds of each other. Contour \#16 occurred a few seconds later. Lotty's contours \#39 to \#42 (Figure 5.9E), which also clustered close together (Figure 5.11), were 
also all produced within a few seconds of each other. Many of the bubblestreams in this sample were produced within a few seconds of other bubblestreams, and the whistles produced in association with those bubblestreams were more similar to each other than whistles that were more separated in time. Therefore, bubblestreams that occur close together in time may be more likely to be associated with similar whistles than bubblestreams that are separated in time. A sample that includes only bubblestreamwhistles is likely to be biased toward a few whistle types because bubblestream-whistles are so rare and often occur in groups. Similar problems occurred with bubblestreams produced by calves. Of the 152 bubblestreams recorded from Nephele's calf, 151 were made within 5 minutes of each other.

\section{Calf Bubblestreams}

One of the best-documented uses of signature whistles is in whistle exchanges between mothers and calves (e.g. Sayigh 1992, Smolker et al. 1993). In such exchanges, the whistles of the mother and calf often are very close together in time, sometimes even overlapping (Sayigh 1992). When whistles are very close together, assigning whistles to recorded bubblestreams can be difficult. The timing of bubblestreams is at best to the nearest second. If more than one whistle occurs in that second, the assignment of the bubblestream may be incorrect (see Figure 5.4). In some cases, therefore, whistles assigned to calf bubblestreams may actually have been produced by the calf's mother. In this way, it might be possible to determine the mother's signature whistle from the incorrectly assigned calf bubblestream-whistles.

All the bubblestream-whistles produced by the calves were compared and categorized by HCA (Figure 5.13). Although MDS is useful for visualizing the positions of a small number of contours, it is less useful for larger sample sizes. From this point on, therefore, only HCA will be used. The cases of this plot are coded by which calf produced the bubblestream. In this case, however, when the lines connecting cases and clusters were color-coded, up to $5 \%$ of the cases in the section were allowed to be from a different group. In this way, sections that were mostly from one group with a small 
Chapter 5: Characterizing the Acoustic Environment

FigURE 5.13: HIERARCHICAL ClUSTER ANALYSIS OF CALF BUBBLESTREAM-WHISTLES

Dotted line indicates the moat index (95 clusters). Contours are examples of contours from that region of the plot. Contours are scaled relative to each other and colored to represent which calf produced the bubblestream-whistle.

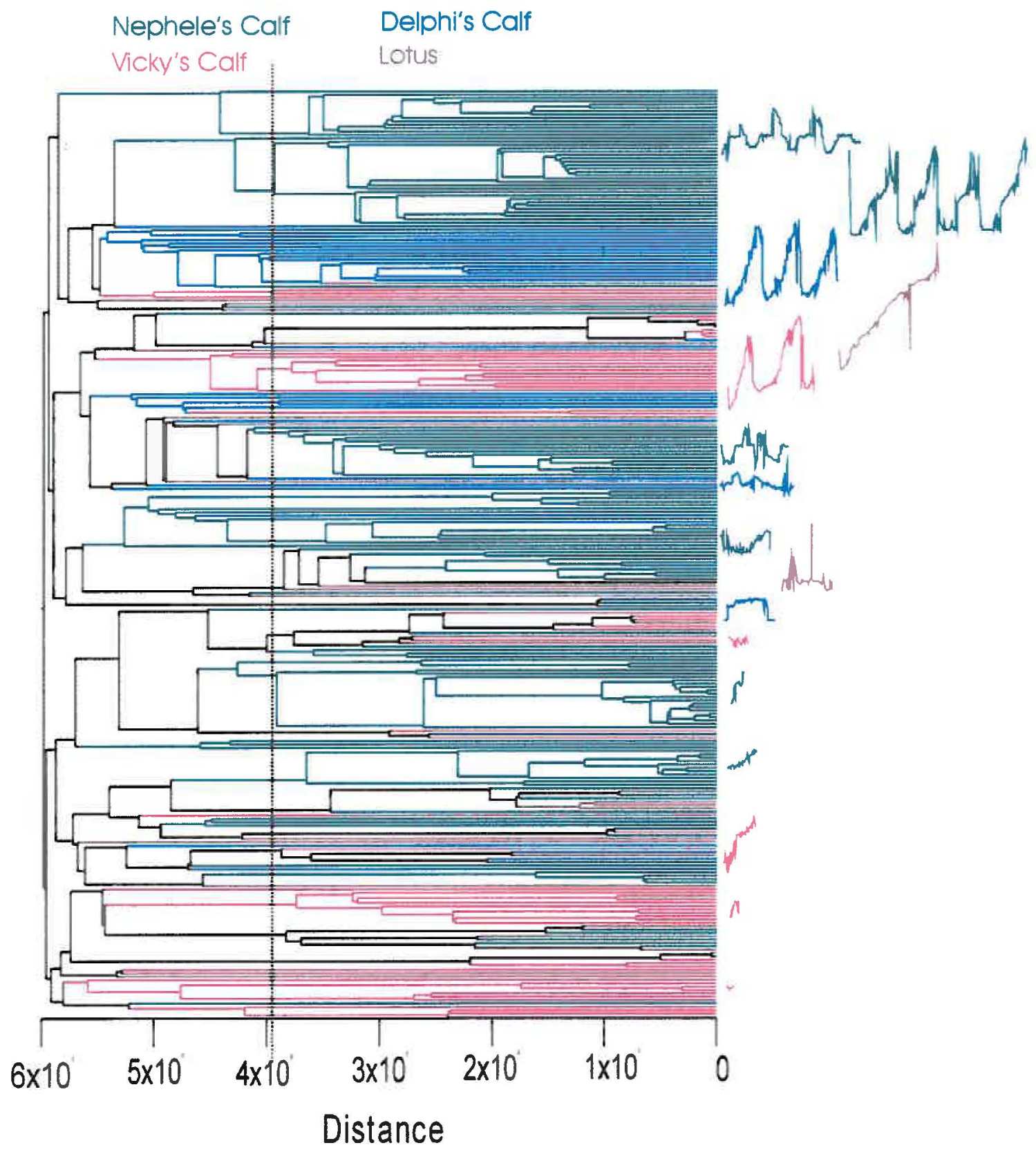


group. In this way, sections that were mostly from one group with a small number of outof-group cases were still colored to reflect the group of the majority of the cases. The moat analysis separated the whistles into 95 categories, varying in size from 1 to 25 whistles. There were 276 calf bubblestream-whistles, which is too many to plot every contour, so only example contours are plotted on the HCA. As before, the contours are colored to reflect which calf produced the bubblestream and all drawn to the same scale. The example contours are positioned near the line representing that contour in the HCA. The contours on this plot appear to have separated in the opposite direction from the contours from Daphne's bubblestream-whistles (Figure 5.7), with the longer contours at the top. The direction in which this is plotted is entirely random, however. The same tree could as easily have been plotted in the other direction, with the long contours at the bottom.

Calf whistles have generally been described as short, quavery, and lacking frequency modulation (e.g. Caldwell et al. 1990). The whistles Lotus produced when he was alone (Lotus Alone), which are all known to be made by a 5-day old calf (Lotus), are quavery and lack frequency modulation (Figure 5.14A). To determine whether calf whistles could be quantitatively separated from adult whistles, the whistles produced by Lotus in Lotus Alone were compared to the adult bubblestreams, which are believed to be all produced by adults. They were also compared to the whistles from the period Before Vicky's Calf, when there were no calves in the group. Two measures were compared, contour duration and quartile bandwidth. The quartile bandwidth is defined as the bandwidth of the contour after removing the lowest $25 \%$ of the frequencies and the highest 25\% (see Appendix 2). The quartile bandwidth of the Lotus Alone contours was significantly lower than the adult contours (ANOVA, $\mathrm{p}<0.001$ ). The mean quartile bandwidth of the contours from Lotus Alone was $1.2 \mathrm{kHz}$, compared to $2.9 \mathrm{kHz}$ for the adult bubblestreams and $2.6 \mathrm{kHz}$ for the Before Vicky's Calf contours. In fact, fewer than $5 \%$ of the contours from Lotus Alone had quartile bandwidth of more than $3 \mathrm{kHz}$, compared to $42 \%$ of the adult bubblestream contours and $35 \%$ of the Before Vicky's 
Chapter 5: Characterizing the Acoustic Environment

FIGURE 5.14: EXAMPLES OF CONTOURS

Contours are scaled the same in both A \& B.

A. KNown Calf Contours: Examples From lotus Alone

These contours were randomly selected from the 245 contours from this period.
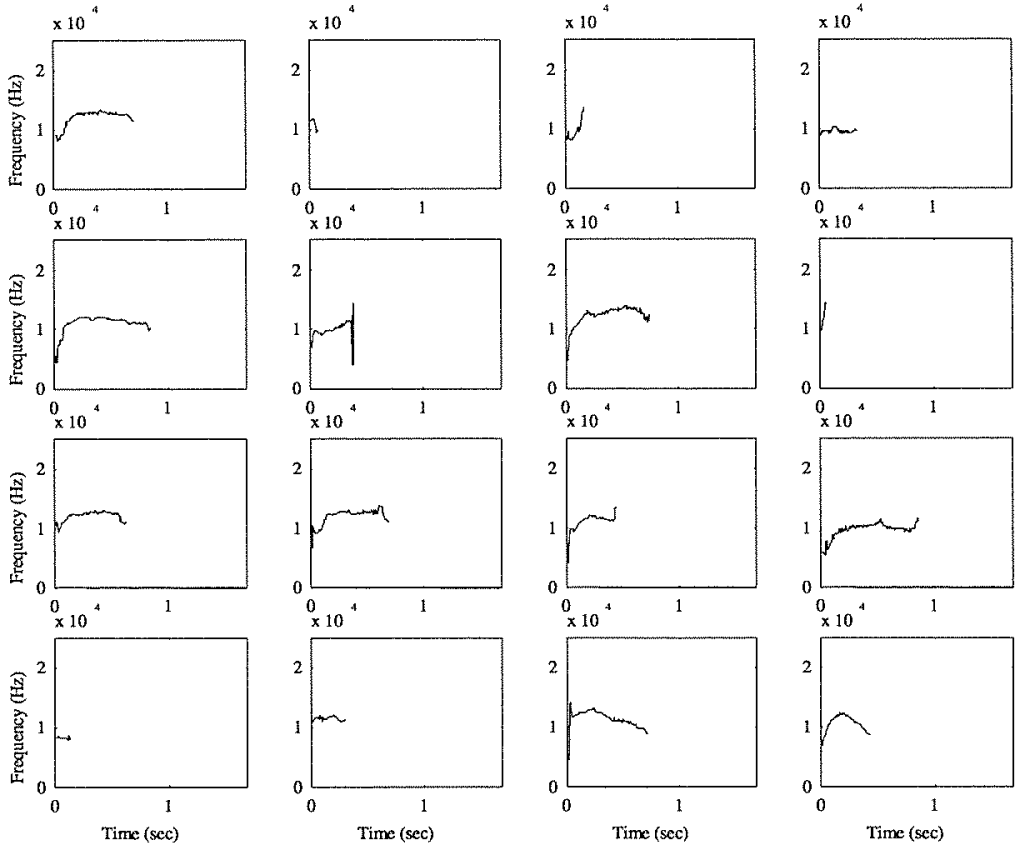

B. Examples of CAlF BubBlestream-Whistle Contours

PROBABLE CALF WHISTLES
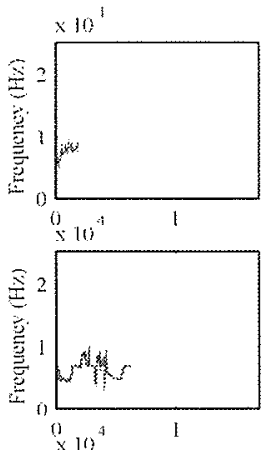

Delphi's calf

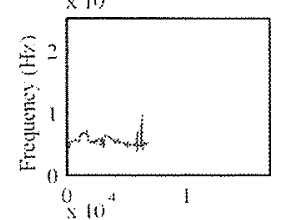

Vicky"s calf

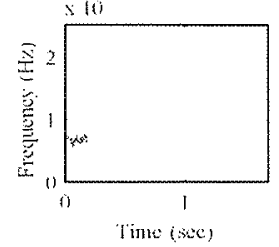

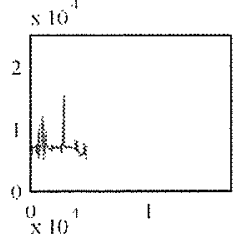
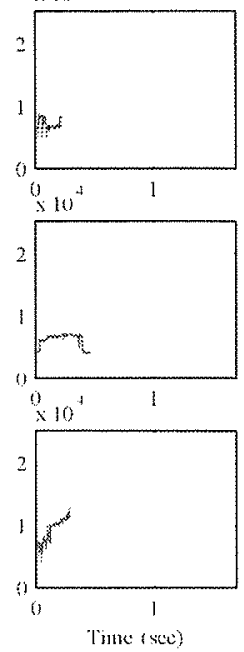

PROBABLE ADULT WHISTLES
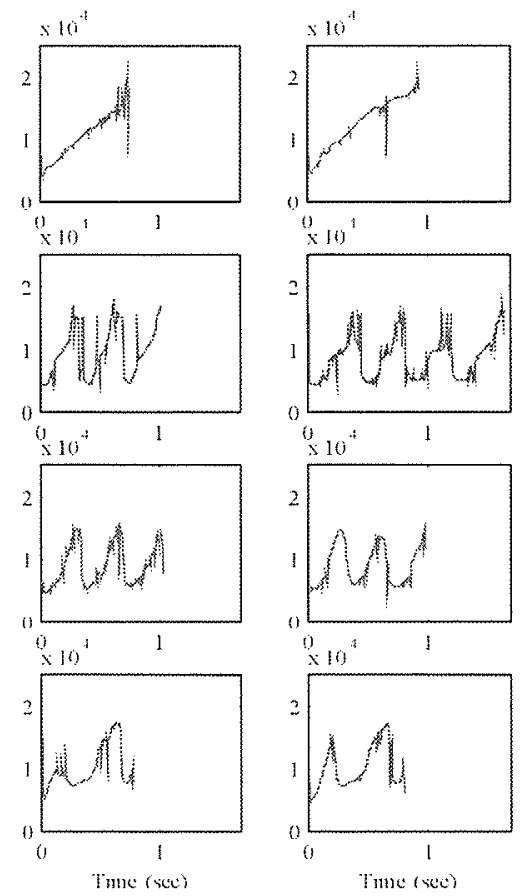
Figure 5.15: Putative Signature Whistles From Mothers of Focal Calves
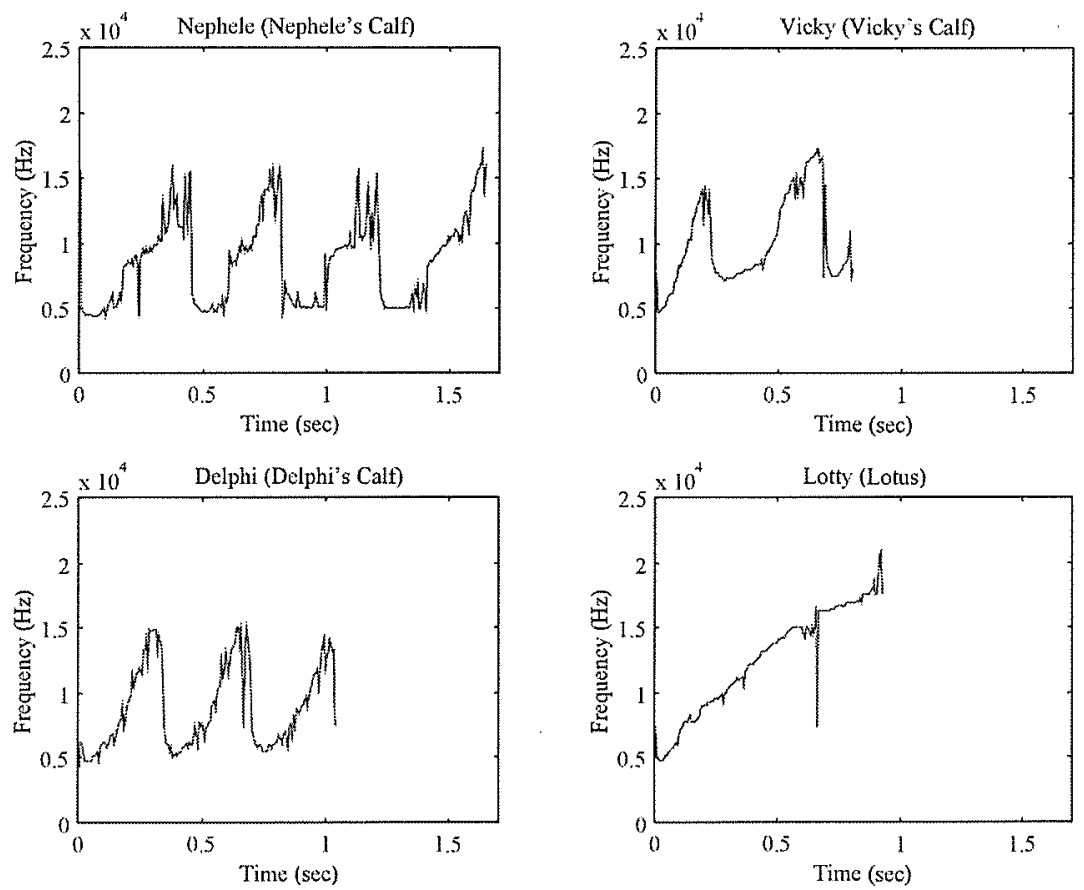

duration of be Before Vicky's Calf contours was actually less than the mean duration found Lotus Alone contours (Lotus Alone: 507 ms; Adult Bubblestreams: 797 ms, Before Vicky's Calf: $426 \mathrm{~ms}$ ). None of the Lotus Alone contours were longer than one second but only $6-7 \%$ of the adult contours were that long. Calf whistles could therefore be separated from adult whistles by their bandwidth but not by their duration.

Some of the contours plotted with the HCA appear to be whistles with low quartile bandwidths (Figure 5.13, 5.14B). However, some of the contours plotted with the HCA are long, and clearly looped, with extensive frequency modulation and high quartile bandwidths (Figure 5.13, 5.14B). These contours are hypothesized to be produced by adults, not calves (Figure 5.14B). In the bubblestream-whistles of each calf, there was one predominant high-bandwidth contour that did not show up in the bubblestream-whistles of the other calves (Figure 5.14B). Because signature whistles are commonly used in whistle exchanges between mothers and their calves (e.g. Sayigh 1992), these can be hypothesized to be the signature whistles of the calves mothers 
Chapter 5: Characterizing the Acoustic Environment

Figure 5.16: COMPARISON OF BUBbleSTREAM-WHISTLES WITH PUTATIVE SigNATURE WHISTLES
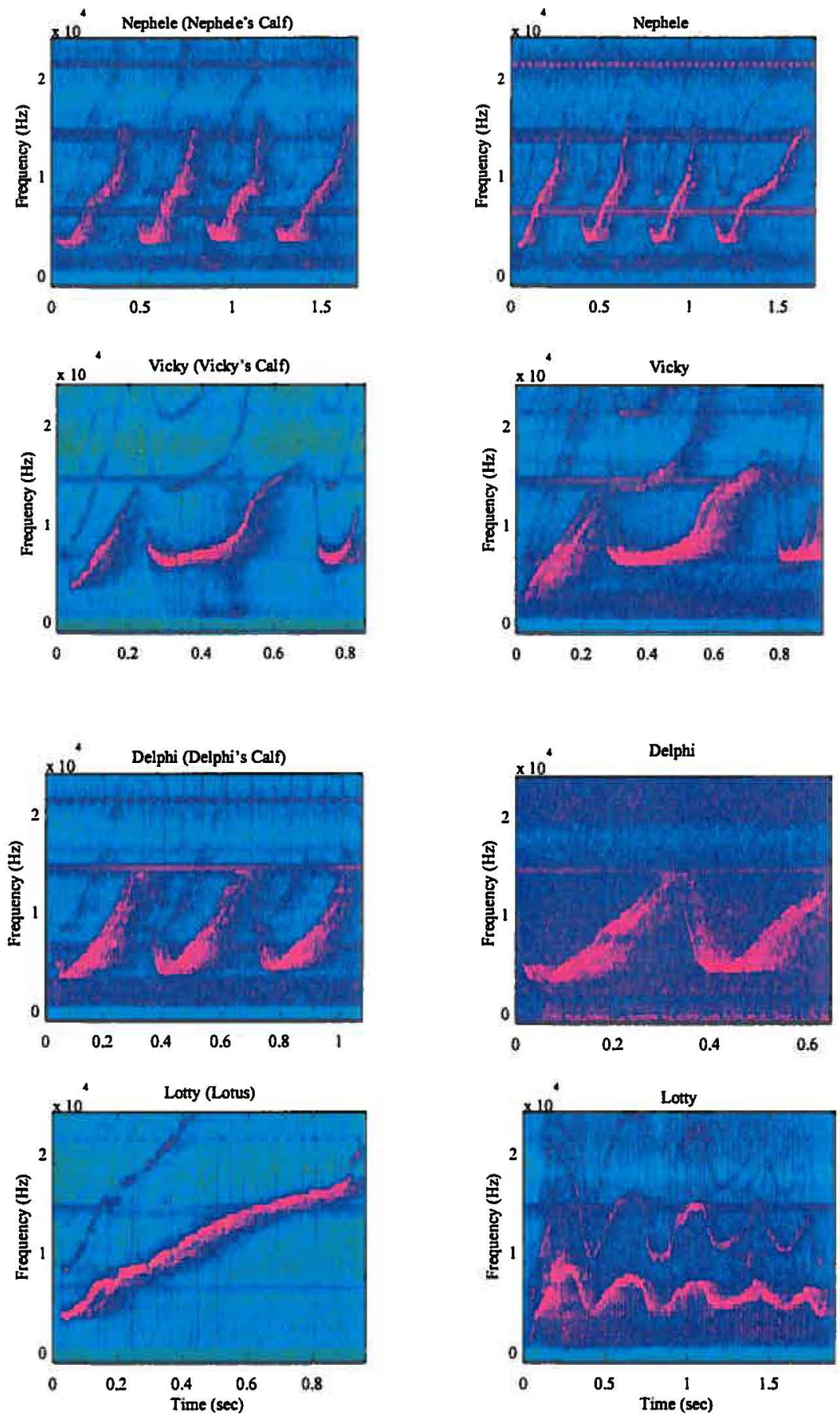
commonly used in whistle exchanges between mothers and their calves (e.g. Sayigh 1992), these can be hypothesized to be the signature whistles of the calves mothers (Figure 5.15). Interestingly, Lotty's signature whistle was only seen among Lotus' bubblestream-whistles on the two days in this sample that Lotty was caring for Lotus: the day Lotus was born and June 10 , Lotus' $6^{\text {th }}$ day. Vicky cared for Lotus in the intervening days (see chapter 3), but no high-bandwidth contours, or contours matching Vicky's putative signature whistle, were seen among Lotus' bubblestream-whistles during that period.

A re-examination of the adult bubblestream-whistles in light of the putative signature whistles is worthwhile. Some of the bubblestream-whistles did match the signature whistles assigned to those adults (Figure 5.16). In particular, several bubblestream-whistles from Delphi, Vicky, and Nephele match their putative signature whistles. This includes a contour produced by Delphi several times (Figure 5.16, 5.9C). However, none of the bubblestream-whistles from Lotty matches her putative signature whistle (Figure 5.16, 5.9E). In fact, Lotty produced one contour several times among her bubblestream-whistles but it was a different contour from her putative signature whistle (Figure 5.16, 5.9E). In addition, one of the bubblestream-whistles from Sharky matches the contour putatively assigned to Vicky (Figure 5.12C/D). This could mean that Sharky's signature whistle is very similar to Vicky's, as Delphi's is to Nephele's (Figure $5.16,5.12 \mathrm{~A} / \mathrm{B})$. Alternatively, Sharky's whistle could be an imitation of Vicky's signature whistle. Since bubblestreams have not clearly been shown to be associated with all the whistles in an animal's repertoire, bubblestreams could be preferentially used in conjunction with such imitation events. A final possibility is that, as with the calf bubblestreams, some of the adult bubblestreams were incorrectly assigned because the animals were involved in a closely timed exchange of whistles. 
Chapter 5: Characterizing the Acoustic Environment

\subsubsection{Changes in Whistle Use}

Vicky's Calf

\section{Contour Analysis}

The results presented in section 5.3.1 demonstrated that some aspects of the dolphins' whistle use changed when Vicky's calf was born. In particular, the dolphins produced more, longer whistles after Vicky's calf was born than before. To further quantify the changes in the whistles produced around the birth of Vicky's calf, the whistles from the periods Before Vicky's calf and With Vicky's calf were compared using HCA (Figure 5.17). This plot is merely a denser version of the previous cluster diagrams and can be read in the same way. The color of each line represents the period that case came from. Colored connecting lines indicate that at least $95 \%$ of the cases within that section are from that period. As with the calves' bubblestream-whistles, the contours on the HCA plot are examples of contours from that region and all drawn to the same scale. The contours are colored to represent the period the whistle came from.

This plot shows that the types of whistles used changed when Vicky's calf was born (Figure 5.17). While there is some overlap between the periods, many of the whistles are grouped with other whistles from the same period. In a plot like this one, the overall structure can be seen by looking for blocks of a single color, particularly blocks that are connected by colored lines farther to the left of the plot. These sections represent groups of whistles from one time period that all clustered together. The moat index separated the plot into two clusters. This had the effect of simply separating off one particularly short whistle (labeled "section 1" on Figure 5.17). This whistle was produced Before Vicky's calf and was only $26 \mathrm{~ms}$ long. However, the second cluster immediately separated into two sections of approximately equal size (labeled "section $2 a$ " and "section $2 b$ " on Figure 5.17). As is expected from the DTW, these two sections separated whistles by duration. Section 2 a consisted of 215 short whistles, averaging 188 ms. Section $2 \mathrm{~b}$ consisted of 284 much longer whistles, averaging $851 \mathrm{~ms}$. Interestingly, $71 \%$ of the whistles in section 2a, with shorter whistles, were from Before Vicky's calf. Section $2 b$, on the other hand, was $66 \%$ whistles from With Vicky's calf. This result is 


\section{FIGURE 5.17: HCA OF THE WEEKS BEFORE AND AFTER VICKY'S CALF WAS BORN}

Vertical dotted line indicates the moat index (2 clusters). Contours are examples of contours from that region of the plot. Contours are scaled relative to each other and colored to represent the appropriate group. The larger cluster is divided into 2 subsections at the next split in the tree.

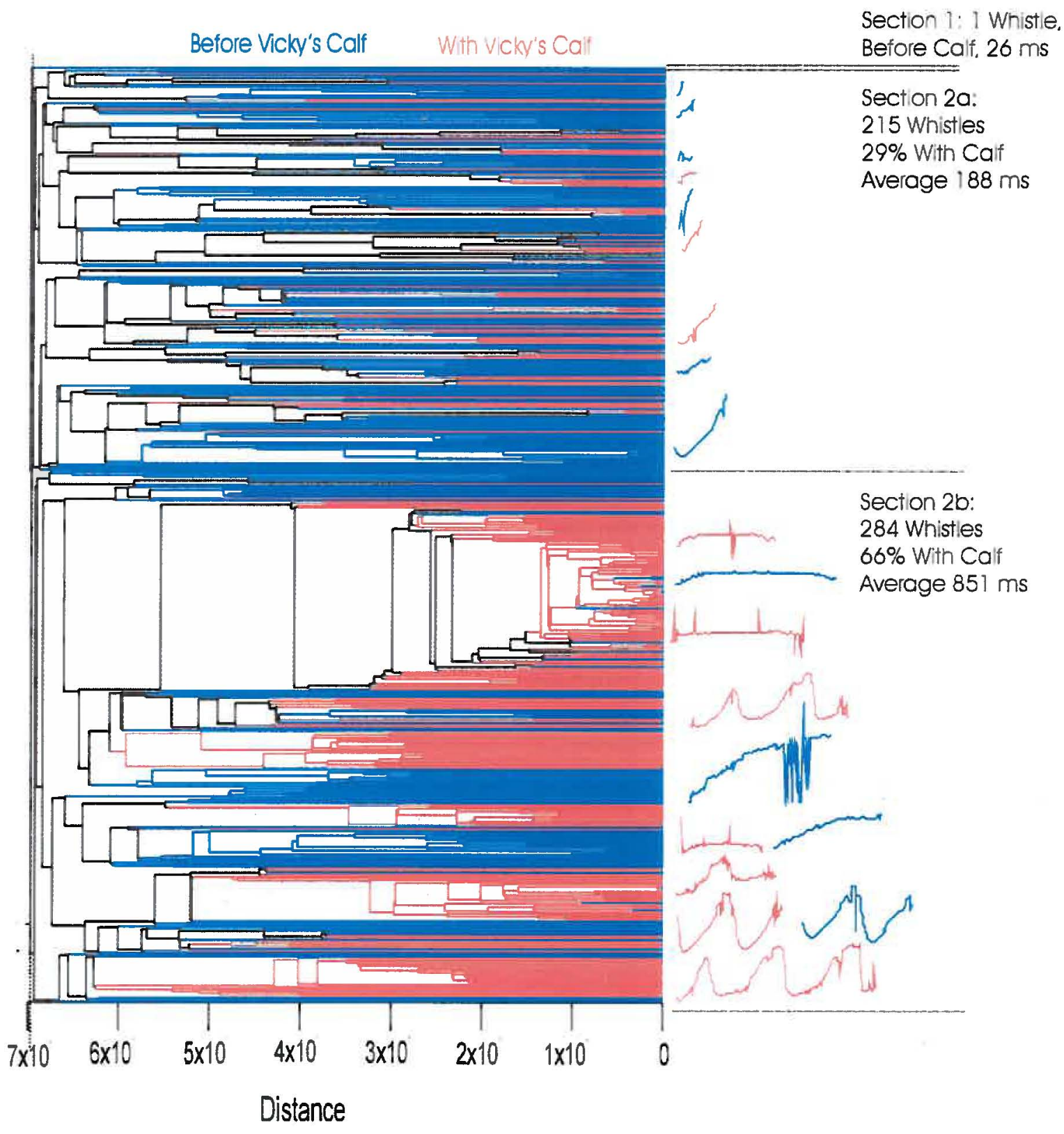


Chapter 5: Characterizing the Acoustic Environment

consistent with the results showing that there was a significant increase in the average duration of whistles when Vicky's calf was born.

This plot suggests that the whistles in each period were more similar to the whistles from the same period than to the whistles from the other period. To evaluate this in another way, the similarity between pairs of whistles was compared based on which period each whistle in the pair came from. The results of DTW depend on which contour is warped and which is held fixed (so the DTW of contour A to contour B is different from contour B to contour A). Therefore, to prevent double counting, the two DTWs for each pair of contours were averaged before the similarities were compared. The pairs were then grouped into three categories: both contours from Before Vicky's Calf, both contours from With Vicky's Calf, and contours from different periods. The contours from With Vicky's Calf were significantly more similar to each other than to the contours from Before Vicky's Calf (ANOVA, Tukey post-hoc test, $\mathrm{p}<0.001$ ).

However, the reverse was not true: the contours from Before Vicky's Calf were no more similar to each other than to the contours from With Vicky's Calf (Tukey test, $p=0.4$ ). This suggests that the change in whistle use seen in the HCA plot is caused by specific whistle types produced after Vicky's calf was born that were not produced before Vicky's calf was born.

\section{$\underline{\text { Limitations of Contour Analysis }}$}

A closer examination of the HCA plot may elucidate some of the changes in the whistle types produced (Figure 5.17). The majority of the whistles in section 2a were from Before Vicky's Calf, which is expected because the whistles Before Vicky's Calf were significantly shorter than the whistles With Vicky's Calf. Within section 2a, only one group of whistles clustered strongly by period: the group of whistles from Before

Vicky's Calf at the bottom of the section () . The other types of short whistles were mostly found in both periods. The use of short whistles therefore did not change substantially between the two periods. However, the use of long whistles did change, as can be seen by a similar examination of section $2 \mathrm{~b}$. Most of the whistles found in section $2 \mathrm{~b}$ are grouped with other whistles from the same period (Figure 5.17). However, it may 
not be possible to determine precisely what types of whistles were produced in each period from the HCA. As was discussed previously, separations based on duration are built into the DTW. Included in this is the separation of whistles with similar contours but different numbers of loops. An example of this is the two contours in figure 5.17 that resemble Vicky's signature whistle, one with 2 loops ( $)$ and one with 3 loops $(4)$, which are separated by the HCA (Figure 5.17). In the past, whistles with similar contours but different numbers of loops have often been considered to be variations of the same whistle (e.g. Caldwell et al. 1990). In addition, the moat index (2) does not aid in the separation of whistles into types. Many contours that are clearly very different are clustered together by the moat index (Figure 5.17). Cluster analysis of contours therefore appears to be more useful for exploring changes in the overall acoustic environment than for separating whistles into categories. The specific types of whistles used need to be determined in another way.

The signature whistles identified for the mothers of the focal calves provide a tool for such fine structure analysis. Although not all the specific types of whistles used can be evaluated by looking for signature whistles, changes in signature whistle use may explain some of the changes in overall whistle use. For the purpose of this pilot study, signature whistle use was evaluated in the traditional manner, by visually classifying spectrograms. The spectrograms for the whistles in the random sub-samples of all the periods, except Lotus Alone, were randomly mixed and then compared to the identified signature whistles (Figure 5.15, 5.8). Each whistle with classified as a non-signature whistle or as the signature whistle identified to Vicky, Lotty, Delphi, Nephele, or Daphne. Because some of the dolphins' signature whistles were not known, such as Sharky's, the overall estimate of signature whistle use will be low. However, a good estimate of signature whistle use by the mothers of the focal calves should be achieved. It is important to note here that this type of analysis does not differentiate between signature whistle use by the mother and imitation of the mother's signature whistle by other animals. To determine that, a method of identifying which animal is whistling is needed (see chapter 1). 
Chapter 5: Characterizing the Acoustic Environment

Signature Whistle Use

There was only a slight increase in the proportion of whistles that could be identified as signature whistles from Before Vicky's Calf to With Vicky's Calf (Before Vicky's Calf: 18\%, With Vicky's Calf: 21\%; see Table 5.7A). However, when signature whistle use was examined by individual, there were two noticeable changes when Vicky's calf was born (see Table 5.7B). In the period Before Vicky's Calf, 11\% of the whistles recorded matched the contour of Lotty's signature whistle and none matched the contour of Vicky's signature whistle. In contrast, in the period With Vicky's Calf, 13\% of the whistles were Vicky's signature whistle and none were Lotty's. One of the changes in whistle use that occurred when Vicky's calf was born was therefore an increase in the occurrence of Vicky's signature whistle and a decrease in Lotty's.

Nephele's Calf

To determine whether a change in whistle use is common to all calf births, the periods Before Nephele's calf and With Nephele's calf were compared in the same way that the periods around the birth of Vicky's calf were compared. Three types of analyses were performed: HCA, a comparison of the mean similarities, and an analysis of signature whistle use. The HCA shows a similar change in whistle use to the one seen when Vicky's calf was born (Figure 5.18). An analysis of the pairwise similarities equivalent to the one done for Vicky's calf found that the contours from both Before Nephele's calf and With Nephele's calf were more similar to the contours from the same period than to contours from the other period (ANOVA, Tukey's test $\mathrm{p}<0.001$ ). However, on average the contours were less similar to each other than the contours for the weeks surrounding Vicky's calf's birth. This is reflected in the moat index of the HCA, which separated the 500 contours into 461 clusters. In the same way that the moat index for Vicky's calf was not useful for separating the whistles into categories, the moat index for this plot was not useful for grouping whistles into categories. The moat index appears to be a better measure for evaluating the overall similarity of the contours than for determining how to categorize the whistles. 
FIGURE 5.18: HCA OF THE WEEKS BEFORE AND AFTER NEPHELE'S CALF WAS BORN Dotted line indicates the moat index (461 clusters). Contours are examples of contours from that region of the plot. Contours are scaled relative to each other and colored to represent the appropriate group.

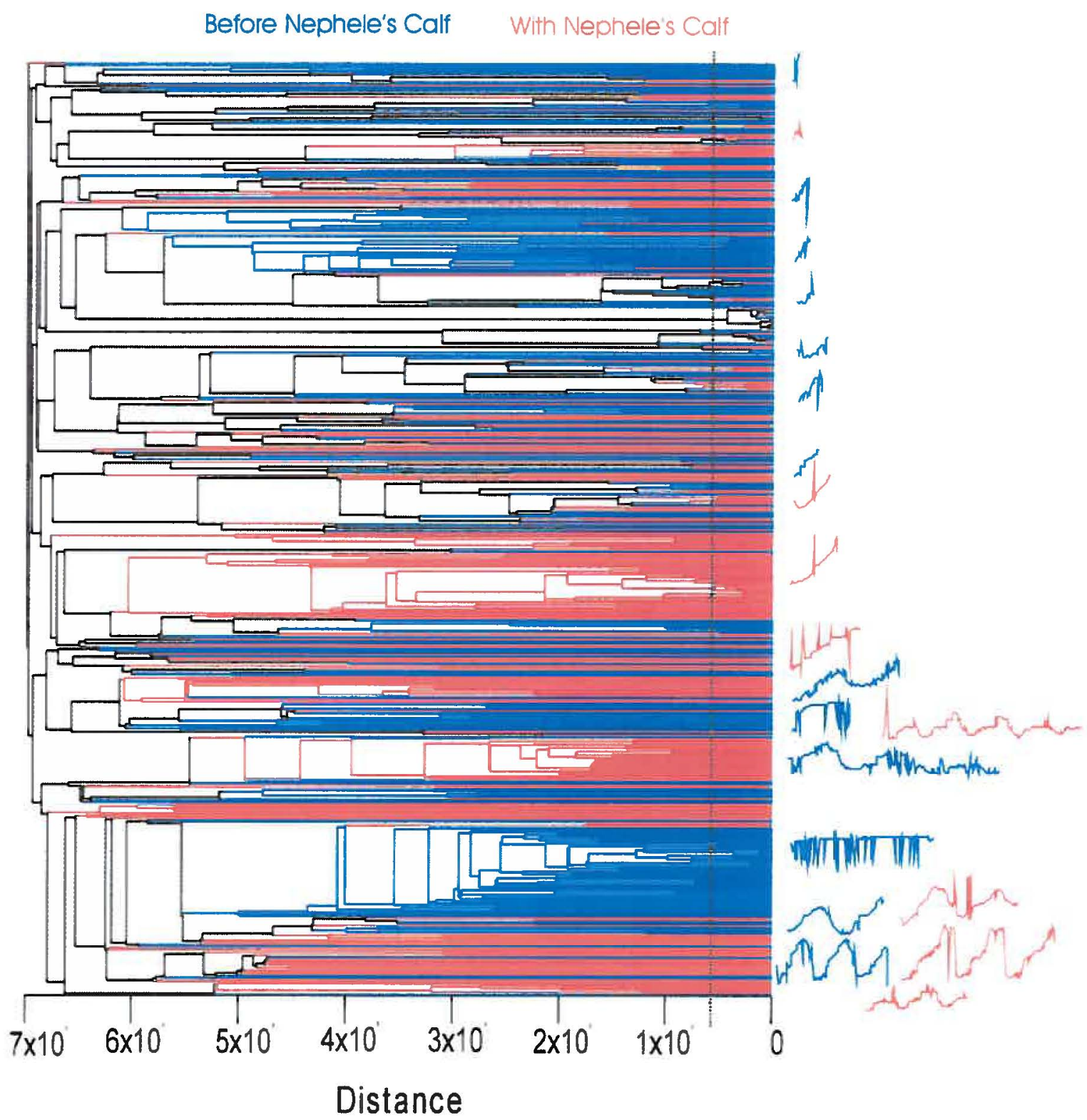


Chapter 5: Characterizing the Acoustic Environment

An examination of the overall structure of the cluster tree shows that the difference in whistle use between the periods was mostly in the longer whistles, as it was for Vicky's calf. The short whistles did not clearly separate by period, with one exception (Figure 5.18). Most of the short whistles from both periods were straight upsweeps $(f)$, but one cluster of contained longer, more curved upsweeps $(J)$. These whistles were almost entirely from With Nephele's calf. These whistles could be fragments of Nephele's signature whistle, similar to the fragments seen of Daphne's signature whistle (see Figures $5.7 \& 5.8$ ). An analysis of signature whistle use in these two periods shows that there was a marked increase in signature whistle use when Nephele's calf was born (from $13 \%$ to $40 \%$, see Table 5.7A). This increase was mostly due to increases in the proportion of Nephele's and Daphne's signature whistles after Nephele's calf was born (see Table 5.7B). The increase in Daphne's signature whistles may be explained by the fact that this was the first time 7-month old Daphne had seen a calf born. The increase in Nephele's signature whistle use, in conjunction with the increase in Vicky's signature whistle use in her calf's first week, suggests that pattern of mothers increasing their signature whistle use when their calves are born.

A substantial change in the acoustic environment occurred both when Nephele's calf was born and when Vicky's calf was born. To determine whether these changes resulted in similar acoustic environments for both calves, the first weeks of the two calves were compared. The HCA clearly shows that the acoustic environment is not the same even for calves born within a month of each other in the same pool (Figure 5.19). The whistles in this comparison separated by period more clearly than in either of the previous two comparisons. Strangely enough, although the whistles from With Vicky's Calf were more similar to each other than to the whistles from With Nephele's calf, the whistles from With Nephele's calf were actually more similar to the whistles from With Vicky's Calf than to each other (ANOVA, Tukey tests, $\mathrm{p}<0.001$ ). This is also reflected in the moat index (203 clusters), which indicates an intermediate level of similarity (Figure 5.19). An examination of the overall structure shows changes in both short and long whistles between the two periods. Several clusters of short whistles produced With 
FIGURE 5.19: HCA OF THE FIRST WEEKS OF NEPHELE'S CALF AND VICKY'S CALF

Dotted line indicates the moat index (203 clusters). Contours are examples of contours from that region of the plot. Contours are scaled relative to each other and colored to represent the appropriate group.

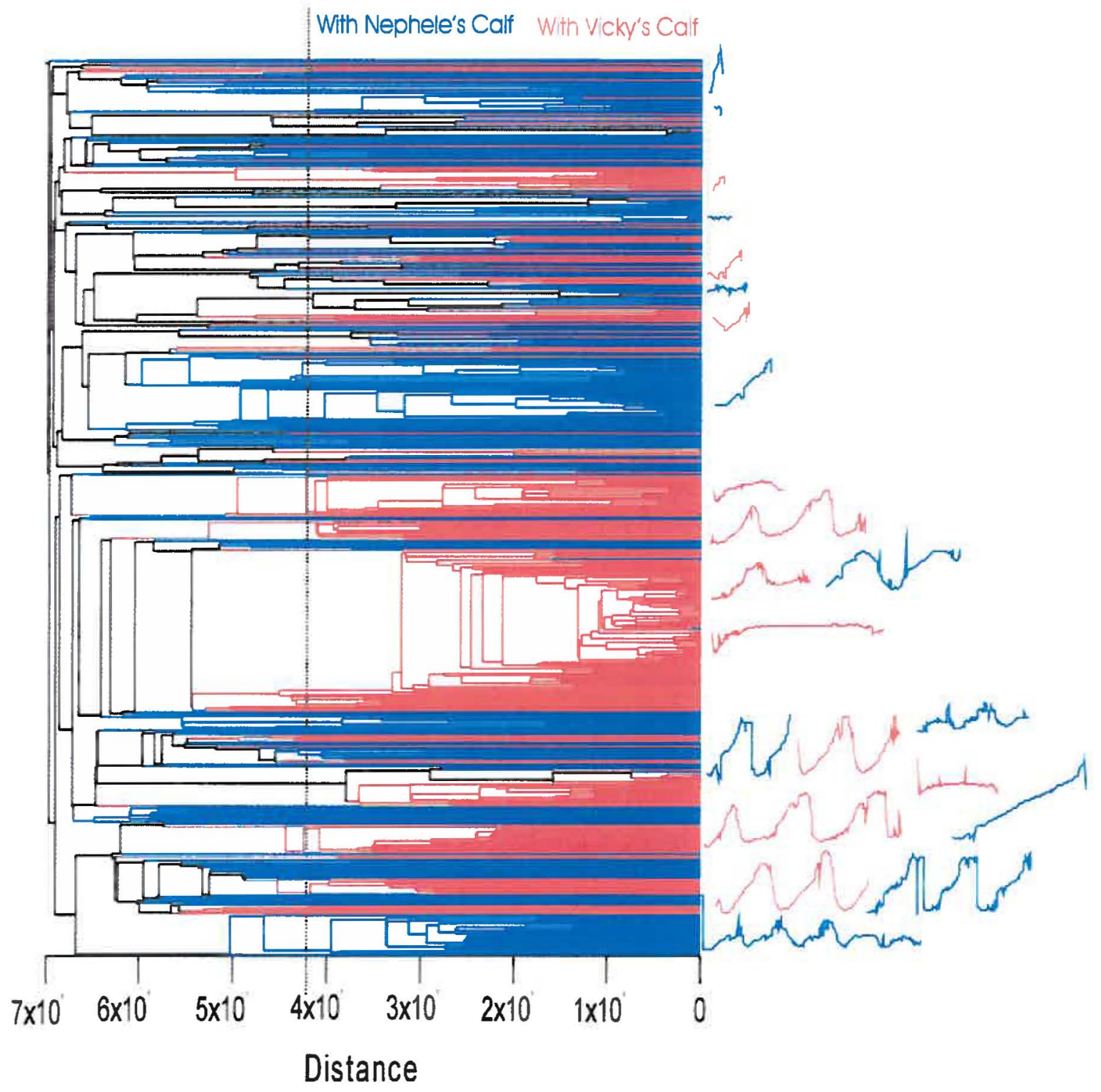


Chapter 5: Characterizing the Acoustic Environment

Nephele's calf separated from the With Vicky's Calf short whistles. These were mostly flat whistles ( $(-m)$ with the exception of one large cluster of curved upsweeps ( These are the same whistles discussed previously as potentially fragments of Nephele's signature whistle. The long whistles used changed more substantially between the periods, including the signature whistles. Almost $20 \%$ of the whistles produced in the period With Nephele's calf were Nephele's signature whistle (Table 5.7B). Only 1\% was Vicky's signature whistle. With Vicky's Calf, 13\% of the whistles were Vicky's signature whistle while only $1 \%$ was Nephele's. The difference in signature whistle use by the mothers in these sections suggests a particular change in signature whistle use by mothers when they give birth. Interestingly, 6\% of the whistles produced With Vicky's Calf were Delphi's signature whistle, compared to only $2 \%$ With Nephele's calf. Delphi's calf was born at the end of the week With Vicky's Calf. This raises the possibility that some mothers actually increase their signature whistle use in the week before they give birth.

\section{Prior to Calf Births}

To test the hypothesis that whistle use changes in the week prior to the birth of a calf, comparisons were done of the periods before the calves were born. First, the week before Nephele's calf was born was compared to a week in March, more than a month before any of the calves were born. Once again, the whistles from the two periods separated to some extent, although they did not separate as clearly as the whistles from before and after the births (Figure 5.20). As with most of the previous comparisons, these data divided into long and short whistles. There was a shift in the use of short whistles, with more short upsweeps Before Nephele's calf than in March. Although most of the short whistles are grouped with whistles from both periods, several sections of short whistles are clustered only with whistles from the same period. Similar clustering can be seen with the longer whistles, although there is also a great deal of overlap in the long whistles used in the two periods. When the mean similarities of the contours are compared, the whistles from March are found to be more similar to each other than to the whistles from Before Nephele's calf (ANOVA, Tukey test $\mathrm{p}<0.001$ ). 
FIGURE 5.20: HCA OF A WEEK IN MARCH VS. THE WEEK BEFORE NEPHELE'S CALF Dotted line indicates the moat index (468 clusters). Contours are examples of contours from that region of the plot. Contours are scaled relative to each other and colored to represent the appropriate group.

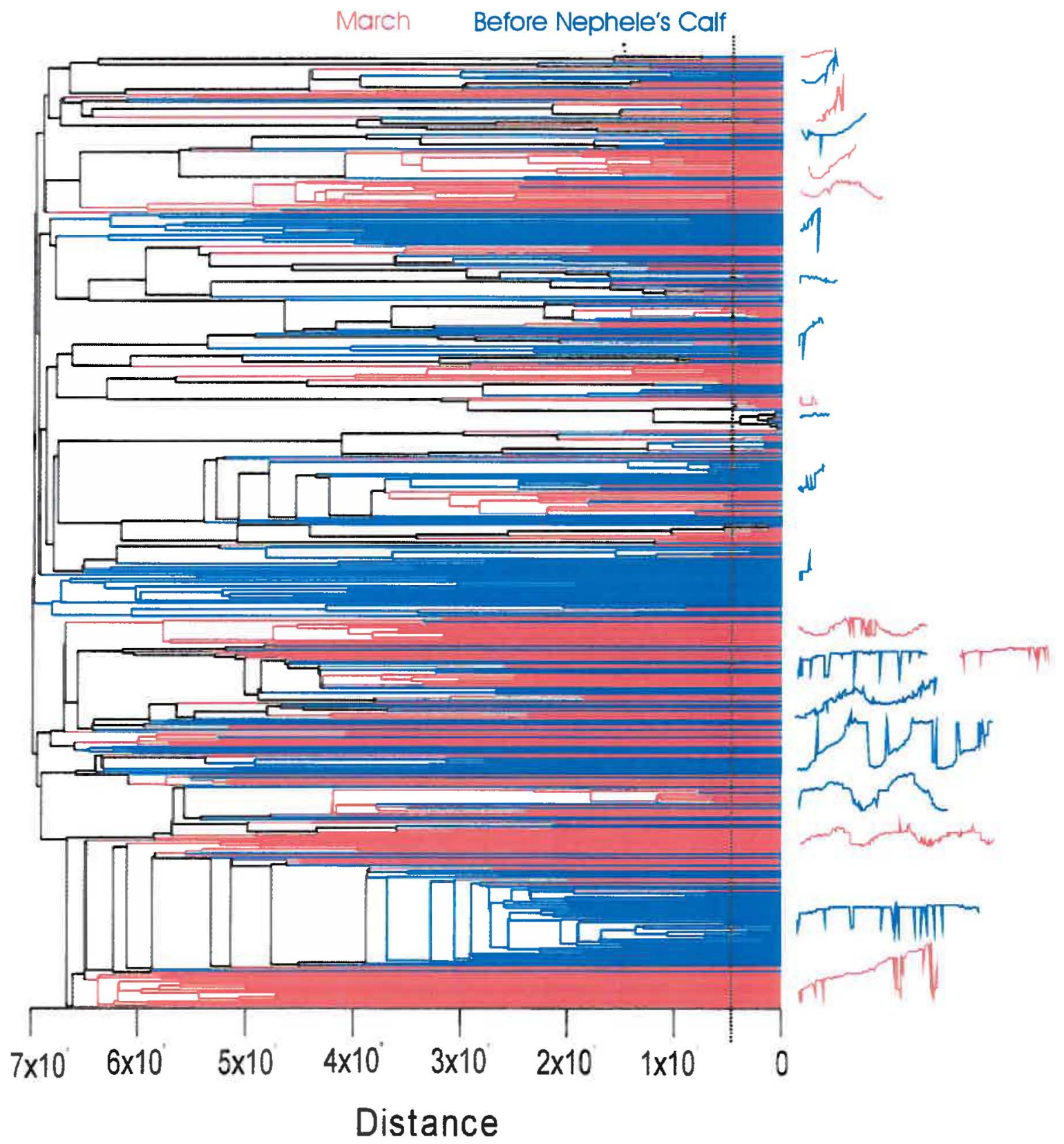


Chapter 5: Characterizing the Acoustic Environment

However, the whistles from Before Nephele's calf are no more similar to each other than to the whistles from March (ANOVA, Tukey test $\mathrm{p}=0.8$ ). This is partly the result of a larger use of signature whistles in March (31\%) compared to Before Nephele's calf (13\%, see Table 5.7A). Because signature whistles are generally stereotyped, the contours from each signature whistle can be expected to be more similar to each other than the contours of non-signature whistles are to each other. The average similarity of a section of signature whistles will therefore be more similar than the average similarity of a section of non-signature whistles. Interestingly, all the adults except Delphi decreased their signature whistle use Before Nephele's calf compared to March (Table 5.7B). Nephele did not increase her signature whistle use in the week before her calf was born.

To determine whether the acoustic environment was similar in the week before the two calves were born, the periods Before Nephele's calf and Before Vicky's Calf were compared. Again, the HCA shows some changes in the two periods but the shift is not as clear as the shift after the calves were born (Figure 5.21). In this case, the contours for each period were significantly more similar to the contours from the same period than to the contours from the other period (ANOVA, Tukey tests $p<0.001$ ). However, the difference between the mean similarity of cases from a single period and the mean similarity of cases from different periods was small. In fact, the short whistles from the two periods were not very different. Little clustering by time period is seen among the short whistles in this plot, except for one cluster primarily of curved upsweeps $(\gamma)$ from Before Vicky's Calf, possibly fragments of Vicky's or Delphi's signature whistle. More separation can be seen among the longer whistles (Figure 5.21). In both periods, less than $20 \%$ of the whistles were signature whistles (Table 5.7A). The biggest difference is any increase in the proportion of whistles that matched Lotty's signature whistle, from $1 \%$ Before Nephele's calf to 11\% Before Vicky's Calf (Table 5.7B). However, none of the whistles Before Vicky's Calf were Vicky's signature whistle, which is actually a decrease from 3\% Before Nephele's calf. No change in the proportion of Nephele's signature whistle was seen between the two periods. These data, therefore, do not 
FIGURE 5.21: HCA OF THE WEEKS BEFORE NEPHELE's CALF VS. BEFORE VICKY'S CALF Dotted line indicates the moat index (292 clusters). Contours are examples of contours from that region of the plot. Contours are scaled relative to each other and colored to represent the appropriate group.

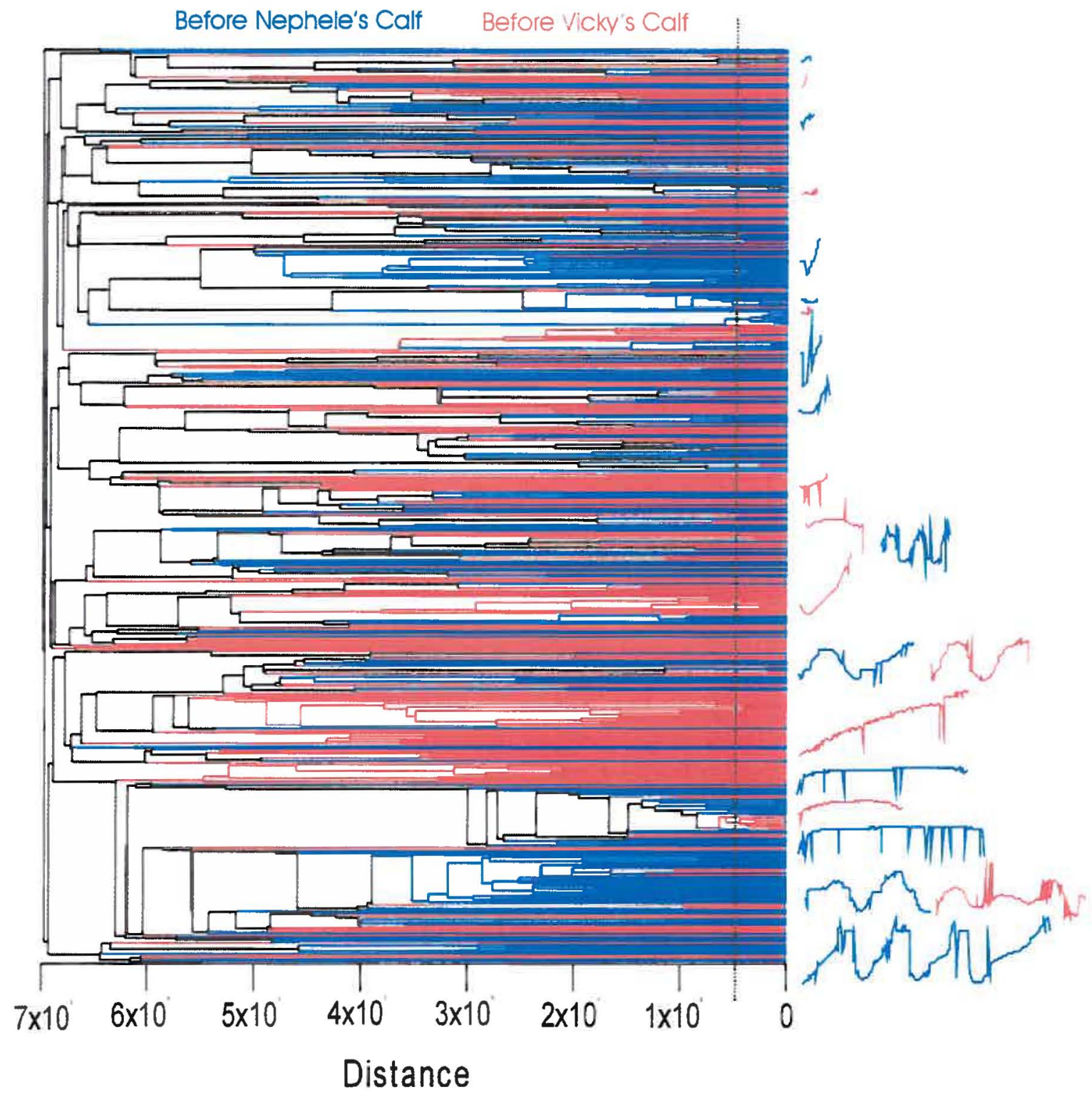


Chapter 5: Characterizing the Acoustic Environment

Table 5.7 Signature Whistle Use by Time Period

Signature whistles as a percent of the whistles produced in each time period.

\section{A. Total Signature Whistle USE}

\begin{tabular}{|c|c|c|}
\hline Time Period & Signature Whistles & Non-Signature Whistles \\
\hline March & $31 \%$ & $69 \%$ \\
\hline Before Nephele's calf & $13 \%$ & $87 \%$ \\
\hline With Nephele's calf & $40 \%$ & $60 \%$ \\
\hline Before Vicky's calf & $18 \%$ & $82 \%$ \\
\hline With Vicky's calf & $21 \%$ & $79 \%$ \\
\hline With Delphi's calf & $21 \%$ & $79 \%$ \\
\hline With Delphi's calf/Lotus & $20 \%$ & $80 \%$ \\
\hline With Lotus & $26 \%$ & $74 \%$ \\
\hline Average & $24 \%$ & $76 \%$ \\
\hline
\end{tabular}

\section{B. Signature Whistle USE By INDividual}

\begin{tabular}{|c|c|c|c|c|c|c|}
\hline Time Period & Nephele & Vicky & Delphi & Lotty & Daphne & Average \\
\hline March & $6 \%$ & $8 \%$ & $0 \%$ & $8 \%$ & $10 \%$ & $6 \%$ \\
\hline Before Nephele's calf & $4 \%$ & $3 \%$ & $2 \%$ & $1 \%$ & $4 \%$ & $3 \%$ \\
\hline With Nephele's calf & $19 \%$ & $1 \%$ & $2 \%$ & $4 \%$ & $14 \%$ & $8 \%$ \\
\hline Before Vicky's Calf & $3 \%$ & $0 \%$ & $2 \%$ & $11 \%$ & $2 \%$ & $4 \%$ \\
\hline With Vicky's Calf & $1 \%$ & $13 \%$ & $6 \%$ & $0 \%$ & $0 \%$ & $4 \%$ \\
\hline With Delphi's calf & $2 \%$ & $6 \%$ & $11 \%$ & $1 \%$ & $0 \%$ & $4 \%$ \\
\hline With Delphi's calf/Lotus & $8 \%$ & $0 \%$ & $5 \%$ & $6 \%$ & $1 \%$ & $4 \%$ \\
\hline With Lotus & $0 \%$ & $0 \%$ & $1 \%$ & $25 \%$ & $0 \%$ & $5 \%$ \\
\hline Average & $5 \%$ & $4 \%$ & $4 \%$ & $7 \%$ & $4 \%$ & $5 \%$ \\
\hline
\end{tabular}

support the hypothesis that mothers increase their signature whistle use in the week before their calves are born.

\section{Signature Whistle Use}

The HCA comparisons of the weeks before and after the births of Nephele's and Vicky's calves showed that the dolphins' whistle use changed when the calves were born. An analysis of the signature whistles used in those periods demonstrated that some of that change was due to an increase in signature whistle production by Nephele and Vicky. Signature whistle use was evaluated in all the time periods (excluding Lotus Alone) to determine how whistle use by each adult changed (Table 5.7). On average, $24 \%$ of the whistles could be classified as signature whistles. This percentage varied considerably between time periods (Table 5.7A). In particular, the percent signature whistles was 
FIGURE 5.22: SigNATURE WHISTLE PRODUCTION BY MOTHERS OF FoCAL CALVES

Points refer to the same dates on all plots, with each plot centered on the birth of that adult's calf.
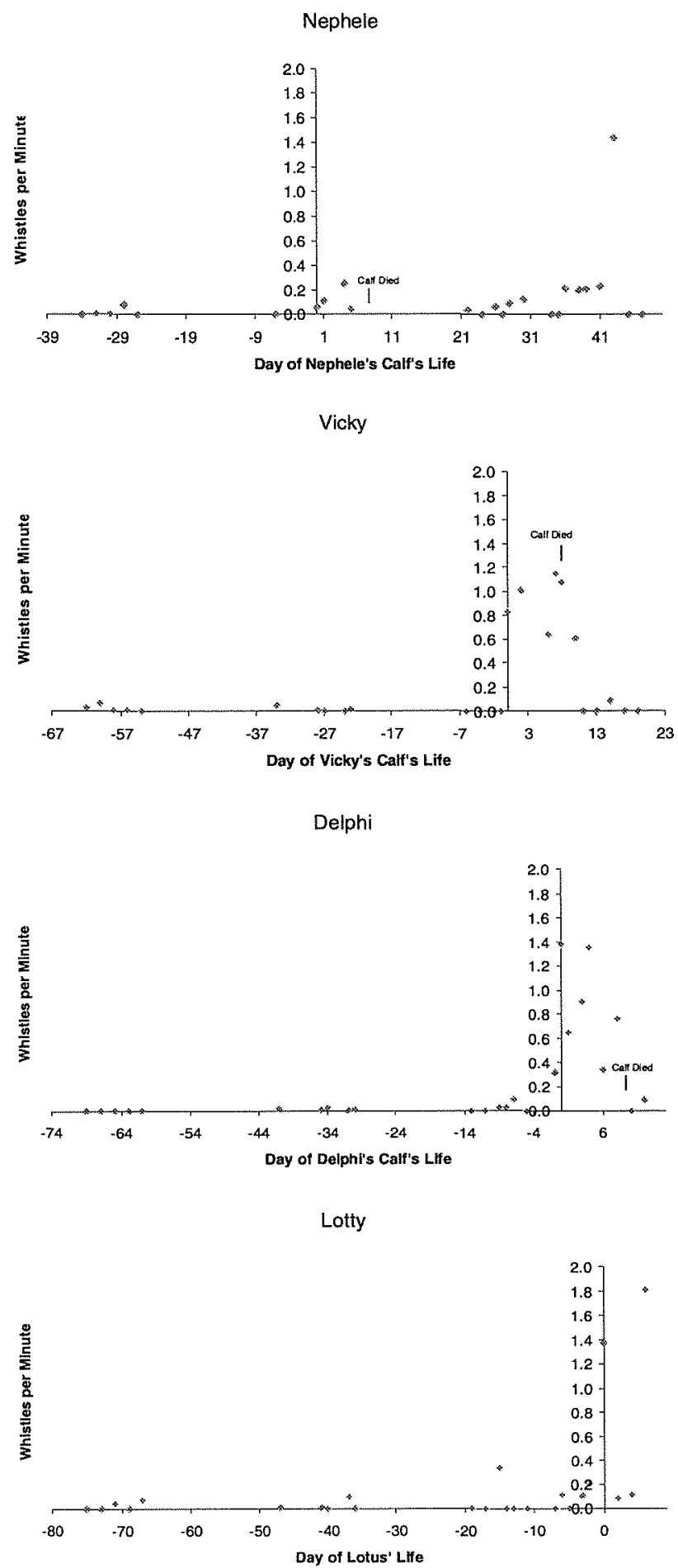
Chapter 5: Characterizing the Acoustic Environment

unusually high in the period With Nephele's calf (40\%) and unusually low Before Nephele's calf (13\%). The low percent Before Nephele's calf was a decrease from March when the percent was relatively high (31\%, Table 5.7A). On average, each of the identified signature whistles made up 5\% of the whistles in the sample (Table 5.7B). Each mother's signature whistle was higher in the period with her calf than in the other periods.

To evaluate the signature whistle use by each mother in the weeks surrounding her calf's birth, the rate at which each mother produced her signature whistle in each period was determined. The total rate of signature whistle production was calculated by multiplying the proportion of signature whistles on each day by the total number of usable whistles produced on that day. Signature whistle production by each mother is plotted with the plot centered on the birth of her calf (Figure 5.22). The plots are lined up so that the dates on all the plots match. The rate of signature whistle production by each mother except Nephele increased markedly when her calf was born (Figure 5.22).

Nephele's increased a smaller amount the week her calf was born, which is a reflection of the lower rate of whistling. Delphi began to increase her signature whistle production the day before her calf was born, as did Lotty (Figure 5.22). Nephele and Vicky only began increasing signature whistle production on the day their calves were born (Figure 5.22). In each case, signature whistle production decreased again when the calf died. These data confirm the hypothesis that the use of mothers' signature whistles increases when their calves are born. The exact timing of that increase appears to vary by individual, with some mothers beginning to increase signature whistle production in the days before their calves are born. Since signature whistles are used in whistle exchanges between mothers and calves (Sayigh 1992), the increase in signature whistle use could represent whistle interactions between the mother and her newborn calf.

Signature whistle use by the mothers is also plotted a percent of the total whistle production on each day (Figure 5.23). The percent of all the whistles that were signature whistles is important to how the signature whistles were perceived by the calf. All the whistles heard in each calf's first week comprise the acoustic environment experienced 


\section{FIgURE 5.23: MOTHER'S SIGNATURE WHISTLES AS PERCENT OF TOTAL}

Points refer to the same dates on all plots, with each plot centered on the birth of that adult's calf.
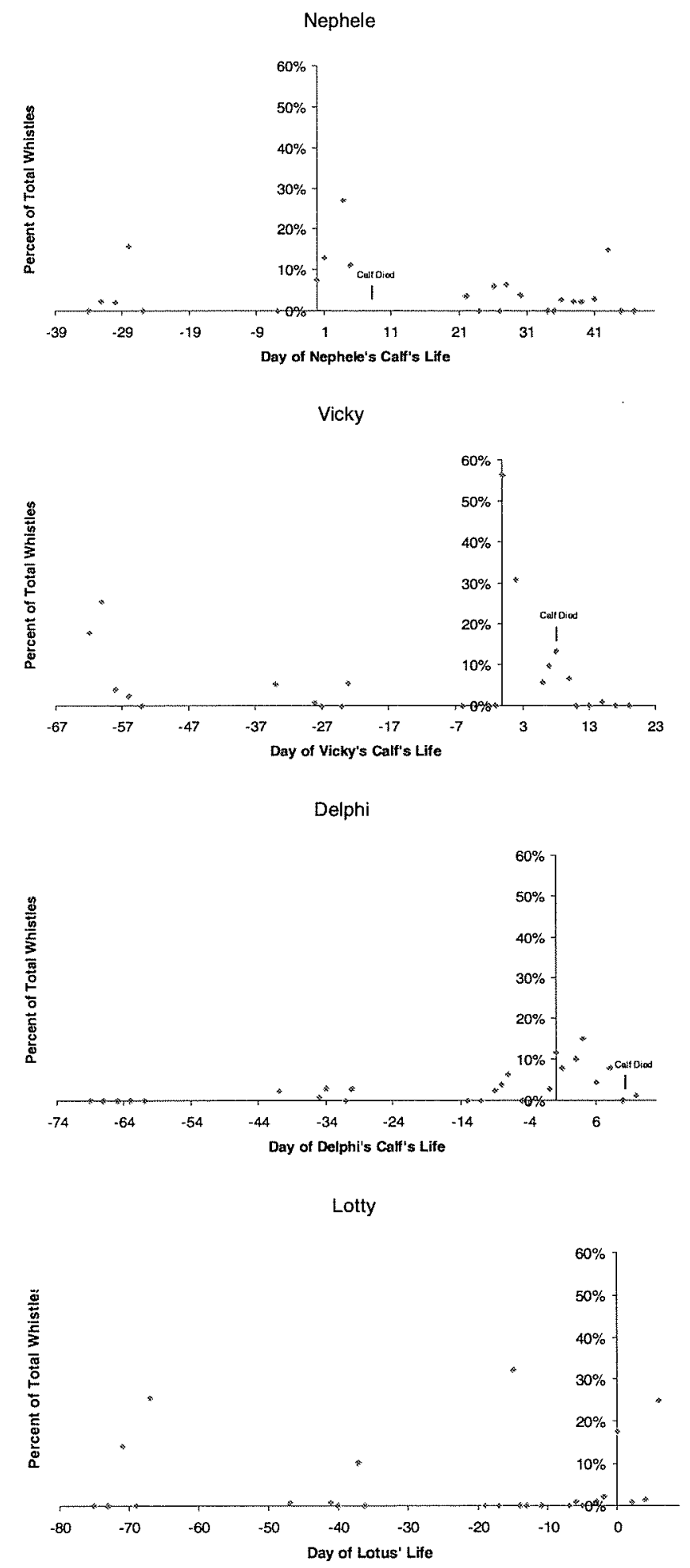
Chapter 5: Characterizing the Acoustic Environment

TAble 5.8 Mother's Whistles as Proportion of The Acoustic Environment

\begin{tabular}{|c|c|c|c|}
\hline Calf & Adult & Days & \% Adult's Signature Whistle \\
\hline Nephele's calf & Nephele & All days & $15 \%$ \\
\hline Vicky's calf & Vicky & All days & $26 \%$ \\
\hline Delphi's calf & Delphi & All days & $10 \%$ \\
\hline \multirow{2}{*}{ Lotus } & \multirow{2}{*}{ Lotty } & All days & $11 \%$ \\
\cline { 3 - 4 } & & Days 0 \& $6^{\ddagger}$ & $21 \%$ \\
\cline { 2 - 4 } & \multirow{2}{*}{ Vicky } & Days 1-5 & $1 \%$ \\
\cline { 3 - 4 } & & All days & $0.2 \%$ \\
\cline { 3 - 4 } & & Days 0 \& $6^{\ddagger}$ & $0 \%$ \\
\cline { 3 - 4 } & & Days 1-5 & $0.4 \%$ \\
\hline Average & Mothers & All days & $15 \%$ \\
\hline
\end{tabular}

${ }^{7}$ The average was calculated including Lotty for all days of Lotus' first week.

$\doteqdot$ On days 1 to 5, Lotus spent most of his time with Vicky. On days 0 and 6, he spent time with Lotty. Lotty was his biological mother.

by that calf in that week. Previous studies have suggested that the proportion of these whistles that are the mother's signature whistle is an important indicator of how the calves' signature whistles will develop (Sayigh 1992). For each calf, a large proportion of the whistles heard in that first week were the calf's mother's signature whistle (Figure 5.23). When viewed in this way, the proportion of whistles in the period With Nephele's calf that were Nephele's signature whistle was as high as the proportions of all the other mothers. All four calves, therefore, experienced a high proportion of their mothers' whistles in their first week (Table 5.8). The overall averages in this table were calculated by averaging the percent for each day (see Figure 5.23). Because the sub-samples for each period did not include the same number of whistles from each day, these averages were weighted differently from the overall average for the time period (compare to Table 5.7). However, to evaluate the mothers' signature whistles as a proportion of their calves' acoustic environments, the average signature whistle use on each day is more appropriate. On average, $15 \%$ of the whistles the calves heard on each day of their first weeks were their mothers' signature whistles (Table 5.8). Vicky's calf heard a slightly higher percent (Table 5.8), particularly on his first day (56\%, Figure 5.23). Delphi's calf heard a slightly lower percent $(10 \%$, Table 5.8$)$. Therefore, there appears to be some individual variation between the mothers, as has been seen in previous studies (Sayigh 1992). 
FIGURE 5.24: SignATURE WHISTLE USE BY VICKY AND LOTTY

Each line starts at the birth of the calf.

A. Signature Whistle Production

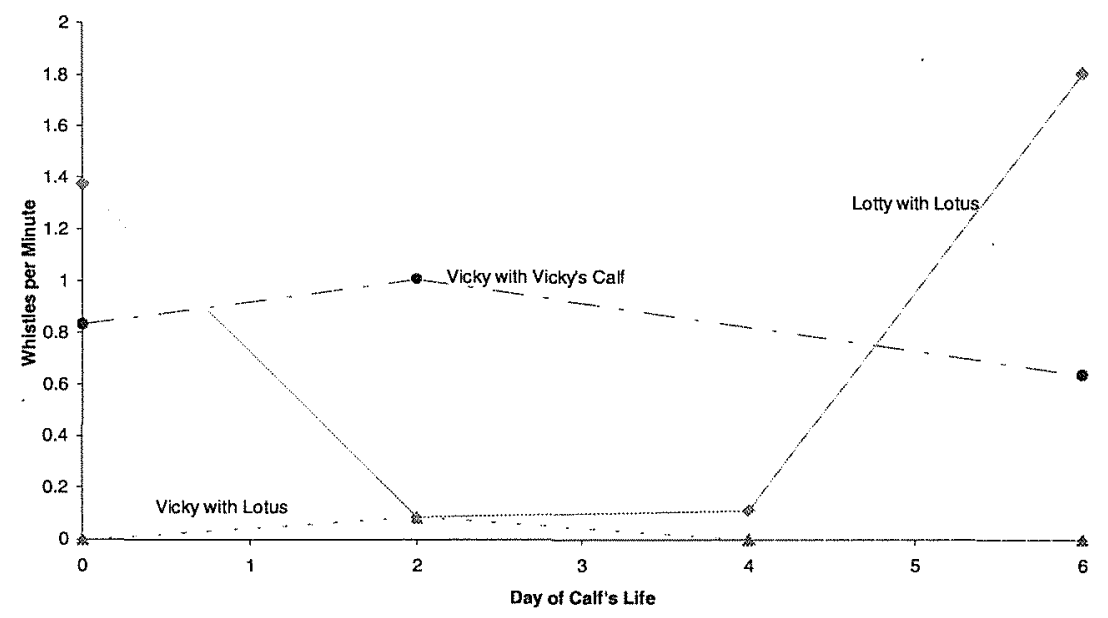

B. As Percent of Total WhISTLes

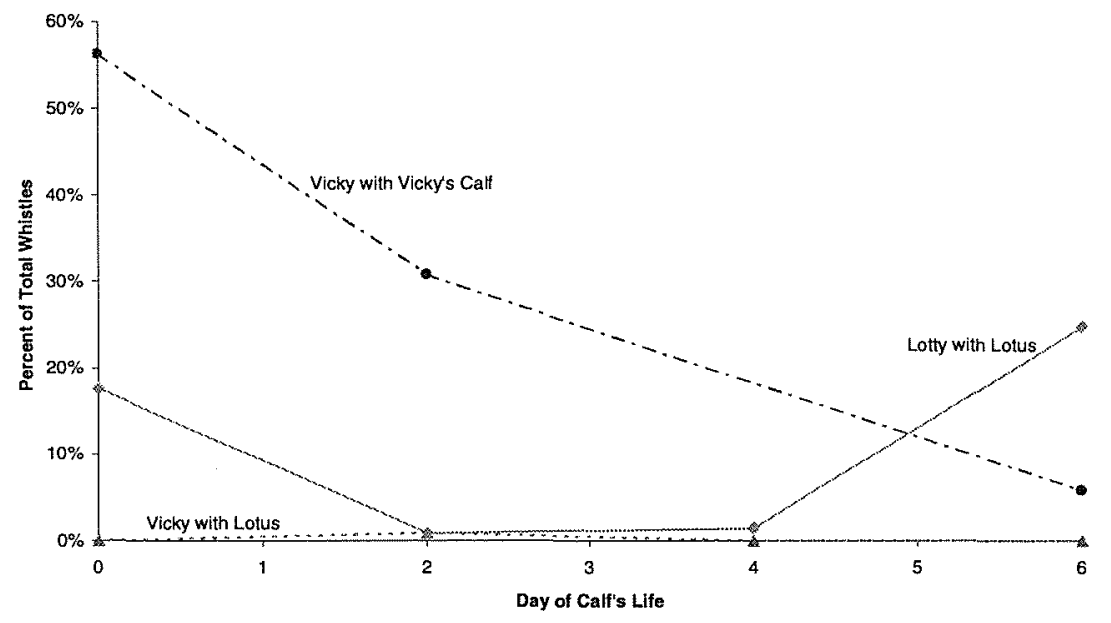

Because Lotus did not spend his first week with his mother Lotty, a closer analysis of his first week is worthwhile. During the days when Vicky was caring for Lotus, Lotty produced her signature whistle rarely (Figure 5.24A). However, Vicky did not increase her signature whistle production during this period either (Figure 5.24A). Particularly compared to Vicky's signature whistle production during her own calf's first week, Vicky's signature whistle production during Lotus' first week is low (Figure 5.24A). Vicky's signature whistle comprised fewer than $1 \%$ of the whistles Lotus heard 
Chapter 5: Characterizing the Acoustic Environment

in his first week (Figure 5.24B, Table 5.8). On the days that Lotty was caring for him (days 0 and 6), Lotty's signature whistle was $21 \%$ of the whistles Lotus heard. However, on the intervening days, when Vicky was caring for Lotus, Lotty's signature whistle was only $1 \%$ of the whistles heard (Table 5.8). The proportion of the acoustic environment made up of Vicky's signature whistles was slightly higher on the days she was caring for Lotus than on the other days, but the difference was minor $(0.4 \%$ vs. $0 \%$, Table 5.8$)$. These results suggest that the increase in signature whistle use by Lotty was related to caring for her calf, rather than simply giving birth. However, although Vicky increased her signature whistle use very slightly when she was acting as Lotus' allomother, the increased use of signature whistles by the mothers in this study appears to have been related to caring for one's own calf, rather than an unrelated calf. Alternatively, if Vicky was treating Lotus as if he were her own calf, who would be 3 weeks old, her signature whistle use could indicate that the mothers' increase in signature whistles is short-lived.

\subsection{DISCUSSION}

A demonstration of vocal learning in a natural repertoire requires infants raised with different acoustic stimuli to match the sounds they hear. In a social species such as dolphins, vocal development must be studied in a normal social setting. For studies of vocal learning to be possible, therefore, the normal acoustic environments of calves must be distinguishable. In this chapter, the early acoustic environments of four calves born in captivity were compared. The whistles heard by each of these calves were distinctive. In particular, a large proportion of the whistles that each calf heard were the signature whistles of his own mother. The acoustic environment of Nephele's calf, who was born a month earlier, was even more different from the environments of Vicky's calf, Delphi's calf, and Lotus, than the latter three calves' environments were from each other. Nephele's calf heard fewer, shorter whistles then the other three calves did (averaging $520 \mathrm{~ms}$, compared to $670 \mathrm{~ms}$ ) and more signature whistles from Nephele and Daphne. This analysis has shown that the acoustic environments of four calves born within a month of each other in the same social group were distinguishable. The acoustic 
environments of calves born into different social groups can be expected to be even more different.

Having established that the normal acoustic environments of calves can be differentiated, the whistles produced by calves born into different acoustic environments need to be compared to those acoustic environments. Because three of these four calves died very young, their later whistles cannot be compared to the environments they experienced. However, the analysis in this chapter has demonstrated how such a comparison could be performed. First, the calves' whistles can be incorporated into HCA comparisons of multiple acoustic environments. The location of each calf's whistles relative to whistles from the calf's own acoustic environment and from the acoustic environments of other calves will indicate whether the calf matched the stimuli it heard. More directly, the mean similarity between each calf's whistles and the whistles from the various acoustic environments can be compared. If the calf matched the whistles from its acoustic environment, the calf's whistles should be significantly more similar to the whistles from its own acoustic environment than to the whistles from other acoustic environments.

\subsubsection{WHISTLE USE IN A CALF'S FIRST WEEK}

\section{Signature Whistles}

After each calf in this study was born, there was an increase in the amount that the signature whistle of that calf's mother was produced. In some cases, the total number of whistles heard in the pool also increased considerably. The precise timing and size of these changes varied between calves. For some mothers, the increase actually began a few days before the calf was born. Wild dolphin mothers in Sarasota, FL also varied in how vocal they were and how often they produced their signature whistle (Sayigh 1992). Individual variation in whistle use is expected in this situation as well. However, the methods available in this study did not distinguish between the mother producing her signature whistle more often and other dolphins imitating the mother's signature whistle. Little is known about the reasons dolphins imitate each others' signature whistles (Tyack 
Chapter 5: Characterizing the Acoustic Environment

1986, Tyack 1993). If signature whistles are imitated as a means of communication with the dolphin whose signature whistle is being imitated, an increase in the imitation of a new mother's signature whistle might be expected. However, in previous studies of signature whistle imitation, no more than $25 \%$ of the signature whistles heard were imitations (Tyack 1986). Therefore, most of the signature whistles produced were likely produced by the mothers themselves.

There are many possible explanations for why mothers would change their whistle production when calves are born. Signature whistles are known to be used in situations where a dolphin is isolated or in distress (Caldwell \& Caldwell 1965, Caldwell et al. 1990, Sayigh 1992). In baboons, when infants are in distress, nearby males produce a particular rhythmic grunt to calm the infant (Smuts 1985). Similarly, human mothers change the frequency contour of their speech when comforting infants (Fernald 1992). New dolphin mothers could therefore be using their signature whistles to comfort their young calves. Signature whistles are also used as contact calls by dolphins mothers and calves when they are separated (Sayigh 1992). These new mothers could be producing their signature whistles to remain in acoustic contact with their calves when their calves wander away from them. Lotus' mother, Lotty, only increased her signature whistle use on the days that she was actually caring for Lotus. On the days that Lotus was being cared for by Vicky, Lotty produced very few signature whistles. This suggests that females only increase their signature whistle use when caring for the calf, which supports the hypotheses that increased signature whistle production by these mothers was related to comforting or remaining in contact with their calves. A further test of this hypothesis could be achieved by comparing the timing of signature whistle production to the behavior of the calves. If signature whistles were produced primarily as comfort or contact calls, they should be more common in situations where a calf is alone or distressed. An alternate hypothesis is that the increased signature whistle production is due to physiological changes that occur in postpartum females. However, if that were the case, Lotty would be expected to increase her signature whistle production even when not caring for Lotus, which did not occur. 
In a preliminary study of signature whistle development, Sayigh (1992) suggested that two of the mothers may have produced whistles that were different from their signature whistles as models for their calves' signature whistle development. Because the identity of whistlers was not known in the current study, the production of such models cannot be explicitly tested for. However, not all of the changes in whistle production seen in the HCA comparisons can be explained by known signature whistles. Some of the contours that were only produced after the calves were born did not match known signature contours of the mothers (see e.g. Figure 5.17). These may have been signature whistles of other dolphins, or whistles produced by the calves. However, the possibility exists that some of those unidentified whistles were produced by the mothers as models for the calves' signature whistle development.

\section{Lotus: The Difference between Acoustic and Behavioral Measures}

Lotus' first week was unusual in that he did not spend most of his time with his mother (Lotty). As soon as Lotus was born, Vicky swam up to him and he remained with her for several days. Lotty made no obvious attempt to regain Lotus until 5 days later when Vicky began to ignore him. Lotty then swam up to Lotus and he remained with her from that time on (see chapters $2 \& 3$ for more detail on this incident). This incident had an interesting affect on Lotus' acoustic environment in that first week. While all three of the other calves heard a large number of their mothers' signature whistles in their first weeks, Lotus heard neither his mother's nor his allomother's (Vicky). Lotty's signature whistle was prevalent on the day Lotus was born and again on the day he returned to her, but was rare in the intervening days. Vicky's signature whistle was rare the entire time. This result is a distinct contrast to the behavioral results (see chapter 3). Behaviorally, Vicky acted toward Lotus as all the mothers acted toward their calves and in the same way that Lotty acted toward Lotus after he returned to her. In chapter 3, this maternal behavior is described as a "caregiver" relationship between the adult and the calf. The acoustic results suggest that the behavioral data alone is incomplete. While the behavioral relationship between Vicky and Lotus was equivalent to the other calves and their mothers, the acoustic relationship was not. Because Lotus' signature whistle is not 
Chapter 5: Characterizing the Acoustic Environment

known, this study cannot determine what aspect of those relationships is most important to shaping a calf's signature whistle. However, these results suggest that behavioral caregiving and acoustic involvement in the raising of a calf are separate.

The low number of Vicky's signature whistles in the period when she was caring for Lotus may be an indication of negligence on Vicky's part. Because Vicky was not Lotus' biological mother, she may not have exchanged whistles with Lotus as often as she did with her own calf, even in separation situations. Smolker et al. (1993) noted that when year-old calves were separated from their mothers, most of the signature whistles heard were produced by the calves, not the mothers. Vicky could have been treating Lotus as she would an older calf who does not need to be watched as carefully. However, Mann and Smuts (1998) found that older calves spent less time within a few meters of their mothers than very young calves did. The time Lotus spent more than a meter away from Vicky was not significantly different from the time the other week-old calves spent away from their mothers (see chapter 3). Previous studies of captive dolphins have indicated that mothers are responsible for maintaining this proximity, not . calves (Reid et al. 1995). Negligence among alloparents has been reported in many species (e.g. primates: Hrdy 1976, Altmann 1980, Shopland \& Altmann 1987; seals: Riedman \& Le Boeuf 1982). However, other than her acoustic behavior, Vicky's behavior does not fit the description of a negligent alloparent (see chapter 3).

Alternatively, Vicky may have been treating Lotus as if he were her own calf, who would have been about 3 weeks old. Vicky's signature whistle use steadily decreased in her calf's first week. Her signature whistle use with Lotus may have merely been a continuation of that pattern. However, Vicky's acoustic behavior may also suggest that signature whistle use by mothers is not solely related to signature whistle use as a comfort or contact call to their calves.

\section{An Alternate Hypothesis}

Vicky's signature whistle use in Lotus' first week may suggest an alternative hypothesis for the increased signature whistle use by mothers of young calves. Unlike the mothers caring for their own calves, Vicky did not substantially increase her signature 
whistle use in the days that she was caring for Lotus. Although the sample size for this is very small, and Vicky's reaction to Lotus could be idiosyncratic, one possible explanation for this result is that females only increase their signature whistle use when caring for their own calves. This suggests an intriguing possibility for why these mothers increased their signature whistle use. These mothers could have been producing their signature whistles as a model for the signature whistles of their calves. All four of these calves were male, and male calves are more likely to develop signature whistles similar to their mothers' signature whistles than are female calves (Sayigh et al. 1990, 1995). Sayigh and her colleagues $(1990,1995)$ suggested that this difference was evolutionarily advantageous because female dolphins remain in their natal groups while males disperse. Females may therefore be under evolutionary pressure to develop signature whistles different from their mothers to avoid confusion within the group. Males' signature whistles, on the other hand, may be shaped by the need for kin-recognition, to avoid inbreeding after the male has not been seen for several years and may not be recognizable visually. Alternatively, males may simply lack the evolutionary pressure to develop a particularly different signature whistle. The present results suggest a possible mechanism for male signature whistle development. The mothers of male calves may increase the production of their signature whistles as models for their calves. Although the signature whistles of male calves tend to be similar to their mothers' signature whistles, every dolphin's signature whistle is unique. However, the present analysis was not detailed enough to determine whether the mothers modified their signature whistles to allow the calves to use them as models.

The hypothesis that the mothers of male calves increase their signature whistle production as a model for their calves has two testable predictions. First, if signature whistles are produced as models rather than as contact or comfort calls, signature whistles should be produced when the calf is near the mother and calm. If signature whistles are produced as comfort or contact calls, they should be produced when the calf is separated from his mother or in distress. The second prediction is that the mothers of female calves should not increase their signature whistle production. None of the calves in the present 
Chapter 5: Characterizing the Acoustic Environment

study was female, so the results of this study cannot be used to test this hypothesis. A preliminary study of vocal learning in wild dolphins included one calf known to be male and one known to be female (Sayigh 1992). Of the four calves in that study, the known male heard the highest proportion of his mother's signature whistle of any of the calves (20\%, Sayigh 1992). The known female heard the lowest proportion (6\%, Sayigh 1992). As expected, the signature whistle of the male was very similar to his mother's while the female's was different. In fact, the mother of this female calf may have produced a model for the calf to use as her signature whistle when the calf was a month old (Sayigh 1992). The calf's final signature whistle was similar to the model produced by her mother, and to the whistle of another female she associated with, but different from her mother's signature whistle. However, with the small sample size in Sayigh's (1992) study, individual variation cannot be discounted as the explanation for the differences in signature whistle production by these two mothers.

This hypothesis suggests a profound difference in maternal behavior toward male and female offspring. Differential treatment of male and female offspring has been widely studied in many species, generally in terms of nutritional investment. Trivers and Willard (1973) hypothesized that females should modify the sex-ratio of their offspring depending on the female's physical condition. Because the reproductive success of males in polygynous species depends heavily on their physical condition, and therefore on the amount of parental investment they get, females in better condition should produce more males than females in poor condition do. To achieve this sex-ratio, females in good condition should invest more in males while females in poor condition should invest more in females (Trivers \& Willard 1973). Since that hypothesis was suggested, many studies have been performed looking for such differential treatment of offspring by mothers (see Byers \& Moodie 1990, Riedman 1990). While some studies have clearly found that mothers do not make such a distinction (Byers \& Moodie 1990, Pélabon et al. 1995, e.g. sea lions: Ono \& Boness 1996), some studies have found clear evidence of differential treatment of offspring by their sex (Byers \& Moodie 1990, Pélabon et al. 1995, e.g. bighorn sheep: Hogg et al. 1992; elephants: Lee \& Moss 1986; pinnipeds: 
Riedman 1990; red deer: Clutton-Brock et al. 1981; Sarahan arrui: Cassinello 1996). Although these studies have focussed on nutritional investment, rather than acoustic contact, they demonstrate that mothers of many species are able to distinguish between male and female offspring and some tend to treat those offspring differently.

\subsubsection{METHODOLOGICAL ISSUES}

\section{Calf Whistles}

One of the goals of this chapter was to find a way to distinguish between calf whistles and adult whistles. Caldwell and Caldwell (1979) reported that calves tend to produce whistles that were shorter and less frequency modulated than adults' whistles. They described the calf whistles as "quavery" (Caldwell \& Caldwell 1979). The known calf whistles in this study, from Lotus Alone, fit that description well (see Figure 5.14A). An analysis of known calf whistles compared to known adult whistles suggested that calf whistles can be distinguished from adult whistles by their quartile bandwidth, i.e. their bandwidth when the extreme values are discounted. Very few of the calf whistles had quartile bandwidths greater than $3 \mathrm{kHz}$, while more than a third of the adult whistles did. This cutoff provides a method for knowing which whistles were probably produced by adults. However, it does not provide a means for determining which whistles were probably produced by calves. Whistles with low quartile bandwidths could be produced by either adults or calves. In addition, these results must be regarded cautiously because the known calf whistles in this sample were all from a particular context, a calf alone with no adults in visual or acoustic contact. The possibility remains, therefore, that calves are capable of producing whistles with high bandwidths in other situations. These results are consistent with a previous report in which Caldwell et al. (1990) showed that there was a significant increase in the number of loops, the frequency modulation, and the average duration of whistles with age.

\section{Bubblestreams}

One of the perennial problems of cetacean research is the inability to identify which animal in a group is producing a sound. Dolphins sometimes provide what 
Chapter 5: Characterizing the Acoustic Environment

appears to be an easy solution to this: a small stream of bubbles in conjunction with a vocalization. Since this sort of "bubblestream" is generally only seen when a dolphin is also whistling, bubblestreams can be a convenient way of identifying which dolphin is whistling. However, bubblestreams are extremely obvious visual signals that are not made with all whistles. In fact, bubblestream-whistles by adults are extremely rare, approximately two-tenths of a percent of the whistles produced in this study. Because bubblestreams are not associated with all whistles, the bubblestream-whistles must be different in some way. Either the dolphin chooses to produce this visual signal along with the acoustic signal in certain contexts, or the dolphin cannot prevent the bubblestream from occurring. The latter hypothesis implies that the physiological or emotional context of bubblestream-whistles is different from other whistles. If the dolphin is producing bubblestreams in certain social or emotional contexts, bubblestreams are likely to be associated with certain whistle types. Bubblestreamwhistles are therefore likely to be a biased sample of the whistles produced. If bubblestreams are produced in certain physiological contexts, such as in association with a change in the pressure in the lungs, the whistles may be randomly associated with the bubblestreams.

Some researchers have assumed that whistles are randomly associated with bubblestreams and used bubblestream-whistles as an unbiased sample of the whistles produced (e.g. McCowan 1995, Herzing 1996). The rarity of bubblestreams suggests that they are unlikely to produce an unbiased sample of the whistles. Before bubblestreamwhistles are used as such a sample, therefore, the hypothesis that they are unbiased must be rigorously tested. This has not been done. Only one study has reported that bubblestream-whistles are an unbiased sample, and that study was based on an extremely small sample (McCowan 1995). If McCowan (1995) used as many whistle types in her bubblestream test as in her final analysis, the sample size was too small for the $\chi^{2}$ test to be valid. In the current study, adult bubblestreams were associated with a number of different contours for each adult. However, a number of other contours, such as Lotty's putative signature whistle, were never associated with bubblestreams from any adult. 
One possible reason for this is that the sample size of bubblestream-whistles was small. However, this fact merely highlights again the problem with using bubblestreamwhistles. The sample of bubblestream-whistles in this study was small but the sample of non-bubblestream whistles was not. The rate of bubblestream-whistles in this study was extremely low, especially compared to the rate of non-bubblestream whistles.

In addition to occurring at a very low rate, bubblestream production in this study did not occur independently. A bubblestream was more likely to be seen shortly after another bubblestream. In addition, the bubblestreams that occurred within a few seconds of each other were often associated with similar contours. Closely timed bubblestreamwhistles, therefore, cannot be treated as an independent sample. This means that bubblestream-whistles cannot be used to determine the relative rates at which different types of whistles are produced. If bubblestreams were a reliable method of identifying whistlers, the lack of independence would simply mean that bubblestreams were only useful for determining what types of whistles dolphins produced, not the rate of production. However, another problem arises when using bubblestreams as the method for identifying whistlers: the error in the timing of the bubblestream compared to the whistle. Any behavioral marking has some error, based on the time it takes to recognize and mark the behavioral event. The error in bubblestream markings in this study was at least a second. The average whistle length in this study, on the other hand, was approximately half a second. More than one whistle could therefore fit into the space of a second, and even more than that if the whistles overlapped each other. Whistles often occur closely spaced or overlapping. There is no way to determine which of the whistles within the marking error of the bubblestream was actually associated with the bubblestream (see Figure 5.4). If whistles cannot be unquestionably associated with bubblestreams, bubblestreams cannot be used to unambiguously determine the identity of whistlers. In fact, in this study, the error in bubblestream markings was itself useful: it allowed the identification of the putative signature whistles of the mothers of the dolphins producing the bubblestreams. 
Chapter 5: Characterizing the Acoustic Environment

\section{The Use of Contours}

Another perennial problem in any acoustic analysis is how to compare sounds. The spectrogram of a sound contains a great deal of information about intensity in sections of frequency and time. However, a spectrogram also contains information about the background noise the sound was made around. In some cases, such as in the recordings in this study, that noise can be a significant problem. Comparisons of the spectrograms of whistles recorded here were more closely related to the characteristics of the background noise than the whistles themselves. In a system with a better signal-tonoise ratio, spectrogram comparisons might be preferable to contour comparisons because they preserve more of the signal (see e.g. Clark et al. 1987). With the signal-tonoise ratio of the sounds in this study, on the other hand, a method is necessary to extract the essential elements of the sound from the background noise.

Contour extraction is one way to solve that problem. The fundamental frequency contour of the sound is determined and extracted from the spectrogram (Buck \& Tyack 1993). This contour can then be used to compare the fundamental frequency patterns of two sounds. Certain elements of the sound are lost in this process, such as information about amplitude modulation. Amplitude modulation could be very important to the animals. However, this lost information is traded for the ability to easily compare the frequency patterns of the two whistles. Contour extraction does not entirely solve the problem of background noise. Noise can still cause spikes in the contours that cannot be removed without significant massaging of the data. However, the analysis in the present study demonstrates that unlike with spectrograms, noise spikes do not interfere with the ability to quantitatively analyze contours. Gaps within the whistles can cause a larger section of noise spikes than is normal in most contours. The comparison between two whistles with such gaps (such as Nephele's and Lotty's in Figure 5.12A) can be difficult. In a similar fashion, some of the fine structure of the whistles are lost when contours are extracted (e.g. compare the contours and spectrograms in Figure 5.12 A \& B). However, comparisons of contours in this study were more robust to noise spikes and changes in fine structure than the equivalent comparisons of spectrograms were. 
The gaps in the whistles in this study are consistent with previous reports that some dolphin whistles appear to have spaces in them (e.g. Caldwell et al. 1990, Sayigh 1992). The shape of the contours, and therefore comparisons between them, will depend on where the whistle falls off and where it begins again. In some cases, there are actual spaces of no sound between the loops of the whistle (see e.g. Figure 5.12A). In others, the sounds in those spaces are merely very low amplitude. How well those are extracted depends on the amplitude gain in the recording system. Because of the space in some whistles, knowing exactly where to separate whistles from each other can be difficult. Some researchers separate whistles at every space that appears on the spectrogram (e.g. McCowan 1995, Smolker \& Pepper in press). The problem with that method is that the breaking point can depend on the gain of the system. Sometimes a whistle that looks broken will look continuous if the gain is increased. In this study, therefore, a correction for the possible error in the gain was built in (see chapter 4). Whistle sections that were separated by less than $100 \mathrm{~ms}$ were considered a single whistle. Nephele's whistle in figure 5.12 is a case in point. On the spectrogram, this whistle appears to be 4 upsweeps separated by small gaps. The noise across those gaps may have been sufficient to fool the automatic extractor into connecting the sections even if they were more than $100 \mathrm{~ms}$ apart. However, in the manual sorting of the spectrograms, the distance between the sections was measured to ensure that it was less than $100 \mathrm{~ms}$.

\section{Dynamic Time Warping \& Hierarchical Cluster Analysis}

The difference in duration between whistles has also been a problem in the comparison of dolphin whistles. Qualitative comparisons of whistles have suggested that whistles of the same contour sometimes vary somewhat in duration (Tyack 1986, Caldwell et al. 1990). In addition to simply extending the whistle's duration, dolphins appear to change the relative lengths of different sections of some of their whistles (Tyack 1986, Buck \& Tyack 1993). These slight differences in structure compromise efforts at frequency correlation. A method for evening out the duration of similar whistles is therefore desirable. Some researchers normalize the duration entirely (e.g. McCowan 1995). There are two problems with this method. First, it does not solve the 
Chapter 5: Characterizing the Acoustic Environment

problem of changes in the relative lengths of different sections. Second, it allows the comparison of whistles with very different durations, sometimes over as much as a factor of ten. The dynamic time warping (DTW) used here attempts to solve both those problems (Buck \& Tyack 1993). DTW solves the first problem by stretching contours to fit each other in a non-linear fashion. The second problem is solved by not allowing comparisons across more than a factor of two. This is actually a relatively lenient estimate compared to those used by others. For instance, Janik (in press) requires the shorter whistle to be at least $75 \%$ the duration of the longer.

The built-in duration limit causes comparisons based on DTW to focus to a large extent on duration. A question arises as to how much of the difference between whistles of different durations is an artifact of the method and how much is a difference in whistle type. Without performing perceptual experiments on dolphins, this question cannot be answered. However, some researchers have suggested that very short whistles are a completely different phenomenon from longer whistles (Caldwell \& Caldwell 1970). In the present study, whistle durations varied continuously from $25 \mathrm{~ms}$ to 3 seconds. If short whistles were a completely different phenomenon, a gap in whistle duration between short and long whistles would be expected. The lack of a gap in the durations suggests that this is not the case. However, the DTW has another limitation: it cannot compare whistles of differing numbers of loops. In some cases, a major difference in duration occurs when two whistles of effectively the same contour have different numbers of loops (see e.g. Figure 5.8). The DTW cannot handle these comparisons, especially if the two whistles differ by more than a factor of two in duration.

The HCA comparisons made in the present study using DTW separated whistles based on duration and frequency structure. Part of this was the artificial limit imposed by the DTW. Duration and frequency structure is, however, exactly the information that contours contain. Because of the constraints on the DTW, this method separates whistles into more categories than human judges might, such as separating one-loop whistles from two-loop whistles. In addition, the moat index for the HCA was useful in determining the overall similarity of the whistles in the sample, but was not particularly useful for 
determining robust categories of whistles. In addition, the maximum sample size is still dictated by the methods, as it was with the more traditional, manual classification. In this case, the maximum sample size is limited by the statistics program. However, because the whistles were sampled in an unbiased manner, a truly unbiased sub-sample can be achieved with these methods. Therefore, although not all the whistles in the sample can be used, a random sub-sample can be counted on to yield equivalent results. A quantitative comparison of the entire whistle repertoire of the dolphins in each period was possible with the techniques demonstrated in this chapter. While the more traditional methods of visual spectrogram classification were useful for signature whistle analysis, they performed poorly on the analysis of non-signature whistles. The DTW and DTW combined with HCA were useful in distinguishing between the whistle samples, and therefore the acoustic environments experienced by the calves. For this analysis, the ability to separate whistles in to categories was not actually necessary. These techniques can also be used to determine how identified whistles produced by calves compare to the whistles produced in their acoustic environments.

\subsection{LITERATURE CITED}

Altmann, J., 1980. Baboon mothers and infants. Harvard University Press, Cambridge, MA.

Buck, J.R. and Tyack, P.L., 1993. "A quantitative measure of similarity for Tursiops truncatus signature whistles." Journal of the Acoustical Society of America, 94 (5), pp. 2497-2506.

Byers, J.A. and Moodie, J.D., 1990. "Sex-specific maternal investment in pronghorn, and the question of a limit on differential provisioning in ungulates." Behavioral Ecology and Sociobiology, 26, pp. 157-164.

Caldwell, M.C. and Caldwell, D.K., 1965. "Individualized whistle contours in bottlenosed dolphins (Tursiops truncatus)." Science, 207, pp. 434-435.

Caldwell, M.C. and Caldwell, D.K., 1970. "Etiology of the chirp sounds emitted by the Atlantic bottlenosed dolphin: A controversial issue." Underwater Naturalist, 6, pp. 6-8.

Caldwell, M.C. and Caldwell, D.K., 1979. "The whistle of the Atlantic bottlenose dolphin (Tursiops truncatus) - ontogeny." Behavior of marine animals, vol. 3 Cetaceans, eds. H.E. Winn and B.L. Olla. Plenum, New York, NY. pp. 369-401.

Caldwell, M.C., Caldwell, D.K., and Tyack, P.L., 1990. "Review of the signature-whistle hypothesis for the Atlantic bottlenose dolphin." The Bottlenose Dolphin, eds. S. Leatherwood and R.R. Reeves. Academic Press, Inc., New York, NY. pp. 199-234.

Cassinello, J., 1996. "High-ranking females bias their investment in favour of male calves in captive Ammotragus lervia." Behavioral Ecology and Sociobiology, 38, pp. 417-424.

Clark, C.W., Marler, P., and Beeman, K., 1987. "Quantitative analysis of animal vocal phonology: An application to swamp sparrow song." Ethology, 76, pp. 101-115.

Clutton-Brock, T.H., Albon, S.D., and Guinness, F.E., 1981. "Parental investment in male and female offspring in polygynous mammals." Nature, 289, pp. 487-489. 
Chapter 5: Characterizing the Acoustic Environment

Fernald, A., 1992. "Human maternal vocalizations to infants as biologically relavant signals: An evolutionary perspective." The adapted mind: Evolutionary psychology and the generation of culture, eds. J.H. Barkow, L. Cosmides, and J. Tooby. Oxford University Press, New York, NY. pp. 392-428.

Herzing, D.L., 1996. "Vocalizations and associated underwater behavior of free-ranging Atlantic spotted dolphins, Stenella frontalis and bottlenose dolphins, Tursiops truncatus." Aquatic Mammals, 22.2, pp. 61-79.

Hogg, J.T., Hass, C.C., and Jenni, D.A., 1992. "Sex-biased maternal expenditure in Rocky Mountain bighorn sheep." Behavioral Ecology and Sociobiology, 31, pp. 243-251.

Hrdy, S.B., 1976. "Care and exploitation of nonhuman primate infants by conspecifics other than the mother." Advances in the Study of Behavior, 6, pp. 101-158.

Janik, V.M. and Slater, P.J.B., 1998. "Context-specific use suggests that bottlenose dolphin signature whistles are cohesion calls." Animal behaviour, 56: 4, pp. 829-838.

Janik, V.M., in press. "Pitfalls in the categorization of behaviour: a comparison of dolphin whistle classification methods." Animal behaviour.

Lee, P.C. and Moss, C.J., 1986. "Early maternal investment in male and female African elephant calves." Behavioral Ecology and Sociobiology, 18, pp. 353-361.

Mann, J. and Smuts, B.B., 1998. "Natal Attraction: Kidnapping, allocare, and separations among wild Bottlenose dolphin (Tursiops truncatus) mothers and infants." Animal behaviour, 55, pp. 10971113.

McCowan, B. and Reiss, D., 1995. "Whistle contour development in captive-born infant bottlenose dolphins (Tursiops truncatus): Role of learning." Journal of Comparative Psychology, 109(3), pp. 242-260.

McCowan, B., 1995. "A new quantitative technique for categorizing whistles using simulated signals and whistles from captive bottlenose dolphins (Delphinidae, Tursiops truncatus)." Ethology, 100, pp. 177-193.

Ono, K.A. and Boness, D.J., 1996. "Sexual dimorphism in sea lion pups: Differential maternal investment, of sex-specific differences in energy allocation?" Behavioral Ecology and Sociobiology, 38, pp. $31-41$.

Pelabon, C., Gaillard, J.M., Loison, A., and Portier, C., 1995. "Is sex-biased maternal care limited by total maternal expenditure in polygynous ungulates?" Behavioral Ecology and Sociobiology, 37, pp. 311-319.

Reid, K., Mann, J., Weiner, J.R., and Hecker, N., 1995. "Infant development in two aquarium bottlenose dolphins." Zoo Biology, 14, pp. 00-00.

Riedman, M., 1990. The pinnipeds: Seals, sea lions, walruses. University of California Press, Berkeley, CA.

Riedman, M.L. and Le Boeuf, B.J., 1982. "Mother-pup separation and adoption in Northern elephant seals." Behavioral Ecology and Sociobiology, 11, pp. 203-215.

Sayigh, L.S., 1992. Development and functions of signature whistles of free-ranging bottlenose dolphins, Tursiops truncatus. Ph.D. Dissertation, MIT/WHOI Joint Program, WHOI 92-37.

Sayigh, L.S., Tyack, P.L., Wells, R.S., and Scott, M.D., 1990. "Signature whistles of free-ranging bottlenose dolphins Tursiops truncatus: Stability and mother-offspring comparisons." Behavioral Ecology and Sociobiology, 26, pp. 247-260.

Sayigh, L.S., Tyack, P.L., Wells, R.S., Scott, M.D., and Irvine, A.B., 1995. "Sex difference in whistle production in free-ranging bottlenose dolphins, Tursiops truncatus." Behavioral Ecology and Sociobiology, 36, pp. 171-177.

Shopland, J.M. and Altmann, J., 1987. "Fatal intragroup kidnapping in yellow baboons." American Journal of Primatology, 13, pp. 61-65.

Smolker, R.A., Mann, J., and Smuts, B.B., 1993. "Use of signature whistles during separations and reunions by wild bottlenose dolphin mothers and infants." Behavioral Ecology and Sociobiology, 33, pp. 393-402.

Smolker, R.S. and Pepper, J., in press. "Whistle convergence among allied male bottlenose dolphins." Ethology. 
Chapter 5: Characterizing the Acoustic Environment

Smuts, B.B., 1985. Sex and friendship in baboons. Gruyter, New York, NY.

Trivers, R.L. and Willard, D.E., 1973. "Natural selection of parental ability to vary the sex ratio of offspring." Science, 179, pp. 90-92.

Tyack, P.L., 1986. "Whistle repertoires of two bottlenosed dolphins, Tursiops truncatus: Mimicry of signature whistles?" Behavioral Ecology and Sociobiology, 18, pp. 251-257.

Tyack, P.L., 1993. "Animal language research needs a broader comparative and evolutionary framework." Language and communication: Comparative perspectives, eds. H.L. Roitblat, L.M. Herman, and P. Nachtigall. Erlbaum, Hillsdale, NJ. pp. 115-52. 


\section{CHAPTER 6: SUMMARY AND CONCLUSIONS}

\subsection{THESIS GOALS}

The goal of this thesis was to develop techniques that would allow a study of vocal learning in dolphin whistle development to be performed. While the ability to learn new sounds is essential to human language development, it is unusual among non-human terrestrial mammals (Janik \& Slater 1997, Seyfarth \& Cheney 1997). Adult and juvenile dolphins have shown the ability to imitate novel sounds both spontaneously and in trained situations (e.g. Caldwell \& Caldwell 1972, Richards et al. 1984). Preliminary evidence suggests that vocal learning is involved in the natural development of dolphin whistles (Sayigh 1992, Tyack \& Sayigh 1997). Several studies have reported that bottlenose dolphin calves developed signature whistles that matched acoustic models in their environment, including the whistles of unrelated animals and man-made whistles (Caldwell \& Caldwell 1979, Sayigh 1992, Tyack 1997). The matching of acoustic models produced by unrelated dolphins or humans can best be explained by vocal learning. However, the methods currently used to study the acoustic and social behavior of dolphins are insufficient to evaluate the role of learning in whistle development and the social influences on that development. The techniques necessary to perform such a study have therefore been developed and tested in this thesis.

A quantitative demonstration of vocal learning requires that the vocalizations of infants raised in different acoustic environments match the vocalizations they heard (see Figure 5.1). To distinguish the role of learning from that of inheritance, the infants must match the vocalizations of unrelated animals, rather than of related animals. Each infant's vocalizations must also be shown not to match vocalizations heard by other infants that this infant did not hear. A test for vocal learning therefore requires the acoustic environments experienced by the different infants to be distinguishable. Methods that have been used in the past to find infants that experienced different acoustic environments were reviewed in chapter 1. A common method has been to look for geographical variation in the calls of wild animals, but this often does not allow learning to be distinguished from inheritance (see chapter 1, Janik \& Slater 1997). Other 
researchers have raised infants in socially or acoustically impoverished environments in an attempt to control the infants' acoustic experiences (see chapter 1, e.g. Marler 1970, Winter et al. 1973, West et al. 1997). In social species, however, infants raised in socially or acoustically impoverished environments often develop abnormally (Harlow \& Harlow 1962, Janik \& Slater 1997, West et al. 1997). Therefore, methods are necessary for evaluating vocal learning in infants raised in normal social groups. If the acoustic environments of infants raised in different social groups is sufficiently different, vocal learning can be evaluated by comparing the vocalizations of infants to the vocalizations in their early acoustic environments. One of the goals of this thesis was to develop methods for making such comparisons in a study of bottlenose dolphin whistles.

Social interactions have been shown to have a profound effect on vocal development in many species (Snowdon \& Hausberger 1997; birds: West et al. 1997; humans: Locke \& Snow 1997; primates: Seyfarth \& Cheney 1997). An understanding of the social influences on vocal learning requires the social interactions between animals to be quantified. Because social interactions are not independent, the social interactions between animals must be understood in terms of the social relationships between the animals (Hinde 1983, Cheney et al. 1986). Although birdsong has been hypothesized to aid in maintaining social bonds, the analysis of social relationships has been best developed for the study of mammals (e.g. Hinde 1983, Brown 1985, Cheney et al. 1986, Hausberger et al. 1995, Whitehead 1997). However, because learning has not been shown to play a large role in the vocal development of most non-human mammals, the study of social relationships has not been applied to the study of vocal learning. Recent data indicate that learning plays a role in the vocal development of bats as well as dolphins (Boughman 1998, Jones \& Ransome 1993). One of the goals of this thesis was to develop methods for quantifying the social relationships between dolphins in order to apply those methods to the study of the social influences on dolphin whistle development. 
Chapter 6: Summary and Conclusions

\subsection{THESIS RESULTS}

\subsubsection{TECHNIQUES FOR SAMPLING ACOUSTIC AND SOCIAL BEHAVIOR}

The first step in quantifying an infant's social and acoustic environment is to record the vocalizations and interactions of the animals in that environment. However, recording and evaluating vocalizations and interactions 24 hours a day is impractical, particularly over the entire course of development for several infants. Therefore, the vocalizations and interactions need to be sampled. The decisions made in designing a strategy for sampling sound and behavior have a profound impact on the conclusions that can be reached (Altmann 1974). The factors influencing the sampling decisions in a study of dolphin whistles were discussed in chapter 2. A sampling strategy was designed for recording the whistles and interactions of dolphin calves born in captivity. This strategy included focal animal samples (Altmann 1974) of mothers and calves in several captive facilities. The samples were to start and end at predetermined times on every day and to record carefully defined behaviors. Acoustic recordings were to be made simultaneously with the behavioral samples. The strategy was then tested in a pilot study of four calves born at Kolmårdens Djurpark in Sweden in the spring of 1995. Three of the four calves died within 10 days of birth.

In the course of the pilot study, a test was performed to determine the number of focal animal samples that were needed to adequately represent the behavior of the entire day (chapter 2). On several days, up to 30 focal animal samples were taken at short, regular intervals throughout the day. The results of using smaller subsets of those samples were compared to the results of using all the samples, which were assumed to adequately represent the behavior of the entire day. For most behaviors, five samples were sufficient to represent the entire day. In addition, the dependence of the results on the time of day that the samples were taken was evaluated. The results of this test depended on how often the behavior occurred. For common behaviors, the time of day had little impact on the results. If the behavior was rare enough to only occur in a few samples, the times chosen for sampling could have a major impact on the results. However, no consistent diurnal pattern was seen for any of the behaviors recorded. 
Therefore, sampling times could not be chosen to consistently record such behaviors. Overall, five focal animal samples spaced throughout the day adequately sampled the dolphins' behavior for the entire day.

In conjunction with the behavioral samples, the vocalizations produced by the dolphins were recorded. However, when these vocalizations were recorded, all the other sounds produced in the pool were recorded with them. The vocalizations of interest, in this case the whistles, had to be separated from the other sounds on the recording. This task has traditionally been done by manually searching the tape for whistles and extracting them (e.g. Tyack 1986, Sayigh et al. 1990, Janik \& Slater 1998). Because whistles are often widely separated on the recordings, this can be very time-consuming task (e.g. Tyack 1986, Sayigh et al. 1990). In addition, manual extraction is fraught with possible biases (see chapter 4). One of the most problematic biases is the bias introduced when choosing which whistles to extract and which to ignore (e.g. Sayigh et al. 1990). To speed up the process and control for these biases, a method of automatically extracting whistles from recordings was developed and tested in chapter 4 . This technique allowed a large, unbiased sample of whistles to be extracted in a short period of time. Because the decisions for how to extract the whistles were pre-programmed, all the sounds on the tape were treated equally and the biases were minimized. Because the extraction took less time than manual extraction, a much larger sample could be gathered. More than 20,000 whistles were extracted from close to 63 hours of recordings. The ability to extract such a large, unbiased sample is essential to the ability to quantify the acoustic environment experienced by the calves.

\subsubsection{TECHNIQUES FOR EVALUATING BEHAVIORAL DATA}

Having established the most appropriate methods for sampling behavioral data, the data collected in the pilot study was used to evaluate techniques for determining social relationships between dolphins (chapter 3). A combination of several multivariate statistical techniques best translated social interactions into social relationships. Loglinear analysis was used to determine which interactions could be used to 


\section{Chapter 6: Summary and Conclusions}

differentiate relationships. Multidimensional scaling and hierarchical cluster analysis were then used to categorize the relationships between pairs of dolphins into types. The analysis in chapter 3 showed that behaviors other than the total association between dolphins were necessary to distinguish relationships. The association between a pair of dolphins often reflected more about the relationships each of the dolphins in the pair had with a third dolphin than the relationship they had with each other. Most studies of freeranging dolphins have used only the association between dolphins to determine those dolphins' relationships (e.g. Wells et al. 1987, Wells 1991, Smolker et al. 1992). The results in chapter 3 indicate that the addition of other measures, such as affiliative contact and calf-protection behaviors, is necessary for more subtle relationships to be evaluated.

Three types of relationships were found between the adults in the pilot study. Most pairs of dolphins had what might be described as a baseline relationship, where the dolphins engaged in few interactions. A few pairs of dolphins had a much closer relationship, characterized by a large amount of association and affiliative contact. Other pairs had intermediate relationships, characterized by association, affiliative contact, and sometimes by agonistic interactions. In some cases, these intermediate relationships appeared to be transitional between the other two relationship types. Similarly, the calves in this study were found to have three types of relationships with the adults. With most adults, the calves had very few interactions. Most of the calves had many interactions with their mothers, including association, calf-protection behaviors, nursing, and affiliative contact. However, the last calf born, Lotus, was taken by a female other than his mother as soon as he was born (see chapter 2). He remained with this "allomother" for five days before returning to his mother. This incident allowed the social relationship between mothers and calves to be distinguished from the genetic relationship. Lotus' relationship with his allomother in those five days was similar to the relationships the other calves had with their mothers and equivalent to Lotus' relationship with his mother after he returned to her. In addition, Lotus had an intermediate relationship with some of the adults, which was similar to the adults' intermediate relationship type. 


\subsubsection{TECHNIQUES FOR EVALUATING ACOUSTIC DATA}

To evaluate the acoustic environments experienced by calves, the whistles produced in each calf's acoustic environment need to be compared. As with whistle extraction, these comparisons have traditionally been done qualitatively (e.g. Tyack 1986, Sayigh et al. 1995, Janik \& Slater 1998). A number of studies have performed quantitative, computer-based comparisons, but each study has used a different method with a unique data set (e.g. Buck \& Tyack 1993, McCowan 1995, Smolker \& Pepper in press). Determining which method was most appropriate for a given problem was not possible from those studies (but see Janik in press). Therefore, a comparison of multiple methods was performed using a single data set (chapter 4). Methods for categorizing whistles by extracted acoustic features were compared to methods for categorizing whistles by extracted frequency contours (Buck \& Tyack 1993, Fristrup \& Watkins 1994). Two methods for comparing extracted contours, cross-correlation and dynamic time warping (Buck \& Tyack 1993), were compared. For extracted features and both methods of contour comparisons, a number of statistical categorization methods, including discriminant analysis, hierarchical cluster analysis, k-means cluster analysis, and multidimensional scaling, were tested and compared to the results of visual analysis of the same whistles. The data set was divided into stereotyped whistles and unstereotyped whistles. The categorization methods were tested first on the stereotyped whistles without the un-stereotyped ones and then on the entire data set. Most of the methods performed well with the stereotyped whistles but poorly when the unstereotyped whistles were added. One statistical technique stood out as good at separating stereotyped whistles and robust to the addition of un-stereotyped whistles: hierarchical cluster analysis of dynamic time warping of extracted contours.

Having developed the methods needed for the quantitative evaluation of acoustic environments, the acoustic environments of the calves in the pilot study were evaluated (chapter 5). The whistles collected in the pilot study were extracted and compared using the automatic extraction and quantitative comparison techniques developed and tested in chapter 4. An analysis of the total whistle production during the weeks surrounding the 
Chapter 6: Summary and Conclusions

births of three of the calves showed that the dolphins increased the number of whistles they produced, and the length of those whistles, after the calves were born. They were also more likely to produce whistles at close to the same time, possibly in response to other dolphins whistling. The hierarchical cluster analysis and dynamic time warping showed that the contours of the whistles produced after the calves were born were quantitatively different from the contours produced before the calves were born. The contours produced in the week immediately prior to the birth of each calf were also slightly different from the contours produced at other times before the calves were born. An analysis of signature whistle production in these periods showed that each new mother's signature whistle was produced more often when her calf was alive than at other times. Interestingly, Lotus' biological mother did not increase her signature whistle use during the prolonged alloparenting episode although she did increase her signature whistle use on the days when she was caring for Lotus. This result suggests that the increased signature whistle production is related to actually caring for the calf. However, Lotus' allomother did not increase her signature whistle use during the time that she was caring for Lotus. Therefore, either acoustic involvement in caring for a calf is separate from behavioral involvement, the signature whistle increase is short-lived, or the increased signature whistle use is not solely due to caring for a calf.

This analysis demonstrated that the acoustic environments of the four calves in the pilot study were different (chapter 5). These four calves were born into the same social group within a few weeks of each other. The ability to distinguish between the acoustic environments of calves in a study of vocal learning is essential. This pilot study has shown that the acoustic environments of dolphin calves born at different times in the same group or into different social groups can be expected to be sufficiently different to be used in a study of vocal learning. Vocal learning in the whistle development of dolphin calves born at different captive facilities can therefore be studied in a quantitative manner using the techniques developed in this thesis.

In addition to the quantitative comparison of acoustic environments, two methodological issues were addressed in chapter 5 . One was a quantitative analysis of 
the difference between whistles produced by calves and whistles produced by adults. Calf whistles have been described as "quavery" and lacking in frequency modulation (Caldwell et al. 1990). Whistles known to have been produced by a five-day old calf (Lotus) were compared to whistles believed to have been produced by adults. The calf whistles were found to have less frequency modulation when the bandwidth was evaluated with the extreme values removed. Most of the whistles whose quartile bandwidth was greater than $3 \mathrm{kHz}$ were produced by adults. However, whistles with lower quartile bandwidths could not be definitively assigned to adults or calves. In addition, the use of the visual cue of a stream of bubbles to identify whistling dolphins was evaluated. Several researchers have used whistles associated with this cue as their entire whistle sample (e.g. McCowan 1995, Herzing 1996). In this study, bubblestreamwhistles were found to be extremely rare and to produce a potentially biased sample of whistle types. In addition, bubblestreams could not be unambiguously assigned to specific whistles. Therefore, bubblestreams could not be used to positively identify which dolphin produced a particular whistle. Overall, these results indicate that bubblestreams should not be used in the attempt to gather a sample of identified whistles.

\subsection{FUTURE RESEARCH}

\subsubsection{A STUdy OF VOCAL LEARNING IN WHISTLE DEVELOPMENT}

The techniques developed in this thesis can now be used to study vocal learning in dolphin whistle development (see Figure 5.1). The behavior and vocalizations of calves born in several locations can be sampled in an unbiased manner using the strategy developed and tested in chapter 2. The behavior of the dolphins in each social group can then be used to evaluate the social relationships between the dolphins with the techniques demonstrated in chapter 3. The whistles produced by each group can be extracted and compared using the techniques developed and tested in chapter 4 . The acoustic environments experienced by the calves can be compared to each other and to the vocalizations of the calves as was done in chapter 5 with the data from the pilot study. Only one piece is still missing: the ability to assign whistles to the dolphins that produced 
Chapter 6: Summary and Conclusions

them. If whistles could be assigned to specific dolphins, the whistles of the calves could be compared to the whistles of each dolphin in the social groups, rather than simply to the overall acoustic environments. The calves' social relationships to the dolphins with the most similar whistles would demonstrate the types of social contact that are important to whistle development. In addition, the ability to identify whistlers is essential to the ability to identify the whistles of the calves themselves. Therefore, while all the other necessary techniques for studying vocal learning in dolphins have been developed in this thesis, the ability to identify whistlers is an essential missing piece.

\subsubsection{ReCEnt Advances in Passive Whistle Localization}

When this thesis was started, no method existed to identify whistlers that could distinguish between the whistles of a mother and calf swimming together (see chapter 1 , section 6). This was a major problem to the ability to perform vocal learning studies with dolphins. Since dolphins' vocal repertoires differ in different contexts, the ability to identify whistlers in an undisturbed social setting is necessary to the quantification of a calf's acoustic environment (Janik \& Slater 1998). However, some recent developments in the passive localization of whistles may soon make identifying whistlers possible even at such close range (e.g. Miller \& Tyack 1998). Several researchers have used the passive localization of sounds with an array of hydrophones to identify vocalizing animals (Freitag \& Tyack 1993, Janik 1998). In the wild, the whistler's location can be determined by the phase shift caused by the angle of arrival at a linear array of hydrophones (Miller \& Tyack 1998). A small linear array can be deployed and towed behind a boat during behavioral observations of wild animals (Miller \& Tyack 1998). Real-time analysis of caller locations by beamforming allows this technology to be coupled with behavioral observations to identify whistlers (Miller \& Tyack 1998). The array is designed to determine the angle within $3-4^{\circ}$, which is equivalent to two animals that are 5-10 meters apart 100 meters from the array. With minor modifications, this exciting new technology may allow whistles to be assigned to individuals even during behavioral observations of dolphin mothers and calves. 


\subsubsection{STUDIES IN CAPTIVITY VS. IN THE WILD}

The techniques developed in this thesis can be used to study calves both in captivity and in the wild. Each type of study has its advantages and its disadvantages. The currently available techniques for identifying which dolphin is whistling are more advanced for studies performed in the wild than for studies performed in captivity. However, studies in captivity have an advantage for behavioral observations over studies in the wild. Many captive locations now have facilities with clear underwater viewing. This allows the dolphins' behavior to be recorded in far more detail than can be achieved when the dolphins are observed from the surface in the wild. Conversely, the behavior of dolphins in captivity is constrained by their human handlers and therefore may be slightly different from the normal behavior of free-ranging dolphins. However, comparisons of captive and free-ranging dolphins have found their social behavior to be similar (Samuels \& Gifford 1997). In fact, the inability of captive dolphins to choose their social group is a distinct advantage to the study vocal learning. Wild dolphin calves grow up in a nursery group with their mothers and several other adult females and their calves, but there is some evidence that some of the females in these groups are related (Duffield \& Wells 1991, Wells 1991). Captive groups are more often composed of unrelated animals. Because learning from a related dolphin is difficult to distinguish from inheriting a call from that dolphin, unrelated associates are important to a study of vocal learning.

Captive dolphins' constrained grouping has another advantage as well. Dolphins in the wild can associate with any other dolphin in the population. Determining all the dolphins that might influence a calf's whistle development is therefore impossible in the wild. Dolphins in captivity, on the other hand, can only interact with dolphins with whom they share a pool. Therefore, all the dolphins a calf in captivity can interact with are known and all the types of whistles he hears can be recorded. The social and acoustic environment experienced by a calf born in captivity can be completely quantified. The same could not be done for a calf born in the wild because free-ranging dolphins have the freedom to associate as they please. In addition, the enforced separation of dolphins from 
Chapter 6: Summary and Conclusions

different captive locations allows the comparison of calves whose acoustic environments are completely separate. Because dolphins collected from the same geographical region are often transported to different aquariums, the dolphins in two aquariums, who therefore have no acoustic contact, are often not genetically distinct. This situation, dolphins that have completely separate acoustic environments but are not genetically distinct, is almost impossible to achieve in the wild. In order to find calves in completely separate acoustic environments in the wild, the calves must be born in widely separated locations. The chances are high that such calves would also be genetically distinct. However, the results of the pilot study suggest that calves born in the same population at different times will experience different acoustic stimuli. Therefore, although there are advantages to studying vocal learning in captive dolphins, vocal learning can also be studied in free-ranging populations using the techniques developed in this thesis.

\subsection{LITERATURE CITED}

Altmann, J., 1974. "Observational study of behavior: Sampling methods." Behaviour, 49, pp. $227-267$. Boughman, J.W., 1998. "Vocal learning in greater spear-nosed bats." Proceeds of the Royal Society of London, Series B, 265, pp. 227-233.

Brown, E.D., 1985. "The role of song and vocal imitation among common crows (Corvus brachyrhynchos)." Zeitschrift fur Tierpsychologie, 68, pp. 115-136.

Buck, J.R. and Tyack, P.L., 1993. "A quantitative measure of similarity for Tursiops truncatus signature whistles." Journal of the Acoustical Society of America, 94 (5), pp. 2497-2506.

Caldwell, M.C. and Caldwell, D.K., 1972. "Vocal mimicry in the whistle mode by an Atlantic bottlenosed dolphin." Cetology, 9, pp. 1-8.

Caldwell, M.C. and Caldwell, D.K., 1979. "The whistle of the Atlantic bottlenose dolphin (Tursiops truncatus) - ontogeny." Behavior of marine animals, vol. 3 Cetaceans, eds. H.E. Winn and B.L. Olla. Plenum, New York, NY. pp. 369-401.

Caldwell, M.C., Caldwell, D.K., and Tyack, P.L., 1990. "Review of the signature-whistle hypothesis for the Atlantic bottlenose dolphin." The Bottlenose Dolphin, eds. S. Leatherwood and R.R. Reeves. Academic Press, Inc., New York, NY. pp. 199-234.

Cheney, D., Seyfarth, R., and Smuts, B., 1986. "Social relationships and social cognition in nonhuman primates." Science, 234, pp. 1361-1366.

Duffield, D.A. and Wells, R.S., 1991. "The combined application of chromosome, protien, and molecular data for the investigation of social unit structure and dynamics in Tursiops truncatus." Reports to the International Whaling Commission, Special issue 13, pp. 155-169.

Freitag, L.E. and Tyack, P.L., 1993. "Passive acoustic localization of the Atlantic bottlenose dolphin using whistles and echolocation clicks." Joumal of the Acoustical Society of America, 93(4), pp. 21972205.

Fristrup, K.M. and Watkins, W.A., 1994. Marine animal sound classification. Woods Hole Oceanographic Institution Technical Report No. 94-13, Woods Hole, MA.

Harlow, H.F. and Harlow, M.K., 1962. "Social deprivation in monkeys." Scientific American, $207, \mathrm{pp.}$ 136-146. 
Chapter 6: Summary and Conclusions

Hausberger, M., Richard, M.A., Henry, L., Lepage, L., and Schmidt, I., 1995. "Song sharing reflects the social organization in a captive group of European starlings (Sturnus vulgaris)." Journal of Comparative Psychology, 109, pp. 222-241.

Herzing, D.L., 1996. "Vocalizations and associated underwater behavior of free-ranging Atlantic spotted dolphins, Stenella frontalis and bottlenose dolphins, Tursiops truncatus." Aquatic Mammals, 22.2, pp. 61-79.

Hinde, R.A., 1983. "A conceptual framework." Primate social relationships, an integrated approach, ed. R.A. Hinde. Sinauer Associates, Inc., Sunderland, MA. pp. 1-7.

Janik, V.M. and Slater, P.J.B., 1997. "Vocal learning in mammals." Advances in the Study of Behavior, 26, pp. 59-99.

Janik, V.M. and Slater, P.J.B., 1998. "Context-specific use suggests that bottlenose dolphin signature whistles are cohesion calls." Animal behaviour, 56: 4, pp. 829-838.

Janik, V.M., 1998. Functional and organizational aspects of vocal repertoires in bottlenose dolphins, Tursiops truncatus. Ph.D. Dissertation, University of St. Andrews.

Janik, V.M., in press. "Pitfalls in the categorization of behaviour: a comparison of dolphin whistle classification methods." Animal Behaviour.

Jones, G. and Ransome, R.D., 1993. "Echolocation calls of bats are influenced by maternal effects and change over a lifetime." Proceeds of the Royal Society of London, Series B, 252, pp. 125-128.

Locke, J.L. and Snow, C., 1997. "Social influences on vocal learning in human and nonhuman primates." Social influences on vocal learning, eds. C.T. Snowdon and M. Hausberger. Cambridge University Press, New York, NY. pp. 274-92.

Marler, P., 1970. "A comparative approach to vocal learning: Song development in white-crowned sparrows." Journal of Comparative Physiology and Psychology, 71, pp. 1-25.

McCowan, B., 1995. "A new quantitative technique for categorizing whistles using simulated signals and whistles from captive bottlenose dolphins (Delphinidae, Tursiops truncatus)." Ethology, 100, pp. 177-193.

Miller, P.J. and Tyack, P.L., 1998. "A small towed beamforming array to identify vocalizing resident killer whales (Orcinus orca) concurrent with focal behavioral observations." Deep Sea Research, 45, pp.1389-1405.

Richards, D.G., Wolz, J.P., and Herman, L.M., 1984. "Vocal mimicry of computer-generated sounds and vocal labeling of objects by a bottlenose dolphin, Tursiops truncatus." Journal of Comparative Psychology, 98(1), pp. 10-28.

Samuels, A. and Gifford, T., 1997. "A quantitative assessment of dominance relations among bottlenose dolphins." Marine Mammal Science, 13, pp. 70-99.

Sayigh, L.S., 1992. Development and functions of signature whistles of free-ranging bottlenose dolphins, Tursiops truncatus. Ph.D. Dissertation, MIT/WHOI Joint Program, WHOI 92-37.

Sayigh, L.S., Tyack, P.L., Wells, R.S., and Scott, M.D., 1990. "Signature whistles of free-ranging bottlenose dolphins Tursiops truncatus: Stability and mother-offspring comparisons." Behavioral Ecology and Sociobiology, 26, pp. 247-260.

Sayigh, L.S., Tyack, P.L., Wells, R.S., Scott, M.D., and Irvine, A.B., 1995. "Sex difference in whistle production in free-ranging bottlenose dolphins, Tursiops truncatus." Behavioral Ecology and Sociobiology, 36, pp. 171-177.

Seyfarth, R.M. and Cheney, D.L., 1997. "Some general features of vocal development in nonhuman primates." Social influences on vocal development, eds. C.T. Snowdon and M. Hausberger. Cambridge University Press, New York, NY. pp. 249-74.

Smolker, R.A., Richards, A.F., Connor, R.C., and Pepper, J.W., 1992. "Sex differences in patterns of association among Indian Ocean bottlenose dolphins." Behaviour, 123(1/2), pp. 38-69.

Smolker, R.S. and Pepper, J., in press. "Whistle convergence among allied male bottlenose dolphins." Ethology.

Snowdon, C.T. and Hausberger, M., 1997. Social influences on vocal development. Cambridge University Press, New York, NY. 
Chapter 6: Summary and Conclusions

Tyack, P.L. and Sayigh, L.S., 1997. "Vocal learning in cetaceans." Social influences on vocal development, eds. C.T. Snowdon and M. Hausberger. Cambridge University Press, New York, NY. pp. 208-33.

Tyack, P.L., 1986. "Whistle repertoires of two bottlenosed dolphins, Tursiops truncatus: Mimicry of signature whistles?" Behavioral Ecology and Sociobiology, 18, pp. 251-257.

Tyack, P.L., 1997. "Development and social functions of signature whistles in bottlenose dolphins Tursiops truncatus." Bioacoustics, 8, pp. 21-46.

Wells, R.S., 1991. "The role of long-term study in understanding the social structure of a bottlenose dolphin community." Dolphin Societies, eds. K. Pryor and K.S. Norris. University of California Press, Berkeley, CA. pp. 199-235.

Wells, R.S., Scott, M.D., and Irvine, A.B., 1987. "The social structure of free-ranging bottlenose dolphins." Current Mammology, ed. H.H. Genoways. Plenum Press, New York, NY. pp. 247-305.

West, M.J., King, A.P., and Freeberg, T.M., 1997. "Building a social agenda for the study of bird song." Social influences on vocal development, eds. C.T. Snowdon and M. Hausberger. Cambridge University Press, New York, NY. pp. 41-56.

Whitehead, H., 1997. "Analyzing animal social structure." Animal behaviour, 53, pp. 1053-1067.

Winter, P., Handley, P., Ploog, D., and Schott, D., 1973. "Ontogeny of squirrel monkey calls under normal conditions and under acoustic isolation." Behaviour, 47, pp. 230-239. 


\section{APPENDIX 1: BEHAVIORAL RECORDINGS}

Because the pilot study was exploratory, many more behaviors were recorded than were used in the final analysis. Some of these behaviors were excluded from the analysis because they could not be recorded reliably. Others were excluded because they happened too infrequently. Still others were not used because they were redundant with the measures being used, such as approaches with time spent together, or did not vary sufficiently between animals. This appendix includes samples of the complete configurations used to record the behavioral data and sample data output of the program (the Observer 3.0). Three example configurations and sample data files are included, two calf configurations: one for Vicky's calf, and one for Lotus; and one adult configuration: for Delphi before her calf was born. In these configurations, the codes are the actual codes that were entered during data collection. When labels that differ from the codes are specified, these are the labels put into the data files by the Observer. When labels are not specified, the labels were the same as the codes.

In addition, a configuration and sample data for the recording of bubblestreams during the digitization of acoustic recordings is included in the final section.

\section{SECTION 1: VICKY'S CALF}

\section{CONFIGURATION}

\subsubsection{Data Collection Methods}

\begin{tabular}{|ll|}
\hline Data Collection & Method Used \\
\hline Sampling & Focal sampling \\
Recording & Continuous \\
Number of Actors & Multiple \\
Maximum duration of observations & 10 minutes \\
Maximum duration based on & Elapsed time \\
Timing resolution & 1 second \\
Timing of duration events & Press for start/end \\
\hline
\end{tabular}


Appendix 1: Behavioral Recordings

\subsubsection{Subjects}

\begin{tabular}{|llll|}
\hline Subject Name & Code & Label & Comments \\
\hline Vicky's calf & $\mathrm{c}$ & vica & Focal calf \\
Vicky & $\mathrm{v}$ & vick & Focal mother \\
Vicky + Calf & $\mathrm{h}$ & v+c & Vicky and Vicky's calf together \\
Lotty & $\mathrm{l}$ & loty & Adult dolphin \\
Delphi & $\mathrm{p}$ & delp & Adult dolphin \\
Nephele & $\mathrm{n}$ & neph & Adult dolphin \\
Trainer & $\mathrm{t}$ & tran & Human trainers \\
Unknown & $\mathrm{u}$ & unkn & For when one of interactors is uncertain \\
None & $\mathrm{x}$ & none & For when behavior is not directed to anyone \\
All & $\mathrm{a}$ & all & For when behavior is directed to all dolphins \\
Snobban & $\mathrm{o}$ & snob & South African fur seal \\
Gate & $\mathrm{g}$ & gate & Dolphins in gate to Holding Pool \\
Window & $\mathrm{w}$ & wind & To windows, generally to people outside pool \\
\hline
\end{tabular}

\subsubsection{Behavioral Elements}

All behavioral elements have a subject and up to two modifiers. The subjects are listed in the previous section. The modifiers are listed in the next section. When the modifier is listed as "subjects", the subject list from section 1.1.2 is used for the modifier. If the modifier column is left blank, that modifier was not used for that element. Behaviors were defined as states, where the duration of the behavior was recorded, or events where only the time of the behavior was recorded. The behaviors were divided into sections.

Approach/Leave

\begin{tabular}{|lccccl|}
\hline Element Name & Code & Type & Modifier 1 & Modifier 2 & Definition \\
\hline Approach & ap & Event & Subjects & Approach to within 1 m of \\
Mutual approach & ma & Event & Subjects & Both dolphins approach at same time \\
Leave & Iv & Event & Subjects & Leave to more than 1 m of \\
Mutual leave & ml & Event & Subjects & Both dolphins leave at same time \\
\hline
\end{tabular}

Breathing

\begin{tabular}{|c|c|c|c|c|c|}
\hline Element Name & Code & Type & Modifier 1 & Modifier 2 & Definition \\
\hline Breathe together & bt & Event & Animals1 & Animals2-2 & Breathe at same time as \\
\hline Follow breath & $\mathrm{fb}$ & Event & Subjects & & $\begin{array}{l}\text { Breathe directly after, when } \\
\text { swimming together }\end{array}$ \\
\hline Before breath & $\mathrm{bb}$ & Event & Subjects & & $\begin{array}{l}\text { Breathe directly before, when } \\
\text { swimming together }\end{array}$ \\
\hline Breathe separately & bs & Event & & & Breathe alone \\
\hline
\end{tabular}


Behaviors

\begin{tabular}{|c|c|c|c|c|c|}
\hline Element Name & Code & Type & Modifier 1 & Modifier 2 & Definition \\
\hline White water & ww & Event & Subjects & & Unidentifiable agonistic interaction \\
\hline Bite & bi & Event & Subjects & Body pos 2 & Bite recipient on marked body part. \\
\hline Hit & hi & Event & Subjects & Body pos 1 & Hit recipient, both body parts marked \\
\hline Pin & pi & Event & Subjects & & $\begin{array}{l}\text { Pin recipient to floor of pool } \\
\text { (generally calves) }\end{array}$ \\
\hline Chase & $\mathrm{ch}$ & Event & Subjects & & Swim after quickly \\
\hline Flee & ba & Event & Subjects & & Swim away from quickly \\
\hline Flinch & fh & Event & Subjects & & Cower away from \\
\hline Thrash & th & Event & Subjects & & Make thrashing movement \\
\hline General threat & gt & Event & Subjects & & Combination of threat behaviors \\
\hline Mutual threat & $\mathrm{tm}$ & Event & Subjects & & $\begin{array}{l}\text { General threat performed } \\
\text { simultaneously by both parties }\end{array}$ \\
\hline Distant threat & $\mathrm{dt}$ & Event & Subjects & & General threat from more than $3 \mathrm{~m}$ \\
\hline $\begin{array}{l}\text { Mutual distant } \\
\text { threat }\end{array}$ & md & Event & Subjects & & $\begin{array}{l}\text { Distant threat performed } \\
\text { simultaneously by both parties }\end{array}$ \\
\hline Tail slap & ts & Event & Subjects & & Slap surface of water with tail \\
\hline Tail flick & tf & Event & Subjects & & Make tail-slap motion under water \\
\hline Head jerk & hj & Event & Subjects & & Nod head sharply at \\
\hline Jaw clap & $\mathrm{jc}$ & Event & Subjects & & $\begin{array}{l}\text { Close mouth sharply, producing a } \\
\text { loud sound }\end{array}$ \\
\hline Mouth open & mo & Event & Subjects & & Open mouth to (threat behavior) \\
\hline Flip & fl & Event & Subjects & & Flip calf out of water with rostrum \\
\hline $\begin{array}{l}\text { Genital } \\
\text { propulsion }\end{array}$ & $\mathrm{gp}$ & Event & Subjects & & $\begin{array}{l}\text { Push calf's genital region with } \\
\text { rostrum so calf is propelled forward }\end{array}$ \\
\hline Carry & $\mathrm{ca}$ & Event & Subjects & Side up & Lift calf out of water on belly \\
\hline Retrieve & rv & Event & Subjects & & $\begin{array}{l}\text { Force calf to come with by herding } \\
\text { or swimming quickly by }\end{array}$ \\
\hline Push & pu & Event & Subjects & Body pos 1 & Push against recipient \\
\hline Wander & wa & Event & Subjects & Distance & $\begin{array}{l}\text { Calf leave nearest neighbor for less } \\
\text { than } 15 \text { seconds }\end{array}$ \\
\hline Nurse & nu & Event & Subjects & & Lock onto mammaries \\
\hline Attempt to nurse & an & Event & Subjects & & Attempt to lock onto mammaries \\
\hline Mammary nudge & $\mathrm{mn}$ & Event & Subjects & & Bump mammaries with head \\
\hline Nuzzle & $\mathrm{nz}$ & Event & Subjects & Body pos 2 & Rub rostrum into marked body part \\
\hline Casual touch & $\mathrm{ct}$ & Event & Subjects & Body pos 1 & Brief touch \\
\hline Rub & $\mathrm{rb}$ & Event & Subjects & Body pos 1 & Gentle moving contact \\
\hline Prolonged rub & $\mathrm{pr}$ & Event & Subjects & Repeated & Rub that lasts more 3 seconds \\
\hline Contact swim & $\operatorname{cs}$ & Event & Subjects & Body pos 1 & Swim while touching \\
\hline Prolonged cs & $\mathrm{pc}$ & Event & Subjects & Body pos 1 & Contact swim lasting more than $10 \mathrm{~s}$. \\
\hline Contact rest & $\mathrm{cr}$ & Event & Subjects & Body pos 1 & Rest while touching \\
\hline Follow swim & fs & Event & Subjects & Distance & $\begin{array}{l}\text { Follow behind while not actually } \\
\text { swimming together }\end{array}$ \\
\hline Ventrum present & $\mathrm{vp}$ & Event & Subjects & & Turn ventrum toward \\
\hline Ventrum away & va & Event & Subjects & & Turn ventrum away from \\
\hline Mount & $\mathrm{mt}$ & Event & Subjects & Body pos 1 & $\begin{array}{l}\text { Rub or touch ventral or genital region } \\
\text { on, often with an erection }\end{array}$ \\
\hline Look at & la & Event & Subjects & Body pos 2 & Look at body part of recipient \\
\hline General look & gl & Event & Subjects & & Look in direction of recipient \\
\hline
\end{tabular}


Appendix 1: Behavioral Recordings

\section{$\underline{\text { States }}$}

\begin{tabular}{|lccccl|}
\hline Element Name & Code & Type & Modifier 1 & Modifier 2 & Definition \\
\hline Swim together & st & State & Animals1 & Animals2 & Swim within 1 m \\
Below ventrum & cv & State & Animals1 & Animals2 & Calf under nearest neighbor \\
At head & ah & State & Animals1 & Animals2 & Calf around nearest neighbor's head \\
Slipstreaming & sl & State & Animals1 & Animals2 & Calf riding next to nearest neighbor, \\
& & & & & without swimming \\
Circling & ir & State & Animals1 & Animals2 & Calf circling nearest neighbor \\
At side & as & State & Animals1 & Animals2 & Calf next to nearest neighbor's side \\
At tail/behind & at & State & Animals1 & Animals2 & Calf behind nearest neighbor \\
At dorsal & cd & State & Animals1 & Animals2 & Calf next to nearest neighbor's dorsal \\
Rest together & rt & State & Animals1 & Animals2 & Rest within 1 m \\
Alone & al & State & Distance & & Swim more than 1 m away from any \\
& & & & & other dolphins \\
Train & tr & State & Animals1 & Animals2-2 & Be trained by same human trainer \\
Socialize & so & State & Animals1 & Animals2 & Interact socially, generaly agonistic \\
Mom swim tog. & ms & State & Animals1 & Animals2-2 & Swim together code for focal mother \\
Mom rest tog. & mr & State & Animals1 & Animals2-2 & Rest together code for focal mother \\
Mom socialize & mc & State & Animals1 & Animals2-2 & Socialize code for focal mother \\
In gate rest tog. & gr & State & Animals1 & Animals2-2 & Rest together in gate to Holding \\
In gate alone & ga & State & & & Rest alone in gate to Holding \\
Lost & ls & State & & & Focal cannot be seen from station \\
\hline
\end{tabular}

Trainer

\begin{tabular}{|c|c|c|c|c|c|}
\hline Element Name & Code & Type & Modifier 1 & Modifier 2 & Definition \\
\hline Present & $==$ & State & & & Human trainer present at pool \\
\hline Absent & -- & State & & & No human trainers present \\
\hline
\end{tabular}

\section{Other}

\begin{tabular}{|c|c|c|c|c|c|}
\hline Element Name & Code & Type & Modifier 1 & Modifier 2 & Definition \\
\hline Unspecified & $\mathrm{xx}$ & Event & Subjects & & Behavior for which no code exists \\
\hline Whistle & wh & Event & & & $\begin{array}{l}\text { Produce a whistle that can be heard } \\
\text { and identified (generally by blowhole } \\
\text { movement) }\end{array}$ \\
\hline Bubblestream & bu & Event & & & $\begin{array}{l}\text { Produce a bubblestream associated } \\
\text { with a whistle }\end{array}$ \\
\hline $\begin{array}{l}\text { Command } \\
\text { behavior }\end{array}$ & $\mathrm{cb}$ & Event & Calf relative & & $\begin{array}{l}\text { Perform a behavior under } \\
\text { instructions of the trainers }\end{array}$ \\
\hline
\end{tabular}




\subsubsection{Modifiers}

Modifiers are lists of possible animals or body parts, or other modifications, on the

behavioral events. Because a modifier list needs to include all the possible modifications, some of these lists are excessively long, such as the list of all possible body parts.

Therefore, for the very long lists, only some of the codes are actually listed.

Animals 1

This list is designed to record whether Delphi, Nephele, and Lotty are swimming with the focal.

\begin{tabular}{|ll|}
\hline Modifier Name & Code \\
\hline None & xxx \\
Lotty & axx \\
Nephele & xax \\
Delphi & xxa \\
Lotty \& Nephele & aax \\
Lotty \& Delphi & axa \\
Nephele \& Delphi & xaa \\
Lotty, Nephele, \& Delphi & aaa \\
\hline
\end{tabular}

\section{Animals 2}

This list is designed to record who Vicky's calf's nearest neighbor is and the distance to Vicky.

\begin{tabular}{|lccl|}
\hline Modifier Name & Code & Nearest & Distance to Vicky \\
\hline Lotty-2 & & Neighbor & Next to, no intervening dolphins \\
Lotty-3 & 12 & Lotty & Next no \\
Lotty-4 & 13 & Lotty & In same group but with another dolphin between them \\
Nephele-2 & 14 & Lotty & Vicky not in group \\
Nephele-3 & $\mathrm{n} 2$ & Nephele & Next to, no intervening dolphins \\
Nephele-4 & $\mathrm{n} 3$ & Nephele & In same group but with another dolphin between them \\
Delphi-2 & $\mathrm{n} 4$ & Nephele & Vicky not in group \\
Delphi-3 & $\mathrm{p} 2$ & Delphi & Next to, no intervening dolphins \\
Delphi-4 & $\mathrm{p} 3$ & Delphi & In same group but with another dolphin between them \\
Vicky-1 & $\mathrm{p} 4$ & Delphi & Vicky not in group \\
\hline
\end{tabular}

\section{Animals 2-2}

This list is designed to record whether Vicky or Vicky's Calf are involved when the nearest neighbor does not need to be recorded (see behavior states).

\begin{tabular}{|lcc|}
\hline Modifier Name & Code & Label \\
\hline Neither & $\mathrm{x}$ & none \\
Vicky's Calf & $\mathrm{c}$ & $\mathrm{c}$ \\
Vicky & $\mathrm{v}$ & $\mathrm{v}$ \\
\hline
\end{tabular}




\section{Body Position 1}

The body parts in this list are arranged so that the first part refers to the subject and the second to the recipient. Only some examples are listed here. The list continues including all recombinations of the body parts that are used in this partial list. There are 99 combinations.

\begin{tabular}{|lc|}
\hline Modifier Name & \\
\hline Back to back & Code \\
Back to fin & bb \\
Back to genital & bf \\
Back to head & bg \\
Back to rostrum & bh \\
Back to side & br \\
Back to tail & bs \\
Back to ventrum & bt \\
Back to unknown & bv \\
Dorsal to genital & bx \\
Dorsal to side & dg \\
Dorsal to tail & ds \\
Dorsal to ventrum & dt \\
\hline
\end{tabular}

\section{Body Position 2}

The body parts in this list refer to the recipient only. These are used for behaviors where the body part used by the subject is part of the definition of the behavior.

\begin{tabular}{|lc|}
\hline Modifier Name & Code \\
\hline To fin & $\mathrm{f}$ \\
To peduncle & $\mathrm{p}$ \\
To head & $\mathrm{h}$ \\
To tail & $\mathrm{t}$ \\
To side & $\mathrm{s}$ \\
To dorsal & $\mathrm{d}$ \\
To ventrum & $\mathrm{v}$ \\
To genitals & $\mathrm{g}$ \\
To chin & $\mathrm{c}$ \\
To rostrum & $\mathrm{r}$ \\
To face & $\mathrm{e}$ \\
To back & $\mathrm{b}$ \\
Unspecified & $\mathrm{x}$ \\
\hline
\end{tabular}

\section{Distance}

\begin{tabular}{|lcc|}
\hline Modifier Name & Code & Label \\
\hline less than $1 \mathrm{~m}$ & 1 & $>1$ \\
$1-3 \mathrm{~m}$ & 2 & 2 \\
more than $3 \mathrm{~m}$ & 3 & $>3$ \\
in different pool & 4 & DP \\
\hline
\end{tabular}


Side up

This is for "carry" only.

\begin{tabular}{|lccl|}
\hline Modifier Name & Code & Label & Definition \\
\hline Upside down & $\mathrm{u}$ & updo & Calf's belly out of the water \\
Right-side up & $\mathrm{r}$ & righ & Calf's head out of the water \\
\hline
\end{tabular}

\section{Calf relative}

This is for "command behavior" only.

\begin{tabular}{|lccl|}
\hline Modifier Name & Code & Label & Definition \\
\hline Calf ignoring & i & ign & Calf ignoring trained behavior \\
Calf following & f & foll & Calf following mother as she performs behavior \\
Calf imitating & m & imit & Calf attempting to imitate behavior \\
Calf circling & c & circ & Calf circling near mother as she is trained \\
\hline
\end{tabular}

\section{Repeated}

This is for "prolonged rub" only.

\begin{tabular}{|lccl|}
\hline Modifier Name & Code & Label & Definition \\
\hline Repeated & $\mathrm{r}$ & rep & The same rub repeated more than 3 times in a row \\
Alternating & $\mathrm{a}$ & alt & The same rub alternating subject and recipient \\
Repeated \& alternating & $\mathrm{c}$ & r/a & $\begin{array}{l}\text { The same rub alternating subject and recipient, repeated } \\
\text { more than } 3 \text { times in a row }\end{array}$ \\
prolonged & p & prol & A rub that lasts more than 3 seconds. \\
\hline
\end{tabular}

\subsubsection{Channels}

The Observer only allows data analysis of classes of behavioral element in combination with subjects that are specified as "channels".

\begin{tabular}{|ll|}
\hline Subject & Behavioral Class \\
\hline Vicky & States \\
Vicky & Approaches/Leaves \\
Vicky & Behaviors \\
Vicky & Other \\
Trainer & Trainer \\
Vicky's calf & States \\
Vicky's calf & Approaches/Leaves \\
Vicky's calf & Breathing \\
Vicky's calf & Behaviors \\
Vicky's calf & Other \\
Vicky \& calf & States \\
Vicky \& calf & Approaches/Leaves \\
Vicky \& calf & Behavior \\
\hline
\end{tabular}


Appendix 1: Behavioral Recordings

\subsubsection{Independent Variables}

The Observer allows variables to be recorded that are only recorded once in each sample.

\begin{tabular}{|c|c|c|}
\hline Variable Name & Label & Definition \\
\hline Lighting/visibility & light & Notes on the ability to observe the dolphins \\
\hline Location & location & $\begin{array}{l}\text { Whether the dolphins are being observed from the } \\
\text { Lagoon or the Holding Pool }\end{array}$ \\
\hline Gate & gate & Whether the gate to Holding is open or closed \\
\hline Holding & holding & What dolphins are in the Holding Pool \\
\hline People & people & Whether people are in the viewing area \\
\hline Listening to hydrophone & list-hy & Whether the observer is listening to the hydrophone \\
\hline Hydrophone recording & hy-recrd & The tape and start time of the sample on the recording \\
\hline Additional comments & comments & Any other notes \\
\hline
\end{tabular}

\section{SAMPLE DATA}

\begin{tabular}{|c|c|c|c|}
\hline \multicolumn{4}{|c|}{ VICKYC.CNF } \\
\hline \multicolumn{4}{|c|}{$05-22-1995$} \\
\hline \multicolumn{4}{|c|}{$09: 02: 25$} \\
\hline \multicolumn{4}{|c|}{ \{indvar $\}$} \\
\hline \multicolumn{4}{|c|}{ partly cloudy } \\
\hline \multicolumn{4}{|l|}{ lag } \\
\hline \multicolumn{4}{|c|}{ in } \\
\hline \multicolumn{4}{|c|}{ closed } \\
\hline \\
\hline \multicolumn{4}{|l|}{$Y$} \\
\hline \multicolumn{4}{|c|}{ tp77 1.22 .55} \\
\hline \multicolumn{4}{|c|}{$\begin{array}{l}\text { C born at } 7: 40 \text { am } \\
\text { start }\end{array}$} \\
\hline 0 & vick, ms & , xax &, $\mathrm{C}$ \\
\hline 0 & tran,-- & & \\
\hline 0 & vica, ca & , $\operatorname{xax}$ &, $\mathrm{v1}$ \\
\hline 0 & vica, $g c$ & & \\
\hline 8 & vica, bt &, $\mathrm{xxx}$ &, $\mathrm{v}$ \\
\hline 15 & vica, bt &, $\mathrm{xxx}$ &, $\mathrm{V}$ \\
\hline 27 & vica, as & , $x a x$ &, $\mathrm{v1}$ \\
\hline 41 & vick, vp & , vica & \\
\hline 50 & vica, an & , vick & \\
\hline 64 & vica, cd & , $\operatorname{xax}$ &, $\mathrm{v1}$ \\
\hline 82 & vica, bt &, $\mathrm{xxx}$ &, $\mathrm{v}$ \\
\hline 92 & vica, bt &,$x \times x$ &, $\mathrm{C}$ \\
\hline 95 & vica, ca &, $\mathrm{xxx}$ & v1 \\
\hline 102 & vick, ms &,$\times \times x$ &, $\mathrm{C}$ \\
\hline 113 & vica, bt & $\mathrm{x} \times \mathrm{x}$ &, $\mathrm{v}$ \\
\hline 132 & neph, ap & , vick & \\
\hline 134 & vick, ms & , $x a x$ &, $\mathrm{C}$ \\
\hline 137 & vica,cd & , xax &,$v 1$ \\
\hline 149 & vica,bt &, $\mathrm{xxx}$ &, $\mathrm{V}$ \\
\hline 163 & vica, bt &,$x a x$ &, $\mathrm{~V}$ \\
\hline 178 & vica, rb & , vick & , ss \\
\hline 181 & vica, bt & , $\operatorname{xax}$ &,$v$ \\
\hline 199 & vica,cv &,$x a x$ &, $\mathrm{v1}$ \\
\hline 206 & vica, as & , xax &,$v 1$ \\
\hline 213 & vica, cd & , xax & v1 \\
\hline
\end{tabular}

\begin{tabular}{|c|c|c|}
\hline 223 & vica,sl & $, \operatorname{xax}, \mathrm{v} 1$ \\
\hline 27 & vica, bt & $, \mathrm{xax}, \mathrm{v}$ \\
\hline 50 & vica, ct & , vick, ss \\
\hline 2 & vica, bt & $, x a x, v$ \\
\hline 8 & vica,bt & $, \mathrm{xxX}, \mathrm{v}$ \\
\hline & vica,cd & $, \mathrm{xax}, \mathrm{v} 1$ \\
\hline 39 & vica, bt & $, \mathrm{XxX}, \mathrm{V}$ \\
\hline & $\mathrm{v}+\mathrm{C}, 1 \mathrm{v}$ & neph \\
\hline 5 & vica,cd & $, \mathrm{xxx}, \mathrm{V} 1$ \\
\hline & vick, ms & $, \mathrm{XxX}, \mathrm{C}$ \\
\hline 2 & vica,bt & $, \mathrm{xxx}, \mathrm{V}$ \\
\hline & vica,bt & $, \mathrm{XxX}, \mathrm{V}$ \\
\hline & $\{$ susp $\}$ & \\
\hline & $\{$ resu $\}$ & \\
\hline & vica,bt & $, \operatorname{xax}, v$ \\
\hline & neph, ap & , vick \\
\hline 34 & vick, ms & $, x a x, c$ \\
\hline & vica, as & $, \operatorname{xax}, \mathrm{v} 1$ \\
\hline & vica,bt & $, \mathrm{Xxx}, \mathrm{V}$ \\
\hline & vica, bt & $, \mathrm{XxX}, \mathrm{V}$ \\
\hline 8 & vica, bt & $, \mathrm{XXX}, \mathrm{V}$ \\
\hline 7 & vica, bt & $, x \times x, v$ \\
\hline 55 & vica, bt & $, \mathrm{xxx}, \mathrm{v}$ \\
\hline & vica, bt & $, x a x, v$ \\
\hline 79 & vica, cs & , vick, ss \\
\hline 6 & vica, cs & , vick, fs \\
\hline 16 & vica, ct & , vick, ss \\
\hline 7 & vica, bt & $, x \times x, V$ \\
\hline 29 & vica, sl & $, \operatorname{xax}, v 1$ \\
\hline 6 & vica, cd & $, \operatorname{xax}, v 1$ \\
\hline 12 & vica,sI & $, \operatorname{xax}, \mathrm{v} 1$ \\
\hline 18 & vica, cs & , vick, Es \\
\hline 3 & vica,bt & $, X \times X, V$ \\
\hline 569 & vica, pc & , vick, fs \\
\hline 572 & vica, bt & $, \mathrm{x} \times \mathrm{x}, \mathrm{v}$ \\
\hline 589 & vica, bt & $, \mathrm{XxX}, \mathrm{V}$ \\
\hline 598 & $\begin{array}{l}\text { neph, IV } \\
\text { \{end\} }\end{array}$ & , vick \\
\hline
\end{tabular}




\section{SECTION 2: LOTUS}

\subsection{CONFIGURATION}

The configurations for the various dolphins are substantially similar. Therefore, only the changes will be listed in this summary of the configuration. This is the configuration for recording Lotus when he was living in the Lagoon with only Lotty and Vicky.

\subsubsection{Data Collection Methods}

The data collection methods were the same as before.

\subsubsection{Subjects}

\begin{tabular}{|llll|}
\hline Subject Name & Code & Label & Comments \\
\hline Lotus & $\mathrm{f}$ & calf & Focal calf \\
Lotty & $\mathrm{l}$ & loty & Focal mother \\
Lotty + Lotus & $\mathrm{i}$ & $\mathrm{l}+\mathrm{f}$ & Lotty and Lotus together \\
Vicky & $\mathrm{v}$ & vick & Adult dolphin \\
Vicky + Lotus & $\mathrm{k}$ & $\mathrm{v}+\mathrm{f}$ & Vicky and Lotus together \\
Lotty + Vicky & $\mathrm{j}$ & $\mathrm{l}+\mathrm{v}$ & Lotty and Vicky together \\
Trainer & $\mathrm{t}$ & tran & Human trainers \\
Unknown & $\mathrm{u}$ & unkn & For when one of interactors is uncertain \\
None & $\mathrm{x}$ & none & For when behavior is not directed to anyone \\
All & $\mathrm{a}$ & all & For when behavior is directed to all dolphins \\
Snobban & $\mathrm{o}$ & snob & South African fur seal \\
Gate & $\mathrm{g}$ & gate & Dolphins in gate to Holding Pool \\
Window & $\mathrm{w}$ & wind & To windows, generally to people outside pool \\
\hline
\end{tabular}

\subsubsection{Behavioral Elements}

Most of the behavioral elements were all exactly the same as in the previous configuration. They were divided into the following classes:
1. Approaches/Leaves
2. Breathing
3. Behaviors
4. States
5. Trainer
6. Other.

The only exception was the modifiers for the states were different in this configuration. 
Appendix 1: Behavioral Recordings

$\underline{\text { States }}$

\begin{tabular}{|lcclll|}
\hline Element Name & Code & Type & Modifier 1 & Modifier 2 & Definition \\
\hline Swim together & st & State & Subjects & Babysitter & Swim within 1 m \\
Below ventrum & cv & State & Subjects & Babysitter & Calf under nearest neighbor \\
At head & ah & State & Subjects & Babysitter & Calf around nearest neighbor's head \\
Slipstreaming & sl & State & Subjects & Babysitter & $\begin{array}{l}\text { Calf riding next to nearest neighbor, } \\
\text { without swimming }\end{array}$ \\
& & & & & \\
Circling & ir & State & Subjects & Babysitter & Calf circling nearest neighbor \\
At side & as & State & Subjects & Babysitter & Calf next to nearest neighbor's side \\
At tail/behind & at & State & Subjects & Babysitter & Calf behind nearest neighbor \\
At dorsal & cd & State & Subjects & Babysitter & Calf next to nearest neighbor's dorsal \\
Rest together & rt & State & Subjects & Babysitter & Rest within 1 m \\
Alone & al & State & Distance & & Swim more than 1 m away from any \\
& & & & & other dolphins \\
Train & tr & State & Subjects & & Be trained by same human trainer \\
Socialize & so & State & Subjects & Babysitter & Interact socially, generaly agonistic \\
Mom swim tog. & ms & State & Subjects & & Swim together code for focal mother \\
Mom rest tog. & mr & State & Subjects & & Rest together code for focal mother \\
Mom socialize & mc & State & Subjects & & Socialize code for focal mother \\
In gate rest tog. & gr & State & Subjects & & Rest together in gate to Holding \\
In gate alone & ga & State & & & Rest alone in gate to Holding \\
Lost & ls & State & & & Focal cannot be seen from station \\
\hline
\end{tabular}

\subsubsection{Modifiers}

The modifiers were divided into the following classes:

1. Body position 1

2. Body position 2

3. Side up

4. Calf relative

5. Distance

6. Repeated

7. Babysitter.

The first 6 classes were the same as in the previous configuration. The $7^{\text {th }}$ was new.

Babysitter (i.e. Nearest Neighbor)

\begin{tabular}{|lcccl|}
\hline Modifier Name & Code & Label & $\begin{array}{c}\text { Nearest } \\
\text { Neighbor }\end{array}$ & Distance to Lotty \\
\hline Lotty & l & loty & Lotty & Lotty is nearest neighbor. \\
Vicky, Lotty-side & i & vbyl & Vicky & Lotus on Lotty's side of Vicky. \\
Vicky, Lotty-away & o & vfrl & Vicky & $\begin{array}{l}\text { Lotus on opposite side of Vicky from } \\
\end{array}$ \\
& & & & Lotty \\
Vicky, under & u & vund & Vicky & Lotus under Vicky, Lotty in group \\
Vicky, no Lotty & v & vick & Vicky & Lotty not in group \\
Both & b & both & Vicky \& Lotty & Lotus equidistant between Vicky \& Lotty \\
\hline
\end{tabular}




\subsubsection{Channels}

\begin{tabular}{|ll|}
\hline Subject & Behavioral Class \\
\hline Lotty & States \\
Lotty & Approaches/Leaves \\
Lotty & Behaviors \\
Lotty & Other \\
Trainer & Trainer \\
Lotus & States \\
Lotus & Approaches/Leaves \\
Lotus & Breathing \\
Lotus & Behaviors \\
Lotus & Other \\
Lotty \& Lotus & States \\
Lotty \& Lotus & Approaches/Leaves \\
Lotty \& Lotus & Behavior \\
\hline
\end{tabular}

\subsubsection{Independent Variables}

The independent variables were the same as in the previous configuration.

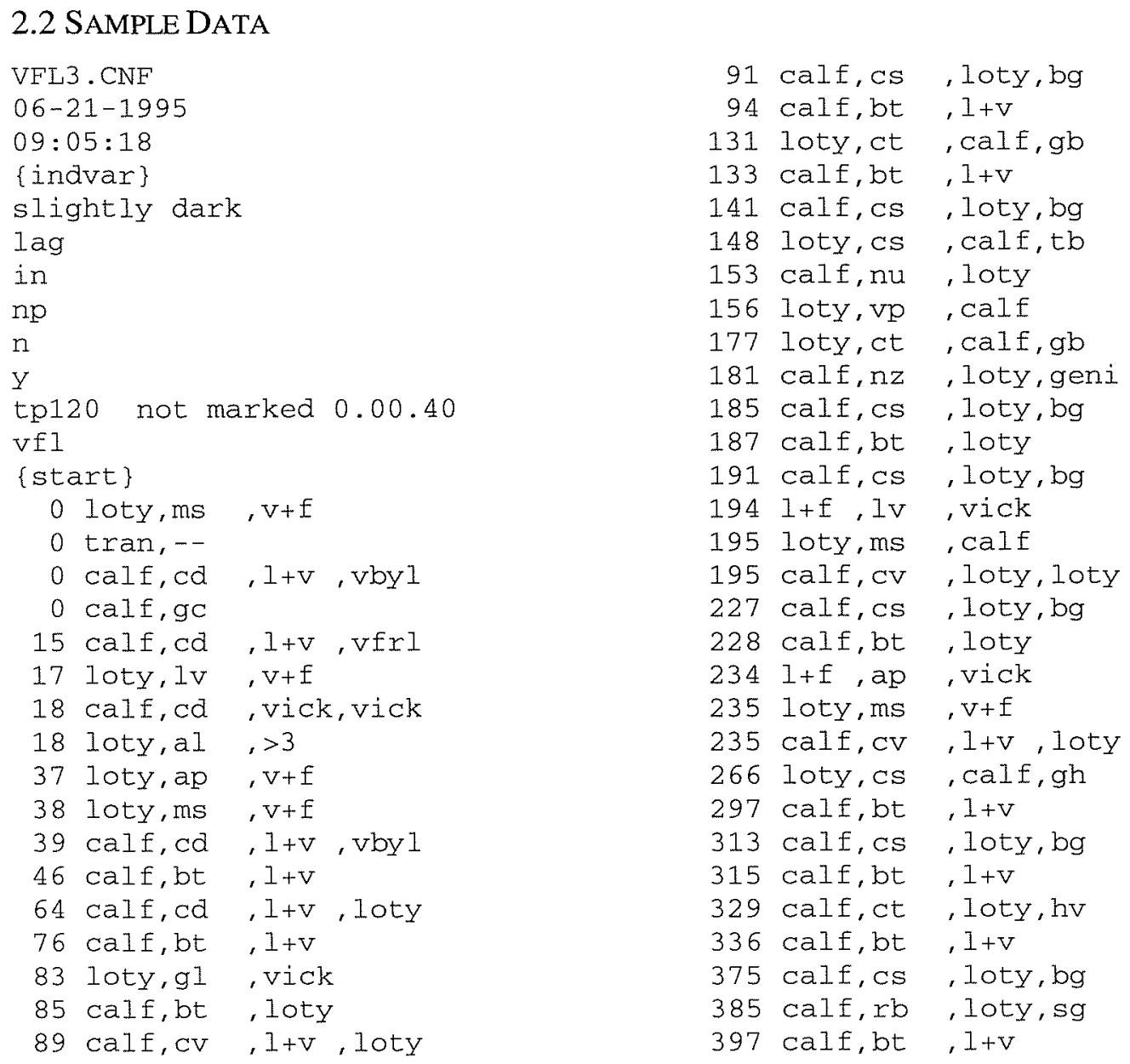


Appendix 1: Behavioral Recordings

\begin{tabular}{|c|c|c|}
\hline 413 & calf, bt &,$I+v$ \\
\hline 35 & calf, bt & $.1+V$ \\
\hline 38 & loty, xb & , vick, bf \\
\hline & loty, rb & , vick, bv \\
\hline & $1+E, I v$ & , vick \\
\hline & loty, ms & . calf \\
\hline & calf, as & , loty, Ioty \\
\hline & calf, ah & , loty, loty \\
\hline & calf, wa & $, \operatorname{lot} y,<1$ \\
\hline & calf, fb & , loty \\
\hline & calf,cd & , Ioty, loty \\
\hline & loty,gl & , vick \\
\hline & calf,rb & , Ioty, fs \\
\hline & calf,rb & , loty, gd \\
\hline & $I+f, a p$ & , vick \\
\hline & loty, ms &, $\mathrm{V}+\mathrm{f}$ \\
\hline & calf,cd & $, 1+v, 10 t y$ \\
\hline & calf, as &, $1+v$, both \\
\hline & $\operatorname{calf}, r \mathrm{~b}$ & , vick, Es \\
\hline & $\operatorname{calf}, \mathrm{a}$ & $, I+v, v b y 1$ \\
\hline & loty, la & , vick, geni \\
\hline & $a \perp f, r b$ & , vick, Es \\
\hline
\end{tabular}

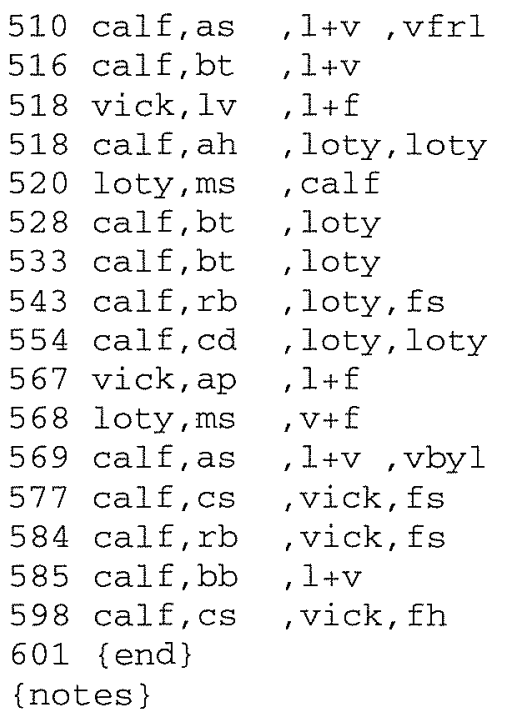

\section{SECTION 3: DELPHI BEFORE HER CALF WAS BORN}

\subsection{CONFIGURATION}

The configurations for the various dolphins are substantially similar. Therefore, only the changes will be listed in this summary of the configuration.

\subsubsection{Data Collection Methods}

The data collection methods were the same as before.

\subsubsection{Subjects}

\begin{tabular}{|llll|}
\hline Subject Name & Code & Label & Comments \\
\hline Delphi & $\mathrm{p}$ & delp & Focal adult \\
Nephele & $\mathrm{n}$ & neph & Adult dolphin \\
Lotty & $\mathrm{l}$ & loty & Adult dolphin \\
Vicky & $\mathrm{v}$ & vick & Adult dolphin \\
Sharky & $\mathrm{s}$ & shar & Adult dolphin \\
Daphne & $\mathrm{d}$ & daph & Sharky's calf \\
Sharky \& Daphne & $\mathrm{e}$ & std & Sharky \& Daphne together \\
Trainer & $\mathrm{t}$ & tran & Human trainers \\
Unknown & $\mathrm{u}$ & unkn & For when one of interactors is uncertain \\
None & $\mathrm{x}$ & none & For when behavior is not directed to anyone \\
All & $\mathrm{a}$ & all & For when behavior is directed to all dolphins \\
Snobban & $\mathrm{o}$ & snob & South African fur seal \\
Gate & $\mathrm{g}$ & gate & Dolphins in gate to Holding Pool \\
Window & $\mathrm{w}$ & wind & To windows, generally to people outside pool \\
Channel & $\mathrm{q}$ & chan & To dolphins in the channel to the Show Pool \\
\hline
\end{tabular}




\subsubsection{Behavioral Elements}

Most of the behavioral elements were all exactly the same as in the previous

configuration. They were divided into the following classes:

1. Approaches/Leaves

2. Breathing

3. Behaviors

4. States

5. Trainer

6. Other

7. Location.

The first 6 classes were the same as in the previous configuration. The $7^{\text {th }}$ was different. Only the states that did not include a calf were possible and the modifiers were as in the configuration for Vicky's calf. In addition, 2 behaviors were added to the "Other" class.

\section{$\underline{\text { States }}$}

\begin{tabular}{|c|c|c|c|c|c|}
\hline Element Name & Code & Type & Modifier 1 & Modifier 2 & Definition \\
\hline Alone & al & State & & & $\begin{array}{l}\text { Swim more than } 1 \mathrm{~m} \text { away from any } \\
\text { other dolphins }\end{array}$ \\
\hline Train & tr & State & Animals1 & Animals2-2 & Be trained by same human trainer \\
\hline Mom swim tog. & $\mathrm{ms}$ & State & Animals1 & Animals $2-2$ & Swim together code for focal mother \\
\hline Mom rest tog. & $\mathrm{mr}$ & State & Animals1 & Animals $2-2$ & Rest together code for focal mother \\
\hline Mom socialize & $\mathrm{mc}$ & State & Animals1 & Animals2-2 & Socialize code for focal mother \\
\hline In gate rest tog. & $\mathrm{gr}$ & State & Animals 1 & Animals2-2 & Rest together in gate to Holding \\
\hline In gate alone & ga & State & & & Rest alone in gate to Holding \\
\hline Channel rest & $\mathrm{qr}$ & state & & & Rest in channel to Show \\
\hline Lost & is & State & & & Focal cannot be seen from station \\
\hline
\end{tabular}

\section{Location}

This was only used in the pre-calf configurations because after the calves were born, the gate to Holding was always closed.

\begin{tabular}{|lccccl|}
\hline Element Name & Code & Type & Modifier 1 & Modifier 2 & Definition \\
\hline In Holding & ih & State & Animals1 & Animals2-2 & In Holding with the gate open \\
In Lagoon & il & State & Animals1 & Animals2-2 & In the Lagoon with the gate open \\
In gate & ig & State & & & $\begin{array}{l}\text { In the gate between Holding and the } \\
\text { Lagoon with the gate open } \\
\text { Gate closed }\end{array}$ \\
gc & State & & & $\begin{array}{l}\text { All dolphins in the Lagoon with the } \\
\text { gate closed }\end{array}$ \\
\hline
\end{tabular}


Appendix 1: Behavioral Recordings

Other

\begin{tabular}{|lccccl|}
\hline Element Name & Code & Type & Modifier 1 & Modifier 2 & Definition \\
\hline Unspecified & $\mathrm{xx}$ & Event & Subjects & Behavior for which no code exists \\
Whistle & wh & Event & & Produce a whistle \\
Bubblestream & bu & Event & & Produce a bubblestream \\
Command & cb & Event & Calf relative & Perform a behavior under \\
behavior & & & & instructions of the trainers \\
Into Holding & ih & Event & & Move into the Holding Pool \\
Out of Holding & oh & Event & & Move out of the Holding Pool \\
\hline
\end{tabular}

\subsubsection{Modifiers}

The modifiers were divided into the following classes:

1. Body position 1

2. Body position 2

3. Distance

4. Repeated

5. Animals 1

6. Animals 2-2.

The first 4 classes were the same as in the previous configuration. Classes 5 and 6 were slightly different.

\section{Animals 1}

This list is designed to record whether Sharky, Daphne, Nephele, and Lotty are with Delphi. This list was extremely long (64 elements) to include all possible combinations of the dolphins. Only examples are given here.

\begin{tabular}{|ll|}
\hline Modifier Name & Code \\
\hline None & $\mathrm{xxx}$ \\
Sharky & $\mathrm{axx}$ \\
Nephele & $\mathrm{xax}$ \\
Lotty & $\mathrm{xxa}$ \\
Daphne & $\mathrm{cxx}$ \\
Sharky \& Daphne & $\mathrm{bxx}$ \\
Sharky \& Nephele & $\mathrm{aax}$ \\
Sharky, Nephele, \& Lotty & aaa \\
Sharky, Daphne, \& Nephele & bax \\
Daphne, Nephele, \& Lotty & caa \\
Daphne \& Lotty & cxa \\
Sharky, Daphne, \& Lotty & bxa \\
\hline
\end{tabular}

Animals 2-2

This list is designed to record whether Vicky was swimming with Delphi.

\begin{tabular}{|lcc|}
\hline Modifier Name & Code & Label \\
\hline No Vicky & $\mathrm{x}$ & none \\
Vicky & $\mathrm{v}$ & $\mathrm{v}$ \\
\hline
\end{tabular}




\subsubsection{Channels}

\begin{tabular}{|ll|}
\hline Subject & Behavioral Class \\
\hline Delphi & States \\
Delphi & Approaches/Leaves \\
Delphi & Breathing \\
Delphi & Location \\
Delphi & Behaviors \\
Delphi & Other \\
Trainer & Trainer \\
\hline
\end{tabular}

\subsubsection{Independent Variables}

The independent variables were the same as in the Vicky's calf configuration.

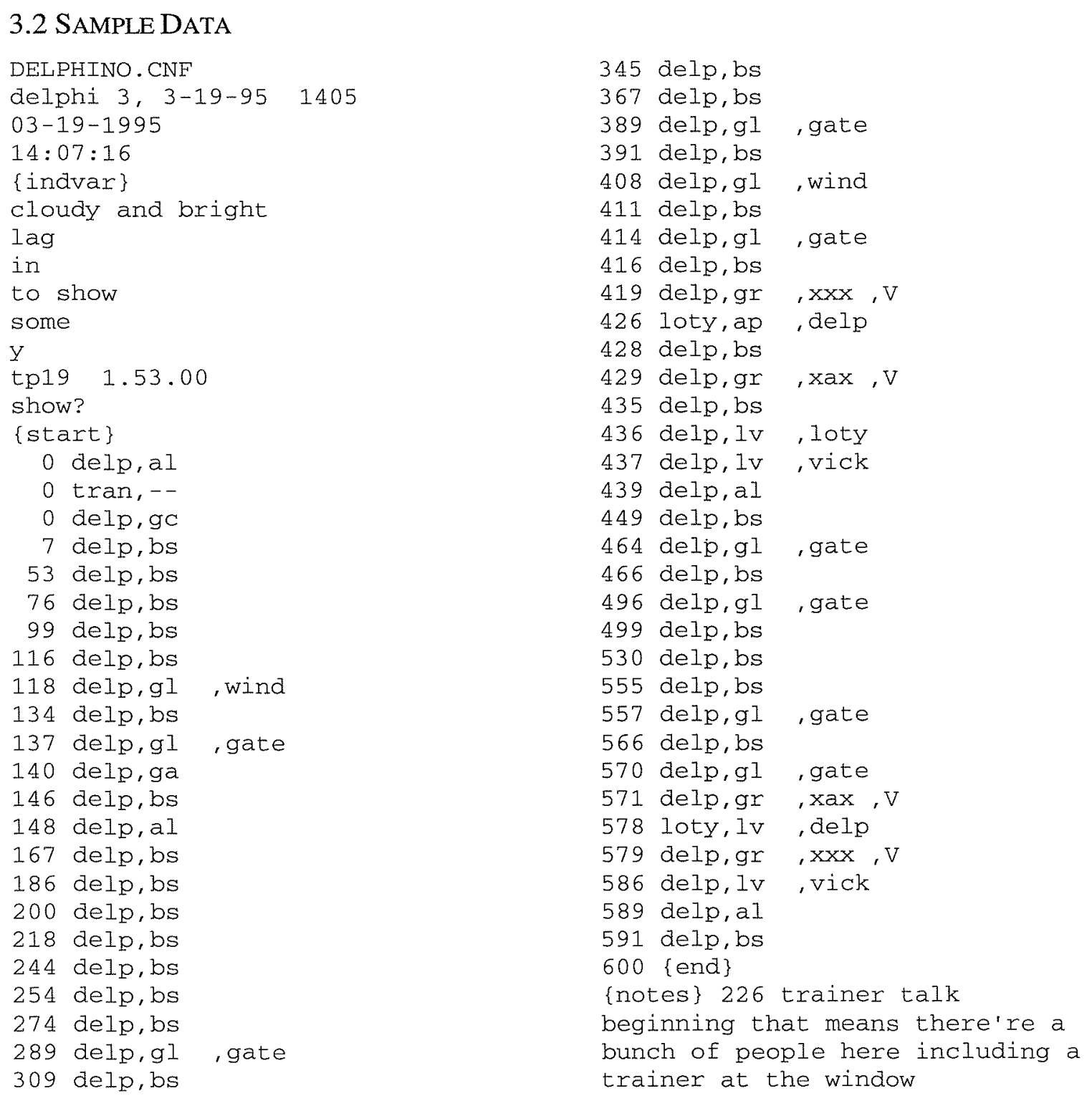


Appendix 1: Behavioral Recordings

\section{SECTION 4: CONFIGURATION FOR BUBBLESTREAM RECORDING}

\subsection{CONFIGURATION}

This configuration was designed for recording bubblestreams that were marked on the second channel of the tape during acoustic recordings.

\subsubsection{Data Collection Methods}

\begin{tabular}{|ll|}
\hline Data Collection & Method Used \\
\hline Sampling & Focal sampling \\
Recording & Continuous \\
Number of Actors & Single \\
Maximum duration of observations & 10 minutes \\
Maximum duration based on & Elapsed time \\
Timing resolution & 1 second \\
Timing of duration events & Press for start/end \\
\hline
\end{tabular}

\subsubsection{Behavioral Elements}

This configuration was designed to merely record the names of animals when the produced a bubblestream. Therefore, there were no subjects, only behavioral elements of the names of the possible dolphins. There were also no modifiers. There were also no channels.

\begin{tabular}{|lccc|}
\hline Element Name & Code & Label & Type \\
\hline Lotty & l & loty & Event \\
Vicky & $\mathrm{v}$ & vick & Event \\
Delphi & $\mathrm{p}$ & delp & Event \\
Nephele & $\mathrm{n}$ & neph & Event \\
Sharky & $\mathrm{s}$ & shar & Event \\
Daphne & $\mathrm{d}$ & daph & Event \\
Lotus & $\mathrm{f}$ & lots & Event \\
Nephele's calf & $\mathrm{y}$ & neca & Event \\
Vicky's calf & $\mathrm{c}$ & vica & Event \\
Delphi's calf & $\mathrm{b}$ & deca & Event \\
Vindy & $\mathrm{i}$ & vind & Event \\
\hline
\end{tabular}




\subsubsection{Independent Variables}

\begin{tabular}{|lll|}
\hline Variable Name & Label & Definition \\
\hline Tape number & tape & The number of the tape being digitized. \\
Focal & focal & The focal dolphin. \\
Sample number & obs & The number (within the day) of the sample session. \\
Observed date & date & The date the sample was taken. \\
Filter at & filter & $\begin{array}{l}\text { The frequency }(\mathrm{kHz}) \text { of the high-pass filtering of the } \\
\text { recording. }\end{array}$ \\
Filter post-gain & gain & The post-filter gain on the digitization. \\
\hline
\end{tabular}

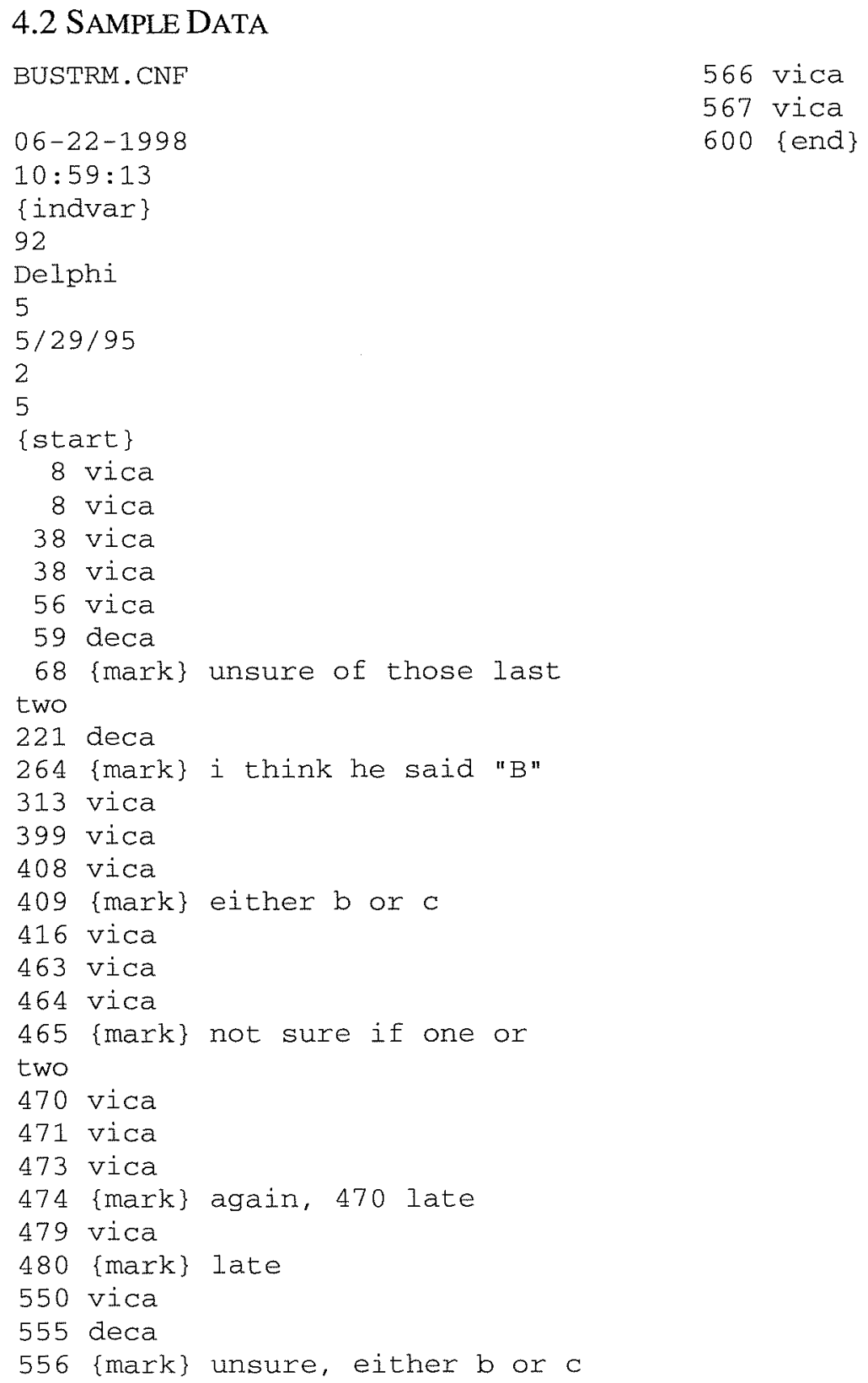

$566 \mathrm{vica}$

567 vica

600 \{end\} 


\section{APPENDIX 2: COMPUTER PROGRAMS}

\section{SECTION 1：TEST OF SAMPLING TIMES}

\subsection{Common to All Programs}

\subsubsection{Setting the Testing Numbers}

function [tot,norm,num] $=$ settest(test)

$\%[$ tot, norm,num] $=$ settest $($ test $)$

$\%$ test $=$ date of test (as number (e.g. 720))

if test $==720$

tot $=30$;

norm $=[6,9,15,20,25]$;

elseif test $==87$

tot $=15$

norm $=[2,4,8,12,15]$;

elseif test $==35$

tot $=11$;

norm $=[3,5,7,11]$;

elseif test $==325$

tot $=9$

norm $=[3,4,6,9]$;

end

num = length(norm);

\subsubsection{Calculation of Differences between Group Means and Overall Mean}

function $\mathrm{d}=\operatorname{diffvect}(\mathrm{X}, \mathrm{m}, \mathrm{n}, \mathrm{tot})$

$\% \mathrm{~d}=\operatorname{diffvect}(\mathrm{X}, \mathrm{m}, \mathrm{n})$

$\%$ gets vector of differences between means for permutations of

\%n sample and total mean of all samples

$\% \mathrm{X}=$ data, $\mathrm{m}=$ number of runs to do, $\mathrm{n}=$ number of samples to test

tot_mean $=$ mean $(\mathrm{X})$

for $\mathrm{i}=1: \mathrm{m}$

$\mathrm{p}=$ randperm(tot);

$\mathrm{y}=\mathrm{p}(1: \mathrm{n})$

for $j=1: n$

end

$$
x(j)=X(y(j)) ;
$$

$\mathrm{d}(\mathrm{i})=$ mean $(\mathrm{x})$ - tot_mean;

clear $\mathrm{y}$

clear $\mathrm{x}$

end

clear $\mathrm{p}$ 


\subsubsection{Calculation of Overall Mean and Standard Deviation}

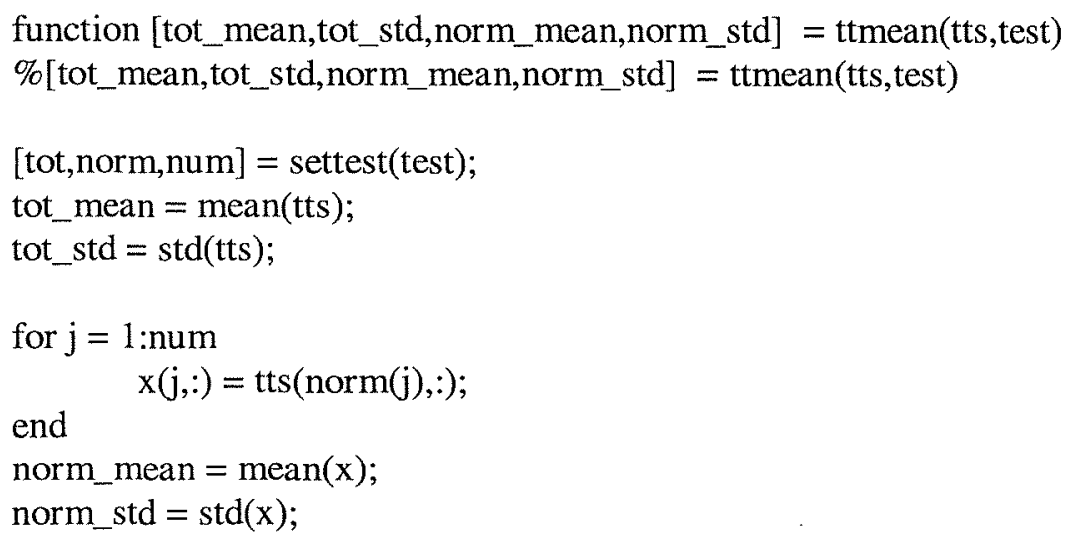

\subsection{Calculation of the PERCENT DeViation ANd CoEfFicient of VARIATION}

\subsubsection{Calculation of Distribution Mean and Standard Deviation}

function $[\mathrm{mn}, \mathrm{s}]=\operatorname{ttdist}(\mathrm{X}, \mathrm{m}, \mathrm{test})$

$\% \mathrm{t}=\mathrm{ttdist}(\mathrm{X}, \mathrm{m}, \mathrm{test})$

\%function to look at distribution of all possible n's in the test

$\% \mathrm{X}=$ data, $\mathrm{m}=$ number of runs, test $=$ which test

$\% \mathrm{mn}=$ mean vector, $\mathrm{s}=$ stdev vector

$[$ tot, norm,num $]=$ settest (test);

for $\mathrm{j}=1$ :tot

$\mathrm{d}=\operatorname{diffvect}(\mathrm{X}, \mathrm{m}, \mathrm{j}, \mathrm{tot})$;

$s(j)=\operatorname{std}(d)$;

$\operatorname{mn}(\mathrm{j})=\operatorname{mean}(\mathrm{d})$;

end

\subsubsection{Calculation of Percent Deviation and Coefficient of Variation}

For each measure for each test

$\mathrm{cv}=\mathrm{mn} / \mathrm{s}$

pd=mn-tot_mean; 


\section{Appendix 2: Computer Programs}

\subsection{COMPARISON OF STANDARD 5 TIMES}

function $[\mathrm{p}, \mathrm{perms}]=\mathrm{ttp} \_$test $(\mathrm{X}, \mathrm{m} 1, \mathrm{~m} 2, \mathrm{test})$

$\%[\mathrm{p}, \mathrm{perms}]=\mathrm{ttp} \_$test $(\mathrm{X}, \mathrm{m} 1, \mathrm{~m} 2$, test $)$

\%function to get times test $\mathrm{p}$ value for normal samples

$\% \mathrm{X}=$ data, $\mathrm{ml}=$ number of runs for diffvect,

$\% \mathrm{~m} 2=$ number of permutations to try here, test $=$ which date

$\%$ is the proportion of the distribution that is greater

$\%$ than the normal samples (as absolute values).

$\%$ so if $\mathrm{p}<0.05$, normal sample is significantly different

[tot,norm,num] = settest(test);

$\mathrm{d}=\operatorname{diffvect}(\mathrm{X}, \mathrm{m} 1$, num,tot $)$;

$\operatorname{ad}=\operatorname{abs}(d)$;

tot_mean $=$ mean $(\mathrm{X})$;

for $\mathrm{i}=1: \mathrm{m} 2$

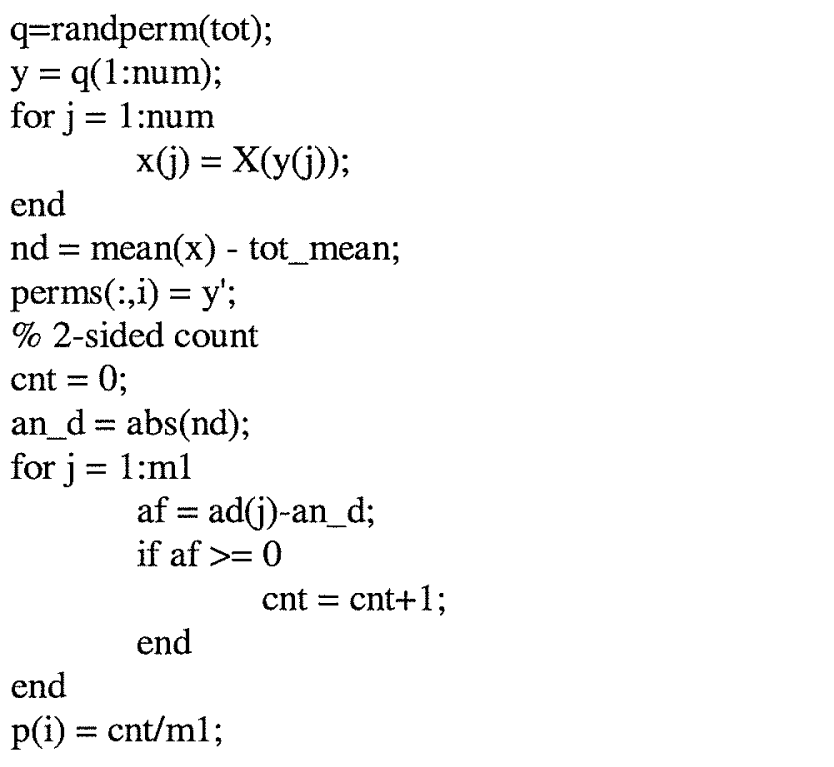

end 


\section{SECTION 2: PROGRAMS FOR WHISTLE EXTRACTION AND SORTING}

\subsection{FINDING THE THRESHOLD}

\subsubsection{Choosing a Noise Segment}

function [nstart,nstop] $=$ find_noise(kayfiles)

$\%$ program to search file and find representative noise segment

$\%$ [nstart,nstop] = find_noise(kayfiles)

$\%$ needs kinput

$\mathrm{n}=\operatorname{size}($ kayfiles, 1$)$

$\mathrm{x}=\left[\begin{array}{ll}0 & 0\end{array}\right]$

for $\mathrm{i}=1: \mathrm{n}$

$\mathrm{k}=\operatorname{kayfiles}(\mathrm{i},:)$;

new_start $=1$;

while isempty(new_start)

start $=$ new_start;

$\mathrm{s}=\operatorname{kinput}(\mathrm{k}, \mathrm{start}, 1 \mathrm{e} 5)$;

$\mathrm{s}=\mathrm{s}$-mean $(\mathrm{s})$;

$\operatorname{specgram}(\mathrm{s},[], 80000) ;$ colormap(hsv)

title(k)

zoom on

end

new_start = input('new start? ');

$\mathrm{q}=$ input('graphical input? ','s');

if isempty (q)

$[\mathrm{x}, \mathrm{y}]=$ ginput;

else

$\mathrm{r}=$ input('repeat? ','s');

if $\sim$ isempty(r)

$\mathrm{x}=$ prev_ $\mathrm{x}$;

else

$\mathrm{x}(1)=$ input('beginning: ');

$x(2)=$ input('end: ');

end

end

prev_x $=\mathrm{x}$;

$x=\operatorname{round}(x * 80000)+$ start;

nstart(i) $=x(1)$;

nstop(i) $=x(2)$;

end 
Appendix 2: Computer Programs

\subsubsection{Reading the Kay File}

NOTE: this program was not written by me.

This program is used in many of the subsequent programs

function [samples,count]=kinput(filename,strt,lngth)

$\%$

$\%$ samples=kread40('filename.kay',starting sample,length)

$\%$ Function to return the samples stored in a Kay file.

$\%$

[fid, message]=fopen(filename,' $r$ ','l');

if fid $==-1$

sprintf('Error opening \%sln', filename)

sprintf('\%sln',message)

return;

end;

if fseek(fid, $512+\operatorname{str}{ }^{*} 2$, 'bof' $\left.^{\prime}\right)==-1$

sprintf('KINPUT error seeking to starting point')

return;

end;

[samples,count]=fread(fid,lngth,'unsigned short');

fclose(fid);

return; 


\subsubsection{Calculating the Threshold}

function thresh=getthrsh(kayfile, start,stop)

$\%$ thresh=getthrsh(kayfile,start,stop)

$\%$ threshold determination for wdetect

\% 7/15/97 DMRF (modified from AMS version)

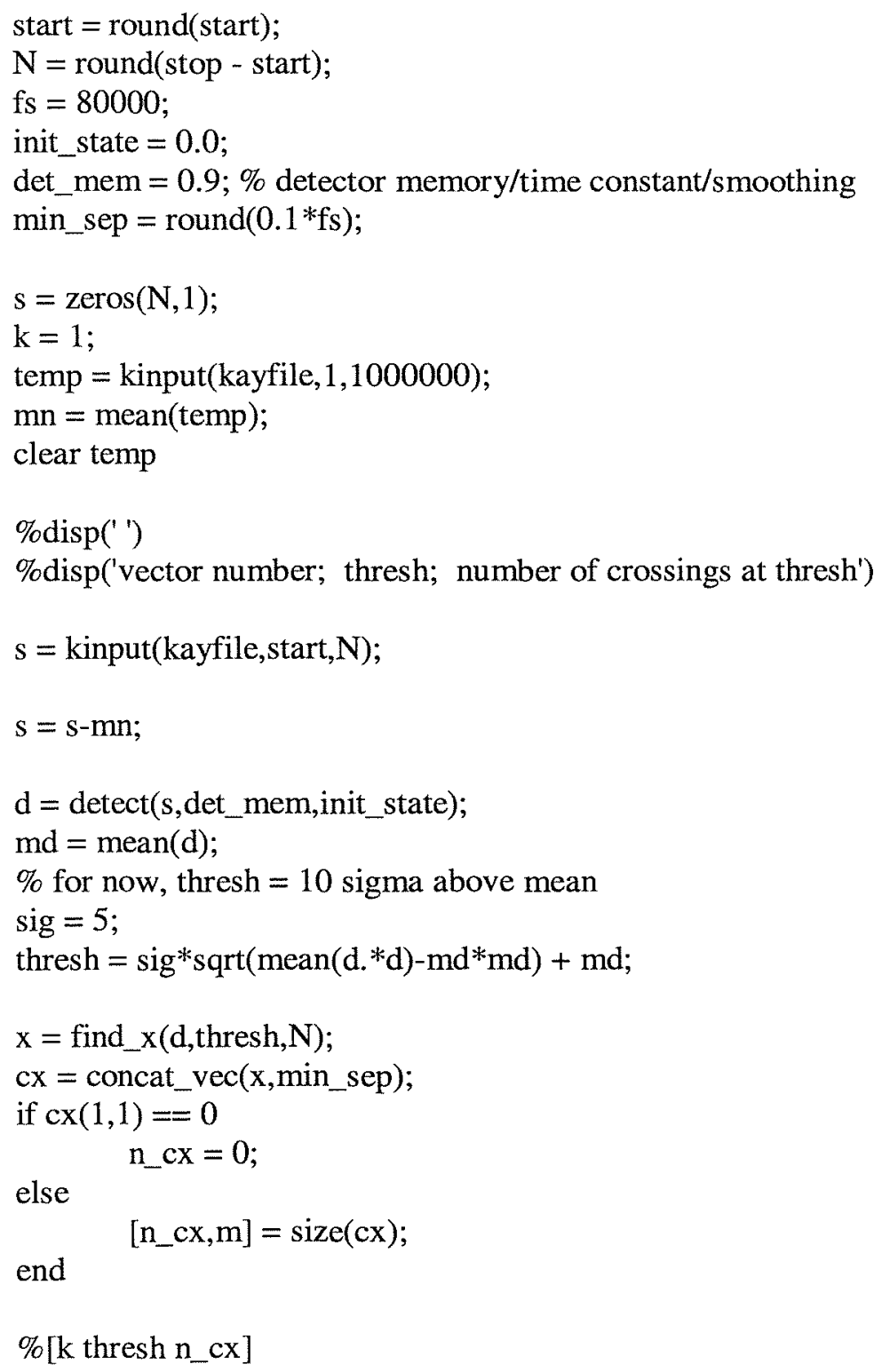




\section{Appendix 2: Computer Programs}

\subsection{THE EXTRACTOR}

\subsubsection{Extraction of Whistles from Multiple Files}

function batch_detect(kayfiles,nstart,nstop,bases,cmts)

$\%$ batch_detect(kayfiles,nstart,nstop,bases,cmts)

$\%$ program to run detector and cut sorter on multiple files

$\%$ needs getthrsh, wdetect, sortcuts 12

$\%$ creates sorts.base for sorting, as well as

$\%$ detection files and pw.base and nw.base = lists of cut \#'s

$\%$ (possible whistles and non-whistles)

$\% * * * *$ warning $* *$ this deletes master files as it goes $* * * *$

$\operatorname{disp}\left({ }^{*} * * *\right.$ Warning: This will delete the master files after using them $\left.* * * '\right)$

$\mathbf{n}=\operatorname{size}($ kayfiles, 1$)$;

$\mathrm{dt}=\mathrm{date}$;

$\mathrm{dt}=[$ datestr(dt,5) '_' datestr(dt,7) '_' datestr(dt,11)];

$\mathrm{dt}=\mathrm{dt}(2: 8) ; \quad \%$ until month $=2$ digits

$\log =\left[\mathrm{dt} \cdot \cdot \log { }^{\prime}\right]$

diary $(\log )$

$\mathrm{fdt}=$ fopen $\left(\mathrm{dt}, \mathrm{w}^{\prime}\right)$;

for $\mathrm{i}=1: \mathrm{n}$

big_cnt $=0$;

clear cnt

clear big

kay $=$ kayfiles $(i,:)$

$\operatorname{disp}([$ 'noise section $=$ ' int2str(nstart(i)) ' to ' int2str(nstop(i))] $)$

thresh $=$ getthrsh(kay,nstart(i),nstop(i))

base $=$ bases $(i,:)$;

$\mathrm{cmt}=\mathrm{cmts}(\mathrm{i},:)$;

cnt $=$ wdetect $($ kay, thresh, base,cmt)

$\operatorname{count}(\mathrm{i})=\mathrm{cnt}$;

script $=[$ 'sorts.' base $]$;

[pw,nw,big] = sortcuts 12 (base, $1, \mathrm{cnt}$, script);

$\mathrm{pf}=[$ 'pw.' base $]$;

nf $=[$ 'nw.' base $]$;

bf $=$ ['big.' base];

save(pf,'pw','-ascii');

save(nf,'nw','-ascii');

save(bf,'big','-ascii');

fprintf(fdt,'\%sln',script);

disp(['Deleting master file: ' kay])

eval(['! rm ' kay]); 


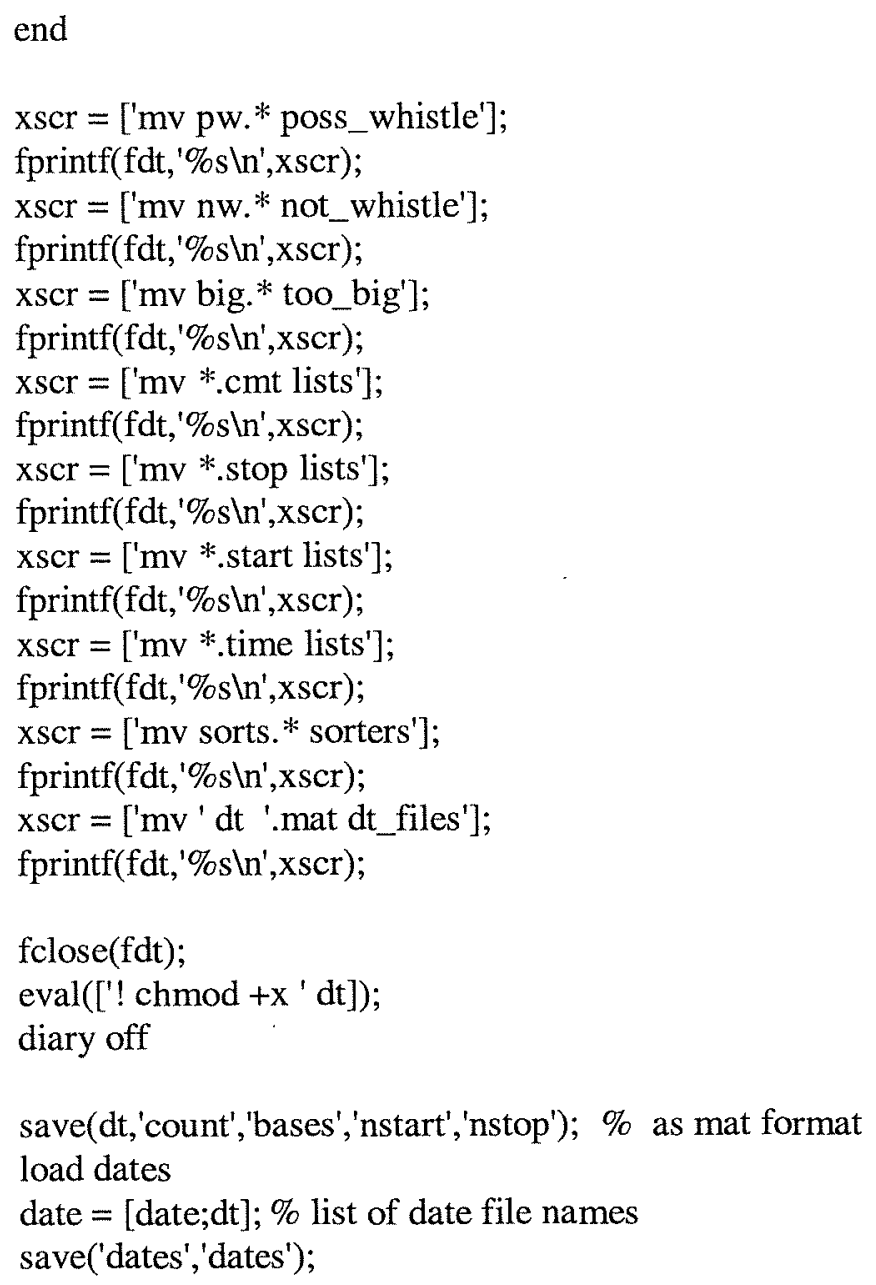


Appendix 2: Computer Programs

\subsubsection{The Extractor Itself}

function $\mathrm{cnt}=$ wdetect(kayfile, thresh, base, $\mathrm{cmt}$ )

$\% \mathrm{cnt}=$ wdetect(kayfile,thresh,base,cmt)

$\%$ whistle detector, modified from AMS version

$\%$ 7/14/97 DMRF

$\%$ for detecting whistles from a kay file in Matlab

$\%$ and saving them as kay files

$\%$ this program calls the following $m$ files:

$\%$ detect, find_x, concat_vec, kinput, kopen

$\mathrm{N} \quad=120000 ; \quad \% 1.5 \mathrm{~s}$

$\%$ Linus Matlab max size $=$ sev vect's of $1,000,000=12.5 \mathrm{sec}$

$\%$ sec $=$ points $/ \mathrm{fs}$

fs $\quad=80000$

init_state $=0.0$

det_mem $=0.9 ; \%$ detector memory/time constant/smoothing

min_dur $=\operatorname{round}(0.050 * \mathrm{fs}) ; \%$ was 0.03

prefix $=\operatorname{round}(0.025 * \mathrm{fs})$;

suffix $=\operatorname{round}(0.025 * \mathrm{fs})$;

min_sep $=$ round $(0.100 * \mathrm{fs}) ; \%$ was 0.05

wav = '.kay'; \%for kay files

len $\quad=10$;

nvec $=\operatorname{round}($ len $* 60 * \mathrm{fs} / \mathrm{N})-1 ; \%$ len (preset) minutes total

file_open $=0$;

check_dur $=0$;

next_suffix $=0$;

prev_up $=0$;

prev_down $=0$;

cmt_file $=[$ base '.cmt' $]$;

$\mathrm{fcmt}=$ fopen $\left(\mathrm{cmt} \_\right.$file, $\left.{ }^{\prime} w \mathrm{tt}^{\prime}\right)$;

$\mathrm{s}=\operatorname{zeros}(\mathrm{N}, 2)$;

temp = kinput(kayfile, 1,1000000);

$\mathrm{mn}=\operatorname{mean}($ temp$)$;

clear temp

cnt $=0$;

$\mathrm{i}=1$;

$\%$ first vector

$\mathrm{s}(:, \mathrm{i})=\operatorname{kinput}($ kayfile, $1, \mathrm{~N})$; 
det $=$ detect(s(:,i)-mn, det_mem, init_state); \% warning: updates init_state $\mathrm{x} \_v e c=$ find_x $($ det, thresh, $\mathrm{N}) ; \%$ at most max_cross crossings

cx_vec $=$ concat_vec $\left(x \_v e c\right.$, min_sep $)$;

clear det;

$\left[\mathrm{n} \_\mathrm{x}, \mathrm{m} \_\mathrm{x}\right]=\operatorname{size}\left(\mathrm{cx} \_\mathrm{vec}\right)$;

$\%$ write first whistle

if $\left(\mathrm{cx}_{\_} \operatorname{vec}(1,1)==0\right)$

else $\% c(1,1) \sim=0$

valid_whis $=0$;

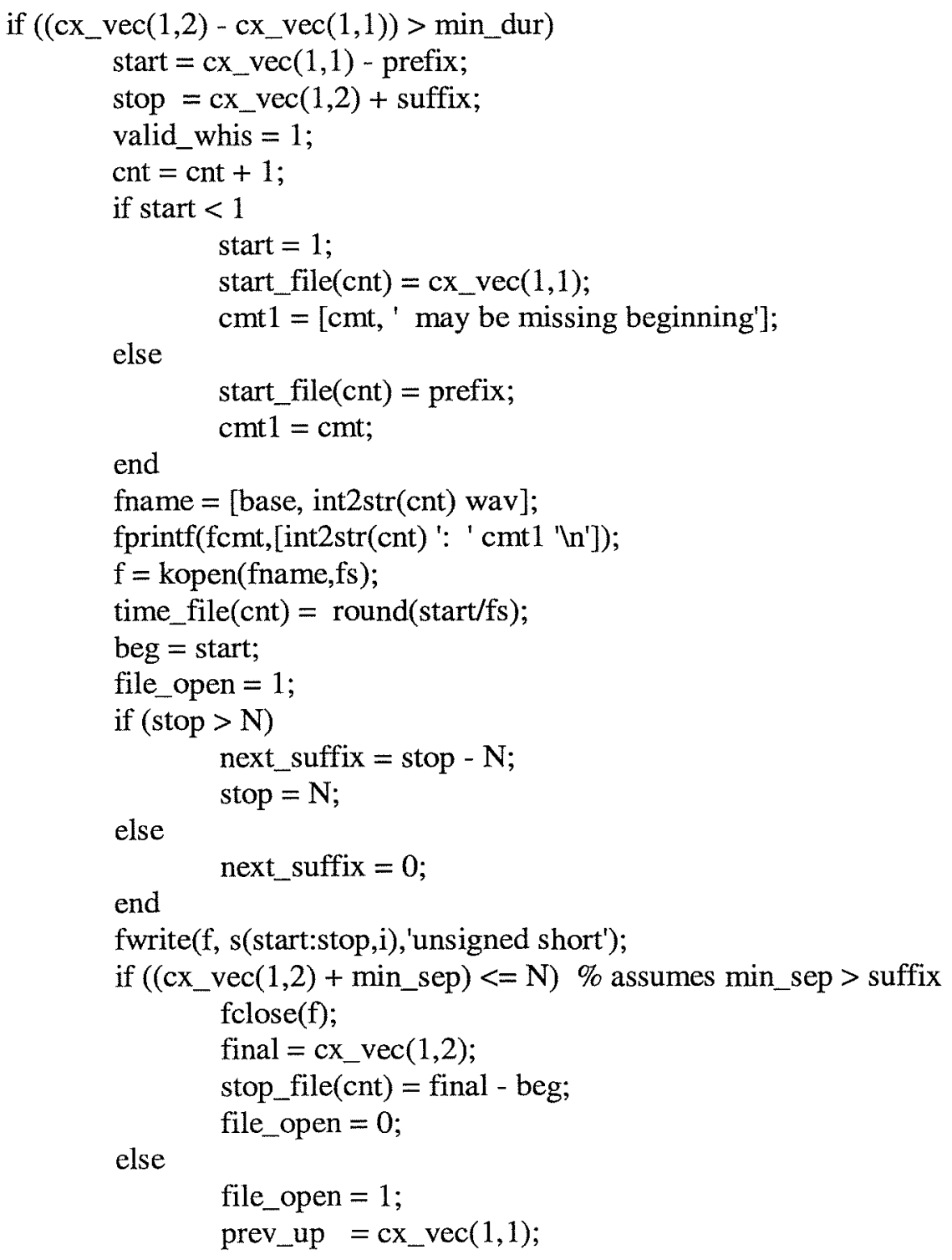


Appendix 2: Computer Programs

$$
\begin{aligned}
& \text { prev_down }=\text { cx_vec }(1,2) \text {; } \\
& \text { end } \\
& \text { if }\left(\left(\mathrm{cx} \_v e c(1,2)+\text { min_sep }\right)>\mathrm{N}\right) \\
& \text { prev_up }=\text { cx_vec }(1,1) \text {; } \\
& \text { prev_down }=\text { cx_vec }(1,2) \text {; } \\
& \text { end } \\
& \text { check_dur }=1 \text {; }
\end{aligned}
$$

end $\quad \% \mathrm{c}(1,1)=0$

$\%$ Write middle whistles (whistles 2 through $n \_x-1$ ).

if $\left(n \_x>=3\right)$

$$
\mathrm{jj}=2 \text {; }
$$

while $\left(\mathrm{jj}<=\left(\mathrm{n} \_\mathrm{x}_{-}-1\right)\right)$

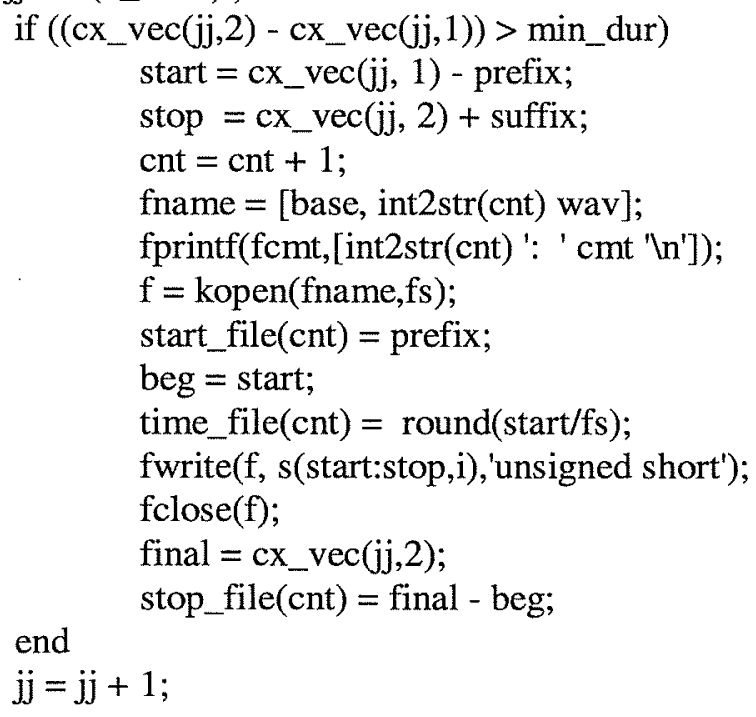

$\%$ Write final whistle of vector.

if $\left(n \_x>=2\right)$

$$
\begin{aligned}
& \text { prev_down }=0 \text {; } \\
& \text { prev_up }=0 \text {; } \\
& \text { start }=\text { cx_vec }\left(n \_x, 1\right)-\text { prefix; } \\
& \text { stop }=\mathrm{cx} \_v e c\left(n \_x, 2\right)+\text { suffix; } \\
& \text { if }\left(\left(\mathrm{cx} \_\operatorname{vec}\left(\mathrm{n} \_\mathrm{x}, 2\right)-\mathrm{cx} \_\operatorname{vec}\left(\mathrm{n} \_\mathrm{x}, 1\right)\right)>\min \_\mathrm{dur}\right) \\
& \text { if }(\text { stop }>N) \\
& \text { next_suffix = stop }-\mathrm{N} \text {; } \\
& \text { stop }=\mathrm{N} \text {; }
\end{aligned}
$$

else 
end

$$
\text { next_suffix }=0 \text {; }
$$

$\mathrm{cnt}=\mathrm{cnt}+1$

fname $=[$ base, int $2 \operatorname{str}(\mathrm{cnt}) \mathrm{wav}]$;

fprintf(fcmt,[int2str(cnt) ': ' cmt 'ln']);

$\mathrm{f}=$ kopen(fname,fs);

start_file $(\mathrm{cnt})=$ prefix;

time_file $(\mathrm{cnt})=\operatorname{round}(\operatorname{start} / \mathrm{fs})$;

beg = start;

fwrite(f, s(start:stop,i), 'unsigned short');

if $\left(\mathrm{cx} \_\right.$vec $\left(\mathrm{n} \_\mathrm{x}, 2\right)+\min \_$sep $)>\mathrm{N}$

file_open $=1$;

prev_up $=$ cx_vec $\left(n \_x, 1\right)$;

else prev_down $=$ cx_vec $\left(\mathrm{n} \_\mathrm{x}, 2\right)$;

fclose(f);

final $=c x \_v e c\left(n \_x, 2\right)$;

stop_file $(\mathrm{cnt})=$ final - beg;

file_open $=0$;

end

else

$$
\text { if } \begin{aligned}
\left(\left(\mathrm{cx} \_v e c\left(n \_x, 2\right)+\min \_s e p\right)\right. & >\mathrm{N}) \\
& \text { check_dur }=1 ; \\
& \text { prev_up }=\mathrm{cx} \_v e c\left(\mathrm{n} \_\mathrm{x}, 1\right) ; \\
& \text { prev_down }=\mathrm{cx} \text { _vec }\left(\mathrm{n} \_\mathrm{x}, 2\right)
\end{aligned}
$$

end

end

end $\% \mathrm{n}>=2$

$\%$ end first vector

$\%$ middle vectors

$\mathrm{k}=1$;

while $(\mathrm{k}<$ nvec $)$

$$
\begin{aligned}
& \mathrm{i}=\mathrm{rem}(\mathrm{i}, 2)+1 \\
& \mathrm{~s}(:, \mathrm{i})=\operatorname{kinput}(\text { kayfile, } \mathrm{k} * \mathrm{~N}+1, \mathrm{~N})
\end{aligned}
$$

$\operatorname{det}=\operatorname{detect}(\mathrm{s}(:, \mathrm{i})-\mathrm{mn}$, det_mem, init_state); \% warning: updates init_state

$\mathrm{x} \_v e c=$ find_x $($ det, thresh, $\mathrm{N}) ; \quad \%$ at most max_cross crossings

cx_vec $=$ concat_vec(x_vec, min_sep);

clear det;

$\left[\mathrm{n} \_\mathrm{x}, \mathrm{m} \_\mathrm{x}\right]=\operatorname{size}\left(\mathrm{cx} \_\mathrm{vec}\right)$;

$\%$ write first whistle

if $\left(\mathrm{cx} \_\operatorname{vec}(1,1)==0\right)$

$$
\text { if (next_suffix } \sim=0 \text { ) } \% \text { possible BUG? }
$$


Appendix 2: Computer Programs

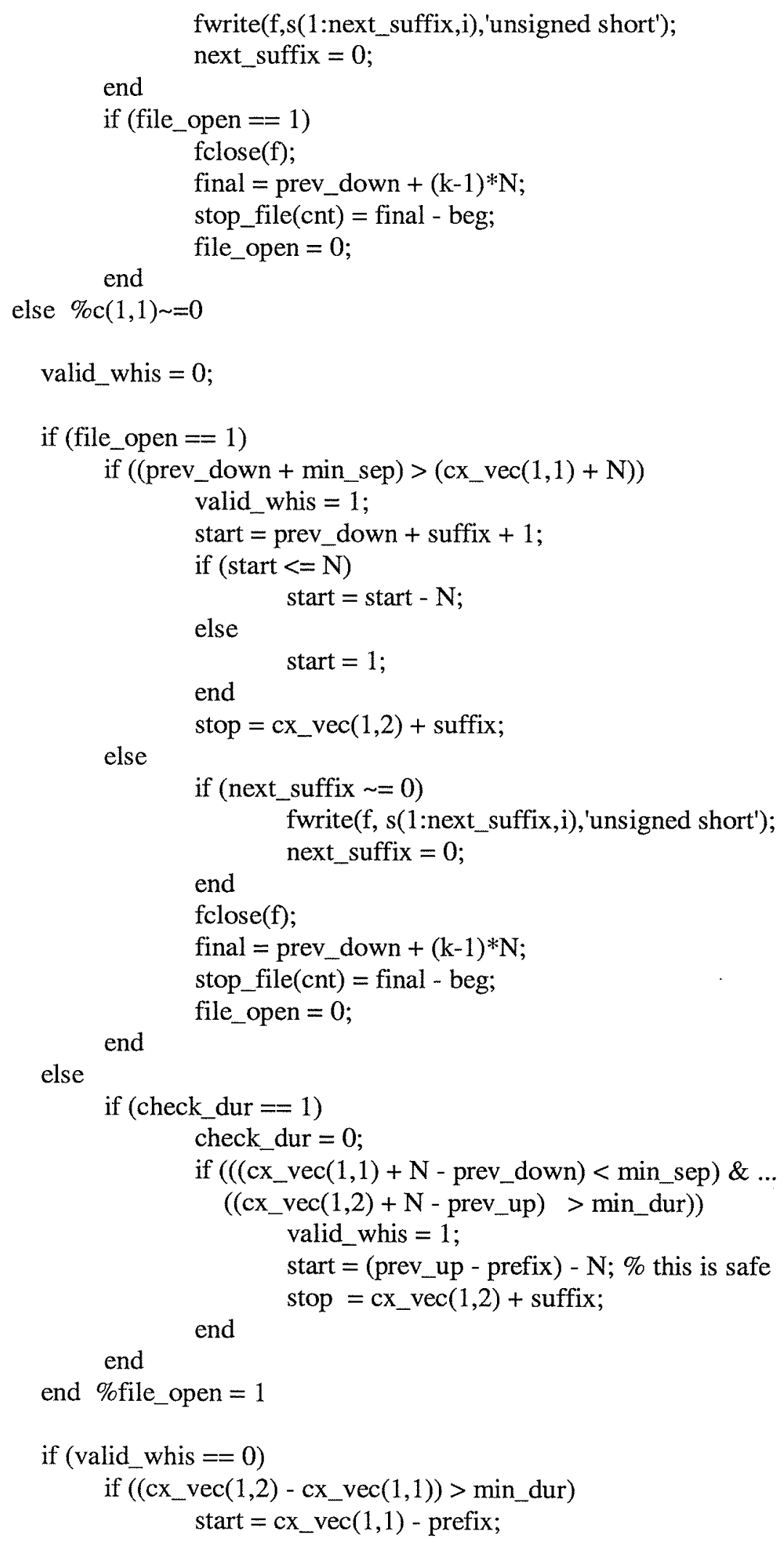




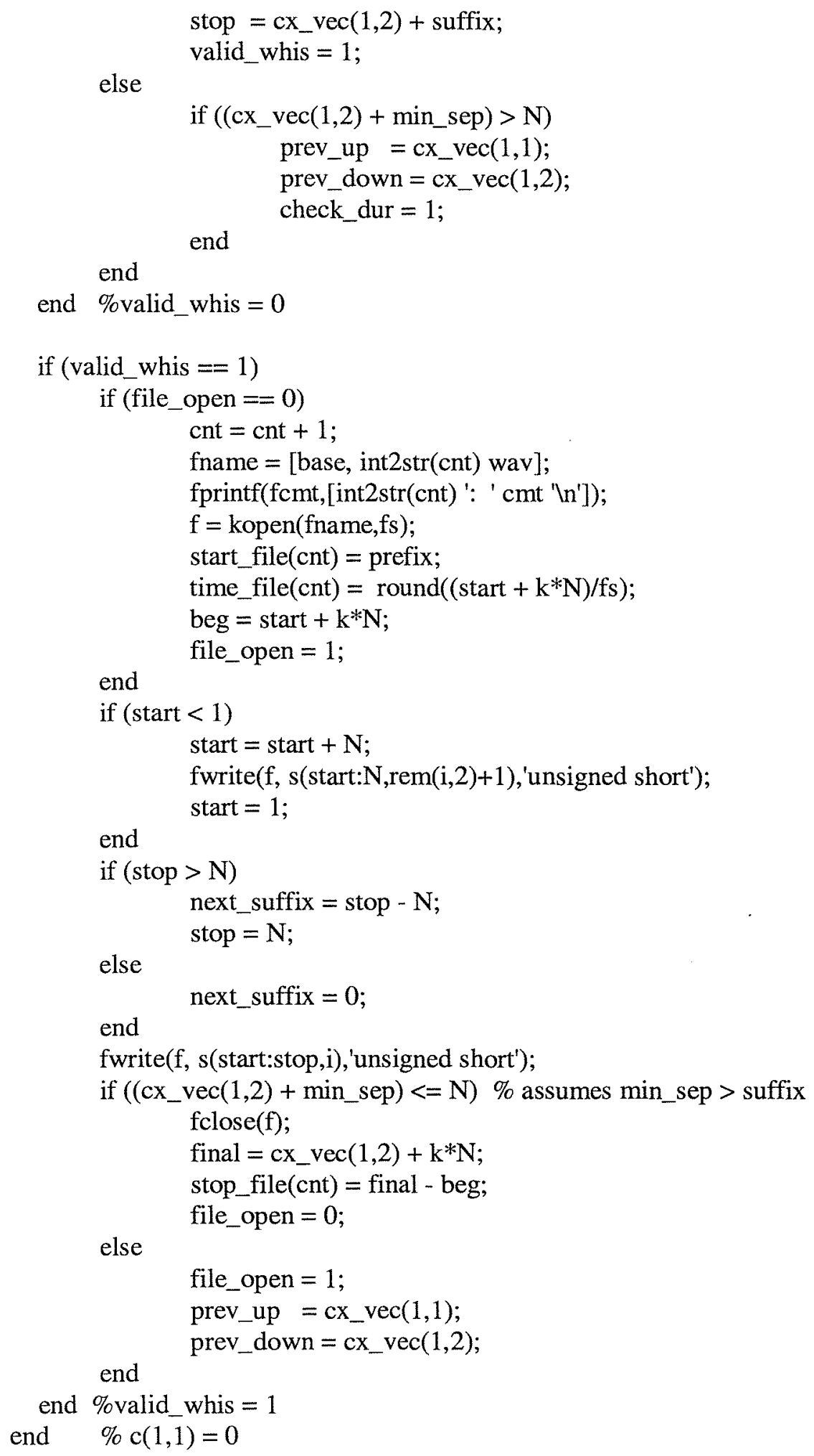


Appendix 2: Computer Programs

$\%$ Write middle whistles (whistles 2 through $n \_x-1$ ).

if $\left(\mathrm{n} \_\mathrm{x}>=3\right)$

$$
\mathrm{jj}=2 \text {; }
$$$$
\text { while }\left(\mathrm{jj}<=\left(\mathrm{n} \_\mathrm{x}-1\right)\right)
$$$$
\text { if }\left(\left(\mathrm{cx} \_ \text {vec }(\mathrm{jj}, 2)-\mathrm{cx} \_v e c(\mathrm{jj}, 1)\right)>\text { min_dur }\right)
$$$$
\text { start }=\text { cx_vec }(\mathrm{jj}, 1) \text { - prefix; }
$$$$
\text { stop }=\text { cx_vec }(j \mathrm{j}, 2)+\text { suffix; }
$$$$
\text { cnt }=\mathrm{cnt}+1
$$

fname $=[$ base, int $2 \operatorname{str}(\mathrm{cnt})$ wav $]$;

fprintf(fcmt,[int2str(cnt) ': ' $\mathrm{cmt}$ 'ln']);

$\mathrm{f}=\operatorname{kopen}($ fname, $\mathrm{fs})$;

start_file $(\mathrm{cnt})=$ prefix;

beg $=$ start $+\mathrm{k} * \mathrm{~N}$;

time_file $(\mathrm{cnt})=\operatorname{round}\left(\left(\operatorname{start}+\mathrm{k}^{*} \mathrm{~N}\right) / \mathrm{fs}\right)$;

fwrite(f, s(start:stop,i),'unsigned short');

fclose(f);

final $=\mathrm{cx} \_$vec $(\mathrm{jj}, 2)+\mathrm{k} * \mathrm{~N}$;

end

stop_file $(\mathrm{cnt})=$ final - beg;

end

$$
\mathrm{jj}=\mathrm{jj}+1 \text {; }
$$

end $\quad \% n>=3$

$\%$ Write final whistle of a given vector.

if ( $\_$_ $\left.x>=2\right)$

$$
\begin{aligned}
& \text { prev_down }=0 \text {; } \\
& \text { prev_up }=0 \text {; } \\
& \text { start }=\text { cx_vec }\left(n_{-} \mathrm{x}, 1\right)-\text { prefix; } \\
& \text { stop }=\mathrm{cx} \_ \text {vec }\left(\mathrm{n}_{-} \mathrm{x}, 2\right)+\text { suffix; } \\
& \text { if }\left(\left(\mathrm{cx} \_\operatorname{vec}\left(\mathrm{n} \_\mathrm{x}, 2\right)-\mathrm{cx} \_\operatorname{vec}\left(\mathrm{n} \_\mathrm{x}, 1\right)\right)>\min \_ \text {dur }\right) \\
& \text { if ( }(\text { top }>N \text { ) } \\
& \text { next_suffix }=\text { stop }-\mathrm{N} \text {; } \\
& \text { stop }=\mathrm{N} \text {; } \\
& \text { next_suffix }=0 \text {; }
\end{aligned}
$$




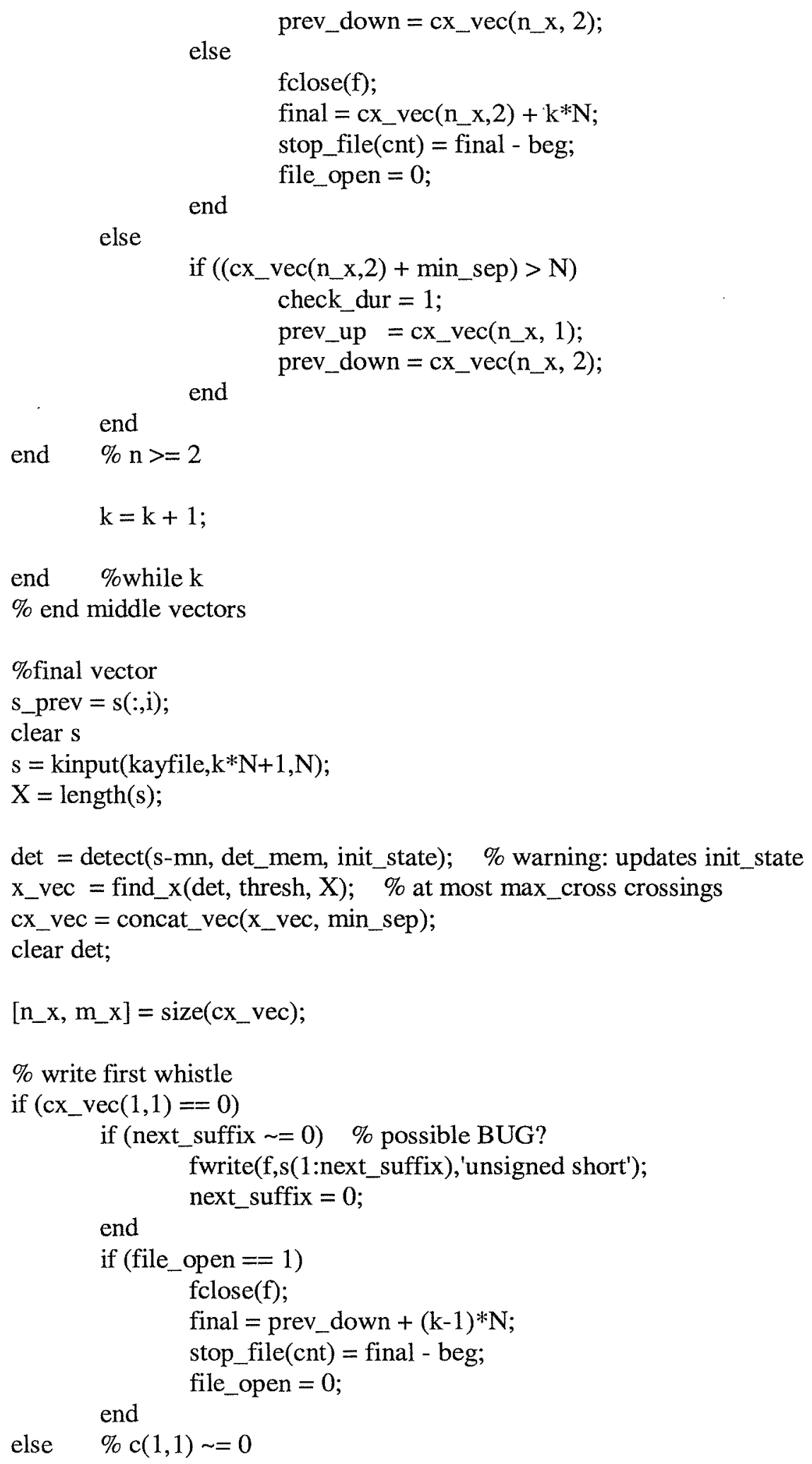


Appendix 2: Computer Programs

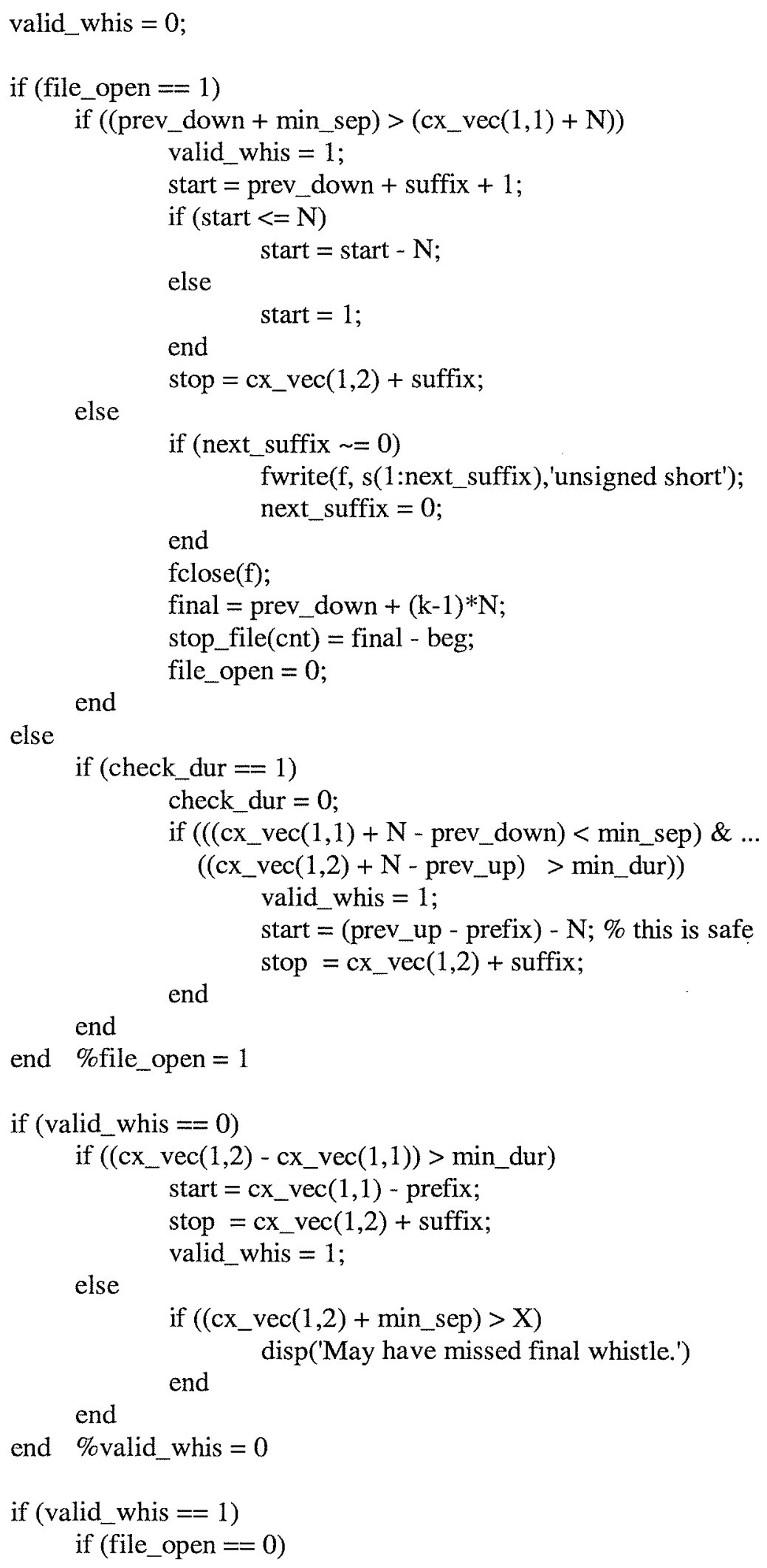




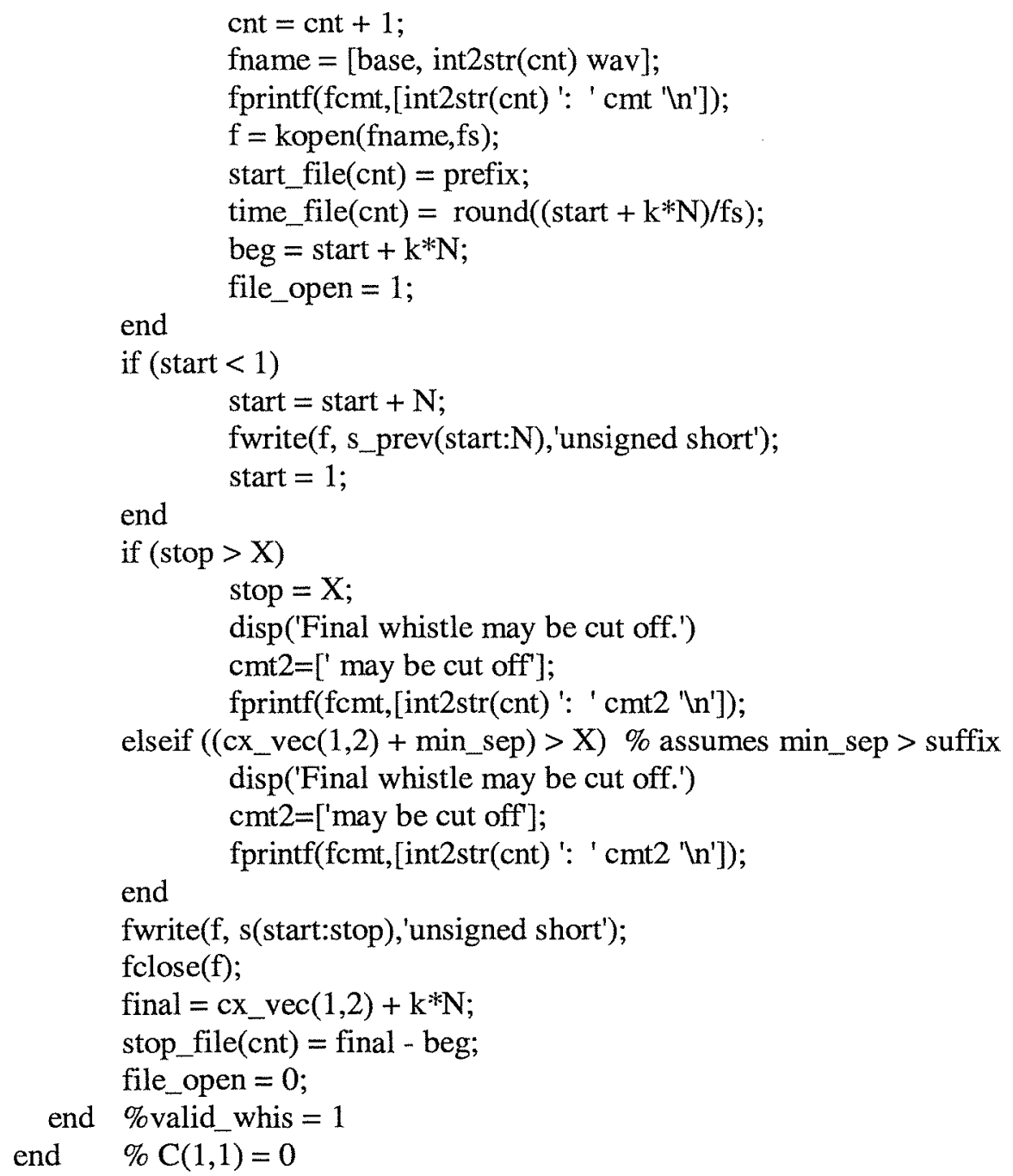

\% Write middle whistles (whistles 2 through $\mathrm{n} \_\mathrm{x}-1$ ).

if $\left(\mathrm{n} \_\mathrm{x}>=3\right)$

$\mathrm{jj}=2$;

while $\left(\mathrm{jj}<=\left(\mathrm{n} \_\mathrm{x}-1\right)\right)$

if $\left(\left(\mathrm{cx} \_v e c(j \mathrm{j}, 2)-\mathrm{cx} \_v e c(j \mathrm{j}, 1)\right)>\min \_d u r\right)$

start $=$ cx_vec(jj, 1) - prefix;

stop $=$ cx_vec $(j \mathrm{j}, 2)+$ suffix;

$\mathrm{cnt}=\mathrm{cnt}+1$

fname $=[$ base, int2str(cnt) wav $]$;

fprintf(fcmt, [int2str(cnt) ': ' cmt 'In']);

$\mathrm{f}=\operatorname{kopen}($ fname, $\mathrm{fs}$ );

start_file $(\mathrm{cnt})=$ prefix;

beg $=$ start $+\mathrm{k}^{*} \mathrm{~N}$;

time_file $($ cnt $)=\operatorname{round}((\operatorname{start}+\mathrm{k} * \mathrm{~N}) / \mathrm{fs})$;

fwrite(f, s(start:stop),'unsigned short'); 
Appendix 2: Computer Programs

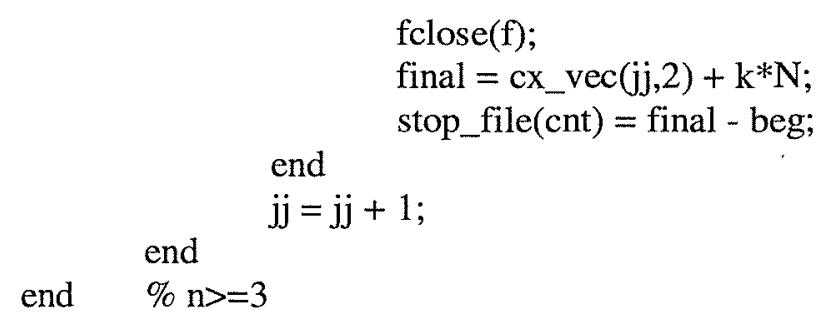

$\%$ Write final whistle of vector.

if $\left(n \_x>=2\right)$

start $=$ cx_vec $\left(n \_x, 1\right)-$ prefix;

stop $=\mathrm{cx} \_v e c\left(n \_x, 2\right)+$ suffix;

if $\left(\left(\mathrm{cx} \_v e c\left(n \_x, 2\right)-c x \_v e c\left(n \_x, 1\right)\right)>\right.$ min_dur $)$

if $($ stop $>X)$

disp('Final whistle may be cut off.')

$\mathrm{cmt}=\left[\mathrm{cmt},{ }^{\prime}\right.$ may be cut off']; stop $=X$;

elseif $\left(\mathrm{cx} \_v e c\left(n \_x, 2\right)+\right.$ min_sep $)>X$ disp('Final whistle may be cut off.')

end $\mathrm{cmt}=\left[\mathrm{cmt},{ }^{\prime}\right.$ may be cut off'];

cnt $=$ cnt +1 ;

fname $=[$ base, int2str(cnt) wav $]$;

fprintf(fcmt,[int2str(cnt) ': ' cmt 'ln']);

$\mathrm{f}=$ kopen (fname, $\mathrm{fs}$ );

start_file $(\mathrm{cnt})=$ prefix;

time_file $($ cnt $)=\operatorname{round}\left(\left(\right.\right.$ start $\left.\left.+\mathrm{k}^{*} \mathrm{~N}\right) / \mathrm{fs}\right)$;

beg $=$ start $+\mathrm{k} * \mathrm{~N}$;

fwrite(f, s(start:stop), 'unsigned short');

fclose(f);

final $=$ cx_vec $\left(n \_x, 2\right)+k * N$;

stop_file $($ cnt $)=$ final - beg;

file_open $=0$;

else

if $\left(\left(\mathrm{cx} \_v e c\left(n \_x, 2\right)+\min \_s e p\right)>X\right)$

disp('May have missed final whistle')

end

end

end $\quad \% \mathrm{n}>=2$

$\%$ end final vector

fclose(fcmt);

start_file = start_file';

stop_file $=$ stop_file';

time_file = time_file';

file $=[$ base '.start' $]$;

save(file,'start_file','-ascii'); 
file $=[$ base '.stop' $]$;

save(file,'stop_file','-ascii');

file $=[$ base '.time' $]$;

save(file,'time_file,'-ascii');

\subsubsection{The Power Calculation}

function det $=$ detect(input,det_mem,init_state)

$\% \operatorname{det}=\operatorname{detect}($ input,det_mem,init_state)

$\%$ power detector function

temp $=$ input. ${ }^{\wedge}$;

temp $=[$ init_state; temp $]$;

mem $=-1$ *det_mem;

fil = filter $([1],[1$, mem $]$,temp $)$;

$\mathrm{n}=$ length(fil);

$\operatorname{det}=\operatorname{fil}(2: n)$;

\subsubsection{The Detection of Above-Threshold Sounds}

function $x=$ find_ $x(v$, thresh, $N)$

$\% \mathrm{x}=$ find_ $\mathrm{x}(\mathrm{v}$, thresh, $\mathrm{N})$

$\%$ function to find threshold crossings

$\mathrm{vt}=\mathrm{v}>\mathrm{thresh}$

$\mathrm{dv}=\operatorname{diff}(\mathrm{vt})$

if $\operatorname{vt}(1)==1$

$\mathrm{dv}=[1 ; \mathrm{dv}]$

else

end

$$
\mathrm{dv}=[0 ; \mathrm{dv}]
$$

$\mathrm{up}=$ find $(\mathrm{dv}==1)$;

down $=$ find $(d v==-1)$;

$\mathrm{nu}=$ length(up);

nd = length(down);

if nu $>$ nd

$$
\text { down }=[\text { down; } N] \text {; }
$$

end

if $n u==0$

$$
\mathrm{x}=\left[\begin{array}{ll}
0 & 0
\end{array}\right] \text {; }
$$

else

$$
\mathrm{x}=[\mathrm{up}, \text { down }] \text {; }
$$

end 
Appendix 2: Computer Programs

\subsubsection{Concatenation of Sections Within $100 \mathrm{~ms}$}

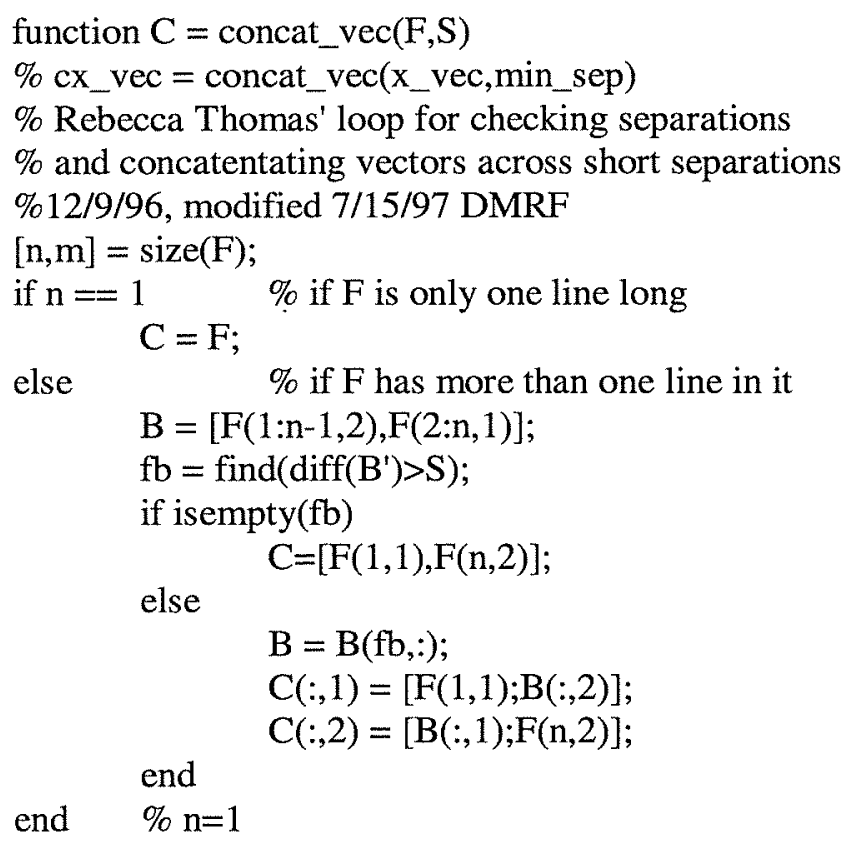

\subsubsection{Opening a New Kay File}

function fid=kopen(filename,samprate)

$\%$

$\%$ kopen('filename',samprate)

$\%$

$\%$ Function to open a Kay format file.

$\%$

[fid message]=fopen(filename, 'wb','l');

if $\mathrm{fid}==-1$

sprintf('Error opening \%s $\ln ^{\prime}$,filename)

sprintf('\%sln',message)

return;

end;

header=zeros $(256,1)$;

header $(61)=2$;

header(62) $=10000 /(1000000 /$ samprate);

fwrite(fid,header,'unsigned short'); 


\subsection{THE AUTOMATIC SORTER}

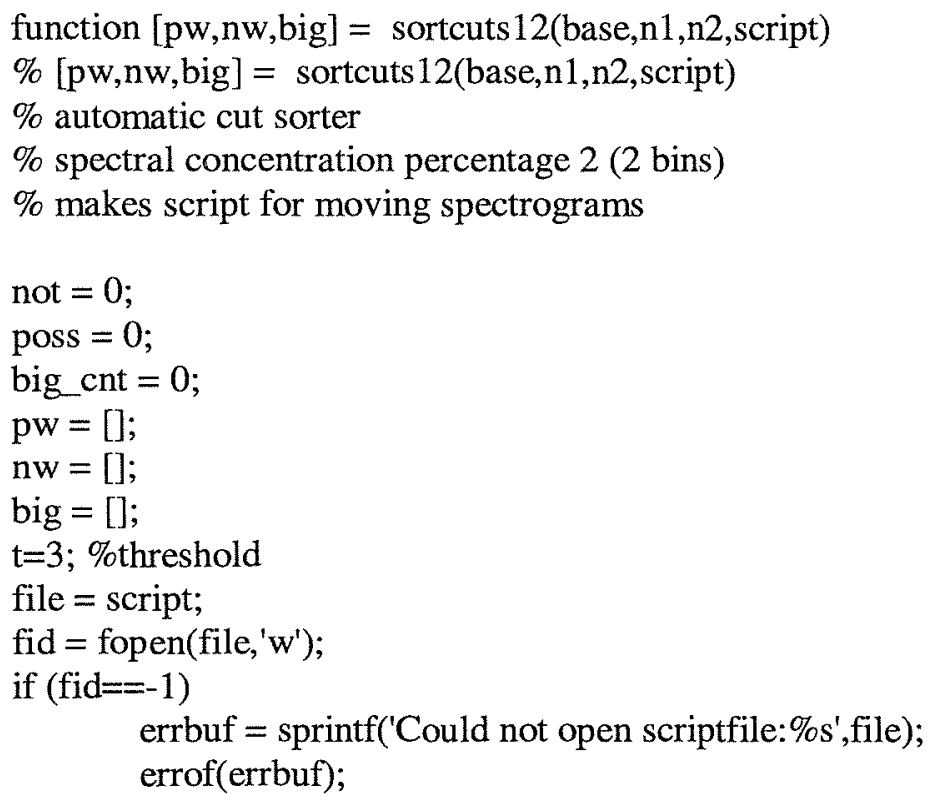

else

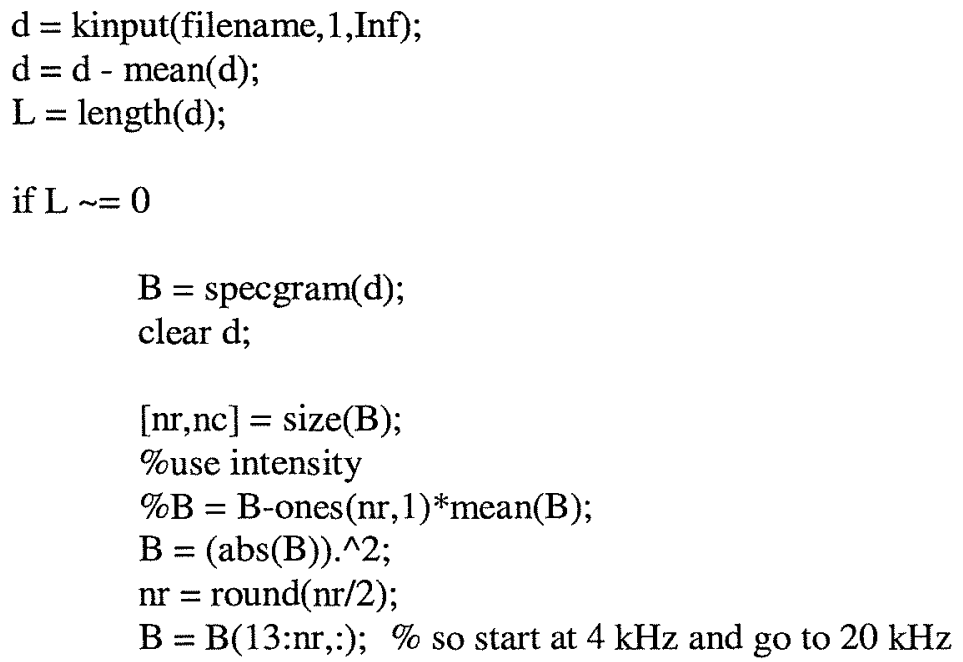


Appendix 2: Computer Programs

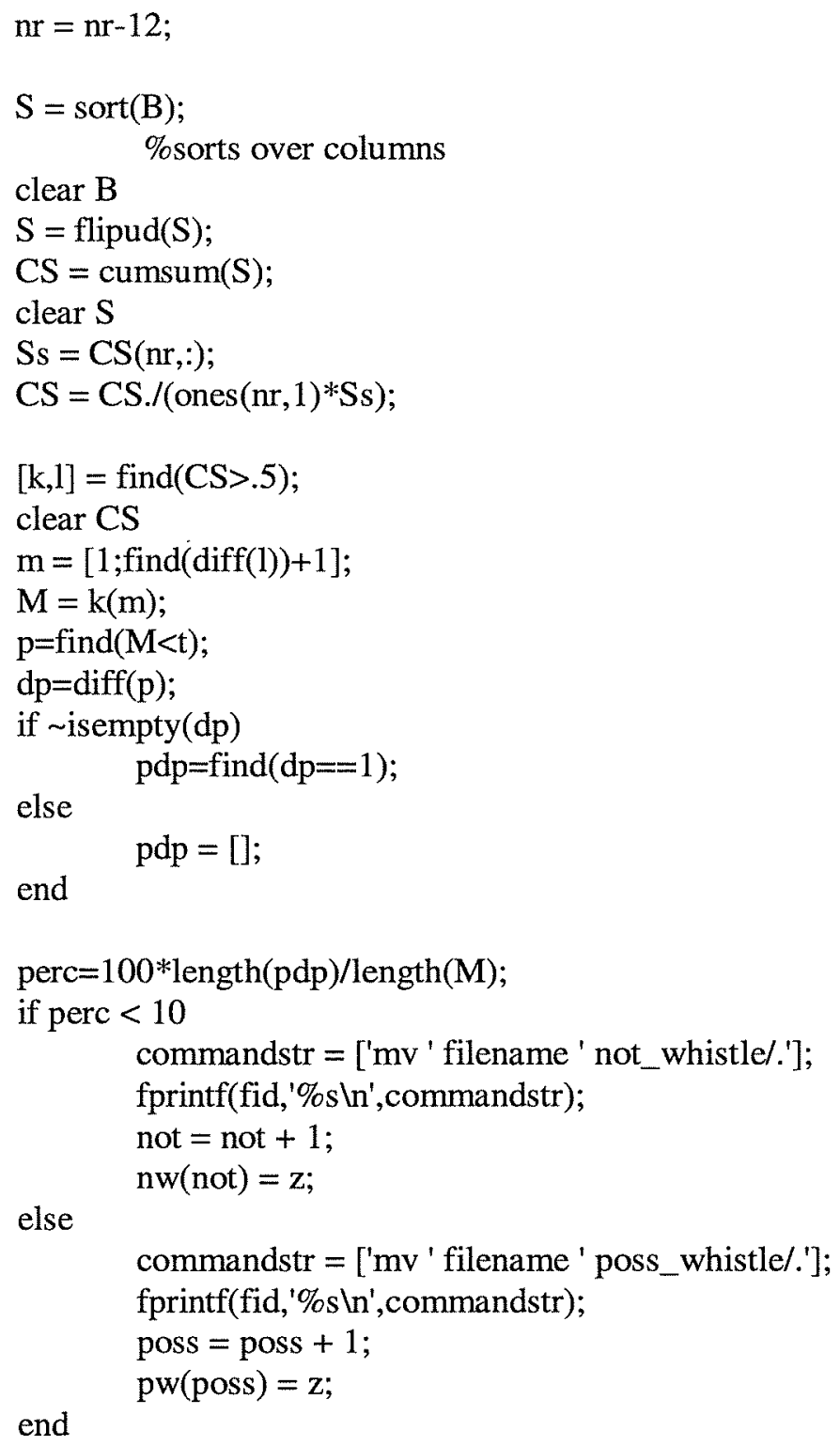




\subsection{THE MANUAL SORTER}

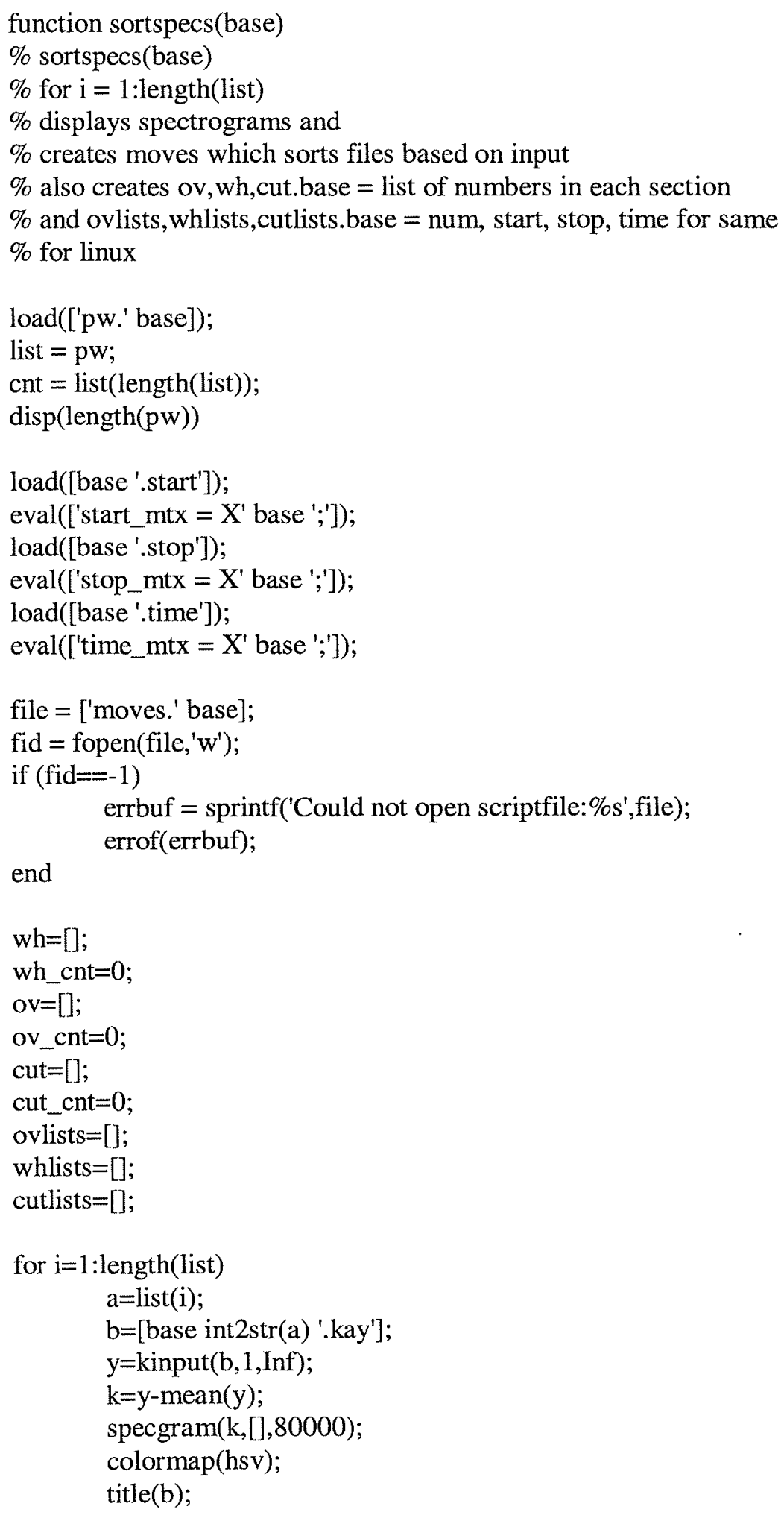


Appendix 2: Computer Programs

\section{zoom on}

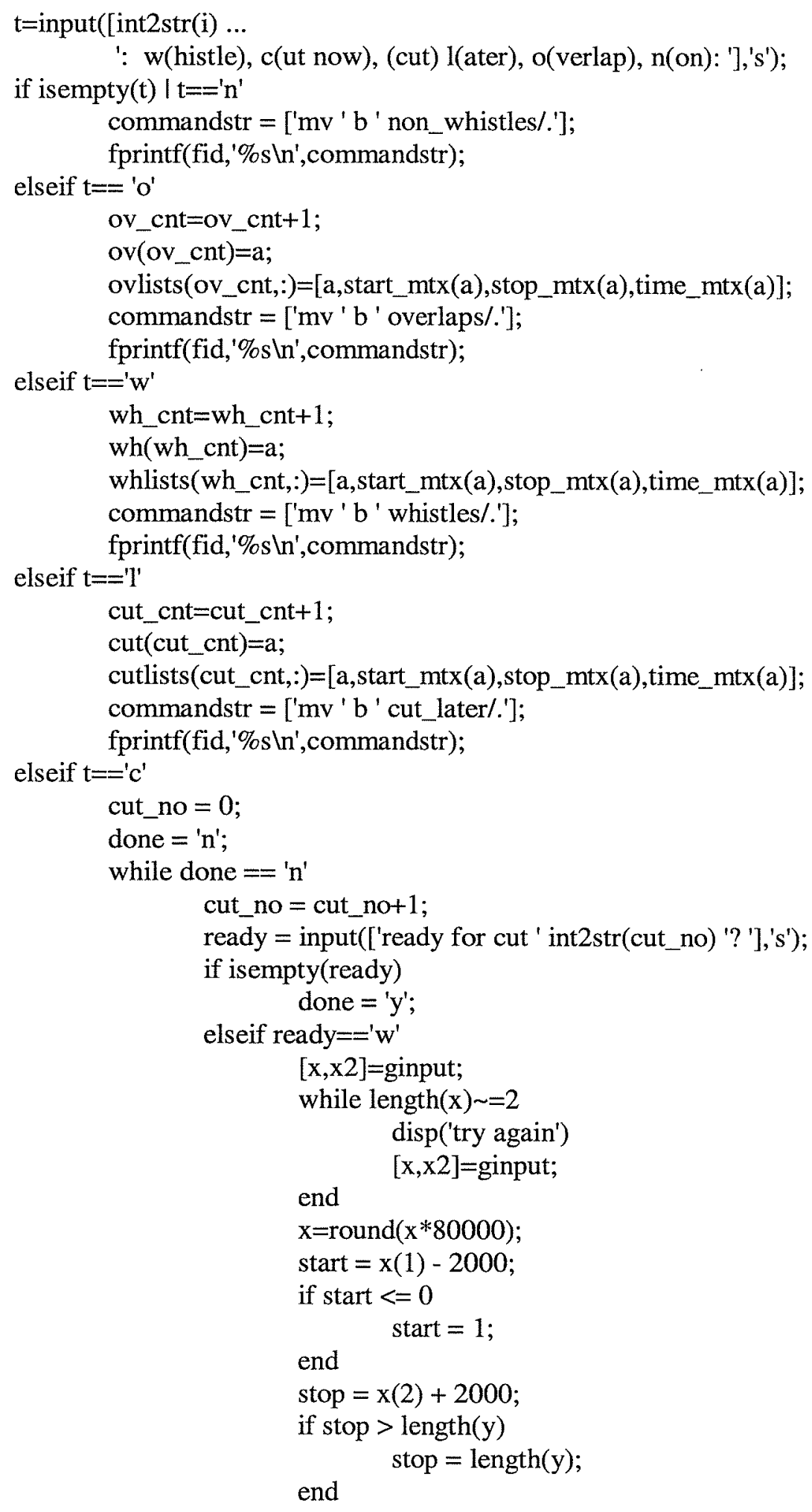




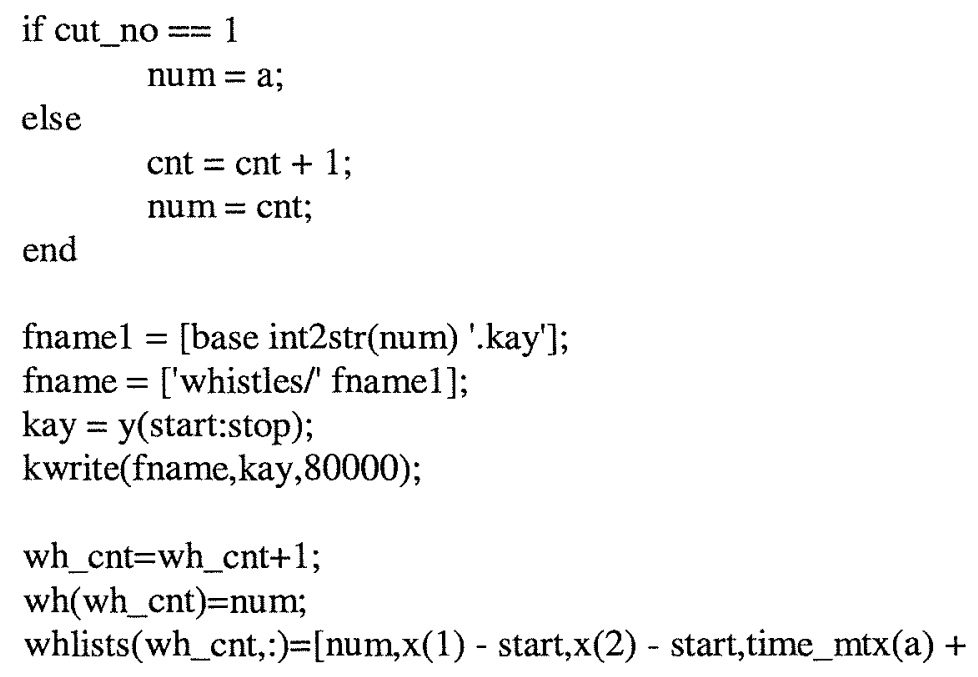

start/80000];

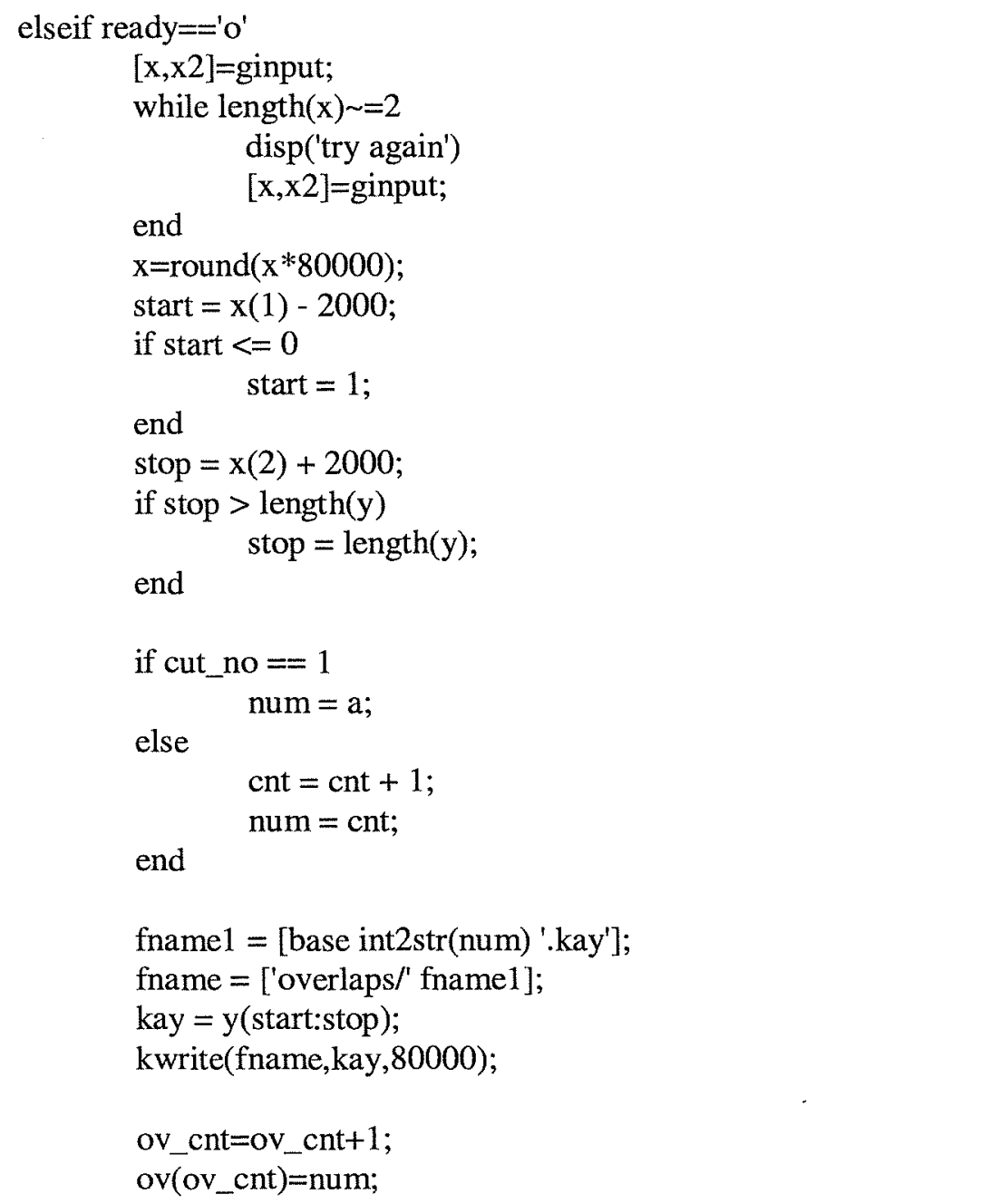




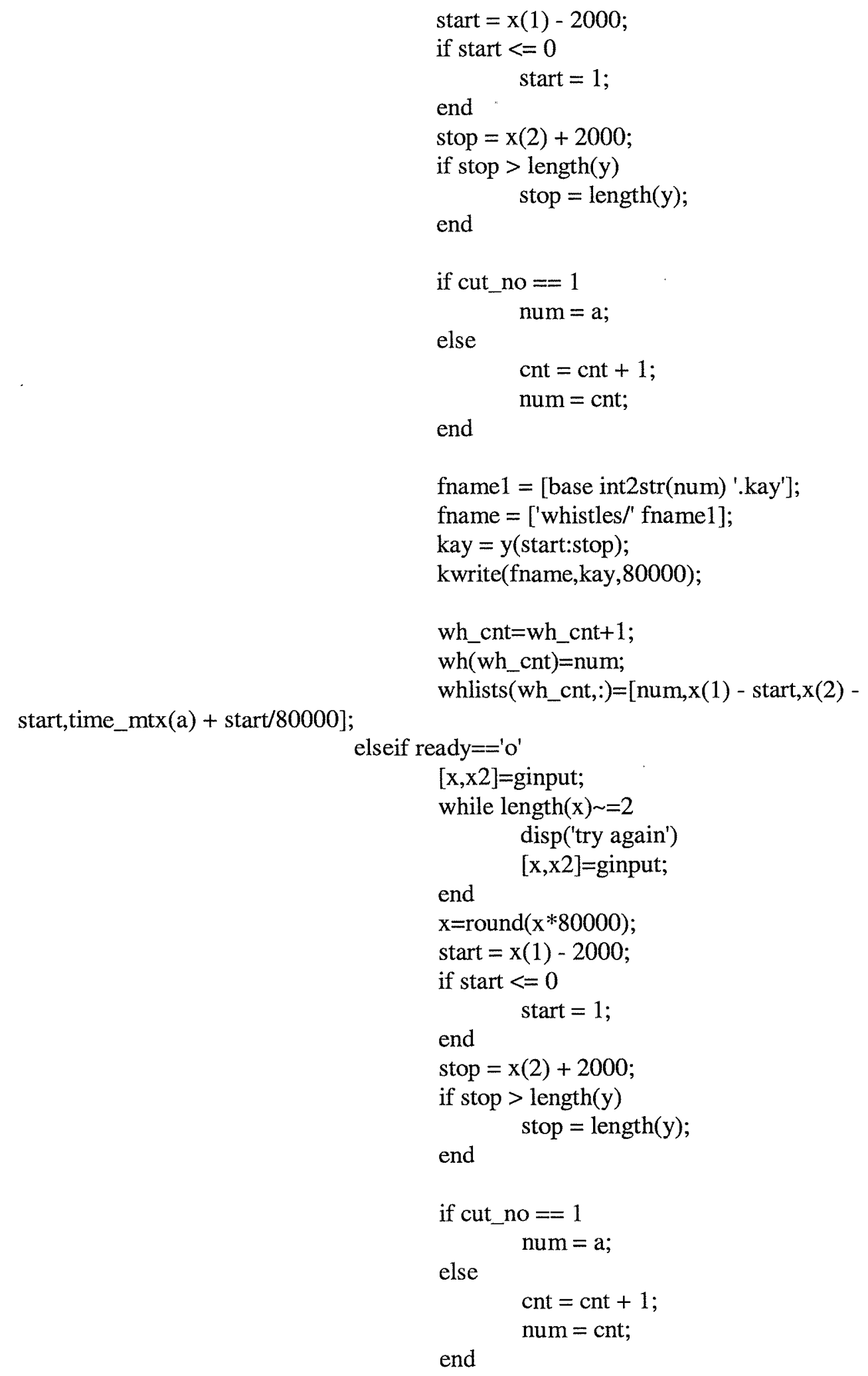


Appendix 2: Computer Programs

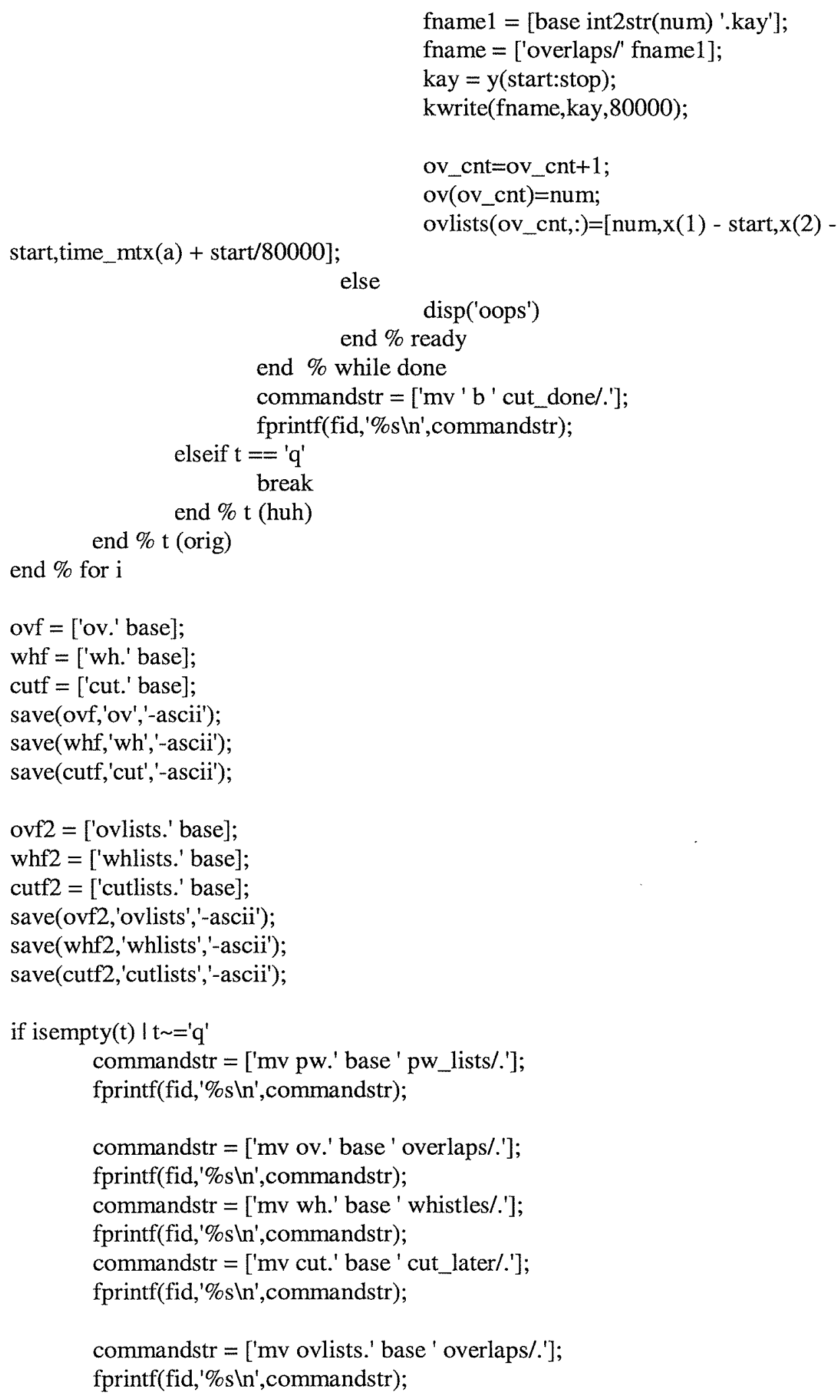


commandstr $=[$ 'mv whlists.' base ' whistles/.']; fprintf(fid,'\%sln',commandstr);

commandstr = ['mv cutlists.' base ' cut_later/.'];

fprintf(fid, '\%s $\backslash n$ ',commandstr);

end

fclose(fid);

eval(['! chmod +x ' file]);

disp(['count: ' int2str(cnt)]);

disp(['whistle count: ' int2str(wh_cnt)]);

disp(['overlap count: ' int2str(ov_cnt)]);

disp(['cut count: ' int2str(cut_cnt)]); 
Appendix 2: Computer Programs

\section{SECTION 3: PROGRAMS FOR CONTOUR COMPARISON}

\subsection{CONTOUR CROSS-CORRELATION}

function $\mathrm{c}=\mathrm{ctrcrln}$ (bases,ind)

$\% \mathrm{c}=\mathrm{ctrcr} \ln ($ bases,ind)

$\%$ bases=column vector of base names

$\%$ ind=matrix of cuts for each base (=row)

$\mathrm{n}=\operatorname{size}($ bases, 1$)$;

$\mathrm{m}=\operatorname{size}($ ind,2);

row $=0$;

for $\mathrm{i}=1: \mathrm{n}$

base $1=$ bases $(\mathrm{i},:)$;

for $\mathrm{k}=1: \mathrm{m}$

col $=0$;

if ind $(\mathrm{i}, \mathrm{k}) \sim=0$

row $=$ row +1 ;

file1=[base1 int2str(ind(i,k)) '.ctf'];

load(file1,'-mat')

$\mathrm{n} 1=\mathrm{ctr}$;

for $\mathrm{jj}=1: \mathrm{n}$

base2=bases(jj,:);

for $\mathrm{kk}=1: \mathrm{m}$

if ind $(\mathrm{jj}, \mathrm{kk}) \sim=0$

$\mathrm{col}=\mathrm{col}+1$

file2=[base2 int2str(ind(jj,kk)) '.ctf'];

load(file2,'-mat')

$\mathrm{n} 2=\mathrm{ctr}$;

$\mathrm{c} 1=\max (\mathrm{xcorr}(\mathrm{n} 1))$;

$\mathrm{c} 2=\max (\operatorname{xcorr}(\mathrm{n} 2))$;

$\mathrm{nm}=\operatorname{sqrt}\left(\mathrm{c} 1 *_{\mathrm{c}} 2\right)$;

end

$\mathrm{c}($ row, col $)=\max (\mathrm{xcorr}(\mathrm{n} 1, \mathrm{n} 2)) / \mathrm{nm}$;

end

end

end

end

end 


\subsection{DICTIONARY CONTOUR COMPARISONS}

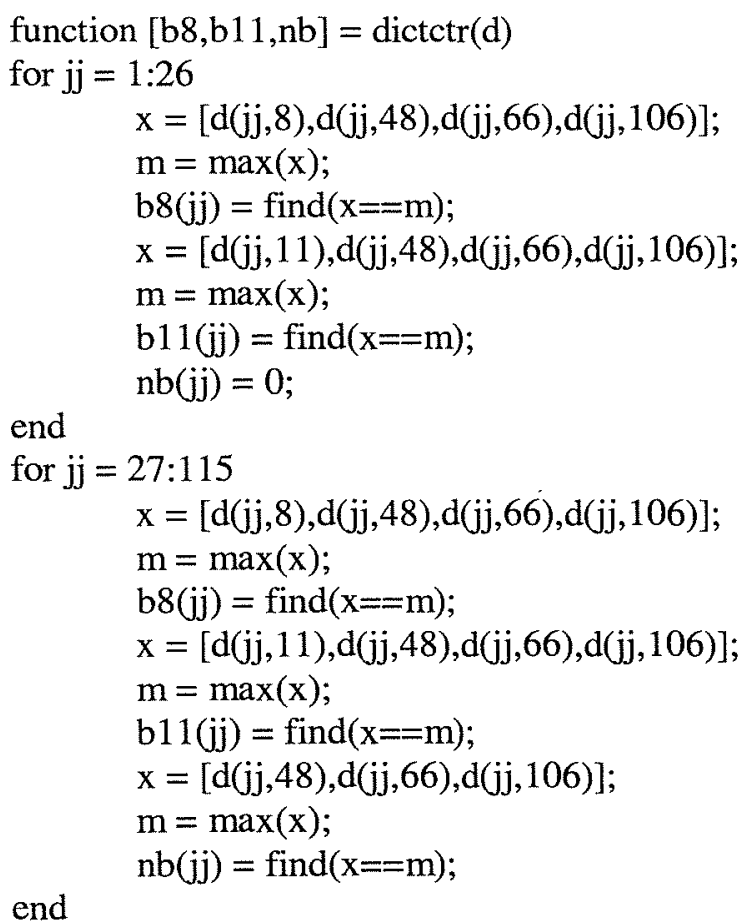


Appendix 2: Computer Programs

\subsection{THE MOAT INDEX}

\subsubsection{Calculating the Cluster Connectivity}

function [last, next $]=$ moatcalc $(\mathrm{m})$

$\% \mathrm{~m}$ is the output of Systat

$$
\begin{aligned}
& \text { len }=\operatorname{size}(m, 1) \\
& \text { for } \mathrm{i}=1 \text { :len } \\
& \mathrm{n} 1=\mathrm{m}(\mathrm{i}, 1) \text {; } \\
& \mathrm{n} 2=\mathrm{m}(\mathrm{i}, 2) \text {; } \\
& \mathrm{f} 11=\text { find }(\mathrm{m}(:, 1)==\mathrm{n} 1) \text {; } \\
& \mathrm{f} 12=\operatorname{find}(\mathrm{m}(:, 2)==\mathrm{n} 1) \text {; } \\
& \mathrm{f} 1=\operatorname{sort}([\mathrm{f} 11 ; \mathrm{f} 12]) \text {; } \\
& \mathrm{lc} 1 \text { = find(f1 == i); } \\
& \mathrm{f} 21=\operatorname{find}(\mathrm{m}(:, 1)==\mathrm{n} 2) \text {; } \\
& \mathrm{f} 22=\text { find }(m(:, 2)==n 2) \text {; } \\
& \mathrm{f} 2=\operatorname{sort}([\mathrm{f} 21 ; \mathrm{f} 22]) \text {; } \\
& \mathrm{lc} 2=\text { find(f2 == i); } \\
& \text { if } 1 \mathrm{c} 1==1 \% \text { Last } \\
& \operatorname{last}(\mathrm{i}, 1)=0 \text {; } \\
& \operatorname{last}(\mathrm{i}, 1)=\mathrm{f} 1(\mathrm{lc} 1-1) \\
& \text { last }(\mathrm{i}, 2)=\mathrm{f} 2(\mathrm{lc} 2-1) \\
& \text { if } \mathrm{lc} 1==\text { length(f1) } \% \text { Next } \\
& \mathrm{x} 1=\mathrm{len}+1 \text {; } \\
& \mathrm{x} 1=\mathrm{f} 1(\mathrm{lc} 1+1) \\
& \operatorname{next}(\mathrm{i})=0 \text {; }
\end{aligned}
$$

end 


\subsubsection{The Moat Index Calculation}

Instructions for Moat Index:

Make an extra copy of the original spreadsheet to keep unchanged

Copy Systat output, and get it into a spreadsheet

It should have 4 columns and $\mathrm{N}-1$ rows, where $\mathrm{N}=$ number of records entered into the cluster analysis

Copy the first 3 columns into the columns B to D of the yellow section of the worksheet

Take the data over to Matlab and run through moatcalc

Return moatcalc numbers to the worksheet and copy to columns $\mathrm{E}$ to $\mathrm{G}$ of the yellow section

Select H8:P8 and drag down to extend these formulas to the last row of data, do the same for A8

Select the cells starting with $\mathrm{A} 7: \mathrm{K} 7$ and extending down through the last row of the aggomeration table.

Assign the name "'BigTable"" to this range.

Read number of clusters and associated moat indices from columns $\mathrm{O}$ and $\mathrm{P}$

Find the maximum moat index in column $\mathrm{P}$ and the associated number of clusters.

Example Table:

\begin{tabular}{|c|c|c|c|c|c|c|c|c|c|c|c|c|c|c|}
\hline \multicolumn{8}{|c|}{ Set name BigTable $=$ area contiguous with imported data } & & & & \multicolumn{4}{|c|}{ This blank column needs to be her } \\
\hline \multicolumn{4}{|c|}{ Yellow is SPSS output } & & & Smallest: & 0.10 & & & & & & & \\
\hline & & & & & & Range: & 0.80 & & & & & & & \\
\hline & \multicolumn{2}{|c|}{$\begin{array}{l}\text { Clusters } \\
\text { combined }\end{array}$} & & \multicolumn{2}{|c|}{$\begin{array}{l}\text { Stage cluster } \\
\text { first appears }\end{array}$} & & & & & & \multicolumn{2}{|c|}{$\begin{array}{c}\text { Old segments } \\
\text { joined }\end{array}$} & & \\
\hline Stage & 1 & 2 & Coeff & 1 & 2 & $\begin{array}{r}\text { Next } \\
\text { stage }\end{array}$ & $\begin{array}{l}\text { Distance: } \\
\text { (or Log } \\
\text { Distance) }\end{array}$ & $\begin{array}{l}\text { Standardized } \\
\text { Distance }\end{array}$ & $\begin{array}{c}\text { Trunk } \\
\text { end }\end{array}$ & Length & $\begin{array}{l}\text { Length } \\
1\end{array}$ & $\begin{array}{l}\text { Length } \\
2\end{array}$ & $\begin{array}{l}\text { Resulting } \\
\text { \# of } \\
\text { clusters }\end{array}$ & $\begin{array}{l}\text { Moat } \\
\text { index }\end{array}$ \\
\hline & & & & & & & & & & & & & & \\
\hline 0 & & & & & & & & 25. & & & & & 4 & 10.00 \\
\hline 1 & 1 & 2 & 0.9 & c & 0 & 3 & 0.100 & 1.0 & 25 & 24.0 & 1 & 1 & 3 & 20.67 \\
\hline 2 & 3 & 4 & 0.3 & & c & 3 & 0.700 & 19.0 & 25 & 6.0 & 19 & 19 & 2 & 15.00 \\
\hline 3 & 1 & 3 & 0.1 & & 2 & c & 0.900 & 25.0 & 25 & 0.0 & 24 & 6 & 1 & 0.00 \\
\hline & & & & & & & & & & & & & & \\
\hline & & & & & & & & & & & & & & \\
\hline & & & & & & & & & & & & & & \\
\hline & & & & & & & & & & & & & & \\
\hline & & & & & & & & & & & & & & \\
\hline & & & & & & & & & & & & & & \\
\hline
\end{tabular}


Appendix 2: Computer Programs

\section{SECTION 4: CALCULATIONS FOR CHAPTER 5}

\subsection{The OVERLAP TeST}

function perc $=$ overlap(rate,runs, dur)

$\%$ rate $=$ number of whistles to use

$\%$ dur $=$ average contour duration

$\%$ runs $=$ number of simulations

dur $=\mathrm{dur} / 1000$

for $\mathrm{k}=1$ :runs

for $m=1$ :rate

$w h(m)=r^{* a n d} * 600$;

end

$\mathrm{s}=$ sort $(\mathrm{wh})$

$\mathrm{d}=\operatorname{diff}(\mathrm{s})$;

$\mathrm{f}=$ find $(\mathrm{d}<$ dur $)$;

len=length $(f)$

$\operatorname{perc}(k)=l e n /$ rate $^{*} 100$;

end

\subsection{THE QUARTILE BANDWIDTH}

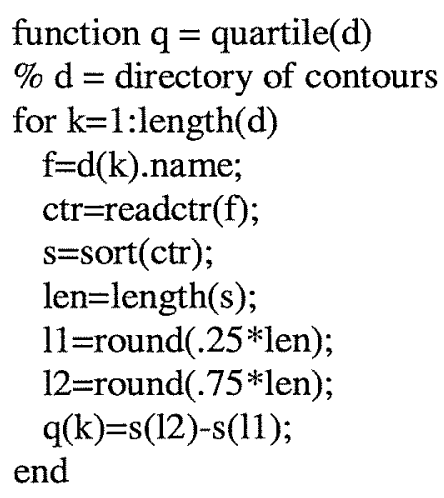

\subsection{READING THE CONTOUR}

NOTE: this program was not written by me.

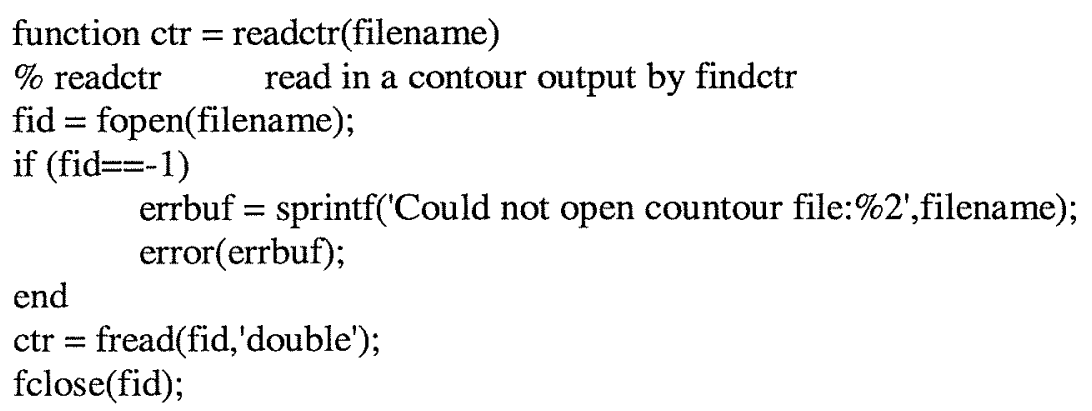




\section{APPENDIX 3: PROGRAMS BY OTHER PEOPLE}

\section{SECTION 1: COMPLETE ACOUSTAT OUTPUT}

\subsection{STATISTICS}

\begin{tabular}{|ll|}
\hline Measurement & Definition \\
\hline Mode & Most common frequency \\
Median & Central frequency \\
Upper & Upper frequency \\
Spread & Difference between highest and lowest frequency \\
Concentration & Minimum bins needed for half the total energy \\
Modewidth & Ratio of total energy to modal energy \\
Asymmetry & (Median - Lower)/(Upper-Lower) \\
\hline
\end{tabular}

\subsection{Spectra Used to Calculate Measurements}

\begin{tabular}{|ll|}
\hline Spectrum & Definition \\
\hline AM5 & 50\% of amplitude modulation spectrum \\
AM7 & 75\% of amplitude modulation spectrum \\
AFM5 & Weighted mean frequency contour, with weights associated with \\
& loudest 50\% of spectrum \\
AFM7 & Weighted mean frequency contour, with weights associated with \\
& loudest 75\% of spectrum \\
TS5 & Average power spectrum, loudest 50\% \\
TS7 & Average power spectrum, loudest 75\% \\
MS5 & Modal frequency, loudest 50\% \\
MS7 & Modal frequency, loudest 75\% \\
ENV5 & Time-amplitude envelope, loudest $50 \%$ \\
ENV7 & Time-amplitude envelope, loudest $75 \%$ \\
FMOD & Modal frequency \\
FMED & Median frequency \\
CONC & Spectral concentration (see 1.1) \\
MODW & Modewidth (see 1.1) \\
FSPRD & Spectral spread (see 1.1) \\
FASYM & Spectral asymmetry \\
\hline
\end{tabular}

1.3 MEASUREMENTS

\begin{tabular}{|c|c|c|}
\hline No. & & Description \\
\hline 0 & $\overline{F N}$ & Tape name \\
\hline 0 & $\mathrm{CN}$ & Cut name \\
\hline 0 & LF & Low frequency cutoff \\
\hline 0 & $\mathrm{HF}$ & High frequency cutoff \\
\hline 0 & Bsize & Block size \\
\hline
\end{tabular}


Appendix 3: Programs by Other People

\begin{tabular}{|c|c|c|}
\hline No. & ID & Description \\
\hline 0 & Xsize & FFT size \\
\hline 0 & Olap & Overlap between adjacent FFTs in a block \\
\hline 0 & CS & Cut size \\
\hline 1 & NumBlocks & Number of data blocks (Bsize) \\
\hline 2 & MaxFlat & Longest section with minimal change in the frequency mode \\
\hline 3 & AM5mode & Mode of AM5 \\
\hline 4 & AM5med & Median of AM5 \\
\hline 5 & AM5upp & Upper frequency of AM5 \\
\hline 6 & AM5sprd & Frequency spread of AM5 \\
\hline 7 & AM5conc & Spectral concentration of AM5 \\
\hline 8 & AM5modw & Modewidth of AM5 \\
\hline 9 & AM5asym & Assymetry of AM5 \\
\hline 10 & AM7mode & Mode of AM7 \\
\hline 11 & AM7med & Median of AM7 \\
\hline 12 & AM7upp & Upper frequency of AM7 \\
\hline 13 & AM7sprd & Frequency spread of AM7 \\
\hline 14 & AM7conc & Spectral concentration of AM7 \\
\hline 15 & AM7modw & Modewidth of AM7 \\
\hline 16 & AM7asym & Asymmetry of AM7 \\
\hline 17 & AFM5mode & Mode of AFM5 \\
\hline 18 & AFM5med & Median of AFM5 \\
\hline 19 & AFM5upp & Upper frequency of AFM5 \\
\hline 20 & AFM5sprd & Frequency spread of AFM5 \\
\hline 21 & AFM5conc & Spectral concentration of AFM5 \\
\hline 22 & AFM5modw & Modewidth of AFM5 \\
\hline 23 & AFM5asym & Asymmetry of AFM5 \\
\hline 24 & AFM7mode & Mode of AFM7 \\
\hline 25 & AFM7med & Median of AFM7 \\
\hline 26 & AFM7upp & Upper frequency of AFM7 \\
\hline 27 & AFM7sprd & Frequency spread of AFM7 \\
\hline 28 & AFM7conc & Spectral concentration of AFM7 \\
\hline 29 & AFM7modw & Modewidth of AFM7 \\
\hline 30 & AFM7asym & Asymmetry of AFM7 \\
\hline 31 & TS5mode & Mode of TS5 \\
\hline 32 & TS5med & Median of TS5 \\
\hline 33 & TS5upp & Upper frequency of TS5 \\
\hline 34 & TS5sprd & Frequency spread of TS5 \\
\hline 35 & TS5conc & Spectral concentration of TS5 \\
\hline 36 & TS5modw & Modewidth of TS5 \\
\hline 37 & TS5asym & Asymmetry of TS5 \\
\hline 38 & TS7mode & Mode of TS7 \\
\hline 39 & TS7med & Median of TS7 \\
\hline 40 & TS7upp & Upper frequency of TS7 \\
\hline
\end{tabular}




\begin{tabular}{|c|c|c|}
\hline No. & ID & Description \\
\hline 41 & TS7sprd & Frequency spread of TS7 \\
\hline 42 & TS7conc & Spectral concentration of TS7 \\
\hline 43 & TS7modw & Modewidth of TS7 \\
\hline 44 & TS7asym & Asymmetry of TS7 \\
\hline 45 & MS5mode & Mode of MS5 \\
\hline 46 & MS5med & Median of MS5 \\
\hline 47 & MS5upp & Upper frequency of MS5 \\
\hline 48 & MS5sprd & Frequency spread of MS5 \\
\hline 49 & MS5conc & Spectral concentration of MS5 \\
\hline 50 & MS5modw & Modewidth of MS5 \\
\hline 51 & MS5asym & Asymmetry of MS5 \\
\hline 52 & MS7mode & Mode of MS7 \\
\hline 53 & MS7med & Median of MS7 \\
\hline 54 & MS7upp & Upper frequency of MS7 \\
\hline 55 & MS7sprd & Frequency spread of MS7 \\
\hline 56 & MS7conc & Spectral concentration of MS7 \\
\hline 57 & MS7modw & Modewidth of MS7 \\
\hline 58 & MS7asym & Asymmetry of MS7 \\
\hline 59 & ERGtot & Total energy after noise removal \\
\hline 60 & ENV5mode & Mode of ENV5 \\
\hline 61 & ENV5med & Median of ENV5 \\
\hline 62 & ENV5upp & Upper frequency of ENV5 \\
\hline 63 & ENV5sprd & Frequency spread of ENV5 \\
\hline 64 & ENV5conc & Spectral concentration of ENV5 \\
\hline 65 & ENV5modw & Modewidth of ENV5 \\
\hline 66 & ENV5asym & Asymmetry of ENV5 \\
\hline 67 & ENV7mode & Mode of ENV7 \\
\hline 68 & ENV7med & Median of ENV7 \\
\hline 69 & ENV7upp & Upper frequency of ENV7 \\
\hline 70 & ENV7sprd & Frequency spread of ENV7 \\
\hline 71 & ENV7conc & Spectral concentration of ENV7 \\
\hline 72 & ENV7modw & Modewidth of ENV7 \\
\hline 73 & ENV7asym & Asymmetry of ENV7 \\
\hline 74 & MNnum & Number of blocks with adjacent, non-zero energy \\
\hline 75 & ATAKfrac & Fraction of 74 with increasing energy \\
\hline 76 & SWPfrac & Fraction of 74 with differing modal frequencies \\
\hline 77 & UPSfrac & Fraction of 74 with increasing frequency \\
\hline 78 & UPSmean & Average increase in frequency from 77 \\
\hline 79 & SWPabsmag & Average absolute difference from 77 \\
\hline 80 & ZERnum & Number of zero blocks \\
\hline 81 & ERGmed & Median energy \\
\hline 82 & ERGcV & Energy coefficient of variance \\
\hline
\end{tabular}


Appendix 3: Programs by Other People

\begin{tabular}{|c|c|c|}
\hline No. & ID & Description \\
\hline 83 & ERGmxmd & Maximum energy/median energy \\
\hline 84 & ERGasym & Energy asymmetry \\
\hline 85 & TFMODr & $\begin{array}{l}\text { Deviation of nonparametric correlation coefficient for time and } \\
\text { modal frequency, in standard deviation }\end{array}$ \\
\hline 86 & TFMBWr & Time-modewidth correlation (as in 85) \\
\hline 87 & AFMBWR & Amplitude-modewidth correlation (as in 85) \\
\hline 88 & Tar & Time-amplitude correlation (as in 85 ) \\
\hline 89 & TFMEDr & Time-median frequency correlation (as in 85 ) \\
\hline 90 & AFMEDr & Amplitude-median frequency correlation (as in 85) \\
\hline 91 & FMODmode & Mode of FMOD \\
\hline 92 & FMODmed & Median of FMOD \\
\hline 93 & FMODsprd & Frequency spread of FMOD \\
\hline 94 & FMODasym & Asymmetry of FMOD \\
\hline 95 & FMEDmode & Mode of FMED \\
\hline 96 & FMEDmed & Median of FMED \\
\hline 97 & FMEDsprd & Frequency spread of FMED \\
\hline 98 & FMEDasym & Asymmetry of FMED \\
\hline 99 & CONCmode & Mode of CONC \\
\hline 100 & CONCmed & Median of CONC \\
\hline 101 & CONCsprd & Frequency spread of CONC \\
\hline 102 & CONCasym & Asymmetry of CONC \\
\hline 103 & MODWmode & Mode of MODW \\
\hline 104 & MODWmed & Median of MODW \\
\hline 105 & MODWsprd & Frequency spread of MODW \\
\hline 106 & MODWasym & Asymmetry of MODW \\
\hline 107 & FSPRDmode & Mode of FSPRD \\
\hline 108 & FSPRDmed & Median of FSPRD \\
\hline 109 & FSPRDsprd & Frequency spread of FSPRD \\
\hline 110 & FSPRDasym & Asymmetry of FSPRD \\
\hline 111 & AFSPRDr & Amplitude-frequency spread correlation (as in 85) \\
\hline 112 & FMEDFSPRDr & Median frequency-spectral spread correlation (as in 85) \\
\hline 113 & TFSPRDr & Time-spectral spread correlation (as in 85 ) \\
\hline 114 & FASYMmode & Mode of FASYM \\
\hline 115 & FASYMmed & Median of FASYM \\
\hline 116 & FASYMsprd & Frequency spread of FASYM \\
\hline 117 & FASYMasym & Asymmetry of FASYM \\
\hline 118 & AFASYMr & Amplitude-spectral asymmetry correlation (as in 85) \\
\hline 119 & FMEDFASYMr & Median frequency-spectral asymmetry correlation (as in 85 ) \\
\hline 120 & TFASYMr & Time-spectral asymmetry correlation (as in 85 ) \\
\hline
\end{tabular}

AcouStat was written by Kurt Fristrup.

Fristrup, K.M. and Watkins, W.A., 1994. "Marine animal sound classification." Woods Hole Oceanographic Institution Technical Report No. 94-13, Woods Hole, MA. 


\section{SECTION 2: CONTOUR EXTRACTION AND COMPARISON}

\subsection{CONTOUR EXTRACTOR}

USAGE: findctr kayfile outfile start stop [-sz block-size] [-st step-size] [-fl floorthreshold] [-pk peak-threshold] [-lowfreq lower bound $(\mathrm{kHz})$ ] [-highfreq upper bound $(\mathrm{kHz})]$ [-matout] [-hm -re -bl] [-fs sampling frequency] [-notchctr notch center $(\mathrm{Hz})]$ [-notchbw notch bandwidth $(\mathrm{Hz})]$

[]$=$ optional

\begin{tabular}{|ll|}
\hline Argument & Definition \\
\hline kayfile & The file with the whistle in question, in kay format. \\
outfile & Name of the file to write the resulting contour to. \\
start & Sample within the kayfile to start extraction \\
stop & Last sample to include in extraction \\
sz & FFT size (default 512) \\
st & FFT step size (default equal to sz). \\
fl & If st is the same as sz, there is no overlap. \\
pk & A peak must exceed the noise floor by at least this factor (default 3) \\
lowfreq & Peak must be within this factor of the strongest peak in the current FFT \\
highfreq & (default 5). \\
matout & Upwer bound of allowed frequencies for contour \\
hm & Specifies that contour is written in Matlab format. \\
bl & Hamming window (default) \\
re & Blackman window \\
fs & Rectangular window \\
notchctr & Sampling frequency (default 81920) \\
notchbw & Center frequency for band-pass filtering. \\
\hline
\end{tabular}

The contour extractor was written by John Buck.

Buck, J.R. and Tyack, P.L., 1993. “A quantitative measure of similarity for Tursiops truncatus signature whistles." Journal of the Acoustical Society of America, 94 (5), pp. 2497-2506. 
Appendix 3: Programs by Other People

\subsection{DYNAMIC TIME WARPING}

\subsubsection{Dynamic Time Warper}

USAGE: ctrdist reference measured [-matin]

[]$=$ optional

\begin{tabular}{|ll|}
\hline Argument & Definition \\
\hline reference & The file containing the reference contour. This is the contour that is \\
& warped. \\
measured & The file containing the measured contour. This contour is held fixed. \\
matin & Flag indicating that the input contours are in Matlab format.
\end{tabular}

The dynamic time warper was written by John Buck.

Buck, J.R. and Tyack, P.L., 1993. "A quantitative measure of similarity for Tursiops truncatus signature whistles." Journal of the Acoustical Society of America, 94 (5), pp. 2497-2506.

\subsubsection{Program to Convert DTW Output into Matrix Form}

This program was written by Jim Partan in Perl. The normal output of the DTW is "the distance obtained by warping [reference] to fit [measured] $=[$ distance]."

\#!/usr/bin/perl

\# extract contour distances from STDIN, and write them to a file.

\# Jim Partan, <partan@ whoi.edu>, 5 May 1997

\# Specifically for use with John Buck's ctrdist.c.

\#

\# usage: ctrdist .... I dist.pl > output.file

\# or

\# usage: ctrdist .... > ctrdist.txt

\# $\quad$ dist.pl $<$ ctrdist.txt $>$ dist.matrix

\# or something equivalent. The second approach might be better as it saves

\# a copy of the results at each stage.

$\$ \mathrm{i}=0 ; \quad$ \# counter index

$\$ \mathrm{~N}=91 ; \quad$ \# the distance matrix is $\mathrm{NxN}$

\$infty $=1 \mathrm{e} 9$; \# effectively infinity

while $(<$ STDIN $>$ ) \{

\# extract the regular expressions in parentheses into $\$ 1, \$ 2, \$ 3$. 


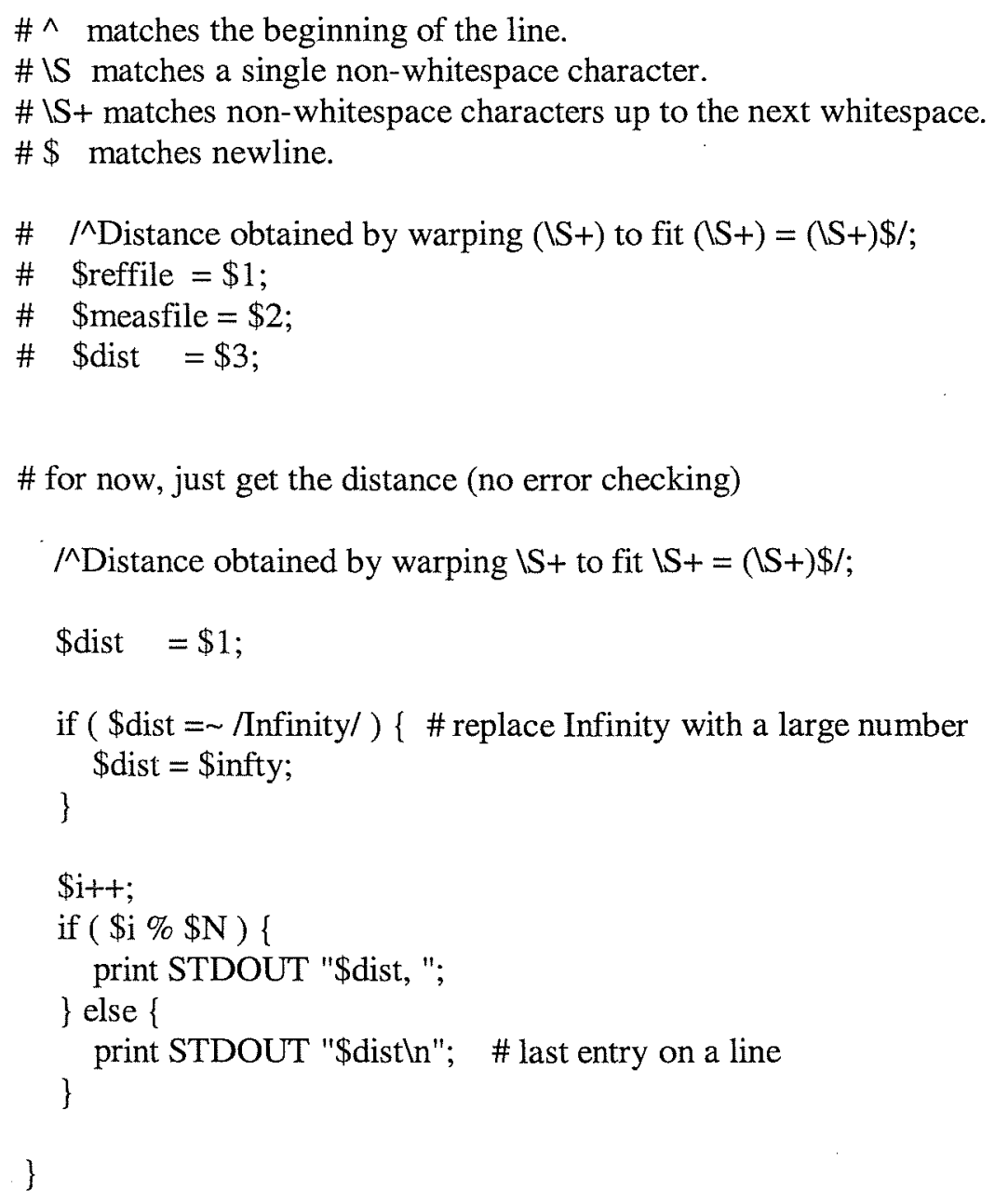




\section{BIOGRAPHICAL NOTE}

The author received a B.S. in Biological Sciences with honors and distinction from Stanford University in 1992. At Stanford, she did an honors thesis on stress and cellular metabolism with Dr. Robert Sapolsky. She entered the MIT/WHOI joint program in Biological Oceanography in the fall of 1992. Her graduate career was funded primarily by a predoctoral fellowship from the Howard Hughes Medical Institute. She was born Deborah Redish and changed her name when she married Michael Fripp in 1996.

During the course of her scientific career, Deborah has worked at a number of locations. Prior to starting her thesis work, she did internships at the National Institutes of Health and at Harvard University. As part of her thesis, she did field work at a number of dolphinariums, including Kolmårdens Djurpark in Sweden, Sea World of San Diego in California, and the Dolphin Connection in Florida.

\section{Grants and Awards}

Howard Hughes Medical Institute Predoctoral Fellowship, 1992-1997

Rinehart Coastal Research Center exploratory grant, 1996-1997

Ocean Ventures Fund thesis grant, 1995-1997

Rinehart Coastal Research Center thesis grant, 1994-1995

Stanford Undergraduate Research Organization undergraduate grant, 1991-1992

\section{Published Papers}

Moss, C.F., Redish, D., Gounden, C., \& Kunz, T.H. 1997. "Ontogeny of Vocal Signals in the Little Brown Bat, Myotis lucifugus," Animal Behaviour, 54: 131-141.

Redish, D.M., Raley-Susman, K.M., \& Sapolsky, R.M., 1993. "Inhibition of Acidification Rate in Cultured Fibroblasts by Glucocorticoids: Application of Silicon Microphysiometry to Endocrinology," Hormone and Metabolic Research, 5(25): 264-267.

Romero, L.M., Raley-Susman, K.M., Redish, D.M., Brook, S.M., Horner, H.C., \& Sapolsky, R.M., 1992. "Possible Mechanisms by Which Stress Accelerates Growth of VirallyDerived Tumors," Proceeds of the National Academy of Science, USA, 89: 11084-11087.

\section{Presented Papers}

Fripp, D.M.R., Tyack, P.L., \& Amundin, M. 1998. "A Case of Prolonged Alloparenting Helps to Define Relationships between Adult and Infant Captive Bottlenose Dolphins, Tursiops truncatus," 12th Biennial Meeting of the Marine Mammal Society, Monaco, January 1998.

Fripp, D., Tyack, P., \& Miksis, J. 1997. “A Comparison of Methods for Categorizing Dolphin Whistles," Northeast Regional Animal Behavior Meeting, Woods Hole, MA, October 1997.

Fripp, D., Tyack, P., Johnson, M., \& Thomas, R. 1997. “An Integrated Approach to the Quantitative Analysis of Dolphin Whistles," 32nd Annual Meeting of the Animal Behavior Society, College Park, MD, June 1997.

Redish, D.M. 1993. "The Development of Signature Whistles in Captive-Born Atlantic Bottlenose Dolphins, Tursiops truncatus," 21st Annual Conference of the International Marine Animal Trainers Association, Kona, HI, November 1993. 